\title{
Development of a [2+2]-Nitroso/Alkene Cycloaddition Using Sodium Tetrakis[3,5-bis(trifluoromethyl)phenyl]borate Catalyst: Controlled Chemoselectivity of Two Equilibrating Isomeric Intermediates
}

\author{
Jia-Xuan Chen ${ }^{\ddagger a}$, Prakash D. Jadhav ${ }^{\ddagger a}$, Ching-Nung Chen and Rai-Shung Liu ${ }^{* a}$
}

Frontier Research Center on Fundamental and Applied Sciences of Matters, Department of Chemistry, National Tsing-Hua University, Hsinchu, Taiwan, ROC.

\section{E-mail: rsliu@mx.nthu.edu.tw}

\section{Content:}

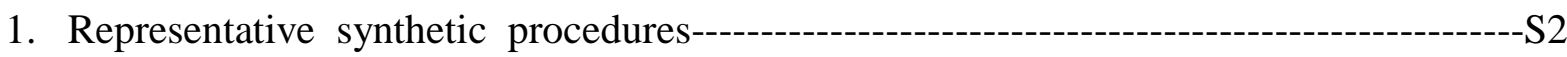

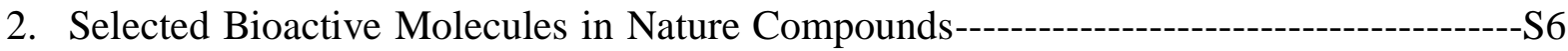

3. Additional results for catalysts screening ---

4. Standard procedures for catalytic operations----------------------------------------S7

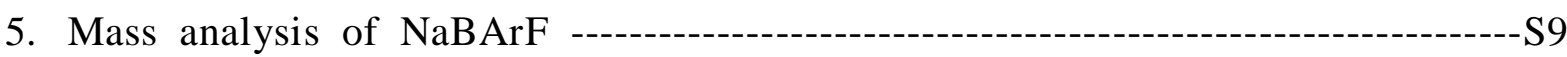

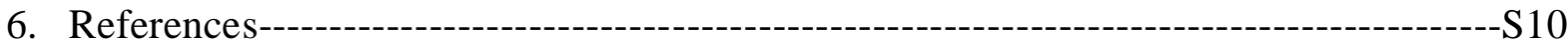

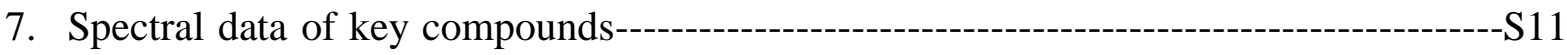

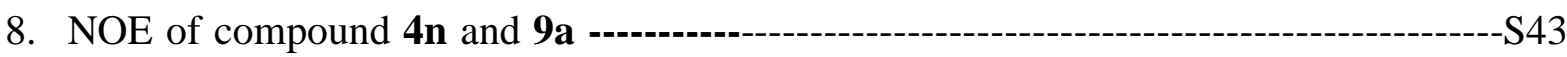

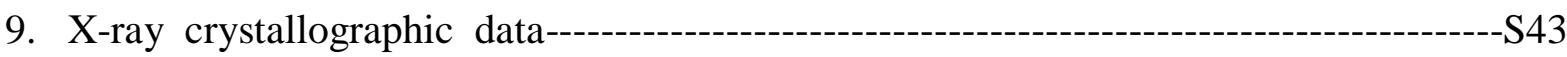

10. The ${ }^{1} \mathrm{H},{ }^{13} \mathrm{C}$ and ${ }^{19} \mathrm{~F}$ NMR of key compounds----------------------------S82 


\section{Representative synthetic procedures.}

\subsection{General methods.}

Unless otherwise noted, all the preparations of the substrates were performed in oven-dried glassware under nitrogen atmosphere with freshly distilled solvents. The catalytic reactions were performed under nitrogen atmosphere. DCM, DCE and toluene were distilled from $\mathrm{CaH}_{2}$ under nitrogen. THF was distilled from Na metal under nitrogen. All other commercial reagents were used without further purification, unless otherwise indicated. 1H NMR and 13C NMR spectra were recorded on a Varian $700 \mathrm{MHz}$, Bruker 400, $600 \mathrm{MHz}$ spectrometers using chloroform-d $\left(\mathrm{CDCl}_{3}\right)$ as the internal standard. High-resolution mass spectral analysis (HRMS) data were measured on JMS-T100LP4G (JEOL) mass spectrometer or a TOF mass analyzer equipped with the ESI source and Magnetic Sector Mass Analyzer (MStation) equipped with the EI source. Melting points of solid compounds were recorded using Buchi melting point B-540. Single-crystal X-ray diffraction intensity data were collected on a Bruker APEX DUO diffractometer equipped with a CCD area detector and Mo $\mathrm{K} \alpha$ radiation ( $\lambda=0.71073 \AA$ ) at $100 \mathrm{~K}$ (The temperature at which the data was collected); all data calculations were performed by using the PC version of the APEX2 program package. Nitroso compound (2b-2l) were prepared according to the reference. ${ }^{[\mathbf{S 1}]}$ Substrates (1a-1p), 1a', 1a-H and (5a-5l) were prepared from procedure reported in the references. ${ }^{[\mathbf{S 2}-\mathbf{S 6}]}$ The catalyst were prepared from procedure reported in the references $\left(\mathrm{HBArF} \cdot\left(\mathrm{EtO}_{2}\right)_{2}\right)_{\text {, }}{ }^{[\mathrm{S} 7]}$ $\left.\left[\mathrm{Ag}\left(\mathrm{NCCH}_{3}\right)_{2}\right]\left[\mathrm{B}\left\{\mathrm{C}_{6} \mathrm{H}_{3}\left(\mathrm{CF}_{3}\right)_{2}\right\}_{4}\right],{ }^{[\mathbf{S 8}]} \mathrm{LiBArF} \cdot\left(\mathrm{EtO}_{2}\right)_{2}\right) .{ }^{[\mathbf{S 9}]}$

\subsection{General synthetic procedure for the preparation of substrates.}

\subsubsection{Preparation of 2-methyl-4-phenylcyclopenta-1,4-dien-1-yl acetate (1a): ${ }^{[\mathrm{S} 2, \mathrm{S6}]}$}

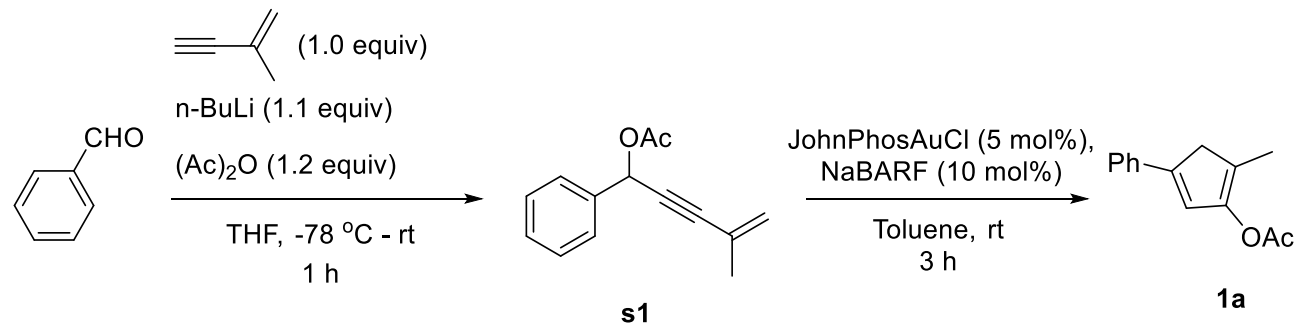

\section{(a) Synthesis of 4-methyl-1-phenylpent-4-en-2-yn-1-yl acetate (s1):}

To a THF (65 mL) solution of 2-methylbut-1-en-3-yne (1.50 g, $22.7 \mathrm{mmol})$ was added $n$ - BuLi (2.5 M in hexane, $10.0 \mathrm{~mL}, 25.0 \mathrm{mmol})$ at $-78{ }^{\circ} \mathrm{C}$, and the mixture was stirred at this temperature for $0.5 \mathrm{~h}$. To this solution was added a THF $(10 \mathrm{~mL})$ solution of benzaldehyde $(2.4 \mathrm{~g}, 22.7 \mathrm{mmol})-78{ }^{\circ} \mathrm{C}$, and the reaction mixture was warmed to room temperature, and stirred for $1 \mathrm{~h}$. The solution was added with acetic anhydride $(2.77 \mathrm{~g}, 27.2 \mathrm{mmol})$ and stirred for $1 \mathrm{~h}$. To this reaction mixture was added, a saturated $\mathrm{NH}_{4} \mathrm{Cl}$ solution, and the solution was extracted with diethyl ether $(2 \times 50 \mathrm{~mL})$ and washed with brine $(30 \mathrm{~mL})$. The combined organic extracts were dried over $\mathrm{MgSO}_{4}$ and concentrated under reduced pressure. The crude 
product was purified by a silica column (EA/hexane $=1 / 5)$ to afford 4-methyl-1-phenylpent-4-en-2-yn-1-ol (s1) (4.17 g, $19.51 \mathrm{mmol}, 86 \%)$ as pale-yellow oil.

\section{(b) Synthesis of 2-methyl-4-phenylcyclopenta-1,4-dien-1-yl acetate (1a):}

A catalytic tube was charged with $\mathrm{P}(\mathrm{t}-\mathrm{Bu})_{2}(\mathrm{o}-$-biphenyl $) \mathrm{AuCl}(36 \mathrm{mg}, 0.066 \mathrm{mmol})$ and NaBARF (61.8 $\mathrm{mg}, 0.069 \mathrm{mmol})$ and to this was added dry toluene $(3 \mathrm{~mL})$ under argon atmosphere. The resulting mixture was stirred at room temperature for $5 \mathrm{~min}$. To this mixture was added a toluene solution ( $3 \mathrm{~mL})$ of 4-methyl-1-phenylpent-4-en-2-yn-1-yl acetate (s1) (300 mg, $1.38 \mathrm{mmol}$ ) for $5 \mathrm{~min}$. After stirring for $3 \mathrm{~h}$, the reaction mixture was concentrated under reduced pressure and purified by silica column eluting with $($ EA/Hexane $=1 / 20)$ to afford 1a (250 mg, $1.17 \mathrm{mmol}, 85 \%)$ as pale-yellow oil.

Substrates $\mathbf{1 b - 1 k}$ and $\mathbf{1 m}$ were synthesized using same as above procedure.

\subsubsection{Preparation of 2-butyl-4-phenylcyclopenta-1,4-dien-1-yl acetate (11):}

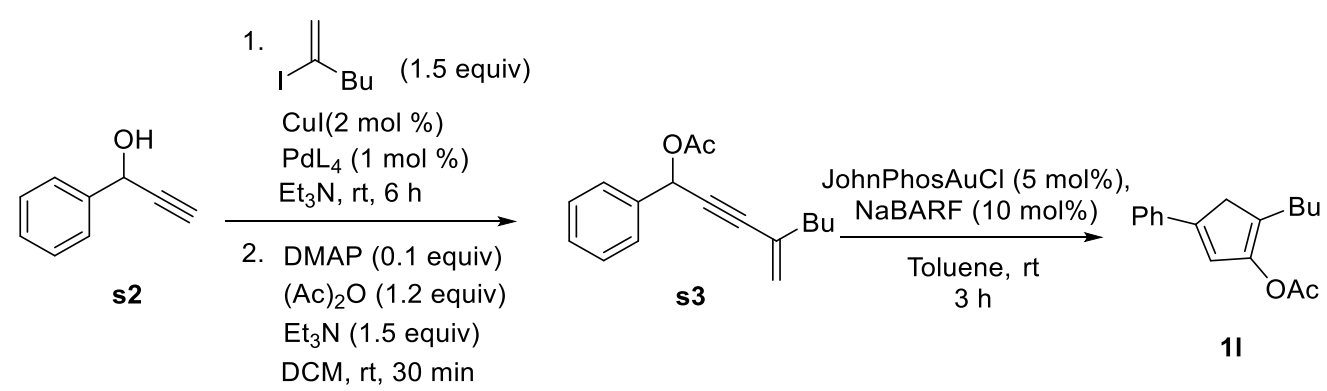

(a) Synthesis of 4-methylene-1-phenyloct-2-yn-1-yl acetate (s3):

Copper (I) iodide $(0.028 \mathrm{~g}, 0.15 \mathrm{mmol})$ and tetrakis(triphenylphosphine) palladium $(0.087 \mathrm{~g}, 0.076 \mathrm{mmol})$ were dissolved in degassed $\mathrm{Et}_{3} \mathrm{~N}(25 \mathrm{~mL})$ and the mixture was cooled to $0{ }^{\circ} \mathrm{C}$. To this solution was added alkyne (s2) $(1.00 \mathrm{~g}, 7.57 \mathrm{mmol})$ and 2-iodohex-1-ene $(2.38 \mathrm{~g}, 11.35 \mathrm{mmol})$, and the mixture was stirred at room temperature until a complete consumption of starting material. The reaction solution was filtered through a celite bed, concentrated and purified by a silica column $(\mathrm{EA} / \mathrm{hexane}=1 / 5)$ to afford 4-methylene-1-phenyloct-2-yn-1-ol (1.07 g, $4.99 \mathrm{mmol}, 66 \%)$ as colorless oil.

To a DCM $(25 \mathrm{~mL})$ solution of $\left(\mathrm{CH}_{3} \mathrm{CO}\right)_{2} \mathrm{O}(610 \mathrm{mg}, 5.98 \mathrm{mmol})$ were added DMAP (61 mg, $0.5 \mathrm{mmol}$ ), and the mixtures were stirred at $0{ }^{\circ} \mathrm{C}$ for $15 \mathrm{~min}$. To this solution was added 4-methylene-1-phenyloct-2-yn-1-ol (1.07 g, $4.99 \mathrm{mmol}_{\text {) }}$ and $\mathrm{Et}_{3} \mathrm{~N}$ (1.04 mL, 7.48 $\mathrm{mmol}$ ), and the mixture was slowly warmed to room temperature with additional stirring for 30 min. The solution was quenched by water, and extracted with DCM ( 2 x $25 \mathrm{~mL})$. The combined organic layer was dried over $\mathrm{MgSO}_{4}$, concentrated, and purified by a silica column $(\mathrm{EA} / \mathrm{hexane}=1 / 7)$ to afford 4-methylene-1-phenyloct-2-yn-1-yl acetate $(\mathbf{s 3})(1.16 \mathrm{~g}, 4.54$ mmol, $91 \%$ ) as pale-yellow oil. 
(b) Synthesis of 2-butyl-4-phenylcyclopenta-1,4-dien-1-yl acetate (11):

The substarte 11 was synthesized using similar synthetic procedure of 1.2.1 substrate 1a from s1.

1.2.3 Preparation of 3,3-dimethyl-4-phenylcyclopenta-1,4-dien-1-yl benzoate (5a): ${ }^{\text {[S2] }}$

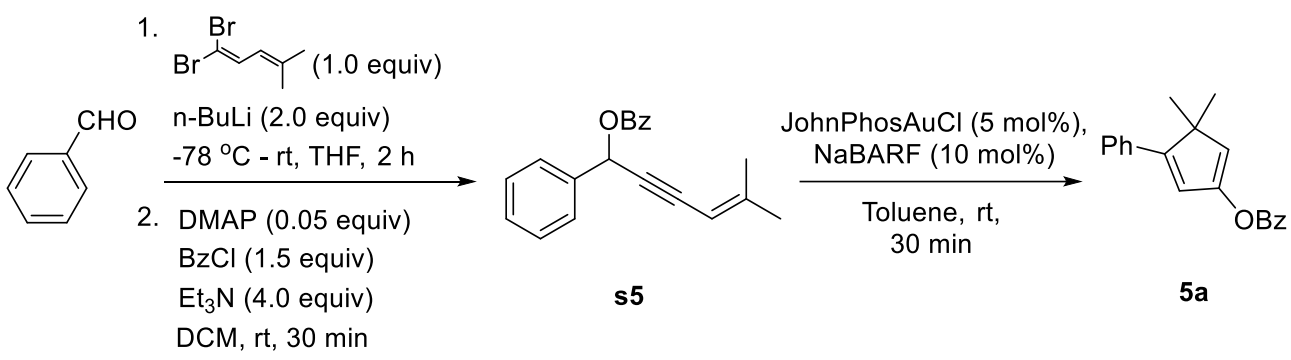

(a) Synthesis of 5-methyl-1-phenylhex-4-en-2-yn-1-yl benzoate (s5):

To a THF (60 mL) solution of 1,1-dibromo-4-methylpenta-1,3-diene (3.30 g, $13.9 \mathrm{mmol})$ was added $n$-BuLi (1.6 M in hexane, $17.4 \mathrm{~mL}, 27.8 \mathrm{mmol}$ ) at $-78{ }^{\circ} \mathrm{C}$; the mixture was stirred at this temperature for $10 \mathrm{mins}$ before warming to room temperature with stirring for additional 30 mins. To this solution was added a THF $(10 \mathrm{~mL})$ solution of benzaldehyde (1.6 $\mathrm{g}, 15.3 \mathrm{mmol})$ at $-78{ }^{\circ} \mathrm{C}$; the solution was warmed to room temperature for $2 \mathrm{~h}$. To this reaction mixture was added a saturated $\mathrm{NH}_{4} \mathrm{Cl}$ solution; the solution was extracted with diethyl ether $(2 \times 50 \mathrm{~mL})$ and washed with brine $(30 \mathrm{~mL})$. The combined organic extracts were dried over $\mathrm{MgSO}_{4}$ and concentrated under reduced pressure. The crude product was purified by a silica column $(\mathrm{EA} / \mathrm{hexane}=1 / 5)$ to afford 5-methyl-1-phenylhex-4en-2-yn-1-ol (s4) (1.93 g, $10.40 \mathrm{mmol}, 75 \%)$ as yellow oil.

To a DCM (30 mL) solution of DMAP (33 mg, $0.3 \mathrm{mmol})$ were added benzoyl chloride (564 mg, $4.03 \mathrm{mmol}$ ); the resulting mixtures were stirred at $0{ }^{\circ} \mathrm{C}$ for $15 \mathrm{~min}$. To this solution was added 5-methyl-1-phenylhex-4-en-2-yn-1-ol (500 mg, $2.69 \mathrm{mmol})$ and $\mathrm{Et}_{3} \mathrm{~N}$ (1.55 mL, $10.75 \mathrm{mmol}$ ), and the mixture was slowly warmed to room temperature with additional stirring for $30 \mathrm{~min}$. The solution was quenched by water, and extracted with DCM $(2 \times 30$ $\mathrm{mL}$ ). The combined organic layer was dried over $\mathrm{MgSO}_{4}$, concentrated, and purified by a silica column $(\mathrm{EA} /$ hexane $=1 / 9)$ to afford 5-methyl-1-phenylhex-4-en-2-yn-1-yl benzoate (s5) (0.61 g, $2.11 \mathrm{mmol}, 79 \%)$ as yellow oil.

\section{(b) Synthesis of 3,3-dimethyl-4-phenylcyclopenta-1,4-dien-1-yl benzoate (5a):}

A catalytic tube was charged with $\mathrm{P}(\mathrm{t}-\mathrm{Bu})_{2}$ (o-biphenyl) $\mathrm{AuCl}(9.2 \mathrm{mg}, 0.018 \mathrm{mmol})$ and NaBARF (30.5 mg, $0.035 \mathrm{mmol}$ ), and to this mixture was added dry toluene $(1 \mathrm{~mL})$ under argon atmosphere. The resulting mixture was stirred at room temperature for $5 \mathrm{~min}$. To this mixture was added a toluene solution $(2 \mathrm{~mL})$ of 5-methyl-1-phenylhex-4-en-2-yn-1-yl benzoate (s5) $(100 \mathrm{mg}, 0.34 \mathrm{mmol})$. After stirring for $30 \mathrm{mins}$, the reaction mixture was filtered through a short celite bed and concentrated under reduced pressure. The residue was purified by a silica column $(\mathrm{EA} / \mathrm{hexane}=1 / 9)$ to afford compound $\mathbf{5 a}(0.092 \mathrm{~g}, 0.317 \mathrm{mmol}$, 
92\%) as yellow oil.

Substrates $\mathbf{5 b - 5 k}$ were synthesized using same as above procedure.

\section{Preparation of 4-phenylspiro[4.5]deca-1,3-dien-2-yl benzoate (5l) :}

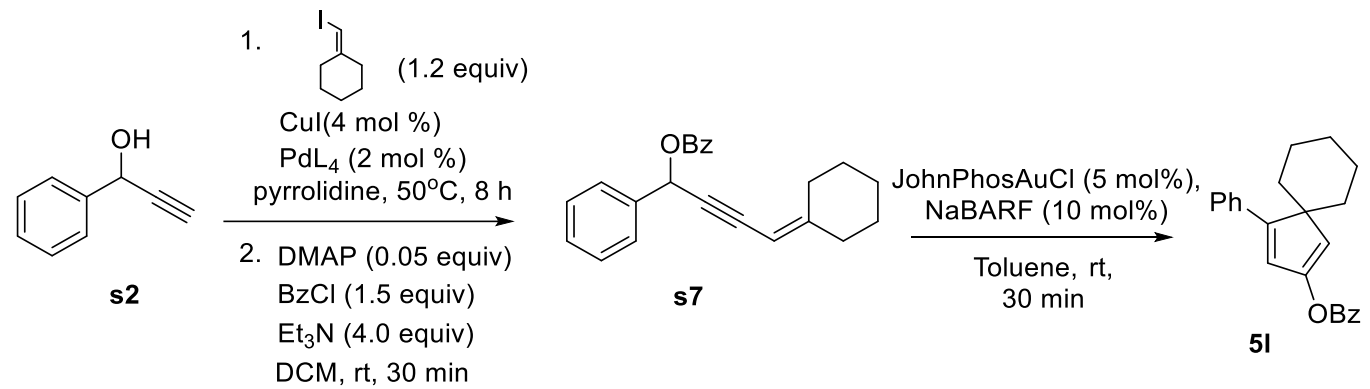

(b) Synthesis of 4-cyclohexylidene-1-phenylbut-2-yn-1-yl benzoate (s7):

Copper (I) iodide $(0.023 \mathrm{~g}, 0.12 \mathrm{mmol})$ and tetrakis(triphenylphosphine) palladium (0.042 $\mathrm{g}, 0.060 \mathrm{mmol})$ were dissolved in degassed pyrrolidine $(6 \mathrm{~mL})$; the mixture was purged with argon for $10 \mathrm{~min}$. To this solution was added (iodomethylene)cyclohexane $(0.80$ $\mathrm{g}, 3.60 \mathrm{mmol})$ and alkyne $(\mathbf{s 2})(0.40 \mathrm{~g}, 3.00 \mathrm{mmol})$, and the mixture was stirred at a $50{ }^{\circ} \mathrm{C}$ oil bath for $8 \mathrm{~h}$. The resulting solution was filtered through a celite bed, concentrated and purified by a silica column $(\mathrm{EA} / \mathrm{hexane}=1 / 5)$ to afford 4-cyclohexylidene-1-phenylbut-2-yn-1-ol (s6) (0.48 g, $2.13 \mathrm{mmol}, 71 \%)$ as colorless oil.

To a DCM (15 mL) solution of DMAP $(0.008 \mathrm{~g}, 0.06 \mathrm{mmol})$ were added benzoyl chloride $(0.279 \mathrm{~g}, 1.99 \mathrm{mmol})$; the mixture was stirred at $0{ }^{\circ} \mathrm{C}$ for $15 \mathrm{~min}$. To this solution was added 4-cyclohexylidene-1-phenylbut-2-yn-1-ol (0.300 mg, $1.33 \mathrm{mmol})$ and $\mathrm{Et}_{3} \mathrm{~N}(0.757$ $\mathrm{mL}, 5.31 \mathrm{mmol}$ ), and the mixture was slowly warmed to room temperature with additional stirring for $30 \mathrm{~min}$. The solution was quenched by water, and extracted with DCM $(2 \times 15$ $\mathrm{mL}$ ). The combined organic layer was dried over $\mathrm{MgSO}_{4}$, concentrated, and purified by a silica column $(\mathrm{EA} / \mathrm{hexane}=1 / 9)$ to afford 4-cyclohexylidene-1-phenylbut-2-yn-1-yl benzoate (s7) (0.350 g, $1.33 \mathrm{mmol}, 80 \%)$ as yellow oil.

(b) Synthesis of 4-phenylspiro[4.5]deca-1,3-dien-2-yl benzoate (5l):

The substarte 5l was synthesized using similar synthetic procedure of 1.2.3 substrate 5a from s5. 


\section{Selected Bioactive Molecules in Nature Compounds.:}

Figure S1. shows several representatives10 that bear 2-aminocyclopentanone (I) and d-lactamecores (II) that are accessible from our [2+2]-nitroso/alkene cycloadditions. Reductiomy-cin (I-1) ${ }^{[\text {S10a] }}$ is active against gram-positive bacteria, fungi, and newcastle disease virus, whereas li-mocrocin $(\mathrm{I}-2)^{[\mathbf{S 1 0 b}]}$ has inhibitory activities against vari-ous kinds of DNA and RNA polymerases. Adalinine (II-1) ${ }^{[\text {S10d] }}$ is a natural alkaloid extracted from the ladybird beetles whereas apixabane $(\mathrm{II}-2)^{[\mathbf{S 1 0 e}]}$ serves as an anticoagu-lant medication to prevent blood clots and stroke. Nota-bly atogepant (II-3) ${ }^{[\text {[10f] }}$ is presently under evaluation in phase III clinical studies as a preventative therapy for episodic migraine.

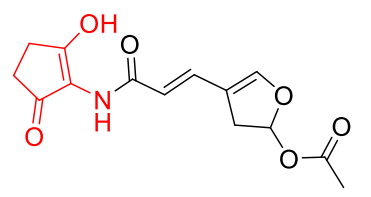

reductiomycin (I-1)

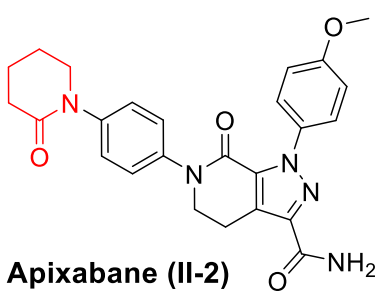<smiles>CC(C)(C)NC1=C(O)CCC1=O</smiles>

limocrocin (I-2)

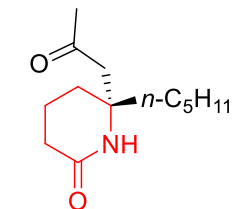

Adalinine (II-1)

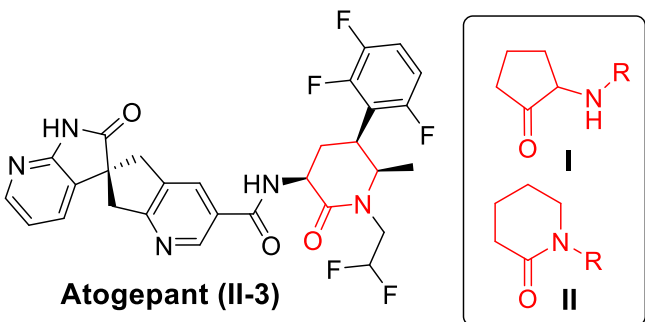

Figure S1. Selected Bioactive Molecules in Nature Compounds

\section{Additional results for catalysts screening:}

Table S1. Solvent screening

2a
(2.0 equiv. $)$

${ }^{a}[1]=0.2 \mathrm{M} .{ }^{b}$ Product yields were obtained after purifi-cation from a silica column. $\mathrm{BArF}=\mathrm{BAr}_{4}{ }_{4}$, $\mathrm{Ar}^{\prime}=\left(\mathrm{CF}_{3}\right)_{2} \mathrm{C}_{6} \mathrm{H}_{3} . \mathrm{ACN}=$ Acetonitrile. 
Table S2 shows some less efficient catalyst conditions. In contrast of our optimized conditions of $\mathrm{NaBArF}$ catalyst (entry 1 ), $\mathrm{HBArF} \mathrm{Et}_{2} \mathrm{O}$ was active only in the hydration of cyclopentadiene 1a (entry 2). We also tested other transition metal catalyst, $\mathrm{Zn}(\mathrm{OTf})_{2}$ and $\mathrm{Cu}(\mathrm{OTf})_{2}(10 \mathrm{~mol} \%)$ were less efficient than $\mathrm{NaBArF}$ whereas byproduct 1a-H was produced in a large proportion (30-37\%, entries 3-4).

Table S2. Other inefficient catalysts

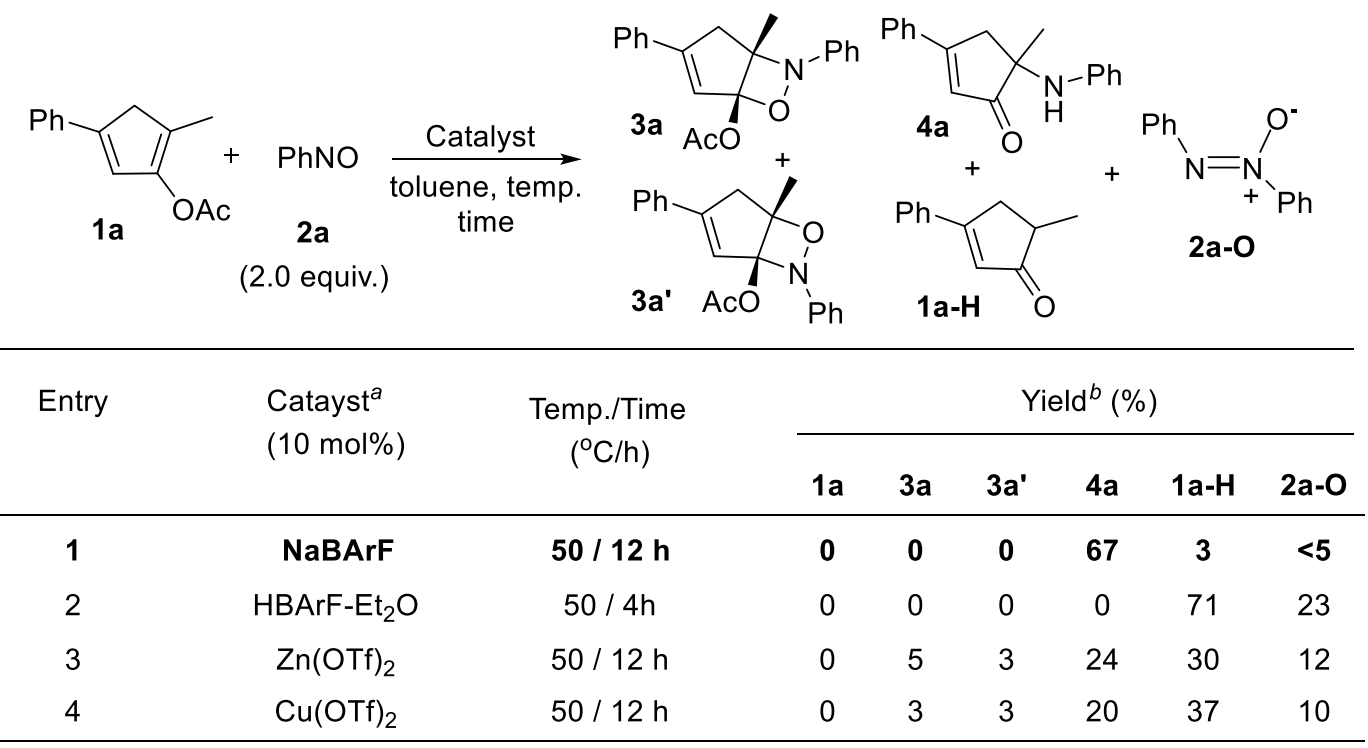

${ }^{a}[1]=0.2 \mathrm{M} .{ }^{b}$ Product yields were obtained after purifi-cation from a silica column.

\section{Standard procedures for catalytic operations:}

\subsection{Typical procedure for synthesis of 5-methyl-3-phenyl-5-(phenylamino)cyclopent} -2-en-1-one (4a):

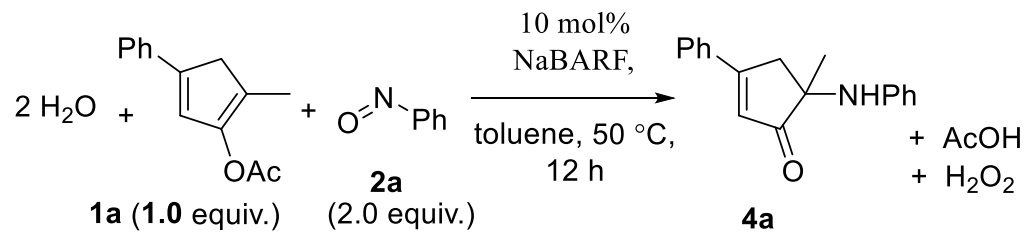

A catalytic tube was charged with $\operatorname{NaBARF}(165.7 \mathrm{mg}, 0.187 \mathrm{mmol})$, and to this mixture was added un-dried toluene $(1.9 \mathrm{~mL})$ under argon. The resulting mixture was stirred at room temperature for $5 \mathrm{~min}$. To this mixture was slowly added an undried toluene solution $(3.8 \mathrm{~mL})$ of 4-methylcyclopenta-1,3-diene-1,3-diyl diacetate (1a) (400.0 mg, $1.87 \mathrm{mmol})$, then a (1.0 $\mathrm{ml}$ ) toluene solution of nitrosobenzene (2a) $(400.2 \mathrm{mg}, 3.74 \mathrm{mmol})$, and the resulting mixture was stirred at $50{ }^{\circ} \mathrm{C}$ oil bath for $12 \mathrm{~h}$ The reaction mixture was filtered over a short celite bed, concentrated under reduced pressure, and purified by a silica column eluting $(\mathrm{EA} / \mathrm{hexane}=$ 1/9) to afford 5-methyl-3-phenyl-5-(phenylamino)cyclopent-2-en-1-one 4a (305.0 mg, 1.16 
mmol, 62\%) as yellow solid.

In the catalytic reaction for less scale, we use the $\mathbf{1 a}(0.49 \mathrm{mmol})$, giving the product $\mathbf{4 a}$ in $67 \%(0.33 \mathrm{mmol})$

\subsection{Typical procedure for synthesis of 6-hydroxy-5,5-dimethyl-1,4-diphenyl-5,6} -dihydropyridin-2(1H)-one (6a):

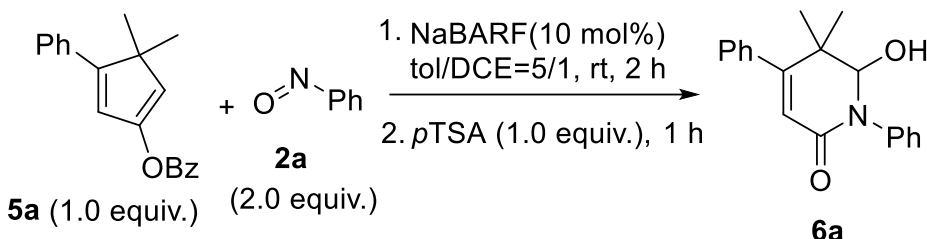

A catalytic tube was charged with $\operatorname{NaBARF}(15.3 \mathrm{mg}, 0.017 \mathrm{mmol})$, and to this mixture was added dried toluene $(0.5 \mathrm{~mL})$ under argon atmosphere. The resulting mixture was stirred at room temperature for $5 \mathrm{~min}$. To this mixture was added a toluene solution $(0.5 \mathrm{~mL})$ of 3,3-dimethyl-4-phenylcyclopenta-1,4-dien-1-yl benzoate (5a) $(50.0 \mathrm{mg}, 0.17 \mathrm{mmol})$ and a toluene $(0.5 \mathrm{ml})$ solution of nitrosobenzene $(\mathbf{2 a})(37.0 \mathrm{mg}, 0.34 \mathrm{mmol})$, the resulting mixture was stirred for $2 \mathrm{~h}$ before treatment with $p$-TSA $(33.0 \mathrm{mg}, 0.17 \mathrm{mmol})$. This final solution was stirred for $1 \mathrm{~h}$. The reaction mixture was added $\mathrm{NEt}_{3}(0.2 \mathrm{~mL})$, filtered over a short celite bed, concentrated under reduced pressure, and purified by a silica column $(\mathrm{EA} / \mathrm{hexane}=1 / 4)$ to afford 6-hydroxy-5,5-dimethyl-1,4-diphenyl-5,6-dihydropyridin-2(1H)-one $\mathbf{6 a}(0.044 \mathrm{~g}$, $0.15 \mathrm{mmol}, 88 \%$ ) as brown solid.

\subsection{Preparation of 5-(hydroxy(phenyl)amino)-5-methyl-3-phenylcyclopent-2-en-1-one} (4a-O):

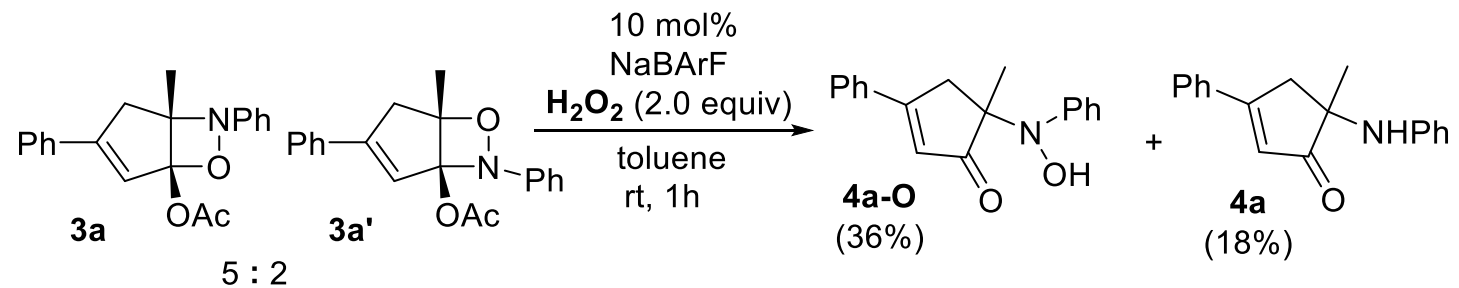

A catalytic tube was charged with $\mathrm{NaBArF}(27.6 \mathrm{mg}, 0.031 \mathrm{mmol})$, and to this mixture was added toluene $(1.0 \mathrm{~mL})$ under argon. The resulting mixture was stirred at room temperature for $5 \mathrm{~min}$. To this mixture was slowly added an toluene solution $(0.6 \mathrm{~mL})$ of mixture of (1S,5R)-1-methyl-3,7-diphenyl-6-oxa-7-azabicyclo[3.2.0]hept-3-en-5-yl acetate (3a) and (1S,5S)-5-methyl-3,7-diphenyl-6-oxa-7-azabicyclo[3.2.0]hept-2-en-1-yl acetate (3a') (100.0 $\mathrm{mg}, 0.311 \mathrm{mmol}$ ), then a $30 \% \mathrm{H}_{2} \mathrm{O}_{2}$ solution $(70 \mu \mathrm{l}$ ) was slowly added to the solution and stirred for $1 \mathrm{~h}$. The reaction mixture was added $\mathrm{NaHCO}_{3}(0.1 \mathrm{~mL})$, filtered over a short celite bed, concentrated under reduced pressure, and purified by a silica column (EA/hexane 
$=1 / 3)$ to afford 5-(hydroxy(phenyl)amino)-5-methyl-3-phenylcyclopent-2-en-1-one (4a-O) (31.3 mg, $0.112 \mathrm{mmol}, 36 \%)$ as yellow oil.

\subsection{Preparation of $(4 a)$ from $(4 a-0)$ :}<smiles></smiles>

(1.0 equiv)<smiles>[O][N+]([O-])=Nc1ccccc1</smiles>

(1.0 equiv)

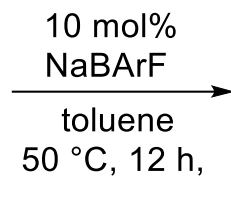<smiles>CC1(Nc2ccccc2)CC(c2ccccc2)=CC1=O</smiles>

A catalytic tube was charged with $\operatorname{NaBARF}(6.4 \mathrm{mg}, 0.007 \mathrm{mmol})$, then un-dried toluene $(0.1 \mathrm{~mL})$ was added under argon. The resulting mixture was stirred at room temperature for $5 \mathrm{~min}$. To this mixture was slowly added an undried toluene solution $(0.1 \mathrm{~mL})$ of 5-(hydroxy(phenyl)amino)-5-methyl-3-phenylcyclopent-2-en-1-one (4a-O) (107.0 mg, $0.49 \mathrm{mmol})$, then a $(0.15 \mathrm{ml})$ toluene solution of diazene oxide (2a-O) $(14.2 \mathrm{mg}, 0.071$ $\mathrm{mmol}$ ), and the resulting mixture was stirred at $50^{\circ} \mathrm{C}$ oil bath for $12 \mathrm{~h}$. The reaction mixture was filtered over a short celite bed, concentrated under reduced pressure, and purified by a silica column eluting $(\mathrm{EA} / \mathrm{h}$ exane $=1 / 5)$ to afford 5-methyl-3-phenyl-5-(phenylamino)cyclopent-2-en-1-one $4 \mathbf{a}$ (10.2 mg, $0.038 \mathrm{mmol}, 54 \%)$ as yellow solid.

\section{Mass analysis of NaBArF:}

With the negative tone of ESI-mass spectra, we observed only the signal of the BARF anion for a mixture of $\mathrm{PhNO}$ with $\mathrm{NaBARF}$ in $\mathrm{MeOH}$ or toluene; no mass signal of $\mathrm{BAr}^{-}$ $\left(\mathrm{Ar} '=3,5-\left(\mathrm{CF}_{3}\right)_{2} \mathrm{C}_{6} \mathrm{H}_{3}\right)$ was observed.

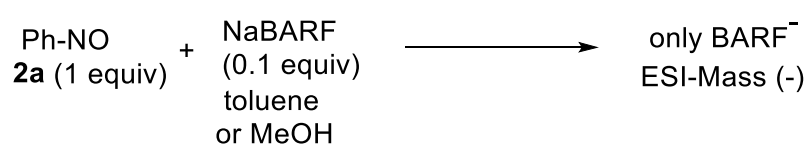




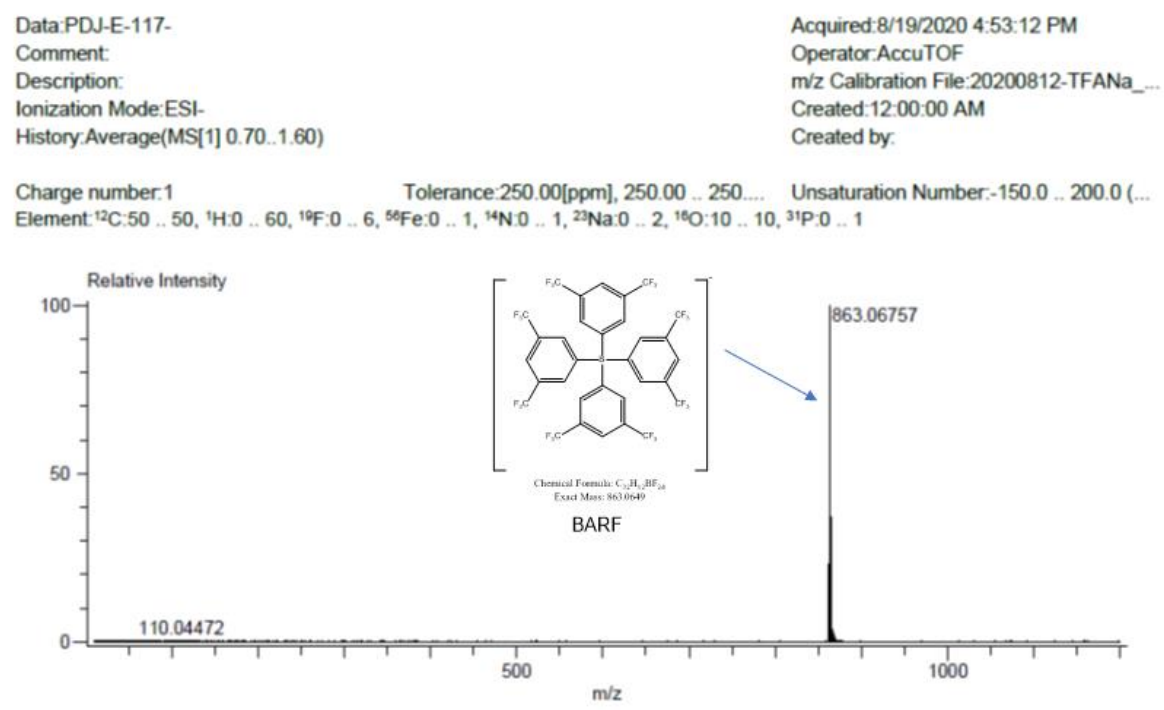

ESI-(-)-MS of mixture of NaBArF and Nitrosobenzene $2 \mathrm{a}$ in Toluene, $\mathrm{rt}, 12 \mathrm{~h}$

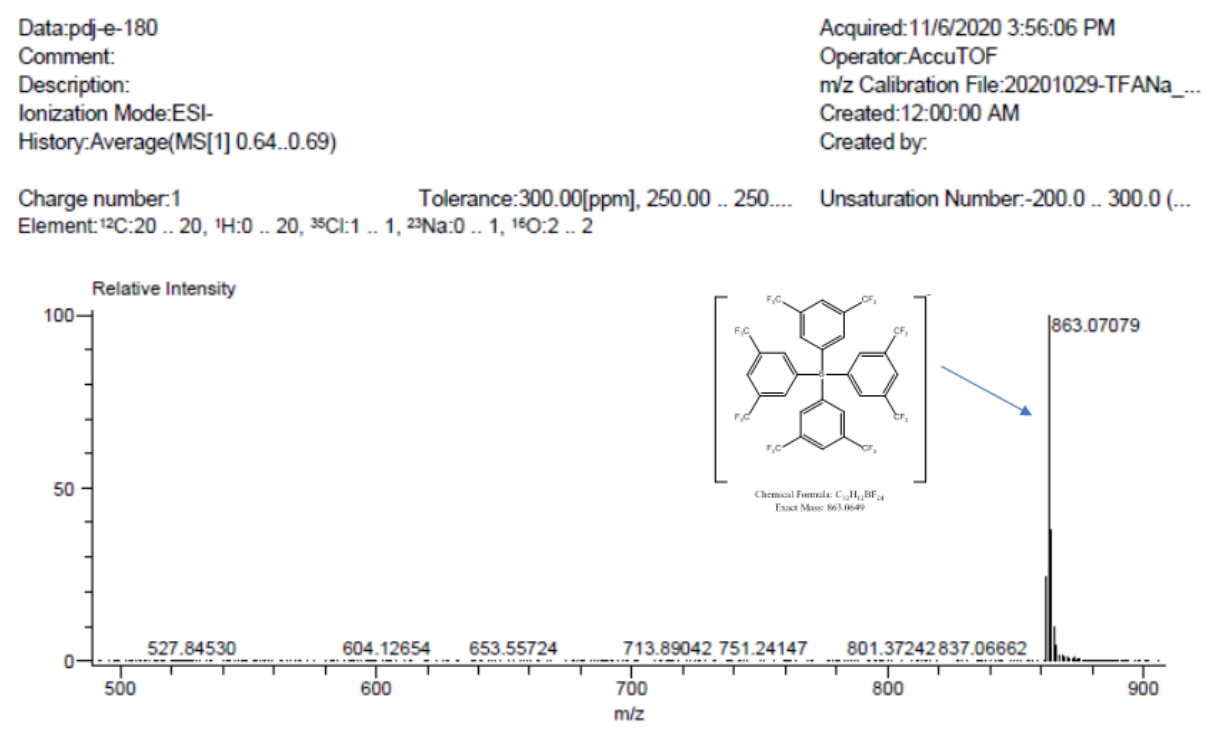

ESI-(-)-MS of mixture of NaBArF and Nitrosobenzene $2 \mathrm{a}$ in $\mathrm{MeOH}, \mathrm{rt}, 12 \mathrm{~h}$

\section{References:}

[S1]. Compound (2b) - (2l): D. Zhao, M. Johansson, J. E. Bäckvall, Eur. J. Org. Chem., 2007, 26, $4431-4436$.

[S2]. B. D. Mokar, P. D. Jadhav, Y. B. Pandit, R.-S. Liu, Chem. Sci., 2018, 9, 4488 - 4492 ;

(b) B. D. Mokar, J. Liu, R.-S. Liu, Org. Lett. 2018, 20, 1038 - 1041.

[S3]. C.-N. Chen, R.-S. Liu, Angew. Chem., Int. Ed. 2019, 58, 9831 - 9835.

[S4]. L. Zhang, S. Wang, J. Am. Chem. Soc. 2006, 128, $1442-1443$. 
[S5]. Compound (10-1p): G. Lemiere, V. Gandon, K. Cariou, A. Hours, T. Fukuyama, A.-L. Dhimane, L. Fensterbank, M. Malacria, J. Am. Chem. Soc. 2009, 131, 2993 - 3006.

[S6] Compound (1n): K. Zhao, Y.-C. Hsu, Z. Yang, R.-S. Liu, L. Zhang, Org. Lett. 2020, 22, $6500-6504$.

[S7]. Catalyst (HBArF. $\left.\left(\mathrm{EtO}_{2}\right)_{2}\right)$ : M. Brookhart, B. Grant, A. F. Volpe, Jr., Organometallics 1992, 11, $3920-3922$.

[S8]. Catalyst $\left[\mathrm{Ag}\left(\mathrm{NCCH}_{3}\right)_{2}\right]\left[\mathrm{B}\left\{\mathrm{C}_{6} \mathrm{H}_{3}\left(\mathrm{CF}_{3}\right)_{2}\right\}_{4}\right]$ : Y. Zhang, A. M. Santos, E. Herdtweck, J. Mink, F. E. Kîhn, New J. Chem. 2005, 29, 366 - 370.

[S9]. Catalyst (LiBArF. $\left.\left(\mathrm{EtO}_{2}\right)_{2}\right)$, KBArF: L. Carreras, L. Rovira, M. Vaquero, I. Mon, E. Martin, J. Benet Buchholz, A. Vidal-Ferran, RSC Adv. 2017, 7, 32833 - 32841.

[S10]. (a) Shimizu, K.; Tamura, G. Reductiomycin, J. Antibiot. 1981, 34(6), 649-653. (b) Hanajima, S.; lshimaru, K.; Sakano, K.; Roy, S.K.; Inouye, Y.; Nakamura, S. J. Antibiot., 1985, 38, 803-805. (c) Munoz, C., Papp, M. Pharmacology Biochemistry and Behavior, 1999, 63, 647-653. (d) Laurent, P.; Lebrun, B.; Braekman, J. C.; Daloze, D.; Pasteels, J. M. Tetrahedron, 2001, 57, 3403-3412. (e) Wong, P. C.; Pinto, D. J. P.; Zhang, D. J. Thromb Thrombolysis. 2011, 31(4), 478-492. (f) Martelletti, P.; Cipolla, F.; Capi, M.; Curto, M.; Lionetto, L. Atogepant. Drugs of the Future, 2020, 45(5), 285.

\section{Spectral data of key compounds:}

Spectral data for 4-(4-chlorophenyl)-2-methylcyclopenta-1,4-dien-1-yl acetate (1b):

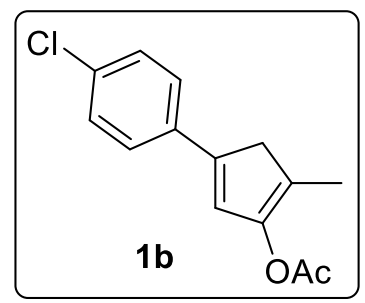

Slightly yellow oil (0.093 g, $0.373 \mathrm{mmol}, 94 \%) ;{ }^{1} \mathrm{H}$ NMR (600 MHz, $\left.\mathrm{CDCl}_{3}\right): \delta 7.32(\mathrm{dd}, J=$ 6.6, $1.8 \mathrm{~Hz}, 2 \mathrm{H}), 7.23$ (dd, $J=6.6,1.8 \mathrm{~Hz}, 2 \mathrm{H}), 6.66(\mathrm{~s}, 1 \mathrm{H}), 3.23(\mathrm{~s}, 2 \mathrm{H}), 2.24(\mathrm{~s}, 3 \mathrm{H}), 1.90$ $(\mathrm{s}, 3 \mathrm{H}) ;{ }^{13} \mathrm{C} \mathrm{NMR}\left(150 \mathrm{MHz}, \mathrm{CDCl}_{3}\right): \delta 168.8,146.5,141.0,134.1,132.3,128.6,125.7$, 124.8, 124.6, 41.4, 20.7, 11.6; HRMS (EI+, m/z) calcd for $\mathrm{C}_{14} \mathrm{H}_{13} \mathrm{ClO}_{2}[\mathrm{M}+]$ : 248.0604, found: 248.0600 .

Spectral data for 4-(4-bromophenyl)-2-methylcyclopenta-1,4-dien-1-yl acetate (1c): 


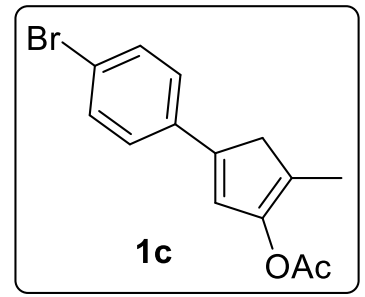

Yellow oil (0.094 g, $0.320 \mathrm{mmol}, 94 \%) ;{ }^{1} \mathrm{H} \mathrm{NMR}\left(600 \mathrm{MHz}, \mathrm{CDCl}_{3}\right): \delta 7.37$ (dd, $J=8.4,1.2$ Hz, 2H), 7.24 (dd, J = 8.4, 1.2 Hz, 2H), 6.67 (s, 1H), 3.21 (s, 2H), 2.23 (s, 3H), 1.89 (s, 3H); ${ }^{13} \mathrm{C} \mathrm{NMR}\left(150 \mathrm{MHz}, \mathrm{CDCl}_{3}\right): \delta 168.8,146.5,141.0,134.5,131.6,126.0,124.9,124.7,120.3$, 41.3, 20.7, 11.6; HRMS (EI+, m/z) calcd for $\mathrm{C}_{14} \mathrm{H}_{13} \mathrm{BrO}_{2}$ [M+]: 292.0099, found: 292.0105.

Spectral data for 2-methyl-4-(4-(trifluoromethyl)phenyl)cyclopenta-1,4-dien-1-yl acetate (1d):

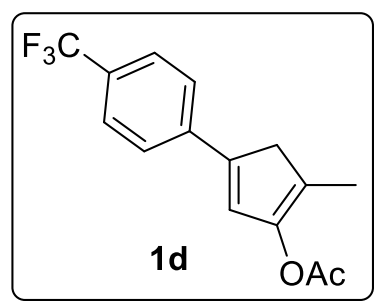

Yellow oil (0.092 g, $0.325 \mathrm{mmol}, 92 \%) ;{ }^{1} \mathrm{H}$ NMR (600 MHz, $\left.\mathrm{CDCl}_{3}\right): \delta 7.50$ (d, $J=7.8 \mathrm{~Hz}$, $2 \mathrm{H}), 7.46(\mathrm{~d}, J=8.4 \mathrm{~Hz}, 2 \mathrm{H}), 6.79(\mathrm{~s}, 1 \mathrm{H}), 3.26(\mathrm{~s}, 2 \mathrm{H}), 2.24(\mathrm{~s}, 3 \mathrm{H}), 1.92(\mathrm{~s}, 3 \mathrm{H}) ;{ }^{13} \mathrm{C} \mathrm{NMR}$ $\left(150 \mathrm{MHz}, \mathrm{CDCl}_{3}\right): \delta 168.8,146.6,140.7,138.8,128.3\left(J_{\mathrm{C}-\mathrm{F}}=68.8,32.4\right), 126.7,125.6\left(J_{\mathrm{C}-\mathrm{F}}\right.$ $=60.8), 125.5,124.5,41.3,20.6,11.6 ;{ }^{19} \mathrm{~F}$ NMR (600 MHz, $\left.\mathrm{CDCl}_{3}\right)$ : -61.81.; HRMS (ESI+, $\mathrm{m} / \mathrm{z}$ ) calcd for $\mathrm{C}_{15} \mathrm{H}_{14} \mathrm{~F}_{3} \mathrm{O}_{2}[\mathrm{M}+\mathrm{H}]: 283.0945$, found: 283.1073 .

Spectral data for 2-methyl-4-(p-tolyl)cyclopenta-1,4-dien-1-yl acetate (1e):

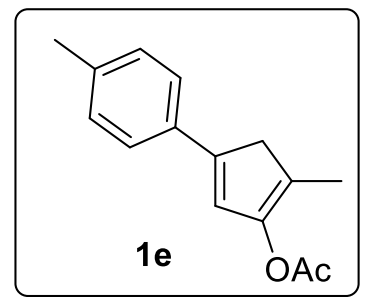

Yellow oil (0.090 g, $0.394 \mathrm{mmol}, 90 \%) ;{ }^{1} \mathrm{H}$ NMR (600 MHz, $\left.\mathrm{CDCl}_{3}\right): \delta 7.31(\mathrm{~d}, J=6.6 \mathrm{~Hz}$, 2H), $7.08(\mathrm{t}, J=7.8 \mathrm{~Hz}, 2 \mathrm{H}), 6.63(\mathrm{~s}, 1 \mathrm{H}), 3.25(\mathrm{~s}, 2 \mathrm{H}), 2.31(\mathrm{~s}, 3 \mathrm{H}), 2.24(\mathrm{~s}, 3 \mathrm{H}), 1.91$ (s, $3 \mathrm{H}) ;{ }^{13} \mathrm{C}$ NMR $\left(150 \mathrm{MHz}, \mathrm{CDCl}_{3}\right): \delta 168.7,146.5,142.4,136.4,132.9,129.1,124.4,123.4$, 123.2, 41.3, 21.0, 20.6, 11.5; HRMS (ESI+, m/z) calcd for $\mathrm{C}_{15} \mathrm{H}_{16} \mathrm{NaO}_{2}[\mathrm{M}+\mathrm{Na}]: 251.1048$, 
found: 251.1048 .

Spectral data for 4-(3-chlorophenyl)-2-methylcyclopenta-1,4-dien-1-yl acetate (1f):

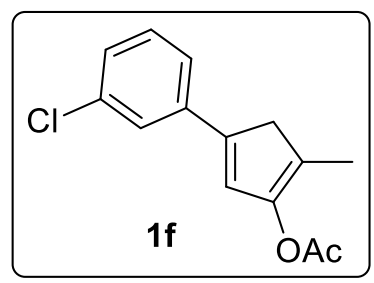

Yellow oil (0.093 g, $0.373 \mathrm{mmol}, 93 \%) ;{ }^{1} \mathrm{H} \mathrm{NMR}\left(600 \mathrm{MHz}, \mathrm{CDCl}_{3}\right): \delta 7.37$ (t, $J=1.8 \mathrm{~Hz}$, $1 \mathrm{H}), 7.28 \sim 7.25(\mathrm{~m}, 1 \mathrm{H}), 7.19(\mathrm{t}, J=7.8 \mathrm{~Hz}, 1 \mathrm{H}), 7.13 \sim 7.11(\mathrm{~m}, 1 \mathrm{H}), 6.70(\mathrm{~s}, 1 \mathrm{H}), 3.24(\mathrm{~s}$, 2H), 2.24 (s, 3H), 1.91 (s, 3H); ${ }^{13} \mathrm{C}$ NMR (150 MHz, $\left.\mathrm{CDCl}_{3}\right): \delta 168.7,146.4,140.8,137.3$, 134.4, 129.7, 126.5, 125.6, 125.1, 124.5, 122.5, 41.3, 20.7, 11.6; HRMS (EI+, m/z) calcd for $\mathrm{C}_{14} \mathrm{H}_{13} \mathrm{ClO}_{2}[\mathrm{M}+]: 248.0604$, found: 248.0603 .

Spectral data for 2-methyl-4-(m-tolyl)cyclopenta-1,4-dien-1-yl acetate (1g):

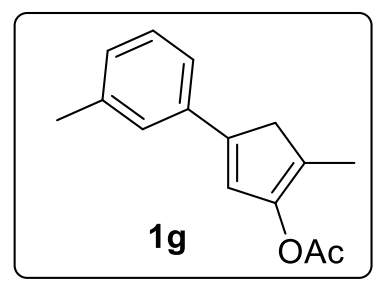

Yellow oil (0.092 g, $0.402 \mathrm{mmol}, 92 \%)$; ${ }^{1} \mathrm{H}$ NMR (600 MHz, $\left.\mathrm{CDCl}_{3}\right): \delta 7.25 \sim 7.22(\mathrm{~m}, 2 \mathrm{H})$, $7.17(\mathrm{t}, J=7.2 \mathrm{~Hz}, 1 \mathrm{H}), 6.99$ (d, $J=7.2 \mathrm{~Hz}, 1 \mathrm{H}), 6.67(\mathrm{~s}, 1 \mathrm{H}), 3.27(\mathrm{~s}, 2 \mathrm{H}), 2.32(\mathrm{~s}, 3 \mathrm{H}), 2.24$ $(\mathrm{d}, J=1.2 \mathrm{~Hz}, 3 \mathrm{H}), 1.91(\mathrm{~s}, 3 \mathrm{H}) ;{ }^{13} \mathrm{C} \mathrm{NMR}\left(150 \mathrm{MHz}, \mathrm{CDCl}_{3}\right): \delta 168.8,146.5,142.4,137.9$, 135.5, 128.4, 127.5, 125.2, 124.0, 123.9, 121.7, 41.4, 21.4, 20.7, 11.5; HRMS (EI+, m/z) calcd for $\mathrm{C}_{15} \mathrm{H}_{16} \mathrm{O}_{2}[\mathrm{M}+]$ : 228.1150, found: 228.1154 .

Spectral data for 2-methyl-4-(o-tolyl)cyclopenta-1,4-dien-1-yl acetate (1h):

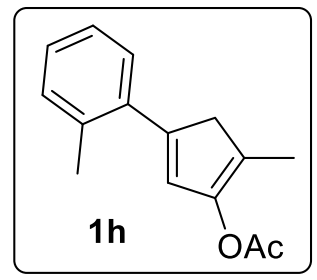


Yellow oil (0.091 g, $0.398 \mathrm{mmol}, 91 \%) ;{ }^{1} \mathrm{H}$ NMR (600 MHz, $\left.\mathrm{CDCl}_{3}\right): \delta 7.29(\mathrm{~d}, J=7.6 \mathrm{~Hz}$, $1 \mathrm{H}), 7.20 \sim 7.10(\mathrm{~m}, 3 \mathrm{H}), 6.47(\mathrm{~s}, 1 \mathrm{H}), 3.32(\mathrm{~s}, 2 \mathrm{H}), 2.44(\mathrm{~s}, 3 \mathrm{H}), 2.26(\mathrm{~s}, 3 \mathrm{H}), 1.93(\mathrm{~s}, 3 \mathrm{H})$; ${ }^{13} \mathrm{C}$ NMR $\left(150 \mathrm{MHz}, \mathrm{CDCl}_{3}\right): \delta 168.8,146.3,142.1,135.6,135.2,131.1,128.0,127.7,126.5$, 125.8, 123.8, 44.3, 22.2, 20.7, 11.4; HRMS (EI+, m/z) calcd for $\mathrm{C}_{15} \mathrm{H}_{16} \mathrm{O}_{2}[\mathrm{M}+]: 228.1150$, found: 228.1152.

Spectral data for 2-methyl-4-(naphthalen-2-yl)cyclopenta-1,4-dien-1-yl acetate (1i):

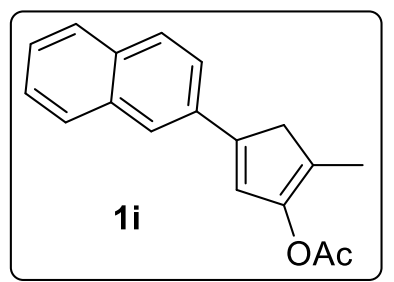

Yellow oil (0.083 g, 0.314 mmol, 83\%); ${ }^{1} \mathrm{H}$ NMR (600 MHz, $\left.\mathrm{CDCl}_{3}\right): \delta 7.78 \sim 7.73(\mathrm{~m}, 4 \mathrm{H})$, $7.59(\mathrm{dd}, J=9.0,1.2 \mathrm{~Hz}, 1 \mathrm{H}), 7.45 \sim 7.38(\mathrm{~m}, 2 \mathrm{H}), 6.81(\mathrm{~s}, 1 \mathrm{H}), 3.41(\mathrm{~s}, 2 \mathrm{H}), 2.27(\mathrm{~s}, 3 \mathrm{H})$, 1.95 (s, 3H); ${ }^{13} \mathrm{C}$ NMR (150 MHz, $\left.\mathrm{CDCl}_{3}\right): \delta 168.9,146.7,142.4,133.7,133.1,132.5,128.0$, 127.9, 127.6, 126.2, 125.5, 124.9, 124.5, 123.3, 122.6, 41.5, 20.7, 11.7; HRMS (EI+, m/z) calcd for $\mathrm{C}_{18} \mathrm{H}_{16} \mathrm{O}_{2}[\mathrm{M}+]$ : 264.1150, found: 264.1142 .

Spectral data for 4-butyl-2-methylcyclopenta-1,4-dien-1-yl acetate (1j):

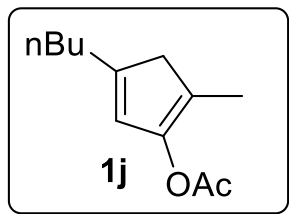

Colourless oil (0.080 g, $0.411 \mathrm{mmol}, 80 \%) ;{ }^{1} \mathrm{H} \mathrm{NMR}\left(600 \mathrm{MHz}, \mathrm{CDCl}_{3}\right): \delta 5.91(\mathrm{~s}, 1 \mathrm{H}), 2.75$ (s, 2H), $2.26(\mathrm{t}, J=7.8 \mathrm{~Hz}, 2 \mathrm{H}), 2.16(\mathrm{~s}, 3 \mathrm{H}), 1.78(\mathrm{~s}, 3 \mathrm{H}), 1.47 \sim 1.42(\mathrm{~m}, 2 \mathrm{H}), 1.33 \sim 1.27$ $(\mathrm{m}, 2 \mathrm{H}), 0.86(\mathrm{t}, J=7.2 \mathrm{~Hz}, 3 \mathrm{H}) ;{ }^{13} \mathrm{C} \mathrm{NMR}\left(150 \mathrm{MHz}, \mathrm{CDCl}_{3}\right): \delta 168.8,146.1,145.5,123.2$, 121.2, 43.4, 31.2, 30.5, 22.4, 20.5, 13.8, 11.2; HRMS (ESI+, m/z) calcd for $\mathrm{C}_{12} \mathrm{H}_{18} \mathrm{NaO}_{2}$ [M+Na]: 217.1204, found: 217.1204 .

Spectral data for 4-isopropyl-2-methylcyclopenta-1,4-dien-1-yl acetate (1k): 


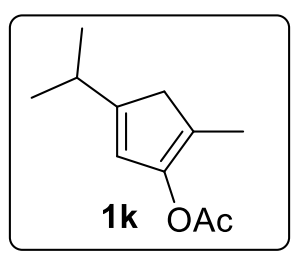

Colorless oil (0.075 g, $0.416 \mathrm{mmol}, 75 \%)$; ${ }^{1} \mathrm{H}$ NMR (700 MHz, $\left.\mathrm{CDCl}_{3}\right): \delta 5.91(\mathrm{~s}, 1 \mathrm{H}), 2.80$ $(\mathrm{s}, 2 \mathrm{H}), 2.59 \sim 2.53(\mathrm{~m}, 1 \mathrm{H}), 2.18(\mathrm{~s}, 3 \mathrm{H}), 1.80(\mathrm{~s}, 3 \mathrm{H}), 1.09(\mathrm{~s}, 3 \mathrm{H}), 1.07(\mathrm{~s}, 3 \mathrm{H}) ;{ }^{13} \mathrm{C} \mathrm{NMR}$ (175 MHz, $\left.\mathrm{CDCl}_{3}\right): \delta 168.9,152.3,145.4,121.5,121.4,41.6,29.6,22.5,22.5,20.7,11.3$; HRMS (ESI+, m/z) calcd for $\mathrm{C}_{11} \mathrm{H}_{16} \mathrm{NaO}_{2}[\mathrm{M}+\mathrm{Na}]: 203.1048$, found: 203.1373.

\section{Spectral data for 2-butyl-4-phenylcyclopenta-1,4-dien-1-yl acetate (11):}

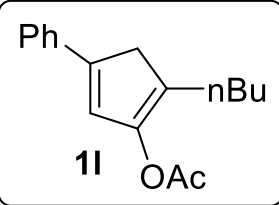

Yellow oil (0.082 g, $0.319 \mathrm{mmol}, 82 \%)$; ${ }^{1} \mathrm{H}$ NMR (600 MHz, $\left.\mathrm{CDCl}_{3}\right): \delta 7.44 \sim 7.42(\mathrm{~m}, 2 \mathrm{H})$, $7.28(\mathrm{t}, J=8.1 \mathrm{~Hz}, 2 \mathrm{H}), 7.16(\mathrm{t}, J=7.4 \mathrm{~Hz}, 1 \mathrm{H}), 6.69(\mathrm{~s}, 1 \mathrm{H}), 3.29(\mathrm{~s}, 2 \mathrm{H}), 2.32(\mathrm{t}, J=7.8$ $\mathrm{Hz}, 2 \mathrm{H}), 2.23(\mathrm{~s}, 3 \mathrm{H}), 1.49(\mathrm{t}, J=7.2 \mathrm{~Hz}, 2 \mathrm{H}), 1.37 \sim 1.31(\mathrm{~m}, 2 \mathrm{H}), 0.92(\mathrm{t}, J=7.8 \mathrm{~Hz}, 3 \mathrm{H})$; ${ }^{13} \mathrm{C}$ NMR $\left(150 \mathrm{MHz}, \mathrm{CDCl}_{3}\right): \delta 168.9,146.3,142.4,135.6,129.0,128.5,126.7,124.5,124.2$, 39.4, 31.4, 25.8, 22.4, 20.7, 13.8; HRMS (ESI+, m/z) calcd for $\mathrm{C}_{17} \mathrm{H}_{20} \mathrm{NaO}_{2}[\mathrm{M}+\mathrm{Na}]$ : 279.1361, found: 279.1907 .

\section{Spectral data for 2-methyl-4-phenylcyclopenta-1,4-dien-1-yl 2-naphthoate (1m):}

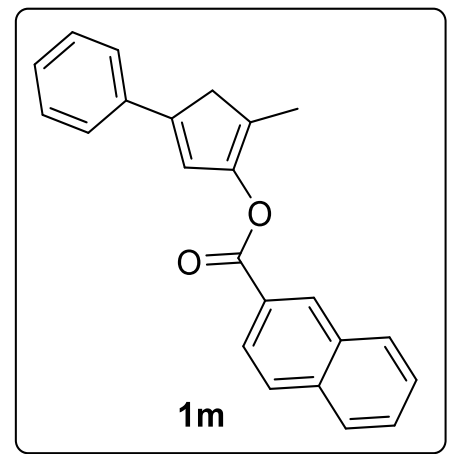

colorless oil, (0.093 g, $0.285 \mathrm{mmol}, 93 \%) ;{ }^{1} \mathrm{H} \mathrm{NMR}\left(600 \mathrm{MHz}, \mathrm{CDCl}_{3}\right): \delta 8.75(\mathrm{~s}, 1 \mathrm{H}), 8.16$ $(\mathrm{dd}, J=8.6,1.6 \mathrm{~Hz}, 1 \mathrm{H}), 7.99(\mathrm{~d}, J=8.1 \mathrm{~Hz}, 1 \mathrm{H}), 7.93$ (d, $J=8.6 \mathrm{~Hz}, 1 \mathrm{H}), 7.90$ (d, $J=1.8$ $\mathrm{Hz}, 1 \mathrm{H}), 7.62(\mathrm{t}, J=7.0 \mathrm{~Hz}, 1 \mathrm{H}), 7.56(\mathrm{t}, J=7.0 \mathrm{~Hz}, 1 \mathrm{H}), 7.47(\mathrm{~d}, J=7.4 \mathrm{~Hz}, 2 \mathrm{H}), 7.30(\mathrm{t}, J$ 
$=7.6 \mathrm{~Hz}, 2 \mathrm{H}), 7.18(\mathrm{t}, J=7.6 \mathrm{~Hz}, 1 \mathrm{H}), 6.85(\mathrm{~s}, 1 \mathrm{H}), 3.38(\mathrm{~s}, 2 \mathrm{H}), 2.03(\mathrm{~s}, 3 \mathrm{H}) ;{ }^{13} \mathrm{C} \mathrm{NMR}$ $\left(150 \mathrm{MHz}, \mathrm{CDCl}_{3}\right): \delta 164.6,146.8,142.5,135.8,135.7,132.5,131.8,129.5,128.6,128.6$, 128.4, 127.8, 126.8, 126.8, 126.6, 125.4, 124.6, 124.6, 124.4, 41.5, 11.8; HRMS (ESI+, m/z) calcd for $\mathrm{C}_{23} \mathrm{H}_{18} \mathrm{NaO}_{2}$ [M+Na]: 349.1204, found: 349.1215.

\section{Spectral data for 3-butylspiro[4.5]deca-1,3-dien-2-yl acetate (1p):}

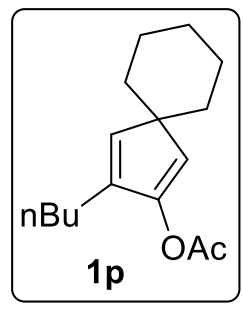

Colorless oil (0.037 g, $0.148 \mathrm{mmol}, 37 \%) ;{ }^{1} \mathrm{H} \mathrm{NMR}\left(600 \mathrm{MHz}, \mathrm{CDCl}_{3}\right): \delta 5.79(\mathrm{~s}, 1 \mathrm{H}), 5.26$ $\sim 5.23(\mathrm{~m}, 1 \mathrm{H}), 2.25(\mathrm{t}, J=1.0 \mathrm{~Hz}, 2 \mathrm{H}), 2.16(\mathrm{~d}, J=1.2 \mathrm{~Hz}, 3 \mathrm{H}), 1.98(\mathrm{q}, J=7.8 \mathrm{~Hz}, 2 \mathrm{H})$, $1.52 \sim 1.36(\mathrm{~m}, 12 \mathrm{H}), 0.89(\mathrm{t}, J=7.8 \mathrm{~Hz}, 3 \mathrm{H}) ;{ }^{13} \mathrm{C} \mathrm{NMR}\left(150 \mathrm{MHz}, \mathrm{CDCl}_{3}\right): \delta 167.9,147.3$, 137.5, 128.0, 117.6, 43.9, 38.2, 37.7, 31.0, 25.7, 23.0, 22.6, 21.0, 13.8, rest of peaks merged with others; HRMS (ESI+, m/z) calcd for $\mathrm{C}_{16} \mathrm{H}_{25} \mathrm{O}_{2}[\mathrm{M}+\mathrm{H}]: 249.1854$, found: 249.1933.

Spectral data for (1S,5R)-1-methyl-3,7-diphenyl-6-oxa-7-azabicyclo[3.2.0]hept-3-en-5-yl acetate (3a):

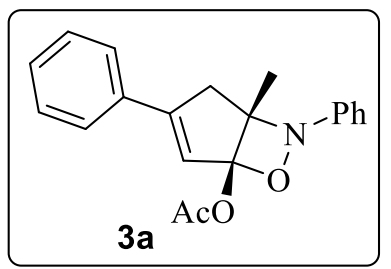

Yellow oil (0.052 g, $0.161 \mathrm{mmol}, 35 \%) ;{ }^{1} \mathrm{H} \mathrm{NMR}\left(600 \mathrm{MHz}, \mathrm{CDCl}_{3}\right): \delta 7.37(\mathrm{dd}, J=7.8,1.8$ $\mathrm{Hz}, 2 \mathrm{H}), 7.30(\mathrm{~d}, J=7.3 \mathrm{~Hz}, 2 \mathrm{H}), 7.26 \sim 7.24(\mathrm{~m}, 3 \mathrm{H}), 6.98(\mathrm{t}, J=7.8 \mathrm{~Hz}, 1 \mathrm{H}), 6.93(\mathrm{~d}, J=$ $7.8 \mathrm{~Hz}, 2 \mathrm{H}), 6.65(\mathrm{t}, J=1.2 \mathrm{~Hz}, 1 \mathrm{H}), 3.17(\mathrm{dd}, J=18.0,2.4 \mathrm{~Hz}, 1 \mathrm{H}), 2.74(\mathrm{dd}, J=17.4,1.8$ $\mathrm{Hz}, 1 \mathrm{H}), 2.18(\mathrm{~s}, 3 \mathrm{H}), 1.60(\mathrm{~s}, 3 \mathrm{H}) ;{ }^{13} \mathrm{C} \mathrm{NMR}\left(150 \mathrm{MHz}, \mathrm{CDCl}_{3}\right): \delta 168.6,150.0,148.1$, 134.1, 129.1, 128.5, 128.4, 127.9, 126.3, 123.3, 121.7, 116.3, 78.2, 42.0, 21.3, 19.5; HRMS $(\mathrm{EI}+, \mathrm{m} / \mathrm{z})$ calcd for $\mathrm{C}_{20} \mathrm{H}_{19} \mathrm{NO}_{3}[\mathrm{M}+]$ : 321.1365, found: 321.1369 .

Spectral data for $(1 S, 5 S)$-5-methyl-3,7-diphenyl-6-oxa-7-azabicyclo[3.2.0]hept-2-en-1-yl acetate (3a'): 


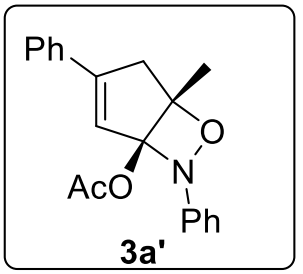

Yellow oil (0.032 g, 0.099 mmol, 22\%); ${ }^{1} \mathrm{H}$ NMR (600 MHz, $\left.\mathrm{CDCl}_{3}\right): \delta 7.30 \sim 7.23(\mathrm{~m}, 7 \mathrm{H})$, $7.04 \sim 7.00(\mathrm{~m}, 3 \mathrm{H}), 5.89$ (t, $J=1.8 \mathrm{~Hz}, 1 \mathrm{H}), 3.13 \sim 3.03(\mathrm{~m}, 2 \mathrm{H}), 2.19$ (s, 3H), 1.83 (s, 3H); ${ }^{13} \mathrm{C} \mathrm{NMR}\left(150 \mathrm{MHz}, \mathrm{CDCl}_{3}\right): \delta 168.3,150.3,147.5,134.3,128.9,128.5,128.4,128.3,126.3$, 123.7, 119.5, 116.9, 89.3, 45.2, 21.3, 19.6; HRMS (ESI+, m/z) calcd for $\mathrm{C}_{20} \mathrm{H}_{19} \mathrm{NNaO}_{3}$ [M+Na]: 344.1262, found: 344.1248.

Spectral data for (1S,5R)-1-methyl-3-(naphthalen-2-yl)-7-phenyl-6-oxa-7-azabicyclo -[3.2.0]hept-3-en-5-yl acetate (3i):

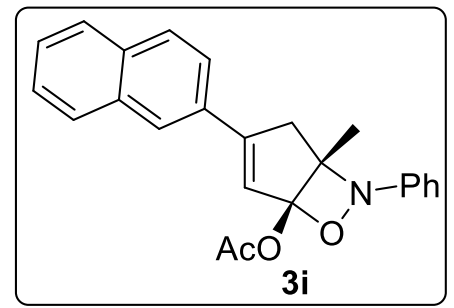

Yellow solid, $\mathrm{mp}=141-142,(0.053 \mathrm{~g}, 0.142 \mathrm{mmol}, 38 \%) ;{ }^{1} \mathrm{H}$ NMR $\left(600 \mathrm{MHz}, \mathrm{CDCl}_{3}\right): \delta$ $7.79 \sim 7.76(\mathrm{~m}, 3 \mathrm{H}), 7.70(\mathrm{~s}, 1 \mathrm{H}), 7.61(\mathrm{dd}, J=9.0,1.8 \mathrm{~Hz}, 1 \mathrm{H}), 7.47 \sim 7.45(\mathrm{~m}, 2 \mathrm{H}), 7.27 \sim$ $7.24(\mathrm{~m}, 2 \mathrm{H}), 7.0 \sim 6.96(\mathrm{~m}, 3 \mathrm{H}), 6.80(\mathrm{~s}, 1 \mathrm{H}), 3.30(\mathrm{dd}, J=17.4,1.8 \mathrm{~Hz}, 1 \mathrm{H}), 2.88(\mathrm{dd}, J=$ 17.4, $1.8 \mathrm{~Hz}, 1 \mathrm{H}), 2.20$ (s, 3H), $1.64(\mathrm{~s}, 3 \mathrm{H}) ;{ }^{13} \mathrm{C} \mathrm{NMR}\left(150 \mathrm{MHz}, \mathrm{CDCl}_{3}\right): \delta 168.6,149.9$, $148.1,133.5,133.0,131.5,128.4,128.3,128.2$, 127.6, 126.7, 126.5, 125.9, 123.9, 123.3, 122.2, 117.0, 116.3, 78.2, 42.0, 21.3, 19.5; HRMS (ESI+, m/z) calcd for $\mathrm{C}_{24} \mathrm{H}_{21} \mathrm{NNaO}_{3}$ [M+Na]: 394.1419, found: 394.1405.

Spectral data for (1S,5S)-5-methyl-3-(naphthalen-2-yl)-7-phenyl-6-oxa-7-azabicyclo -[3.2.0]hept-2-en-1-yl acetate (3i'):

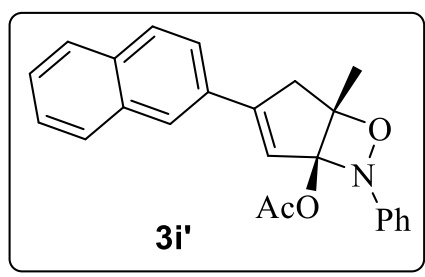

Yellow solid $\mathrm{mp}=148-149{ }^{\circ} \mathrm{C},(0.032 \mathrm{~g}, 0.086 \mathrm{mmol}, 23 \%)$, recrystallation by $(\mathrm{MeCN} /$ 
$\mathrm{DCM} /$ pentane $=1: 1: 10) ;{ }^{1} \mathrm{H}$ NMR $\left(600 \mathrm{MHz}, \mathrm{CDCl}_{3}\right): \delta 7.78 \sim 7.75(\mathrm{~m}, 2 \mathrm{H}), 7.72(\mathrm{~s}, 1 \mathrm{H})$, $7.70(\mathrm{~d}, J=8.6 \mathrm{~Hz}, 1 \mathrm{H}), 7.46 \sim 7.44(\mathrm{~m}, 2 \mathrm{H}), 7.41(\mathrm{dd}, J=1.7,8.6 \mathrm{~Hz}, 1 \mathrm{H}), 7.24(\mathrm{t}, J=7.8$ $\mathrm{Hz}, 2 \mathrm{H}), 7.61(\mathrm{~d}, J=7.8 \mathrm{~Hz}, 2 \mathrm{H}), 7.01(\mathrm{t}, J=7.2 \mathrm{~Hz}, 1 \mathrm{H}), 6.03(\mathrm{~s}, 1 \mathrm{H}), 3.26(\mathrm{dd}, J=17.4$, $1.8 \mathrm{~Hz}, 1 \mathrm{H}), 3.16(\mathrm{dd}, J=17.4,2.4 \mathrm{~Hz}, 1 \mathrm{H}), 2.21(\mathrm{~s}, 3 \mathrm{H}), 1.87(\mathrm{~s}, 3 \mathrm{H}) ;{ }^{13} \mathrm{C}$ NMR $(150 \mathrm{MHz}$, $\left.\mathrm{CDCl}_{3}\right): \delta 168.4,150.2,147.5,133.4,133.1,131.6,128.3,128.3,128.0,127.6,126.6,126.4$, 125.9, 123.9, 123.8, 120.1, 117.0, 107.0, 89.3, 45.3, 21.4, 19.7; HRMS (ESI+, m/z) calcd for $\mathrm{C}_{24} \mathrm{H}_{21} \mathrm{NNaO}_{3}[\mathrm{M}+\mathrm{Na}]: 394.1419$, found: 394.1404.

Spectral data for (1S,5R)-1-methyl-3,7-diphenyl-6-oxa-7-azabicyclo[3.2.0]hept-3-en-5-yl 2-naphthoate (3m):

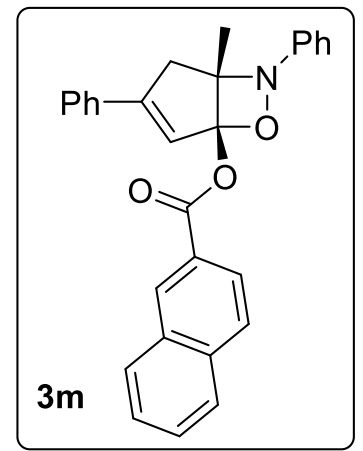

Orange solid $\mathrm{mp}=78-79{ }^{\circ} \mathrm{C},(0.015 \mathrm{~g}, 0.034 \mathrm{mmol}, 11 \%)$, recrystallation by $(\mathrm{MeCN} / \mathrm{DCM} /$ pentane $=1: 1: 10) ;{ }^{1} \mathrm{H} \mathrm{NMR}\left(400 \mathrm{MHz}, \mathrm{CDCl}_{3}\right): \delta 8.68(\mathrm{~s}, 1 \mathrm{H}), 8.10(\mathrm{dd}, J=8.6,1.6 \mathrm{~Hz}$, 1H), 7.97 (d, $J=8.2 \mathrm{~Hz}, 1 \mathrm{H}), 7.89$ (t, $J=8.9 \mathrm{~Hz}, 2 \mathrm{H}), 7.61(\mathrm{t}, J=7.0 \mathrm{~Hz}, 1 \mathrm{H}), 7.55$ (t, $J=$ $7.0 \mathrm{~Hz}, 1 \mathrm{H}), 7.43(\mathrm{~d}, J=6.5 \mathrm{~Hz}, 2 \mathrm{H}), 7.34 \sim 7.26(\mathrm{~m}, 5 \mathrm{H}), 7.01(\mathrm{~d}, J=6.8 \mathrm{~Hz}, 3 \mathrm{H}), 6.83(\mathrm{~s}$, $1 \mathrm{H}), 3.29$ (d, $J=17.5 \mathrm{~Hz}, 1 \mathrm{H}), 2.89$ (d, $J=17.5 \mathrm{~Hz}, 1 \mathrm{H}), 1.79(\mathrm{~s}, 3 \mathrm{H}) ;{ }^{13} \mathrm{C}$ NMR $(100 \mathrm{MHz}$, $\left.\mathrm{CDCl}_{3}\right): \delta 164.1,150.1,148.2,135.8,134.2,132.4,131.8,129.5,129.1,128.6,128.5,128.5$, $128.5,128.4,127.8,126.8,126.7,126.4,125.2,123.3,121.9,116.4,78.6,42.3,19.5$; HRMS $(\mathrm{ESI}+, \mathrm{m} / \mathrm{z})$ calcd for $\mathrm{C}_{29} \mathrm{H}_{33} \mathrm{NNaO}_{3}[\mathrm{M}+\mathrm{Na}]: 456.1576$, found: 456.1568

Spectral data for 5-methyl-3-phenyl-5-(phenylamino)cyclopent-2-en-1-one (4a):

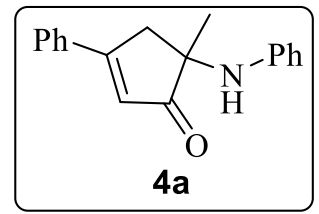

Yellow solid, $\mathrm{mp}=141-142{ }^{\circ} \mathrm{C},(0.088 \mathrm{~g}, 0.33 \mathrm{mmol}, 67 \%) ;{ }^{1} \mathrm{H} \mathrm{NMR}\left(600 \mathrm{MHz}, \mathrm{CDCl}_{3}\right): \delta$ $7.68(\mathrm{dd}, J=6.6,1.8 \mathrm{~Hz}, 2 \mathrm{H}), 7.50 \sim 7.45(\mathrm{~m}, 3 \mathrm{H}), 7.13(\mathrm{t}, J=7.4 \mathrm{~Hz}, 2 \mathrm{H}), 6.71(\mathrm{t}, J=7.2$ $\mathrm{Hz}, 1 \mathrm{H}), 6.66(\mathrm{t}, J=7.2 \mathrm{~Hz}, 1 \mathrm{H}), 6.56 \sim 6.54(\mathrm{~m}, 2 \mathrm{H}), 4.22(\mathrm{bs}, 1 \mathrm{H}), 3.58(\mathrm{dd}, J=18.0,1.8$ $\mathrm{Hz}, 1 \mathrm{H}), 3.12(\mathrm{dd}, J=18.0,1.8 \mathrm{~Hz}, 1 \mathrm{H}), 1.48(\mathrm{~s}, 3 \mathrm{H}) ;{ }^{13} \mathrm{C} \mathrm{NMR}\left(150 \mathrm{MHz}, \mathrm{CDCl}_{3}\right): \delta 209.3$, 
170.4, 145.2, 133.6, 131.8, 129.3, 129.0, 127.1, 123.8, 118.1, 114.2, 61.6, 42.4, 25.0; HRMS (ESI+, m/z) calcd for $\mathrm{C}_{18} \mathrm{H}_{18} \mathrm{NO}[\mathrm{M}+\mathrm{H}]: 264.1388$, found: 264.1400 .

Spectral data for 5-(hydroxy(phenyl)amino)-5-methyl-3-phenylcyclopent-2-en-1-one (4a-O):

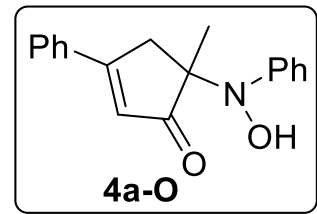

Yellow oil (0.010 g, $0.04 \mathrm{mmol}, 54 \%) ;{ }^{1} \mathrm{H} \mathrm{NMR}\left(400 \mathrm{MHz}, \mathrm{CDCl}_{3}\right): \delta 7.63(\mathrm{~d}, J=7.2 \mathrm{~Hz}$, 2H), $7.43(\mathrm{~d}, J=7.6 \mathrm{~Hz}, 3 \mathrm{H}), 7.34 \sim 7.25(\mathrm{~m}, 4 \mathrm{H}), 7.13(\mathrm{t}, J=7.6 \mathrm{~Hz}, 1 \mathrm{H}), 6.54(\mathrm{~s}, 1 \mathrm{H}), 6.36$ (bs, 1H), $3.74(\mathrm{~d}, J=17.6 \mathrm{~Hz}, 1 \mathrm{H}), 2.50(\mathrm{~d}, J=17.6 \mathrm{~Hz}, 1 \mathrm{H}), 1.32(\mathrm{~s}, 3 \mathrm{H}) ;{ }^{13} \mathrm{C}$ NMR $(100$ $\left.\mathrm{MHz}, \mathrm{CDCl}_{3}\right): \delta 208.3,171.5,149.0,133.8,131.5,128.9,128.2,127.0,125.4,124.3,123.2$, 72.5, 36.3, 24.1; HRMS (ESI+, m/z) calcd for $\mathrm{C}_{18} \mathrm{H}_{17} \mathrm{NNaO}_{2}$ [M+Na]: 302.1157, found: 302.1162 .

Spectral data for 3-(4-chlorophenyl)-5-methyl-5-(phenylamino)cyclopent-2-en-1-one (4b):

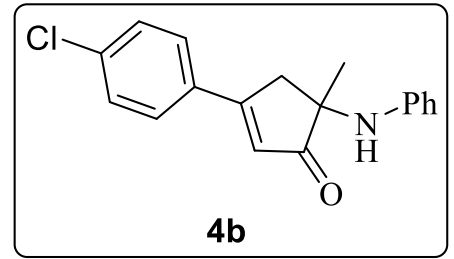

Yellow solid $\mathrm{mp}=145-146{ }^{\circ} \mathrm{C},(0.088 \mathrm{~g}, 0.295 \mathrm{mmol}, 74 \%) ;{ }^{1} \mathrm{H} \mathrm{NMR}\left(600 \mathrm{MHz}, \mathrm{CDCl}_{3}\right): \delta$ $7.60(\mathrm{~d}, J=9.0 \mathrm{~Hz}, 2 \mathrm{H}), 7.43(\mathrm{~d}, J=9.0 \mathrm{~Hz}, 2 \mathrm{H}), 7.13(\mathrm{dd}, J=9.0,1.8 \mathrm{~Hz}, 2 \mathrm{H}), 6.73 \sim 6.70$ $(\mathrm{m}, 1 \mathrm{H}), 6.62(\mathrm{t}, J=1.8 \mathrm{~Hz}, 1 \mathrm{H}), 6.54 \sim 6.53(\mathrm{~m}, 2 \mathrm{H}), 4.20(\mathrm{bs}, 1 \mathrm{H}), 3.51(\mathrm{dd}, J=18.0,1.8$ $\mathrm{Hz}, 1 \mathrm{H}), 3.08(\mathrm{dd}, J=17.6,1.8 \mathrm{~Hz}, 1 \mathrm{H}), 1.47(\mathrm{~s}, 3 \mathrm{H}) ;{ }^{13} \mathrm{C} \mathrm{NMR}\left(150 \mathrm{MHz}, \mathrm{CDCl}_{3}\right): \delta 209.0$, 168.8, 145.2, 137.9, 132.1, 129.3, 128.3, 124.2, 118.2, 114.2, 61.7, 42.4, 24.9, one carbon merged with others; HRMS (ESI+, m/z) calcd for $\mathrm{C}_{18} \mathrm{H}_{16} \mathrm{ClNNaO}$ [M+Na]: 320.0818, found: 320.0801 .

Spectral data for 3-(4-bromophenyl)-5-methyl-5-(phenylamino)cyclopent-2-en-1-one (4c): 


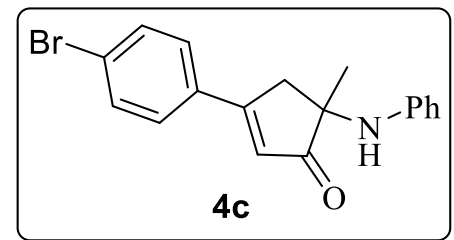

Yellow solid $\mathrm{mp}=152-153{ }^{\circ} \mathrm{C},(0.088 \mathrm{~g}, 0.257 \mathrm{mmol}, 76 \%) ;{ }^{1} \mathrm{H} \mathrm{NMR}\left(600 \mathrm{MHz}, \mathrm{CDCl}_{3}\right): \delta$ $7.59(\mathrm{~d}, J=9.0 \mathrm{~Hz}, 2 \mathrm{H}), 7.53(\mathrm{dd}, J=9.0,1.8 \mathrm{~Hz}, 2 \mathrm{H}), 7.13(\mathrm{t}, J=7.8 \mathrm{~Hz}, 2 \mathrm{H}), 6.73 \sim 6.70$ $(\mathrm{m}, 1 \mathrm{H}), 6.64(\mathrm{~s}, 1 \mathrm{H}), 6.53(\mathrm{~d}, J=8.6 \mathrm{~Hz}, 2 \mathrm{H}), 4.21(\mathrm{bs}, 1 \mathrm{H}), 3.51(\mathrm{dd}, J=18.6,1.8 \mathrm{~Hz}, 1 \mathrm{H})$, $3.08(\mathrm{~d}, J=17.4, \mathrm{~Hz}, 1 \mathrm{H}), 1.47$ (s, 3H); ${ }^{13} \mathrm{C} \mathrm{NMR}\left(150 \mathrm{MHz}, \mathrm{CDCl}_{3}\right): \delta 209.0,168.9,145.1$, 132.5, 132.3, 129.3, 128.4, 126.3, 124.2, 118.2, 114.2, 61.6, 42.4, 24.9; HRMS (ESI+, m/z) calcd for $\mathrm{C}_{18} \mathrm{H}_{16} \mathrm{BrNNaO}$ [M+Na]: 364.0313, found: 364.0312.

Spectral data for 5-methyl-5-(phenylamino)-3-(4-(trifluoromethyl)phenyl)cyclopent-2en-1-one (4d):

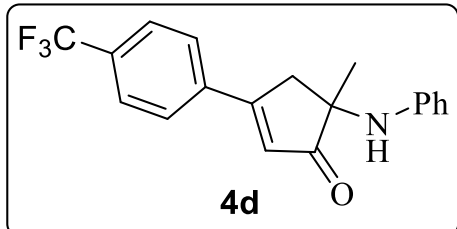

Yellow solid $\mathrm{mp}=164-165{ }^{\circ} \mathrm{C},(0.085 \mathrm{~g}, 0.258 \mathrm{mmol}, 73 \%) ;{ }^{1} \mathrm{H} \mathrm{NMR}\left(600 \mathrm{MHz}, \mathrm{CDCl}_{3}\right): \delta$ $7.78(\mathrm{~d}, J=8.4 \mathrm{~Hz}, 2 \mathrm{H}), 7.71(\mathrm{~d}, J=8.4 \mathrm{~Hz}, 2 \mathrm{H}), 7.13(\mathrm{dd}, J=8.0,1.8 \mathrm{~Hz}, 2 \mathrm{H}), 6.74 \sim 6.71$ (m, 2H), 6.53 (dd, $J=9.0,1.8 \mathrm{~Hz}, 2 \mathrm{H}), 4.19$ (bs, 1H), 3.57 (dd, $J=18.0,2.4 \mathrm{~Hz}, 1 \mathrm{H}), 3.11$ $(\mathrm{dd}, J=17.4,1.2 \mathrm{~Hz}, 1 \mathrm{H}), 1.49$ (s, 3H); ${ }^{13} \mathrm{C} \mathrm{NMR}\left(150 \mathrm{MHz}, \mathrm{CDCl}_{3}\right): \delta 209.0,168.3,145.1$, $136.9,133.0\left(J_{\mathrm{C}-\mathrm{F}}=33.0\right), 129.4,127.3,126.0,125.8\left(J_{\mathrm{C}-\mathrm{F}}=23.1\right), 118.3,114.3,61.8,42.5$, 24.9, one carbon merged with others; ${ }^{19} \mathrm{~F}$ NMR (600 MHz, $\left.\mathrm{CDCl}_{3}\right)$ : -62.4 HRMS (ESI+, m/z) calcd for $\mathrm{C}_{19} \mathrm{H}_{17} \mathrm{~F}_{3} \mathrm{NO}[\mathrm{M}+\mathrm{H}]:$ 332.1262, found: 332.1262 .

Spectral data for 5-methyl-5-(phenylamino)-3-(p-tolyl)cyclopent-2-en-1-one (4e):

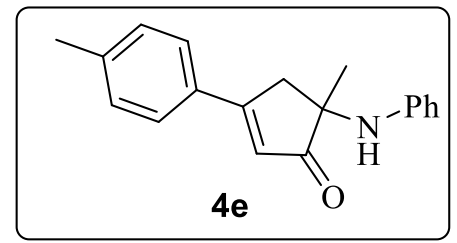

Yellow solid mp $=167-168{ }^{\circ} \mathrm{C},(0.098 \mathrm{~g}, 0.353 \mathrm{mmol}, 81 \%) ;{ }^{1} \mathrm{H} \mathrm{NMR}\left(600 \mathrm{MHz}, \mathrm{CDCl}_{3}\right): \delta$ $7.58(\mathrm{~d}, J=8.4 \mathrm{~Hz}, 2 \mathrm{H}), 7.26(\mathrm{~d}, J=8.2 \mathrm{~Hz}, 2 \mathrm{H}), 7.14 \sim 7.11(\mathrm{~m}, 2 \mathrm{H}), 6.71(\mathrm{t}, J=7.2 \mathrm{~Hz}$, $1 \mathrm{H}), 6.60(\mathrm{~s}, 1 \mathrm{H}), 6.55(\mathrm{~d}, J=8.5 \mathrm{~Hz}, 2 \mathrm{H}), 4.23(\mathrm{bs}, 1 \mathrm{H}), 3.52(\mathrm{dd}, J=17.4,1.2 \mathrm{~Hz}, 1 \mathrm{H})$, $3.11(\mathrm{dd}, J=17.4,1.2 \mathrm{~Hz}, 1 \mathrm{H}), 2.41(\mathrm{~s}, 3 \mathrm{H}), 1.47(\mathrm{~s}, 3 \mathrm{H}) ;{ }^{13} \mathrm{C} \mathrm{NMR}\left(150 \mathrm{MHz}, \mathrm{CDCl}_{3}\right): \delta$ 209.3, 170.5, 145.3, 142.5, 130.9, 129.7, 129.3, 127.1, 122.9, 118.0, 114.2, 61.5, 42.5, 24.9, 21.6; HRMS (ESI+, m/z) calcd for $\mathrm{C}_{19} \mathrm{H}_{20} \mathrm{NO}[\mathrm{M}+\mathrm{H}]: 278.1544$, found: 278.1544 . 

(4f):

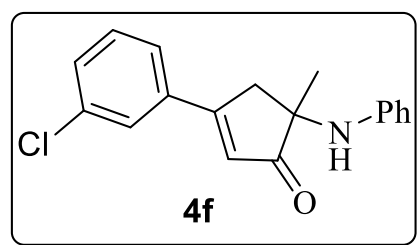

Yellow solid $\mathrm{mp}=160-161{ }^{\circ} \mathrm{C},(0.090 \mathrm{~g}, 0.305 \mathrm{mmol}, 76 \%) ;{ }^{1} \mathrm{H} \mathrm{NMR}\left(700 \mathrm{MHz}, \mathrm{CDCl}_{3}\right): \delta$ $7.63(\mathrm{~s}, 1 \mathrm{H}), 7.56(\mathrm{~d}, J=6.7 \mathrm{~Hz}, 1 \mathrm{H}), 7.46(\mathrm{~d}, J=7.0 \mathrm{~Hz}, 1 \mathrm{H}), 7.40(\mathrm{t}, J=6.7 \mathrm{~Hz}, 1 \mathrm{H}), 7.13$ $(\mathrm{t}, J=7.0 \mathrm{~Hz}, 2 \mathrm{H}), 6.72(\mathrm{t}, J=7.0 \mathrm{~Hz}, 1 \mathrm{H}), 6.65(\mathrm{~s}, 1 \mathrm{H}), 6.53(\mathrm{~d}, J=7.7 \mathrm{~Hz}, 2 \mathrm{H}), 3.52(\mathrm{dd}, J$ $=17.4,1.8 \mathrm{~Hz}, 1 \mathrm{H}), 3.08(\mathrm{dd}, J=17.4,1.2 \mathrm{~Hz}, 1 \mathrm{H}), 1.48(\mathrm{~s}, 3 \mathrm{H})$, one proton not clearly visible; ${ }^{13} \mathrm{C}$ NMR $\left(150 \mathrm{MHz}, \mathrm{CDCl}_{3}\right): \delta$ 209.0, 168.6, 145.1, 135.4, 135.2, 131.5, 130.3, 129.3, 127.0, 125.1, 124.9, 118.2, 114.2, 61.7, 42.4, 24.9; HRMS (ESI+, m/z) calcd for $\mathrm{C}_{18} \mathrm{H}_{17} \mathrm{ClNO}[\mathrm{M}+\mathrm{H}]: 298.0998$, found: 298.0995 .

\section{Spectral data for 5-methyl-5-(phenylamino)-3-(m-tolyl)cyclopent-2-en-1-one (4g):}

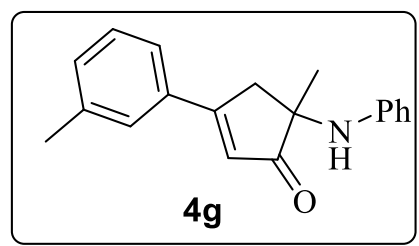

Yellow solid mp $=164-165{ }^{\circ} \mathrm{C},(0.095 \mathrm{~g}, 0.346 \mathrm{mmol}, 79 \%) ;{ }^{1} \mathrm{H} \mathrm{NMR}\left(600 \mathrm{MHz}, \mathrm{CDCl}_{3}\right): \delta$ $7.49(\mathrm{~d}, J=8.4 \mathrm{~Hz}, 2 \mathrm{H}), 7.35(\mathrm{t}, J=7.8 \mathrm{~Hz}, 1 \mathrm{H}), 7.30(\mathrm{~d}, J=7.2 \mathrm{~Hz}, 1 \mathrm{H}), 7.14 \sim 7.11(\mathrm{~m}$, $2 \mathrm{H}), 6.72 \sim 6.69(\mathrm{~m}, 1 \mathrm{H}), 6.63(\mathrm{t}, J=1.2 \mathrm{~Hz}, 1 \mathrm{H}), 6.56 \sim 6.53(\mathrm{~m}, 2 \mathrm{H}), 4.21(\mathrm{bs}, 1 \mathrm{H}), 3.54$ $(\mathrm{dd}, J=18.0,1.8 \mathrm{~Hz}, 1 \mathrm{H}), 3.11(\mathrm{dd}, J=17.4,1.2 \mathrm{~Hz}, 1 \mathrm{H}), 2.41(\mathrm{~s}, 3 \mathrm{H}), 1.47(\mathrm{~s}, 3 \mathrm{H}) ;{ }^{13} \mathrm{C}$ NMR $\left(150 \mathrm{MHz}, \mathrm{CDCl}_{3}\right): \delta 209.3,170.6,145.3,138.8,133.6,132.6,129.3,128.9,127.7$, 124.2, 123.7, 118.0, 114.2, 61.6, 42.5, 25.0, 21.4; HRMS (ESI+, m/z) calcd for $\mathrm{C}_{19} \mathrm{H}_{20} \mathrm{NO}$ $[\mathrm{M}+\mathrm{H}]: 278.1544$, found: 278.1544 .

Spectral data for 5-methyl-5-(phenylamino)-3-(o-tolyl)cyclopent-2-en-1-one (4h):

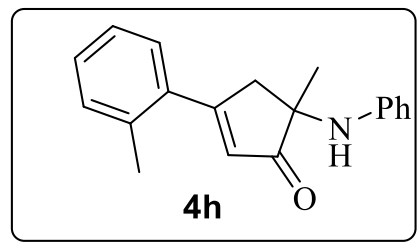

Yellow solid $\mathrm{mp}=155-156{ }^{\circ} \mathrm{C},(0.091 \mathrm{~g}, 0.328 \mathrm{mmol}, 75 \%) ;{ }^{1} \mathrm{H} \mathrm{NMR}\left(600 \mathrm{MHz}, \mathrm{CDCl}_{3}\right): \delta$ $7.36(\mathrm{~d}, J=7.7 \mathrm{~Hz}, 1 \mathrm{H}), 7.32(\mathrm{t}, J=7.4 \mathrm{~Hz}, 1 \mathrm{H}), 7.29 \sim 7.26(\mathrm{~m}, 2 \mathrm{H}), 7.14(\mathrm{t}, J=7.2 \mathrm{~Hz}$, 2H), $6.74 \sim 6.71(\mathrm{~m}, 1 \mathrm{H}), 6.57(\mathrm{t}, J=6.5 \mathrm{~Hz}, 2 \mathrm{H}), 6.40(\mathrm{~s}, 1 \mathrm{H}), 4.22(\mathrm{bs}, 1 \mathrm{H}), 3.61(\mathrm{~d}, J=$ 
17.4 Hz, 1H), $3.03(\mathrm{~d}, J=17.4 \mathrm{~Hz}, 1 \mathrm{H}), 2.45(\mathrm{~s}, 3 \mathrm{H}), 1.50(\mathrm{~s}, 3 \mathrm{H}) ;{ }^{13} \mathrm{C}$ NMR $(150 \mathrm{MHz}$, $\left.\mathrm{CDCl}_{3}\right): \delta 209.5,172.3,145.3,136.4,134.8,131.5,130.1,129.3,128.5,127.4,126.2,118.1$, 114.3, 61.3, 46.1, 24.7, 21.6; HRMS (ESI+, m/z) calcd for $\mathrm{C}_{19} \mathrm{H}_{20} \mathrm{NO}[\mathrm{M}+\mathrm{H}]: 278.1544$, found: 278.1546 .

Spectral data for 5-methyl-3-(naphthalen-2-yl)-5-(phenylamino)cyclopent-2-en-1-one (4i):

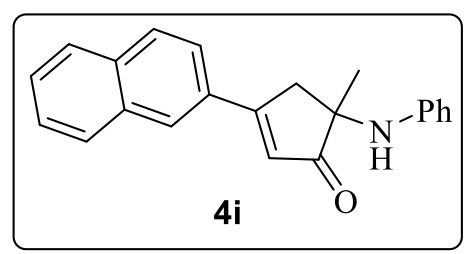

Yellow solid $\mathrm{mp}=130-131{ }^{\circ} \mathrm{C},(0.092 \mathrm{~g}, 0.295 \mathrm{mmol}, 78 \%)$, recrystallation by $(\mathrm{MeCN} /$ $\mathrm{DCM} /$ pentane $=1: 1: 10) ;{ }^{1} \mathrm{H}$ NMR $\left(600 \mathrm{MHz}, \mathrm{CDCl}_{3}\right): \delta 8.12(\mathrm{~s}, 1 \mathrm{H}), 7.90(\mathrm{~d}, J=9.0 \mathrm{~Hz}$, 2H), $7.86(\mathrm{~d}, J=7.8 \mathrm{~Hz}, 1 \mathrm{H}), 7.79 \sim 7.77(\mathrm{~m}, 1 \mathrm{H}), 7.58 \sim 7.53(\mathrm{~m}, 2 \mathrm{H}), 7.16 \sim 7.14(\mathrm{~m}, 2 \mathrm{H})$, $6.77(\mathrm{t}, J=1.8 \mathrm{~Hz}, 1 \mathrm{H}), 6.74 \sim 6.71(\mathrm{~m}, 1 \mathrm{H}), 6.60 \sim 6.58(\mathrm{~m}, 2 \mathrm{H}), 4.25(\mathrm{bs}, 1 \mathrm{H}), 3.68(\mathrm{~d}, J=$ $17.4 \mathrm{~Hz}, 1 \mathrm{H}), 3.27(\mathrm{dd}, J=17.4,1.2 \mathrm{~Hz}, 1 \mathrm{H}), 1.52(\mathrm{~s}, 3 \mathrm{H}) ;{ }^{13} \mathrm{C} \mathrm{NMR}\left(150 \mathrm{MHz}, \mathrm{CDCl}_{3}\right): \delta$ 209.3, 170.1, 145.3, 134.8, 132.9, 131.0, 129.3, 129.0, 128.8, 128.0, 127.8, 127.4, 127.0, 124.2, 123.8, 118.1, 114.3, 61.6, 42.5, 25.0; HRMS (ESI+, m/z) calcd for $\mathrm{C}_{22} \mathrm{H}_{19} \mathrm{NNaO}$ $[\mathrm{M}+\mathrm{Na}]: 336.1364$, found: 336.0042 .

Spectral data for 3-butyl-5-methyl-5-(phenylamino)cyclopent-2-en-1-one (4j):

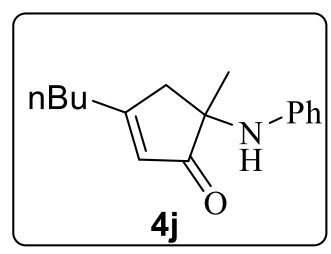

Yellow oil (0.085 g, $0.349 \mathrm{mmol}, 68 \%)$; ${ }^{1} \mathrm{H}$ NMR (600 MHz, $\left.\mathrm{CDCl}_{3}\right): \delta 7.13 \sim 7.10(\mathrm{~m}, 2 \mathrm{H})$, $6.71 \sim 6.68(\mathrm{~m}, 1 \mathrm{H}), 6.51 \sim 6.49(\mathrm{~m}, 2 \mathrm{H}), 6.01 \sim 6.00(\mathrm{~m}, 1 \mathrm{H}), 4.11(\mathrm{bs}, 1 \mathrm{H}), 3.10(\mathrm{~d}, J=$ $18.0 \mathrm{~Hz}, 1 \mathrm{H}), 2.67 \sim 2.64(\mathrm{~m}, 1 \mathrm{H}), 2.44 \sim 2.41(\mathrm{~m}, 2 \mathrm{H}), 1.60 \sim 1.55(\mathrm{~m}, 2 \mathrm{H}), 1.39 \sim 1.35(\mathrm{~m}$, $5 \mathrm{H}), 0.93(\mathrm{t}, J=7.2 \mathrm{~Hz}, 3 \mathrm{H}) ;{ }^{13} \mathrm{C} \mathrm{NMR}\left(150 \mathrm{MHz}, \mathrm{CDCl}_{3}\right): \delta 209.9,180.1,145.4,129.2$, 125.9, 117.9, 114.3, 61.4, 45.6, 33.4, 28.9, 24.3, 22.4, 13.8; HRMS (ESI+, m/z) calcd for $\mathrm{C}_{16} \mathrm{H}_{22} \mathrm{NO}[\mathrm{M}+\mathrm{H}]: 244.1701$, found: 244.1701 .

Spectral data for 3-isopropyl-5-methyl-5-(phenylamino)cyclopent-2-en-1-one (4k): 


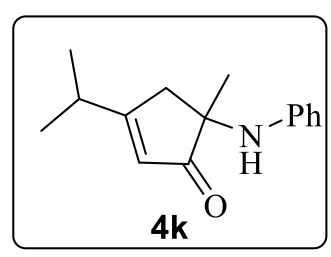

Yellow oil (0.081 g, $0.355 \mathrm{mmol}, 64 \%) ;{ }^{1} \mathrm{H}$ NMR (600 MHz, $\left.\mathrm{CDCl}_{3}\right): \delta 7.14 \sim 7.11(\mathrm{~m}, 2 \mathrm{H})$, $6.71 \sim 6.69(\mathrm{~m}, 1 \mathrm{H}), 6.51(\mathrm{dd}, J=7.8,1.2 \mathrm{~Hz}, 2 \mathrm{H}), 5.99(\mathrm{q}, J=1.2 \mathrm{~Hz}, 1 \mathrm{H}), 4.12(\mathrm{bs}, 1 \mathrm{H})$, $3.12(\mathrm{dd}, J=18.0,1.8 \mathrm{~Hz}, 1 \mathrm{H}), 2.71 \sim 2.63(\mathrm{~m}, 2 \mathrm{H}), 1.37(\mathrm{~s}, 3 \mathrm{H}), 1.19(\mathrm{~d}, J=6.6 \mathrm{~Hz}, 3 \mathrm{H})$, $1.18(\mathrm{~d}, J=7.2 \mathrm{~Hz}, 3 \mathrm{H}) ;{ }^{13} \mathrm{C} \mathrm{NMR}\left(150 \mathrm{MHz}, \mathrm{CDCl}_{3}\right): \delta 210.0,185.2,145.4,129.2,124.1$, 117.9, 114.3, 61.4, 43.7, 32.3, 24.3, 20.6, 20.5; HRMS (ESI+, m/z) calcd for $\mathrm{C}_{15} \mathrm{H}_{19} \mathrm{NO}[\mathrm{M}+]$ : 229.1466, found: 229.0980 .

Spectral data for 5-butyl-3-phenyl-5-(phenylamino)cyclopent-2-en-1-one (4I):

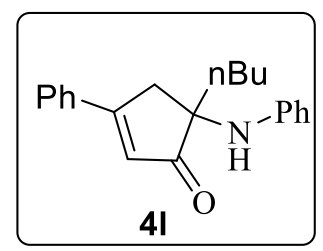

Yellow solid $\mathrm{mp}=142-143{ }^{\circ} \mathrm{C},(0.078 \mathrm{~g}, 0.257 \mathrm{mmol}, 66 \%) ;{ }^{1} \mathrm{H}$ NMR $\left(400 \mathrm{MHz}, \mathrm{CDCl}_{3}\right): \delta$ $7.69(\mathrm{~d}, J=6.4 \mathrm{~Hz}, 2 \mathrm{H}), 7.50 \sim 7.45(\mathrm{~m}, 3 \mathrm{H}), 7.12(\mathrm{t}, J=7.6 \mathrm{~Hz}, 2 \mathrm{H}), 6.69(\mathrm{t}, J=7.3 \mathrm{~Hz}$, $1 \mathrm{H}), 6.65(\mathrm{~s}, 1 \mathrm{H}), 6.55(\mathrm{~d}, J=8.0 \mathrm{~Hz}, 2 \mathrm{H}), 4.25(\mathrm{bs}, 1 \mathrm{H}), 3.49(\mathrm{~d}, J=17.6 \mathrm{~Hz}, 1 \mathrm{H}), 3.15$ (d, $J=17.6 \mathrm{~Hz}, 1 \mathrm{H}), 1.86(\mathrm{~d}, J=8.9 \mathrm{~Hz}, 2 \mathrm{H}), 1.27 \sim 1.22(\mathrm{~m}, 4 \mathrm{H}), 0.88 \sim 0.81(\mathrm{~m}, 3 \mathrm{H}) ;{ }^{13} \mathrm{C}$ NMR (100 MHz, $\left.\mathrm{CDCl}_{3}\right): \delta 209.1,170.7,145.3,133.5,131.7,129.3,129.0,127.0,124.9$, 117.9, 114.2, 64.5, 40.9, 37.7, 25.8, 22.9, 13.8; HRMS (ESI+, m/z) calcd for $\mathrm{C}_{21} \mathrm{H}_{24} \mathrm{NO}$ $[\mathrm{M}+\mathrm{H}]: 306.1857$, found: 306.1855 .

Spectral data for 5-((4-chlorophenyl)amino)-5-methyl-3-phenylcyclopent-2-en-1-one (4m):

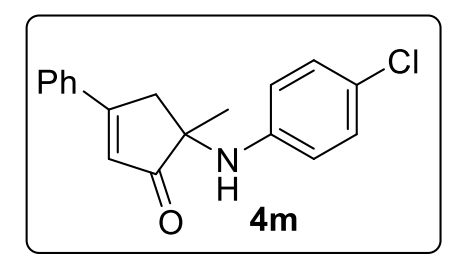

Yellow solid $\mathrm{mp}=181-182{ }^{\circ} \mathrm{C},(0.098 \mathrm{~g}, 0.331 \mathrm{mmol}, 71 \%) ;{ }^{1} \mathrm{H} \mathrm{NMR}\left(600 \mathrm{MHz}, \mathrm{CDCl}_{3}\right): \delta$ $7.68(\mathrm{dd}, J=7.8,1.8 \mathrm{~Hz}, 2 \mathrm{H}), 7.50 \sim 7.45(\mathrm{~m}, 3 \mathrm{H}), 7.07(\mathrm{~d}, J=8.9 \mathrm{~Hz}, 2 \mathrm{H}), 6.65(\mathrm{t}, J=1.8$ $\mathrm{Hz}, 1 \mathrm{H}), 6.45(\mathrm{~d}, J=8.9 \mathrm{~Hz}, 2 \mathrm{H}), 4.23(\mathrm{bs}, 1 \mathrm{H}), 3.48(\mathrm{dd}, J=18.0,1.8 \mathrm{~Hz}, 1 \mathrm{H}), 3.11$ (dd, $J=$ 17.4, $1.2 \mathrm{~Hz}, 1 \mathrm{H}), 1.46(\mathrm{~s}, 3 \mathrm{H}) ;{ }^{13} \mathrm{C} \mathrm{NMR}\left(150 \mathrm{MHz}, \mathrm{CDCl}_{3}\right): \delta 208.9,170.4,143.9,133.5$, 131.9, 129.2, 129.0, 127.1, 123.8, 122.8, 115.3, 61.6, 42.3, 24.9; HRMS (ESI+, m/z) calcd for $\mathrm{C}_{18} \mathrm{H}_{17} \mathrm{ClNO}[\mathrm{M}+\mathrm{H}]$ : 298.0998, found: 298.0989 . 
Spectral data for 5-((4-fluorophenyl)amino)-5-methyl-3-phenylcyclopent-2-en-1-one (4n):

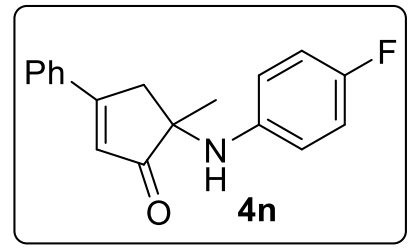

Yellow solid $\mathrm{mp}=174-175{ }^{\circ} \mathrm{C},(0.095 \mathrm{~g}, 0.340 \mathrm{mmol}, 73 \%) ;{ }^{1} \mathrm{H} \mathrm{NMR}\left(700 \mathrm{MHz}, \mathrm{CDCl}_{3}\right): \delta$ $7.68(\mathrm{~d}, J=7.0 \mathrm{~Hz}, 2 \mathrm{H}), 7.47 \sim 7.45(\mathrm{~m}, 3 \mathrm{H}), 6.83(\mathrm{t}, J=8.6 \mathrm{~Hz}, 2 \mathrm{H}), 6.65(\mathrm{~s}, 1 \mathrm{H}), 6.49$ 6.47 (m, 2H), 4.09 (bs, 1H), 3.49 (dd, $J=17.5,1.4 \mathrm{~Hz}, 1 \mathrm{H}), 3.09$ (dd, $J=17.5 \mathrm{~Hz}, 1.4 \mathrm{~Hz}$, $1 \mathrm{H}), 1.45(\mathrm{~s}, 3 \mathrm{H}) ;{ }^{13} \mathrm{C}$ NMR $\left(175 \mathrm{MHz}, \mathrm{CDCl}_{3}\right): \delta 209.3,170.4,156.9,155.6,141.5\left(J_{\mathrm{C}-\mathrm{F}}=\right.$ $1.9 \mathrm{~Hz}), 133.5,131.8,129.1\left(J_{\mathrm{C}-\mathrm{F}}=13.9\right), 126.8\left(J_{\mathrm{C}-\mathrm{F}}=63.6\right), 123.9,115.6\left(J_{\mathrm{C}-\mathrm{F}}=64.1,22.2\right)$, 61.9, 42.3, 25.1; ${ }^{19} \mathrm{~F}$ NMR (600 $\left.\mathrm{MHz}, \mathrm{CDCl}_{3}\right)$ : -126.7.; HRMS (ESI+, m/z) calcd for $\mathrm{C}_{18} \mathrm{H}_{17} \mathrm{FNO}[\mathrm{M}+\mathrm{H}]: 282.1294$, found: 282.1317 .

Spectral data for 5-((4-bromophenyl)amino)-5-methyl-3-phenylcyclopent-2-en-1-one (40):

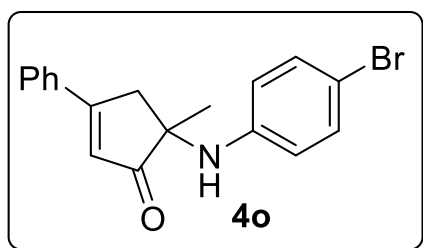

Yellow solid $\mathrm{mp}=184-185{ }^{\circ} \mathrm{C},(0.119 \mathrm{~g}, 0.347 \mathrm{mmol}, 75 \%) ;{ }^{1} \mathrm{H}$ NMR $\left(600 \mathrm{MHz}, \mathrm{CDCl}_{3}\right): \delta$ $7.68 \sim 7.67(\mathrm{~m}, 2 \mathrm{H}), 7.50 \sim 7.45(\mathrm{~m}, 3 \mathrm{H}), 7.20(\mathrm{dd}, J=7.2,1.8 \mathrm{~Hz}, 2 \mathrm{H}), 6.64(\mathrm{t}, J=1.8 \mathrm{~Hz}$, $1 \mathrm{H}), 6.41$ (dd, $J=7.2,1.8 \mathrm{~Hz}, 2 \mathrm{H}), 4.23$ (bs, 1H), 3.48 (dd, $J=18.0,1.8 \mathrm{~Hz}, 1 \mathrm{H}), 3.11$ (dd, $J$ $=17.4,1.2 \mathrm{~Hz}, 1 \mathrm{H}), 1.46(\mathrm{~s}, 3 \mathrm{H}) ;{ }^{13} \mathrm{C} \mathrm{NMR}\left(150 \mathrm{MHz}, \mathrm{CDCl}_{3}\right): \delta 208.8,170.4,144.3,133.5$, 132.1, 131.9, 130.6, 129.1, 127.1, 123.8, 115.8, 61.6, 42.3, 24.9; HRMS (ESI+, m/z) calcd for $\mathrm{C}_{18} \mathrm{H}_{17} \mathrm{BrNO}[\mathrm{M}+\mathrm{H}]:$ 342.0493, found: 342.0503 .

Spectral data for 5-methyl-3-phenyl-5-(p-tolylamino)cyclopent-2-en-1-one (4p):

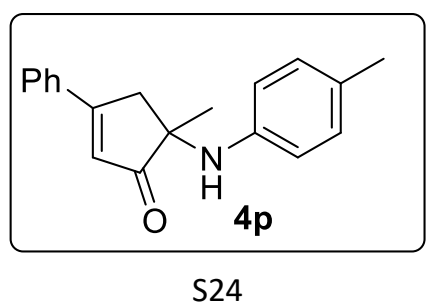


Yellow solid mp $=188-189{ }^{\circ} \mathrm{C},(0.106 \mathrm{~g}, 0.382 \mathrm{mmol}, 82 \%) ;{ }^{1} \mathrm{H} \mathrm{NMR}\left(600 \mathrm{MHz}, \mathrm{CDCl}_{3}\right): \delta$ $7.68 \sim 7.67(\mathrm{~m}, 2 \mathrm{H}), 7.48 \sim 7.44(\mathrm{~m}, 3 \mathrm{H}), 6.99(\mathrm{dd}, J=8.4 \mathrm{~Hz}, 2 \mathrm{H}), 6.63(\mathrm{t}, J=1.8 \mathrm{~Hz}, 1 \mathrm{H})$, $6.48(\mathrm{~d}, J=8.4 \mathrm{~Hz}, 2 \mathrm{H}), 4.07$ (bs, $1 \mathrm{H}), 3.54$ (dd, $J=17.4,1.2 \mathrm{~Hz}, 1 \mathrm{H}), 3.10$ (dd, $J=17.4,1.2$ $\mathrm{Hz}, 1 \mathrm{H}), 2.20(\mathrm{~s}, 3 \mathrm{H}), \quad 1.45$ (s, 3H); ${ }^{13} \mathrm{C} \mathrm{NMR}\left(175 \mathrm{MHz}, \mathrm{CDCl}_{3}\right): \delta 209.6,170.4,142.9$, $133.7,131.7,129.8,129.0,127.5,127.1,123.9$, 114.6, 61.8, 42.6, 25.0, 20.3.; HRMS (EI+, $\mathrm{m} / \mathrm{z}$ ) calcd for $\mathrm{C}_{19} \mathrm{H}_{19} \mathrm{NO}[\mathrm{M}+]$ : 277.1467, found: 277.1468 .

Spectral data for ethyl 4-((1-methyl-2-oxo-4-phenylcyclopent-3-en-1-yl)amino)benzoate (4q):

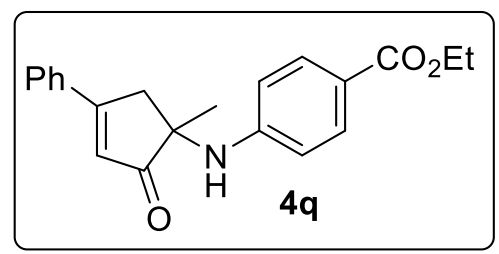

Yellow solid mp $=193-194{ }^{\circ} \mathrm{C},(0.101 \mathrm{~g}, 0.303 \mathrm{mmol}, 65 \%) ;{ }^{1} \mathrm{H} \mathrm{NMR}\left(600 \mathrm{MHz}, \mathrm{CDCl}_{3}\right): \delta$ $7.83(\mathrm{~d}, J=8.4 \mathrm{~Hz}, 2 \mathrm{H}), 7.68(\mathrm{dd}, J=8.4,1.2 \mathrm{~Hz}, 2 \mathrm{H}), 7.52 \sim 7.46(\mathrm{~m}, 3 \mathrm{H}), 6.65(\mathrm{t}, J=1.8$ $\mathrm{Hz}, 1 \mathrm{H}), 6.51$ (dd, $J=6.6,1.8 \mathrm{~Hz}, 2 \mathrm{H}), 4.63(\mathrm{bs}, 1 \mathrm{H}), 4.28(\mathrm{q}, J=7.2 \mathrm{~Hz}, 2 \mathrm{H}), 3.51$ (dd, $J=$ 17.4, $1.8 \mathrm{~Hz}, 1 \mathrm{H}), 3.18(\mathrm{dd}, J=18.0,1.8 \mathrm{~Hz}, 1 \mathrm{H}), 1.51(\mathrm{~s}, 3 \mathrm{H}), 1.32(\mathrm{t}, J=7.2 \mathrm{~Hz}, 3 \mathrm{H}) ;{ }^{13} \mathrm{C}$ NMR (150 MHz, $\left.\mathrm{CDCl}_{3}\right): \delta$ 208.2, 170.4, 166.6, 149.2, 133.4, 132.0, 131.5, 129.1, 127.1, 123.6, 119.5, 112.8, 61.4, 60.2, 42.4, 24.9, 14.4; HRMS (ESI+, m/z) calcd for $\mathrm{C}_{21} \mathrm{H}_{22} \mathrm{NO}_{3}$ $[\mathrm{M}+\mathrm{H}]: 336.1599$, found: 336.1703 .

Spectral data for 5-((3-chlorophenyl)amino)-5-methyl-3-phenylcyclopent-2-en-1-one (4r):

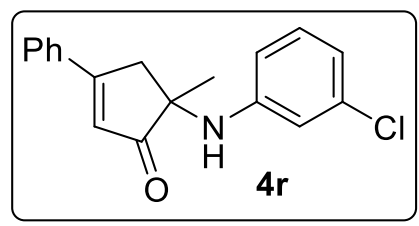

Yellow solid mp $=140-141{ }^{\circ} \mathrm{C},(0.102 \mathrm{~g}, 0.345 \mathrm{mmol}, 74 \%) ;{ }^{1} \mathrm{H} \mathrm{NMR}\left(700 \mathrm{MHz}, \mathrm{CDCl}_{3}\right): \delta$ $7.69(\mathrm{~d}, J=7.7 \mathrm{~Hz}, 2 \mathrm{H}), 7.51 \sim 7.46(\mathrm{~m}, 3 \mathrm{H}), 7.03(\mathrm{t}, J=8.4 \mathrm{~Hz}, 1 \mathrm{H}), 6.67(\mathrm{dd}, J=7.7,1.0$ $\mathrm{Hz}, 2 \mathrm{H}), 6.65$ (s, 1H), $6.54(\mathrm{t}, J=2.1 \mathrm{~Hz}, 1 \mathrm{H}), 6.39$ (dd, $J=7.7,2.1 \mathrm{~Hz}, 1 \mathrm{H}), 4.32(\mathrm{bs}, 1 \mathrm{H})$, $3.50(\mathrm{t}, J=16.1 \mathrm{~Hz}, 1 \mathrm{H}), 3.15(\mathrm{~d}, J=17.5 \mathrm{~Hz}, 1 \mathrm{H}), 1.47(\mathrm{~s}, 3 \mathrm{H}) ;{ }^{13} \mathrm{C}$ NMR $(175 \mathrm{MHz}$, $\left.\mathrm{CDCl}_{3}\right): \delta 208.7,170.5,146.5,134.9,133.4,131.9,130.3,129.0,127.1,123.6,117.9,113.9$, 112.3, 61.5, 42.5, 24.7; HRMS (ESI+, m/z) calcd for $\mathrm{C}_{18} \mathrm{H}_{17} \mathrm{ClNO}[\mathrm{M}+\mathrm{H}]$ : 298.0998, found: 298.0998 . 

(4s):

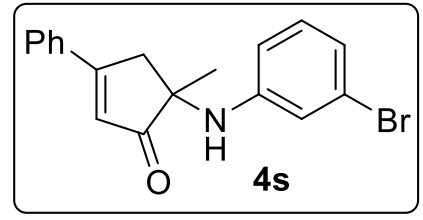

Yellow solid mp $=144-145{ }^{\circ} \mathrm{C},(0.121 \mathrm{~g}, 0.354 \mathrm{mmol}, 76 \%) ;{ }^{1} \mathrm{H} \mathrm{NMR}\left(700 \mathrm{MHz}, \mathrm{CDCl}_{3}\right): \delta$ $7.69(\mathrm{~d}, J=7.7 \mathrm{~Hz}, 2 \mathrm{H}), 7.50 \sim 7.46(\mathrm{~m}, 3 \mathrm{H}), 6.97(\mathrm{t}, J=8.4 \mathrm{~Hz}, 1 \mathrm{H}), 6.82(\mathrm{t}, J=2.1 \mathrm{~Hz}$, $1 \mathrm{H}), 6.71(\mathrm{t}, J=2.1 \mathrm{~Hz}, 1 \mathrm{H}), 6.64(\mathrm{~s}, 1 \mathrm{H}), 6.43(\mathrm{dd}, J=7.7,1.4 \mathrm{~Hz}, 1 \mathrm{H}), 4.29(\mathrm{bs}, 1 \mathrm{H}), 3.48$ $(\mathrm{dd}, J=17.5,1.4 \mathrm{~Hz}, 1 \mathrm{H}), 3.15(\mathrm{dd}, J=17.5,1.0 \mathrm{~Hz}, 1 \mathrm{H}), 1.47(\mathrm{~s}, 3 \mathrm{H}) ;{ }^{13} \mathrm{C}$ NMR $(175 \mathrm{MHz}$, $\left.\mathrm{CDCl}_{3}\right): \delta 208.6,170.6,146.7,133.4,131.9,130.6,129.1,127.1,123.6,123.2,120.8,116.9$, 112.6, 61.5, 42.5, 24.7; HRMS (ESI+, m/z) calcd for $\mathrm{C}_{18} \mathrm{H}_{17} \mathrm{BrNO}[\mathrm{M}+\mathrm{H}]: 342.0493$, found: 342.0480 .

Spectral data for 5-methyl-3-phenyl-5-(m-tolylamino)cyclopent-2-en-1-one (4t):

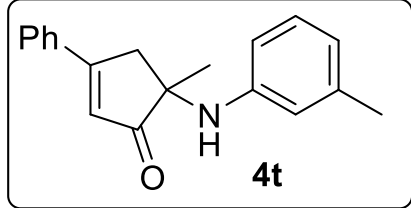

Yellow solid $\mathrm{mp}=157-158{ }^{\circ} \mathrm{C},(0.103 \mathrm{~g}, 0.373 \mathrm{mmol}, 80 \%) ;{ }^{1} \mathrm{H}$ NMR $\left(700 \mathrm{MHz}, \mathrm{CDCl}_{3}\right): \delta$ $7.69(\mathrm{~d}, J=7.7 \mathrm{~Hz}, 2 \mathrm{H}), 7.50 \sim 7.46(\mathrm{~m}, 3 \mathrm{H}), 7.03(\mathrm{t}, J=7.7 \mathrm{~Hz}, 1 \mathrm{H}), 6.65(\mathrm{~s}, 1 \mathrm{H}), 6.55(\mathrm{~d}, J$ $=7.7 \mathrm{~Hz}, 1 \mathrm{H}), 6.41(\mathrm{~s}, 1 \mathrm{H}), 6.36(\mathrm{~d}, J=8.4 \mathrm{~Hz}, 1 \mathrm{H}), 4.18(\mathrm{bs}, 1 \mathrm{H}), 3.56(\mathrm{~d}, J=17.5 \mathrm{~Hz}, 1 \mathrm{H})$, $3.13(\mathrm{~d}, J=17.5 \mathrm{~Hz}, 1 \mathrm{H}), 2.24(\mathrm{~s}, 3 \mathrm{H}), 1.48(\mathrm{~s}, 3 \mathrm{H}) ;{ }^{13} \mathrm{C} \mathrm{NMR}\left(175 \mathrm{MHz}, \mathrm{CDCl}_{3}\right): \delta 209.3$, 170.4, 145.3, 139.0, 133.6, 131.7, 129.1, 129.0, 127.0, 123.8, 119.0, 115.3, 111.0, 61.6, 42.6, 24.9, 21.6; HRMS (ESI+, m/z) calcd for $\mathrm{C}_{19} \mathrm{H}_{20} \mathrm{NO}$ [M+H]: 278.1544, found: 278.1542 .

Spectral data for 5-((2-chlorophenyl)amino)-5-methyl-3-phenylcyclopent-2-en-1-one (4u):

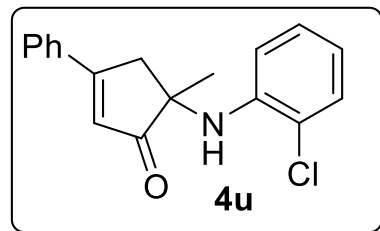

Yellow solid $\mathrm{mp}=154-155{ }^{\circ} \mathrm{C},(0.061 \mathrm{~g}, 0.205 \mathrm{mmol}, 44 \%) ;{ }^{1} \mathrm{H} \mathrm{NMR}\left(700 \mathrm{MHz}, \mathrm{CDCl}_{3}\right): \delta$ $7.64(\mathrm{~d}, J=7.7 \mathrm{~Hz}, 2 \mathrm{H}), 7.48 \sim 7.42(\mathrm{~m}, 3 \mathrm{H}), 7.38(\mathrm{~s}, 1 \mathrm{H}), 7.32$ (d, J = 8.4 Hz, 1H), 7.19 (d, $J=7.9 \mathrm{~Hz}, 1 \mathrm{H}), 6.87 \sim 6.85(\mathrm{~m}, 1 \mathrm{H}), 6.59(\mathrm{~s}, 1 \mathrm{H}), 3.35(\mathrm{~d}, J=17.3 \mathrm{~Hz}, 1 \mathrm{H}), 3.03(\mathrm{dd}, J=$ 17.3, $1.4 \mathrm{~Hz}, 1 \mathrm{H}), 1.51(\mathrm{~s}, 3 \mathrm{H})$, one proton not clearly visible; ${ }^{13} \mathrm{C} \mathrm{NMR}\left(175 \mathrm{MHz}, \mathrm{CDCl}_{3}\right)$ : 
$\delta$ 209.0, 168.7, 145.1, 135.4, 135.2, 131.5, 130.3, 129.3, 127.1, 125.1, 124.9, 118.2, 114.2, 61.7, 42.4, 24.9; HRMS (EI+, m/z) calcd for $\mathrm{C}_{18} \mathrm{H}_{16} \mathrm{ClNO}$ [M+]: 297.0920, found: 297.0925.

Spectral data for (3aS,7aR)-3-phenyl-7a-(phenylamino)-3a,4,5,6,7,7a-hexahydro -1H-inden-1-one (4v):

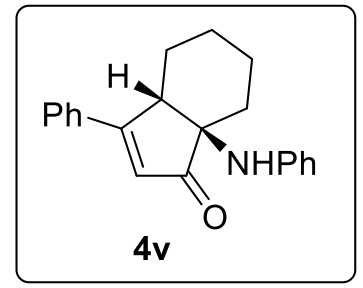

Yellow oil, (0.047 g, $0.157 \mathrm{mmol}, 40 \%) ;{ }^{1} \mathrm{H}$ NMR $\left(600 \mathrm{MHz}, \mathrm{CDCl}_{3}\right): \delta 7.53 \sim 7.52(\mathrm{~m}, 2 \mathrm{H})$, $7.46 \sim 7.45(\mathrm{~m}, 3 \mathrm{H}), 7.10 \sim 7.07(\mathrm{~m}, 2 \mathrm{H}), 6.70 \sim 6.68(\mathrm{~m}, 1 \mathrm{H}), 6.53(\mathrm{~d}, J=8.4,1.2 \mathrm{~Hz}, 1 \mathrm{H})$, $6.45 \sim 6.43(\mathrm{~m}, 2 \mathrm{H}), 4.10(\mathrm{bs}, 1 \mathrm{H}), 3.88(\mathrm{t}, J=4.2 \mathrm{~Hz}, 1 \mathrm{H}), 1.86 \sim 1.78(\mathrm{~m}, 4 \mathrm{H}), 1.62 \sim 1.57$ $(\mathrm{m}, 2 \mathrm{H}), 1.53 \sim 1.49(\mathrm{~m}, 2 \mathrm{H}), 1.31 \sim 1.23(\mathrm{~m}, 2 \mathrm{H}) ;{ }^{13} \mathrm{C} \mathrm{NMR}\left(175 \mathrm{MHz}, \mathrm{CDCl}_{3}\right): \delta 209.6$, 176.0, 144.9, 134.0, 130.8, 129.3, 129.0, 127.9, 127.5, 118.2, 114.1, 64.2, 42.9, 31.8, 23.1, 18.9, 17.9; HRMS (ESI+, m/z) calcd for $\mathrm{C}_{21} \mathrm{H}_{22} \mathrm{NO}[\mathrm{M}+\mathrm{H}]$ : 304.1701, found: 304.1701 .

Spectral data for 3-methyl-1-(phenylamino)spiro[4.5]dec-3-en-2-one (4w):

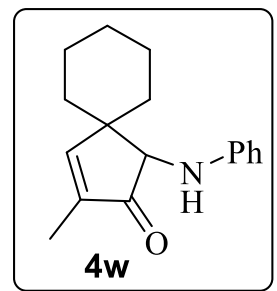

Yellow oil, (0.049 g, $0.193 \mathrm{mmol}, 40 \%) ;{ }^{1} \mathrm{H}$ NMR $\left(600 \mathrm{MHz}, \mathrm{CDCl}_{3}\right): \delta 7.59(\mathrm{~s}, 1 \mathrm{H}), 7.15(\mathrm{t}$, $J=7.4 \mathrm{~Hz}, 2 \mathrm{H}), 6.72 \sim 6.68(\mathrm{~m}, 3 \mathrm{H}), 4.06(\mathrm{bs}, 1 \mathrm{H}), 3.78(\mathrm{~s}, 1 \mathrm{H}), 1.81(\mathrm{~d}, J=1.2 \mathrm{~Hz}, 3 \mathrm{H})$, $1.72 \sim 1.63(\mathrm{~m}, 6 \mathrm{H}), 1.46 \sim 1.39(\mathrm{~m}, 2 \mathrm{H}), 1.23 \sim 1.12(\mathrm{~m}, 2 \mathrm{H}) ;{ }^{13} \mathrm{C} \mathrm{NMR}\left(150 \mathrm{MHz}, \mathrm{CDCl}_{3}\right)$ : $\delta 206.7,161.5,148.2,137.9,129.3,117.9,113.1,69.6,47.8,36.0,35.2,25.7,22.6,22.5,10.7$; HRMS (ESI+, m/z) calcd for $\mathrm{C}_{17} \mathrm{H}_{21} \mathrm{NNaO}$ [M+Na]: 278.1520, found: 278.1523 .

Spectral data for 1-hydroxy-4-methyl-2-phenyl-2-azaspiro[5.5] undec-4-en-3-one (4w'): 


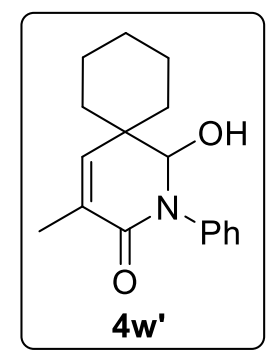

Yellow oil (0.028 g, $0.106 \mathrm{mmol}, 22 \%) ;{ }^{1} \mathrm{H} \mathrm{NMR}\left(700 \mathrm{MHz}, \mathrm{CDCl}_{3}\right): \delta 7.37$ (t, $J=7.7 \mathrm{~Hz}$, $2 \mathrm{H}), 7.32(\mathrm{~d}, J=7.7 \mathrm{~Hz}, 2 \mathrm{H}), 7.25(\mathrm{t}, J=7.7 \mathrm{~Hz}, 1 \mathrm{H}), 6.30(\mathrm{~s}, 1 \mathrm{H}), 4.84(\mathrm{~d}, J=7.0 \mathrm{~Hz}, 1 \mathrm{H})$, $2.67(\mathrm{~s}, 1 \mathrm{H}), 1.93(\mathrm{~d}, J=1.4 \mathrm{~Hz}, 3 \mathrm{H}), 1.76(\mathrm{~d}, J=4.8 \mathrm{~Hz}, 1 \mathrm{H}), 1.64(\mathrm{~d}, J=7.0 \mathrm{~Hz}, 1 \mathrm{H}), 1.60$ $\sim 1.53(\mathrm{~m}, 5 \mathrm{H}), 1.45(\mathrm{~d}, J=8.4 \mathrm{~Hz}, 1 \mathrm{H}), 1.38(\mathrm{~d}, J=16.1 \mathrm{~Hz}, 2 \mathrm{H}) ;{ }^{13} \mathrm{C}$ NMR $(175 \mathrm{MHz}$, $\left.\mathrm{CDCl}_{3}\right): \delta 163.7,142.0,141.0,129.7,128.7,126.9,126.6,89.6,40.5,36.0,31.7,25.5,22.1$, 21.1, 17.1; HRMS (ESI+, m/z) calcd for $\mathrm{C}_{17} \mathrm{H}_{22} \mathrm{NO}_{2}[\mathrm{M}+\mathrm{H}]: 272.1650$, found: 272.1658.

\section{Spectral data for 3-butyl-1-(phenylamino)spiro[4.5]dec-3-en-2-one (4x):}

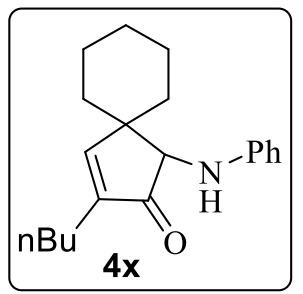

Yellow oil, (0.050 g, $0.169 \mathrm{mmol}, 42 \%) ;{ }^{1} \mathrm{H} \mathrm{NMR}\left(600 \mathrm{MHz}, \mathrm{CDCl}_{3}\right): \delta 7.14(\mathrm{~s}, 1 \mathrm{H}), 7.10$ $\sim 7.08(\mathrm{~m}, 2 \mathrm{H}), 6.65 \sim 6.62(\mathrm{~m}, 1 \mathrm{H}), 6.49(\mathrm{~d}, J=8.4,1.2 \mathrm{~Hz}, 2 \mathrm{H}), 4.13(\mathrm{t}, J=6.6 \mathrm{~Hz}, 1 \mathrm{H})$, 4.03 (bs, 1H), 2.24 (q, $J=15.0 \mathrm{~Hz}, 2 \mathrm{H}), 1.77 \sim 1.70(\mathrm{~m}, 2 \mathrm{H}), 1.67 \sim 1.59(\mathrm{~m}, 4 \mathrm{H}), 1.40 \sim$ $1.22(\mathrm{~m}, 8 \mathrm{H}), 0.90(\mathrm{t}, J=7.8 \mathrm{~Hz}, 3 \mathrm{H}) ;{ }^{13} \mathrm{C} \mathrm{NMR}\left(150 \mathrm{MHz}, \mathrm{CDCl}_{3}\right): \delta 208.6,167.9,147.2$, $143.5,129.1,117.4,113.5,50.4,48.4,43.3,36.9,36.8,36.7,25.3,23.3,19.4,13.9$, rest of carbon merged with others; HRMS (ESI+, m/z) calcd for $\mathrm{C}_{20} \mathrm{H}_{28} \mathrm{NO}[\mathrm{M}+\mathrm{H}]: 298.2170$, found: 298.2186 .

Spectral data for 3,3-dimethyl-4-phenylcyclopenta-1,4-dien-1-yl benzoate (5a):

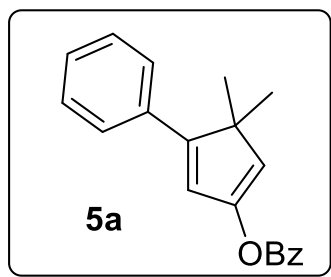

Yellow oil, (0.092 g, $0.317 \mathrm{mmol}, 92 \%) ;{ }^{1} \mathrm{H} \mathrm{NMR}\left(400 \mathrm{MHz}, \mathrm{CDCl}_{3}\right): \delta 8.15$ (d, $J=7.0 \mathrm{~Hz}$, 2H), $7.63 \sim 7.58(\mathrm{~m}, 1 \mathrm{H}), 7.53 \sim 7.47(\mathrm{~m}, 4 \mathrm{H}), 7.36(\mathrm{t}, J=7.4 \mathrm{~Hz}, 2 \mathrm{H}), 7.29 \sim 7.24(\mathrm{~m}, 1 \mathrm{H})$, $6.64(\mathrm{~d}, J=1.8 \mathrm{~Hz}, 1 \mathrm{H}), 6.16(\mathrm{~d}, J=1.8 \mathrm{~Hz}, 1 \mathrm{H}), 1.43(\mathrm{~s}, 6 \mathrm{H}) ;{ }^{13} \mathrm{C} \mathrm{NMR}\left(100 \mathrm{MHz}, \mathrm{CDCl}_{3}\right)$ : 
$\delta 164.1,154.5,147.8,135.1,133.4,130.0,129.6,128.5,128.5,128.3,127.1,126.6,123.0$, 50.5, 23.2, 23.2; HRMS (ESI+, m/z) calcd for $\mathrm{C}_{20} \mathrm{H}_{18} \mathrm{NaO}_{2}[\mathrm{M}+\mathrm{Na}]$ : 313.1204, found: 313.1216 .

Spectral data for 4-(4-chlorophenyl)-2,3,3-trimethylcyclopenta-1,4-dien-1-yl benzoate (5b):

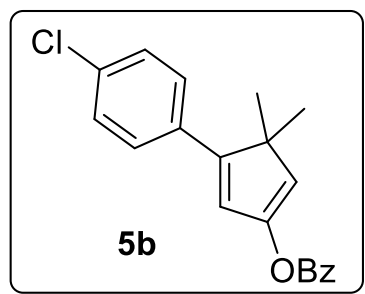

Yellow oil, (0.092 g, $0.283 \mathrm{mmol}, 92 \%) ;{ }^{1} \mathrm{H} \mathrm{NMR}\left(600 \mathrm{MHz}, \mathrm{CDCl}_{3}\right): \delta 8.12(\mathrm{dd}, J=8.4$, $1.2 \mathrm{~Hz}, 2 \mathrm{H}), 7.61 \sim 7.58(\mathrm{~m}, 1 \mathrm{H}), 7.49 \sim 7.46(\mathrm{~m}, 2 \mathrm{H}), 7.42(\mathrm{dd}, J=7.7,1.8 \mathrm{~Hz}, 2 \mathrm{H}), 7.31$ $(\mathrm{dd}, J=6.6,1.8 \mathrm{~Hz}, 2 \mathrm{H}), 6.61(\mathrm{~d}, J=1.7 \mathrm{~Hz}, 1 \mathrm{H}), 6.14(\mathrm{~d}, J=1.7 \mathrm{~Hz}, 1 \mathrm{H}), 1.39(\mathrm{~s}, 6 \mathrm{H}) ;{ }^{13} \mathrm{C}$ NMR (150 MHz, $\left.\mathrm{CDCl}_{3}\right): \delta 164.1,153.3,147.8,133.5,135.5,132.9,130.0,128.8,128.5$, 127.9, 123.6, 50.4, 23.1, rest of peaks merged with others; HRMS (EI+, m/z) calcd for $\mathrm{C}_{20} \mathrm{H}_{17} \mathrm{ClO}_{2}[\mathrm{M}+]: 324.0917$, found: 324.0913.

Spectral data for 4-(4-bromophenyl)-3,3-dimethylcyclopenta-1,4-dien-1-yl benzoate (5c):

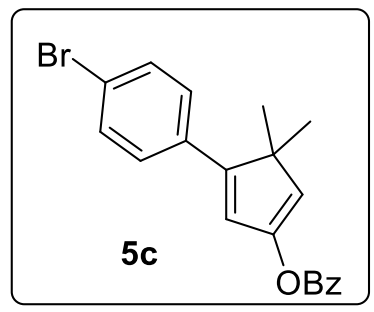

Yellow oil (0.086 g, $0.234 \mathrm{mmol}, 86 \%) ;{ }^{1} \mathrm{H}$ NMR (400 MHz, $\left.\mathrm{CDCl}_{3}\right): \delta 8.18 \sim 8.15(\mathrm{~m}, 2 \mathrm{H})$, $7.61 \sim 7.57(\mathrm{~m}, 1 \mathrm{H}), 7.49 \sim 7.46(\mathrm{~m}, 4 \mathrm{H}), 7.39 \sim 7.36(\mathrm{~m}, 2 \mathrm{H}), 6.67(\mathrm{~d}, J=1.8 \mathrm{~Hz}, 1 \mathrm{H}), 6.20$ $(\mathrm{d}, J=1.8 \mathrm{~Hz}, 1 \mathrm{H}), 1.41(\mathrm{~s}, 6 \mathrm{H}) ;{ }^{13} \mathrm{C} \mathrm{NMR}\left(100 \mathrm{MHz}, \mathrm{CDCl}_{3}\right): \delta 163.8,153.0,147.6,133.7$, 133.3, 131.4, 129.8, 129.3, 128.7, 128.4, 128.0, 123.6, 121.0, 50.2, 22.9, 22.9; HRMS (EI+, $\mathrm{m} / \mathrm{z}$ ) calcd for $\mathrm{C}_{20} \mathrm{H}_{17} \mathrm{BrO}_{2}[\mathrm{M}+]$ : 368.0412, found: 368.0413 .

Spectral data for 3,3-dimethyl-4-(p-tolyl)cyclopenta-1,4-dien-1-yl benzoate (5d): 


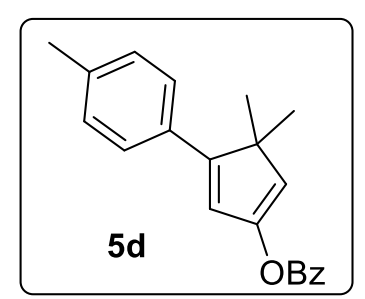

Yellow oil (0.086 g, $0.283 \mathrm{mmol}, 86 \%) ;{ }^{1} \mathrm{H} \mathrm{NMR}\left(400 \mathrm{MHz}, \mathrm{CDCl}_{3}\right): \delta 8.15(\mathrm{~d}, J=6.1 \mathrm{~Hz}$, 2H), 7.60 (t, $J=7.5 \mathrm{~Hz}, 1 \mathrm{H}), 7.48(\mathrm{t}, J=7.7 \mathrm{~Hz}, 2 \mathrm{H}), 7.43(\mathrm{~d}, J=6.1 \mathrm{~Hz}, 2 \mathrm{H}), 7.17$ (d, $J=$ $7.3 \mathrm{~Hz}, 2 \mathrm{H}), 6.60(\mathrm{~s}, 1 \mathrm{H}), 6.13(\mathrm{~s}, 1 \mathrm{H}), 2.36(\mathrm{~s}, 3 \mathrm{H}), 1.43$ (s, 3H), 1.42 (s, 3H); ${ }^{13} \mathrm{C} \mathrm{NMR}$ $\left(100 \mathrm{MHz}, \mathrm{CDCl}_{3}\right): \delta 164.1,154.5,147.9,136.9,133.4,132.2,130.0,129.6,129.1,128.5$, 128.1, 126.5, 122.2, 50.4, 23.2, 23.2, 21.1; HRMS (EI+, m/z) calcd for $\mathrm{C}_{21} \mathrm{H}_{20} \mathrm{O}_{2}[\mathrm{M}+]$ : 304.1463, found: 304.1464 .

Spectral data for 4-(4-methoxyphenyl)-3,3-dimethylcyclopenta-1,4-dien-1-yl benzoate (5e):

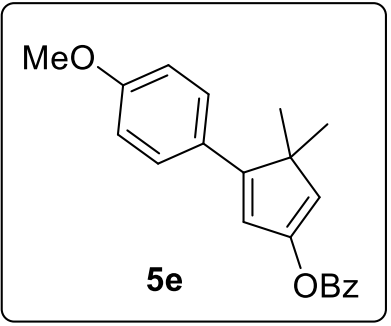

Yellow oil (0.089 g, $0.278 \mathrm{mmol}, 89 \%) ;{ }^{1} \mathrm{H} \mathrm{NMR}\left(400 \mathrm{MHz}, \mathrm{CDCl}_{3}\right): \delta 8.15(\mathrm{~d}, J=7.8 \mathrm{~Hz}$, 2H), $7.60(\mathrm{t}, J=7.4 \mathrm{~Hz}, 1 \mathrm{H}), 7.50 \sim 7.47(\mathrm{~m}, 4 \mathrm{H}), 6.90(\mathrm{~d}, J=8.8 \mathrm{~Hz}, 2 \mathrm{H}), 6.53(\mathrm{~d}, J=1.8$ $\mathrm{Hz}, 1 \mathrm{H}), 6.10(\mathrm{~d}, J=1.8 \mathrm{~Hz}, 1 \mathrm{H}), 3.82(\mathrm{~s}, 3 \mathrm{H}), 1.41(\mathrm{~s}, 6 \mathrm{H}) ;{ }^{13} \mathrm{C} \mathrm{NMR}\left(100 \mathrm{MHz}, \mathrm{CDCl}_{3}\right): \delta$ 164.1, 158.7, 154.2, 147.9, 133.4, 129.9, 129.6, 128.5, 127.8, 127.6, 121.3, 113.8, 55.2, 50.3, 50.3, 23.3, 23.3; HRMS (EI+, m/z) calcd for $\mathrm{C}_{21} \mathrm{H}_{20} \mathrm{O}_{3}$ [M+]: 320.1412, found: 320.1415 .

Spectral data for 4-(3-chlorophenyl)-2,3,3-trimethylcyclopenta-1,4-dien-1-yl benzoate (5f):

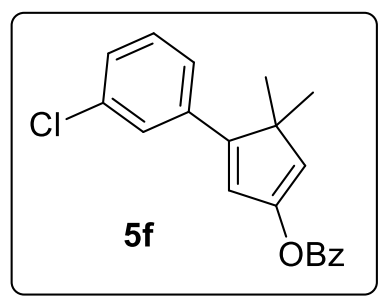

Yellow oil, (0.088 g, $0.270 \mathrm{mmol}, 88 \%) ;{ }^{1} \mathrm{H}$ NMR (600 MHz, $\left.\mathrm{CDCl}_{3}\right): \delta 8.14 \sim 8.12(\mathrm{~m}, 2 \mathrm{H})$, $7.61 \sim 7.59(\mathrm{~m}, 1 \mathrm{H}), 7.49 \sim 7.46(\mathrm{~m}, 3 \mathrm{H}), 7.37 \sim 7.36(\mathrm{~m}, 1 \mathrm{H}), 7.27(\mathrm{t}, J=8.4 \mathrm{~Hz}, 1 \mathrm{H}), 7.23$ $\sim 7.21(\mathrm{~m}, 1 \mathrm{H}), 6.64(\mathrm{~d}, J=1.8 \mathrm{~Hz}, 1 \mathrm{H}), 6.15(\mathrm{~d}, J=1.8 \mathrm{~Hz}, 1 \mathrm{H}), 1.40(\mathrm{~s}, 6 \mathrm{H}) ;{ }^{13} \mathrm{C} \mathrm{NMR}$ 
$\left(150 \mathrm{MHz}, \mathrm{CDCl}_{3}\right): \delta 164.1,153.1,147.7,136.9,134.3,133.5,130.0,129.6,129.5,129.2$, 128.6, 127.1, 126.7, 124.7, 124.3, 50.5, 23.1, one carbon merged with others; HRMS (ESI+, $\mathrm{m} / \mathrm{z}$ ) calcd for HRMS (EI+, m/z) calcd for $\mathrm{C}_{20} \mathrm{H}_{17} \mathrm{ClO}_{2}$ [M+]: 324.0917, found: 324.0913.

Spectral data for 3,3-dimethyl-4-(m-tolyl)cyclopenta-1,4-dien-1-yl benzoate (5g):

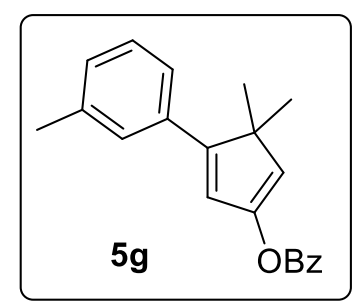

Yellow oil (0.085 g, $0.279 \mathrm{mmol}, 85 \%) ;{ }^{1} \mathrm{H}$ NMR (400 MHz, $\left.\mathrm{CDCl}_{3}\right): \delta 8.17 \sim 8.14(\mathrm{~m}, 2 \mathrm{H})$, $7.63 \sim 7.58(\mathrm{~m}, 1 \mathrm{H}), 7.49(\mathrm{t}, J=7.8 \mathrm{~Hz}, 2 \mathrm{H}), 7.33(\mathrm{~d}, J=5.0 \mathrm{~Hz}, 2 \mathrm{H}), 7.26(\mathrm{t}, J=7.5 \mathrm{~Hz}$, 1H), $7.09(\mathrm{~d}, J=3.7 \mathrm{~Hz}, 1 \mathrm{H}), 6.63(\mathrm{~d}, J=1.8 \mathrm{~Hz}, 1 \mathrm{H}), 6.15(\mathrm{~d}, J=1.8 \mathrm{~Hz}, 1 \mathrm{H}), 2.38(\mathrm{~s}, 3 \mathrm{H})$, $1.44(\mathrm{~s}, 6 \mathrm{H}) ;{ }^{13} \mathrm{C} \mathrm{NMR}\left(100 \mathrm{MHz}, \mathrm{CDCl}_{3}\right): \delta 164.1,154.7,147.8,137.8,135.0,133.4,129.9$, $129.6,128.5,128.3,128.2,127.9,127.4,123.7,122.9,50.5,23.2$, 23.2, 21.6; HRMS (EI+, $\mathrm{m} / \mathrm{z}$ ) calcd for $\mathrm{C}_{21} \mathrm{H}_{20} \mathrm{O}_{2}[\mathrm{M}+]$ : 304.1463, found: 304.1460 .

Spectral data for 4-(3-methoxyphenyl)-3,3-dimethylcyclopenta-1,4-dien-1-yl benzoate (5h):

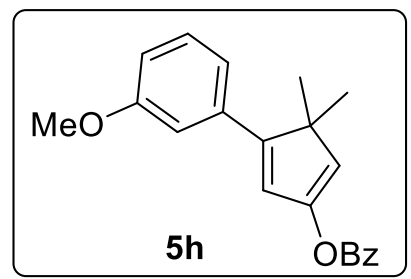

Yellow oil (0.087 g, $0.271 \mathrm{mmol}, 87 \%) ;{ }^{1} \mathrm{H} \mathrm{NMR}\left(600 \mathrm{MHz}, \mathrm{CDCl}_{3}\right): \delta 8.15(\mathrm{~d}, J=8.3 \mathrm{~Hz}$, 2H), $7.60(\mathrm{t}, J=8.0 \mathrm{~Hz}, 1 \mathrm{H}), 7.48(\mathrm{t}, J=8.0 \mathrm{~Hz}, 2 \mathrm{H}), 7.27(\mathrm{t}, J=8.0 \mathrm{~Hz}, 1 \mathrm{H}), 7.12(\mathrm{~d}, J=$ $7.7 \mathrm{~Hz}, 1 \mathrm{H}), 7.05(\mathrm{~d}, J=2.2 \mathrm{~Hz}, 1 \mathrm{H}), 6.82(\mathrm{dd}, J=8.2,1.3 \mathrm{~Hz}, 1 \mathrm{H}), 6.63(\mathrm{t}, J=1.8 \mathrm{~Hz}, 1 \mathrm{H})$, $6.15(\mathrm{t}, J=1.8 \mathrm{~Hz}, 1 \mathrm{H}), 3.83(\mathrm{~s}, 3 \mathrm{H}), 1.43(\mathrm{~s}, 6 \mathrm{H}) ;{ }^{13} \mathrm{C} \mathrm{NMR}\left(150 \mathrm{MHz}, \mathrm{CDCl}_{3}\right): \delta 164.1$, $159.5,154.4,147.8,136.5,133.4,129.9$, 129.6, 129.2, 128.6, 128.5, 123.3, 119.3, 112.6, 112.4, 55.2, 50.5, 23.2, 23.2; HRMS (EI+, m/z) calcd for $\mathrm{C}_{21} \mathrm{H}_{20} \mathrm{O}_{3}[\mathrm{M}+]$ : 320.1412, found: 320.1410 .

Spectral data for 4-(2-chlorophenyl)-2,3,3-trimethylcyclopenta-1,4-dien-1-yl benzoate (5i): 


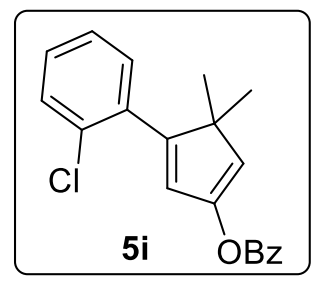

Yellow oil, (0.085 g, $0.261 \mathrm{mmol}, 85 \%) ;{ }^{1} \mathrm{H} \mathrm{NMR}\left(600 \mathrm{MHz}, \mathrm{CDCl}_{3}\right): \delta 8.13(\mathrm{dd}, J=8.4$, $1.2 \mathrm{~Hz}, 2 \mathrm{H}),), 7.60 \sim 7.57(\mathrm{~m}, 1 \mathrm{H}), 7.46(\mathrm{~d}, J=7.6 \mathrm{~Hz}, 2 \mathrm{H}), 7.45 \sim 7.43(\mathrm{~m}, 2 \mathrm{H}), 7.24 \sim$ $7.22(\mathrm{~m}, 2 \mathrm{H}), 6.26(\mathrm{~d}, J=1.8 \mathrm{~Hz}, 1 \mathrm{H}), 6.17(\mathrm{~d}, J=1.8 \mathrm{~Hz}, 1 \mathrm{H}), 1.28(\mathrm{~s}, 6 \mathrm{H}) ;{ }^{13} \mathrm{C}$ NMR $(150$ $\left.\mathrm{MHz}, \mathrm{CDCl}_{3}\right): \delta 164.1,153.0,147.8,135.3,133.4,130.8,130.0,129.8,129.6,128.6,128.5$, 128.4, 126.5, 126.2, 126.0, 52.9, 22.5, carbon merged with others; HRMS (EI+, m/z) calcd for $\mathrm{C}_{20} \mathrm{H}_{17} \mathrm{ClO}_{2}[\mathrm{M}+]$ : 324.0917 , found: 324.0920 .

Spectral data for 3,3-dimethyl-4-(o-tolyl)cyclopenta-1,4-dien-1-yl benzoate (5j):

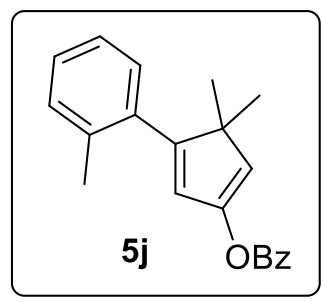

Yellow oil (0.088 g, $0.289 \mathrm{mmol}, 88 \%) ;{ }^{1} \mathrm{H} \mathrm{NMR}\left(600 \mathrm{MHz}, \mathrm{CDCl}_{3}\right): \delta 8.14(\mathrm{~d}, J=8.4 \mathrm{~Hz}$, 2H), 7.59 (t, $J=7.6 \mathrm{~Hz}, 1 \mathrm{H}), 7.48(\mathrm{t}, J=8.0 \mathrm{~Hz}, 2 \mathrm{H}), 7.25$ (d, $J=3.8 \mathrm{~Hz}, 1 \mathrm{H}), 7.21$ (td, $J=$ $7.2,1.5 \mathrm{~Hz}, 1 \mathrm{H}), 7.17(\mathrm{t}, J=7.3 \mathrm{~Hz}, 1 \mathrm{H}), 7.14(\mathrm{~d}, J=6.2 \mathrm{~Hz}, 1 \mathrm{H}), 6.14(\mathrm{~d}, J=6.7 \mathrm{~Hz}, 2 \mathrm{H})$, $2.28(\mathrm{~s}, 3 \mathrm{H}), 1.27(\mathrm{~s}, 6 \mathrm{H}) ;{ }^{13} \mathrm{C} \mathrm{NMR}\left(150 \mathrm{MHz}, \mathrm{CDCl}_{3}\right): \delta 164.1,155.7,148.0,136.8,135.9$, 133.4, 130.3, 130.0, 129.7, 129.2, 128.5, 127.2, 125.6, 125.0, 125.0, 52.8, 22.5, 22.5, 20.4; HRMS (EI+, m/z) calcd for $\mathrm{C}_{21} \mathrm{H}_{20} \mathrm{O}_{2}[\mathrm{M}+]$ : 304.1463, found: 304.1466 .

Spectral data for 3,3-dimethyl-4-(thiophen-2-yl)cyclopenta-1,4-dien-1-yl benzoate (5k):

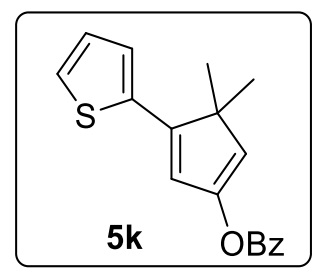

Yellow oil (0.091g, $0.307 \mathrm{mmol}, 91 \%) ;{ }^{1} \mathrm{H} \mathrm{NMR}\left(600 \mathrm{MHz}, \mathrm{CDCl}_{3}\right): \delta 8.16(\mathrm{dd}, J=8.4,1.3$ $\mathrm{Hz}, 2 \mathrm{H}), 7.60(\mathrm{tt}, J=7.4,1.3 \mathrm{~Hz}, 1 \mathrm{H}), 7.48(\mathrm{t}, J=7.5 \mathrm{~Hz}, 2 \mathrm{H}), 7.22(\mathrm{dd}, J=5.2,1.0 \mathrm{~Hz}, 1 \mathrm{H})$, $7.14(\mathrm{dd}, J=3.7,1.0 \mathrm{~Hz}, 1 \mathrm{H}), 7.04(\mathrm{dd}, J=5.2,3.7 \mathrm{~Hz}, 1 \mathrm{H}), 6.65(\mathrm{~d}, J=1.7 \mathrm{~Hz}, 1 \mathrm{H}), 6.19$ $(\mathrm{d}, J=1.7 \mathrm{~Hz}, 1 \mathrm{H}), 1.44(\mathrm{~s}, 6 \mathrm{H}) ;{ }^{13} \mathrm{C} \mathrm{NMR}\left(150 \mathrm{MHz}, \mathrm{CDCl}_{3}\right): \delta 163.9,148.8,147.8,138.2$, 133.4, 129.9, 129.4, 128.4, 127.6, 127.4, 124.0, 123.7, 121.7, 50.4, 23.5, 23.5; HRMS (EI+, 
m/z) calcd for $\mathrm{C}_{18} \mathrm{H}_{16} \mathrm{O}_{2} \mathrm{~S}$ [M+]: 296.0871, found: 296.0873 .

Spectral data for 4-phenylspiro[4.5]deca-1,3-dien-2-yl benzoate (51):

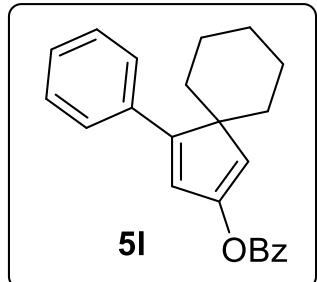

Yellow oil (0.087 g, $0.263 \mathrm{mmol}, 87 \%) ;{ }^{1} \mathrm{H}$ NMR (400 MHz, $\left.\mathrm{CDCl}_{3}\right): \delta 8.14(\mathrm{~d}, J=7.1 \mathrm{~Hz}$, $2 \mathrm{H}), 7.60(\mathrm{t}, J=7.4 \mathrm{~Hz}, 1 \mathrm{H}), 7.50 \sim 7.44(\mathrm{~m}, 4 \mathrm{H}), 7.35(\mathrm{t}, J=7.8 \mathrm{~Hz}, 2 \mathrm{H}), 7.27(\mathrm{t}, J=7.4 \mathrm{~Hz}$, $1 \mathrm{H}), 6.69(\mathrm{~d}, J=1.7 \mathrm{~Hz}, 1 \mathrm{H}), 6.52(\mathrm{~d}, J=1.7 \mathrm{~Hz}, 1 \mathrm{H}), 1.97(\mathrm{td}, J=13.2,3.5 \mathrm{~Hz}, 2 \mathrm{H}), 1.83$ $(\mathrm{d}, J=9.7 \mathrm{~Hz}, 3 \mathrm{H}), 1.64 \sim 1.54(\mathrm{~m}, 2 \mathrm{H}), 1.42(\mathrm{~d}, J=12.3 \mathrm{~Hz}, 2 \mathrm{H}), 1.34 \sim 1.24(\mathrm{~m}, 1 \mathrm{H}) ;{ }^{13} \mathrm{C}$ NMR $\left(100 \mathrm{MHz}, \mathrm{CDCl}_{3}\right): \delta 164.2,155.9,149.5,135.9,133.4,130.0,129.6,128.5,128.2$, 127.6, 127.1, 123.7, 122.9, 55.8, 32.2, 32.2, 25.7, 25.0, 25.0; HRMS (ESI+, m/z) calcd for $\mathrm{C}_{23} \mathrm{H}_{22} \mathrm{NaO}_{2}[\mathrm{M}+\mathrm{Na}]: 353.1517$, found: 353.1518 .

Spectral data for 3,3-dimethyl-6-oxo-1,4-diphenyl-1,2,3,6-tetrahydropyridin-2-yl benzoate (6a'):

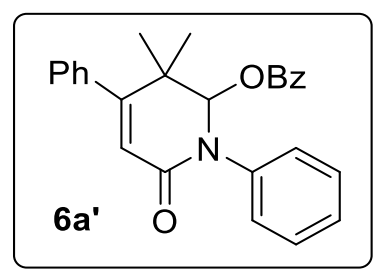

Gray solid (0.12 g, $0.303 \mathrm{mmol}, 71 \%) \mathrm{mp}=120-121{ }^{\circ} \mathrm{C} ;{ }^{1} \mathrm{H}$ NMR $\left(400 \mathrm{MHz}, \mathrm{CDCl}_{3}\right): \delta$ $8.04(\mathrm{~d}, J=7.2 \mathrm{~Hz}, 2 \mathrm{H}), 7.60(\mathrm{t}, J=7.2 \mathrm{~Hz}, 1 \mathrm{H}), 7.47(\mathrm{t}, J=7.8 \mathrm{~Hz}, 2 \mathrm{H}), 7.39 \sim 7.36(\mathrm{~m}$, $5 \mathrm{H}), 7.32 \sim 7.26(\mathrm{~m}, 5 \mathrm{H}), 6.48(\mathrm{~s}, 1 \mathrm{H}), 6.12(\mathrm{~s}, 1 \mathrm{H}), 1.71(\mathrm{~s}, 3 \mathrm{H}), 1.05(\mathrm{~s}, 3 \mathrm{H}) ;{ }^{13} \mathrm{C} \mathrm{NMR}$ $\left(100 \mathrm{MHz}, \mathrm{CDCl}_{3}\right): \delta 165.3,164.0,159.5,140.5,137.8,133.6,129.8,129.4,129.2,128.6$, 128.5, 128.3, 127.6, 127.5, 126.6, 121.7, 91.0, 40.7, 26.8, 21.9; HRMS (EI+, m/z) calcd for $\mathrm{C}_{26} \mathrm{H}_{23} \mathrm{NO}_{3}[\mathrm{M}+]: 397.1678$, found: 397.1684 .

Spectral data for 1-(4-chlorophenyl)-3,3-dimethyl-6-oxo-4-phenyl-1,2,3,6-tetrahydro -pyridin-2-yl benzoate (7a'): 


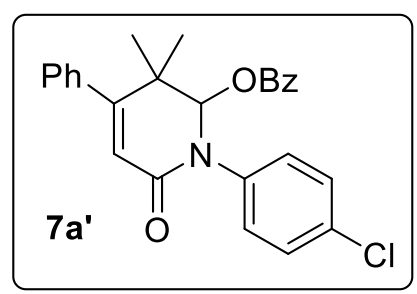

Orange solid $(0.11 \mathrm{~g}, 0.255 \mathrm{mmol}, 74 \%) ; \mathrm{mp}=130-131{ }^{\circ} \mathrm{C}$, recrystallation by $(\mathrm{MeCN} /$ DCM/ pentane = $1: 1: 10) ;{ }^{1} \mathrm{H}$ NMR (400 MHz, d $\mathrm{d}_{6}$-acetone): $\delta 8.05(\mathrm{~d}, J=7.2 \mathrm{~Hz}, 2 \mathrm{H}), 7.67$ $(\mathrm{t}, J=7.4 \mathrm{~Hz}, 1 \mathrm{H}), 7.54(\mathrm{t}, J=7.6 \mathrm{~Hz}, 2 \mathrm{H}), 7.47 \sim 7.42(\mathrm{~m}, 7 \mathrm{H}), 7.38 \sim 7.35(\mathrm{~m}, 2 \mathrm{H}), 6.53(\mathrm{~s}$, 1H), $5.99(\mathrm{~s}, 1 \mathrm{H}), 1.80(\mathrm{~s}, 3 \mathrm{H}), 1.05(\mathrm{~s}, 3 \mathrm{H}) ;{ }^{13} \mathrm{C}$ NMR (100 MHz, d6-acetone): $\delta$ 165.8, 163.6, $160.8,140.7,138.8,134.5,132.9,130.5,130.1,129.9$, 129.7, 129.4, 129.4, 129.2, 128.4, 122.0, 91.7, 41.5, 27.2, 22.2; HRMS (ESI+, m/z) calcd for $\mathrm{C}_{26} \mathrm{H}_{23} \mathrm{ClNO}_{3}[\mathrm{M}+\mathrm{H}]: 432.1366$, found: 432.1333

Spectral data for 6-hydroxy-5,5-dimethyl-1,4-diphenyl-5,6-dihydropyridin-2(1H)-one (6a):

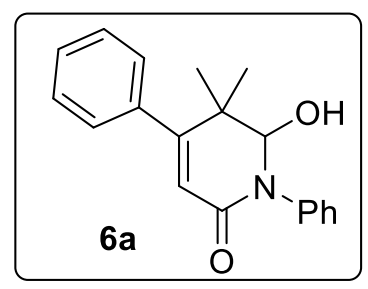

Brown solid $(0.044 \mathrm{~g}, 0.15 \mathrm{mmol}, 88 \%) ; \mathrm{mp}=167-168{ }^{\circ} \mathrm{C}$, recrystallation by (Acetone/ $\mathrm{DCM} /$ pentane $=1: 1: 10) ;{ }^{1} \mathrm{H}$ NMR $\left(600 \mathrm{MHz}, \mathrm{CDCl}_{3}\right): \delta 7.46(\mathrm{~d}, J=8.3 \mathrm{~Hz}, 2 \mathrm{H}), 7.37(\mathrm{t}$, $J=7.5 \mathrm{~Hz}, 2 \mathrm{H}), 7.33(\mathrm{t}, J=7.4 \mathrm{~Hz}, 1 \mathrm{H}), 7.28(\mathrm{~d}, J=7.4 \mathrm{~Hz}, 3 \mathrm{H}), 7.14(\mathrm{~d}, J=3.8 \mathrm{~Hz}, 2 \mathrm{H})$, $5.91(\mathrm{~s}, 1 \mathrm{H}), 4.75(\mathrm{~s}, 1 \mathrm{H}), 1.49$ (s, 3H), 1.07 (s, 3H); ${ }^{13} \mathrm{C}$ NMR (150 MHz, $\left.\mathrm{CDCl}_{3}\right): \delta 163.3$, 159.2, 141.4, 138.1, 129.1, 128.1, 128.1, 127.4, 126.7, 125.8, 121.8, 92.4, 41.5, 27.2, 22.5; HRMS (ESI+, m/z) calcd for $\mathrm{C}_{19} \mathrm{H}_{20} \mathrm{NO}_{2}[\mathrm{M}+\mathrm{H}]: 294.1494$, found: 294.1497.

Spectral data for 4-(4-chlorophenyl)-6-hydroxy-5,5-dimethyl-1-phenyl-5,6-dihydro -pyridin-2(1H)-one (6b):

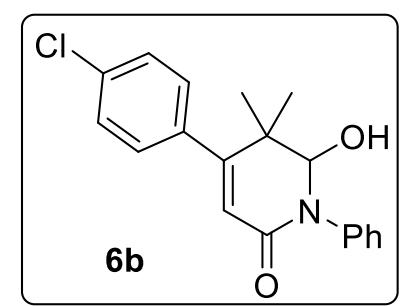

Yellow solid mp $=185-186{ }^{\circ} \mathrm{C},(0.062 \mathrm{~g}, 0.190 \mathrm{mmol}, 62 \%) ;{ }^{1} \mathrm{H} \mathrm{NMR}\left(600 \mathrm{MHz}, \mathrm{CDCl}_{3}\right): \delta$ $7.42 \sim 7.41(\mathrm{~m}, 4 \mathrm{H}), 7.33(\mathrm{~d}, J=8.4 \mathrm{~Hz}, 2 \mathrm{H}),), 7.31 \sim 7.29(\mathrm{~m}, 1 \mathrm{H}), 7.18(\mathrm{~d}, J=8.4 \mathrm{~Hz}, 2 \mathrm{H})$, 
$5.97(\mathrm{~s}, 1 \mathrm{H}), 4.81(\mathrm{~d}, J=6.1 \mathrm{~Hz}, 1 \mathrm{H}), 2.95(\mathrm{~d}, J=8.2 \mathrm{~Hz}, 1 \mathrm{H}), 1.50(\mathrm{~s}, 3 \mathrm{H}), 1.1(\mathrm{~s}, 3 \mathrm{H}) ;{ }^{13} \mathrm{C}$ NMR (150 MHz, $\left.\mathrm{CDCl}_{3}\right): \delta 162.7,157.8,141.1,136.5,134.6,129.3,128.9,128.5,127.1$, 126.2, 122.4, 92.2, 41.5, 27.2, 22.6; HRMS (ESI+, m/z) calcd for $\mathrm{C}_{19} \mathrm{H}_{19} \mathrm{ClNO}_{2}[\mathrm{M}+\mathrm{H}]$ : 328.1104, found: 328.1108 .

Spectral data for 4-(4-bromophenyl)-6-hydroxy-5,5-dimethyl-1-phenyl-5,6-dihydro -pyridin-2(1H)-one (6c):

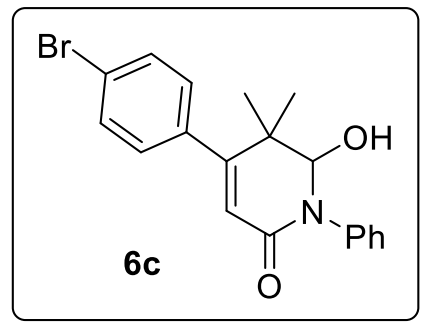

Gray solid (0.056 g, $0.152 \mathrm{mmol}, 56 \%) ; \mathrm{mp}=189-190{ }^{\circ} \mathrm{C} ;{ }^{1} \mathrm{H}$ NMR $\left(600 \mathrm{MHz}, \mathrm{CDCl}_{3}\right): \delta$ $7.44 \sim 7.42(\mathrm{~m}, 4 \mathrm{H}), 7.39(\mathrm{t}, J=8.2 \mathrm{~Hz}, 2 \mathrm{H}), 7.29(\mathrm{t}, J=7.3 \mathrm{~Hz}, 1 \mathrm{H}), 7.03(\mathrm{~d}, J=8.4 \mathrm{~Hz}$, 2H), $5.91(\mathrm{~s}, 1 \mathrm{H}), 4.77(\mathrm{~s}, 1 \mathrm{H}), 3.59(\mathrm{bs}, 1 \mathrm{H}), 1.48(\mathrm{~s}, 3 \mathrm{H}), 1.07(\mathrm{~s}, 3 \mathrm{H}) ;{ }^{13} \mathrm{C} \mathrm{NMR}(150 \mathrm{MHz}$, $\left.\mathrm{CDCl}_{3}\right): \delta 162.9,157.9,141.3,136.9,131.4,129.2,129.1,126.9,125.8,122.6,122.3,92.3$, 41.4, 27.2, 22.5; HRMS (EI+, m/z) calcd for $\mathrm{C}_{19} \mathrm{H}_{18} \mathrm{BrNO}$ [M+]: 371.0521, found: 371.0520 .

Spectral data for 6-hydroxy-5,5-dimethyl-1-phenyl-4-(p-tolyl)-5,6-dihydropyridin -2(1H)-one (6d):

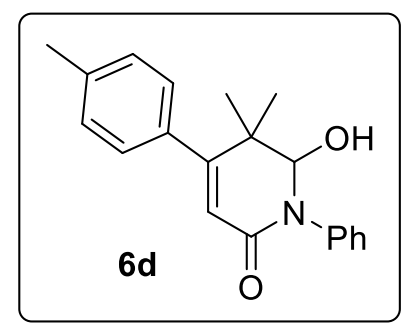

Green solid (0.044 g, $0.148 \mathrm{mmol}, 45 \%) ; \mathrm{mp}=152-153{ }^{\circ} \mathrm{C} ;{ }^{1} \mathrm{H}$ NMR $\left(600 \mathrm{MHz}, \mathrm{CDCl}_{3}\right): \delta$ $7.44 \sim 7.38(\mathrm{~m}, 4 \mathrm{H}), 7.27(\mathrm{t}, J=7.3 \mathrm{~Hz}, 1 \mathrm{H}), 7.13(\mathrm{q}, J=15.5,8.2 \mathrm{~Hz}, 4 \mathrm{H}), 5.96(\mathrm{~s}, 1 \mathrm{H})$, $4.78(\mathrm{~s}, 1 \mathrm{H}), 3.17(\mathrm{~s}, 1 \mathrm{H}), 2.36(\mathrm{~s}, 3 \mathrm{H}), 1.50(\mathrm{~s}, 3 \mathrm{H}), 1.11(\mathrm{~s}, 3 \mathrm{H}) ;{ }^{13} \mathrm{C} \mathrm{NMR}(150 \mathrm{MHz}$, $\left.\mathrm{CDCl}_{3}\right): \delta 163.0,159.1,141.4,138.3,135.2,129.2,128.9,127.4,126.9,126.0,121.7,92.4$, 41.6, 27.2, 22.6, 21.2; HRMS (ESI+, m/z) calcd for $\mathrm{C}_{20} \mathrm{H}_{22} \mathrm{NO}_{2}[\mathrm{M}+\mathrm{H}]: 308.1650$, found: 308.1650 .

Spectral data for 6-hydroxy-4-(4-methoxyphenyl)-5,5-dimethyl-1-phenyl-5,6-dihydro -pyridin-2(1H)-one (6e): 


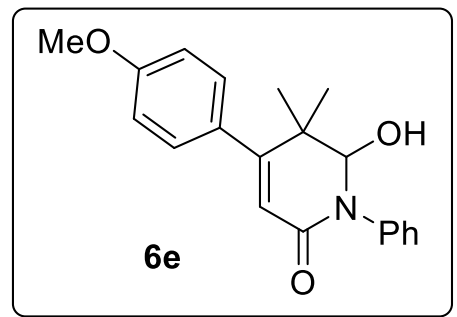

Orange solid (0.039 g, $122 \mathrm{mmol}, 39 \%) ; \mathrm{mp}=208-209{ }^{\circ} \mathrm{C} ;{ }^{1} \mathrm{H} \mathrm{NMR}\left(600 \mathrm{MHz}, \mathrm{CDCl}_{3}\right): \delta$ $7.45 \sim 7.38(\mathrm{~m}, 4 \mathrm{H}), 7.30 \sim 7.26(\mathrm{~m}, 1 \mathrm{H}), 7.17(\mathrm{~d}, J=4.3 \mathrm{~Hz}, 2 \mathrm{H}), 6.86(\mathrm{~d}, J=4.3 \mathrm{~Hz}, 2 \mathrm{H})$, $5.96(\mathrm{~s}, 1 \mathrm{H}), 4.77(\mathrm{~s}, 1 \mathrm{H}), 3.82(\mathrm{~s}, 3 \mathrm{H}), 1.50(\mathrm{~s}, 3 \mathrm{H}), 1.13(\mathrm{~s}, 3 \mathrm{H}) ;{ }^{13} \mathrm{C}$ NMR $(150 \mathrm{MHz}$, $\mathrm{d}_{6}$-DMSO): $\delta 162.3,159.2,158.3,142.0,130.7,128.8,128.5,126.1,125.9,120.7,113.6$, 91.0, 55.1, 40.9, 26.8, 22.4; HRMS (EI+, m/z) calcd for $\mathrm{C}_{20} \mathrm{H}_{21} \mathrm{NO}_{3}[\mathrm{M}+]$ : 323.1521, found: 323.1520 .

Spectral data for 4-(3-chlorophenyl)-6-hydroxy-5,5-dimethyl-1-phenyl-5,6dihydropyridin-2(1H)-one (6f):

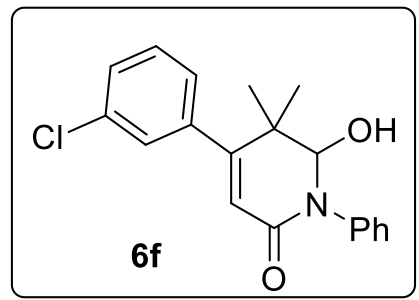

Orange solid mp $=190-191^{\circ} \mathrm{C},(0.068 \mathrm{~g}, 0.207 \mathrm{mmol}, 68 \%) ;{ }^{1} \mathrm{H}$ NMR $\left(600 \mathrm{MHz}, \mathrm{CDCl}_{3}\right): \delta$ $7.45 \sim 7.44(\mathrm{~m}, 3 \mathrm{H}), 7.40(\mathrm{t}, J=8.2 \mathrm{~Hz}, 2 \mathrm{H})$, ), $7.30 \sim 7.26(\mathrm{~m}, 3 \mathrm{H}), 7.17(\mathrm{dd}, J=7.2,1.8 \mathrm{~Hz}$, 1H), $5.98(\mathrm{~s}, 1 \mathrm{H}), 4.80(\mathrm{~s}, 1 \mathrm{H}), 3.16(\mathrm{bs}, 1 \mathrm{H}), 1.50(\mathrm{~s}, 3 \mathrm{H}), 1.05(\mathrm{~s}, 3 \mathrm{H}) ;{ }^{13} \mathrm{C} \mathrm{NMR}(150 \mathrm{MHz}$, $\left.\mathrm{CDCl}_{3}\right): \delta 162.0,155.9,141.6,136.4,132.6,130.0,129.5,129.2,126.9,126.6,126.2,124.6$, 92.3, 42.7, 27.5, 21.4, one carbon merged with others; HRMS (ESI+, m/z) calcd for $\mathrm{C}_{19} \mathrm{H}_{19} \mathrm{ClNO}_{2}[\mathrm{M}+\mathrm{H}]: 328.1104$, found: 328.1114.

Spectral data for 6-hydroxy-5,5-dimethyl-1-phenyl-4-(m-tolyl)-5,6-dihydro -pyridin-2(1H)-one (6g):

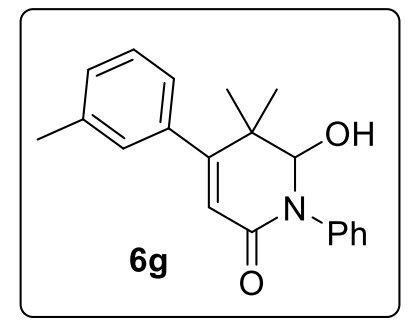


Brown solid (0.079 g, $0.266 \mathrm{mmol}, 81 \%) ; \mathrm{mp}=159-160{ }^{\circ} \mathrm{C} ;{ }^{1} \mathrm{H}$ NMR $\left(600 \mathrm{MHz}, \mathrm{CDCl}_{3}\right): \delta$ $7.43(\mathrm{~d}, J=7.4 \mathrm{~Hz}, 2 \mathrm{H}), 7.37(\mathrm{t}, J=7.5 \mathrm{~Hz}, 2 \mathrm{H}), 7.28 \sim 7.25(\mathrm{~m}, 1 \mathrm{H}), 7.20(\mathrm{t}, J=7.6 \mathrm{~Hz}$, 1H), $7.16(\mathrm{~d}, J=7.6 \mathrm{~Hz}, 1 \mathrm{H}), 7.04(\mathrm{~s}, 1 \mathrm{H}), 6.98(\mathrm{~d}, J=7.6 \mathrm{~Hz}, 1 \mathrm{H}), 5.93(\mathrm{~s}, 1 \mathrm{H}), 4.76$ (s, 1H), $3.76(\mathrm{~s}, 1 \mathrm{H}), 2.35$ (s, 3H), 1.49 (s, 3H), 1.09 (s, 3H); $\left.{ }^{13} \mathrm{C} \mathrm{NMR} \mathrm{(150} \mathrm{MHz,} \mathrm{CDCl}_{3}\right): \delta$ 163.2, 159.4, 141.4, 138.1, 137.7, 129.1, 129.0, 128.1, 128.0, 126.7, 126.0, 124.6, 121.7, 92.3, 41.5, 27.2, 22.5, 21.4; HRMS (ESI+, m/z) calcd for $\mathrm{C}_{20} \mathrm{H}_{22} \mathrm{NO}_{2}[\mathrm{M}+\mathrm{H}]$ : 308.1650, found:308.1647

Spectral data for 6-hydroxy-4-(3-methoxyphenyl)-5,5-dimethyl-1-phenyl-5,6-dihydro -pyridin-2(1H)-one (6h):

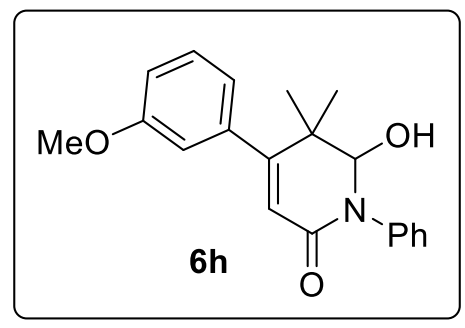

Brown solid (0.063 g, $197 \mathrm{mmol}, 63 \%) ; \mathrm{mp}=128-129{ }^{\circ} \mathrm{C} ;{ }^{1} \mathrm{H} \mathrm{NMR}\left(400 \mathrm{MHz}, \mathrm{CDCl}_{3}\right): \delta$ $7.42 \sim 7.34(\mathrm{~m}, 4 \mathrm{H}), 7.27 \sim 7.20(\mathrm{~m}, 2 \mathrm{H}), 6.89 \sim 6.86(\mathrm{~m}, 1 \mathrm{H}), 6.77 \sim 6.75(\mathrm{~m}, 2 \mathrm{H}), 5.94(\mathrm{~s}$, $1 \mathrm{H}), 4.74(\mathrm{~s}, 1 \mathrm{H}), 3.78(\mathrm{~s}, 3 \mathrm{H}), 1.48(\mathrm{~s}, 3 \mathrm{H}), 1.08(\mathrm{~s}, 3 \mathrm{H}) ;{ }^{13} \mathrm{C} \mathrm{NMR}\left(100 \mathrm{MHz}, \mathrm{CDCl}_{3}\right): \delta$ 163.1, 159.1, 159.0, 141.3, 139.4, 129.2, 129.1, 126.8, 126.0, 121.8, 120.0, 113.5, 113.4, 92.2, 55.2, 41.5, 27.2, 22.4; HRMS (EI+, $\mathrm{m} / \mathrm{z}$ ) calcd for $\mathrm{C}_{20} \mathrm{H}_{21} \mathrm{NO}_{3}[\mathrm{M}+]$ : 323.1521, found: 323.1522 .

Spectral data for 4-(2-chlorophenyl)-6-hydroxy-5,5-dimethyl-1-phenyl-5,6dihydropyridin-2(1H)-one (6i):

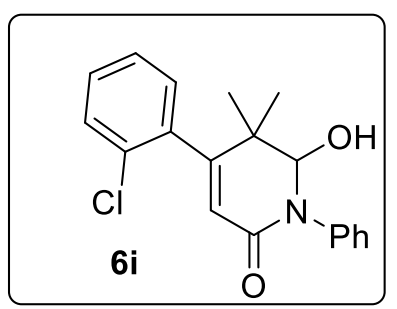

Yellow solid $\mathrm{mp}=135-136{ }^{\circ} \mathrm{C},(0.064 \mathrm{~g}, 0.195 \mathrm{mmol}, 64 \%) ;{ }^{1} \mathrm{H} \mathrm{NMR}\left(600 \mathrm{MHz}, \mathrm{CDCl}_{3}\right): \delta$ $7.42(\mathrm{~d}, J=8.5 \mathrm{~Hz}, 2 \mathrm{H}), 7.37(\mathrm{t}, J=7.1 \mathrm{~Hz}, 2 \mathrm{H}), 7.32 \sim 7.31(\mathrm{~m}, 1 \mathrm{H}), 7.28 \sim 7.21(\mathrm{~m}, 3 \mathrm{H})$, $7.03(\mathrm{~d}, J=7.7 \mathrm{~Hz}, 1 \mathrm{H}), 5.91(\mathrm{~s}, 1 \mathrm{H}), 4.76(\mathrm{~s}, 1 \mathrm{H}), 1.48(\mathrm{~s}, 3 \mathrm{H}), 1.07(\mathrm{~s}, 3 \mathrm{H}) ;{ }^{13} \mathrm{C} \mathrm{NMR}(150$ $\left.\mathrm{MHz}, \mathrm{CDCl}_{3}\right): \delta 162.9,157.6,141.2,139.8,134.1,129.6,129.2,128.4,127.5,127.0,125.9$, 125.8, 122.5, 92.2, 41.5, 27.1, 22.4; HRMS (ESI+, m/z) calcd for $\mathrm{C}_{19} \mathrm{H}_{19} \mathrm{ClNO}_{2}[\mathrm{M}+\mathrm{H}]$ : 328.1104, found: 328.1113 . 
Spectral data for 6-hydroxy-5,5-dimethyl-1-phenyl-4-(m-tolyl)-5,6-dihydro -pyridin-2(1H)-one (6j):

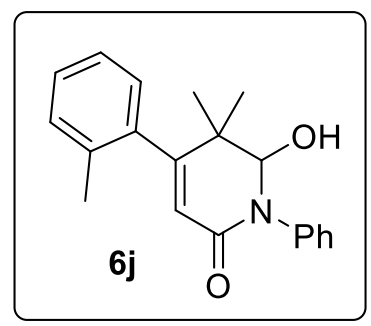

White solid (0.064 g, $0.217 \mathrm{mmol}, 66 \%) ; \mathrm{mp}=88-89{ }^{\circ} \mathrm{C} ;{ }^{1} \mathrm{H} \mathrm{NMR}\left(400 \mathrm{MHz}, \mathrm{CDCl}_{3}\right): \delta$ $7.40(\mathrm{~d}, J=4.3 \mathrm{~Hz}, 4 \mathrm{H}), 7.28$ (quintet, $J=4.4 \mathrm{~Hz}, 1 \mathrm{H}), 7.23(\mathrm{~d}, J=4.9 \mathrm{~Hz}, 2 \mathrm{H}), 7.19 \sim 7.15$ $(\mathrm{m}, 1 \mathrm{H}), 7.05$ (d, J = 7.4 Hz, 1H), $5.88(\mathrm{~s}, 1 \mathrm{H}), 4.84(\mathrm{~s}, 1 \mathrm{H}), 3.02$ (s, 1H), $2.31(\mathrm{~s}, 3 \mathrm{H}), 1.48$ $(\mathrm{s}, 3 \mathrm{H}), 0.96(\mathrm{~s}, 3 \mathrm{H}) ;{ }^{13} \mathrm{C} \mathrm{NMR}\left(150 \mathrm{MHz}, \mathrm{CDCl}_{3}\right): \delta 163.0,141.3,137.1,135.2,130.5$, 129.2, 127.9, 127.0, 126.4, 125.2, 122.6, 92.1, 77.3, 42.3, 27.4, 21.2, 20.1, 20.1; HRMS (EI+, $\mathrm{m} / \mathrm{z}$ ) calcd for $\mathrm{C}_{20} \mathrm{H}_{21} \mathrm{NO}_{2}[\mathrm{M}+]$ : 307.1572, found:307.1575

Spectral data for 6-hydroxy-5,5-dimethyl-1-phenyl-4-(thiophen-2-yl)-5,6-dihydro -pyridin-2(1H)-one (6k):

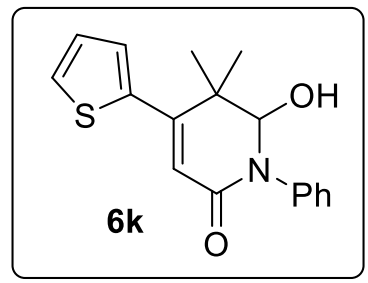

White solid (0.045 g, $152 \mathrm{mmol}, 45 \%) ; \mathrm{mp}=217-218{ }^{\circ} \mathrm{C} ;{ }^{1} \mathrm{H}$ NMR $\left(400 \mathrm{MHz}, \mathrm{CDCl}_{3}\right): \delta$ $7.41(\mathrm{~d}, J=4.4 \mathrm{~Hz}, 4 \mathrm{H}), 7.37(\mathrm{dd}, J=5.1,1.1 \mathrm{~Hz}, 1 \mathrm{H}), 7.28$ (quintet, $J=4.2 \mathrm{~Hz}, 1 \mathrm{H}), 7.19$ $(\mathrm{dd}, J=3.7,1.0 \mathrm{~Hz}, 1 \mathrm{H}), 7.06(\mathrm{dd}, J=5.1,3.8 \mathrm{~Hz}, 1 \mathrm{H}), 6.25(\mathrm{~s}, 1 \mathrm{H}), 4.81(\mathrm{~d}, J=8.4 \mathrm{~Hz}, 1 \mathrm{H})$, $2.77(\mathrm{~d}, J=8.5 \mathrm{~Hz}, 1 \mathrm{H}), 1.52(\mathrm{~s}, 3 \mathrm{H}), 1.38(\mathrm{~s}, 3 \mathrm{H}) ;{ }^{13} \mathrm{C} \mathrm{NMR}\left(100 \mathrm{MHz}, \mathrm{CDCl}_{3}\right): \delta 162.4$, $151.4,141.1,139.0,129.2$, 127.5, 127.0, 127.0, 126.1, 126.1, 121.5, 92.3, 41.6, 26.9, 23.3; HRMS (EI+, m/z) calcd for $\mathrm{C}_{17} \mathrm{H}_{17} \mathrm{NO}_{2} \mathrm{~S}[\mathrm{M}+]$ : 299.0980, found:299.0979

Spectral data for 1-hydroxy-2,5-diphenyl-2-azaspiro[5.5] undec-4-en-3-one (61): 


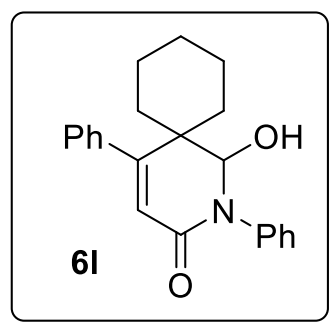

Gray solid (0.069 g, $0.206 \mathrm{mmol}, 68 \%) ; \mathrm{mp}=217-218{ }^{\circ} \mathrm{C} ;{ }^{1} \mathrm{H} \mathrm{NMR}\left(600 \mathrm{MHz}, \mathrm{CDCl}_{3}\right): \delta$ $7.39 \sim 7.25(\mathrm{~m}, 8 \mathrm{H}), 7.15(\mathrm{~d}, J=7.8 \mathrm{~Hz}, 2 \mathrm{H}), 5.86(\mathrm{~s}, 1 \mathrm{H}), 5.38(\mathrm{~s}, 1 \mathrm{H}), 3.92(\mathrm{bs}, 1 \mathrm{H}), 2.01$ $(\mathrm{d}, J=6.8 \mathrm{~Hz}, 1 \mathrm{H}), 1.94(\mathrm{~d}, J=6.3 \mathrm{~Hz}, 1 \mathrm{H}), 1.80(\mathrm{td}, J=13.7,4.1 \mathrm{~Hz}, 1 \mathrm{H}), 1.61(\mathrm{t}, J=14.7$ $\mathrm{Hz}, 2 \mathrm{H}), 1.47 \sim 1.41(\mathrm{~m}, 2 \mathrm{H}), 1.34 \sim 1.21(\mathrm{~m}, 2 \mathrm{H}), 1.13 \sim 1.07(\mathrm{~m}, 1 \mathrm{H}) ;{ }^{13} \mathrm{C}$ NMR $(150 \mathrm{MHz}$, $\left.\mathrm{CDCl}_{3}\right): \delta 163.2,160.4,141.7,137.9,129.1,127.9,126.9,126.3,122.5,85.4,44.6,33.6,29.8$, 25.0, 22.0, 20.6, rest of carbon merged with others; HRMS (ESI+, m/z) calcd for $\mathrm{C}_{22} \mathrm{H}_{24} \mathrm{NO}_{2}$ $[\mathrm{M}+\mathrm{H}]: 334.1807$, found:334.1812

Spectral data for 1-(4-chlorophenyl)-6-hydroxy-5,5-dimethyl-4-phenyl-5,6-dihydro -pyridin-2(1H)-one (6m):

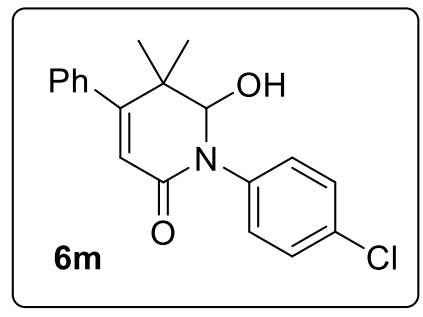

Yellow solid (0.074 g, 0.227mmol, 66\%); mp = 210-211 ${ }^{\circ} \mathrm{C} ;{ }^{1} \mathrm{H} \mathrm{NMR}\left(600 \mathrm{MHz}, \mathrm{CDCl}_{3}\right): \delta$ $7.41(\mathrm{~d}, J=8.7 \mathrm{~Hz}, 2 \mathrm{H}), 7.35 \sim 7.30(\mathrm{~m}, 5 \mathrm{H}), 7.14(\mathrm{~d}, J=6.8 \mathrm{~Hz}, 2 \mathrm{H}), 5.89(\mathrm{~s}, 1 \mathrm{H}), 4.73$ (s, $1 \mathrm{H}), 1.48$ (s, 3H), 1.09 (s, 3H); ${ }^{13} \mathrm{C} \mathrm{NMR}\left(150 \mathrm{MHz}, \mathrm{CDCl}_{3}\right): \delta 163.0,159.6,139.9,137.9$, 132.2, 129.2, 128.4, 128.2, 127.4, 127.0, 121.8, 92.4, 41.7, 27.2, 22.5; HRMS (ESI+, m/z) calcd for $\mathrm{C}_{19} \mathrm{H}_{19} \mathrm{ClNO}_{2}[\mathrm{M}+\mathrm{H}]: 328.1104$, found: 328.1103 .

Spectral data for 1-(4-bromophenyl)-6-hydroxy-5,5-dimethyl-4-phenyl-5,6-dihydro -pyridin-2(1H)-one (6n):

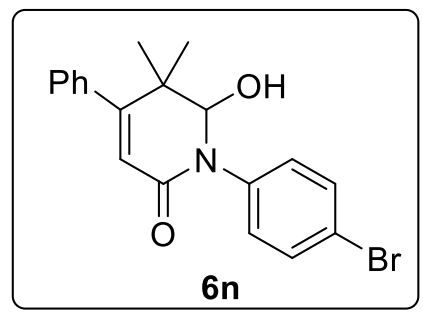


Yellow solid (0.089 g, $0.241 \mathrm{mmol}, 70 \%) ; \mathrm{mp}=197-198{ }^{\circ} \mathrm{C} ;{ }^{1} \mathrm{H}$ NMR $\left(600 \mathrm{MHz}, \mathrm{CDCl}_{3}\right): \delta$ $7.47(\mathrm{~d}, J=7.7 \mathrm{~Hz}, 2 \mathrm{H}), 7.35(\mathrm{~d}, J=7.7 \mathrm{~Hz}, 3 \mathrm{H}), 7.30$ (t, $J=7.1 \mathrm{~Hz}, 2 \mathrm{H}), 7.09$ (d, $J=7.7 \mathrm{~Hz}$, 2H), $5.85(\mathrm{~s}, 1 \mathrm{H}), 4.70(\mathrm{~s}, 1 \mathrm{H}), 4.14(\mathrm{~s}, 1 \mathrm{H}), 1.46(\mathrm{~s}, 3 \mathrm{H}), 1.06(\mathrm{~s}, 3 \mathrm{H}) ;{ }^{13} \mathrm{C} \mathrm{NMR}(150 \mathrm{MHz}$, $\left.\mathrm{CDCl}_{3}\right): \delta 163.1,159.7,140.5,137.9,132.1,128.4,128.2,127.3,127.2,121.7,120.1,92.4$, 41.7, 27.2, 22.5; HRMS (EI+, $\mathrm{m} / \mathrm{z}$ ) calcd for $\mathrm{C}_{19} \mathrm{H}_{18} \mathrm{BrNO}_{2}[\mathrm{M}+]$ : 371.0521, found: 371.0519 .

Spectral data for 6-hydroxy-5,5-dimethyl-4-phenyl-1-(p-tolyl)-5,6-dihydro -pyridin-2(1H)-one (6o):

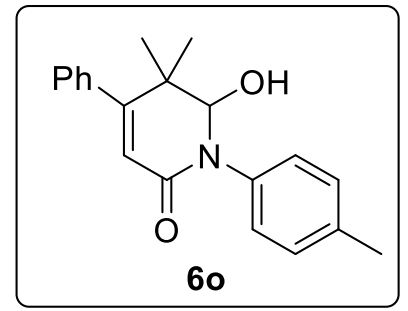

Yellow solid (0.053 g, $0.172 \mathrm{mmol}, 50 \%) ; \mathrm{mp}=198-199{ }^{\circ} \mathrm{C} ;{ }^{1} \mathrm{H}$ NMR $\left(600 \mathrm{MHz}, \mathrm{CDCl}_{3}\right): \delta$ $7.33 \sim 7.31(\mathrm{~m}, 3 \mathrm{H}), 7.28(\mathrm{t}, J=7.1 \mathrm{~Hz}, 2 \mathrm{H}), 7.18 \sim 7.14(\mathrm{~m}, 4 \mathrm{H}), 5.91(\mathrm{~s}, 1 \mathrm{H}), 4.72(\mathrm{~s}, 1 \mathrm{H})$, $3.81(\mathrm{~s}, 1 \mathrm{H}), 2.36(\mathrm{~s}, 3 \mathrm{H}), 1.48(\mathrm{~s}, 3 \mathrm{H}), 1.06(\mathrm{~s}, 3 \mathrm{H}) ;{ }^{13} \mathrm{C}$ NMR $\left(150 \mathrm{MHz}, \mathrm{CDCl}_{3}\right): \delta 163.2$, 158.9, 138.9, 138.2, 136.5, 129.7, 128.1, 128.1, 127.5, 125.7, 122.0, 92.5, 41.5, 27.2, 22.5, 21.1; HRMS (ESI+, m/z) calcd for $\mathrm{C}_{20} \mathrm{H}_{22} \mathrm{NO}_{2}[\mathrm{M}+\mathrm{H}]$ : 308.1650 , found: 308.1658 .

Spectral data for 6-hydroxy-1-(4-isopropylphenyl)-5,5-dimethyl-4-phenyl-5,6-dihydro -pyridin-2(1H)-one (6p):

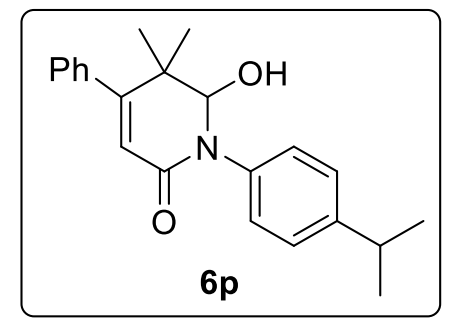

Yellow solid (0.069 g, $0.207 \mathrm{mmol}, 60 \%) ; \mathrm{mp}=195-196{ }^{\circ} \mathrm{C} ;{ }^{1} \mathrm{H} \mathrm{NMR}\left(400 \mathrm{MHz}, \mathrm{CDCl}_{3}\right): \delta$ $7.35 \sim 7.23(\mathrm{~m}, 5 \mathrm{H}), 7.20(\mathrm{t}, J=7.3 \mathrm{~Hz}, 4 \mathrm{H}), 5.94(\mathrm{~s}, 1 \mathrm{H}), 4.73(\mathrm{~s}, 1 \mathrm{H}), 3.92(\mathrm{bs}, 1 \mathrm{H}), 2.96 \sim$ $2.86(\mathrm{~m}, 1 \mathrm{H}), 1.48(\mathrm{~s}, 3 \mathrm{H}), 1.25(\mathrm{~d}, J=1.1 \mathrm{~Hz}, 3 \mathrm{H}), 1.24(\mathrm{~d}, J=1.1 \mathrm{~Hz}, 3 \mathrm{H}), 1.06(\mathrm{~s}, 3 \mathrm{H})$; ${ }^{13} \mathrm{C}$ NMR $\left(100 \mathrm{MHz}, \mathrm{CDCl}_{3}\right): \delta 163.3,159.0,147.3,139.0,138.2,128.1,128.1,127.5,127.1$, 125.8, 121.9, 92.4, 41.5, 33.7, 27.2, 23.9, 23.9, 22.5; HRMS (EI+, m/z) calcd for $\mathrm{C}_{22} \mathrm{H}_{25} \mathrm{NO}_{2}$ [M+]: 335.1885, found: 335.1882.

Spectral data for ethyl 4-(2-hydroxy-3,3-dimethyl-6-oxo-4-phenyl-3,6-dihydro 
-pyridin-1(2H)-yl)benzoate (6q):

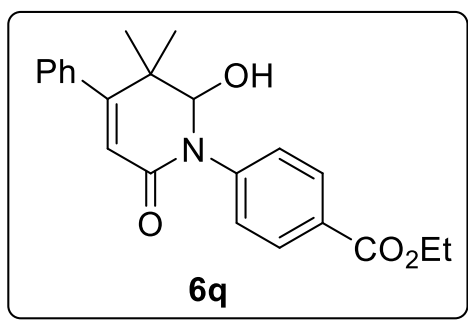

Brown solid (0.053 g, $0.145 \mathrm{mmol}, 42 \%) ; \mathrm{mp}=178-179{ }^{\circ} \mathrm{C} ;{ }^{1} \mathrm{H} \mathrm{NMR}\left(600 \mathrm{MHz}, \mathrm{CDCl}_{3}\right): \delta$ $8.03(\mathrm{~d}, J=8.5 \mathrm{~Hz}, 2 \mathrm{H}), 7.55(\mathrm{~d}, J=8.5 \mathrm{~Hz}, 2 \mathrm{H}), 7.30(\mathrm{~d}, J=7.3 \mathrm{~Hz}, 1 \mathrm{H}), 7.27(\mathrm{t}, J=7.3 \mathrm{~Hz}$, 2H), $7.11(\mathrm{~d}, J=7.6 \mathrm{~Hz}, 2 \mathrm{H}), 5.87(\mathrm{~s}, 1 \mathrm{H}), 4.79(\mathrm{~s}, 1 \mathrm{H}), 4.38(\mathrm{q}, J=7.1 \mathrm{~Hz}, 2 \mathrm{H}), 4.12$ (bs, $1 \mathrm{H}), 1.46(\mathrm{~s}, 3 \mathrm{H}), 1.39(\mathrm{t}, J=7.1 \mathrm{~Hz}, 3 \mathrm{H}), 1.09(\mathrm{~s}, 3 \mathrm{H}) ;{ }^{13} \mathrm{C} \mathrm{NMR}\left(150 \mathrm{MHz}, \mathrm{CDCl}_{3}\right): \delta$ 166.0, 163.0, 159.8, 145.5, 137.9, 130.4, 128.4, 128.3, 128.1, 127.3, 124.9, 121.7, 92.2, 61.0, 41.8, 27.2, 22.5, 14.3; HRMS (EI+, m/z) calcd for $\mathrm{C}_{22} \mathrm{H}_{23} \mathrm{NO}_{4}[\mathrm{M}+]$ : 365.1627, found: 365.1630 .

Spectral data for 1-(3-chlorophenyl)-6-hydroxy-5,5-dimethyl-4-phenyl-5,6-dihydro -pyridin-2(1H)-one (6r):

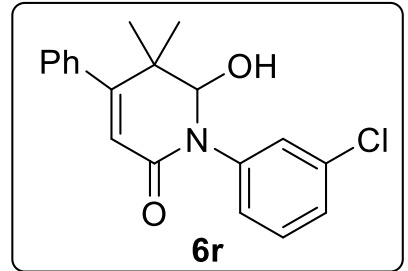

Purple solid (0.050 g, $\left.0.153 \mathrm{mmol}, 32 \%) ;{ }^{1} \mathrm{H} \mathrm{NMR} \mathrm{(600} \mathrm{MHz,} \mathrm{CDCl}_{3}\right): \delta 7.50(\mathrm{t}, J=1.9 \mathrm{~Hz}$, 1H), $7.38 \sim 7.29(\mathrm{~m}, 5 \mathrm{H}), 7.25(\mathrm{~d}, J=1.4 \mathrm{~Hz}, 1 \mathrm{H}), 7.17(\mathrm{~d}, J=7.5 \mathrm{~Hz}, 2 \mathrm{H}), 5.91(\mathrm{~s}, 1 \mathrm{H})$, 4.76 (s, $1 \mathrm{H}), 3.78$ (bs, $1 \mathrm{H}), 1.49$ (s, 3H), 1.10 (s, 3H); $\left.{ }^{13} \mathrm{C} \mathrm{NMR} \mathrm{(150} \mathrm{MHz,} \mathrm{CDCl}_{3}\right)$ : $\delta$ 163.0, 159.7, 142.5, 137.9, 134.5, 130.0, 128.4, 128.2, 127.4, 126.9, 125.9, 123.9, 121.7, 92.3, 41.7, 27.2, 22.5; HRMS (ESI+, m/z) calcd for $\mathrm{C}_{19} \mathrm{H}_{19} \mathrm{ClNO}_{2}[\mathrm{M}+\mathrm{H}]: 328.1104$, found: 328.1104

Spectral data for 6-hydroxy-5,5-dimethyl-4-phenyl-1-(m-tolyl)-5,6-dihydro -pyridin-2(1H)-one (6s):

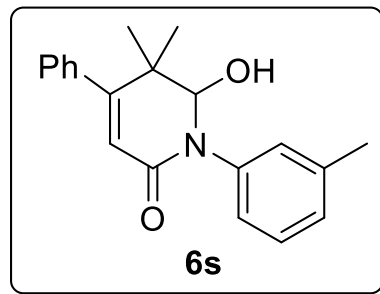


Green solid (0.077 g, $0.252 \mathrm{mmol}, 73 \%) ; \mathrm{mp}=162-163{ }^{\circ} \mathrm{C} ;{ }^{1} \mathrm{H}$ NMR $\left(600 \mathrm{MHz}, \mathrm{CDCl}_{3}\right): \delta$ $7.32(\mathrm{t}, J=7.3 \mathrm{~Hz}, 1 \mathrm{H}), 7.29 \sim 7.25(\mathrm{~m}, 5 \mathrm{H}), 7.12(\mathrm{~d}, J=7.3 \mathrm{~Hz}, 2 \mathrm{H}), 7.09(\mathrm{t}, J=3.5 \mathrm{~Hz}$, $1 \mathrm{H}), 5.91(\mathrm{~s}, 1 \mathrm{H}), 4.76(\mathrm{~s}, 1 \mathrm{H}), 4.35(\mathrm{~s}, 1 \mathrm{H}), 2.32(\mathrm{~s}, 3 \mathrm{H}), 1.49(\mathrm{~s}, 3 \mathrm{H}), 1.07(\mathrm{~s}, 3 \mathrm{H}) ;{ }^{13} \mathrm{C}$ NMR (150 MHz, $\left.\mathrm{CDCl}_{3}\right): \delta 163.3,159.0,141.4,138.9,138.2,128.8,128.0,128.0,127.5$, 127.4, 126.2, 122.8, 121.9, 92.4, 41.5, 27.1, 22.4, 21.3; HRMS (ESI+, m/z) calcd for $\mathrm{C}_{20} \mathrm{H}_{22} \mathrm{NO}_{2}[\mathrm{M}+\mathrm{H}]: 308.1650$, found: 308.1662 .

Spectral data for 1-(2-chlorophenyl)-6-hydroxy-5,5-dimethyl-4-phenyl-5,6-dihydro -pyridin-2(1H)-one (6t):

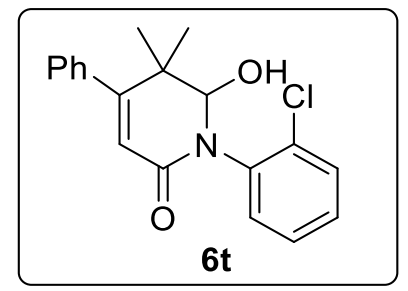

Brown solid $\mathrm{mp}=177-178{ }^{\circ} \mathrm{C},(0.103 \mathrm{~g}, 0.317 \mathrm{mmol}, 92 \%) ;{ }^{1} \mathrm{H} \mathrm{NMR}\left(600 \mathrm{MHz}, \mathrm{CDCl}_{3}\right.$, Temp .= 312.4K): $\delta 7.56(\mathrm{~s}, 1 \mathrm{H}), 7.45(\mathrm{~s}, 1 \mathrm{H}), 7.34(\mathrm{~s}, 3 \mathrm{H}), 7.25(\mathrm{~s}, 4 \mathrm{H}), 5.94(\mathrm{~s}, 1 \mathrm{H}), 4.70(\mathrm{~s}$, $1 \mathrm{H}), 3.41(\mathrm{~s}, 1 \mathrm{H}), 1.58(\mathrm{~s}, 3 \mathrm{H}), 1.10(\mathrm{~s}, 3 \mathrm{H}) ;{ }^{13} \mathrm{C} \mathrm{NMR}\left(150 \mathrm{MHz}, \mathrm{CDCl}_{3}, \mathrm{Temp} .=312.4 \mathrm{~K}\right)$ : $\delta 160.0,138.4,130.3,130.2,128.9,128.3,128.1,127.7,121.5,91.2,27.6,22.6$, Other carbons are merged; HRMS $(\mathrm{EI}+\mathrm{m} / \mathrm{z})$ calcd for $\mathrm{C}_{19} \mathrm{H}_{18} \mathrm{ClNO}_{2}[\mathrm{M}+]$ : 327.1026, found: 327.1033 .

Spectral data for 6-hydroxy-5,5-dimethyl-4-phenyl-1-(o-tolyl)-5,6-dihydro -pyridin-2(1H)-one (6u):

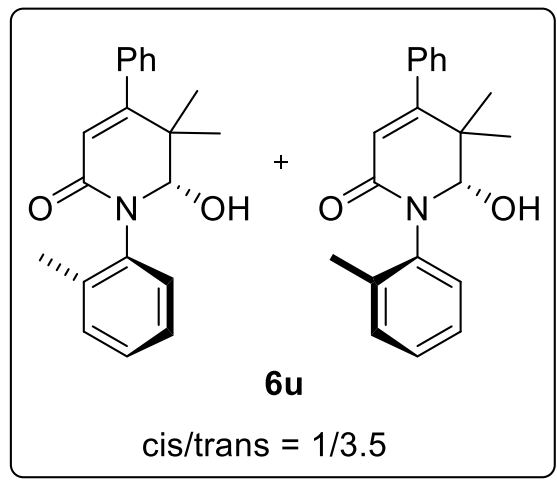

Black solid mp $=145-146{ }^{\circ} \mathrm{C},(0.075 \mathrm{~g}, 0.245 \mathrm{mmol}, 71 \%) ;{ }^{1} \mathrm{H} \mathrm{NMR}\left(600 \mathrm{MHz}, \mathrm{CDCl}_{3}\right)$, atropisomer, cis/trans $=1 / 3.5$, major isomer : $\delta 7.56(\mathrm{~d}, J=7.0 \mathrm{~Hz}, 1 \mathrm{H}), 7.43(\mathrm{~d}, J=7.3 \mathrm{~Hz}$, $1 \mathrm{H}), 7.36 \sim 7.28(\mathrm{~m}, 4 \mathrm{H}), 7.23 \sim 7.18(\mathrm{~m}, 3 \mathrm{H}), 5.90(\mathrm{~s}, 1 \mathrm{H}), 4.63(\mathrm{~s}, 1 \mathrm{H}), 4.00(\mathrm{~s}, 1 \mathrm{H}), 1.57$ $(\mathrm{s}, 3 \mathrm{H}), 1.05(\mathrm{~s}, 3 \mathrm{H}) ;{ }^{13} \mathrm{C} \mathrm{NMR}\left(150 \mathrm{MHz}, \mathrm{CDCl}_{3}\right): \delta 163.2,159.2,140.0,135.8,131.0$, $128.3,128.2$, 127.8, 127.6, 127.5, 127.2, 126.9, 122.0, 91.7, 41.7, 27.8, 22.7, 18.6; minor 
isomer : $\delta 7.48(\mathrm{~d}, J=7.3 \mathrm{~Hz}, 1 \mathrm{H}), 5.96(\mathrm{~s}, 1 \mathrm{H}), 4.77(\mathrm{~s}, 1 \mathrm{H}), 3.11(\mathrm{~s}, 1 \mathrm{H}), 1.52(\mathrm{~s}, 3 \mathrm{~h}), 1.11$ $(\mathrm{s}, 3 \mathrm{H})$, other protons are merged; ${ }^{13} \mathrm{C} \mathrm{NMR}\left(150 \mathrm{MHz}, \mathrm{CDCl}_{3}\right): \delta 162.1,158.9,138.2,137.6$, 127.9, 127.1, 121.7, 92.7, 41.3, 27.5, 22.3, 18.9, other carbons are merged; HRMS (EI+, m/z) calcd for $\mathrm{C}_{20} \mathrm{H}_{21} \mathrm{NO}_{2}[\mathrm{M}+]$ : 307.1572 , found: 307.1571

\section{NOE of key compound (4v):}

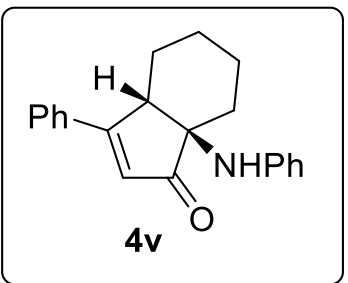

\begin{tabular}{|l|l|l|}
\hline Sr. No. & Irradition & Intensity increase \% (Key peaks) \\
\hline 1. & $\mathrm{Hc}(\delta 3.88)$ & $\mathrm{He}(\delta 7.53,15.46 \%)$ \\
\hline 2. & $\mathrm{He}(\delta 6.44)$ & $\mathrm{H}_{\mathrm{c}}(\delta 4.85,6.39 \%), \mathrm{Hh}(\delta 6.68,12.12 \%)$ \\
\hline
\end{tabular}

\section{X-ray crystallographic structures and data:}

\subsection{X-ray structure of 3i':}
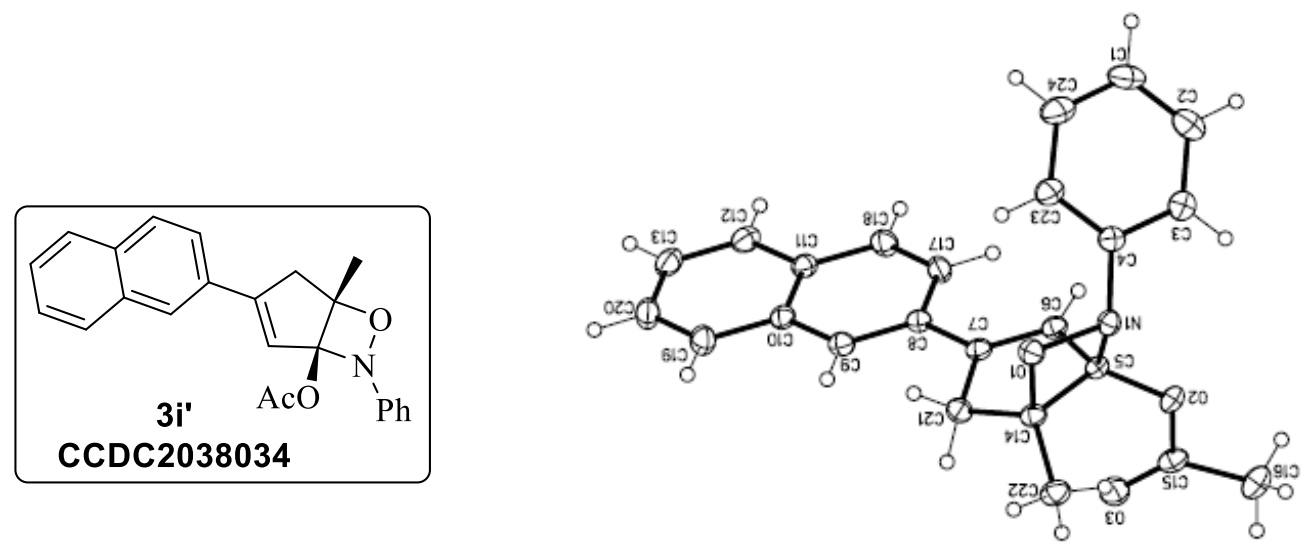

Figure S2. X-ray structure of 3i'. Displacement ellipsoids are shown at the $50 \%$ probability level.

Table S3. Crystal data and structure refinement for 200460lt.

Identification code

Empirical formula

Formula weight

Temperature

Wavelength
200460LT

C24 H21 N O3

371.42

$100(2) \mathrm{K}$

$0.71073 \AA$ 
Crystal system

Space group

Unit cell dimensions

Volume

$\mathrm{Z}$

Density (calculated)

Absorption coefficient

$\mathrm{F}(000)$

Crystal size

Theta range for data collection

Index ranges

Reflections collected

Independent reflections

Completeness to theta $=25.242^{\circ}$

Absorption correction

Max. and min. transmission

Refinement method

Data / restraints / parameters

Goodness-of-fit on $\mathrm{F}^{2}$

Final $\mathrm{R}$ indices $[\mathrm{I}>2 \operatorname{sigma}(\mathrm{I})]$

$\mathrm{R}$ indices (all data)

Extinction coefficient

Largest diff. peak and hole
Monoclinic

$\mathrm{P} 21 / \mathrm{c}$

$\mathrm{a}=16.2544(8) \AA$

$\alpha=90^{\circ}$.

$\mathrm{b}=9.9945(5) \AA$

$\beta=93.721(3)^{\circ}$.

$\mathrm{c}=11.5863(5) \AA$

$\gamma=90^{\circ}$.

1878.28(15) $\AA^{3}$

4

$1.313 \mathrm{Mg} / \mathrm{m}^{3}$

$0.087 \mathrm{~mm}^{-1}$

784

$0.15 \times 0.15 \times 0.10 \mathrm{~mm}^{3}$

2.394 to $26.408^{\circ}$.

$-20<=\mathrm{h}<=19,-12<=\mathrm{k}<=12,-14<=\mathrm{l}<=14$

16204

$3859[\mathrm{R}(\mathrm{int})=0.0418]$

$99.9 \%$

Semi-empirical from equivalents

0.7454 and 0.6936

Full-matrix least-squares on $\mathrm{F}^{2}$

$3859 / 0 / 255$

1.026

$\mathrm{R} 1=0.0411, \mathrm{wR} 2=0.0934$

$\mathrm{R} 1=0.0569, \mathrm{wR} 2=0.1023$

$\mathrm{n} / \mathrm{a}$

0.290 and -0.210 e. $\AA^{-3}$

Table S4. Atomic coordinates $\left(\mathrm{x} 10^{4}\right)$ and equivalent isotropic displacement parameters $\left(\AA^{2} \times 10^{3}\right)$ for 200460lt. $\mathrm{U}(\mathrm{eq})$ is defined as one third of the trace of the orthogonalized $\mathrm{U}^{\mathrm{ij}}$ tensor.

\begin{tabular}{lrrrr}
\hline & $\mathrm{x}$ & $\mathrm{y}$ & $\mathrm{z}$ & $\mathrm{U}(\mathrm{eq})$ \\
\hline $\mathrm{O}(1)$ & $8377(1)$ & $1135(1)$ & $1926(1)$ & $19(1)$ \\
$\mathrm{O}(2)$ & $8360(1)$ & $284(1)$ & $-809(1)$ & $19(1)$ \\
$\mathrm{O}(3)$ & $7002(1)$ & $184(1)$ & $-1343(1)$ & $26(1)$ \\
$\mathrm{N}(1)$ & $8936(1)$ & $1067(1)$ & $963(1)$ & $17(1)$ \\
$\mathrm{C}(1)$ & $10705(1)$ & $4114(2)$ & $1020(2)$ & $32(1)$ \\
$\mathrm{C}(2)$ & $10660(1)$ & $3159(2)$ & $155(1)$ & $31(1)$ \\
$\mathrm{C}(3)$ & $10077(1)$ & $2153(2)$ & $154(1)$ & $25(1)$
\end{tabular}




$\begin{array}{lrrrr}\mathrm{C}(4) & 9520(1) & 2132(1) & 1013(1) & 18(1) \\ \mathrm{C}(5) & 8206(1) & 1162(1) & 126(1) & 16(1) \\ \mathrm{C}(6) & 7909(1) & 2521(1) & -230(1) & 16(1) \\ \mathrm{C}(7) & 7264(1) & 2905(1) & 351(1) & 16(1) \\ \mathrm{C}(8) & 6822(1) & 4190(1) & 241(1) & 17(1) \\ \mathrm{C}(9) & 6205(1) & 4496(1) & 954(1) & 18(1) \\ \mathrm{C}(10) & 5776(1) & 5728(1) & 874(1) & 18(1) \\ \mathrm{C}(11) & 5977(1) & 6664(1) & 17(1) & 18(1) \\ \mathrm{C}(12) & 5543(1) & 7893(2) & -72(1) & 23(1) \\ \mathrm{C}(13) & 4942(1) & 8180(2) & 666(1) & 25(1) \\ \mathrm{C}(14) & 7672(1) & 767(1) & 1118(1) & 17(1) \\ \mathrm{C}(15) & 7701(1) & -107(1) & -1511(1) & 20(1) \\ \mathrm{C}(16) & 7986(1) & -944(2) & -2478(1) & 28(1) \\ \mathrm{C}(17) & 7017(1) & 5142(2) & -609(1) & 20(1) \\ \mathrm{C}(18) & 6610(1) & 6332(2) & -718(1) & 22(1) \\ \mathrm{C}(19) & 5146(1) & 6052(2) & 1616(1) & 24(1) \\ \mathrm{C}(20) & 4740(1) & 7245(2) & 1509(1) & 26(1) \\ \mathrm{C}(21) & 7009(1) & 1826(1) & 1175(1) & 18(1) \\ \mathrm{C}(22) & 7446(1) & -678(2) & 1262(1) & 23(1) \\ \mathrm{C}(23) & 9564(1) & 3077(2) & 1887(1) & 26(1) \\ \mathrm{C}(24) & 10167(1) & 4061(2) & 1889(2) & 34(1) \\ & & & & \end{array}$

Table S5. Bond lengths $[\AA]$ and angles $\left[{ }^{\circ}\right]$ for 2004601 .

\begin{tabular}{ll}
\hline $\mathrm{O}(1)-\mathrm{C}(14)$ & $1.4793(16)$ \\
$\mathrm{O}(1)-\mathrm{N}(1)$ & $1.4852(14)$ \\
$\mathrm{O}(2)-\mathrm{C}(15)$ & $1.3599(17)$ \\
$\mathrm{O}(2)-\mathrm{C}(5)$ & $1.4292(16)$ \\
$\mathrm{O}(3)-\mathrm{C}(15)$ & $1.2003(18)$ \\
$\mathrm{N}(1)-\mathrm{C}(4)$ & $1.4249(18)$ \\
$\mathrm{N}(1)-\mathrm{C}(5)$ & $1.4871(18)$ \\
$\mathrm{C}(1)-\mathrm{C}(24)$ & $1.376(2)$ \\
$\mathrm{C}(1)-\mathrm{C}(2)$ & $1.383(2)$ \\
$\mathrm{C}(1)-\mathrm{H}(1)$ & 0.9500 \\
$\mathrm{C}(2)-\mathrm{C}(3)$ & $1.382(2)$ \\
$\mathrm{C}(2)-\mathrm{H}(21)$ & 0.9500 \\
$\mathrm{C}(3)-\mathrm{C}(4)$ & $1.388(2)$
\end{tabular}




\begin{tabular}{|c|c|}
\hline $\mathrm{C}(3)-\mathrm{H}(3)$ & 0.9500 \\
\hline$C(4)-C(23)$ & $1.383(2)$ \\
\hline$C(5)-C(6)$ & $1.4904(19)$ \\
\hline$C(5)-C(14)$ & $1.5358(19)$ \\
\hline$C(6)-C(7)$ & $1.339(2)$ \\
\hline $\mathrm{C}(6)-\mathrm{H}(7)$ & 0.9500 \\
\hline$C(7)-C(8)$ & $1.473(2)$ \\
\hline$C(7)-C(21)$ & $1.5164(19)$ \\
\hline $\mathrm{C}(8)-\mathrm{C}(9)$ & $1.3743(19)$ \\
\hline C(8)-C(17) & $1.420(2)$ \\
\hline C(9)-C(10) & $1.415(2)$ \\
\hline $\mathrm{C}(9)-\mathrm{H}(8)$ & 0.9500 \\
\hline$C(10)-C(19)$ & $1.417(2)$ \\
\hline$C(10)-C(11)$ & $1.418(2)$ \\
\hline $\mathrm{C}(11)-\mathrm{C}(18)$ & $1.416(2)$ \\
\hline $\mathrm{C}(11)-\mathrm{C}(12)$ & $1.417(2)$ \\
\hline$C(12)-C(13)$ & $1.369(2)$ \\
\hline $\mathrm{C}(12)-\mathrm{H}(9)$ & 0.9500 \\
\hline$C(13)-C(20)$ & $1.406(2)$ \\
\hline $\mathrm{C}(13)-\mathrm{H}(2)$ & 0.9500 \\
\hline$C(14)-C(22)$ & $1.502(2)$ \\
\hline$C(14)-C(21)$ & $1.514(2)$ \\
\hline$C(15)-C(16)$ & $1.496(2)$ \\
\hline $\mathrm{C}(16)-\mathrm{H}(6)$ & 0.9800 \\
\hline $\mathrm{C}(16)-\mathrm{H}(4)$ & 0.9800 \\
\hline $\mathrm{C}(16)-\mathrm{H}(5)$ & 0.9800 \\
\hline$C(17)-C(18)$ & $1.363(2)$ \\
\hline $\mathrm{C}(17)-\mathrm{H}(13)$ & 0.9500 \\
\hline $\mathrm{C}(18)-\mathrm{H}(12)$ & 0.9500 \\
\hline$C(19)-C(20)$ & $1.364(2)$ \\
\hline C(19)-H(11) & 0.9500 \\
\hline $\mathrm{C}(20)-\mathrm{H}(10)$ & 0.9500 \\
\hline $\mathrm{C}(21)-\mathrm{H}(15)$ & 0.9900 \\
\hline $\mathrm{C}(21)-\mathrm{H}(14)$ & 0.9900 \\
\hline $\mathrm{C}(22)-\mathrm{H}(16)$ & 0.9800 \\
\hline $\mathrm{C}(22)-\mathrm{H}(18)$ & 0.9800 \\
\hline $\mathrm{C}(22)-\mathrm{H}(17)$ & 0.9800 \\
\hline $\mathrm{C}(23)-\mathrm{C}(24)$ & $1.388(2)$ \\
\hline
\end{tabular}




\begin{tabular}{|c|c|}
\hline $\mathrm{C}(23)-\mathrm{H}(19)$ & 0.9500 \\
\hline $\mathrm{C}(24)-\mathrm{H}(20)$ & 0.9500 \\
\hline $\mathrm{C}(14)-\mathrm{O}(1)-\mathrm{N}(1)$ & $90.04(9)$ \\
\hline $\mathrm{C}(15)-\mathrm{O}(2)-\mathrm{C}(5)$ & $117.36(11)$ \\
\hline $\mathrm{C}(4)-\mathrm{N}(1)-\mathrm{O}(1)$ & $111.93(10)$ \\
\hline $\mathrm{C}(4)-\mathrm{N}(1)-\mathrm{C}(5)$ & $118.70(11)$ \\
\hline $\mathrm{O}(1)-\mathrm{N}(1)-\mathrm{C}(5)$ & $89.12(9)$ \\
\hline $\mathrm{C}(24)-\mathrm{C}(1)-\mathrm{C}(2)$ & $119.86(15)$ \\
\hline $\mathrm{C}(24)-\mathrm{C}(1)-\mathrm{H}(1)$ & 120.1 \\
\hline $\mathrm{C}(2)-\mathrm{C}(1)-\mathrm{H}(1)$ & 120.1 \\
\hline$C(3)-C(2)-C(1)$ & $120.45(15)$ \\
\hline $\mathrm{C}(3)-\mathrm{C}(2)-\mathrm{H}(21)$ & 119.8 \\
\hline $\mathrm{C}(1)-\mathrm{C}(2)-\mathrm{H}(21)$ & 119.8 \\
\hline $\mathrm{C}(2)-\mathrm{C}(3)-\mathrm{C}(4)$ & $119.27(15)$ \\
\hline $\mathrm{C}(2)-\mathrm{C}(3)-\mathrm{H}(3)$ & 120.4 \\
\hline $\mathrm{C}(4)-\mathrm{C}(3)-\mathrm{H}(3)$ & 120.4 \\
\hline $\mathrm{C}(23)-\mathrm{C}(4)-\mathrm{C}(3)$ & $120.64(14)$ \\
\hline $\mathrm{C}(23)-\mathrm{C}(4)-\mathrm{N}(1)$ & $122.89(13)$ \\
\hline $\mathrm{C}(3)-\mathrm{C}(4)-\mathrm{N}(1)$ & $116.43(13)$ \\
\hline $\mathrm{O}(2)-\mathrm{C}(5)-\mathrm{N}(1)$ & $106.42(11)$ \\
\hline $\mathrm{O}(2)-\mathrm{C}(5)-\mathrm{C}(6)$ & $114.78(11)$ \\
\hline$N(1)-C(5)-C(6)$ & $117.99(12)$ \\
\hline $\mathrm{O}(2)-\mathrm{C}(5)-\mathrm{C}(14)$ & $122.99(11)$ \\
\hline $\mathrm{N}(1)-\mathrm{C}(5)-\mathrm{C}(14)$ & $87.83(10)$ \\
\hline$C(6)-C(5)-C(14)$ & $104.71(11)$ \\
\hline$C(7)-C(6)-C(5)$ & $111.79(12)$ \\
\hline $\mathrm{C}(7)-\mathrm{C}(6)-\mathrm{H}(7)$ & 124.1 \\
\hline $\mathrm{C}(5)-\mathrm{C}(6)-\mathrm{H}(7)$ & 124.1 \\
\hline$C(6)-C(7)-C(8)$ & $126.88(13)$ \\
\hline$C(6)-C(7)-C(21)$ & $111.59(12)$ \\
\hline$C(8)-C(7)-C(21)$ & $121.53(12)$ \\
\hline$C(9)-C(8)-C(17)$ & $118.13(13)$ \\
\hline$C(9)-C(8)-C(7)$ & $120.87(12)$ \\
\hline $\mathrm{C}(17)-\mathrm{C}(8)-\mathrm{C}(7)$ & $121.00(12)$ \\
\hline$C(8)-C(9)-C(10)$ & $122.03(13)$ \\
\hline $\mathrm{C}(8)-\mathrm{C}(9)-\mathrm{H}(8)$ & 119.0 \\
\hline $\mathrm{C}(10)-\mathrm{C}(9)-\mathrm{H}(8)$ & 119.0 \\
\hline $\mathrm{C}(9)-\mathrm{C}(10)-\mathrm{C}(19)$ & $122.16(13)$ \\
\hline
\end{tabular}




\begin{tabular}{|c|c|}
\hline $\mathrm{C}(9)-\mathrm{C}(10)-\mathrm{C}(11)$ & $118.98(13)$ \\
\hline $\mathrm{C}(19)-\mathrm{C}(10)-\mathrm{C}(11)$ & $118.86(13)$ \\
\hline $\mathrm{C}(18)-\mathrm{C}(11)-\mathrm{C}(12)$ & $122.52(13)$ \\
\hline $\mathrm{C}(18)-\mathrm{C}(11)-\mathrm{C}(10)$ & $118.40(13)$ \\
\hline $\mathrm{C}(12)-\mathrm{C}(11)-\mathrm{C}(10)$ & $119.07(13)$ \\
\hline $\mathrm{C}(13)-\mathrm{C}(12)-\mathrm{C}(11)$ & $120.60(14)$ \\
\hline $\mathrm{C}(13)-\mathrm{C}(12)-\mathrm{H}(9)$ & 119.7 \\
\hline $\mathrm{C}(11)-\mathrm{C}(12)-\mathrm{H}(9)$ & 119.7 \\
\hline$C(12)-C(13)-C(20)$ & $120.16(14)$ \\
\hline $\mathrm{C}(12)-\mathrm{C}(13)-\mathrm{H}(2)$ & 119.9 \\
\hline $\mathrm{C}(20)-\mathrm{C}(13)-\mathrm{H}(2)$ & 119.9 \\
\hline $\mathrm{O}(1)-\mathrm{C}(14)-\mathrm{C}(22)$ & $110.72(11)$ \\
\hline $\mathrm{O}(1)-\mathrm{C}(14)-\mathrm{C}(21)$ & $108.81(11)$ \\
\hline $\mathrm{C}(22)-\mathrm{C}(14)-\mathrm{C}(21)$ & $119.19(12)$ \\
\hline $\mathrm{O}(1)-\mathrm{C}(14)-\mathrm{C}(5)$ & $87.51(10)$ \\
\hline $\mathrm{C}(22)-\mathrm{C}(14)-\mathrm{C}(5)$ & $119.00(12)$ \\
\hline $\mathrm{C}(21)-\mathrm{C}(14)-\mathrm{C}(5)$ & $106.91(11)$ \\
\hline $\mathrm{O}(3)-\mathrm{C}(15)-\mathrm{O}(2)$ & $123.41(13)$ \\
\hline $\mathrm{O}(3)-\mathrm{C}(15)-\mathrm{C}(16)$ & $126.80(14)$ \\
\hline $\mathrm{O}(2)-\mathrm{C}(15)-\mathrm{C}(16)$ & $109.78(13)$ \\
\hline $\mathrm{C}(15)-\mathrm{C}(16)-\mathrm{H}(6)$ & 109.5 \\
\hline $\mathrm{C}(15)-\mathrm{C}(16)-\mathrm{H}(4)$ & 109.5 \\
\hline $\mathrm{H}(6)-\mathrm{C}(16)-\mathrm{H}(4)$ & 109.5 \\
\hline $\mathrm{C}(15)-\mathrm{C}(16)-\mathrm{H}(5)$ & 109.5 \\
\hline $\mathrm{H}(6)-\mathrm{C}(16)-\mathrm{H}(5)$ & 109.5 \\
\hline $\mathrm{H}(4)-\mathrm{C}(16)-\mathrm{H}(5)$ & 109.5 \\
\hline $\mathrm{C}(18)-\mathrm{C}(17)-\mathrm{C}(8)$ & $121.37(13)$ \\
\hline $\mathrm{C}(18)-\mathrm{C}(17)-\mathrm{H}(13)$ & 119.3 \\
\hline $\mathrm{C}(8)-\mathrm{C}(17)-\mathrm{H}(13)$ & 119.3 \\
\hline $\mathrm{C}(17)-\mathrm{C}(18)-\mathrm{C}(11)$ & 121.07(13) \\
\hline $\mathrm{C}(17)-\mathrm{C}(18)-\mathrm{H}(12)$ & 119.5 \\
\hline $\mathrm{C}(11)-\mathrm{C}(18)-\mathrm{H}(12)$ & 119.5 \\
\hline$C(20)-C(19)-C(10)$ & $120.60(14)$ \\
\hline $\mathrm{C}(20)-\mathrm{C}(19)-\mathrm{H}(11)$ & 119.7 \\
\hline $\mathrm{C}(10)-\mathrm{C}(19)-\mathrm{H}(11)$ & 119.7 \\
\hline $\mathrm{C}(19)-\mathrm{C}(20)-\mathrm{C}(13)$ & $120.70(14)$ \\
\hline $\mathrm{C}(19)-\mathrm{C}(20)-\mathrm{H}(10)$ & 119.6 \\
\hline $\mathrm{C}(13)-\mathrm{C}(20)-\mathrm{H}(10)$ & 119.6 \\
\hline
\end{tabular}




$\begin{array}{ll}\mathrm{C}(14)-\mathrm{C}(21)-\mathrm{C}(7) & 104.20(11) \\ \mathrm{C}(14)-\mathrm{C}(21)-\mathrm{H}(15) & 110.9 \\ \mathrm{C}(7)-\mathrm{C}(21)-\mathrm{H}(15) & 110.9 \\ \mathrm{C}(14)-\mathrm{C}(21)-\mathrm{H}(14) & 110.9 \\ \mathrm{C}(7)-\mathrm{C}(21)-\mathrm{H}(14) & 110.9 \\ \mathrm{H}(15)-\mathrm{C}(21)-\mathrm{H}(14) & 108.9 \\ \mathrm{C}(14)-\mathrm{C}(22)-\mathrm{H}(16) & 109.5 \\ \mathrm{C}(14)-\mathrm{C}(22)-\mathrm{H}(18) & 109.5 \\ \mathrm{H}(16)-\mathrm{C}(22)-\mathrm{H}(18) & 109.5 \\ \mathrm{C}(14)-\mathrm{C}(22)-\mathrm{H}(17) & 109.5 \\ \mathrm{H}(16)-\mathrm{C}(22)-\mathrm{H}(17) & 109.5 \\ \mathrm{H}(18)-\mathrm{C}(22)-\mathrm{H}(17) & 109.5 \\ \mathrm{C}(4)-\mathrm{C}(23)-\mathrm{C}(24) & 119.25(14) \\ \mathrm{C}(4)-\mathrm{C}(23)-\mathrm{H}(19) & 120.4 \\ \mathrm{C}(24)-\mathrm{C}(23)-\mathrm{H}(19) & 120.4 \\ \mathrm{C}(1)-\mathrm{C}(24)-\mathrm{C}(23) & 120.49(15) \\ \mathrm{C}(1)-\mathrm{C}(24)-\mathrm{H}(20) & 119.8 \\ \mathrm{C}(23)-\mathrm{C}(24)-\mathrm{H}(20) & 119.8 \\ & \\ & \end{array}$

Symmetry transformations used to generate equivalent atoms:

Table S6. Anisotropic displacement parameters $\left(\AA^{2} \times 10^{3}\right)$ for 200460lt. The anisotropic displacement factor exponent takes the form: $\quad-2 \pi^{2}\left[h^{2} a^{* 2} U^{11}+\ldots+2 h k a^{*} b^{*} U^{12}\right]$

\begin{tabular}{lllllll}
\hline & $\mathrm{U}^{11}$ & $\mathrm{U}^{22}$ & $\mathrm{U}^{33}$ & $\mathrm{U}^{23}$ & $\mathrm{U}^{13}$ & $\mathrm{U}^{12}$ \\
\hline $\mathrm{O}(1)$ & $20(1)$ & $22(1)$ & $15(1)$ & $0(1)$ & $1(1)$ & $-2(1)$ \\
$\mathrm{O}(2)$ & $23(1)$ & $17(1)$ & $17(1)$ & $-4(1)$ & $0(1)$ & $1(1)$ \\
$\mathrm{O}(3)$ & $24(1)$ & $30(1)$ & $23(1)$ & $-4(1)$ & $-1(1)$ & $-3(1)$ \\
$\mathrm{N}(1)$ & $19(1)$ & $18(1)$ & $14(1)$ & $-1(1)$ & $1(1)$ & $1(1)$ \\
$\mathrm{C}(1)$ & $23(1)$ & $28(1)$ & $44(1)$ & $3(1)$ & $-3(1)$ & $-9(1)$ \\
$\mathrm{C}(2)$ & $25(1)$ & $35(1)$ & $34(1)$ & $6(1)$ & $7(1)$ & $-4(1)$ \\
$\mathrm{C}(3)$ & $26(1)$ & $25(1)$ & $25(1)$ & $-3(1)$ & $4(1)$ & $0(1)$ \\
$\mathrm{C}(4)$ & $16(1)$ & $17(1)$ & $19(1)$ & $3(1)$ & $-3(1)$ & $1(1)$ \\
$\mathrm{C}(5)$ & $18(1)$ & $14(1)$ & $16(1)$ & $-2(1)$ & $0(1)$ & $-1(1)$ \\
$\mathrm{C}(6)$ & $19(1)$ & $15(1)$ & $15(1)$ & $1(1)$ & $0(1)$ & $-2(1)$ \\
$\mathrm{C}(7)$ & $18(1)$ & $16(1)$ & $15(1)$ & $-1(1)$ & $-1(1)$ & $-3(1)$ \\
$\mathrm{C}(8)$ & $16(1)$ & $16(1)$ & $17(1)$ & $-1(1)$ & $0(1)$ & $-2(1)$ \\
$\mathrm{C}(9)$ & $20(1)$ & $18(1)$ & $16(1)$ & $1(1)$ & $1(1)$ & $-2(1)$ \\
& & & & &
\end{tabular}




\begin{tabular}{lllllll}
$\mathrm{C}(10)$ & $17(1)$ & $19(1)$ & $16(1)$ & $-4(1)$ & $-2(1)$ & $-1(1)$ \\
$\mathrm{C}(11)$ & $17(1)$ & $17(1)$ & $21(1)$ & $-1(1)$ & $-1(1)$ & $-1(1)$ \\
$\mathrm{C}(12)$ & $23(1)$ & $17(1)$ & $27(1)$ & $-2(1)$ & $-2(1)$ & $0(1)$ \\
$\mathrm{C}(13)$ & $22(1)$ & $21(1)$ & $32(1)$ & $-8(1)$ & $-3(1)$ & $4(1)$ \\
$\mathrm{C}(14)$ & $19(1)$ & $17(1)$ & $15(1)$ & $0(1)$ & $-1(1)$ & $-2(1)$ \\
$\mathrm{C}(15)$ & $27(1)$ & $15(1)$ & $16(1)$ & $2(1)$ & $-2(1)$ & $-3(1)$ \\
$\mathrm{C}(16)$ & $37(1)$ & $25(1)$ & $22(1)$ & $-6(1)$ & $-1(1)$ & $2(1)$ \\
$\mathrm{C}(17)$ & $18(1)$ & $20(1)$ & $24(1)$ & $1(1)$ & $6(1)$ & $1(1)$ \\
$\mathrm{C}(18)$ & $23(1)$ & $19(1)$ & $24(1)$ & $5(1)$ & $6(1)$ & $-1(1)$ \\
$\mathrm{C}(19)$ & $25(1)$ & $27(1)$ & $20(1)$ & $-3(1)$ & $4(1)$ & $1(1)$ \\
$\mathrm{C}(20)$ & $23(1)$ & $30(1)$ & $27(1)$ & $-9(1)$ & $5(1)$ & $3(1)$ \\
$\mathrm{C}(21)$ & $19(1)$ & $19(1)$ & $18(1)$ & $2(1)$ & $2(1)$ & $-1(1)$ \\
$\mathrm{C}(22)$ & $30(1)$ & $17(1)$ & $22(1)$ & $2(1)$ & $2(1)$ & $-4(1)$ \\
$\mathrm{C}(23)$ & $27(1)$ & $26(1)$ & $25(1)$ & $-6(1)$ & $2(1)$ & $-5(1)$ \\
$\mathrm{C}(24)$ & $34(1)$ & $32(1)$ & $35(1)$ & $-9(1)$ & $-1(1)$ & $-10(1)$ \\
& & & & & & \\
\hline
\end{tabular}

Table S7. Hydrogen coordinates ( $\left.\times 10^{4}\right)$ and isotropic displacement parameters $\left(\AA^{2} \times 10^{3}\right)$ for 200460lt.

\begin{tabular}{|c|c|c|c|c|}
\hline & $\mathrm{x}$ & $\mathrm{y}$ & $\mathrm{z}$ & $\mathrm{U}(\mathrm{eq})$ \\
\hline $\mathrm{H}(1)$ & 11106 & 4805 & 1015 & 38 \\
\hline $\mathrm{H}(21)$ & 11033 & 3194 & -442 & 37 \\
\hline $\mathrm{H}(3)$ & 10057 & 1483 & -429 & 30 \\
\hline $\mathrm{H}(7)$ & 8149 & 3051 & -801 & 19 \\
\hline $\mathrm{H}(8)$ & 6063 & 3861 & 1517 & 22 \\
\hline $\mathrm{H}(9)$ & 5670 & 8523 & -648 & 27 \\
\hline $\mathrm{H}(2)$ & 4661 & 9013 & 607 & 30 \\
\hline $\mathrm{H}(6)$ & 7507 & -1320 & -2922 & 42 \\
\hline $\mathrm{H}(4)$ & 8334 & -1673 & -2159 & 42 \\
\hline $\mathrm{H}(5)$ & 8303 & -388 & -2986 & 42 \\
\hline $\mathrm{H}(13)$ & 7439 & 4946 & -1112 & 24 \\
\hline $\mathrm{H}(12)$ & 6752 & 6949 & -1296 & 26 \\
\hline $\mathrm{H}(11)$ & 5004 & 5433 & 2193 & 28 \\
\hline $\mathrm{H}(10)$ & 4317 & 7446 & 2010 & 32 \\
\hline $\mathrm{H}(15)$ & 6991 & 2182 & 1971 & 22 \\
\hline
\end{tabular}




\begin{tabular}{lrrrr}
$\mathrm{H}(14)$ & 6461 & 1458 & 925 & 22 \\
$\mathrm{H}(16)$ & 7935 & -1237 & 1186 & 35 \\
$\mathrm{H}(18)$ & 7018 & -928 & 666 & 35 \\
$\mathrm{H}(17)$ & 7238 & -812 & 2029 & 35 \\
$\mathrm{H}(19)$ & 9187 & 3053 & 2479 & 31 \\
$\mathrm{H}(20)$ & 10208 & 4703 & 2494 & 40 \\
\hline
\end{tabular}

\subsection{X-ray structure of $3 \mathrm{~m}$ :}
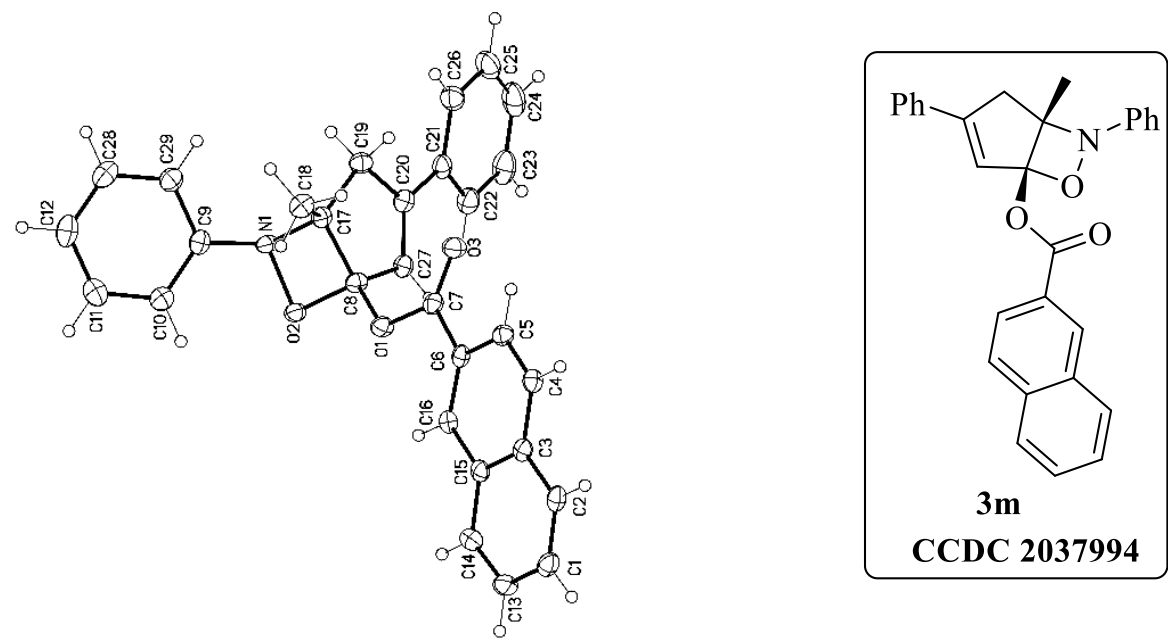

Figure S3. X-ray structure of 3m. Displacement ellipsoids are shown at the $50 \%$ probability level.

Table S8. Crystal data and structure refinement for mo_200528lt_0m.

Identification code

Empirical formula

Formula weight

Temperature

Wavelength

Crystal system

Space group

Unit cell dimensions

Volume

Z

Density (calculated)

Absorption coefficient
mo_200528LT_0m

C29 H23 N O3

433.48

$100(2) \mathrm{K}$

$0.71073 \AA$

Monoclinic

$\mathrm{P} 21$

$\mathrm{a}=10.0647(11) \AA \quad \alpha=90^{\circ}$.

$\mathrm{b}=5.9175(6) \AA$

$\beta=104.726(4)^{\circ}$.

$\mathrm{c}=19.388(2) \AA$

$\gamma=90^{\circ}$.

1116.8(2) $\AA^{3}$

2

$1.289 \mathrm{Mg} / \mathrm{m}^{3}$

$0.083 \mathrm{~mm}^{-1}$ 
$\mathrm{F}(000)$

Crystal size

Theta range for data collection

Index ranges

Reflections collected

Independent reflections

Completeness to theta $=25.242^{\circ}$

Absorption correction

Max. and min. transmission

Refinement method

Data / restraints / parameters

Goodness-of-fit on $\mathrm{F}^{2}$

Final $\mathrm{R}$ indices [I>2sigma(I)]

$\mathrm{R}$ indices (all data)

Absolute structure parameter

Extinction coefficient

Largest diff. peak and hole
456

$0.20 \times 0.18 \times 0.18 \mathrm{~mm}^{3}$

1.086 to $26.573^{\circ}$.

$-12<=\mathrm{h}<=12,-5<=\mathrm{k}<=7,-24<=\mathrm{l}<=24$

17081

$4108[\mathrm{R}(\mathrm{int})=0.0500]$

$100.0 \%$

Semi-empirical from equivalents

0.7454 and 0.5446

Full-matrix least-squares on $\mathrm{F}^{2}$

4108 / 1 / 299

1.045

$\mathrm{R} 1=0.0350, \mathrm{wR} 2=0.0811$

$\mathrm{R} 1=0.0419, \mathrm{wR} 2=0.0841$

$-0.1(7)$

$\mathrm{n} / \mathrm{a}$

0.210 and -0.185 e. $\AA^{-3}$

Table S9. Atomic coordinates $\left(\mathrm{x} 10^{4}\right)$ and equivalent isotropic displacement parameters $\left(\AA^{2} \times 10^{3}\right)$ for mo_2005281t_0m. U(eq) is defined as one third of the trace of the orthogonalized $\mathrm{U}^{\mathrm{ij}}$ tensor.

\begin{tabular}{|c|c|c|c|c|}
\hline & $\mathrm{x}$ & $\mathrm{y}$ & $\mathrm{z}$ & $\mathrm{U}(\mathrm{eq})$ \\
\hline $\mathrm{O}(1)$ & $5806(2)$ & 9783(3) & $2265(1)$ & $20(1)$ \\
\hline $\mathrm{O}(2)$ & $6171(1)$ & $7428(3)$ & $3218(1)$ & $19(1)$ \\
\hline $\mathrm{O}(3)$ & $4115(2)$ & 12388(3) & 1946(1) & $22(1)$ \\
\hline $\mathrm{N}(1)$ & $5611(2)$ & $8119(3)$ & $3833(1)$ & $18(1)$ \\
\hline $\mathrm{C}(1)$ & $8322(2)$ & $12737(5)$ & $-648(1)$ & $24(1)$ \\
\hline$C(2)$ & $7475(2)$ & $14182(4)$ & $-411(1)$ & $21(1)$ \\
\hline$C(3)$ & $6958(2)$ & $13588(4)$ & 183(1) & $18(1)$ \\
\hline $\mathrm{C}(4)$ & $6026(2)$ & $15008(4)$ & $422(1)$ & $19(1)$ \\
\hline$C(5)$ & $5472(2)$ & 14329(4) & $963(1)$ & 19(1) \\
\hline$C(6)$ & $5849(2)$ & $12215(4)$ & $1305(1)$ & $17(1)$ \\
\hline$C(7)$ & $5152(2)$ & 11526(4) & $1862(1)$ & $18(1)$ \\
\hline $\mathrm{C}(8)$ & $5147(2)$ & $8834(4)$ & $2768(1)$ & $18(1)$ \\
\hline $\mathrm{C}(9)$ & $6684(2)$ & $8438(4)$ & $4462(1)$ & $20(1)$ \\
\hline$C(10)$ & $8011(2)$ & $7662(5)$ & $4533(1)$ & $25(1)$ \\
\hline $\mathrm{C}(11)$ & $8979(3)$ & $7941(5)$ & $5181(1)$ & $31(1)$ \\
\hline
\end{tabular}




\begin{tabular}{lrrrr}
$\mathrm{C}(12)$ & $8646(3)$ & $9006(5)$ & $5746(1)$ & $31(1)$ \\
$\mathrm{C}(13)$ & $8715(2)$ & $10669(5)$ & $-301(1)$ & $25(1)$ \\
$\mathrm{C}(14)$ & $8252(2)$ & $10051(4)$ & $278(1)$ & $21(1)$ \\
$\mathrm{C}(15)$ & $7346(2)$ & $11483(4)$ & $528(1)$ & $18(1)$ \\
$\mathrm{C}(16)$ & $6785(2)$ & $10851(4)$ & $1104(1)$ & $17(1)$ \\
$\mathrm{C}(17)$ & $4923(2)$ & $10171(4)$ & $3410(1)$ & $18(1)$ \\
$\mathrm{C}(18)$ & $5676(2)$ & $12377(4)$ & $3594(1)$ & $22(1)$ \\
$\mathrm{C}(19)$ & $3380(2)$ & $10139(4)$ & $3333(1)$ & $20(1)$ \\
$\mathrm{C}(20)$ & $2861(2)$ & $8330(4)$ & $2778(1)$ & $19(1)$ \\
$\mathrm{C}(21)$ & $1437(2)$ & $7475(5)$ & $2629(1)$ & $20(1)$ \\
$\mathrm{C}(22)$ & $1047(2)$ & $5458(5)$ & $2257(1)$ & $25(1)$ \\
$\mathrm{C}(23)$ & $-298(3)$ & $4684(6)$ & $2124(1)$ & $34(1)$ \\
$\mathrm{C}(24)$ & $-1265(3)$ & $5922(6)$ & $2365(1)$ & $36(1)$ \\
$\mathrm{C}(25)$ & $-893(2)$ & $7909(5)$ & $2734(1)$ & $34(1)$ \\
$\mathrm{C}(26)$ & $449(2)$ & $8692(5)$ & $2867(1)$ & $26(1)$ \\
$\mathrm{C}(27)$ & $3816(2)$ & $7675(4)$ & $2452(1)$ & $18(1)$ \\
$\mathrm{C}(28)$ & $7314(3)$ & $9770(5)$ & $5672(1)$ & $27(1)$ \\
$\mathrm{C}(29)$ & $6330(2)$ & $9473(5)$ & $5036(1)$ & $23(1)$ \\
& & & & \\
\hline
\end{tabular}

Table S10. Bond lengths $[\AA ̊]$ and angles $\left[{ }^{\circ}\right]$ for mo_2005281t_0m.

\begin{tabular}{ll}
\hline $\mathrm{O}(1)-\mathrm{C}(7)$ & $1.359(3)$ \\
$\mathrm{O}(1)-\mathrm{C}(8)$ & $1.427(2)$ \\
$\mathrm{O}(2)-\mathrm{C}(8)$ & $1.435(3)$ \\
$\mathrm{O}(2)-\mathrm{N}(1)$ & $1.499(2)$ \\
$\mathrm{O}(3)-\mathrm{C}(7)$ & $1.209(3)$ \\
$\mathrm{N}(1)-\mathrm{C}(9)$ & $1.422(3)$ \\
$\mathrm{N}(1)-\mathrm{C}(17)$ & $1.529(3)$ \\
$\mathrm{C}(1)-\mathrm{C}(2)$ & $1.367(3)$ \\
$\mathrm{C}(1)-\mathrm{C}(13)$ & $1.404(4)$ \\
$\mathrm{C}(1)-\mathrm{H}(1)$ & 0.9500 \\
$\mathrm{C}(2)-\mathrm{C}(3)$ & $1.424(3)$ \\
$\mathrm{C}(2)-\mathrm{H}(18)$ & 0.9500 \\
$\mathrm{C}(3)-\mathrm{C}(15)$ & $1.421(3)$ \\
$\mathrm{C}(3)-\mathrm{C}(4)$ & $1.421(3)$ \\
$\mathrm{C}(4)-\mathrm{C}(5)$ & $1.368(3)$ \\
$\mathrm{C}(4)-\mathrm{H}(19)$ & 0.9500
\end{tabular}




\begin{tabular}{|c|c|}
\hline$C(5)-C(6)$ & $1.422(3)$ \\
\hline $\mathrm{C}(5)-\mathrm{H}(20)$ & 0.9500 \\
\hline$C(6)-C(16)$ & $1.370(3)$ \\
\hline$C(6)-C(7)$ & $1.486(3)$ \\
\hline$C(8)-C(27)$ & $1.491(3)$ \\
\hline$C(8)-C(17)$ & $1.538(3)$ \\
\hline $\mathrm{C}(9)-\mathrm{C}(10)$ & $1.385(3)$ \\
\hline $\mathrm{C}(9)-\mathrm{C}(29)$ & $1.394(3)$ \\
\hline$C(10)-C(11)$ & $1.391(3)$ \\
\hline $\mathrm{C}(10)-\mathrm{H}(17)$ & 0.9500 \\
\hline $\mathrm{C}(11)-\mathrm{C}(12)$ & $1.377(4)$ \\
\hline $\mathrm{C}(11)-\mathrm{H}(16)$ & 0.9500 \\
\hline$C(12)-C(28)$ & $1.388(4)$ \\
\hline $\mathrm{C}(12)-\mathrm{H}(2)$ & 0.9500 \\
\hline$C(13)-C(14)$ & $1.370(3)$ \\
\hline $\mathrm{C}(13)-\mathrm{H}(23)$ & 0.9500 \\
\hline C(14)-C(15) & $1.417(3)$ \\
\hline $\mathrm{C}(14)-\mathrm{H}(22)$ & 0.9500 \\
\hline$C(15)-C(16)$ & $1.424(3)$ \\
\hline $\mathrm{C}(16)-\mathrm{H}(21)$ & 0.9500 \\
\hline C(17)-C(18) & $1.506(3)$ \\
\hline C(17)-C(19) & $1.523(3)$ \\
\hline $\mathrm{C}(18)-\mathrm{H}(4)$ & 0.9800 \\
\hline $\mathrm{C}(18)-\mathrm{H}(5)$ & 0.9800 \\
\hline $\mathrm{C}(18)-\mathrm{H}(3)$ & 0.9800 \\
\hline C(19)-C(20) & $1.515(3)$ \\
\hline $\mathrm{C}(19)-\mathrm{H}(12)$ & 0.9900 \\
\hline C(19)-H(13) & 0.9900 \\
\hline $\mathrm{C}(20)-\mathrm{C}(27)$ & $1.334(3)$ \\
\hline$C(20)-C(21)$ & $1.478(3)$ \\
\hline $\mathrm{C}(21)-\mathrm{C}(22)$ & $1.398(4)$ \\
\hline$C(21)-C(26)$ & $1.398(3)$ \\
\hline$C(22)-C(23)$ & $1.390(3)$ \\
\hline $\mathrm{C}(22)-\mathrm{H}(10)$ & 0.9500 \\
\hline $\mathrm{C}(23)-\mathrm{C}(24)$ & $1.390(4)$ \\
\hline $\mathrm{C}(23)-\mathrm{H}(9)$ & 0.9500 \\
\hline$C(24)-C(25)$ & $1.378(4)$ \\
\hline $\mathrm{C}(24)-\mathrm{H}(8)$ & 0.9500 \\
\hline
\end{tabular}




\begin{tabular}{|c|c|}
\hline$C(25)-C(26)$ & $1.389(3)$ \\
\hline $\mathrm{C}(25)-\mathrm{H}(7)$ & 0.9500 \\
\hline $\mathrm{C}(26)-\mathrm{H}(6)$ & 0.9500 \\
\hline $\mathrm{C}(27)-\mathrm{H}(11)$ & 0.9500 \\
\hline $\mathrm{C}(28)-\mathrm{C}(29)$ & $1.382(3)$ \\
\hline $\mathrm{C}(28)-\mathrm{H}(14)$ & 0.9500 \\
\hline $\mathrm{C}(29)-\mathrm{H}(15)$ & 0.9500 \\
\hline $\mathrm{C}(7)-\mathrm{O}(1)-\mathrm{C}(8)$ & $116.76(17)$ \\
\hline $\mathrm{C}(8)-\mathrm{O}(2)-\mathrm{N}(1)$ & $88.17(14)$ \\
\hline $\mathrm{C}(9)-\mathrm{N}(1)-\mathrm{O}(2)$ & $111.15(15)$ \\
\hline $\mathrm{C}(9)-\mathrm{N}(1)-\mathrm{C}(17)$ & $119.76(19)$ \\
\hline $\mathrm{O}(2)-\mathrm{N}(1)-\mathrm{C}(17)$ & $90.01(13)$ \\
\hline $\mathrm{C}(2)-\mathrm{C}(1)-\mathrm{C}(13)$ & $120.5(2)$ \\
\hline $\mathrm{C}(2)-\mathrm{C}(1)-\mathrm{H}(1)$ & 119.7 \\
\hline $\mathrm{C}(13)-\mathrm{C}(1)-\mathrm{H}(1)$ & 119.7 \\
\hline $\mathrm{C}(1)-\mathrm{C}(2)-\mathrm{C}(3)$ & $120.3(2)$ \\
\hline $\mathrm{C}(1)-\mathrm{C}(2)-\mathrm{H}(18)$ & 119.8 \\
\hline $\mathrm{C}(3)-\mathrm{C}(2)-\mathrm{H}(18)$ & 119.8 \\
\hline$C(15)-C(3)-C(4)$ & $119.4(2)$ \\
\hline $\mathrm{C}(15)-\mathrm{C}(3)-\mathrm{C}(2)$ & $118.9(2)$ \\
\hline $\mathrm{C}(4)-\mathrm{C}(3)-\mathrm{C}(2)$ & $121.6(2)$ \\
\hline $\mathrm{C}(5)-\mathrm{C}(4)-\mathrm{C}(3)$ & $120.5(2)$ \\
\hline $\mathrm{C}(5)-\mathrm{C}(4)-\mathrm{H}(19)$ & 119.8 \\
\hline $\mathrm{C}(3)-\mathrm{C}(4)-\mathrm{H}(19)$ & 119.8 \\
\hline $\mathrm{C}(4)-\mathrm{C}(5)-\mathrm{C}(6)$ & $120.3(2)$ \\
\hline $\mathrm{C}(4)-\mathrm{C}(5)-\mathrm{H}(20)$ & 119.9 \\
\hline $\mathrm{C}(6)-\mathrm{C}(5)-\mathrm{H}(20)$ & 119.9 \\
\hline$C(16)-C(6)-C(5)$ & $120.42(19)$ \\
\hline$C(16)-C(6)-C(7)$ & $122.0(2)$ \\
\hline$C(5)-C(6)-C(7)$ & $117.5(2)$ \\
\hline $\mathrm{O}(3)-\mathrm{C}(7)-\mathrm{O}(1)$ & $123.5(2)$ \\
\hline $\mathrm{O}(3)-\mathrm{C}(7)-\mathrm{C}(6)$ & $124.4(2)$ \\
\hline $\mathrm{O}(1)-\mathrm{C}(7)-\mathrm{C}(6)$ & $112.13(18)$ \\
\hline $\mathrm{O}(1)-\mathrm{C}(8)-\mathrm{O}(2)$ & $104.88(16)$ \\
\hline $\mathrm{O}(1)-\mathrm{C}(8)-\mathrm{C}(27)$ & $115.17(17)$ \\
\hline $\mathrm{O}(2)-\mathrm{C}(8)-\mathrm{C}(27)$ & $114.52(19)$ \\
\hline $\mathrm{O}(1)-\mathrm{C}(8)-\mathrm{C}(17)$ & $122.64(19)$ \\
\hline $\mathrm{O}(2)-\mathrm{C}(8)-\mathrm{C}(17)$ & $92.10(15)$ \\
\hline
\end{tabular}




\begin{tabular}{|c|c|}
\hline$C(27)-C(8)-C(17)$ & $105.54(17)$ \\
\hline$C(10)-C(9)-C(29)$ & $120.1(2)$ \\
\hline $\mathrm{C}(10)-\mathrm{C}(9)-\mathrm{N}(1)$ & $123.0(2)$ \\
\hline $\mathrm{C}(29)-\mathrm{C}(9)-\mathrm{N}(1)$ & $116.8(2)$ \\
\hline$C(9)-C(10)-C(11)$ & $119.1(2)$ \\
\hline $\mathrm{C}(9)-\mathrm{C}(10)-\mathrm{H}(17)$ & 120.4 \\
\hline $\mathrm{C}(11)-\mathrm{C}(10)-\mathrm{H}(17)$ & 120.4 \\
\hline $\mathrm{C}(12)-\mathrm{C}(11)-\mathrm{C}(10)$ & $121.1(2)$ \\
\hline $\mathrm{C}(12)-\mathrm{C}(11)-\mathrm{H}(16)$ & 119.5 \\
\hline $\mathrm{C}(10)-\mathrm{C}(11)-\mathrm{H}(16)$ & 119.5 \\
\hline $\mathrm{C}(11)-\mathrm{C}(12)-\mathrm{C}(28)$ & $119.5(2)$ \\
\hline $\mathrm{C}(11)-\mathrm{C}(12)-\mathrm{H}(2)$ & 120.3 \\
\hline $\mathrm{C}(28)-\mathrm{C}(12)-\mathrm{H}(2)$ & 120.3 \\
\hline $\mathrm{C}(14)-\mathrm{C}(13)-\mathrm{C}(1)$ & $120.8(2)$ \\
\hline $\mathrm{C}(14)-\mathrm{C}(13)-\mathrm{H}(23)$ & 119.6 \\
\hline $\mathrm{C}(1)-\mathrm{C}(13)-\mathrm{H}(23)$ & 119.6 \\
\hline $\mathrm{C}(13)-\mathrm{C}(14)-\mathrm{C}(15)$ & $120.2(2)$ \\
\hline $\mathrm{C}(13)-\mathrm{C}(14)-\mathrm{H}(22)$ & 119.9 \\
\hline $\mathrm{C}(15)-\mathrm{C}(14)-\mathrm{H}(22)$ & 119.9 \\
\hline$C(14)-C(15)-C(3)$ & $119.2(2)$ \\
\hline $\mathrm{C}(14)-\mathrm{C}(15)-\mathrm{C}(16)$ & $121.9(2)$ \\
\hline$C(3)-C(15)-C(16)$ & $118.9(2)$ \\
\hline$C(6)-C(16)-C(15)$ & $120.5(2)$ \\
\hline $\mathrm{C}(6)-\mathrm{C}(16)-\mathrm{H}(21)$ & 119.8 \\
\hline $\mathrm{C}(15)-\mathrm{C}(16)-\mathrm{H}(21)$ & 119.8 \\
\hline$C(18)-C(17)-C(19)$ & $118.2(2)$ \\
\hline $\mathrm{C}(18)-\mathrm{C}(17)-\mathrm{N}(1)$ & $115.41(18)$ \\
\hline $\mathrm{C}(19)-\mathrm{C}(17)-\mathrm{N}(1)$ & $110.70(18)$ \\
\hline $\mathrm{C}(18)-\mathrm{C}(17)-\mathrm{C}(8)$ & $118.04(18)$ \\
\hline$C(19)-C(17)-C(8)$ & $105.69(18)$ \\
\hline $\mathrm{N}(1)-\mathrm{C}(17)-\mathrm{C}(8)$ & $83.46(16)$ \\
\hline $\mathrm{C}(17)-\mathrm{C}(18)-\mathrm{H}(4)$ & 109.5 \\
\hline $\mathrm{C}(17)-\mathrm{C}(18)-\mathrm{H}(5)$ & 109.5 \\
\hline $\mathrm{H}(4)-\mathrm{C}(18)-\mathrm{H}(5)$ & 109.5 \\
\hline $\mathrm{C}(17)-\mathrm{C}(18)-\mathrm{H}(3)$ & 109.5 \\
\hline $\mathrm{H}(4)-\mathrm{C}(18)-\mathrm{H}(3)$ & 109.5 \\
\hline $\mathrm{H}(5)-\mathrm{C}(18)-\mathrm{H}(3)$ & 109.5 \\
\hline $\mathrm{C}(20)-\mathrm{C}(19)-\mathrm{C}(17)$ & $103.66(18)$ \\
\hline
\end{tabular}




\begin{tabular}{|c|c|}
\hline $\mathrm{C}(20)-\mathrm{C}(19)-\mathrm{H}(12)$ & 111.0 \\
\hline $\mathrm{C}(17)-\mathrm{C}(19)-\mathrm{H}(12)$ & 111.0 \\
\hline $\mathrm{C}(20)-\mathrm{C}(19)-\mathrm{H}(13)$ & 111.0 \\
\hline $\mathrm{C}(17)-\mathrm{C}(19)-\mathrm{H}(13)$ & 111.0 \\
\hline $\mathrm{H}(12)-\mathrm{C}(19)-\mathrm{H}(13)$ & 109.0 \\
\hline$C(27)-C(20)-C(21)$ & $126.0(2)$ \\
\hline$C(27)-C(20)-C(19)$ & $112.50(19)$ \\
\hline$C(21)-C(20)-C(19)$ & $121.47(19)$ \\
\hline$C(22)-C(21)-C(26)$ & $118.7(2)$ \\
\hline$C(22)-C(21)-C(20)$ & $121.6(2)$ \\
\hline$C(26)-C(21)-C(20)$ & $119.7(2)$ \\
\hline$C(23)-C(22)-C(21)$ & $120.7(2)$ \\
\hline $\mathrm{C}(23)-\mathrm{C}(22)-\mathrm{H}(10)$ & 119.7 \\
\hline $\mathrm{C}(21)-\mathrm{C}(22)-\mathrm{H}(10)$ & 119.7 \\
\hline $\mathrm{C}(22)-\mathrm{C}(23)-\mathrm{C}(24)$ & $119.7(3)$ \\
\hline $\mathrm{C}(22)-\mathrm{C}(23)-\mathrm{H}(9)$ & 120.2 \\
\hline $\mathrm{C}(24)-\mathrm{C}(23)-\mathrm{H}(9)$ & 120.2 \\
\hline$C(25)-C(24)-C(23)$ & $120.3(2)$ \\
\hline $\mathrm{C}(25)-\mathrm{C}(24)-\mathrm{H}(8)$ & 119.9 \\
\hline $\mathrm{C}(23)-\mathrm{C}(24)-\mathrm{H}(8)$ & 119.9 \\
\hline $\mathrm{C}(24)-\mathrm{C}(25)-\mathrm{C}(26)$ & $120.2(3)$ \\
\hline $\mathrm{C}(24)-\mathrm{C}(25)-\mathrm{H}(7)$ & 119.9 \\
\hline $\mathrm{C}(26)-\mathrm{C}(25)-\mathrm{H}(7)$ & 119.9 \\
\hline$C(25)-C(26)-C(21)$ & $120.4(3)$ \\
\hline $\mathrm{C}(25)-\mathrm{C}(26)-\mathrm{H}(6)$ & 119.8 \\
\hline $\mathrm{C}(21)-\mathrm{C}(26)-\mathrm{H}(6)$ & 119.8 \\
\hline $\mathrm{C}(20)-\mathrm{C}(27)-\mathrm{C}(8)$ & $110.6(2)$ \\
\hline $\mathrm{C}(20)-\mathrm{C}(27)-\mathrm{H}(11)$ & 124.7 \\
\hline $\mathrm{C}(8)-\mathrm{C}(27)-\mathrm{H}(11)$ & 124.7 \\
\hline $\mathrm{C}(29)-\mathrm{C}(28)-\mathrm{C}(12)$ & $120.3(2)$ \\
\hline $\mathrm{C}(29)-\mathrm{C}(28)-\mathrm{H}(14)$ & 119.8 \\
\hline $\mathrm{C}(12)-\mathrm{C}(28)-\mathrm{H}(14)$ & 119.8 \\
\hline $\mathrm{C}(28)-\mathrm{C}(29)-\mathrm{C}(9)$ & $119.8(2)$ \\
\hline $\mathrm{C}(28)-\mathrm{C}(29)-\mathrm{H}(15)$ & 120.1 \\
\hline $\mathrm{C}(9)-\mathrm{C}(29)-\mathrm{H}(15)$ & 120.1 \\
\hline
\end{tabular}

Symmetry transformations used to generate equivalent atoms: 
Table 11. Anisotropic displacement parameters $\left(\AA^{2} \times 10^{3}\right)$ for mo_2005281t_0m. The anisotropic displacement factor exponent takes the form: $\quad-2 \pi^{2}\left[\mathrm{~h}^{2} \mathrm{a}^{* 2} \mathrm{U}^{11}+\ldots \quad+2 \mathrm{hk} \mathrm{a}^{*} \mathrm{~b}^{*} \mathrm{U}^{12}\right]$

\begin{tabular}{|c|c|c|c|c|c|c|}
\hline & $\mathrm{U}^{11}$ & $\mathrm{U}^{22}$ & $\mathrm{U}^{33}$ & $\mathrm{U}^{23}$ & $\mathrm{U}^{13}$ & $\mathrm{U}^{12}$ \\
\hline $\mathrm{O}(1)$ & 21(1) & $20(1)$ & $20(1)$ & $5(1)$ & $10(1)$ & $2(1)$ \\
\hline $\mathrm{O}(2)$ & 21(1) & $19(1)$ & $18(1)$ & $1(1)$ & $8(1)$ & $3(1)$ \\
\hline $\mathrm{O}(3)$ & $24(1)$ & $22(1)$ & $24(1)$ & $0(1)$ & $9(1)$ & $3(1)$ \\
\hline $\mathrm{N}(1)$ & $22(1)$ & $18(1)$ & $17(1)$ & $-2(1)$ & $9(1)$ & $2(1)$ \\
\hline $\mathrm{C}(1)$ & $19(1)$ & $36(2)$ & $17(1)$ & $0(1)$ & $6(1)$ & $-6(1)$ \\
\hline $\mathrm{C}(2)$ & $20(1)$ & $26(1)$ & $16(1)$ & $2(1)$ & $1(1)$ & $-6(1)$ \\
\hline $\mathrm{C}(3)$ & $15(1)$ & $20(1)$ & $16(1)$ & $-1(1)$ & $2(1)$ & $-6(1)$ \\
\hline $\mathrm{C}(4)$ & $22(1)$ & $15(1)$ & $18(1)$ & $2(1)$ & $2(1)$ & $0(1)$ \\
\hline $\mathrm{C}(5)$ & $22(1)$ & $17(1)$ & $18(1)$ & $-2(1)$ & $4(1)$ & 1(1) \\
\hline $\mathrm{C}(6)$ & $16(1)$ & 19(1) & $14(1)$ & $0(1)$ & $1(1)$ & $-4(1)$ \\
\hline $\mathrm{C}(7)$ & 21(1) & $16(1)$ & $16(1)$ & $-2(1)$ & $3(1)$ & $-1(1)$ \\
\hline $\mathrm{C}(8)$ & 21(1) & $16(1)$ & $18(1)$ & $2(1)$ & $9(1)$ & $0(1)$ \\
\hline $\mathrm{C}(9)$ & $23(1)$ & $18(1)$ & $18(1)$ & $4(1)$ & $5(1)$ & $-3(1)$ \\
\hline$C(10)$ & $26(1)$ & $28(2)$ & $23(1)$ & $0(1)$ & $7(1)$ & $0(1)$ \\
\hline $\mathrm{C}(11)$ & $24(1)$ & $35(2)$ & $31(1)$ & $1(1)$ & $3(1)$ & $2(1)$ \\
\hline $\mathrm{C}(12)$ & $33(1)$ & $31(2)$ & $22(1)$ & $1(1)$ & $-2(1)$ & $-7(1)$ \\
\hline$C(13)$ & $18(1)$ & $33(2)$ & $26(1)$ & $-3(1)$ & $8(1)$ & $1(1)$ \\
\hline$C(14)$ & $18(1)$ & $20(1)$ & $25(1)$ & $-1(1)$ & $5(1)$ & $1(1)$ \\
\hline$C(15)$ & $14(1)$ & $20(1)$ & $17(1)$ & $-1(1)$ & $1(1)$ & $-3(1)$ \\
\hline$C(16)$ & $16(1)$ & $17(1)$ & $17(1)$ & $2(1)$ & $1(1)$ & $-1(1)$ \\
\hline$C(17)$ & $20(1)$ & $16(1)$ & $17(1)$ & $2(1)$ & $5(1)$ & 1(1) \\
\hline $\mathrm{C}(18)$ & $27(1)$ & $17(1)$ & $24(1)$ & $-1(1)$ & $8(1)$ & $-4(1)$ \\
\hline$C(19)$ & 21(1) & $18(1)$ & $22(1)$ & $0(1)$ & $8(1)$ & $0(1)$ \\
\hline$C(20)$ & $22(1)$ & $17(1)$ & $18(1)$ & $3(1)$ & $5(1)$ & $-2(1)$ \\
\hline $\mathrm{C}(21)$ & $19(1)$ & $24(1)$ & $17(1)$ & $5(1)$ & $3(1)$ & $-2(1)$ \\
\hline$C(22)$ & $24(1)$ & $30(2)$ & 21(1) & $2(1)$ & $5(1)$ & $-3(1)$ \\
\hline$C(23)$ & $31(1)$ & $38(2)$ & $27(1)$ & $0(1)$ & $-2(1)$ & $-14(1)$ \\
\hline$C(24)$ & $20(1)$ & $53(2)$ & $32(1)$ & $10(1)$ & $-1(1)$ & $-9(1)$ \\
\hline$C(25)$ & $19(1)$ & $49(2)$ & $33(1)$ & $8(1)$ & $6(1)$ & $1(1)$ \\
\hline$C(26)$ & $23(1)$ & $32(2)$ & $26(1)$ & $4(1)$ & $7(1)$ & 1(1) \\
\hline $\mathrm{C}(27)$ & $20(1)$ & $18(1)$ & $16(1)$ & $1(1)$ & $4(1)$ & $-2(1)$ \\
\hline$C(28)$ & $39(1)$ & $22(1)$ & $20(1)$ & $-1(1)$ & $9(1)$ & $-2(1)$ \\
\hline $\mathrm{C}(29)$ & $25(1)$ & $25(1)$ & 21(1) & $3(1)$ & $8(1)$ & $-1(1)$ \\
\hline
\end{tabular}


Table S12. Hydrogen coordinates ( x 10 $)$ and isotropic displacement parameters $\left(\AA^{2} \times 10^{3}\right)$ for mo_2005281t_0m.

$\begin{array}{llll}\mathrm{x} & \mathrm{y} & \mathrm{U} & \mathrm{u}\end{array}$

\begin{tabular}{|c|c|c|c|c|}
\hline $\mathrm{H}(1)$ & 8646 & 13133 & -1051 & 28 \\
\hline $\mathrm{H}(18)$ & 7231 & 15591 & -644 & 25 \\
\hline $\mathrm{H}(19)$ & 5787 & 16439 & 204 & 23 \\
\hline $\mathrm{H}(20)$ & 4833 & 15275 & 1111 & 23 \\
\hline $\mathrm{H}(17)$ & 8255 & 6949 & 4144 & 30 \\
\hline $\mathrm{H}(16)$ & 9886 & 7388 & 5235 & 37 \\
\hline $\mathrm{H}(2)$ & 9322 & 9216 & 6183 & 37 \\
\hline $\mathrm{H}(23)$ & 9311 & 9687 & -469 & 30 \\
\hline $\mathrm{H}(22)$ & 8538 & 8658 & 512 & 25 \\
\hline $\mathrm{H}(21)$ & 7061 & 9474 & 1351 & 20 \\
\hline $\mathrm{H}(4)$ & 5240 & 13535 & 3249 & 33 \\
\hline $\mathrm{H}(5)$ & 6635 & 12187 & 3580 & 33 \\
\hline $\mathrm{H}(3)$ & 5641 & 12844 & 4074 & 33 \\
\hline $\mathrm{H}(12)$ & 3172 & 9746 & 3791 & 24 \\
\hline $\mathrm{H}(13)$ & 2963 & 11624 & 3171 & 24 \\
\hline $\mathrm{H}(10)$ & 1709 & 4608 & 2094 & 30 \\
\hline $\mathrm{H}(9)$ & -556 & 3315 & 1869 & 40 \\
\hline $\mathrm{H}(8)$ & -2185 & 5397 & 2274 & 44 \\
\hline $\mathrm{H}(7)$ & -1558 & 8746 & 2898 & 41 \\
\hline $\mathrm{H}(6)$ & 697 & 10065 & 3121 & 32 \\
\hline $\mathrm{H}(11)$ & 3668 & 6618 & 2072 & 22 \\
\hline $\mathrm{H}(14)$ & 7076 & 10500 & 6061 & 32 \\
\hline $\mathrm{H}(15)$ & 5414 & 9972 & 4991 & 28 \\
\hline
\end{tabular}




\subsection{X-ray structure of $4 \mathrm{i}$ :}
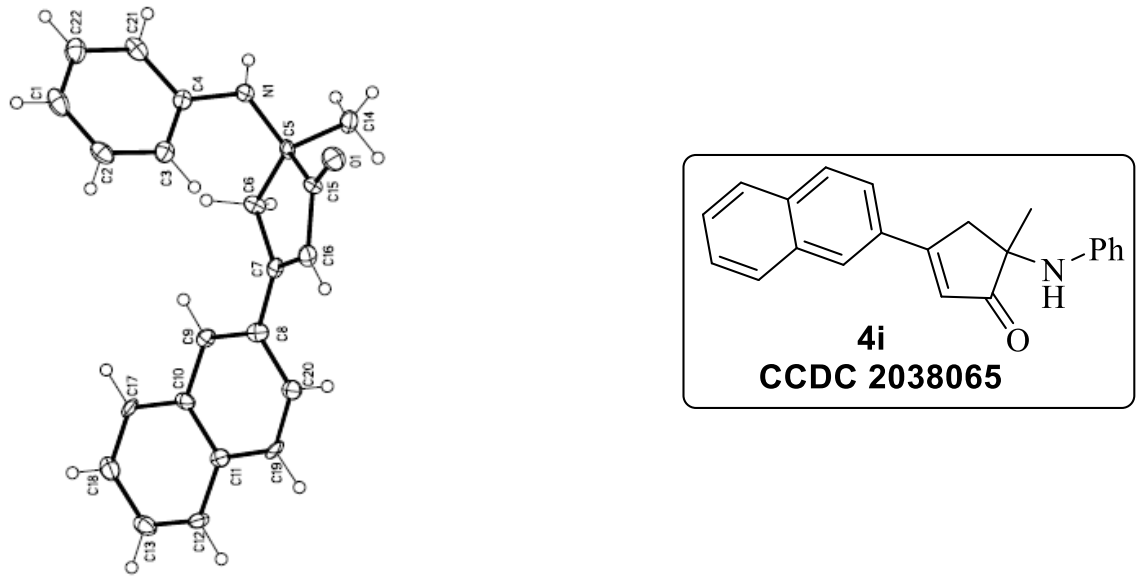

Figure S4. X-ray structure of $\mathbf{4 i}$. Displacement ellipsoids are shown at the $50 \%$ probability level.

Table S13. Crystal data and structure

refinement for twin5.

Identification code

twin5

Empirical formula

C22 H19 N O

Formula weight

313.38

Temperature

$100(2) \mathrm{K}$

Wavelength

$0.71073 \AA$

Crystal system

Orthorhombic

Space group

Unit cell dimensions

$\mathrm{P} 2{ }_{1} 2_{1}$

$\mathrm{a}=6.0284(13) \AA$ $\alpha=90^{\circ}$.

$\mathrm{b}=11.610(3) \AA$ $\beta=90^{\circ}$.

$c=22.775(6) \AA$ $\gamma=90^{\circ}$.

Volume

1594.0(7) $\AA^{3}$

Z

4

Density (calculated)

$1.306 \mathrm{Mg} / \mathrm{m}^{3}$

Absorption coefficient

$0.080 \mathrm{~mm}^{-1}$

$\mathrm{F}(000)$

664

Crystal size

$0.08 \times 0.01 \times 0.01 \mathrm{~mm}^{3}$

Theta range for data collection

1.788 to $26.508^{\circ}$.

Index ranges

$0<=\mathrm{h}<=7,0<=\mathrm{k}<=14,0<=1<=28$

Reflections collected

1947

Independent reflections

$1947[\mathrm{R}(\mathrm{int})=0.0665]$

Completeness to theta $=25.242^{\circ}$

$100.0 \%$

Absorption correction

Semi-empirical from equivalents

Max. and min. transmission

0.7454 and 0.6273 
Refinement method

Data / restraints / parameters

Goodness-of-fit on $\mathrm{F}^{2}$

Final $\mathrm{R}$ indices [I $>2 \operatorname{sigma}(\mathrm{I})]$

$\mathrm{R}$ indices (all data)

Absolute structure parameter

Extinction coefficient

Largest diff. peak and hole
Full-matrix least-squares on $\mathrm{F}^{2}$

1947 / 198 / 219

1.213

$\mathrm{R} 1=0.0957, \mathrm{wR} 2=0.1467$

$\mathrm{R} 1=0.1554, \mathrm{wR} 2=0.1628$

2(5)

$\mathrm{n} / \mathrm{a}$

0.411 and -0.408 e. $\AA^{-3}$

Table S14. Atomic coordinates ( x 10 $)$ and equivalent isotropic displacement parameters $\left(\AA^{2} \times 10^{3}\right)$ for twin5. $U(e q)$ is defined as one third of the trace of the orthogonalized $U^{i j}$ tensor.

\begin{tabular}{|c|c|c|c|c|}
\hline & $\mathrm{x}$ & $\mathrm{y}$ & $\mathrm{z}$ & $\mathrm{U}(\mathrm{eq})$ \\
\hline $\mathrm{O}(1)$ & $7969(7)$ & $-954(4)$ & $2640(2)$ & $23(1)$ \\
\hline $\mathrm{N}(1)$ & $3205(9)$ & $-1052(4)$ & $2290(2)$ & $16(1)$ \\
\hline $\mathrm{C}(1)$ & $3219(12)$ & $-3111(6)$ & $769(3)$ & $24(2)$ \\
\hline $\mathrm{C}(2)$ & $5006(12)$ & $-2399(6)$ & $881(3)$ & $20(2)$ \\
\hline $\mathrm{C}(3)$ & $5038(11)$ & $-1687(6)$ & $1376(3)$ & $17(1)$ \\
\hline $\mathrm{C}(4)$ & $3251(11)$ & $-1680(5)$ & $1765(3)$ & $14(1)$ \\
\hline$C(5)$ & $4474(10)$ & $14(5)$ & $2373(3)$ & $14(1)$ \\
\hline$C(6)$ & $4162(11)$ & $945(6)$ & 1891(3) & $16(1)$ \\
\hline$C(7)$ & $6469(11)$ & $1374(5)$ & $1754(3)$ & $15(1)$ \\
\hline$C(8)$ & $6844(11)$ & $2335(5)$ & $1342(3)$ & $16(1)$ \\
\hline $\mathrm{C}(9)$ & $5249(11)$ & $2618(5)$ & $936(3)$ & $14(1)$ \\
\hline $\mathrm{C}(10)$ & $5639(11)$ & $3499(5)$ & $505(3)$ & $14(1)$ \\
\hline $\mathrm{C}(11)$ & $7693(10)$ & 4098(5) & $513(3)$ & $15(1)$ \\
\hline$C(12)$ & $8092(12)$ & 4953(6) & $78(3)$ & $18(2)$ \\
\hline$C(13)$ & $6530(12)$ & $5189(6)$ & $-339(3)$ & $21(2)$ \\
\hline$C(14)$ & $3869(11)$ & $475(6)$ & $2985(2)$ & $18(2)$ \\
\hline$C(15)$ & $7017(11)$ & $-175(5)$ & $2384(3)$ & $16(1)$ \\
\hline$C(16)$ & $8019(11)$ & $744(5)$ & $2035(3)$ & $17(1)$ \\
\hline$C(17)$ & $4055(11)$ & $3756(5)$ & $64(3)$ & $16(1)$ \\
\hline$C(18)$ & $4477(12)$ & $4584(5)$ & $-353(3)$ & $21(2)$ \\
\hline$C(19)$ & $9278(10)$ & $3801(5)$ & $952(3)$ & $15(1)$ \\
\hline $\mathrm{C}(20)$ & 8894(11) & $2939(5)$ & $1344(3)$ & $16(1)$ \\
\hline $\mathrm{C}(21)$ & $1415(11)$ & $-2391(5)$ & $1640(3)$ & $18(2)$ \\
\hline $\mathrm{C}(22)$ & $1428(12)$ & $-3097(6)$ & $1147(3)$ & $22(2)$ \\
\hline
\end{tabular}


Table S15. Bond lengths $[\AA]$ and angles $\left[{ }^{\circ}\right]$ for twin5.

\begin{tabular}{|c|c|}
\hline $\mathrm{O}(1)-\mathrm{C}(15)$ & $1.219(7)$ \\
\hline $\mathrm{N}(1)-\mathrm{C}(4)$ & $1.400(7)$ \\
\hline $\mathrm{N}(1)-\mathrm{C}(5)$ & $1.468(8)$ \\
\hline $\mathrm{N}(1)-\mathrm{H}(4)$ & 1.0389 \\
\hline $\mathrm{C}(1)-\mathrm{C}(2)$ & $1.382(10)$ \\
\hline$C(1)-C(22)$ & $1.382(9)$ \\
\hline $\mathrm{C}(1)-\mathrm{H}(1)$ & 0.9500 \\
\hline$C(2)-C(3)$ & $1.399(9)$ \\
\hline $\mathrm{C}(2)-\mathrm{H}(20)$ & 0.9500 \\
\hline$C(3)-C(4)$ & $1.394(9)$ \\
\hline $\mathrm{C}(3)-\mathrm{H}(3)$ & 0.9500 \\
\hline$C(4)-C(21)$ & $1.410(9)$ \\
\hline$C(5)-C(14)$ & $1.537(8)$ \\
\hline$C(5)-C(15)$ & $1.549(9)$ \\
\hline$C(5)-C(6)$ & $1.552(8)$ \\
\hline$C(6)-C(7)$ & $1.510(9)$ \\
\hline $\mathrm{C}(6)-\mathrm{H}(9)$ & 0.9900 \\
\hline $\mathrm{C}(6)-\mathrm{H}(10)$ & 0.9900 \\
\hline$C(7)-C(16)$ & $1.347(9)$ \\
\hline$C(7)-C(8)$ & $1.476(8)$ \\
\hline$C(8)-C(9)$ & $1.373(9)$ \\
\hline$C(8)-C(20)$ & $1.421(9)$ \\
\hline$C(9)-C(10)$ & $1.437(8)$ \\
\hline $\mathrm{C}(9)-\mathrm{H}(16)$ & 0.9500 \\
\hline$C(10)-C(17)$ & $1.418(8)$ \\
\hline $\mathrm{C}(10)-\mathrm{C}(11)$ & $1.421(8)$ \\
\hline $\mathrm{C}(11)-\mathrm{C}(12)$ & $1.423(8)$ \\
\hline $\mathrm{C}(11)-\mathrm{C}(19)$ & $1.426(8)$ \\
\hline$C(12)-C(13)$ & $1.365(9)$ \\
\hline $\mathrm{C}(12)-\mathrm{H}(13)$ & 0.9500 \\
\hline $\mathrm{C}(13)-\mathrm{C}(18)$ & $1.423(10)$ \\
\hline $\mathrm{C}(13)-\mathrm{H}(2)$ & 0.9500 \\
\hline $\mathrm{C}(14)-\mathrm{H}(6)$ & 0.9800 \\
\hline $\mathrm{C}(14)-\mathrm{H}(7)$ & 0.9800 \\
\hline $\mathrm{C}(14)-\mathrm{H}(8)$ & 0.9800 \\
\hline
\end{tabular}




\begin{tabular}{|c|c|}
\hline$C(15)-C(16)$ & $1.462(9)$ \\
\hline $\mathrm{C}(16)-\mathrm{H}(17)$ & 0.9500 \\
\hline $\mathrm{C}(17)-\mathrm{C}(18)$ & $1.376(8)$ \\
\hline $\mathrm{C}(17)-\mathrm{H}(15)$ & 0.9500 \\
\hline $\mathrm{C}(18)-\mathrm{H}(14)$ & 0.9500 \\
\hline$C(19)-C(20)$ & $1.361(8)$ \\
\hline $\mathrm{C}(19)-\mathrm{H}(12)$ & 0.9500 \\
\hline $\mathrm{C}(20)-\mathrm{H}(11)$ & 0.9500 \\
\hline $\mathrm{C}(21)-\mathrm{C}(22)$ & $1.390(9)$ \\
\hline $\mathrm{C}(21)-\mathrm{H}(18)$ & 0.9500 \\
\hline $\mathrm{C}(22)-\mathrm{H}(19)$ & 0.9500 \\
\hline $\mathrm{C}(4)-\mathrm{N}(1)-\mathrm{C}(5)$ & $122.6(5)$ \\
\hline $\mathrm{C}(4)-\mathrm{N}(1)-\mathrm{H}(4)$ & 116.1 \\
\hline $\mathrm{C}(5)-\mathrm{N}(1)-\mathrm{H}(4)$ & 107.8 \\
\hline $\mathrm{C}(2)-\mathrm{C}(1)-\mathrm{C}(22)$ & $119.1(6)$ \\
\hline $\mathrm{C}(2)-\mathrm{C}(1)-\mathrm{H}(1)$ & 120.4 \\
\hline $\mathrm{C}(22)-\mathrm{C}(1)-\mathrm{H}(1)$ & 120.4 \\
\hline $\mathrm{C}(1)-\mathrm{C}(2)-\mathrm{C}(3)$ & $120.9(6)$ \\
\hline $\mathrm{C}(1)-\mathrm{C}(2)-\mathrm{H}(20)$ & 119.5 \\
\hline $\mathrm{C}(3)-\mathrm{C}(2)-\mathrm{H}(20)$ & 119.5 \\
\hline $\mathrm{C}(4)-\mathrm{C}(3)-\mathrm{C}(2)$ & $120.3(6)$ \\
\hline $\mathrm{C}(4)-\mathrm{C}(3)-\mathrm{H}(3)$ & 119.8 \\
\hline $\mathrm{C}(2)-\mathrm{C}(3)-\mathrm{H}(3)$ & 119.8 \\
\hline $\mathrm{C}(3)-\mathrm{C}(4)-\mathrm{N}(1)$ & $124.1(6)$ \\
\hline $\mathrm{C}(3)-\mathrm{C}(4)-\mathrm{C}(21)$ & $118.4(6)$ \\
\hline$N(1)-C(4)-C(21)$ & $117.4(6)$ \\
\hline $\mathrm{N}(1)-\mathrm{C}(5)-\mathrm{C}(14)$ & $106.7(5)$ \\
\hline $\mathrm{N}(1)-\mathrm{C}(5)-\mathrm{C}(15)$ & $113.5(5)$ \\
\hline$C(14)-C(5)-C(15)$ & $105.6(5)$ \\
\hline$N(1)-C(5)-C(6)$ & $115.6(5)$ \\
\hline$C(14)-C(5)-C(6)$ & $111.8(5)$ \\
\hline$C(15)-C(5)-C(6)$ & $103.3(5)$ \\
\hline$C(7)-C(6)-C(5)$ & $105.3(5)$ \\
\hline $\mathrm{C}(7)-\mathrm{C}(6)-\mathrm{H}(9)$ & 110.7 \\
\hline $\mathrm{C}(5)-\mathrm{C}(6)-\mathrm{H}(9)$ & 110.7 \\
\hline $\mathrm{C}(7)-\mathrm{C}(6)-\mathrm{H}(10)$ & 110.7 \\
\hline $\mathrm{C}(5)-\mathrm{C}(6)-\mathrm{H}(10)$ & 110.7 \\
\hline $\mathrm{H}(9)-\mathrm{C}(6)-\mathrm{H}(10)$ & 108.8 \\
\hline
\end{tabular}




\begin{tabular}{|c|c|}
\hline$C(16)-C(7)-C(8)$ & $127.3(6)$ \\
\hline$C(16)-C(7)-C(6)$ & $111.3(5)$ \\
\hline $\mathrm{C}(8)-\mathrm{C}(7)-\mathrm{C}(6)$ & $121.4(6)$ \\
\hline$C(9)-C(8)-C(20)$ & $119.6(6)$ \\
\hline C(9)-C(8)-C(7) & $120.1(6)$ \\
\hline $\mathrm{C}(20)-\mathrm{C}(8)-\mathrm{C}(7)$ & $120.3(6)$ \\
\hline$C(8)-C(9)-C(10)$ & $121.0(6)$ \\
\hline $\mathrm{C}(8)-\mathrm{C}(9)-\mathrm{H}(16)$ & 119.5 \\
\hline $\mathrm{C}(10)-\mathrm{C}(9)-\mathrm{H}(16)$ & 119.5 \\
\hline $\mathrm{C}(17)-\mathrm{C}(10)-\mathrm{C}(11)$ & $119.5(6)$ \\
\hline $\mathrm{C}(17)-\mathrm{C}(10)-\mathrm{C}(9)$ & $121.6(6)$ \\
\hline $\mathrm{C}(11)-\mathrm{C}(10)-\mathrm{C}(9)$ & $118.9(6)$ \\
\hline$C(10)-C(11)-C(12)$ & $118.8(6)$ \\
\hline $\mathrm{C}(10)-\mathrm{C}(11)-\mathrm{C}(19)$ & $118.3(6)$ \\
\hline $\mathrm{C}(12)-\mathrm{C}(11)-\mathrm{C}(19)$ & $123.0(6)$ \\
\hline$C(13)-C(12)-C(11)$ & $120.5(6)$ \\
\hline $\mathrm{C}(13)-\mathrm{C}(12)-\mathrm{H}(13)$ & 119.7 \\
\hline $\mathrm{C}(11)-\mathrm{C}(12)-\mathrm{H}(13)$ & 119.7 \\
\hline $\mathrm{C}(12)-\mathrm{C}(13)-\mathrm{C}(18)$ & $121.0(6)$ \\
\hline $\mathrm{C}(12)-\mathrm{C}(13)-\mathrm{H}(2)$ & 119.5 \\
\hline $\mathrm{C}(18)-\mathrm{C}(13)-\mathrm{H}(2)$ & 119.5 \\
\hline $\mathrm{C}(5)-\mathrm{C}(14)-\mathrm{H}(6)$ & 109.5 \\
\hline $\mathrm{C}(5)-\mathrm{C}(14)-\mathrm{H}(7)$ & 109.5 \\
\hline $\mathrm{H}(6)-\mathrm{C}(14)-\mathrm{H}(7)$ & 109.5 \\
\hline $\mathrm{C}(5)-\mathrm{C}(14)-\mathrm{H}(8)$ & 109.5 \\
\hline $\mathrm{H}(6)-\mathrm{C}(14)-\mathrm{H}(8)$ & 109.5 \\
\hline $\mathrm{H}(7)-\mathrm{C}(14)-\mathrm{H}(8)$ & 109.5 \\
\hline $\mathrm{O}(1)-\mathrm{C}(15)-\mathrm{C}(16)$ & $127.4(6)$ \\
\hline $\mathrm{O}(1)-\mathrm{C}(15)-\mathrm{C}(5)$ & $125.4(6)$ \\
\hline$C(16)-C(15)-C(5)$ & $107.3(6)$ \\
\hline$C(7)-C(16)-C(15)$ & $111.5(6)$ \\
\hline $\mathrm{C}(7)-\mathrm{C}(16)-\mathrm{H}(17)$ & 124.2 \\
\hline $\mathrm{C}(15)-\mathrm{C}(16)-\mathrm{H}(17)$ & 124.2 \\
\hline $\mathrm{C}(18)-\mathrm{C}(17)-\mathrm{C}(10)$ & $120.8(6)$ \\
\hline $\mathrm{C}(18)-\mathrm{C}(17)-\mathrm{H}(15)$ & 119.6 \\
\hline $\mathrm{C}(10)-\mathrm{C}(17)-\mathrm{H}(15)$ & 119.6 \\
\hline$C(17)-C(18)-C(13)$ & $119.4(7)$ \\
\hline $\mathrm{C}(17)-\mathrm{C}(18)-\mathrm{H}(14)$ & 120.3 \\
\hline
\end{tabular}




$\begin{array}{ll}\mathrm{C}(13)-\mathrm{C}(18)-\mathrm{H}(14) & 120.3 \\ \mathrm{C}(20)-\mathrm{C}(19)-\mathrm{C}(11) & 121.6(6) \\ \mathrm{C}(20)-\mathrm{C}(19)-\mathrm{H}(12) & 119.2 \\ \mathrm{C}(11)-\mathrm{C}(19)-\mathrm{H}(12) & 119.2 \\ \mathrm{C}(19)-\mathrm{C}(20)-\mathrm{C}(8) & 120.6(6) \\ \mathrm{C}(19)-\mathrm{C}(20)-\mathrm{H}(11) & 119.7 \\ \mathrm{C}(8)-\mathrm{C}(20)-\mathrm{H}(11) & 119.7 \\ \mathrm{C}(22)-\mathrm{C}(21)-\mathrm{C}(4) & 120.2(6) \\ \mathrm{C}(22)-\mathrm{C}(21)-\mathrm{H}(18) & 119.9 \\ \mathrm{C}(4)-\mathrm{C}(21)-\mathrm{H}(18) & 119.9 \\ \mathrm{C}(1)-\mathrm{C}(22)-\mathrm{C}(21) & 121.0(7) \\ \mathrm{C}(1)-\mathrm{C}(22)-\mathrm{H}(19) & 119.5 \\ \mathrm{C}(21)-\mathrm{C}(22)-\mathrm{H}(19) & 119.5\end{array}$

Symmetry transformations used to generate equivalent atoms:

Table S16. Anisotropic displacement parameters $\left(\AA^{2} \times 10^{3}\right)$ for twin5. The anisotropic displacement factor exponent takes the form: $\quad-2 \pi^{2}\left[h^{2} a^{* 2} U^{11}+\ldots+2 h k a^{*} b^{*} U^{12}\right]$

\begin{tabular}{|c|c|c|c|c|c|c|}
\hline & $\mathrm{U}^{11}$ & $\mathrm{U}^{22}$ & $\mathrm{U}^{33}$ & $\mathrm{U}^{23}$ & $\mathrm{U}^{13}$ & $\mathrm{U}^{12}$ \\
\hline $\mathrm{O}(1)$ & $24(2)$ & $24(2)$ & $22(2)$ & $5(2)$ & $-4(2)$ & $2(2)$ \\
\hline $\mathrm{N}(1)$ & $20(3)$ & $15(2)$ & $13(3)$ & $0(2)$ & $1(2)$ & $-2(2)$ \\
\hline $\mathrm{C}(1)$ & $34(4)$ & $20(3)$ & $16(3)$ & $-1(3)$ & $-1(3)$ & $7(3)$ \\
\hline$C(2)$ & $28(4)$ & $17(3)$ & $15(3)$ & $3(3)$ & $2(3)$ & $7(3)$ \\
\hline$C(3)$ & $19(3)$ & $16(3)$ & $17(3)$ & $1(3)$ & $-2(3)$ & $3(3)$ \\
\hline$C(4)$ & $18(3)$ & $10(3)$ & $15(3)$ & $2(2)$ & $-3(2)$ & $4(3)$ \\
\hline$C(5)$ & $15(3)$ & $16(3)$ & $10(3)$ & $-3(2)$ & $1(2)$ & $0(2)$ \\
\hline$C(6)$ & $15(3)$ & $17(3)$ & $16(3)$ & $2(3)$ & $2(2)$ & 1(3) \\
\hline$C(7)$ & $17(3)$ & $15(3)$ & $14(3)$ & $-4(2)$ & $0(2)$ & $-1(2)$ \\
\hline$C(8)$ & $15(3)$ & $14(3)$ & $18(3)$ & $-2(2)$ & $3(2)$ & $2(2)$ \\
\hline $\mathrm{C}(9)$ & $12(3)$ & $14(3)$ & $16(3)$ & $-2(2)$ & $2(2)$ & $0(3)$ \\
\hline$C(10)$ & $18(3)$ & $12(3)$ & $13(3)$ & $-3(2)$ & $5(2)$ & $-1(2)$ \\
\hline $\mathrm{C}(11)$ & $17(3)$ & $13(3)$ & $15(3)$ & $-5(2)$ & $5(2)$ & $-1(2)$ \\
\hline$C(12)$ & $19(3)$ & $15(3)$ & $18(3)$ & $0(3)$ & $4(3)$ & $-5(3)$ \\
\hline$C(13)$ & $30(3)$ & $15(3)$ & $16(3)$ & $-1(3)$ & $5(3)$ & $-1(3)$ \\
\hline$C(14)$ & $17(4)$ & $23(3)$ & $16(3)$ & $-6(3)$ & $0(3)$ & $-2(3)$ \\
\hline$C(15)$ & $17(3)$ & $19(3)$ & $11(3)$ & $0(3)$ & $2(2)$ & $-2(2)$ \\
\hline
\end{tabular}




\begin{tabular}{lllllll}
$\mathrm{C}(16)$ & $12(3)$ & $23(3)$ & $15(3)$ & $-1(3)$ & $1(3)$ & $0(3)$ \\
$\mathrm{C}(17)$ & $18(3)$ & $13(3)$ & $17(3)$ & $-3(2)$ & $-2(3)$ & $-5(3)$ \\
$\mathrm{C}(18)$ & $28(4)$ & $17(3)$ & $16(3)$ & $0(3)$ & $-1(3)$ & $1(3)$ \\
$\mathrm{C}(19)$ & $9(3)$ & $17(3)$ & $19(3)$ & $-3(3)$ & $1(2)$ & $-5(3)$ \\
$\mathrm{C}(20)$ & $17(3)$ & $15(3)$ & $16(3)$ & $-2(3)$ & $2(3)$ & $0(3)$ \\
$\mathrm{C}(21)$ & $22(3)$ & $17(3)$ & $16(3)$ & $1(3)$ & $0(3)$ & $4(3)$ \\
$\mathrm{C}(22)$ & $28(4)$ & $19(3)$ & $20(3)$ & $-3(3)$ & $-3(3)$ & $2(3)$ \\
\hline
\end{tabular}

Table S17. Hydrogen coordinates ( x 10 $)$ and isotropic displacement parameters $\left(\AA^{2} \times 10^{3}\right)$ for twin5.

\begin{tabular}{|c|c|c|c|c|}
\hline & $\mathrm{x}$ & $\mathrm{y}$ & $\mathrm{z}$ & $\mathrm{U}(\mathrm{eq})$ \\
\hline $\mathrm{H}(1)$ & 3221 & -3603 & 435 & 28 \\
\hline $\mathrm{H}(20)$ & 6230 & -2394 & 618 & 24 \\
\hline $\mathrm{H}(3)$ & 6284 & -1206 & 1448 & 21 \\
\hline $\mathrm{H}(9)$ & 3465 & 609 & 1537 & 19 \\
\hline $\mathrm{H}(10)$ & 3216 & 1581 & 2036 & 19 \\
\hline $\mathrm{H}(16)$ & 3867 & 2224 & 940 & 17 \\
\hline $\mathrm{H}(13)$ & 9455 & 5363 & 77 & 21 \\
\hline $\mathrm{H}(2)$ & 6818 & 5766 & -625 & 25 \\
\hline $\mathrm{H}(6)$ & 2262 & 592 & 3009 & 28 \\
\hline $\mathrm{H}(7)$ & 4630 & 1209 & 3052 & 28 \\
\hline $\mathrm{H}(8)$ & 4330 & -83 & 3284 & 28 \\
\hline $\mathrm{H}(17)$ & 9570 & 881 & 2008 & 20 \\
\hline $\mathrm{H}(15)$ & 2686 & 3351 & 57 & 19 \\
\hline $\mathrm{H}(14)$ & 3408 & 4751 & -647 & 25 \\
\hline $\mathrm{H}(12)$ & 10635 & 4216 & 972 & 18 \\
\hline $\mathrm{H}(11)$ & 10007 & 2739 & 1621 & 19 \\
\hline $\mathrm{H}(18)$ & 164 & -2388 & 1894 & 22 \\
\hline $\mathrm{H}(19)$ & 187 & -3578 & 1070 & 27 \\
\hline $\mathrm{H}(4)$ & 1653 & -935 & 2479 & 80 \\
\hline
\end{tabular}




\subsection{X-ray structure of 7a':}
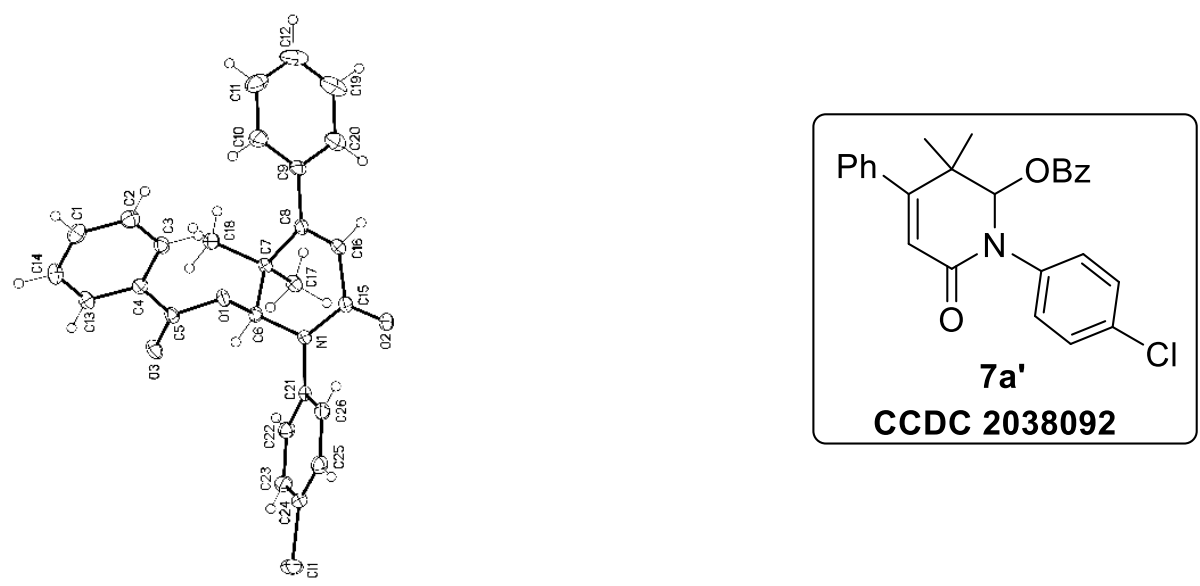

Figure S5. X-ray structure of 7a'. Displacement ellipsoids are shown at the $50 \%$ probability level.

Table S18. Crystal data and structure refinement for mo_200817lt_0m_a.

Identification code

Empirical formula

Formula weight

Temperature

Wavelength

Crystal system

Space group

Unit cell dimensions

Volume

$\mathrm{Z}$

Density (calculated)

Absorption coefficient

$\mathrm{F}(000)$

Crystal size

Theta range for data collection

Index ranges

Reflections collected

Independent reflections
mo_200817LT_0m_a

$\mathrm{C} 26 \mathrm{H} 22 \mathrm{ClN} \mathrm{O} 3$

431.89

100(2) K

$0.71073 \AA$

Monoclinic

$\mathrm{P} 21 / \mathrm{c}$

$a=9.6980(5) \AA$

$\alpha=90^{\circ}$

$\mathrm{b}=21.5874(11) \AA$

$\beta=108.115(2)^{\circ}$.

$c=10.7583(6) \AA$

$\gamma=90^{\circ}$

2140.7(2) $\AA^{3}$

$1.340 \mathrm{Mg} / \mathrm{m}^{3}$

$0.207 \mathrm{~mm}^{-1}$

904

$0.26 \times 0.09 \times 0.08 \mathrm{~mm}^{3}$

1.887 to $26.512^{\circ}$.

$-12<=\mathrm{h}<=12,-22<=\mathrm{k}<=27,-13<=\mathrm{k}<=13$

27031

$4424[\mathrm{R}($ int $)=0.0428]$ 
Completeness to theta $=25.242^{\circ}$

Absorption correction

Max. and min. transmission

Refinement method

Data / restraints / parameters

Goodness-of-fit on $\mathrm{F}^{2}$

Final $\mathrm{R}$ indices $[\mathrm{I}>2 \operatorname{sigma}(\mathrm{I})]$

$\mathrm{R}$ indices (all data)

Extinction coefficient

Largest diff. peak and hole
$100.0 \%$

Semi-empirical from equivalents

0.7454 and 0.7126

Full-matrix least-squares on $\mathrm{F}^{2}$

4424 / 0 / 282

1.033

$\mathrm{R} 1=0.0360, \mathrm{wR} 2=0.0800$

$\mathrm{R} 1=0.0506, \mathrm{wR} 2=0.0863$

$\mathrm{n} / \mathrm{a}$

0.302 and -0.320 e. $\AA^{-3}$

Table S19. Atomic coordinates ( $\left.\mathrm{x} 10^{4}\right)$ and equivalent isotropic displacement parameters $\left(\AA^{2} \times 10^{3}\right)$ for mo_2008171t_0m_a. U(eq) is defined as one third of the trace of the orthogonalized $\mathrm{U}^{\mathrm{ij}}$ tensor.

\begin{tabular}{|c|c|c|c|c|}
\hline & $\mathrm{x}$ & $\mathrm{y}$ & $\mathrm{z}$ & $\mathrm{U}(\mathrm{eq})$ \\
\hline $\mathrm{Cl}(1)$ & $1574(1)$ & $7946(1)$ & $716(1)$ & $31(1)$ \\
\hline $\mathrm{O}(1)$ & $2848(1)$ & $4788(1)$ & 2059(1) & $16(1)$ \\
\hline $\mathrm{O}(2)$ & $3245(1)$ & $5832(1)$ & $5374(1)$ & $24(1)$ \\
\hline $\mathrm{O}(3)$ & 1595(1) & $4908(1)$ & $-82(1)$ & $21(1)$ \\
\hline $\mathrm{N}(1)$ & $2008(1)$ & $5496(1)$ & $3316(1)$ & $15(1)$ \\
\hline $\mathrm{C}(1)$ & $6142(2)$ & $3786(1)$ & $-50(2)$ & $22(1)$ \\
\hline$C(2)$ & $6181(2)$ & $3870(1)$ & $1240(2)$ & $21(1)$ \\
\hline$C(3)$ & $5066(2)$ & $4175(1)$ & $1528(2)$ & $18(1)$ \\
\hline $\mathrm{C}(4)$ & $3892(2)$ & $4400(1)$ & $513(1)$ & $15(1)$ \\
\hline$C(5)$ & $2658(2)$ & $4727(1)$ & $763(1)$ & $15(1)$ \\
\hline$C(6)$ & $1586(2)$ & 4973(1) & $2437(1)$ & $14(1)$ \\
\hline$C(7)$ & $1095(2)$ & $4415(1)$ & $3072(1)$ & $15(1)$ \\
\hline $\mathrm{C}(8)$ & $2263(2)$ & $4281(1)$ & $4374(1)$ & $16(1)$ \\
\hline $\mathrm{C}(9)$ & $2527(2)$ & $3638(1)$ & 4909(1) & $18(1)$ \\
\hline $\mathrm{C}(10)$ & $3373(2)$ & $3228(1)$ & $4456(2)$ & $25(1)$ \\
\hline $\mathrm{C}(11)$ & $3678(2)$ & $2640(1)$ & $5001(2)$ & $32(1)$ \\
\hline$C(12)$ & $3151(2)$ & $2460(1)$ & $5998(2)$ & $34(1)$ \\
\hline$C(13)$ & $3848(2)$ & 4311(1) & $-782(1)$ & $19(1)$ \\
\hline$C(14)$ & $4967(2)$ & $4004(1)$ & $-1057(2)$ & $22(1)$ \\
\hline$C(15)$ & $2786(2)$ & $5403(1)$ & $4618(1)$ & $17(1)$ \\
\hline$C(16)$ & $3004(2)$ & $4753(1)$ & $5055(1)$ & $18(1)$ \\
\hline $\mathrm{C}(17)$ & $-329(2)$ & $4586(1)$ & $3335(1)$ & $18(1)$ \\
\hline
\end{tabular}




\begin{tabular}{lrlll}
$\mathrm{C}(18)$ & $789(2)$ & $3866(1)$ & $2116(1)$ & $19(1)$ \\
$\mathrm{C}(19)$ & $2315(2)$ & $2863(1)$ & $6452(2)$ & $34(1)$ \\
$\mathrm{C}(20)$ & $2004(2)$ & $3450(1)$ & $5914(2)$ & $26(1)$ \\
$\mathrm{C}(21)$ & $1873(2)$ & $6106(1)$ & $2752(1)$ & $15(1)$ \\
$\mathrm{C}(22)$ & $577(2)$ & $6284(1)$ & $1839(1)$ & $18(1)$ \\
$\mathrm{C}(23)$ & $455(2)$ & $6856(1)$ & $1224(2)$ & $21(1)$ \\
$\mathrm{C}(24)$ & $1638(2)$ & $7248(1)$ & $1543(2)$ & $20(1)$ \\
$\mathrm{C}(25)$ & $2926(2)$ & $7086(1)$ & $2484(2)$ & $20(1)$ \\
$\mathrm{C}(26)$ & $3040(2)$ & $6514(1)$ & $3085(1)$ & $17(1)$ \\
\hline
\end{tabular}

Table S20. Bond lengths $[\AA]$ and angles $\left[{ }^{\circ}\right]$ for mo_200817lt_0m_a.

\begin{tabular}{ll}
\hline $\mathrm{Cl}(1)-\mathrm{C}(24)$ & $1.7413(15)$ \\
$\mathrm{O}(1)-\mathrm{C}(5)$ & $1.3553(17)$ \\
$\mathrm{O}(1)-\mathrm{C}(6)$ & $1.4601(17)$ \\
$\mathrm{O}(2)-\mathrm{C}(15)$ & $1.2225(17)$ \\
$\mathrm{O}(3)-\mathrm{C}(5)$ & $1.2087(17)$ \\
$\mathrm{N}(1)-\mathrm{C}(15)$ & $1.3838(18)$ \\
$\mathrm{N}(1)-\mathrm{C}(21)$ & $1.4388(18)$ \\
$\mathrm{N}(1)-\mathrm{C}(6)$ & $1.4483(17)$ \\
$\mathrm{C}(1)-\mathrm{C}(14)$ & $1.388(2)$ \\
$\mathrm{C}(1)-\mathrm{C}(2)$ & $1.388(2)$ \\
$\mathrm{C}(1)-\mathrm{H}(1)$ & 0.9500 \\
$\mathrm{C}(2)-\mathrm{C}(3)$ & $1.381(2)$ \\
$\mathrm{C}(2)-\mathrm{H}(22)$ & 0.9500 \\
$\mathrm{C}(3)-\mathrm{C}(4)$ & $1.397(2)$ \\
$\mathrm{C}(3)-\mathrm{H}(3)$ & 0.9500 \\
$\mathrm{C}(4)-\mathrm{C}(13)$ & $1.394(2)$ \\
$\mathrm{C}(4)-\mathrm{C}(5)$ & $1.484(2)$ \\
$\mathrm{C}(6)-\mathrm{C}(7)$ & $1.532(2)$ \\
$\mathrm{C}(6)-\mathrm{H}(21)$ & 1.0000 \\
$\mathrm{C}(7)-\mathrm{C}(8)$ & $1.5305(19)$ \\
$\mathrm{C}(7)-\mathrm{C}(18)$ & $1.5360(19)$ \\
$\mathrm{C}(7)-\mathrm{C}(17)$ & $1.538(2)$ \\
$\mathrm{C}(8)-\mathrm{C}(16)$ & $1.328(2)$ \\
$\mathrm{C}(8)-\mathrm{C}(9)$ & $1.493(2)$ \\
$\mathrm{C}(9)-\mathrm{C}(20)$ & $1.389(2)$ \\
& \\
&
\end{tabular}




\begin{tabular}{|c|c|}
\hline$C(9)-C(10)$ & $1.394(2)$ \\
\hline $\mathrm{C}(10)-\mathrm{C}(11)$ & $1.391(2)$ \\
\hline $\mathrm{C}(10)-\mathrm{H}(16)$ & 0.9500 \\
\hline$C(11)-C(12)$ & $1.380(3)$ \\
\hline $\mathrm{C}(11)-\mathrm{H}(15)$ & 0.9500 \\
\hline$C(12)-C(19)$ & $1.377(3)$ \\
\hline $\mathrm{C}(12)-\mathrm{H}(2)$ & 0.9500 \\
\hline $\mathrm{C}(13)-\mathrm{C}(14)$ & $1.381(2)$ \\
\hline $\mathrm{C}(13)-\mathrm{H}(5)$ & 0.9500 \\
\hline $\mathrm{C}(14)-\mathrm{H}(4)$ & 0.9500 \\
\hline$C(15)-C(16)$ & $1.475(2)$ \\
\hline $\mathrm{C}(16)-\mathrm{H}(6)$ & 0.9500 \\
\hline $\mathrm{C}(17)-\mathrm{H}(8)$ & 0.9800 \\
\hline $\mathrm{C}(17)-\mathrm{H}(7)$ & 0.9800 \\
\hline $\mathrm{C}(17)-\mathrm{H}(9)$ & 0.9800 \\
\hline $\mathrm{C}(18)-\mathrm{H}(11)$ & 0.9800 \\
\hline $\mathrm{C}(18)-\mathrm{H}(10)$ & 0.9800 \\
\hline $\mathrm{C}(18)-\mathrm{H}(12)$ & 0.9800 \\
\hline $\mathrm{C}(19)-\mathrm{C}(20)$ & $1.388(2)$ \\
\hline $\mathrm{C}(19)-\mathrm{H}(14)$ & 0.9500 \\
\hline $\mathrm{C}(20)-\mathrm{H}(13)$ & 0.9500 \\
\hline $\mathrm{C}(21)-\mathrm{C}(22)$ & $1.387(2)$ \\
\hline $\mathrm{C}(21)-\mathrm{C}(26)$ & $1.390(2)$ \\
\hline $\mathrm{C}(22)-\mathrm{C}(23)$ & $1.388(2)$ \\
\hline $\mathrm{C}(22)-\mathrm{H}(20)$ & 0.9500 \\
\hline $\mathrm{C}(23)-\mathrm{C}(24)$ & $1.381(2)$ \\
\hline $\mathrm{C}(23)-\mathrm{H}(19)$ & 0.9500 \\
\hline$C(24)-C(25)$ & $1.386(2)$ \\
\hline$C(25)-C(26)$ & $1.381(2)$ \\
\hline $\mathrm{C}(25)-\mathrm{H}(18)$ & 0.9500 \\
\hline $\mathrm{C}(26)-\mathrm{H}(17)$ & 0.9500 \\
\hline $\mathrm{C}(5)-\mathrm{O}(1)-\mathrm{C}(6)$ & $117.13(11)$ \\
\hline $\mathrm{C}(15)-\mathrm{N}(1)-\mathrm{C}(21)$ & $120.66(12)$ \\
\hline$C(15)-N(1)-C(6)$ & $120.09(11)$ \\
\hline $\mathrm{C}(21)-\mathrm{N}(1)-\mathrm{C}(6)$ & $117.94(11)$ \\
\hline $\mathrm{C}(14)-\mathrm{C}(1)-\mathrm{C}(2)$ & $119.84(15)$ \\
\hline $\mathrm{C}(14)-\mathrm{C}(1)-\mathrm{H}(1)$ & 120.1 \\
\hline $\mathrm{C}(2)-\mathrm{C}(1)-\mathrm{H}(1)$ & 120.1 \\
\hline
\end{tabular}




\begin{tabular}{|c|c|}
\hline $\mathrm{C}(3)-\mathrm{C}(2)-\mathrm{C}(1)$ & $120.39(15)$ \\
\hline $\mathrm{C}(3)-\mathrm{C}(2)-\mathrm{H}(22)$ & 119.8 \\
\hline $\mathrm{C}(1)-\mathrm{C}(2)-\mathrm{H}(22)$ & 119.8 \\
\hline $\mathrm{C}(2)-\mathrm{C}(3)-\mathrm{C}(4)$ & $119.69(14)$ \\
\hline $\mathrm{C}(2)-\mathrm{C}(3)-\mathrm{H}(3)$ & 120.2 \\
\hline $\mathrm{C}(4)-\mathrm{C}(3)-\mathrm{H}(3)$ & 120.2 \\
\hline$C(13)-C(4)-C(3)$ & $119.88(14)$ \\
\hline$C(13)-C(4)-C(5)$ & $118.05(13)$ \\
\hline$C(3)-C(4)-C(5)$ & $122.06(13)$ \\
\hline $\mathrm{O}(3)-\mathrm{C}(5)-\mathrm{O}(1)$ & $123.58(14)$ \\
\hline $\mathrm{O}(3)-\mathrm{C}(5)-\mathrm{C}(4)$ & $124.45(13)$ \\
\hline $\mathrm{O}(1)-\mathrm{C}(5)-\mathrm{C}(4)$ & $111.96(12)$ \\
\hline $\mathrm{N}(1)-\mathrm{C}(6)-\mathrm{O}(1)$ & $107.66(11)$ \\
\hline $\mathrm{N}(1)-\mathrm{C}(6)-\mathrm{C}(7)$ & $112.74(11)$ \\
\hline $\mathrm{O}(1)-\mathrm{C}(6)-\mathrm{C}(7)$ & $108.25(11)$ \\
\hline $\mathrm{N}(1)-\mathrm{C}(6)-\mathrm{H}(21)$ & 109.4 \\
\hline $\mathrm{O}(1)-\mathrm{C}(6)-\mathrm{H}(21)$ & 109.4 \\
\hline $\mathrm{C}(7)-\mathrm{C}(6)-\mathrm{H}(21)$ & 109.4 \\
\hline$C(8)-C(7)-C(6)$ & $108.22(12)$ \\
\hline$C(8)-C(7)-C(18)$ & $113.60(12)$ \\
\hline$C(6)-C(7)-C(18)$ & $109.31(12)$ \\
\hline$C(8)-C(7)-C(17)$ & $109.15(12)$ \\
\hline$C(6)-C(7)-C(17)$ & $108.32(12)$ \\
\hline $\mathrm{C}(18)-\mathrm{C}(7)-\mathrm{C}(17)$ & $108.11(12)$ \\
\hline$C(16)-C(8)-C(9)$ & $120.07(13)$ \\
\hline$C(16)-C(8)-C(7)$ & $118.67(13)$ \\
\hline $\mathrm{C}(9)-\mathrm{C}(8)-\mathrm{C}(7)$ & $121.20(12)$ \\
\hline$C(20)-C(9)-C(10)$ & $118.91(14)$ \\
\hline$C(20)-C(9)-C(8)$ & $120.75(14)$ \\
\hline$C(10)-C(9)-C(8)$ & $120.24(14)$ \\
\hline $\mathrm{C}(11)-\mathrm{C}(10)-\mathrm{C}(9)$ & $120.24(17)$ \\
\hline $\mathrm{C}(11)-\mathrm{C}(10)-\mathrm{H}(16)$ & 119.9 \\
\hline $\mathrm{C}(9)-\mathrm{C}(10)-\mathrm{H}(16)$ & 119.9 \\
\hline $\mathrm{C}(12)-\mathrm{C}(11)-\mathrm{C}(10)$ & $120.13(17)$ \\
\hline $\mathrm{C}(12)-\mathrm{C}(11)-\mathrm{H}(15)$ & 119.9 \\
\hline $\mathrm{C}(10)-\mathrm{C}(11)-\mathrm{H}(15)$ & 119.9 \\
\hline $\mathrm{C}(19)-\mathrm{C}(12)-\mathrm{C}(11)$ & $120.00(16)$ \\
\hline $\mathrm{C}(19)-\mathrm{C}(12)-\mathrm{H}(2)$ & 120.0 \\
\hline
\end{tabular}




\begin{tabular}{|c|c|}
\hline $\mathrm{C}(11)-\mathrm{C}(12)-\mathrm{H}(2)$ & 120.0 \\
\hline$C(14)-C(13)-C(4)$ & $119.89(14)$ \\
\hline $\mathrm{C}(14)-\mathrm{C}(13)-\mathrm{H}(5)$ & 120.1 \\
\hline $\mathrm{C}(4)-\mathrm{C}(13)-\mathrm{H}(5)$ & 120.1 \\
\hline $\mathrm{C}(13)-\mathrm{C}(14)-\mathrm{C}(1)$ & $120.28(14)$ \\
\hline $\mathrm{C}(13)-\mathrm{C}(14)-\mathrm{H}(4)$ & 119.9 \\
\hline $\mathrm{C}(1)-\mathrm{C}(14)-\mathrm{H}(4)$ & 119.9 \\
\hline $\mathrm{O}(2)-\mathrm{C}(15)-\mathrm{N}(1)$ & $122.28(13)$ \\
\hline $\mathrm{O}(2)-\mathrm{C}(15)-\mathrm{C}(16)$ & $121.54(13)$ \\
\hline $\mathrm{N}(1)-\mathrm{C}(15)-\mathrm{C}(16)$ & $116.17(12)$ \\
\hline$C(8)-C(16)-C(15)$ & $123.68(13)$ \\
\hline $\mathrm{C}(8)-\mathrm{C}(16)-\mathrm{H}(6)$ & 118.2 \\
\hline $\mathrm{C}(15)-\mathrm{C}(16)-\mathrm{H}(6)$ & 118.2 \\
\hline $\mathrm{C}(7)-\mathrm{C}(17)-\mathrm{H}(8)$ & 109.5 \\
\hline $\mathrm{C}(7)-\mathrm{C}(17)-\mathrm{H}(7)$ & 109.5 \\
\hline $\mathrm{H}(8)-\mathrm{C}(17)-\mathrm{H}(7)$ & 109.5 \\
\hline $\mathrm{C}(7)-\mathrm{C}(17)-\mathrm{H}(9)$ & 109.5 \\
\hline $\mathrm{H}(8)-\mathrm{C}(17)-\mathrm{H}(9)$ & 109.5 \\
\hline $\mathrm{H}(7)-\mathrm{C}(17)-\mathrm{H}(9)$ & 109.5 \\
\hline $\mathrm{C}(7)-\mathrm{C}(18)-\mathrm{H}(11)$ & 109.5 \\
\hline $\mathrm{C}(7)-\mathrm{C}(18)-\mathrm{H}(10)$ & 109.5 \\
\hline $\mathrm{H}(11)-\mathrm{C}(18)-\mathrm{H}(10)$ & 109.5 \\
\hline $\mathrm{C}(7)-\mathrm{C}(18)-\mathrm{H}(12)$ & 109.5 \\
\hline $\mathrm{H}(11)-\mathrm{C}(18)-\mathrm{H}(12)$ & 109.5 \\
\hline $\mathrm{H}(10)-\mathrm{C}(18)-\mathrm{H}(12)$ & 109.5 \\
\hline $\mathrm{C}(12)-\mathrm{C}(19)-\mathrm{C}(20)$ & $120.25(17)$ \\
\hline $\mathrm{C}(12)-\mathrm{C}(19)-\mathrm{H}(14)$ & 119.9 \\
\hline $\mathrm{C}(20)-\mathrm{C}(19)-\mathrm{H}(14)$ & 119.9 \\
\hline$C(19)-C(20)-C(9)$ & $120.47(17)$ \\
\hline $\mathrm{C}(19)-\mathrm{C}(20)-\mathrm{H}(13)$ & 119.8 \\
\hline $\mathrm{C}(9)-\mathrm{C}(20)-\mathrm{H}(13)$ & 119.8 \\
\hline$C(22)-C(21)-C(26)$ & $119.56(13)$ \\
\hline $\mathrm{C}(22)-\mathrm{C}(21)-\mathrm{N}(1)$ & $119.68(13)$ \\
\hline $\mathrm{C}(26)-\mathrm{C}(21)-\mathrm{N}(1)$ & $120.73(13)$ \\
\hline $\mathrm{C}(21)-\mathrm{C}(22)-\mathrm{C}(23)$ & $120.49(14)$ \\
\hline $\mathrm{C}(21)-\mathrm{C}(22)-\mathrm{H}(20)$ & 119.8 \\
\hline $\mathrm{C}(23)-\mathrm{C}(22)-\mathrm{H}(20)$ & 119.8 \\
\hline $\mathrm{C}(24)-\mathrm{C}(23)-\mathrm{C}(22)$ & $119.07(14)$ \\
\hline
\end{tabular}




$\begin{array}{ll}\mathrm{C}(24)-\mathrm{C}(23)-\mathrm{H}(19) & 120.5 \\ \mathrm{C}(22)-\mathrm{C}(23)-\mathrm{H}(19) & 120.5 \\ \mathrm{C}(23)-\mathrm{C}(24)-\mathrm{C}(25) & 121.12(14) \\ \mathrm{C}(23)-\mathrm{C}(24)-\mathrm{Cl}(1) & 120.59(12) \\ \mathrm{C}(25)-\mathrm{C}(24)-\mathrm{Cl}(1) & 118.23(12) \\ \mathrm{C}(26)-\mathrm{C}(25)-\mathrm{C}(24) & 119.37(14) \\ \mathrm{C}(26)-\mathrm{C}(25)-\mathrm{H}(18) & 120.3 \\ \mathrm{C}(24)-\mathrm{C}(25)-\mathrm{H}(18) & 120.3 \\ \mathrm{C}(25)-\mathrm{C}(26)-\mathrm{C}(21) & 120.33(14) \\ \mathrm{C}(25)-\mathrm{C}(26)-\mathrm{H}(17) & 119.8 \\ \mathrm{C}(21)-\mathrm{C}(26)-\mathrm{H}(17) & 119.8\end{array}$

Symmetry transformations used to generate equivalent atoms:

Table S21. Anisotropic displacement parameters $\left(\AA^{2} \times 10^{3}\right)$ for mo_200817lt_0m_a. The anisotropic displacement factor exponent takes the form: $\quad-2 \pi^{2}\left[h^{2} a^{* 2} U^{11}+\ldots+2 h k a^{*} b^{*} U^{12}\right]$

\begin{tabular}{|c|c|c|c|c|c|c|}
\hline & $\mathrm{U}^{11}$ & $\mathrm{U}^{22}$ & $\mathrm{U}^{33}$ & $\mathrm{U}^{23}$ & $\mathrm{U}^{13}$ & $\mathrm{U}^{12}$ \\
\hline $\mathrm{Cl}(1)$ & $44(1)$ & $16(1)$ & $28(1)$ & $6(1)$ & $5(1)$ & $-1(1)$ \\
\hline $\mathrm{O}(1)$ & $15(1)$ & $22(1)$ & $10(1)$ & $-1(1)$ & $4(1)$ & $2(1)$ \\
\hline $\mathrm{O}(2)$ & $36(1)$ & $18(1)$ & $14(1)$ & $-4(1)$ & $1(1)$ & $-1(1)$ \\
\hline $\mathrm{O}(3)$ & $17(1)$ & $31(1)$ & $13(1)$ & $2(1)$ & $2(1)$ & $4(1)$ \\
\hline $\mathrm{N}(1)$ & $20(1)$ & $13(1)$ & 11(1) & $0(1)$ & $2(1)$ & $1(1)$ \\
\hline $\mathrm{C}(1)$ & $19(1)$ & $22(1)$ & $27(1)$ & $-4(1)$ & $9(1)$ & 1(1) \\
\hline $\mathrm{C}(2)$ & $18(1)$ & $21(1)$ & $23(1)$ & $0(1)$ & $3(1)$ & $1(1)$ \\
\hline$C(3)$ & $20(1)$ & $18(1)$ & $15(1)$ & $-1(1)$ & $4(1)$ & $-3(1)$ \\
\hline$C(4)$ & $15(1)$ & $14(1)$ & $16(1)$ & $0(1)$ & $5(1)$ & $-3(1)$ \\
\hline$C(5)$ & $17(1)$ & $16(1)$ & $12(1)$ & $0(1)$ & $4(1)$ & $-4(1)$ \\
\hline$C(6)$ & $14(1)$ & $16(1)$ & $12(1)$ & $-2(1)$ & $3(1)$ & 1(1) \\
\hline$C(7)$ & $17(1)$ & $16(1)$ & $12(1)$ & $0(1)$ & $3(1)$ & 1(1) \\
\hline $\mathrm{C}(8)$ & $17(1)$ & $18(1)$ & $12(1)$ & $1(1)$ & $5(1)$ & $3(1)$ \\
\hline $\mathrm{C}(9)$ & $17(1)$ & $16(1)$ & $14(1)$ & $1(1)$ & $-3(1)$ & $-1(1)$ \\
\hline$C(10)$ & $27(1)$ & $21(1)$ & $21(1)$ & $0(1)$ & $2(1)$ & $3(1)$ \\
\hline $\mathrm{C}(11)$ & $29(1)$ & $20(1)$ & $34(1)$ & $-4(1)$ & $-6(1)$ & $6(1)$ \\
\hline$C(12)$ & $28(1)$ & $20(1)$ & $38(1)$ & $11(1)$ & $-11(1)$ & $-6(1)$ \\
\hline$C(13)$ & $18(1)$ & $23(1)$ & $15(1)$ & $1(1)$ & $3(1)$ & $-1(1)$ \\
\hline$C(14)$ & $23(1)$ & $28(1)$ & $18(1)$ & $-4(1)$ & $9(1)$ & $0(1)$ \\
\hline
\end{tabular}




\begin{tabular}{lllllll}
$\mathrm{C}(15)$ & $19(1)$ & $18(1)$ & $12(1)$ & $-1(1)$ & $4(1)$ & $1(1)$ \\
$\mathrm{C}(16)$ & $21(1)$ & $19(1)$ & $11(1)$ & $1(1)$ & $1(1)$ & $3(1)$ \\
$\mathrm{C}(17)$ & $18(1)$ & $21(1)$ & $15(1)$ & $2(1)$ & $4(1)$ & $1(1)$ \\
$\mathrm{C}(18)$ & $22(1)$ & $17(1)$ & $16(1)$ & $-1(1)$ & $3(1)$ & $0(1)$ \\
$\mathrm{C}(19)$ & $31(1)$ & $35(1)$ & $30(1)$ & $15(1)$ & $1(1)$ & $-6(1)$ \\
$\mathrm{C}(20)$ & $26(1)$ & $27(1)$ & $22(1)$ & $5(1)$ & $3(1)$ & $0(1)$ \\
$\mathrm{C}(21)$ & $20(1)$ & $14(1)$ & $11(1)$ & $-1(1)$ & $5(1)$ & $2(1)$ \\
$\mathrm{C}(22)$ & $18(1)$ & $16(1)$ & $18(1)$ & $-2(1)$ & $3(1)$ & $-1(1)$ \\
$\mathrm{C}(23)$ & $22(1)$ & $19(1)$ & $18(1)$ & $1(1)$ & $1(1)$ & $5(1)$ \\
$\mathrm{C}(24)$ & $30(1)$ & $13(1)$ & $17(1)$ & $0(1)$ & $7(1)$ & $2(1)$ \\
$\mathrm{C}(25)$ & $22(1)$ & $18(1)$ & $19(1)$ & $-3(1)$ & $6(1)$ & $-4(1)$ \\
$\mathrm{C}(26)$ & $16(1)$ & $20(1)$ & $15(1)$ & $-2(1)$ & $4(1)$ & $1(1)$ \\
& & & & & & \\
\hline
\end{tabular}

Table S22. Hydrogen coordinates ( $\left.\times 10^{4}\right)$ and isotropic displacement parameters $\left(\AA^{2} \times 10^{3}\right)$ for mo_200817lt_0m_a.

$\mathrm{X}$

y $\quad$ z $\quad$ U(eq)

$\begin{array}{lrrrr}\mathrm{H}(1) & 6917 & 3580 & -242 & 27 \\ \mathrm{H}(22) & 6979 & 3715 & 1929 & 26 \\ \mathrm{H}(3) & 5099 & 4233 & 2412 & 21 \\ \mathrm{H}(21) & 784 & 5100 & 1640 & 17 \\ \mathrm{H}(16) & 3743 & 3351 & 3773 & 30 \\ \mathrm{H}(15) & 4251 & 2361 & 4685 & 38 \\ \mathrm{H}(2) & 3365 & 2059 & 6372 & 40 \\ \mathrm{H}(5) & 3049 & 4463 & -1474 & 23 \\ \mathrm{H}(4) & 4933 & 3941 & -1941 & 27 \\ \mathrm{H}(6) & 3715 & 4666 & 5870 & 22 \\ \mathrm{H}(8) & -618 & 4246 & 3806 & 28 \\ \mathrm{H}(7) & -1091 & 4656 & 2502 & 28 \\ \mathrm{H}(9) & -185 & 4964 & 3864 & 28 \\ \mathrm{H}(11) & 1691 & 3740 & 1958 & 29 \\ \mathrm{H}(10) & 76 & 3989 & 1288 & 29 \\ \mathrm{H}(12) & 406 & 3518 & 2493 & 29 \\ \mathrm{H}(14) & 1950 & 2738 & 7137 & 41 \\ \mathrm{H}(13) & 1430 & 3726 & 6234 & 31\end{array}$




$\begin{array}{rrrrr}H(20) & -233 & 6012 & 1632 & 22 \\ H(19) & -431 & 6976 & 594 & 25 \\ H(18) & 3722 & 7365 & 2714 & 24 \\ H(17) & 3919 & 6400 & 3730 & 21\end{array}$

\subsection{X-ray structure of 6a:}
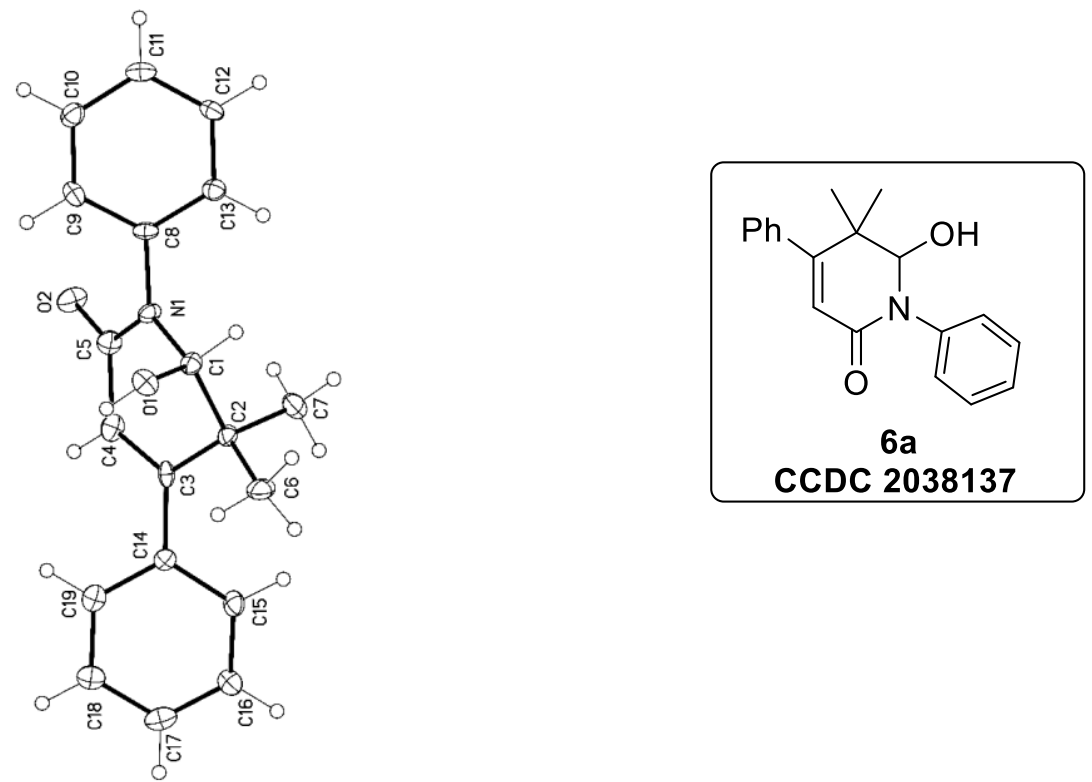

Figure S6. X-ray structure of 6a. Displacement ellipsoids are shown at the $50 \%$ probability level.

Table S23. Crystal data and structure refinement for 2007471t_a.

Identification code

Empirical formula

Formula weight

Temperature

Wavelength

Crystal system

Space group

Unit cell dimensions

Volume

Z

Density (calculated)

Absorption coefficient
200747LT_a

C19 H19 N O2

293.35

100(2) K

$0.71073 \AA$

Monoclinic

$\mathrm{P} 21$

$\mathrm{a}=9.697(2) \AA$

$\alpha=90^{\circ}$.

$\mathrm{b}=7.9555(17) \AA$

$\beta=115.199(6)^{\circ}$.

$c=10.763(3) \AA$ $\gamma=90^{\circ}$
751.3(3) $\AA^{3}$

2

$1.297 \mathrm{Mg} / \mathrm{m}^{3}$

$0.084 \mathrm{~mm}^{-1}$ 
$\mathrm{F}(000)$

Crystal size

Theta range for data collection

Index ranges

Reflections collected

Independent reflections

Completeness to theta $=25.242^{\circ}$

Absorption correction

Max. and min. transmission

Refinement method

Data / restraints / parameters

Goodness-of-fit on $\mathrm{F}^{2}$

Final $\mathrm{R}$ indices [I>2sigma(I)]

$\mathrm{R}$ indices (all data)

Absolute structure parameter

Extinction coefficient

Largest diff. peak and hole
312

$0.13 \times 0.08 \times 0.03 \mathrm{~mm}^{3}$

2.091 to $26.515^{\circ}$.

$-12<=\mathrm{h}<=12,-9<=\mathrm{k}<=9,-13<=\mathrm{l}<=13$

10513

$2922[\mathrm{R}(\mathrm{int})=0.0399]$

$99.9 \%$

Semi-empirical from equivalents

0.7454 and 0.6427

Full-matrix least-squares on $\mathrm{F}^{2}$

2922 / 1 / 202

1.617

$\mathrm{R} 1=0.1110, \mathrm{wR} 2=0.3515$

$\mathrm{R} 1=0.1177, \mathrm{wR} 2=0.3564$

2.4(9)

$\mathrm{n} / \mathrm{a}$

1.086 and -0.511 e. $\AA^{-3}$

Table S24. Atomic coordinates $\left(\mathrm{x} 10^{4}\right)$ and equivalent isotropic displacement parameters $\left(\AA^{2} \mathrm{x} 10^{3}\right)$ for 200747lt_a. U(eq) is defined as one third of the trace of the orthogonalized $\mathrm{U}^{\mathrm{ij}}$ tensor.

\begin{tabular}{|c|c|c|c|c|}
\hline & $\mathrm{x}$ & $\mathrm{y}$ & $\mathrm{z}$ & $\mathrm{U}(\mathrm{eq})$ \\
\hline $\mathrm{C}(1)$ & $6002(8)$ & $4521(10)$ & $3117(7)$ & $15(2)$ \\
\hline $\mathrm{C}(2)$ & $4479(8)$ & $5378(10)$ & $2147(7)$ & $14(2)$ \\
\hline$C(3)$ & $3370(8)$ & $5328(10)$ & $2802(7)$ & $13(2)$ \\
\hline$C(4)$ & $3948(9)$ & $5430(11)$ & $4176(7)$ & $17(2)$ \\
\hline$C(5)$ & 5581(9) & $5589(10)$ & $5077(7)$ & $17(2)$ \\
\hline$C(6)$ & $3920(9)$ & $4456(13)$ & $755(8)$ & $23(2)$ \\
\hline$C(7)$ & $4825(9)$ & $7230(12)$ & 1969(9) & $25(2)$ \\
\hline $\mathrm{C}(8)$ & $8171(8)$ & $5170(10)$ & $5350(7)$ & $13(2)$ \\
\hline$C(9)$ & $8797(9)$ & $4277(10)$ & $6574(8)$ & $17(2)$ \\
\hline$C(10)$ & 10371(9) & $4277(10)$ & $7331(8)$ & $18(2)$ \\
\hline $\mathrm{C}(11)$ & $11325(9)$ & $5159(11)$ & $6901(8)$ & $19(2)$ \\
\hline$C(12)$ & $10666(8)$ & $6035(11)$ & $5670(7)$ & $16(2)$ \\
\hline$C(13)$ & $9088(8)$ & $6049(10)$ & $4900(7)$ & $15(2)$ \\
\hline$C(14)$ & $1688(8)$ & $5221(10)$ & 1992(7) & $13(2)$ \\
\hline$C(15)$ & $913(9)$ & $6081(11)$ & $738(8)$ & $17(2)$ \\
\hline
\end{tabular}




\begin{tabular}{lrlrl}
$\mathrm{C}(16)$ & $-670(8)$ & $6029(10)$ & $51(8)$ & $16(2)$ \\
$\mathrm{C}(17)$ & $-1493(9)$ & $5147(11)$ & $561(8)$ & $19(2)$ \\
$\mathrm{C}(18)$ & $-768(9)$ & $4255(11)$ & $1791(8)$ & $18(2)$ \\
$\mathrm{C}(19)$ & $824(9)$ & $4290(11)$ & $2492(7)$ & $18(2)$ \\
$\mathrm{N}(1)$ & $6543(7)$ & $5177(9)$ & $4525(6)$ & $14(1)$ \\
$\mathrm{O}(1)$ & $5959(6)$ & $2778(8)$ & $3082(5)$ & $17(1)$ \\
$\mathrm{O}(2)$ & $6049(6)$ & $6079(9)$ & $6282(5)$ & $20(1)$ \\
\hline
\end{tabular}

Table S25. Bond lengths $\left[\AA ̊\right.$ ] and angles $\left[{ }^{\circ}\right]$ for 200747lt_a.

\begin{tabular}{ll}
\hline $\mathrm{C}(1)-\mathrm{O}(1)$ & $1.388(10)$ \\
$\mathrm{C}(1)-\mathrm{N}(1)$ & $1.472(9)$ \\
$\mathrm{C}(1)-\mathrm{C}(2)$ & $1.557(10)$ \\
$\mathrm{C}(1)-\mathrm{H}(1 \mathrm{~A})$ & 1.0000 \\
$\mathrm{C}(2)-\mathrm{C}(3)$ & $1.517(10)$ \\
$\mathrm{C}(2)-\mathrm{C}(7)$ & $1.541(12)$ \\
$\mathrm{C}(2)-\mathrm{C}(6)$ & $1.545(11)$ \\
$\mathrm{C}(3)-\mathrm{C}(4)$ & $1.342(10)$ \\
$\mathrm{C}(3)-\mathrm{C}(14)$ & $1.489(10)$ \\
$\mathrm{C}(4)-\mathrm{C}(5)$ & $1.468(11)$ \\
$\mathrm{C}(4)-\mathrm{H}(4)$ & 0.9500 \\
$\mathrm{C}(5)-\mathrm{O}(2)$ & $1.240(9)$ \\
$\mathrm{C}(5)-\mathrm{N}(1)$ & $1.343(10)$ \\
$\mathrm{C}(6)-\mathrm{H}(6 \mathrm{~A})$ & 0.9800 \\
$\mathrm{C}(6)-\mathrm{H}(6 \mathrm{~B})$ & 0.9800 \\
$\mathrm{C}(6)-\mathrm{H}(6 \mathrm{C})$ & 0.9800 \\
$\mathrm{C}(7)-\mathrm{H}(7 \mathrm{~A})$ & 0.9800 \\
$\mathrm{C}(7)-\mathrm{H}(7 \mathrm{~B})$ & 0.9800 \\
$\mathrm{C}(7)-\mathrm{H}(7 \mathrm{C})$ & 0.9800 \\
$\mathrm{C}(8)-\mathrm{C}(13)$ & $1.372(10)$ \\
$\mathrm{C}(8)-\mathrm{C}(9)$ & $1.388(11)$ \\
$\mathrm{C}(8)-\mathrm{N}(1)$ & $1.444(9)$ \\
$\mathrm{C}(9)-\mathrm{C}(10)$ & $1.391(11)$ \\
$\mathrm{C}(9)-\mathrm{H}(9)$ & 0.9500 \\
$\mathrm{C}(10)-\mathrm{C}(11)$ & $1.388(11)$ \\
$\mathrm{C}(10)-\mathrm{H}(10)$ & 0.9500 \\
$\mathrm{C}(11)-\mathrm{C}(12)$ & $1.389(11)$ \\
$\mathrm{C}(11)-\mathrm{H}(11)$ & 0.9500 \\
&
\end{tabular}




\begin{tabular}{|c|c|}
\hline $\mathrm{C}(12)-\mathrm{C}(13)$ & $1.396(10)$ \\
\hline $\mathrm{C}(12)-\mathrm{H}(12)$ & 0.9500 \\
\hline $\mathrm{C}(13)-\mathrm{H}(13)$ & 0.9500 \\
\hline $\mathrm{C}(14)-\mathrm{C}(19)$ & $1.387(11)$ \\
\hline $\mathrm{C}(14)-\mathrm{C}(15)$ & $1.411(10)$ \\
\hline$C(15)-C(16)$ & $1.392(10)$ \\
\hline $\mathrm{C}(15)-\mathrm{H}(15)$ & 0.9500 \\
\hline$C(16)-C(17)$ & $1.343(11)$ \\
\hline $\mathrm{C}(16)-\mathrm{H}(16)$ & 0.9500 \\
\hline $\mathrm{C}(17)-\mathrm{C}(18)$ & $1.400(11)$ \\
\hline $\mathrm{C}(17)-\mathrm{H}(17)$ & 0.9500 \\
\hline $\mathrm{C}(18)-\mathrm{C}(19)$ & $1.400(11)$ \\
\hline C(18)-H(18) & 0.9500 \\
\hline C(19)-H(19) & 0.9500 \\
\hline $\mathrm{O}(1)-\mathrm{H}(1)$ & 0.8400 \\
\hline $\mathrm{O}(1)-\mathrm{C}(1)-\mathrm{N}(1)$ & $112.1(6)$ \\
\hline $\mathrm{O}(1)-\mathrm{C}(1)-\mathrm{C}(2)$ & $114.2(7)$ \\
\hline $\mathrm{N}(1)-\mathrm{C}(1)-\mathrm{C}(2)$ & $110.8(6)$ \\
\hline $\mathrm{O}(1)-\mathrm{C}(1)-\mathrm{H}(1 \mathrm{~A})$ & 106.4 \\
\hline $\mathrm{N}(1)-\mathrm{C}(1)-\mathrm{H}(1 \mathrm{~A})$ & 106.4 \\
\hline $\mathrm{C}(2)-\mathrm{C}(1)-\mathrm{H}(1 \mathrm{~A})$ & 106.4 \\
\hline $\mathrm{C}(3)-\mathrm{C}(2)-\mathrm{C}(7)$ & $108.6(6)$ \\
\hline $\mathrm{C}(3)-\mathrm{C}(2)-\mathrm{C}(6)$ & $114.4(6)$ \\
\hline$C(7)-C(2)-C(6)$ & $109.8(7)$ \\
\hline $\mathrm{C}(3)-\mathrm{C}(2)-\mathrm{C}(1)$ & $109.6(6)$ \\
\hline $\mathrm{C}(7)-\mathrm{C}(2)-\mathrm{C}(1)$ & $107.9(6)$ \\
\hline$C(6)-C(2)-C(1)$ & $106.5(6)$ \\
\hline $\mathrm{C}(4)-\mathrm{C}(3)-\mathrm{C}(14)$ & $119.2(6)$ \\
\hline $\mathrm{C}(4)-\mathrm{C}(3)-\mathrm{C}(2)$ & $117.7(6)$ \\
\hline$C(14)-C(3)-C(2)$ & $123.1(6)$ \\
\hline $\mathrm{C}(3)-\mathrm{C}(4)-\mathrm{C}(5)$ & $124.0(7)$ \\
\hline $\mathrm{C}(3)-\mathrm{C}(4)-\mathrm{H}(4)$ & 118.0 \\
\hline $\mathrm{C}(5)-\mathrm{C}(4)-\mathrm{H}(4)$ & 118.0 \\
\hline $\mathrm{O}(2)-\mathrm{C}(5)-\mathrm{N}(1)$ & 121.7(7) \\
\hline $\mathrm{O}(2)-\mathrm{C}(5)-\mathrm{C}(4)$ & $122.0(7)$ \\
\hline $\mathrm{N}(1)-\mathrm{C}(5)-\mathrm{C}(4)$ & $116.3(6)$ \\
\hline $\mathrm{C}(2)-\mathrm{C}(6)-\mathrm{H}(6 \mathrm{~A})$ & 109.5 \\
\hline $\mathrm{C}(2)-\mathrm{C}(6)-\mathrm{H}(6 \mathrm{~B})$ & 109.5 \\
\hline
\end{tabular}




\begin{tabular}{|c|c|}
\hline $\mathrm{H}(6 \mathrm{~A})-\mathrm{C}(6)-\mathrm{H}(6 \mathrm{~B})$ & 109.5 \\
\hline $\mathrm{C}(2)-\mathrm{C}(6)-\mathrm{H}(6 \mathrm{C})$ & 109.5 \\
\hline $\mathrm{H}(6 \mathrm{~A})-\mathrm{C}(6)-\mathrm{H}(6 \mathrm{C})$ & 109.5 \\
\hline $\mathrm{H}(6 \mathrm{~B})-\mathrm{C}(6)-\mathrm{H}(6 \mathrm{C})$ & 109.5 \\
\hline $\mathrm{C}(2)-\mathrm{C}(7)-\mathrm{H}(7 \mathrm{~A})$ & 109.5 \\
\hline $\mathrm{C}(2)-\mathrm{C}(7)-\mathrm{H}(7 \mathrm{~B})$ & 109.5 \\
\hline $\mathrm{H}(7 \mathrm{~A})-\mathrm{C}(7)-\mathrm{H}(7 \mathrm{~B})$ & 109.5 \\
\hline $\mathrm{C}(2)-\mathrm{C}(7)-\mathrm{H}(7 \mathrm{C})$ & 109.5 \\
\hline $\mathrm{H}(7 \mathrm{~A})-\mathrm{C}(7)-\mathrm{H}(7 \mathrm{C})$ & 109.5 \\
\hline $\mathrm{H}(7 \mathrm{~B})-\mathrm{C}(7)-\mathrm{H}(7 \mathrm{C})$ & 109.5 \\
\hline $\mathrm{C}(13)-\mathrm{C}(8)-\mathrm{C}(9)$ & $120.6(7)$ \\
\hline $\mathrm{C}(13)-\mathrm{C}(8)-\mathrm{N}(1)$ & $119.0(6)$ \\
\hline $\mathrm{C}(9)-\mathrm{C}(8)-\mathrm{N}(1)$ & $120.5(7)$ \\
\hline$C(8)-C(9)-C(10)$ & $119.0(7)$ \\
\hline $\mathrm{C}(8)-\mathrm{C}(9)-\mathrm{H}(9)$ & 120.5 \\
\hline $\mathrm{C}(10)-\mathrm{C}(9)-\mathrm{H}(9)$ & 120.5 \\
\hline $\mathrm{C}(11)-\mathrm{C}(10)-\mathrm{C}(9)$ & $121.7(7)$ \\
\hline $\mathrm{C}(11)-\mathrm{C}(10)-\mathrm{H}(10)$ & 119.2 \\
\hline $\mathrm{C}(9)-\mathrm{C}(10)-\mathrm{H}(10)$ & 119.2 \\
\hline $\mathrm{C}(10)-\mathrm{C}(11)-\mathrm{C}(12)$ & $118.1(7)$ \\
\hline $\mathrm{C}(10)-\mathrm{C}(11)-\mathrm{H}(11)$ & 121.0 \\
\hline $\mathrm{C}(12)-\mathrm{C}(11)-\mathrm{H}(11)$ & 121.0 \\
\hline $\mathrm{C}(11)-\mathrm{C}(12)-\mathrm{C}(13)$ & $120.9(7)$ \\
\hline $\mathrm{C}(11)-\mathrm{C}(12)-\mathrm{H}(12)$ & 119.6 \\
\hline $\mathrm{C}(13)-\mathrm{C}(12)-\mathrm{H}(12)$ & 119.6 \\
\hline $\mathrm{C}(8)-\mathrm{C}(13)-\mathrm{C}(12)$ & $119.9(7)$ \\
\hline $\mathrm{C}(8)-\mathrm{C}(13)-\mathrm{H}(13)$ & 120.1 \\
\hline $\mathrm{C}(12)-\mathrm{C}(13)-\mathrm{H}(13)$ & 120.1 \\
\hline$C(19)-C(14)-C(15)$ & $117.8(7)$ \\
\hline$C(19)-C(14)-C(3)$ & $119.7(6)$ \\
\hline$C(15)-C(14)-C(3)$ & $122.4(7)$ \\
\hline$C(16)-C(15)-C(14)$ & $120.7(7)$ \\
\hline $\mathrm{C}(16)-\mathrm{C}(15)-\mathrm{H}(15)$ & 119.6 \\
\hline $\mathrm{C}(14)-\mathrm{C}(15)-\mathrm{H}(15)$ & 119.6 \\
\hline$C(17)-C(16)-C(15)$ & $120.8(7)$ \\
\hline $\mathrm{C}(17)-\mathrm{C}(16)-\mathrm{H}(16)$ & 119.6 \\
\hline $\mathrm{C}(15)-\mathrm{C}(16)-\mathrm{H}(16)$ & 119.6 \\
\hline$C(16)-C(17)-C(18)$ & $120.3(7)$ \\
\hline
\end{tabular}




$\begin{array}{ll}\mathrm{C}(16)-\mathrm{C}(17)-\mathrm{H}(17) & 119.8 \\ \mathrm{C}(18)-\mathrm{C}(17)-\mathrm{H}(17) & 119.8 \\ \mathrm{C}(17)-\mathrm{C}(18)-\mathrm{C}(19) & 119.6(8) \\ \mathrm{C}(17)-\mathrm{C}(18)-\mathrm{H}(18) & 120.2 \\ \mathrm{C}(19)-\mathrm{C}(18)-\mathrm{H}(18) & 120.2 \\ \mathrm{C}(14)-\mathrm{C}(19)-\mathrm{C}(18) & 120.8(7) \\ \mathrm{C}(14)-\mathrm{C}(19)-\mathrm{H}(19) & 119.6 \\ \mathrm{C}(18)-\mathrm{C}(19)-\mathrm{H}(19) & 119.6 \\ \mathrm{C}(5)-\mathrm{N}(1)-\mathrm{C}(8) & 120.8(6) \\ \mathrm{C}(5)-\mathrm{N}(1)-\mathrm{C}(1) & 122.1(6) \\ \mathrm{C}(8)-\mathrm{N}(1)-\mathrm{C}(1) & 116.7(6) \\ \mathrm{C}(1)-\mathrm{O}(1)-\mathrm{H}(1) & 109.5\end{array}$

Symmetry transformations used to generate equivalent atoms:

Table S26. Anisotropic displacement parameters $\quad\left(\AA^{2} \times 10^{3}\right)$ for 200747lt_a. The anisotropic displacement factor exponent takes the form: $\quad-2 \pi^{2}\left[h^{2} a^{* 2} U^{11}+\ldots+2 h k a^{*} b^{*} U^{12}\right]$

\begin{tabular}{|c|c|c|c|c|c|c|}
\hline & $\mathrm{U}^{11}$ & $\mathrm{U}^{22}$ & $\mathrm{U}^{33}$ & $\mathrm{U}^{23}$ & $\mathrm{U}^{13}$ & $\mathrm{U}^{12}$ \\
\hline $\mathrm{C}(1)$ & $13(3)$ & $17(4)$ & $14(3)$ & $2(3)$ & $6(3)$ & $3(3)$ \\
\hline $\mathrm{C}(2)$ & $11(3)$ & 18(4) & $14(3)$ & $5(3)$ & $6(3)$ & $3(3)$ \\
\hline$C(3)$ & $20(4)$ & $7(3)$ & $16(3)$ & $3(3)$ & 11(3) & 1(3) \\
\hline $\mathrm{C}(4)$ & $22(4)$ & 18(4) & $15(3)$ & $-2(3)$ & 11(3) & $3(3)$ \\
\hline$C(5)$ & $19(4)$ & $15(4)$ & $15(3)$ & $-4(3)$ & $6(3)$ & $-2(3)$ \\
\hline$C(6)$ & $11(4)$ & $39(5)$ & $18(4)$ & $1(3)$ & $5(3)$ & 1(4) \\
\hline $\mathrm{C}(7)$ & $17(4)$ & $28(5)$ & $29(4)$ & $9(3)$ & $10(3)$ & $-2(3)$ \\
\hline $\mathrm{C}(8)$ & $8(3)$ & $13(4)$ & $16(3)$ & $-3(3)$ & $3(3)$ & $2(3)$ \\
\hline $\mathrm{C}(9)$ & $17(4)$ & $15(4)$ & $22(4)$ & $1(3)$ & 11(3) & $-1(3)$ \\
\hline$C(10)$ & $21(4)$ & $16(4)$ & $14(3)$ & $0(3)$ & $4(3)$ & $-1(3)$ \\
\hline $\mathrm{C}(11)$ & $13(3)$ & $20(4)$ & $20(3)$ & $-6(3)$ & $2(3)$ & $-2(3)$ \\
\hline $\mathrm{C}(12)$ & $12(3)$ & $14(4)$ & $20(3)$ & $-1(3)$ & $6(3)$ & $-3(3)$ \\
\hline $\mathrm{C}(13)$ & $13(3)$ & 13(4) & $17(3)$ & $0(3)$ & $5(3)$ & 1(3) \\
\hline $\mathrm{C}(14)$ & $15(4)$ & 11(4) & $13(3)$ & $-2(3)$ & $5(3)$ & $0(3)$ \\
\hline$C(15)$ & $19(4)$ & $12(4)$ & $20(3)$ & $5(3)$ & $9(3)$ & $3(3)$ \\
\hline$C(16)$ & $17(4)$ & $10(4)$ & $24(4)$ & $2(3)$ & $10(3)$ & $3(3)$ \\
\hline $\mathrm{C}(17)$ & $18(4)$ & $16(4)$ & $19(3)$ & $-8(3)$ & $3(3)$ & $2(3)$ \\
\hline $\mathrm{C}(18)$ & $18(4)$ & $15(4)$ & $20(4)$ & $-6(3)$ & $7(3)$ & $-1(3)$ \\
\hline
\end{tabular}




\begin{tabular}{lllllll}
$\mathrm{C}(19)$ & $22(4)$ & $15(4)$ & $16(3)$ & $-1(3)$ & $9(3)$ & $2(3)$ \\
$\mathrm{N}(1)$ & $12(3)$ & $22(4)$ & $9(3)$ & $-3(3)$ & $4(2)$ & $0(3)$ \\
$\mathrm{O}(1)$ & $18(3)$ & $15(3)$ & $23(3)$ & $-3(2)$ & $13(2)$ & $2(2)$ \\
$\mathrm{O}(2)$ & $18(3)$ & $30(3)$ & $13(2)$ & $-6(2)$ & $7(2)$ & $4(3)$ \\
\hline
\end{tabular}

Table S27. Hydrogen coordinates ( $\left.\times 10^{4}\right)$ and isotropic displacement parameters $\left(\AA^{2} \times 10^{3}\right)$ for 200747lt_a.

$\begin{array}{llll}\mathrm{x} & \mathrm{y} & \mathrm{z} & \mathrm{U}\end{array}$

$\begin{array}{lrrrr}\mathrm{H}(1 \mathrm{~A}) & 6776 & 4865 & 2781 & 18 \\ \mathrm{H}(4) & 3258 & 5397 & 4591 & 21 \\ \mathrm{H}(6 \mathrm{~A}) & 3547 & 3336 & 838 & 34 \\ \mathrm{H}(6 \mathrm{~B}) & 4765 & 4348 & 489 & 34 \\ \mathrm{H}(6 \mathrm{C}) & 3092 & 5100 & 53 & 34 \\ \mathrm{H}(7 \mathrm{~A}) & 3884 & 7794 & 1349 & 37 \\ \mathrm{H}(7 \mathrm{~B}) & 5572 & 7282 & 1580 & 37 \\ \mathrm{H}(7 \mathrm{C}) & 5239 & 7792 & 2864 & 37 \\ \mathrm{H}(9) & 8161 & 3676 & 6889 & 20 \\ \mathrm{H}(10) & 10804 & 3657 & 8165 & 22 \\ \mathrm{H}(11) & 12397 & 5163 & 7434 & 23 \\ \mathrm{H}(12) & 11298 & 6632 & 5347 & 19 \\ \mathrm{H}(13) & 8650 & 6666 & 4065 & 18 \\ \mathrm{H}(15) & 1479 & 6702 & 359 & 20 \\ \mathrm{H}(16) & -1173 & 6625 & -787 & 20 \\ \mathrm{H}(17) & -2573 & 5128 & 84 & 23 \\ \mathrm{H}(18) & -1352 & 3629 & 2149 & 22 \\ \mathrm{H}(19) & 1317 & 3670 & 3319 & 21 \\ \mathrm{H}(1) & 5327 & 2434 & 3368 & 25 \\ & & & & \end{array}$


10. The ${ }^{1} \mathrm{H},{ }^{13} \mathrm{C}$ and ${ }^{19} \mathrm{~F}$ NMR of Key compounds:

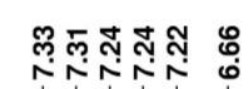

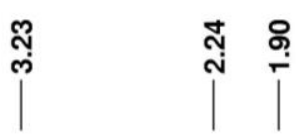
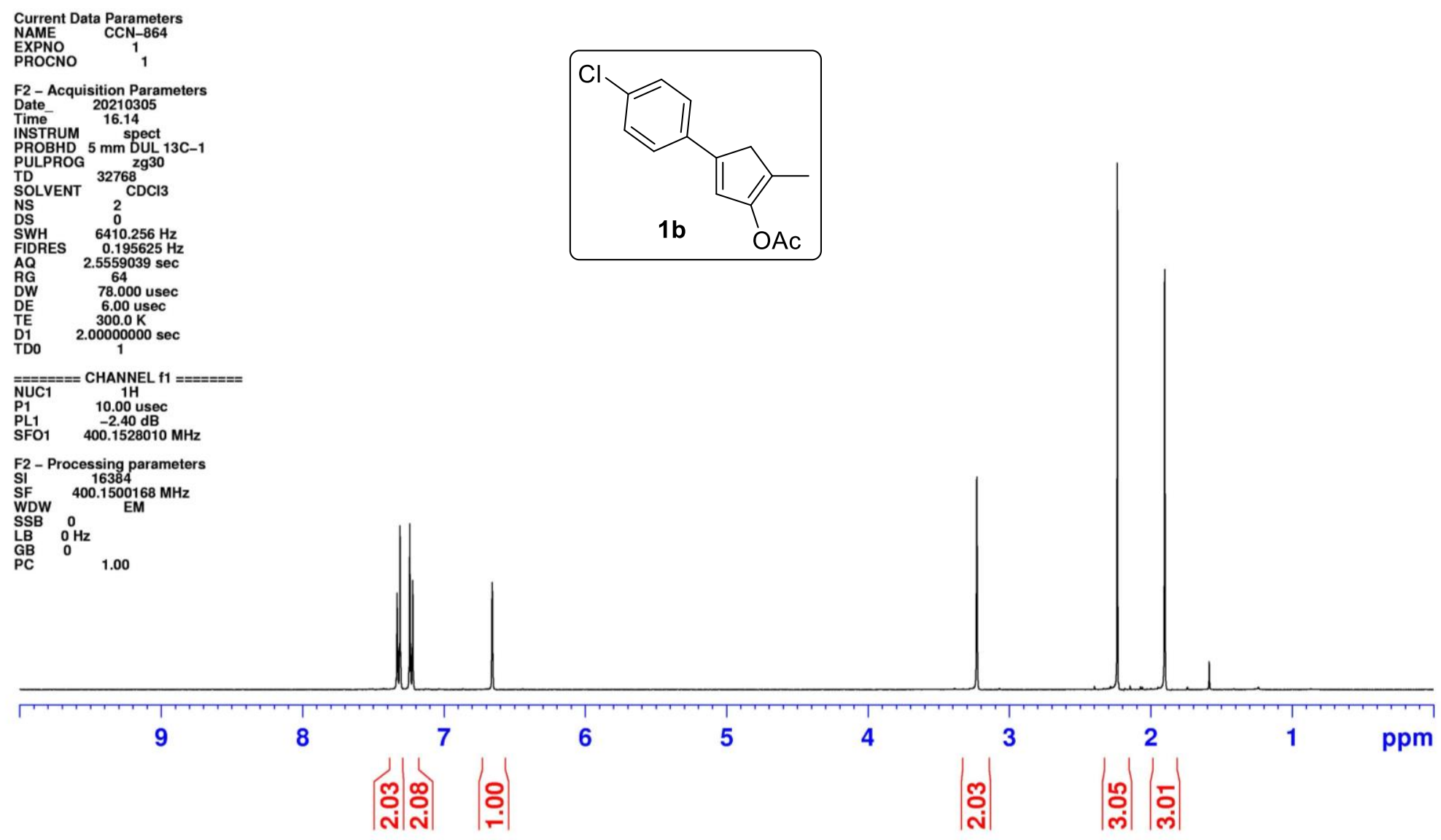


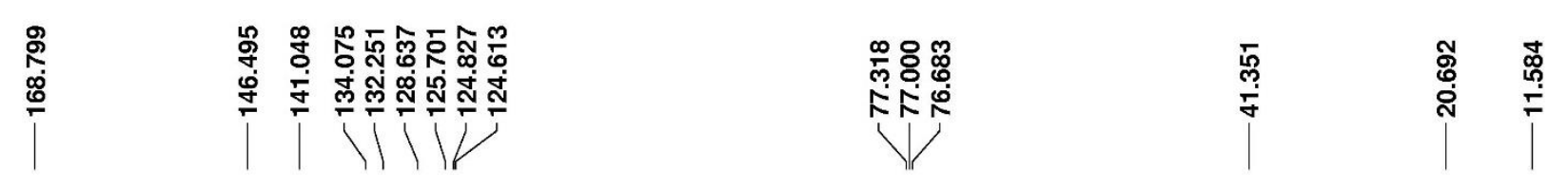

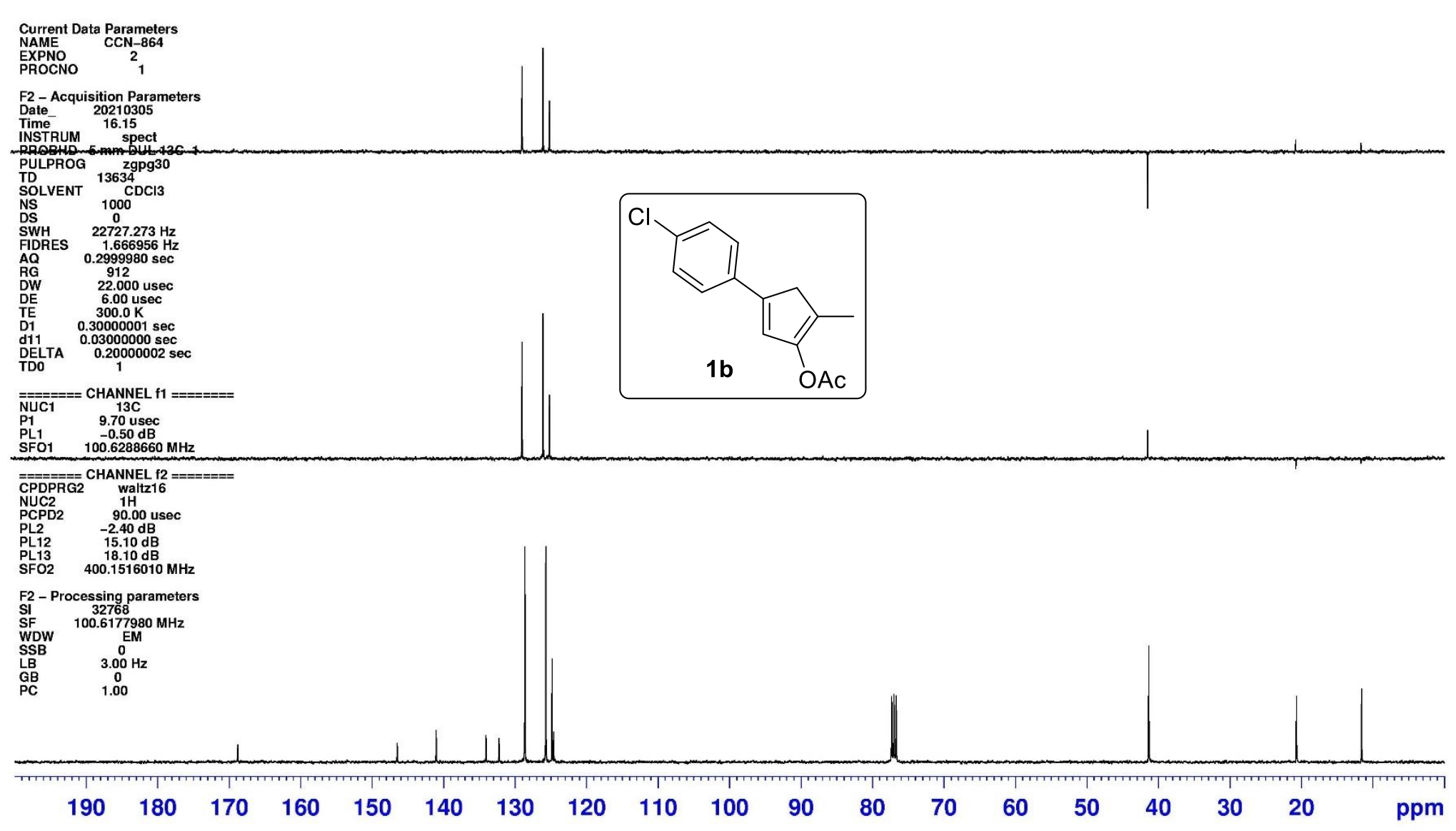




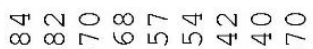

$\therefore \operatorname{ararar6}$

vivi
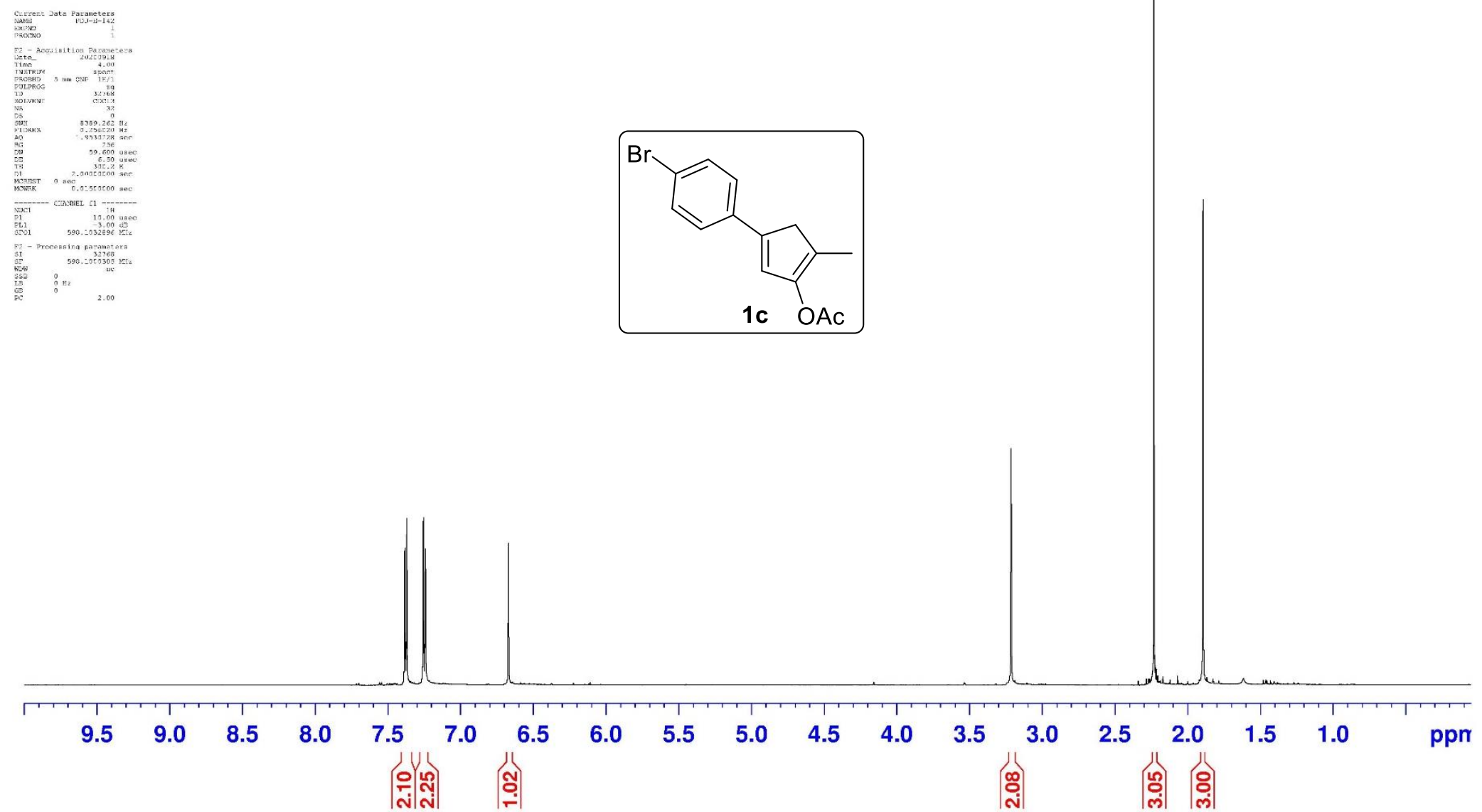


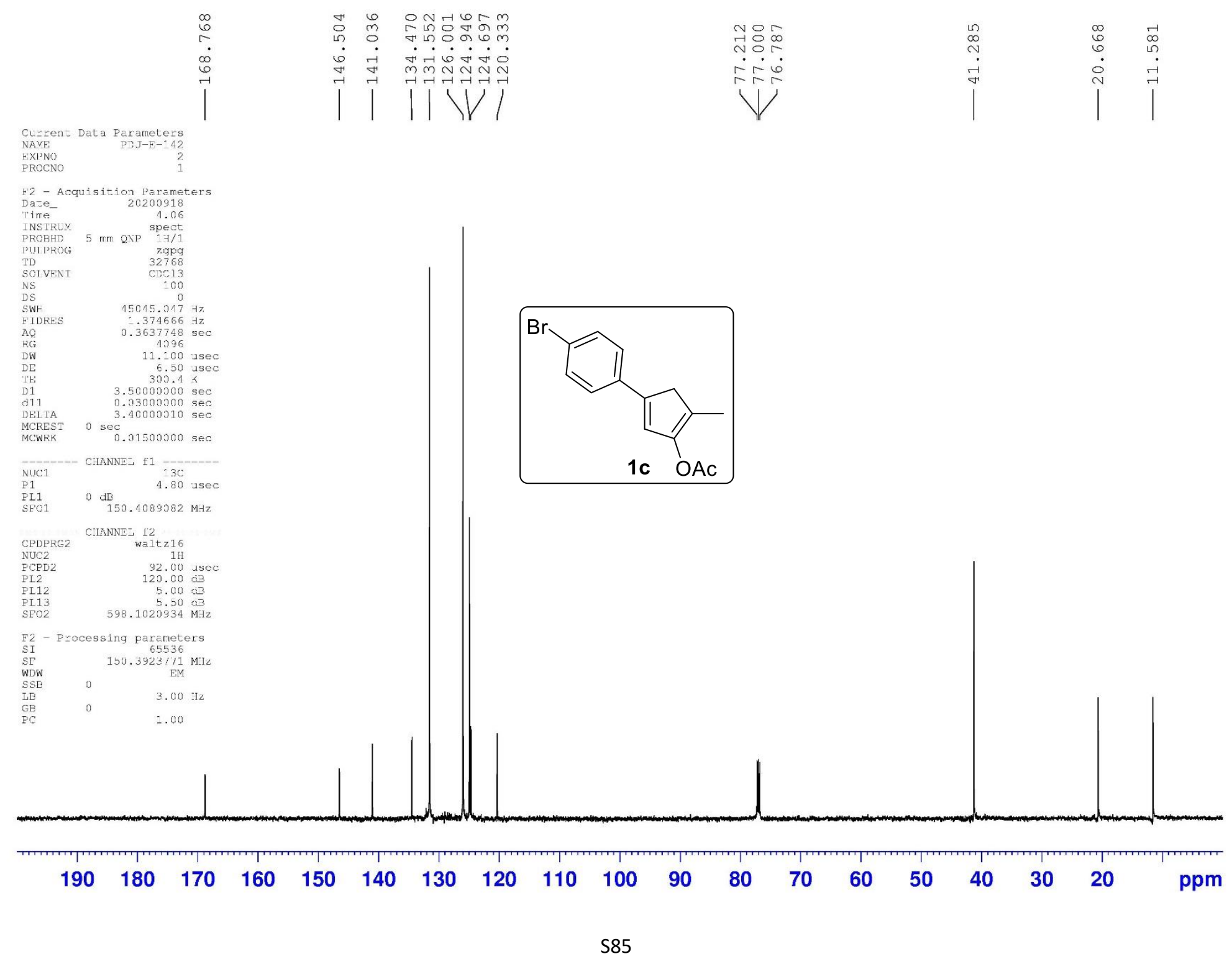




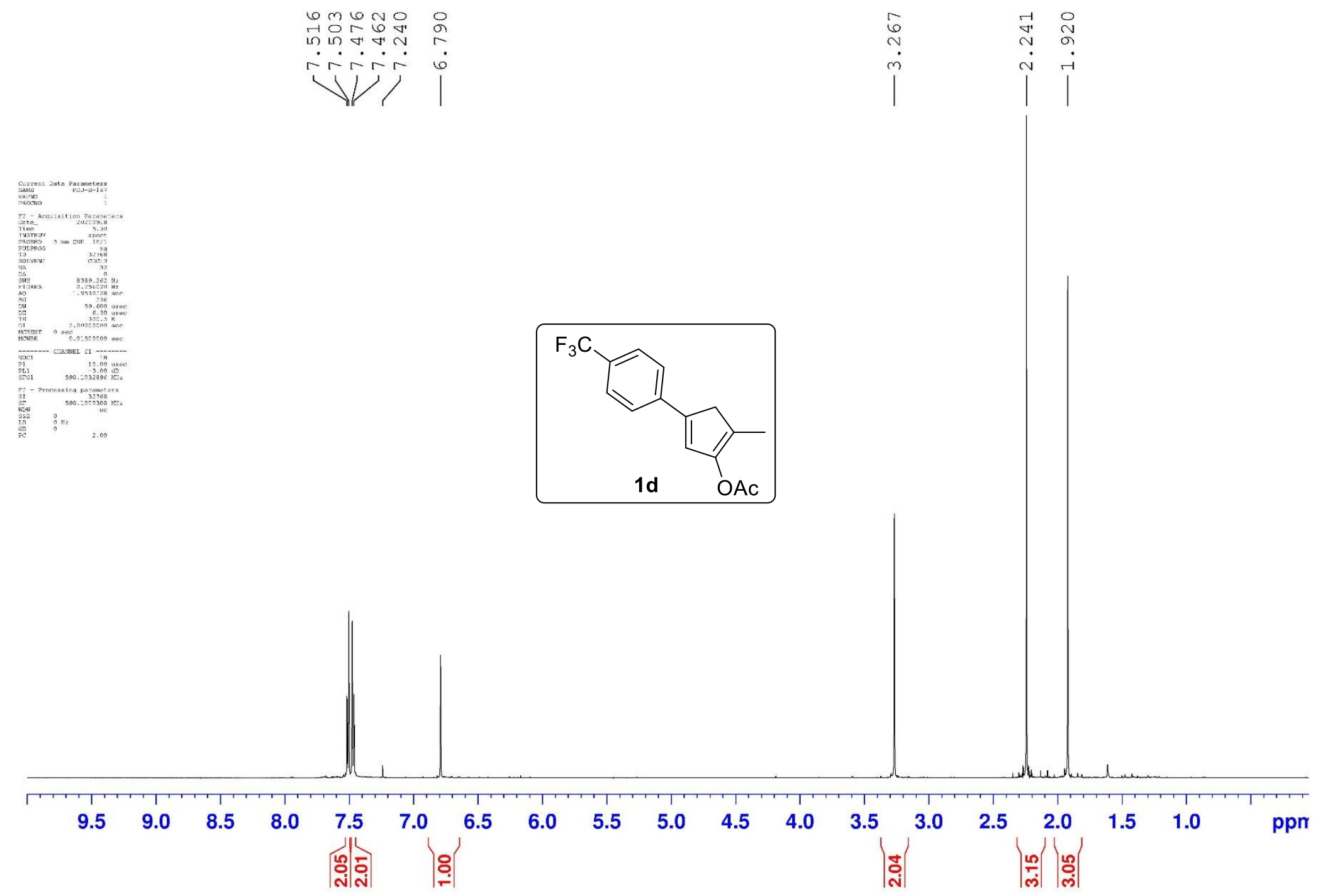




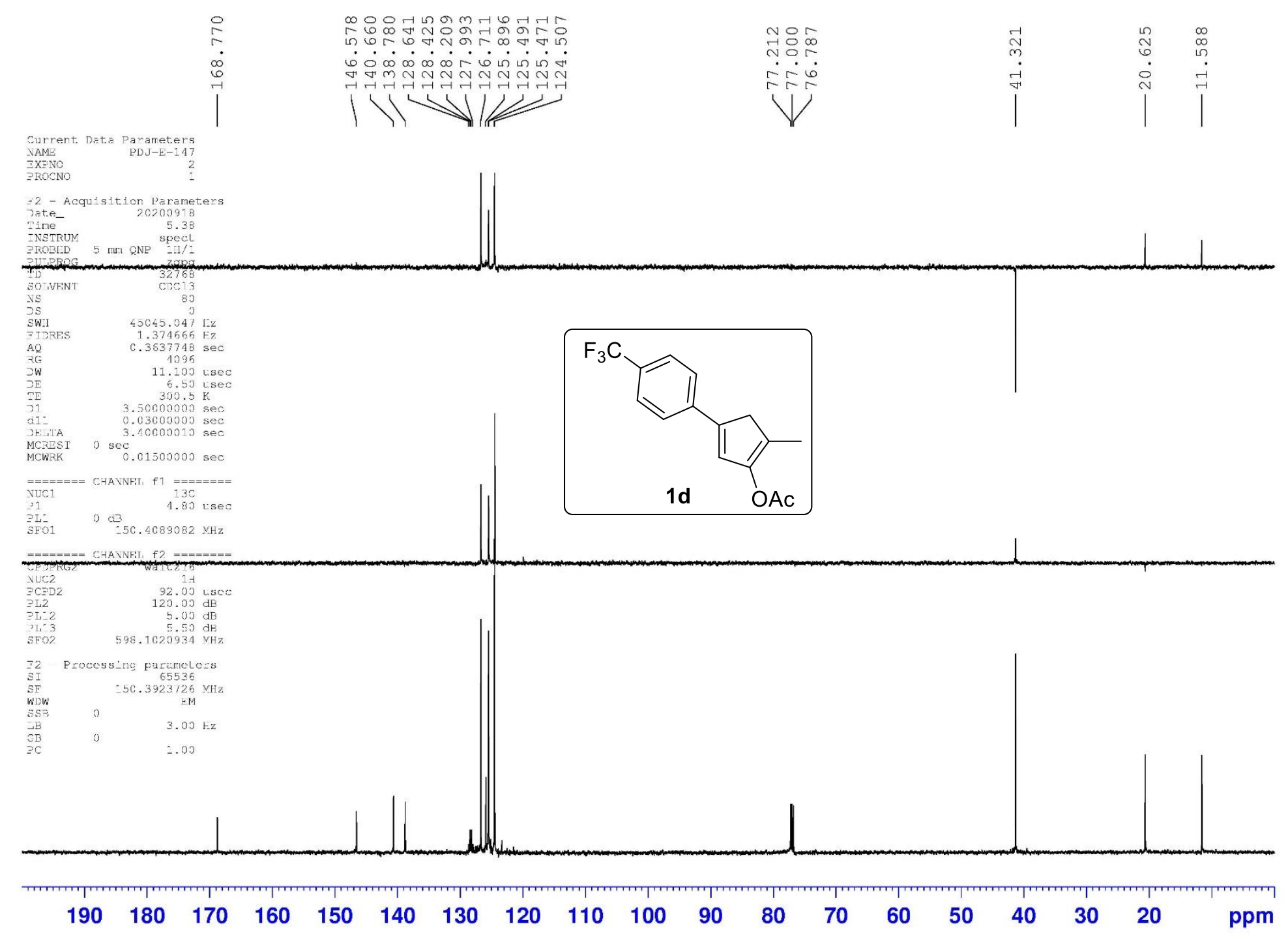


Current Data Parameters
NIAME
PDJ-E-147-F19

EXPNO
PROCNO

F2 - Acquisition Paraneters
Date- 20200917

Date-
Tire-
Tire

INSTRUM
PROBHD $5 \mathrm{~mm}$ ONP $\begin{gathered}11.3 \mathrm{spect} \\ 1 \mathrm{H} / 1\end{gathered}$

Pll $1 \mathrm{H} / \mathrm{s}$

TD SOLVENT

$\begin{array}{lr}\text { DS } & 0 \\ \text { SWH } & 100000.000\end{array}$

$\begin{array}{lr}100000.000 ~ \mathrm{~Hz} \\ \text { FIDRES } & 6.103516 \mathrm{~Hz}\end{array}$

$\begin{array}{lr}\text { AQ } & 6.0819700 \mathrm{sec} \\ \text { RG } & 4096 \\ \text { DW } & 5.000 \mathrm{usec}\end{array}$

$\begin{array}{lr}\text { DE } & 6.00 \mathrm{usec} \\ \text { TE } & 297.9 \mathrm{~K} \\ \mathrm{D} 1 & 2.00000000 \mathrm{sec}\end{array}$

$\begin{array}{lrl}\text { MCREST } & 0 \mathrm{sec} \\ \text { MCWRK } & 0.01500000 \mathrm{sec}\end{array}$

$\begin{array}{ll}======\text { CHANNEL } & \text { f1 } 1====== \\ \text { NUC1 } & 19 \mathrm{~F} \\ \text { P1 } & 2.00 \text { usec }\end{array}$

$\begin{array}{lr}\text { P11 } & 2.00 \text { usec } \\ \text { PL1 } & 5.00 \mathrm{~dB} \\ \text { SF01 } & 562.9358871 \mathrm{MHz}\end{array}$

F2 - Processing parameters

SI $\quad 562.9759353 \mathrm{MHz}$

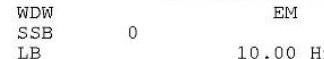

$\begin{array}{ll}\mathrm{CB} & 0 \\ \mathrm{PC} & \end{array}$
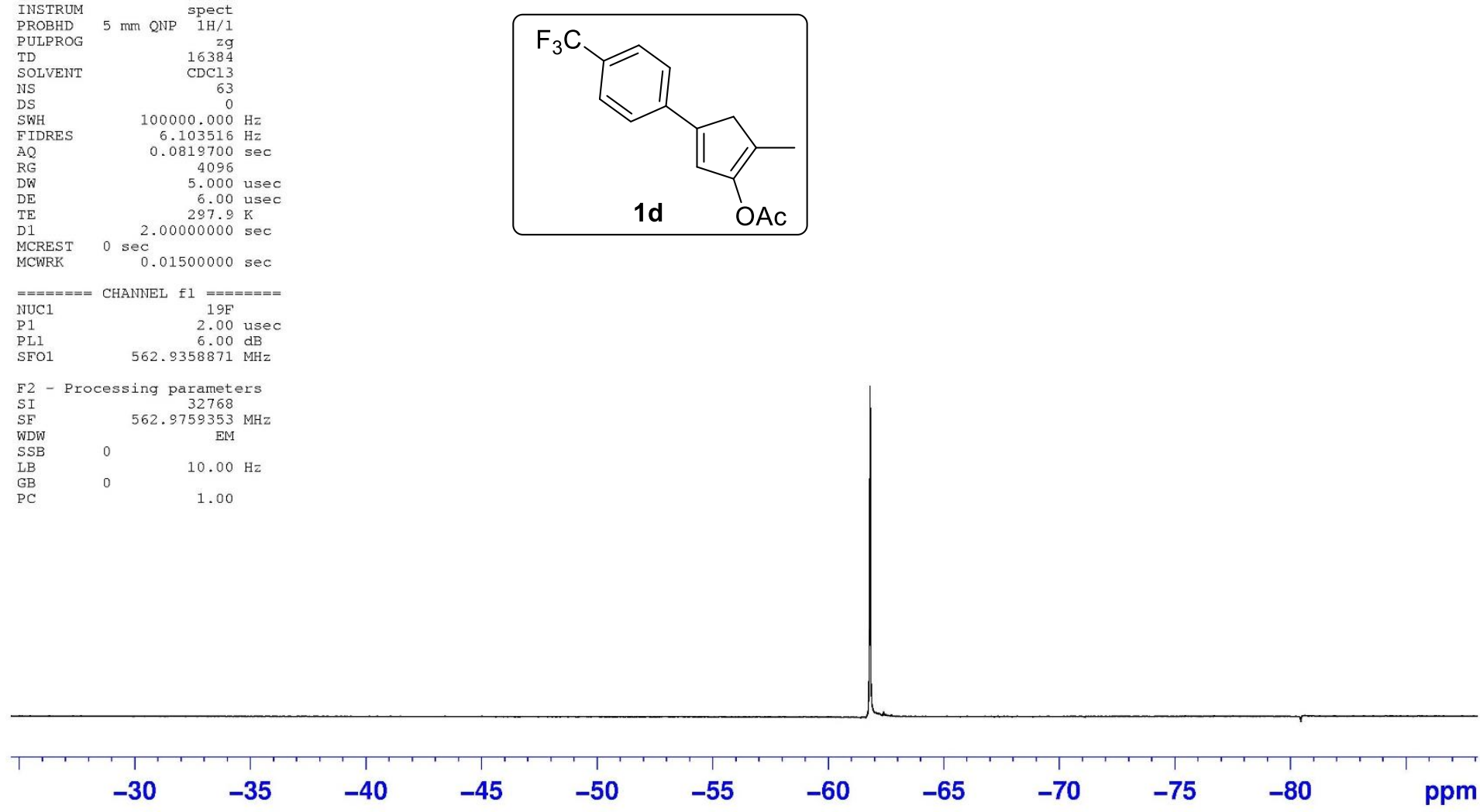


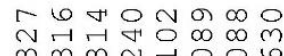

inirisi

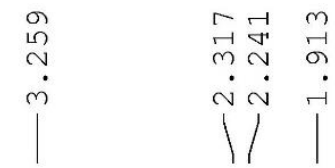
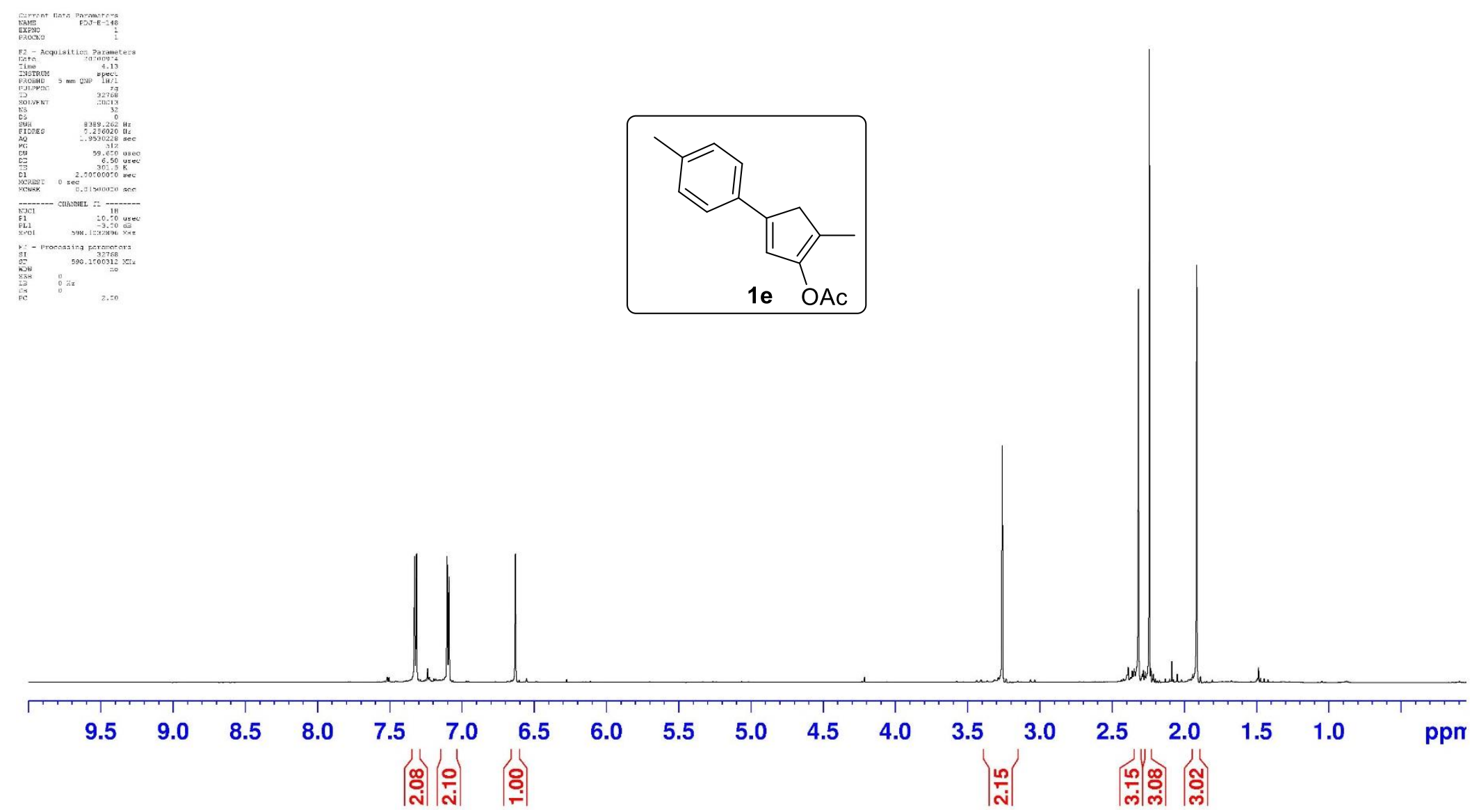


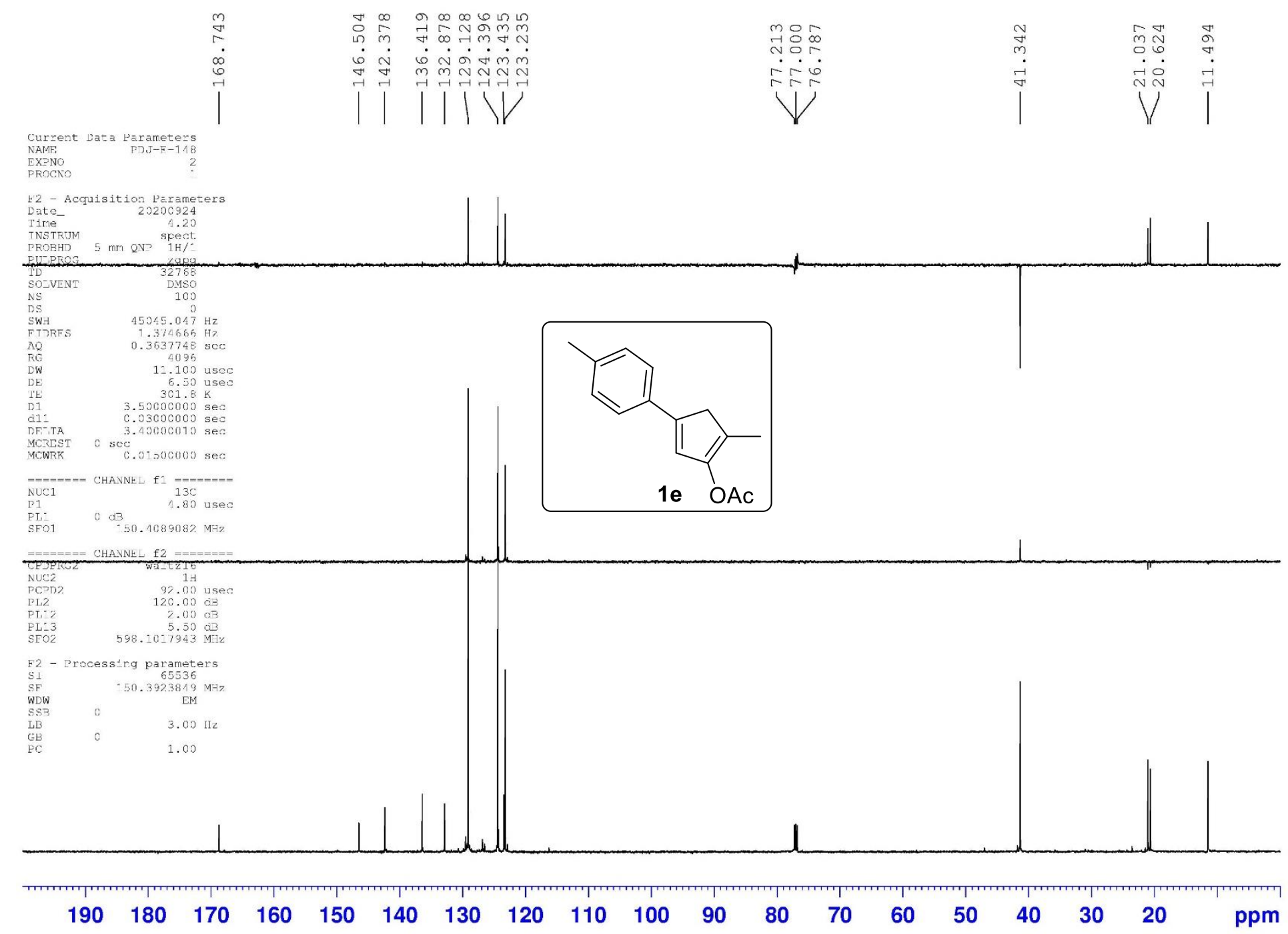



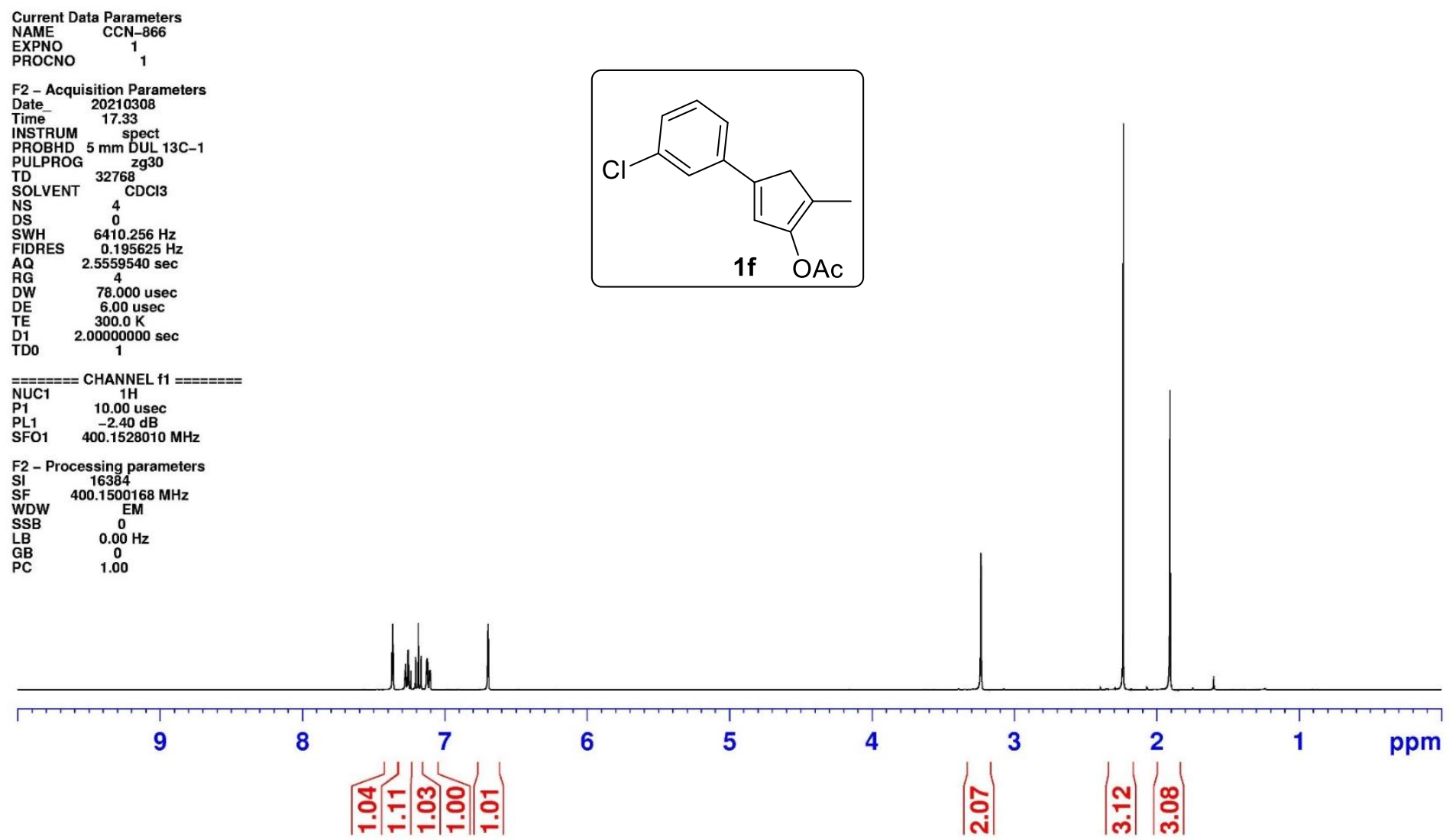

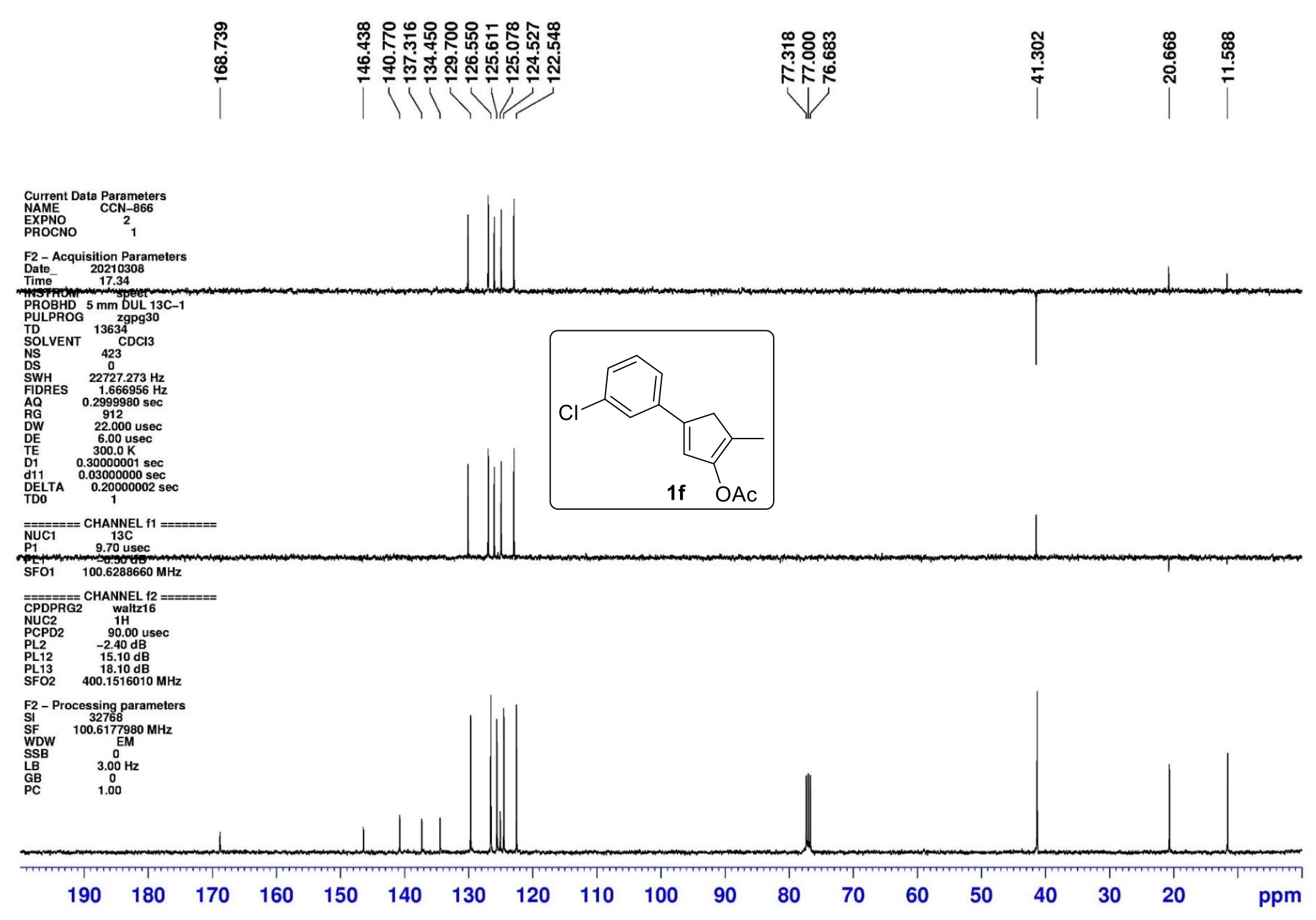
구유의

กำ

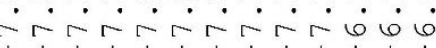

$\longrightarrow$
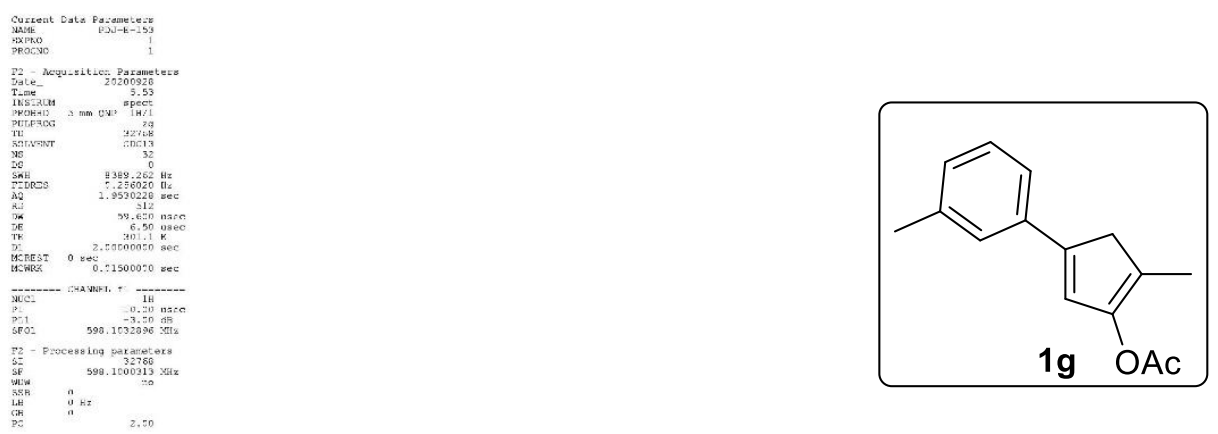

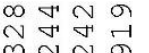

(1)

$\iint^{2}$
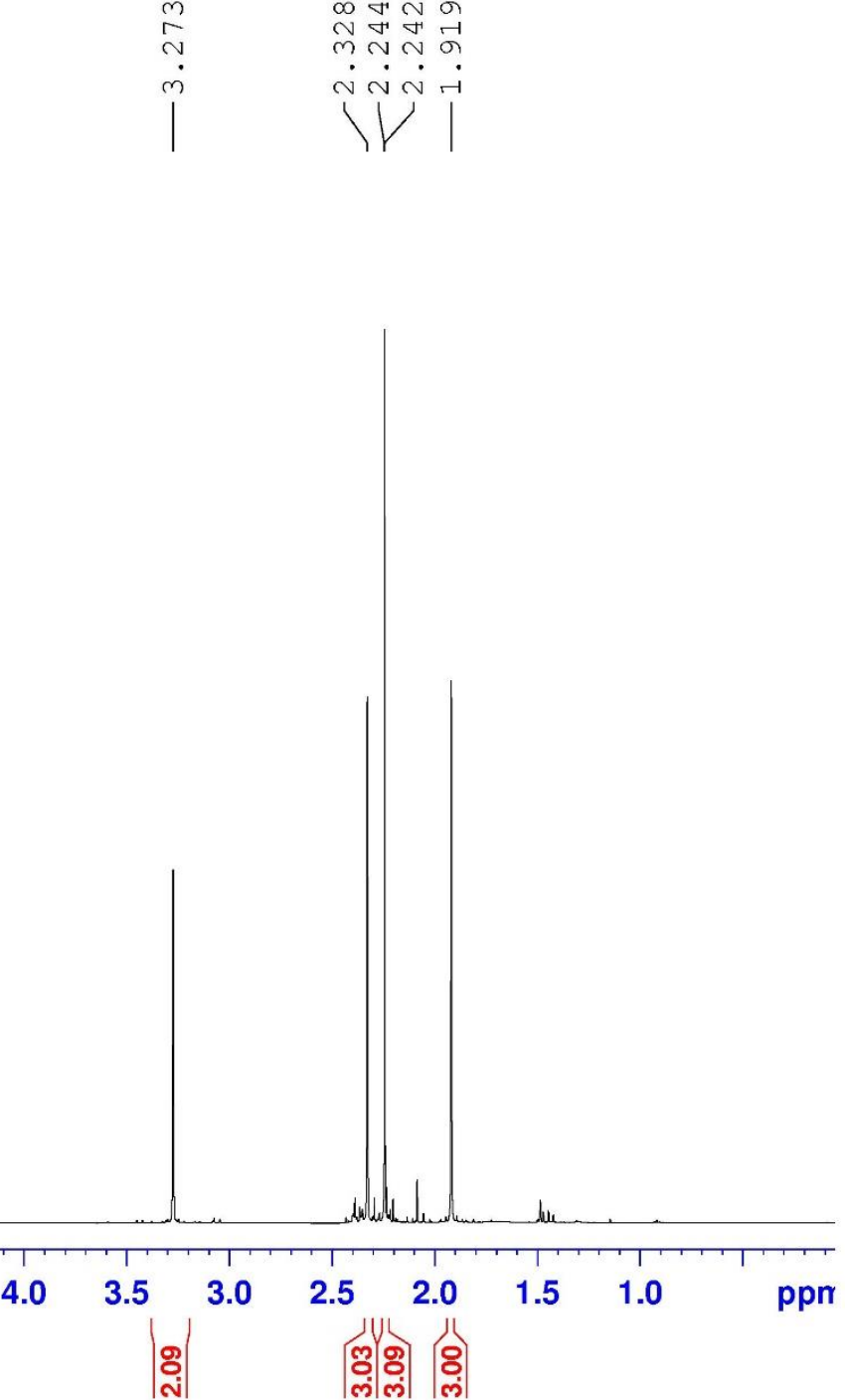


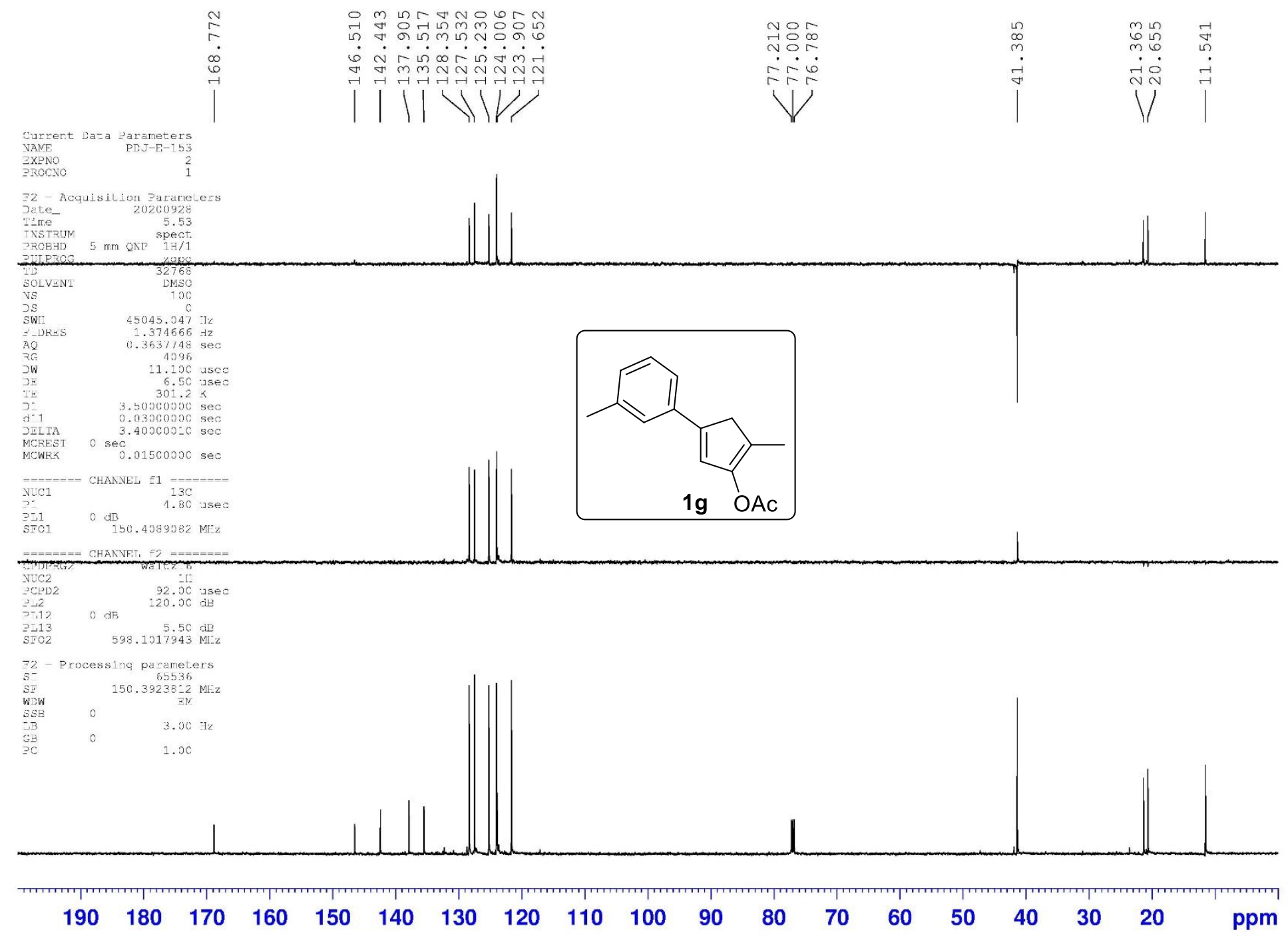




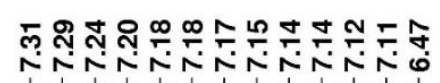

|

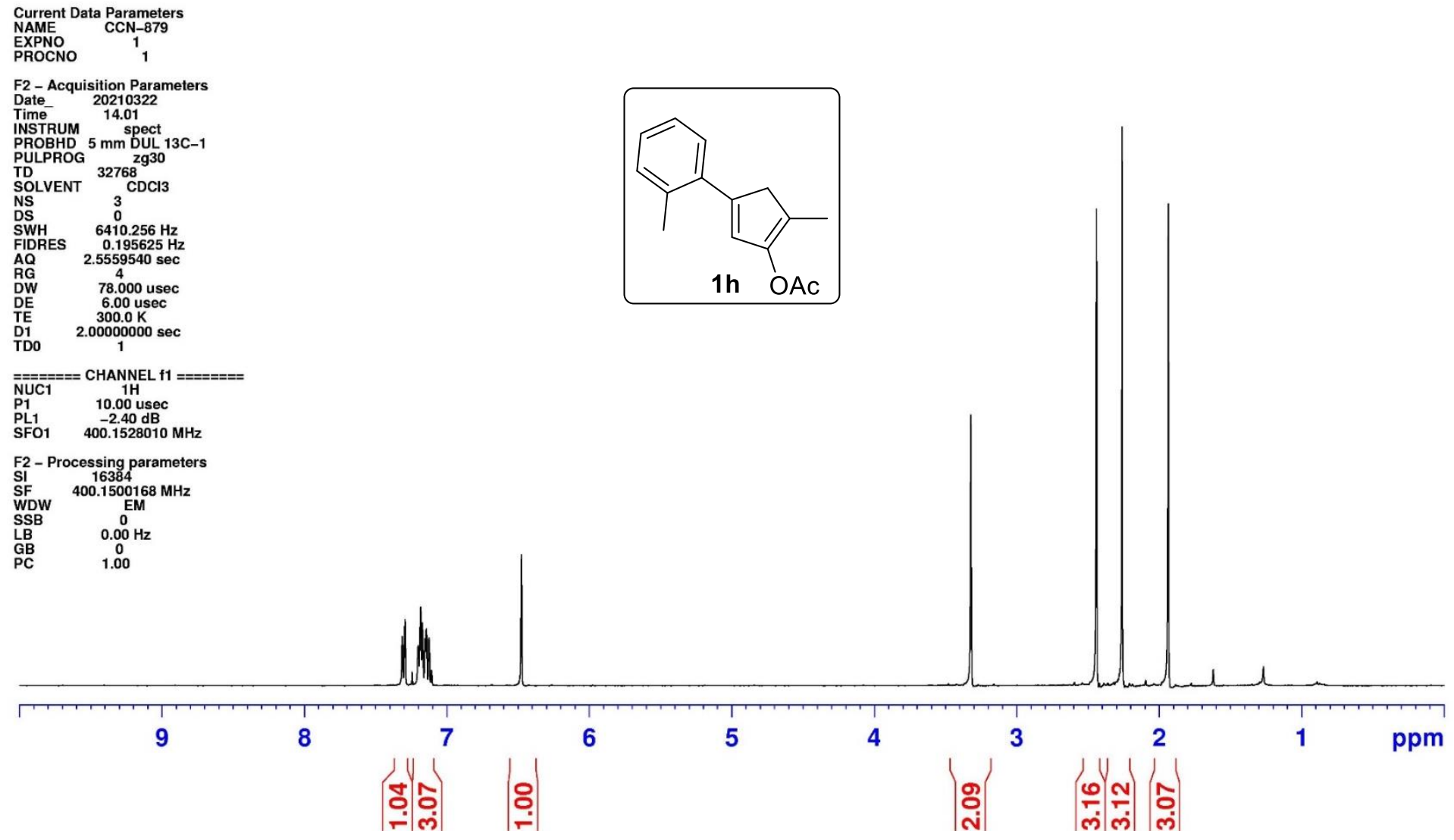




|

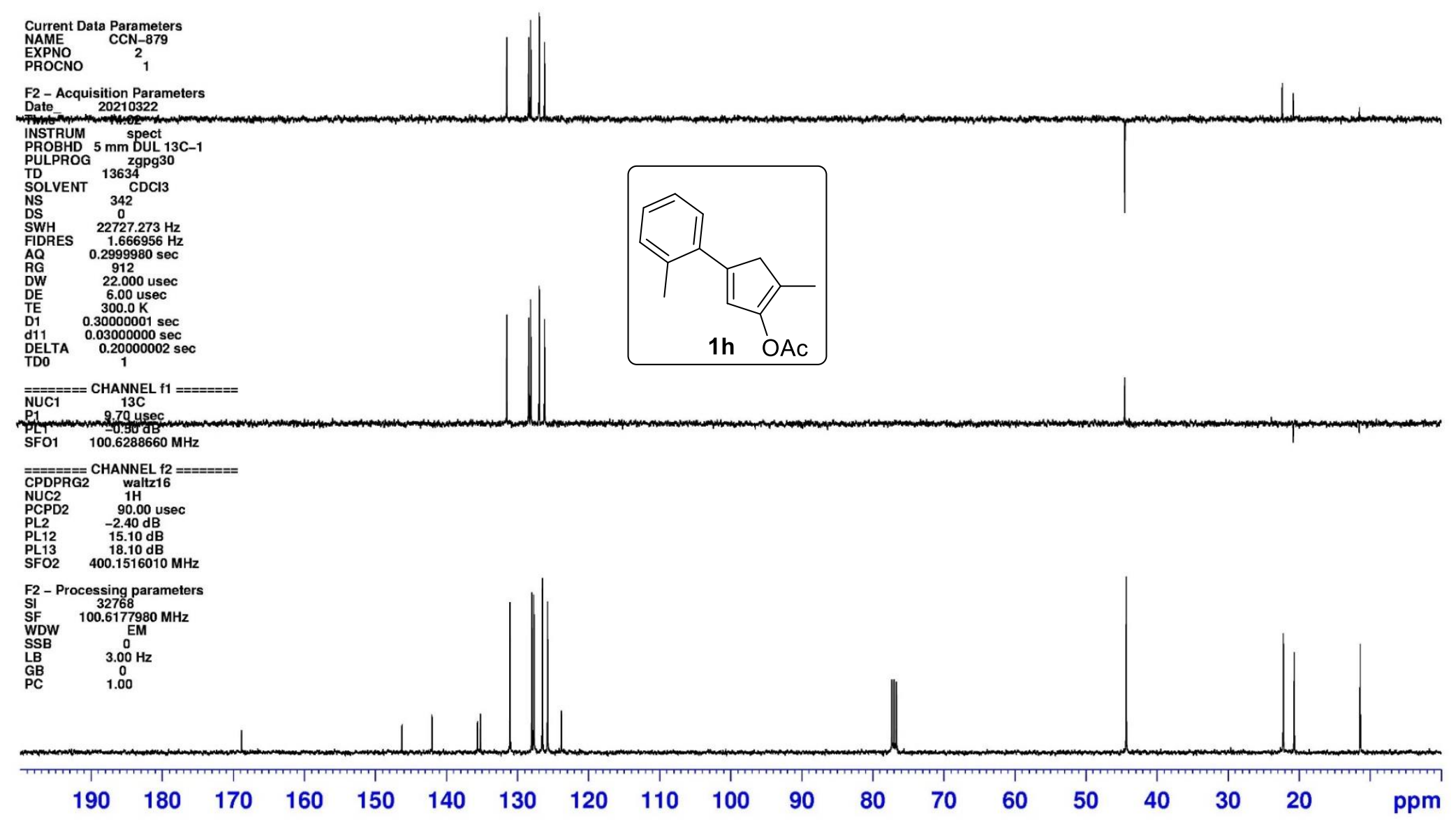




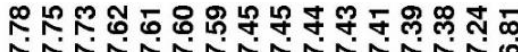

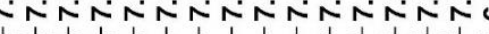

‡

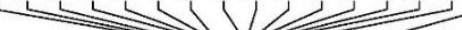

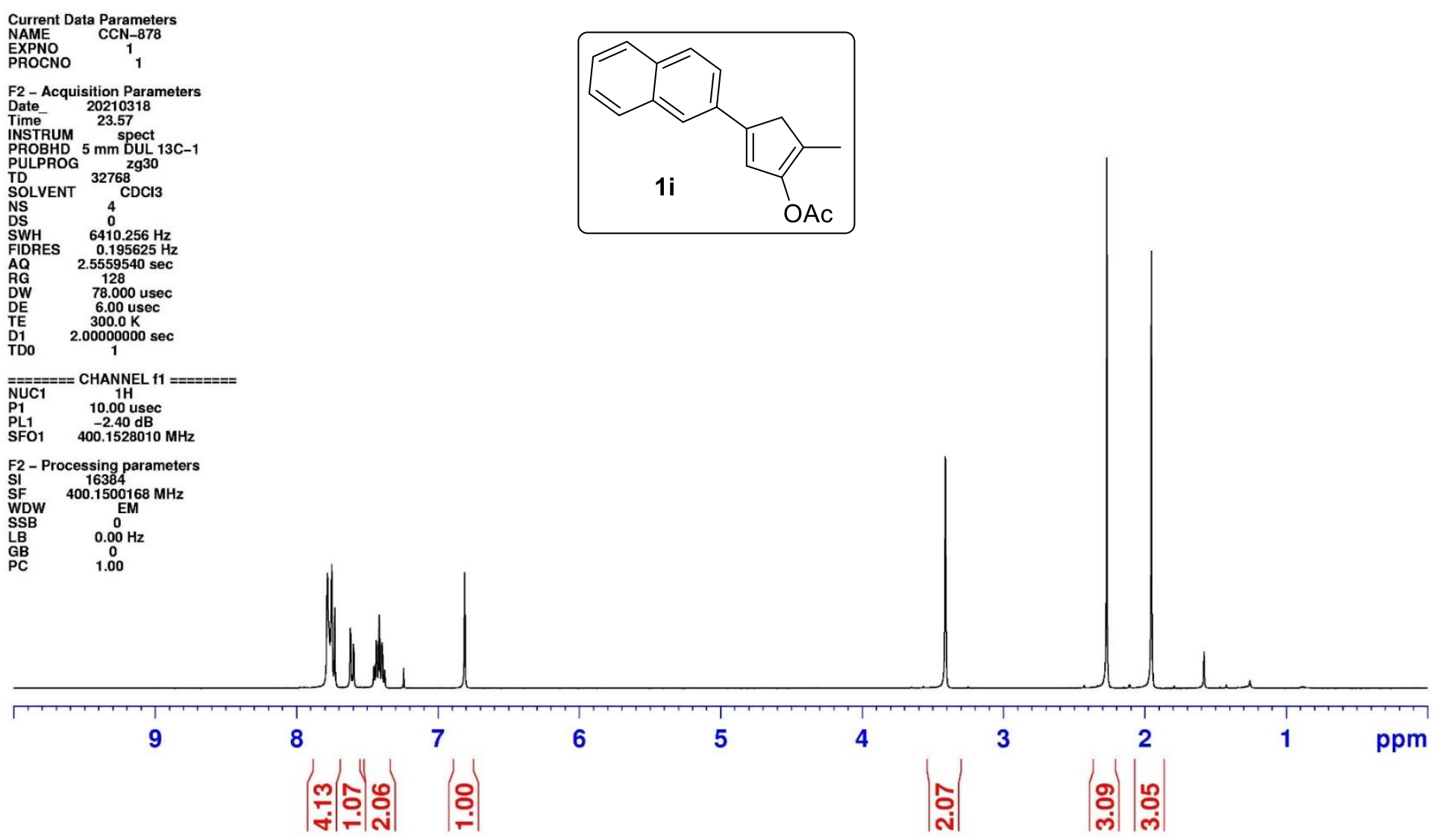



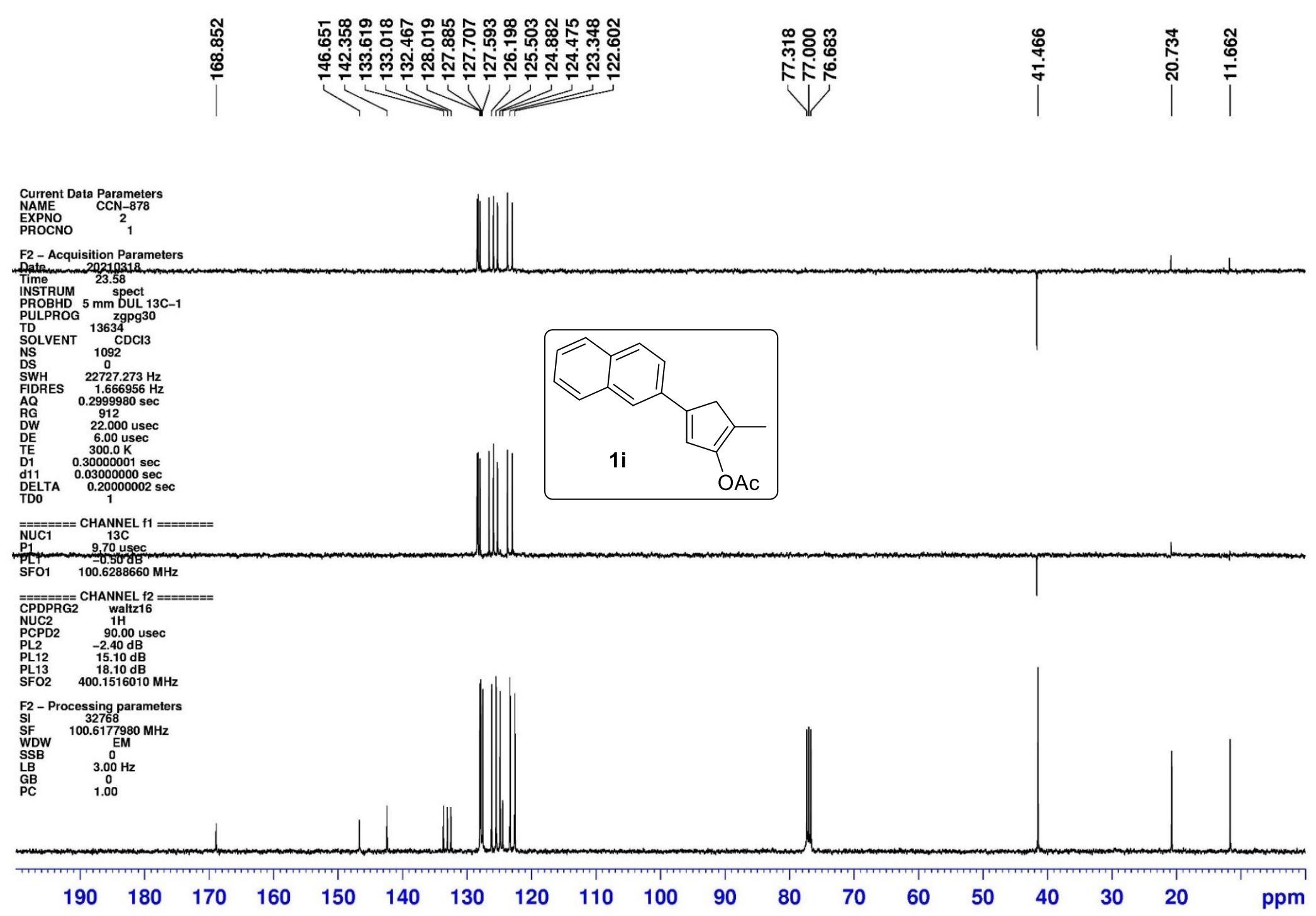


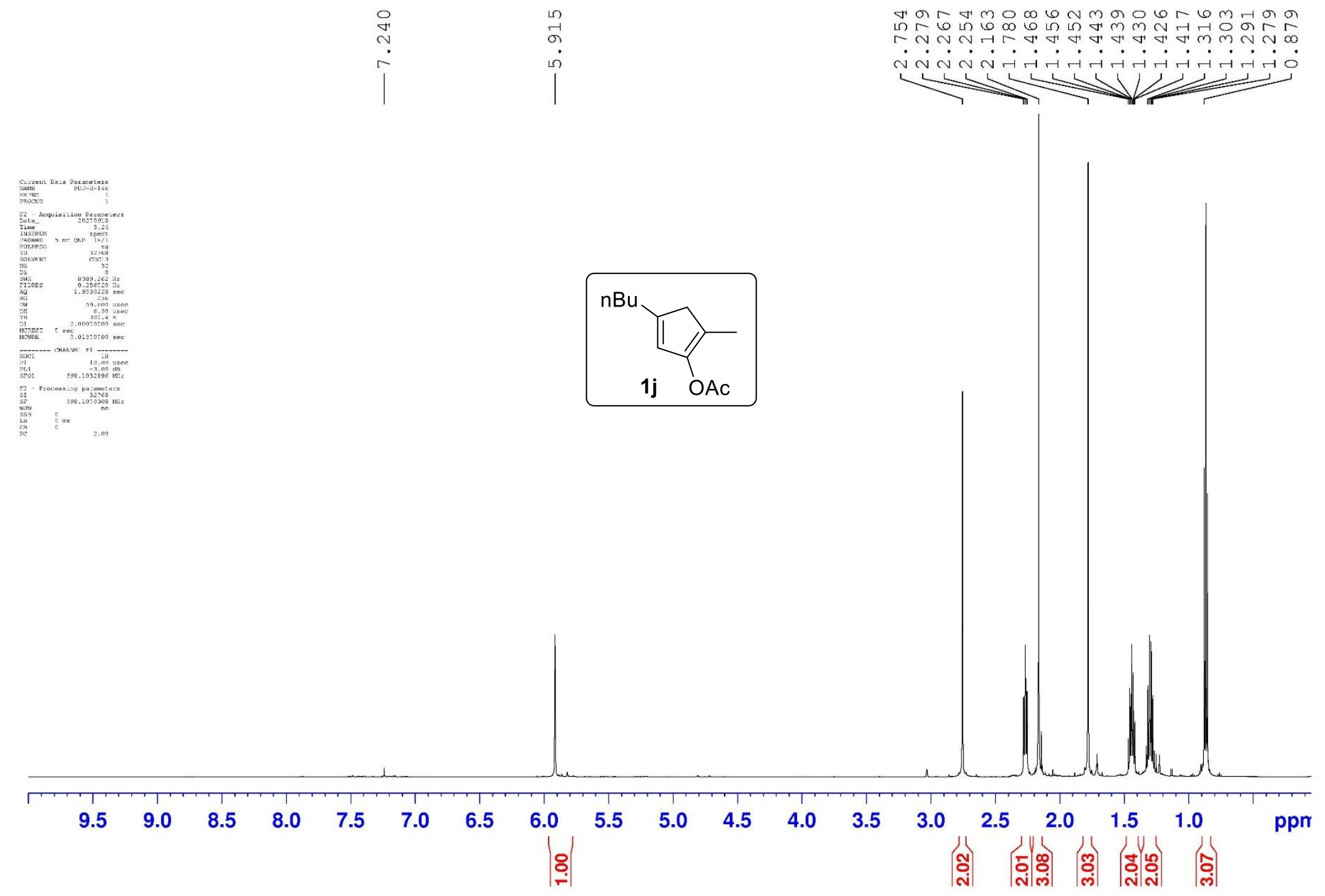




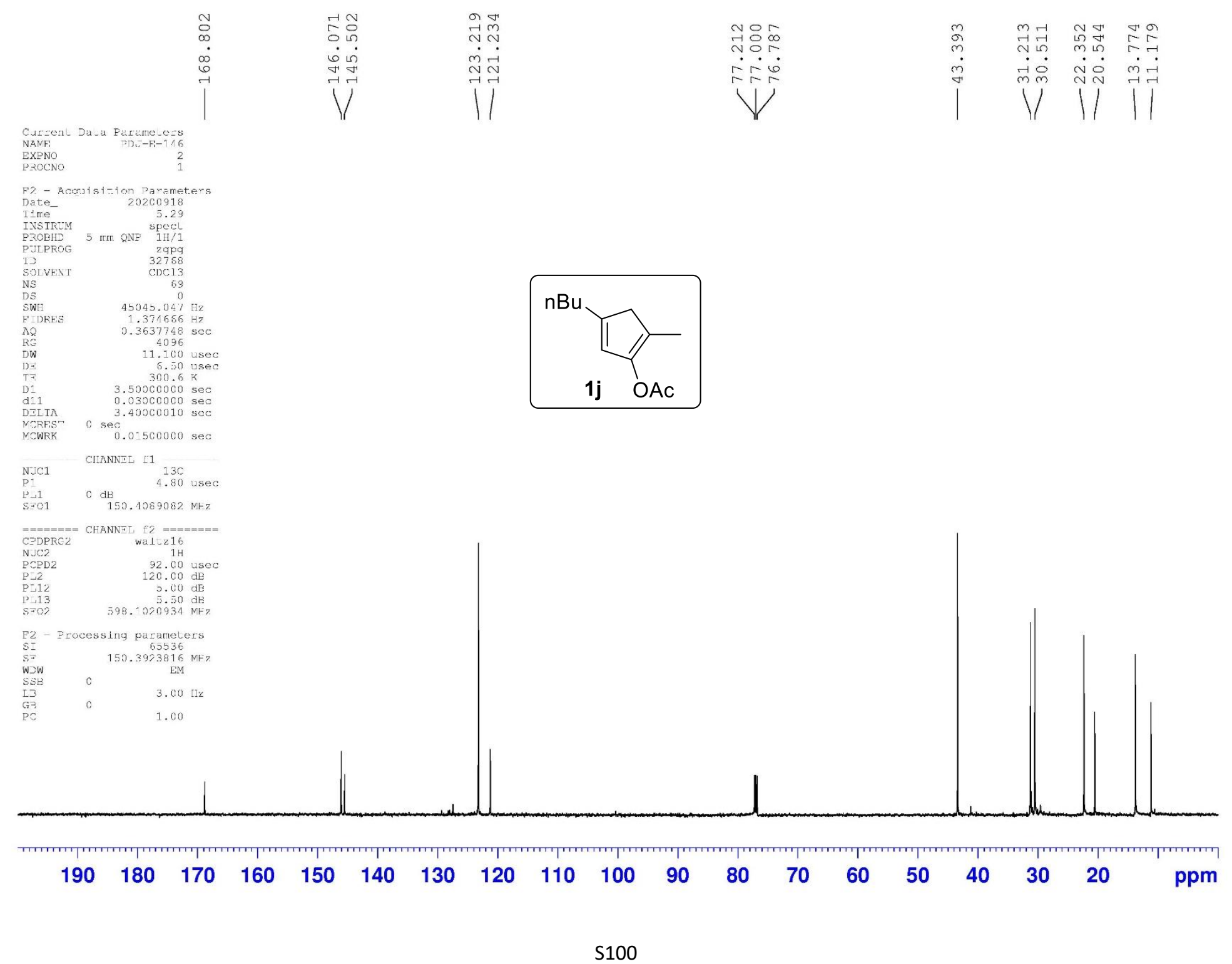




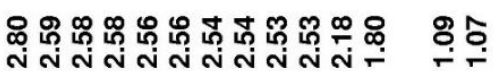

$\underbrace{\sqrt{3}}$

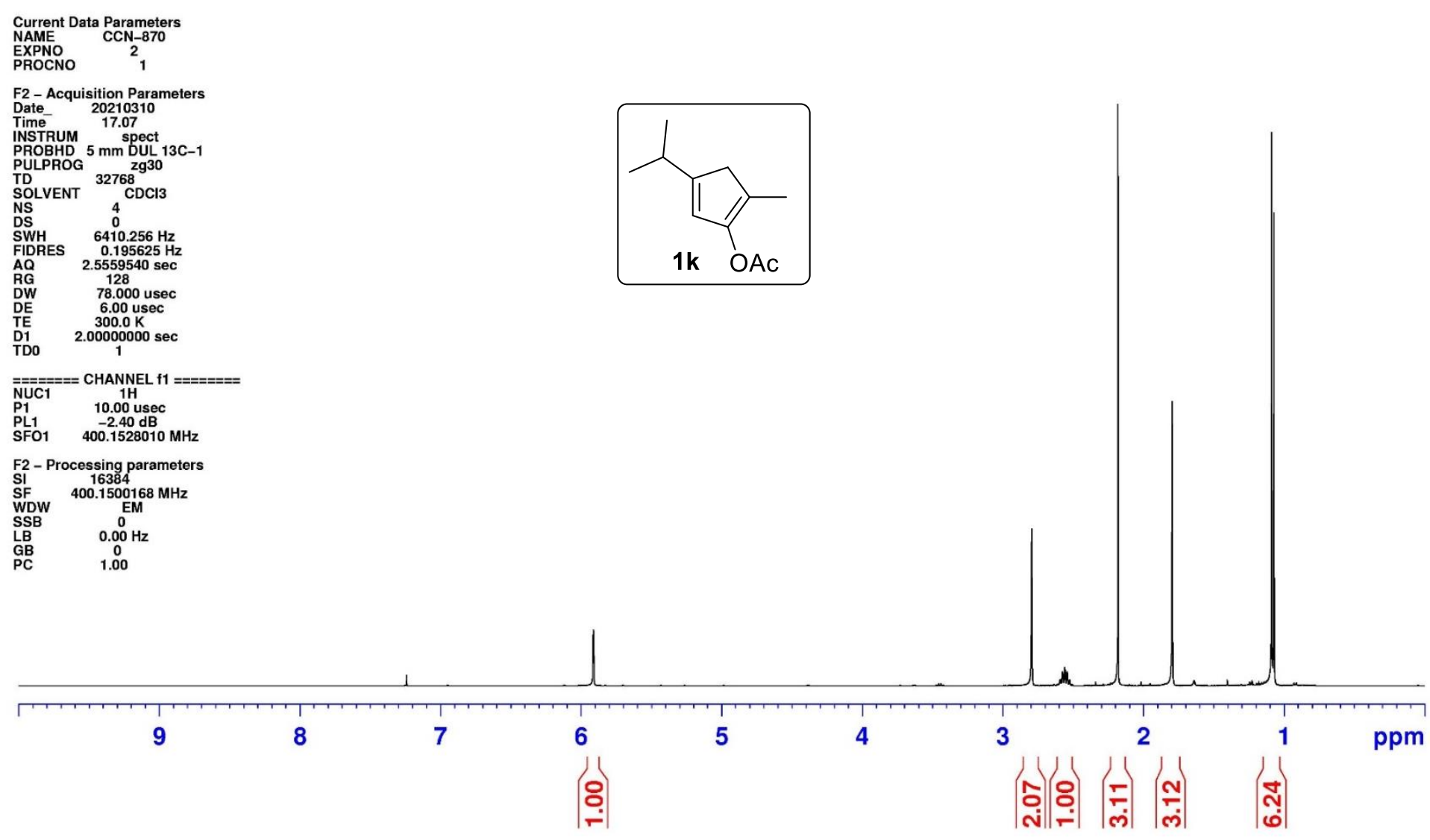



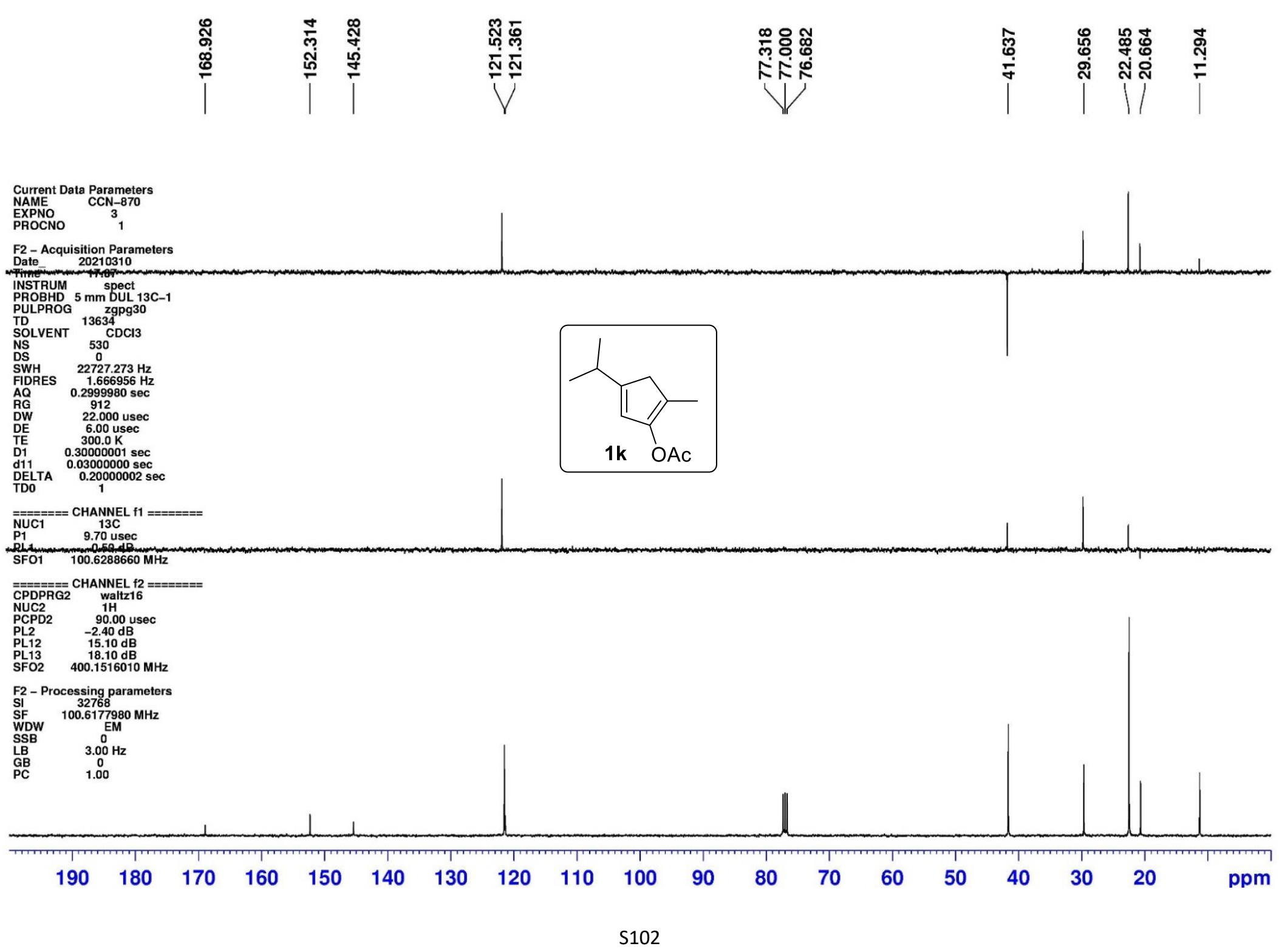

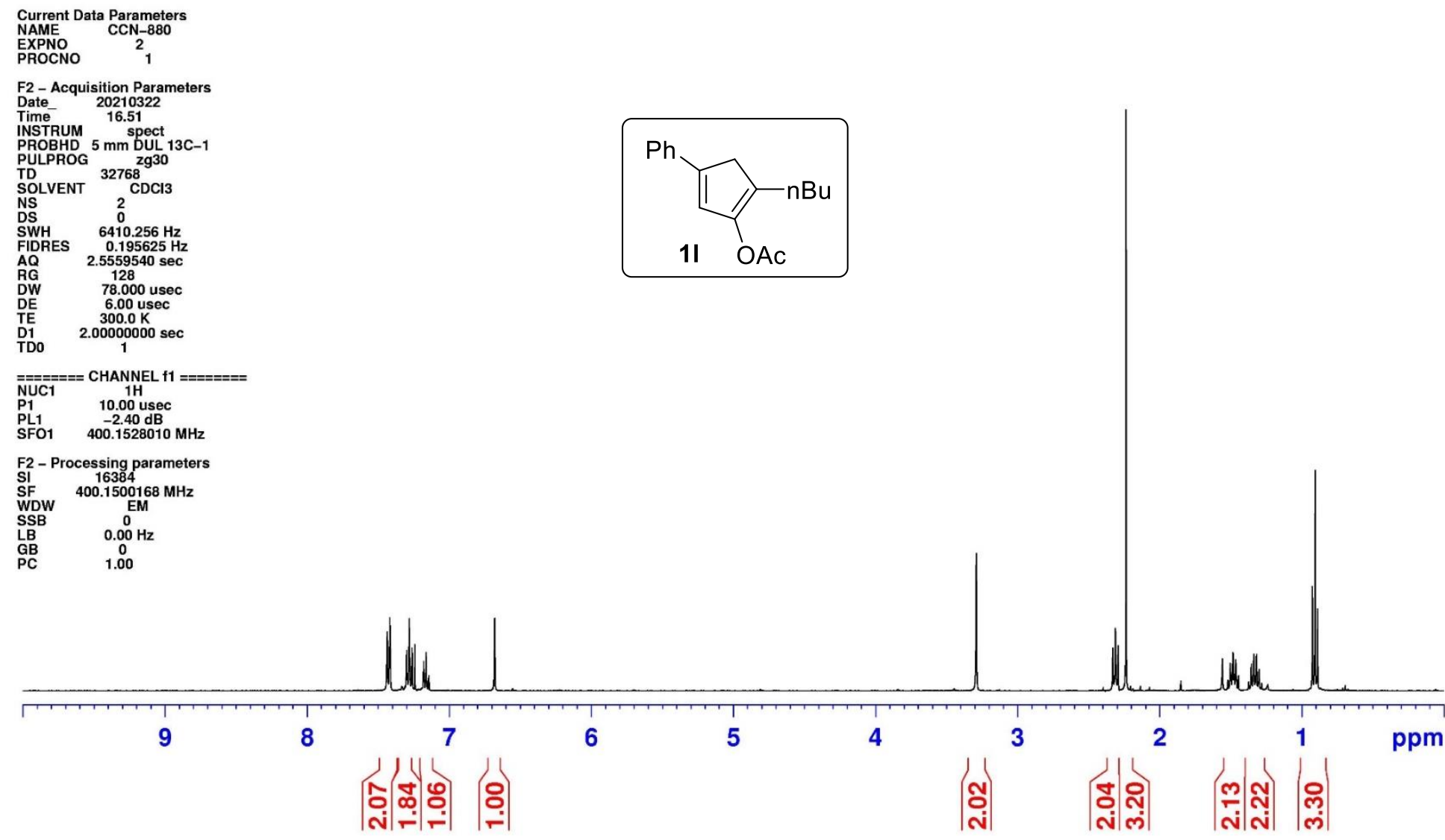

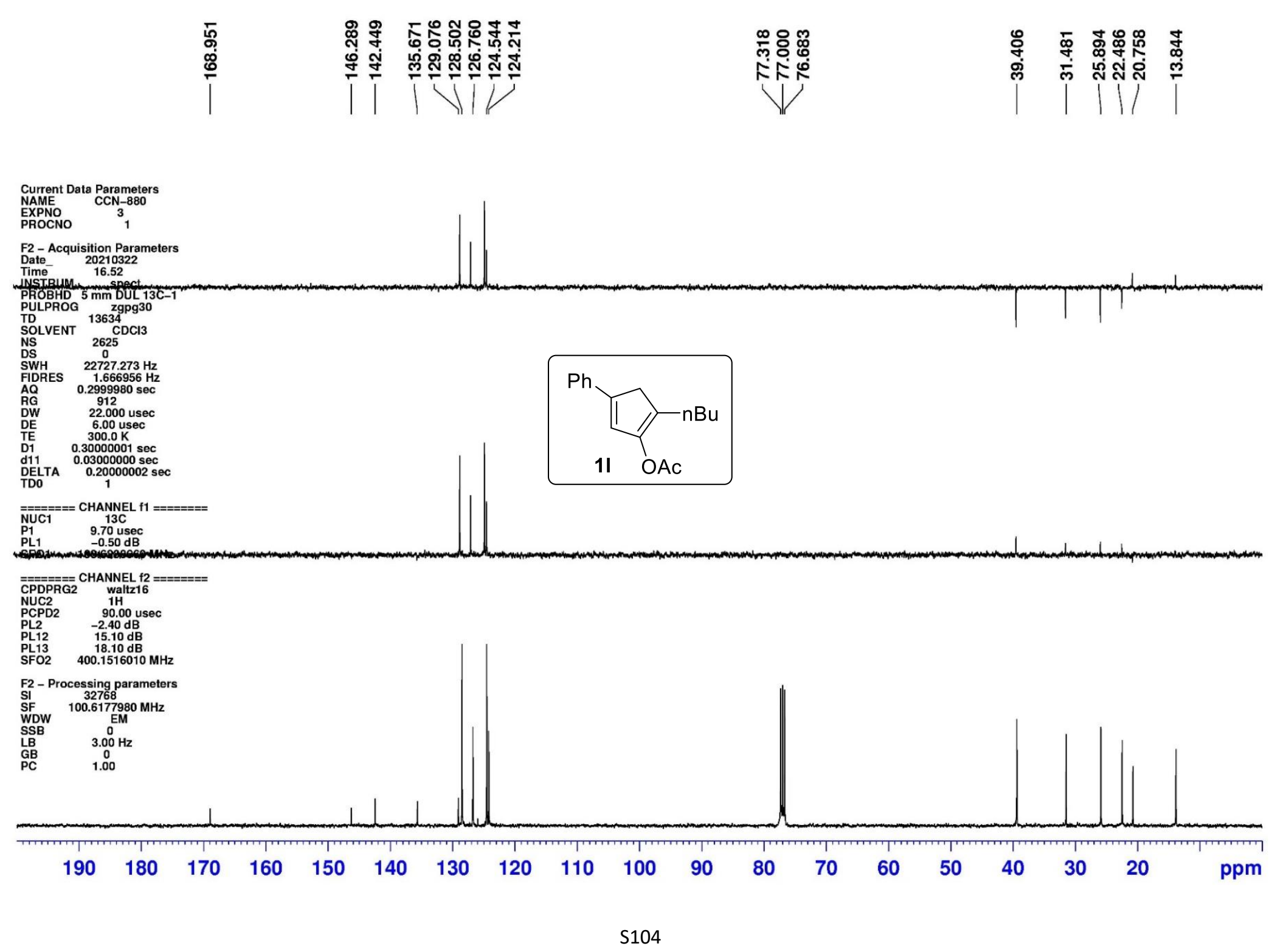

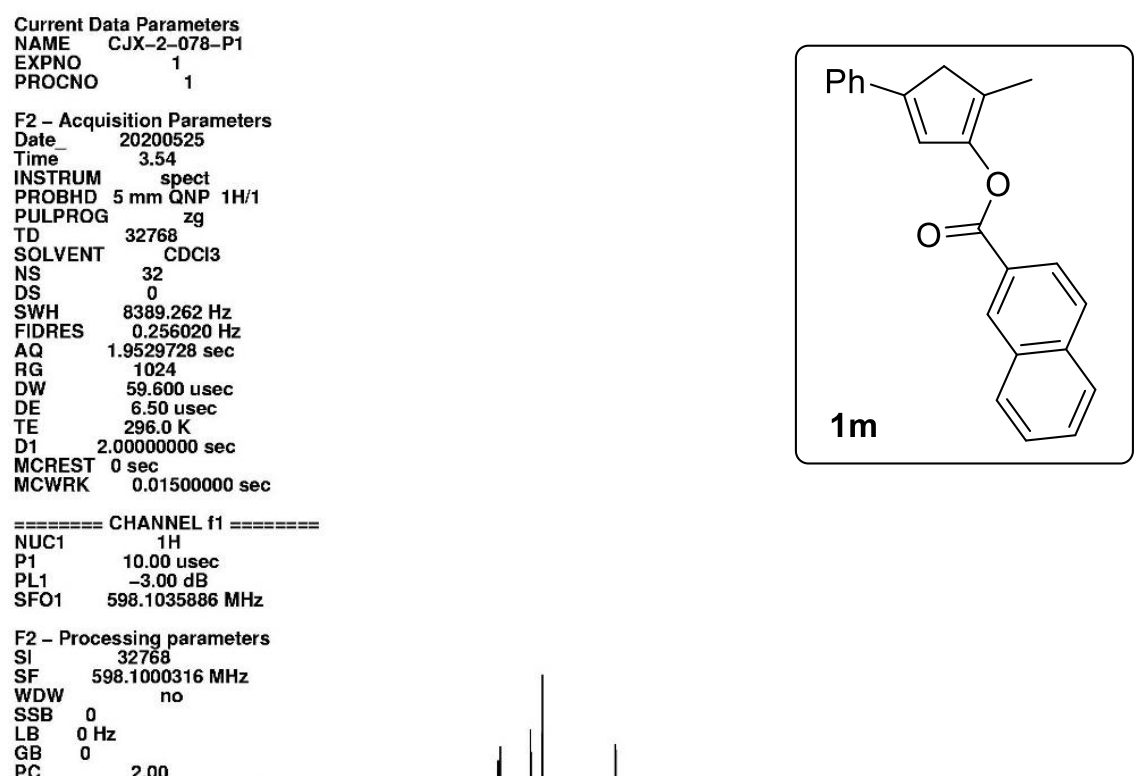

$\begin{array}{lll}\mathrm{GB} & & \\ \mathrm{PC} & & \end{array}$

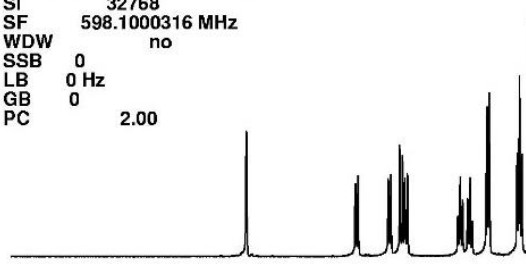

|ำ
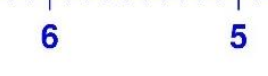

đั

ppm

लํ. 

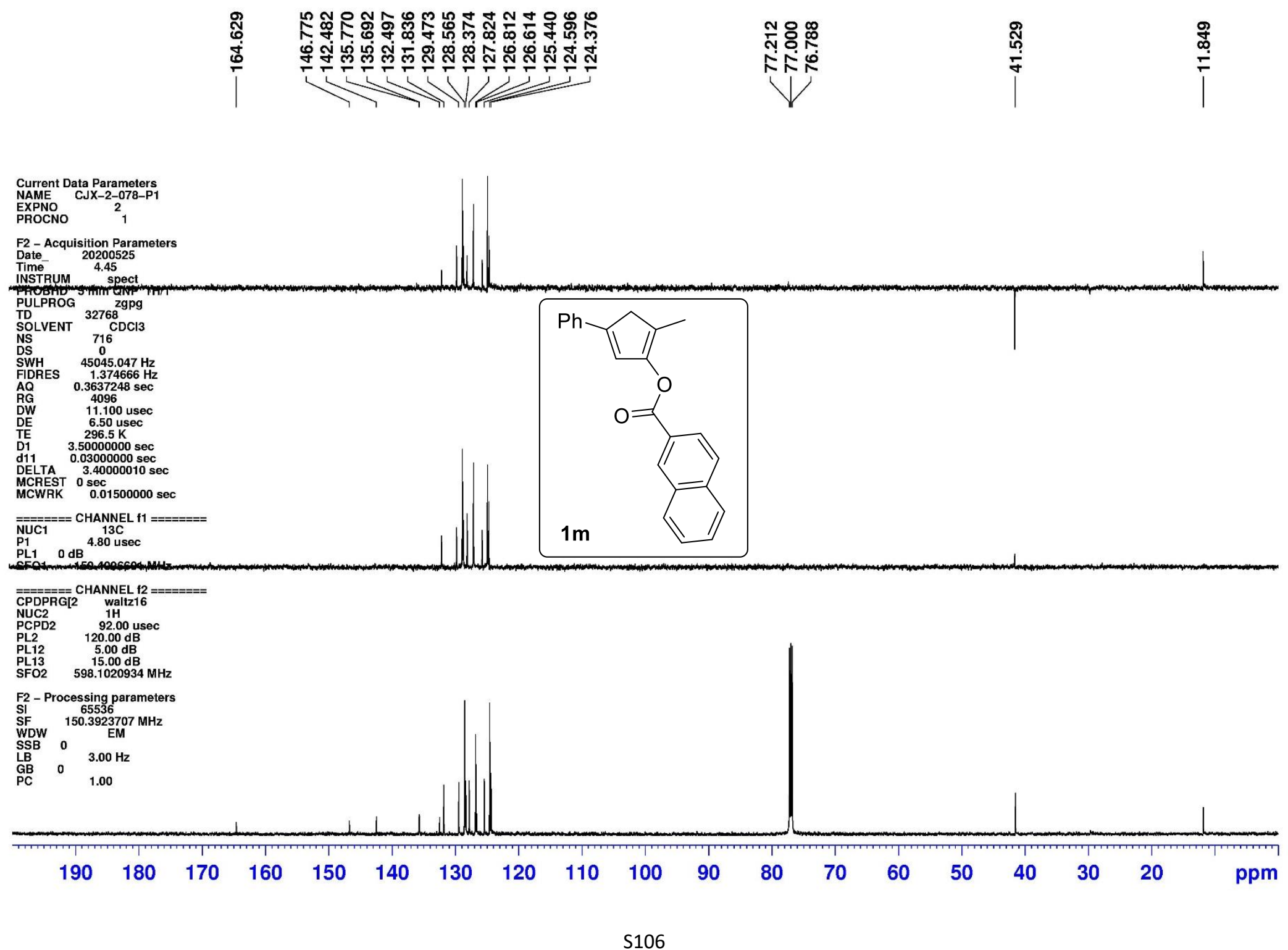


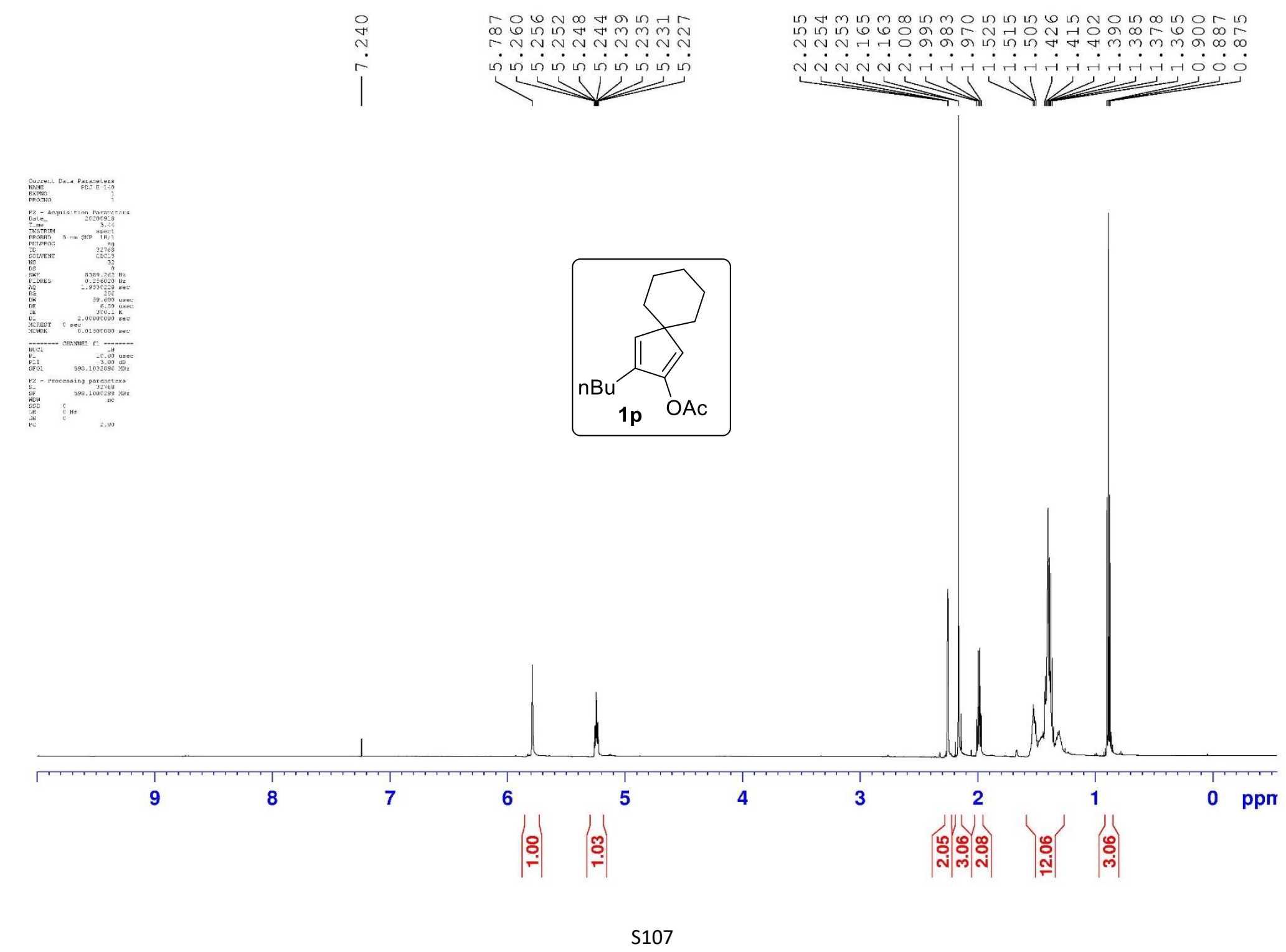




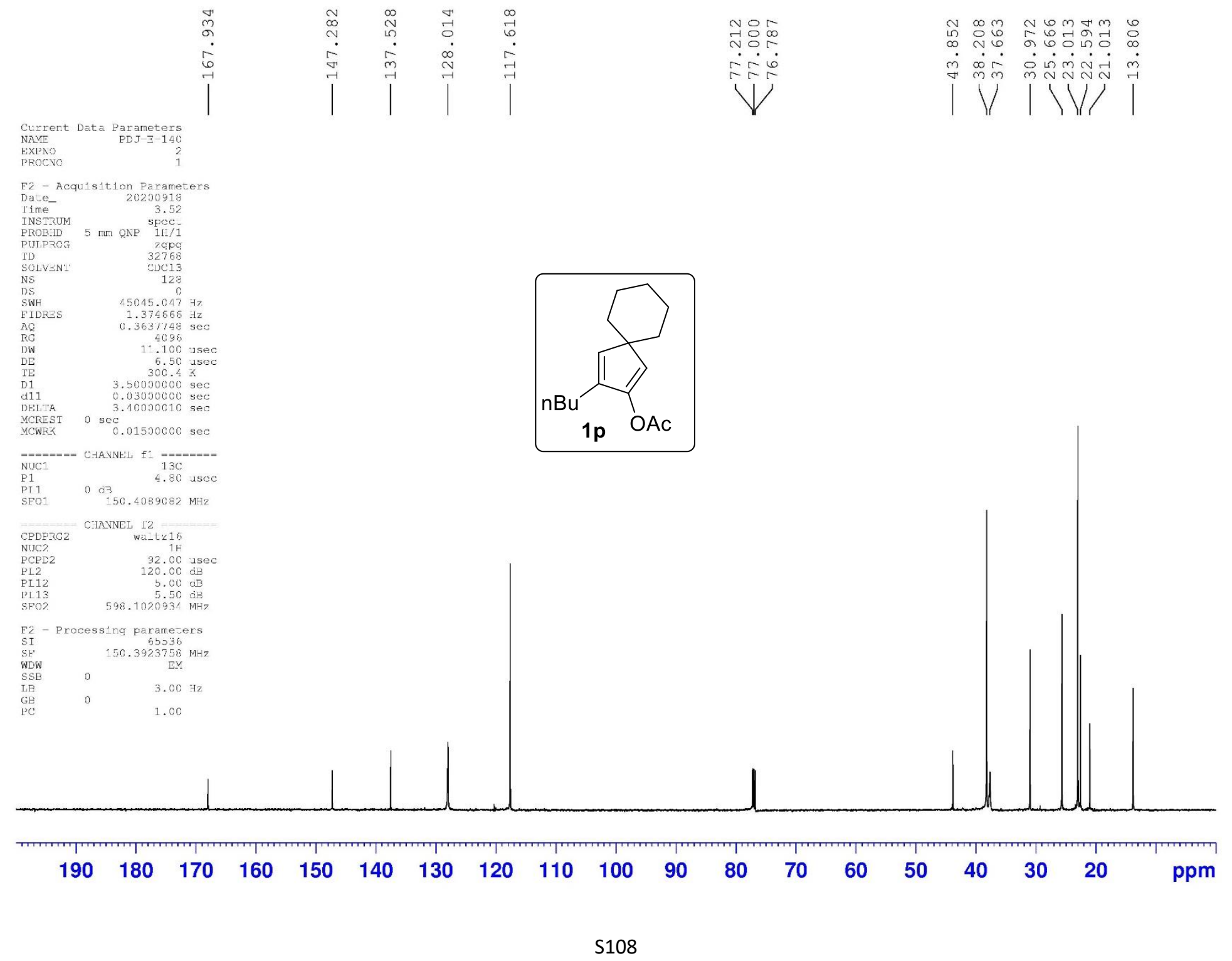


め ๘

mmmmmm N N N

rrarararar

$\ldots$

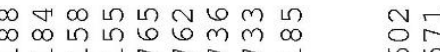

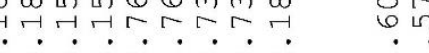

$\sqrt{V^{2}}$
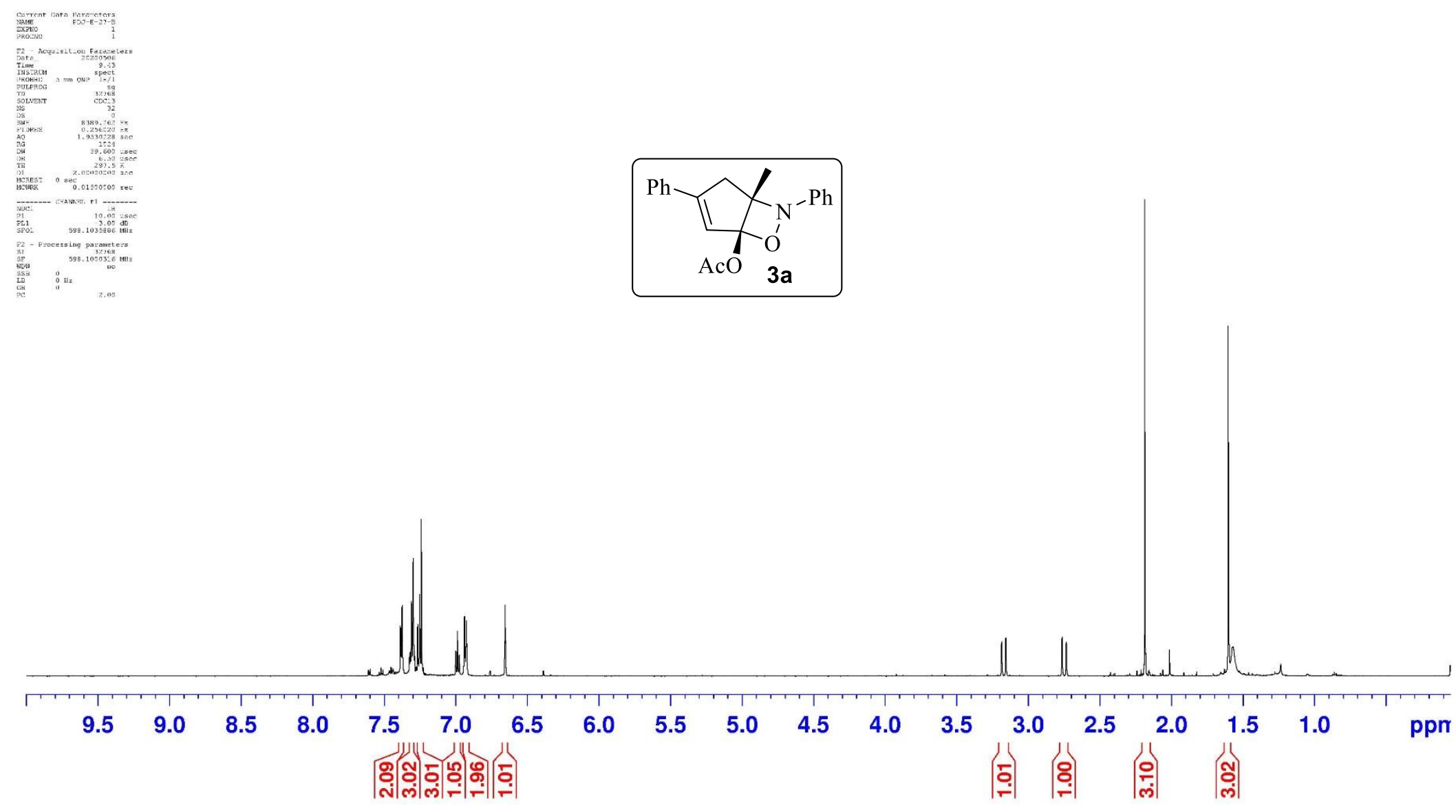


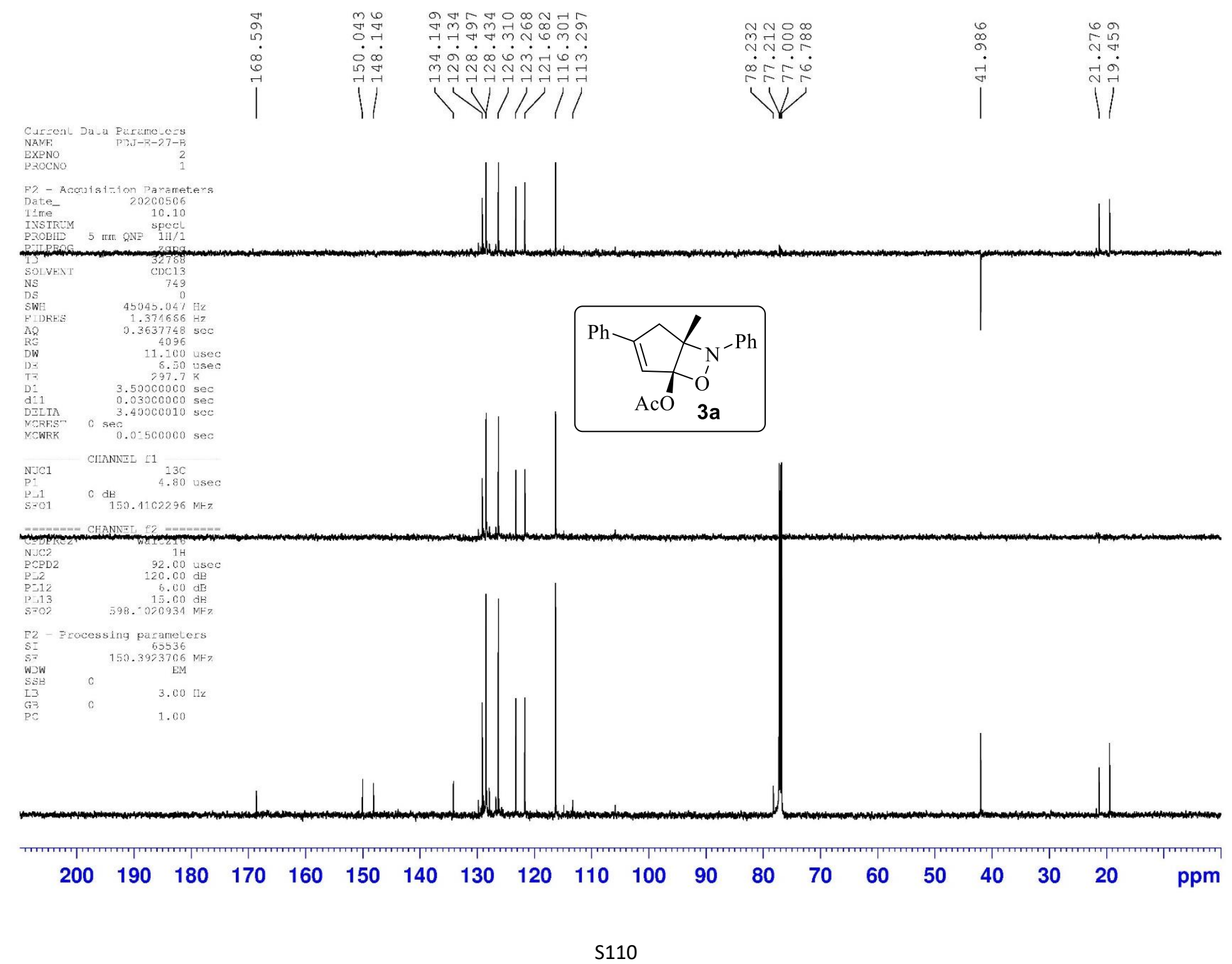



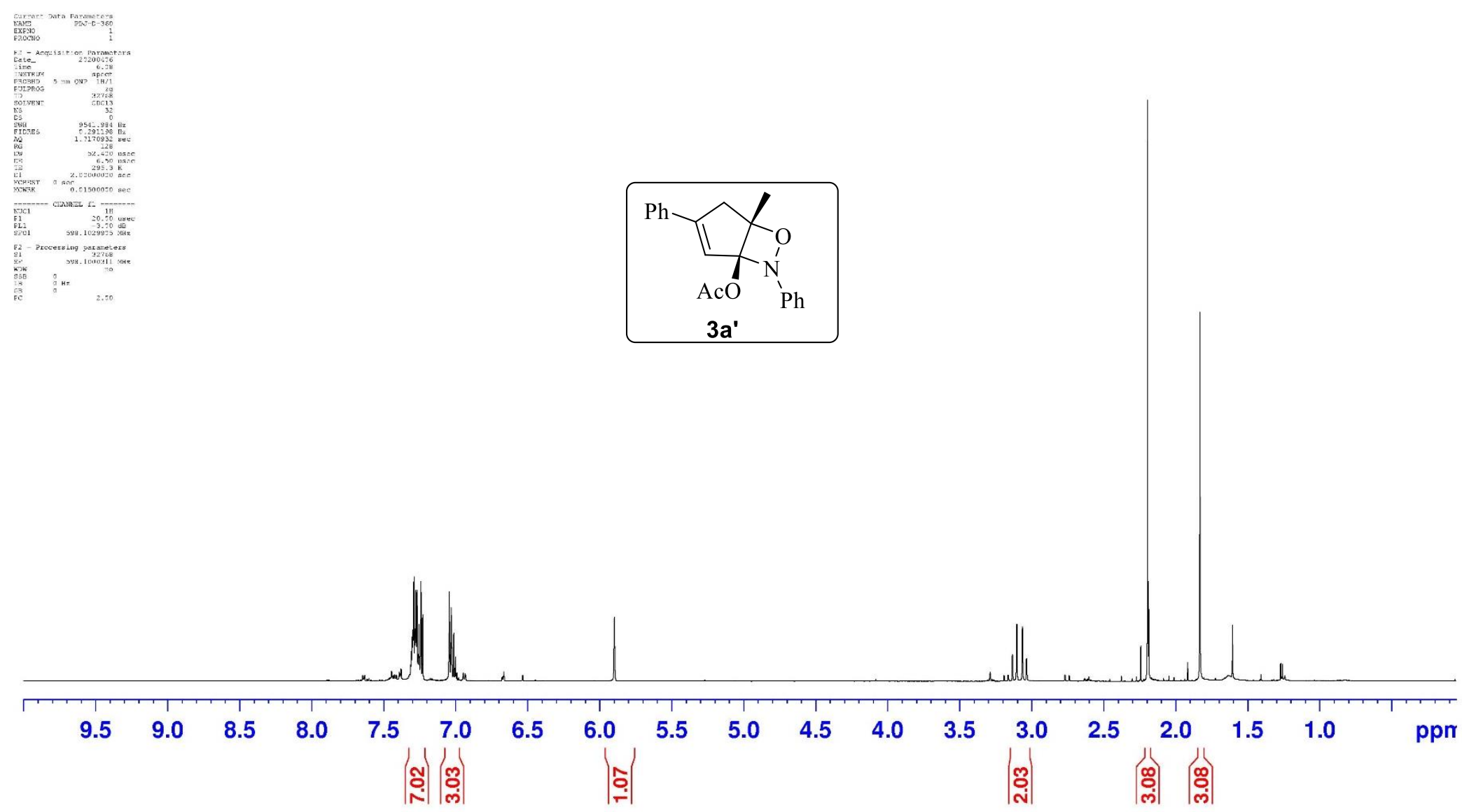


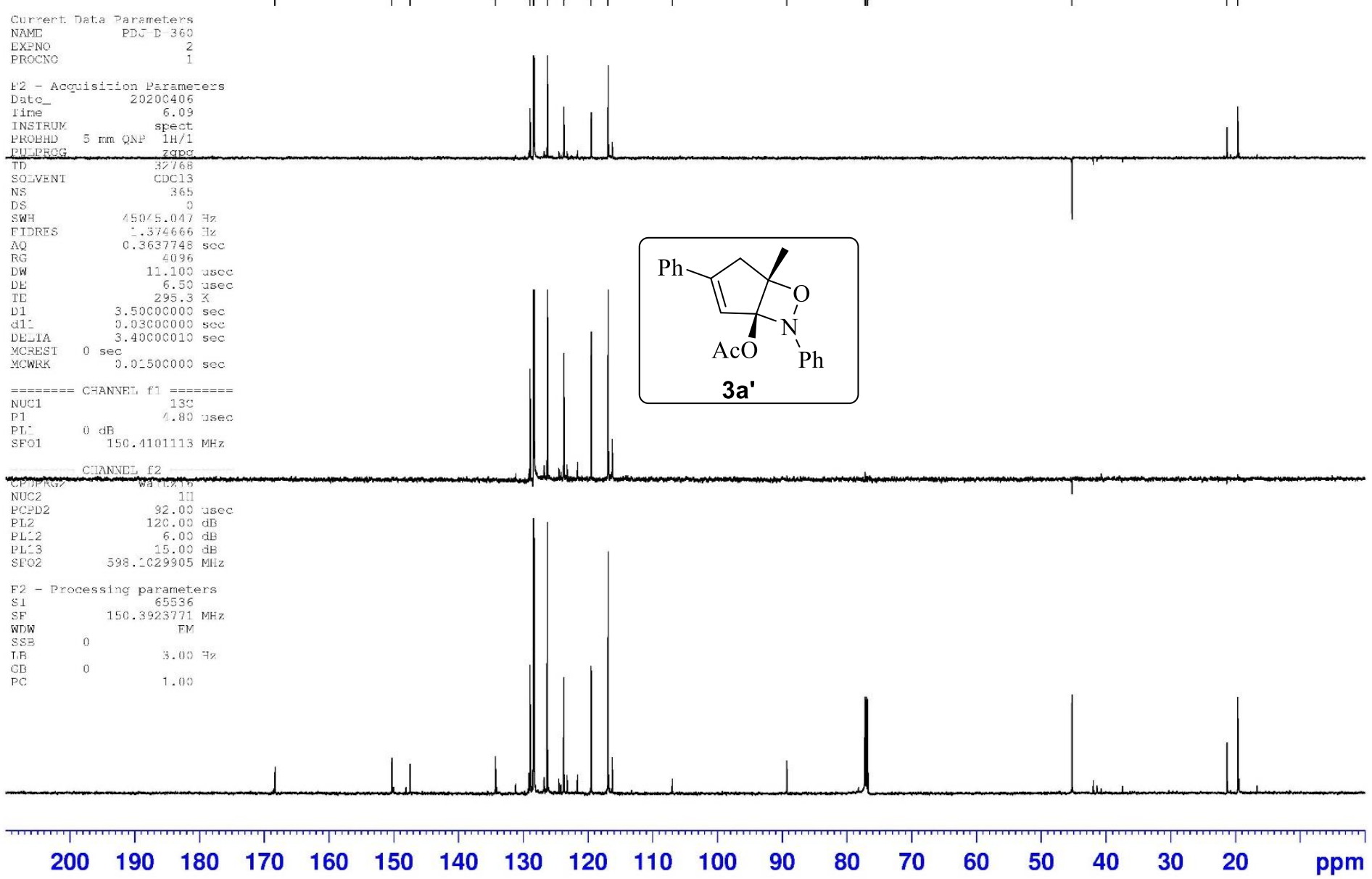



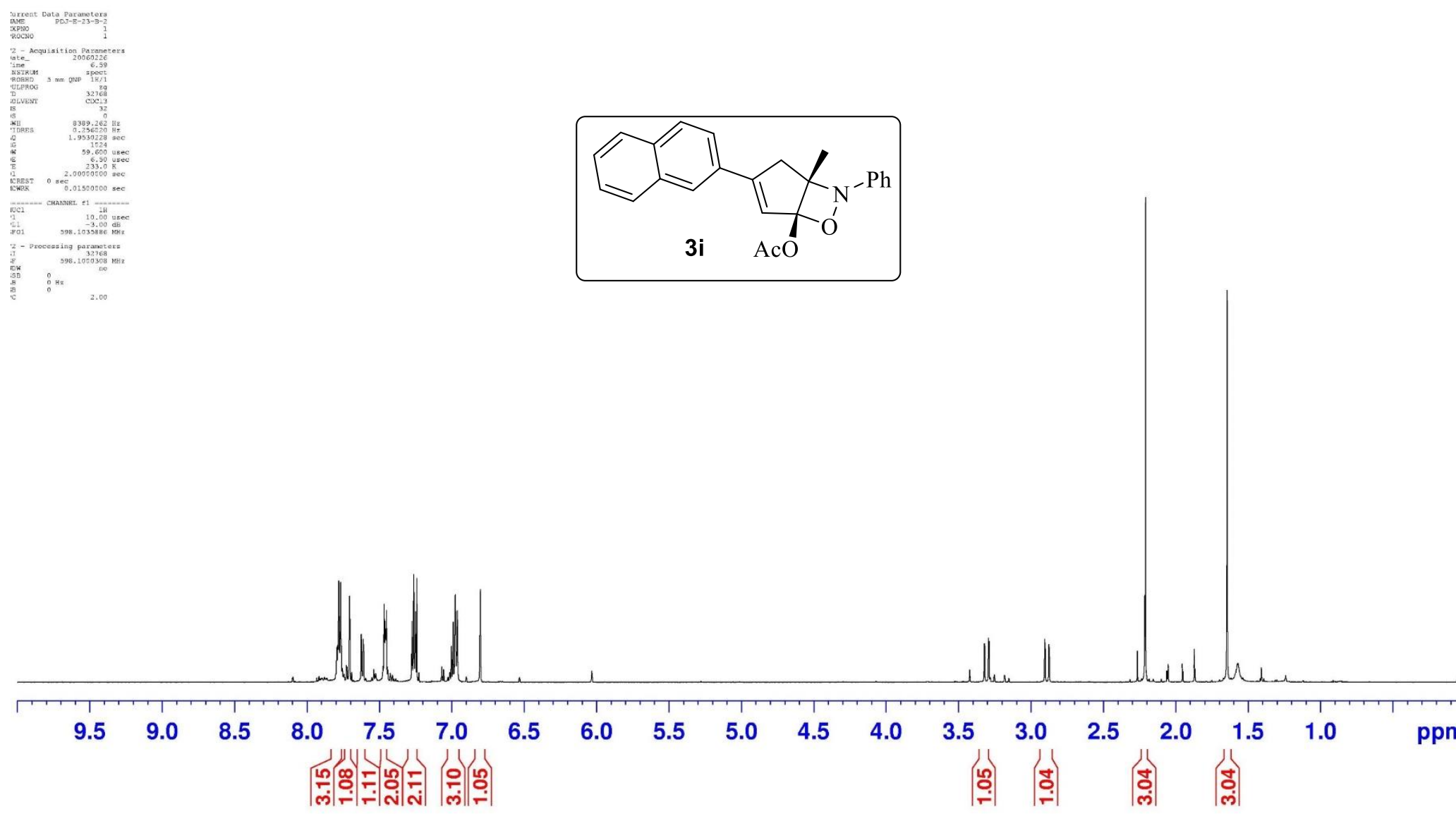


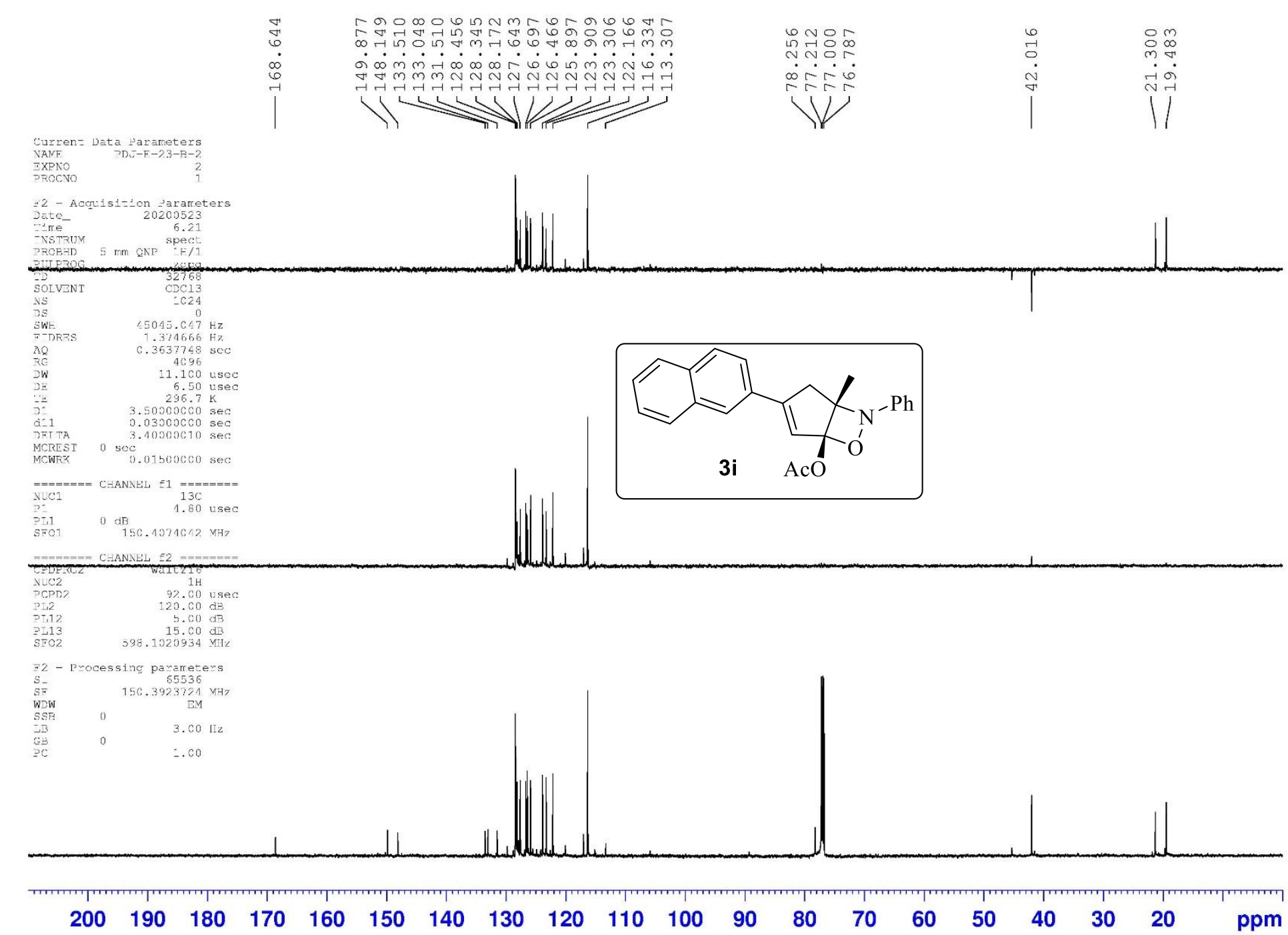




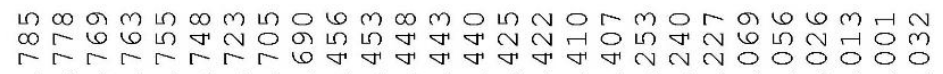

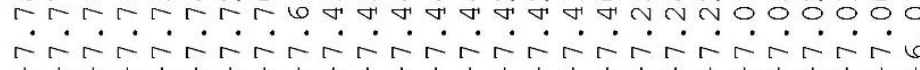

moํㅐำ

Im
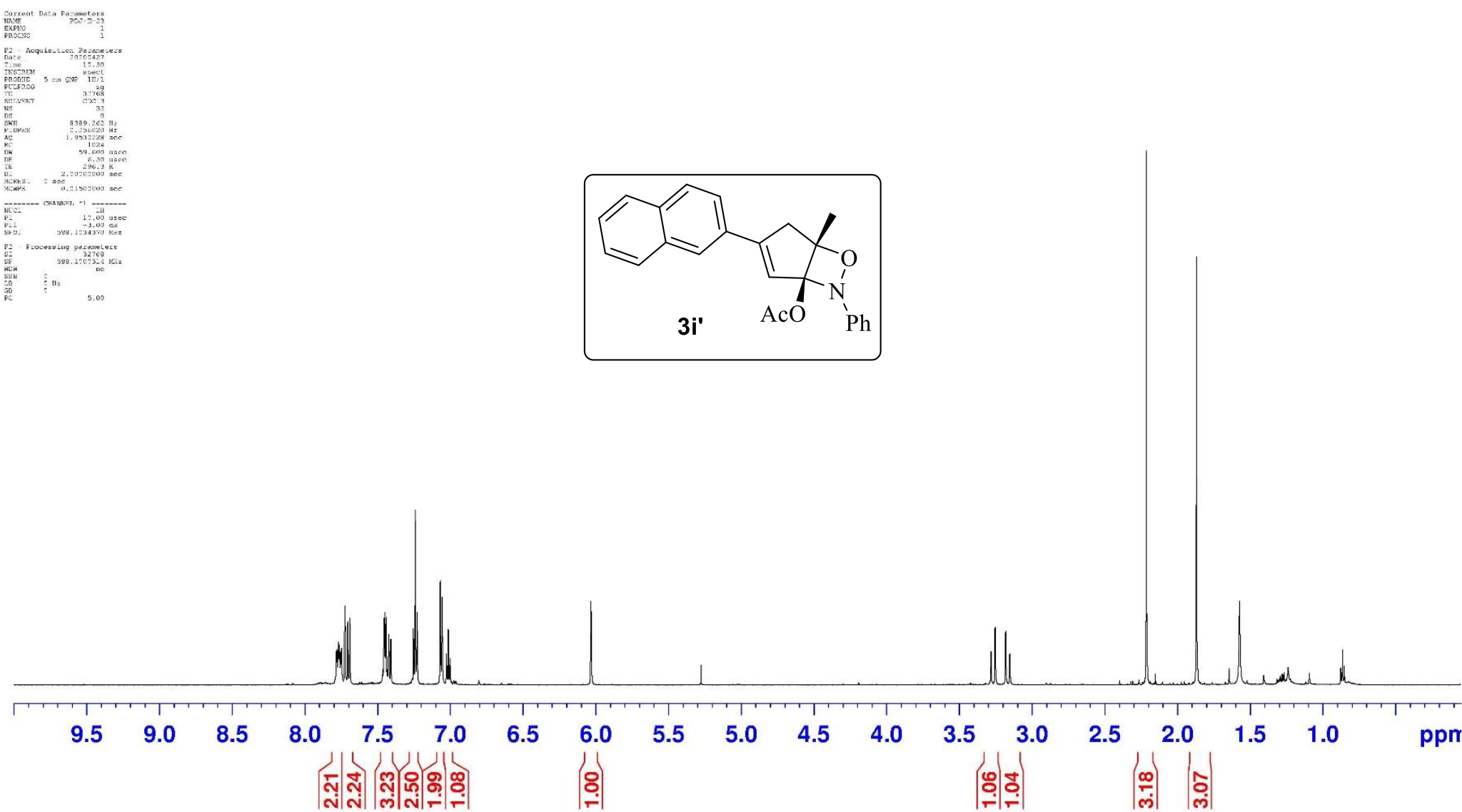


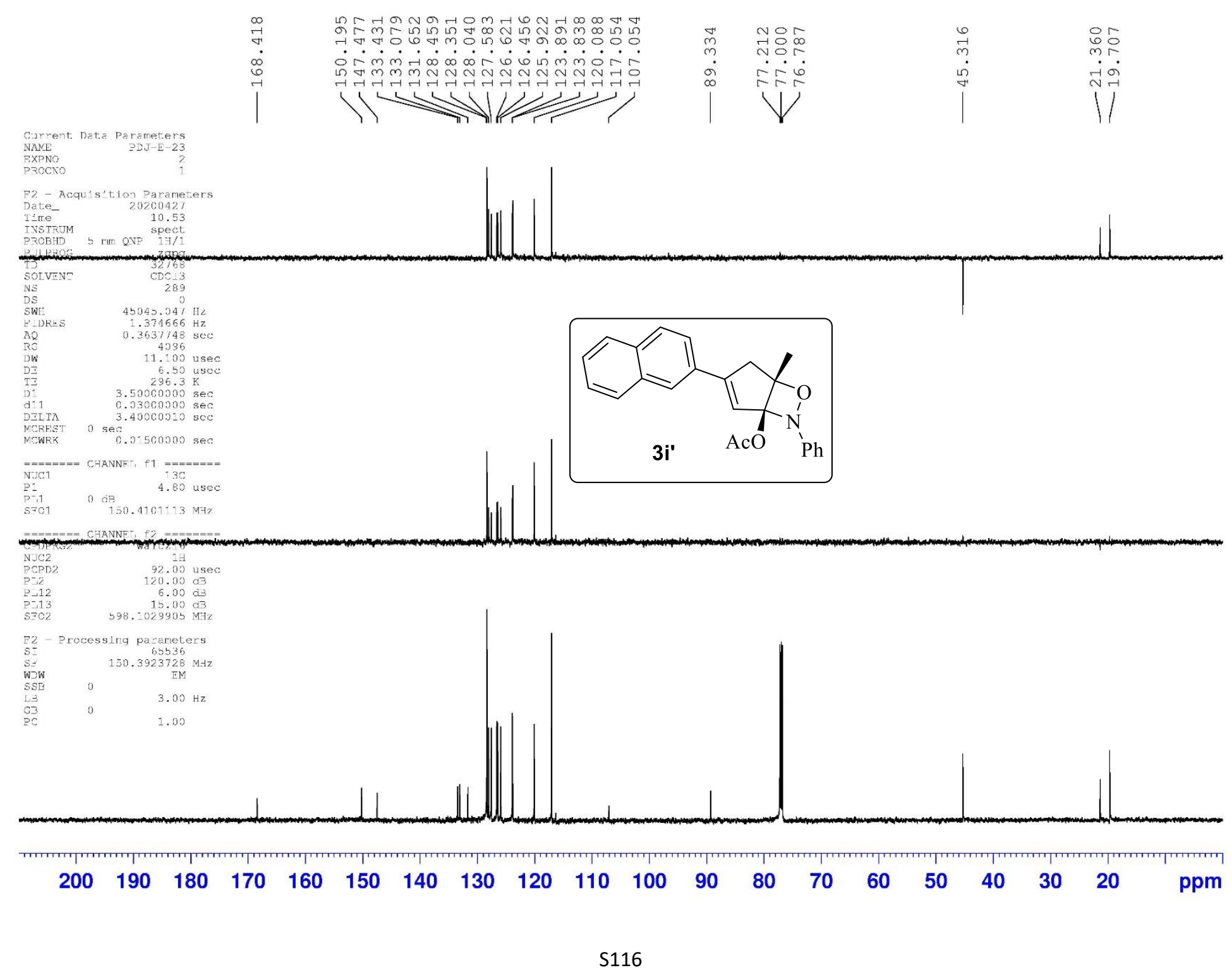




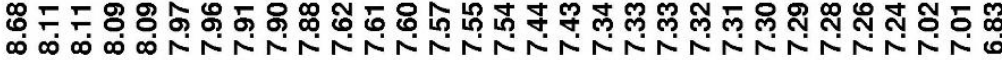

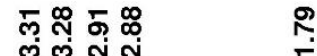

ग

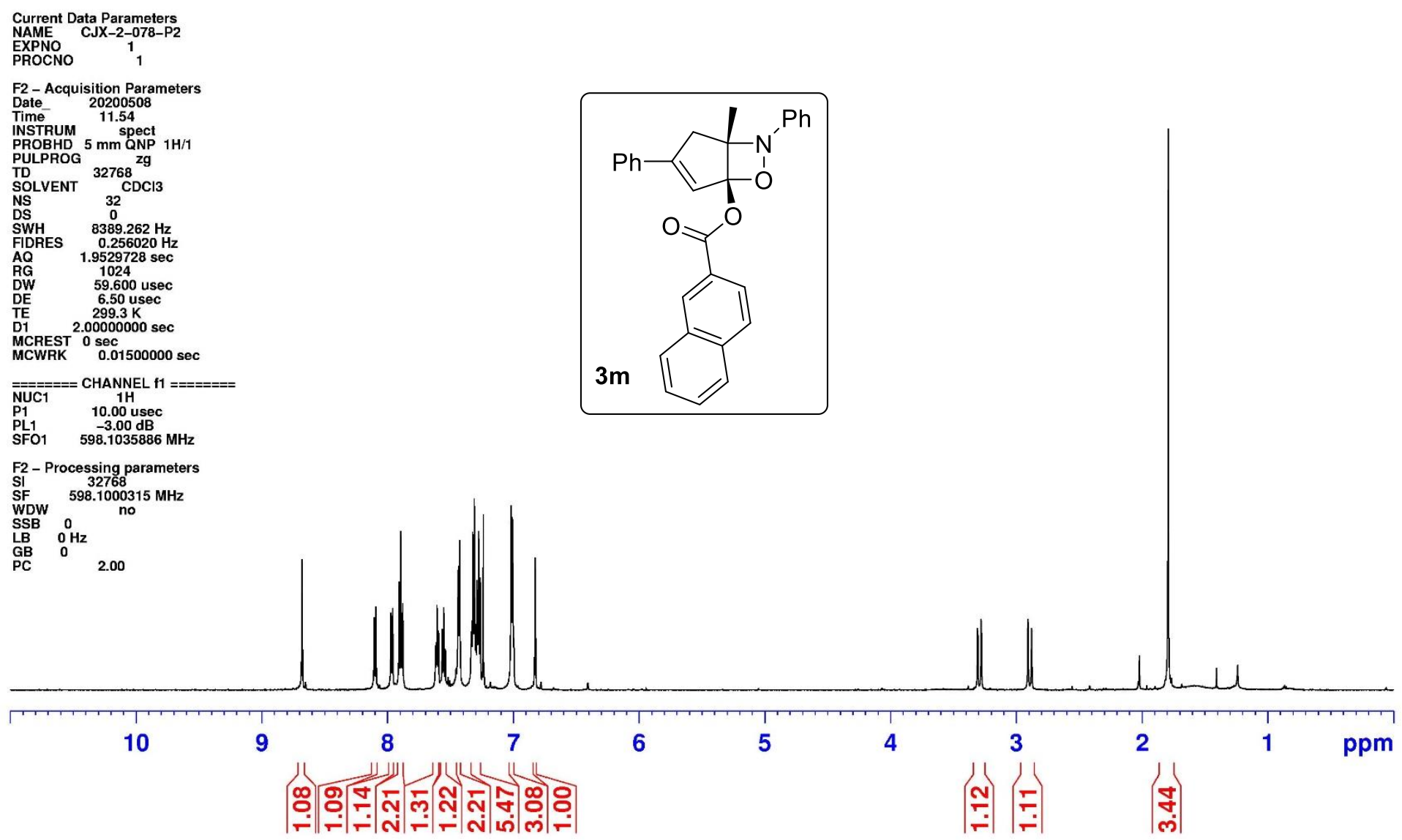




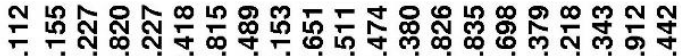

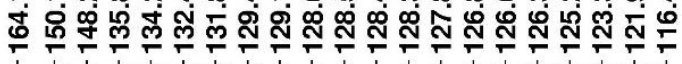 \\ 웅 꾸ำ

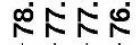 \\ |}

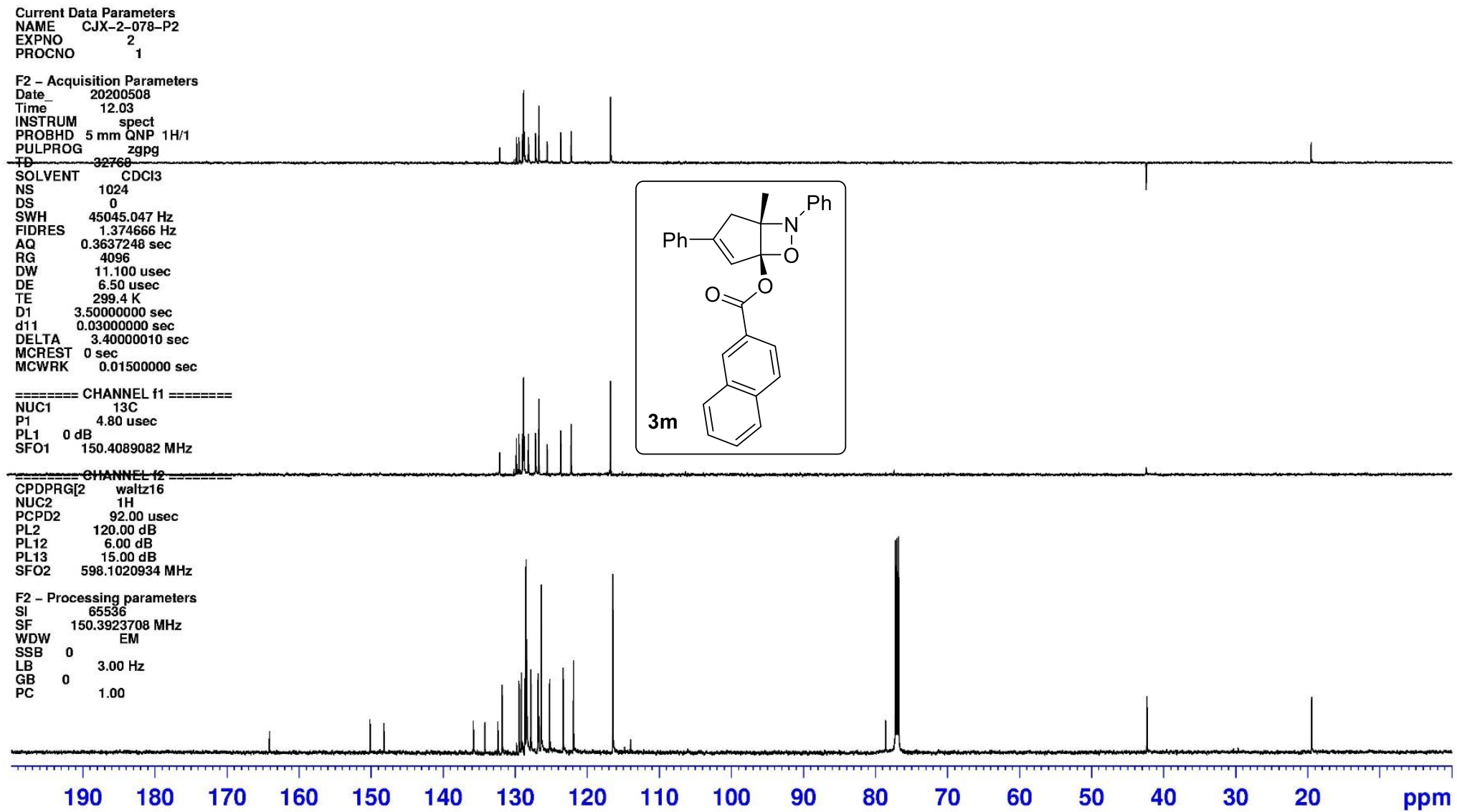



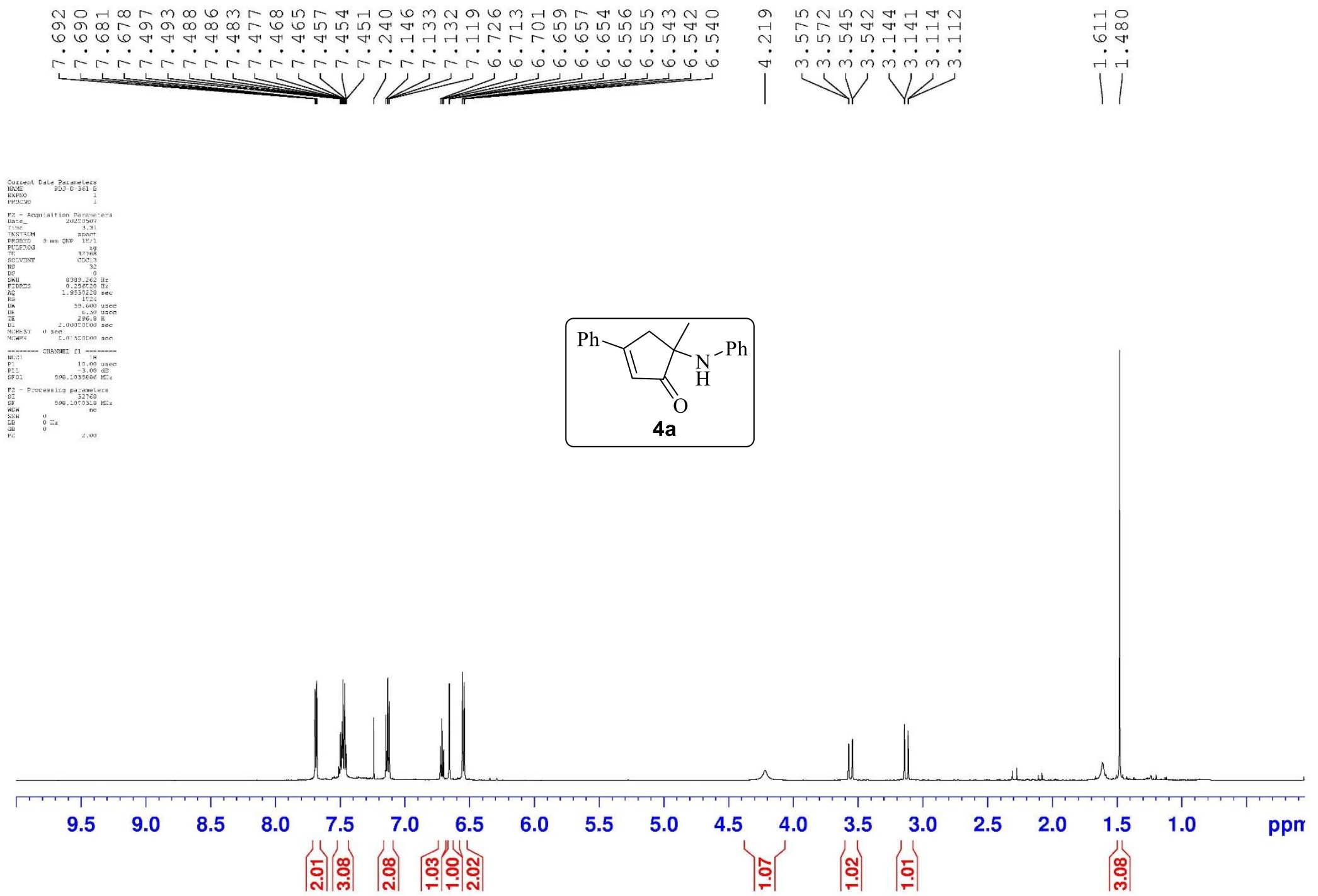


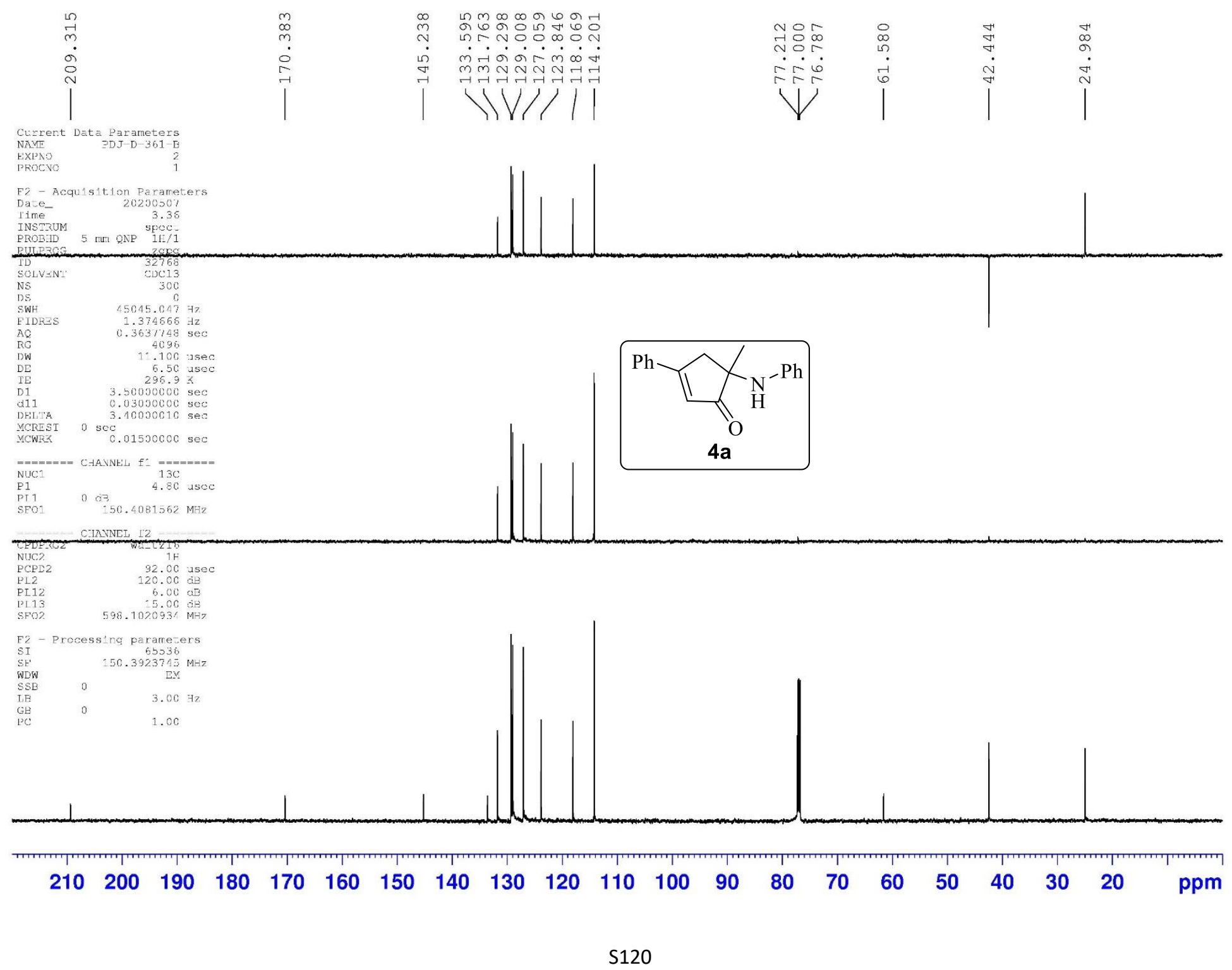



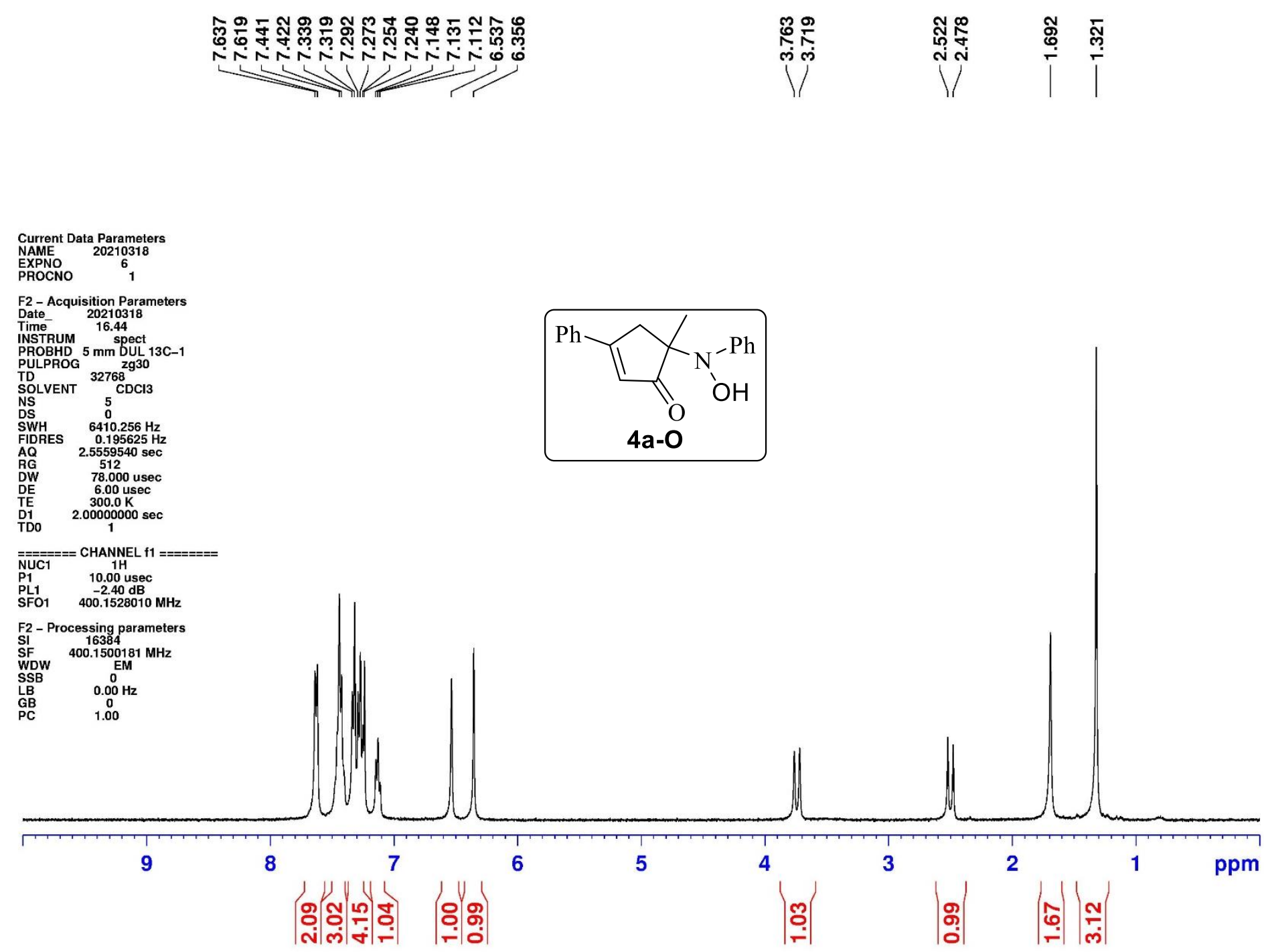


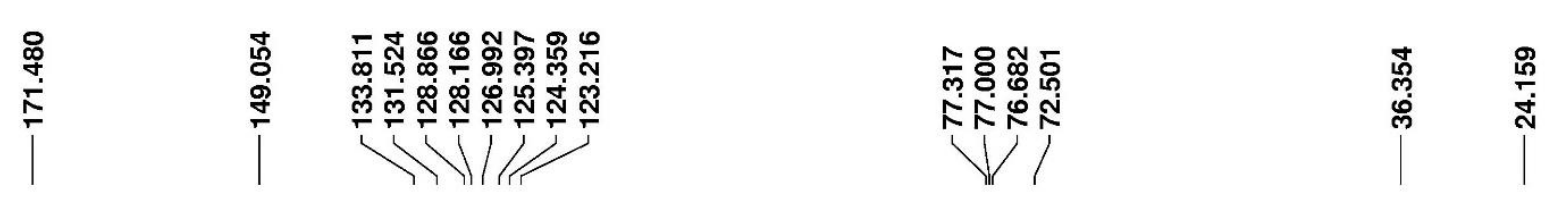

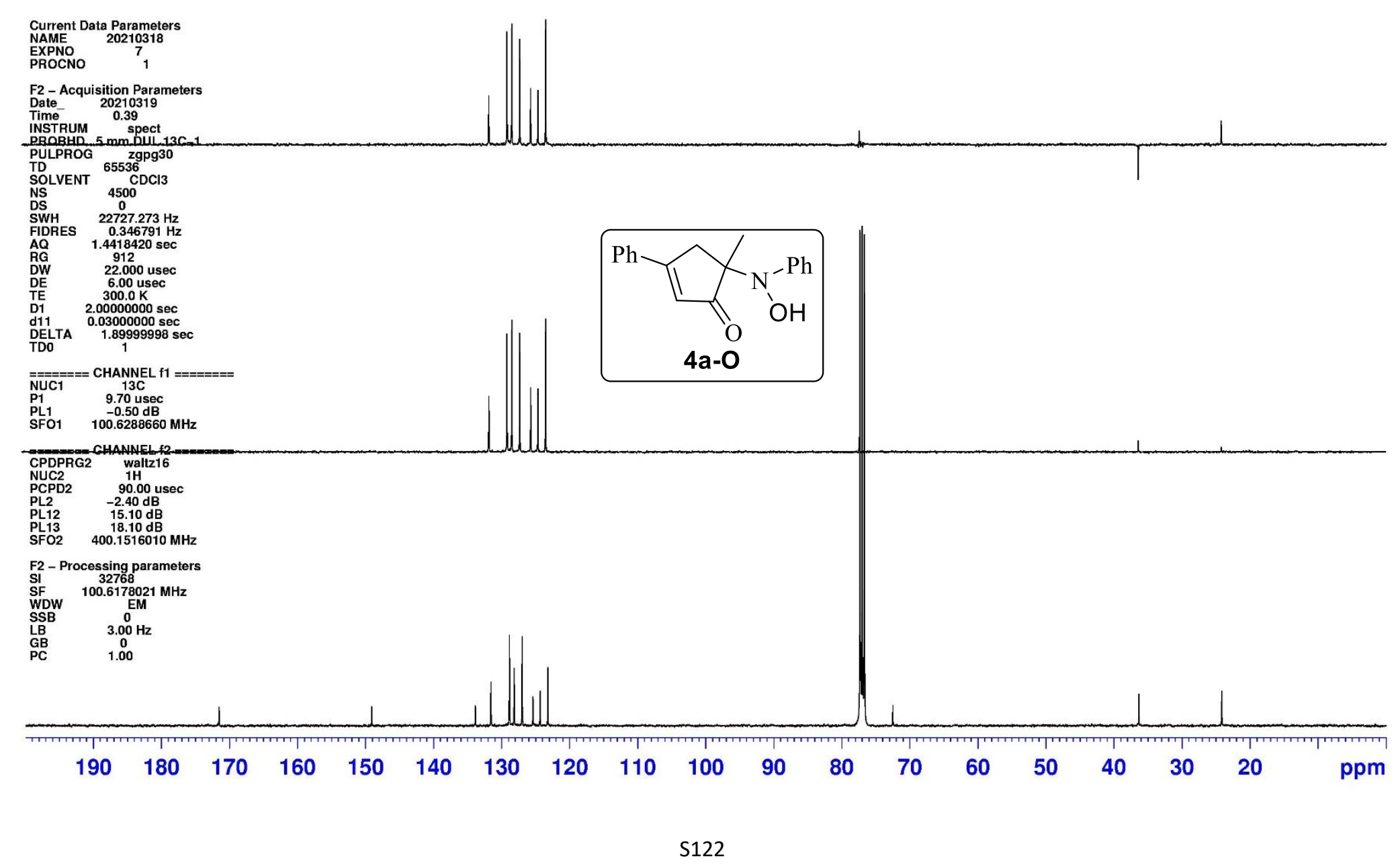



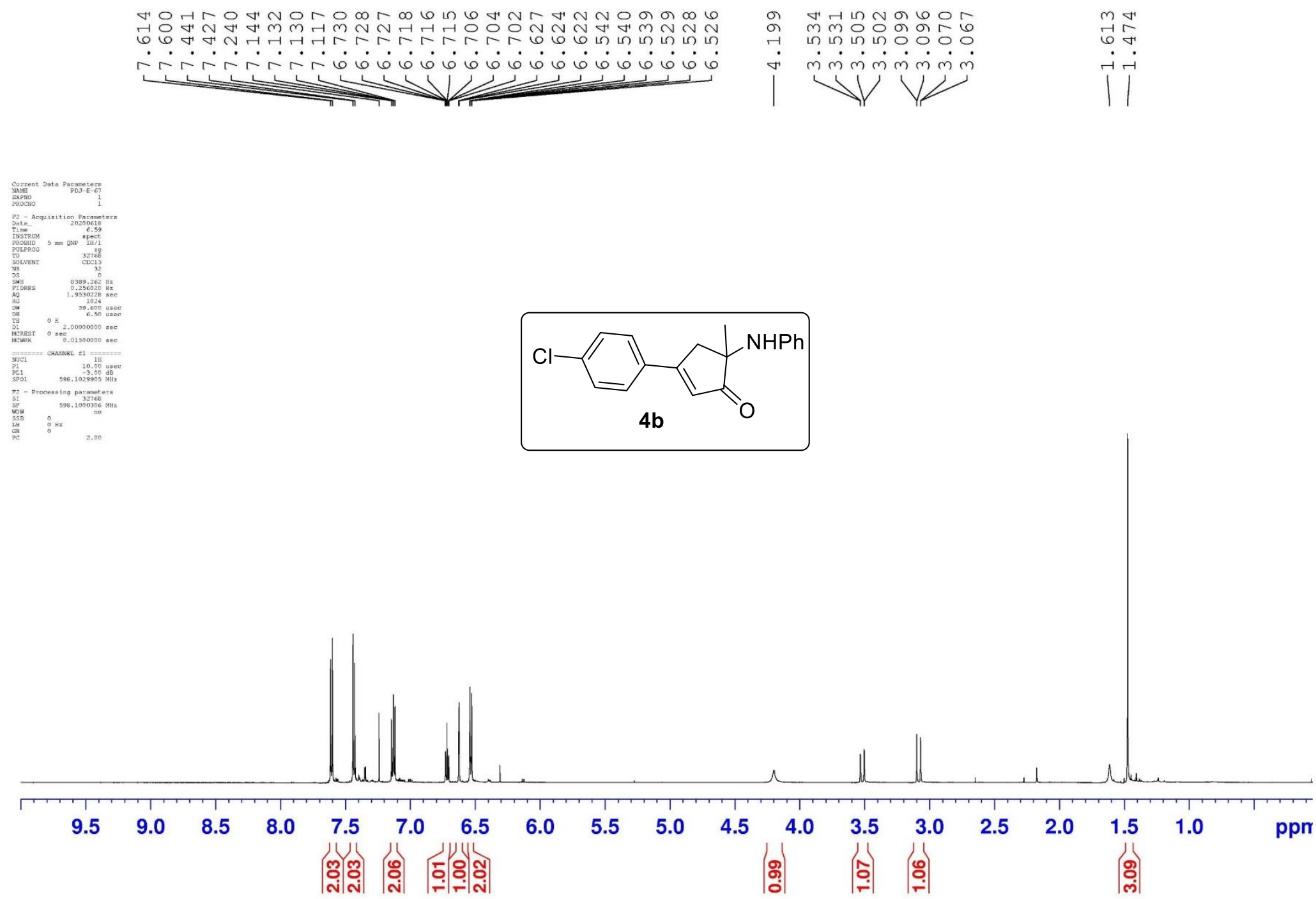


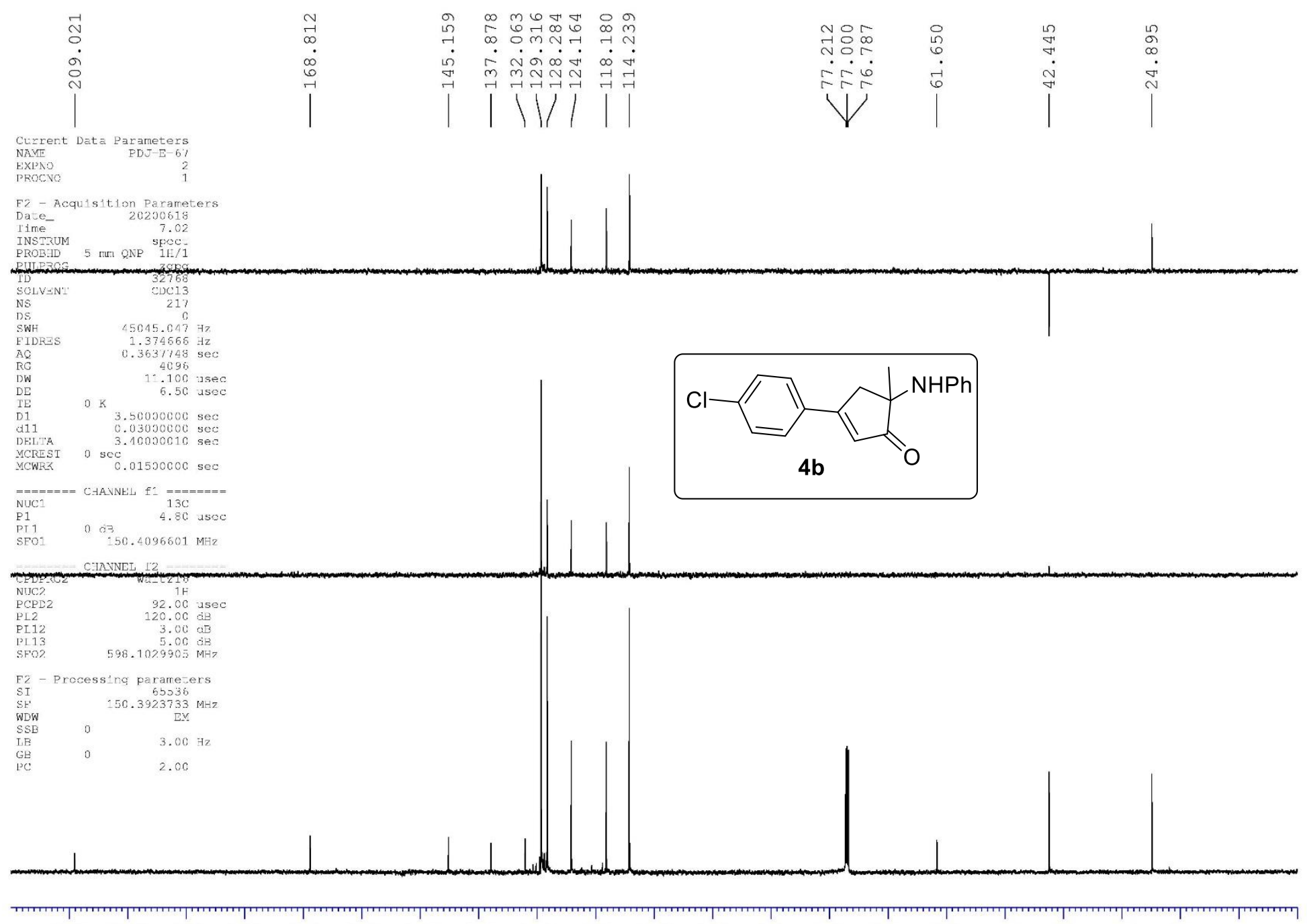

$\begin{array}{llllllllllllllllllllll}210 & 200 & 190 & 180 & 170 & 160 & 150 & 140 & 130 & 120 & 110 & 100 & 90 & 80 & 70 & 60 & 50 & 40 & 30 & 20 & \text { ppm }\end{array}$ 


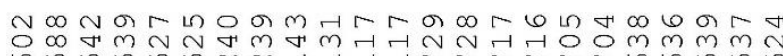

rirrirrir

10
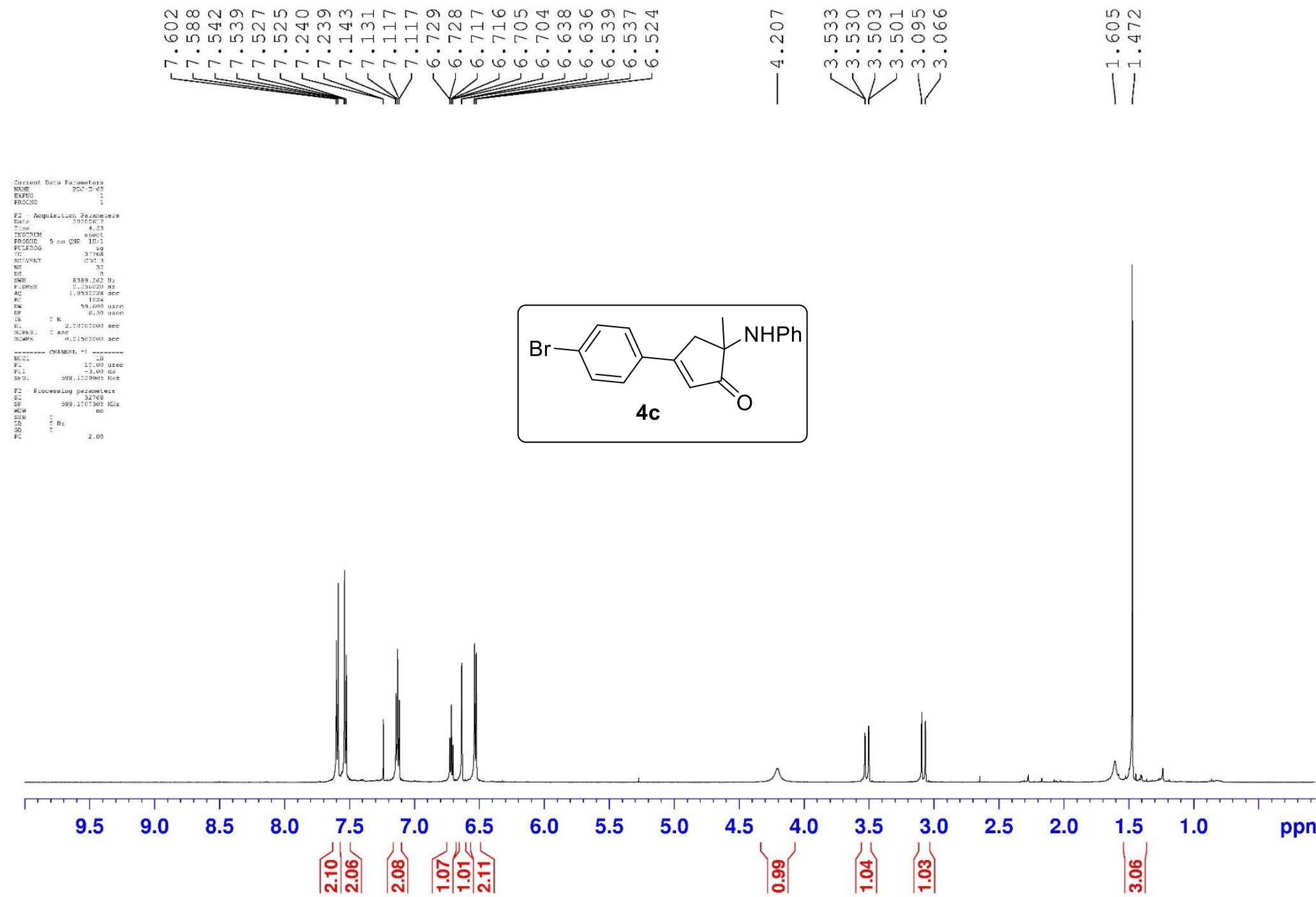


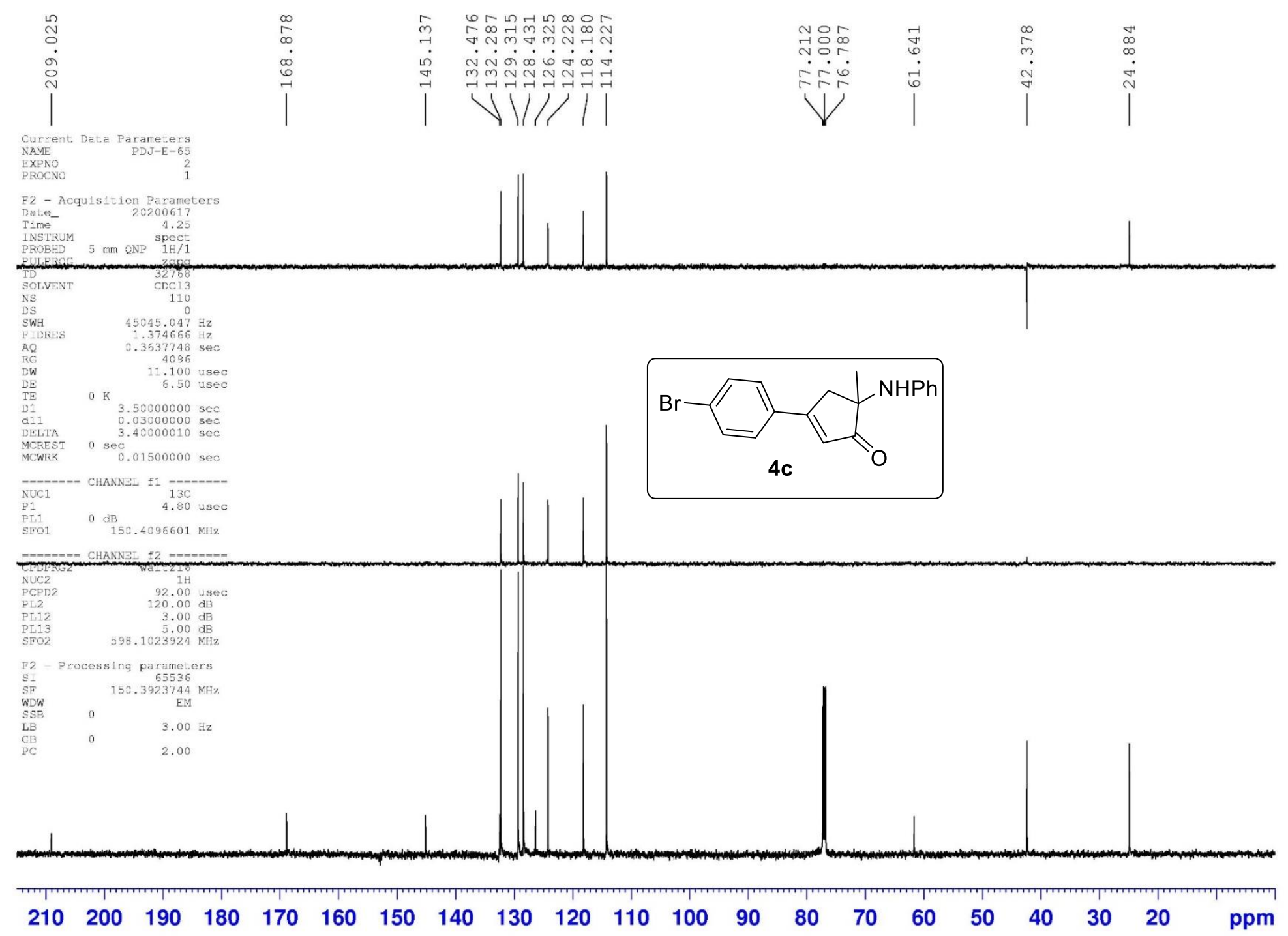



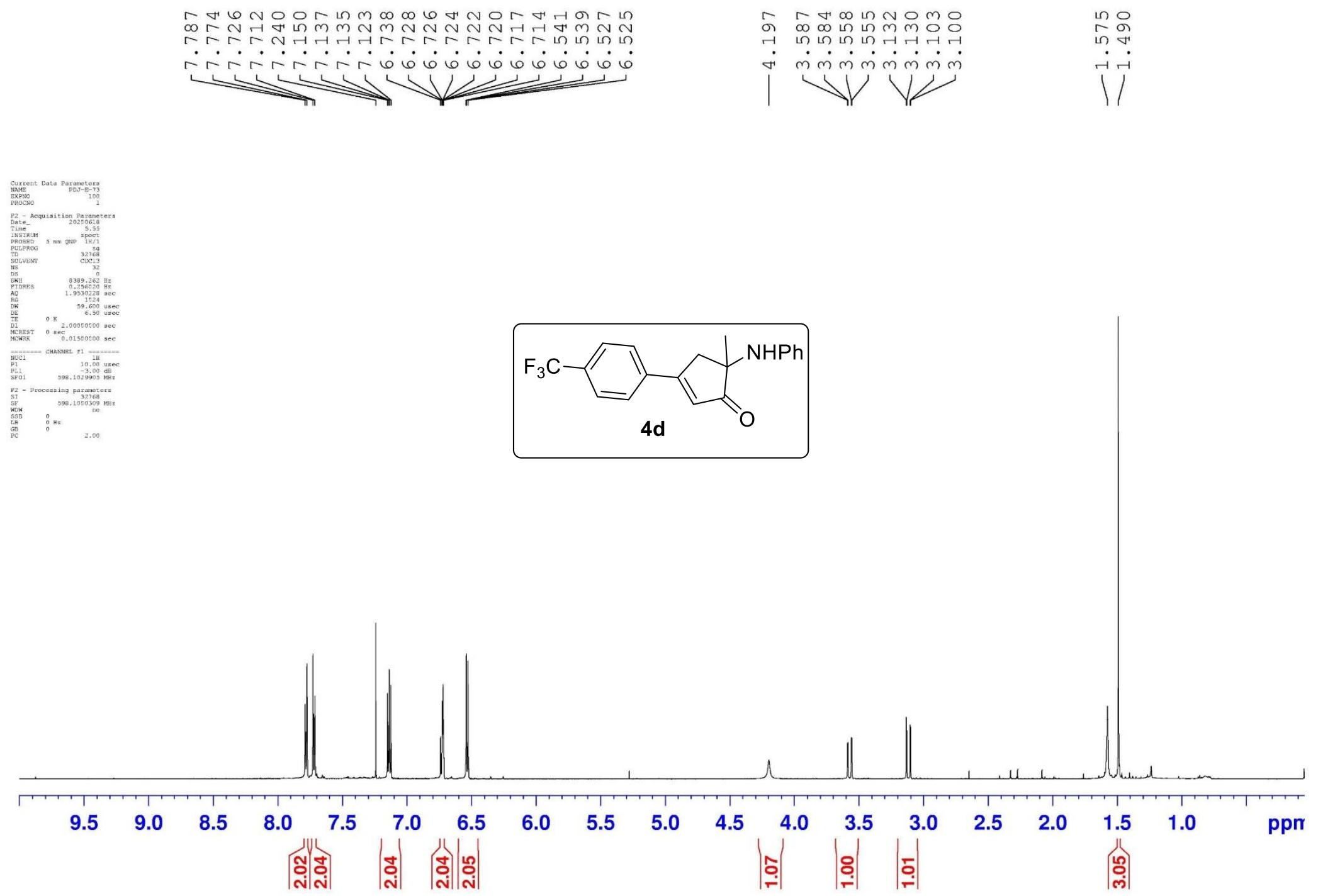

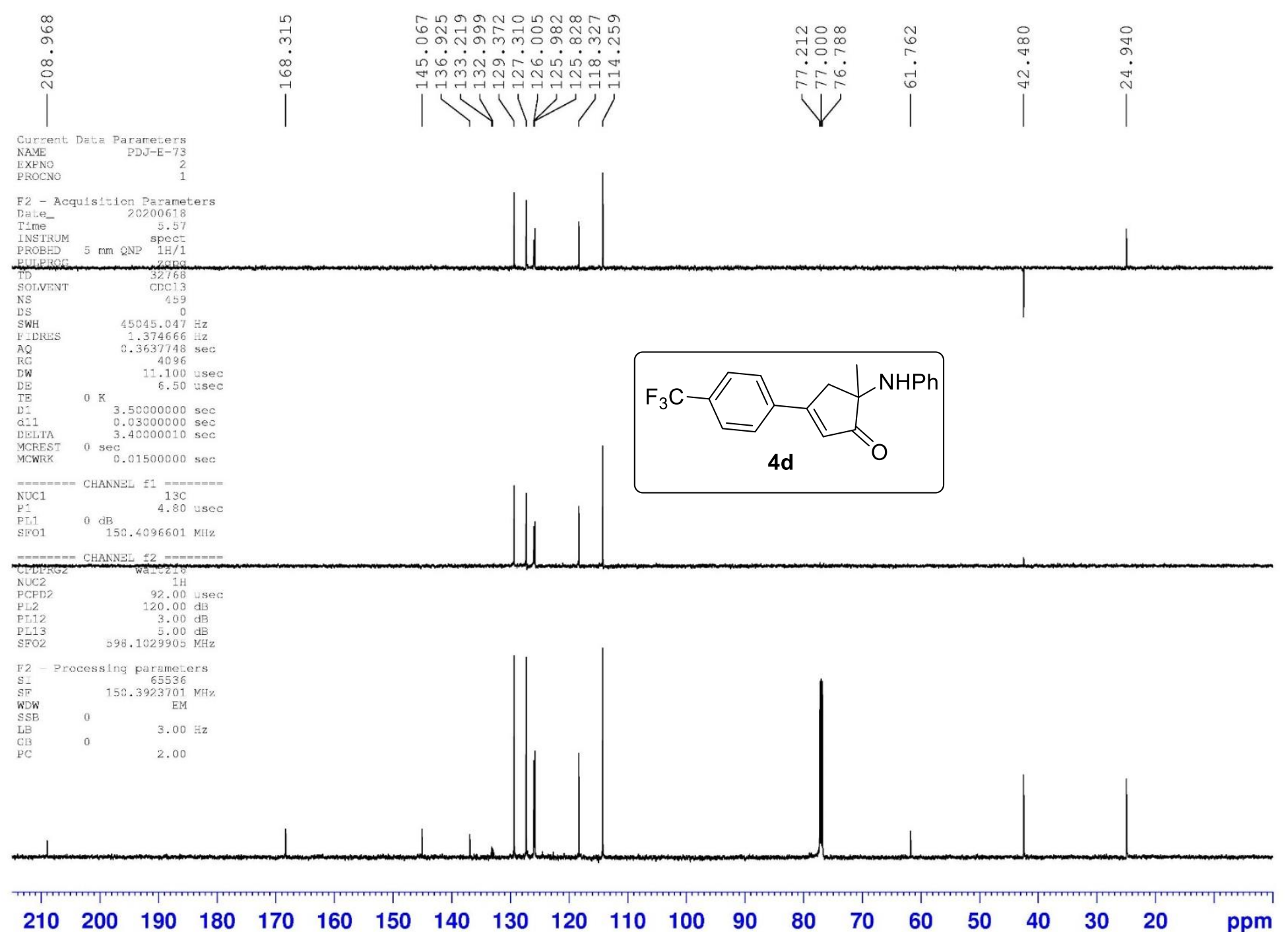


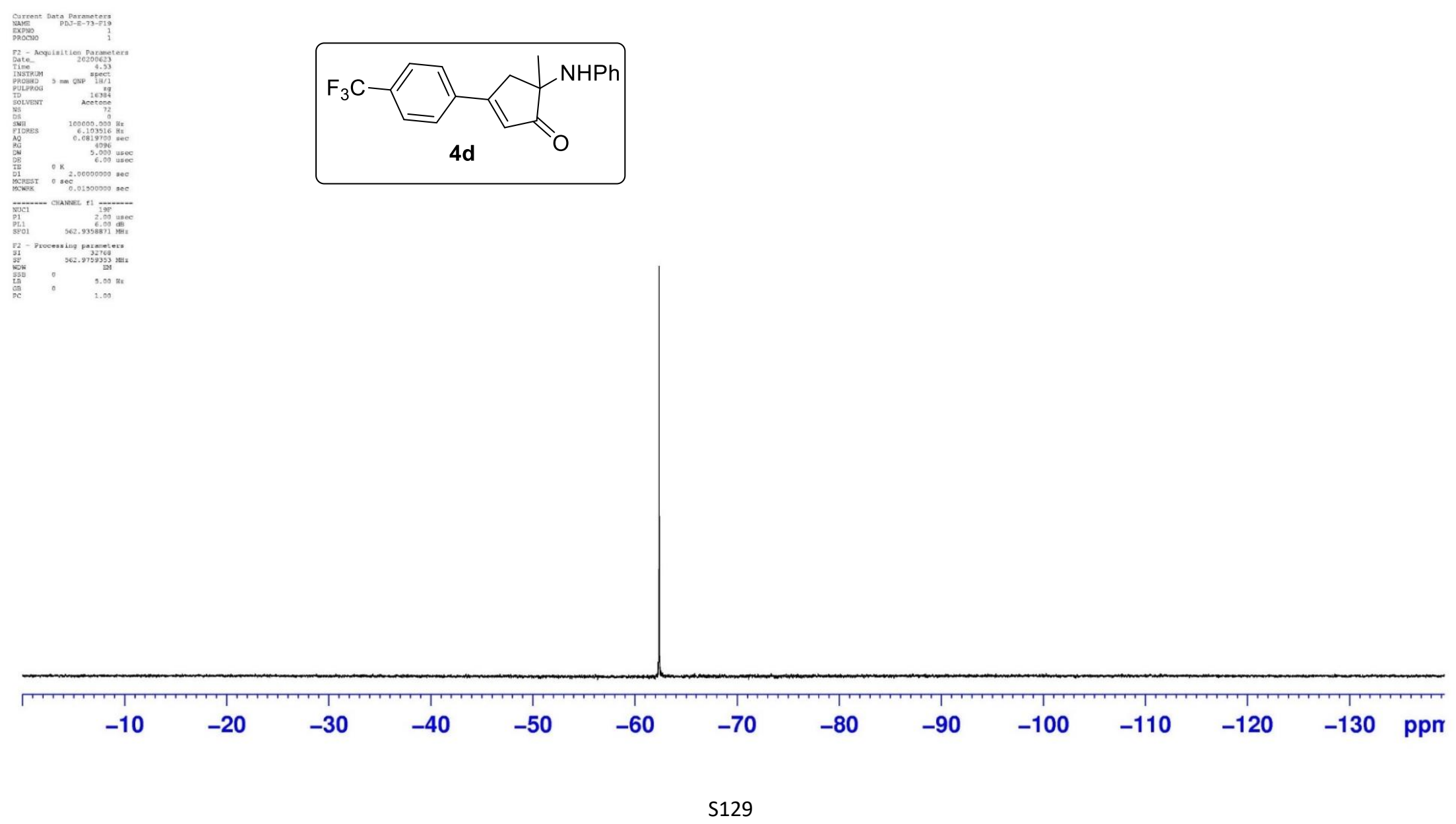




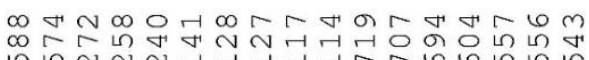

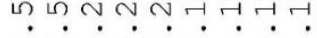

arararámo

$\longrightarrow$

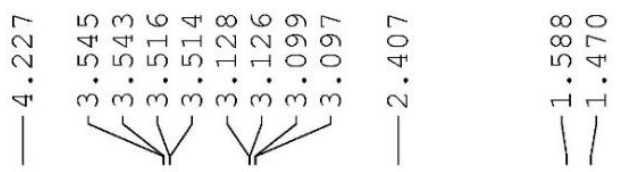
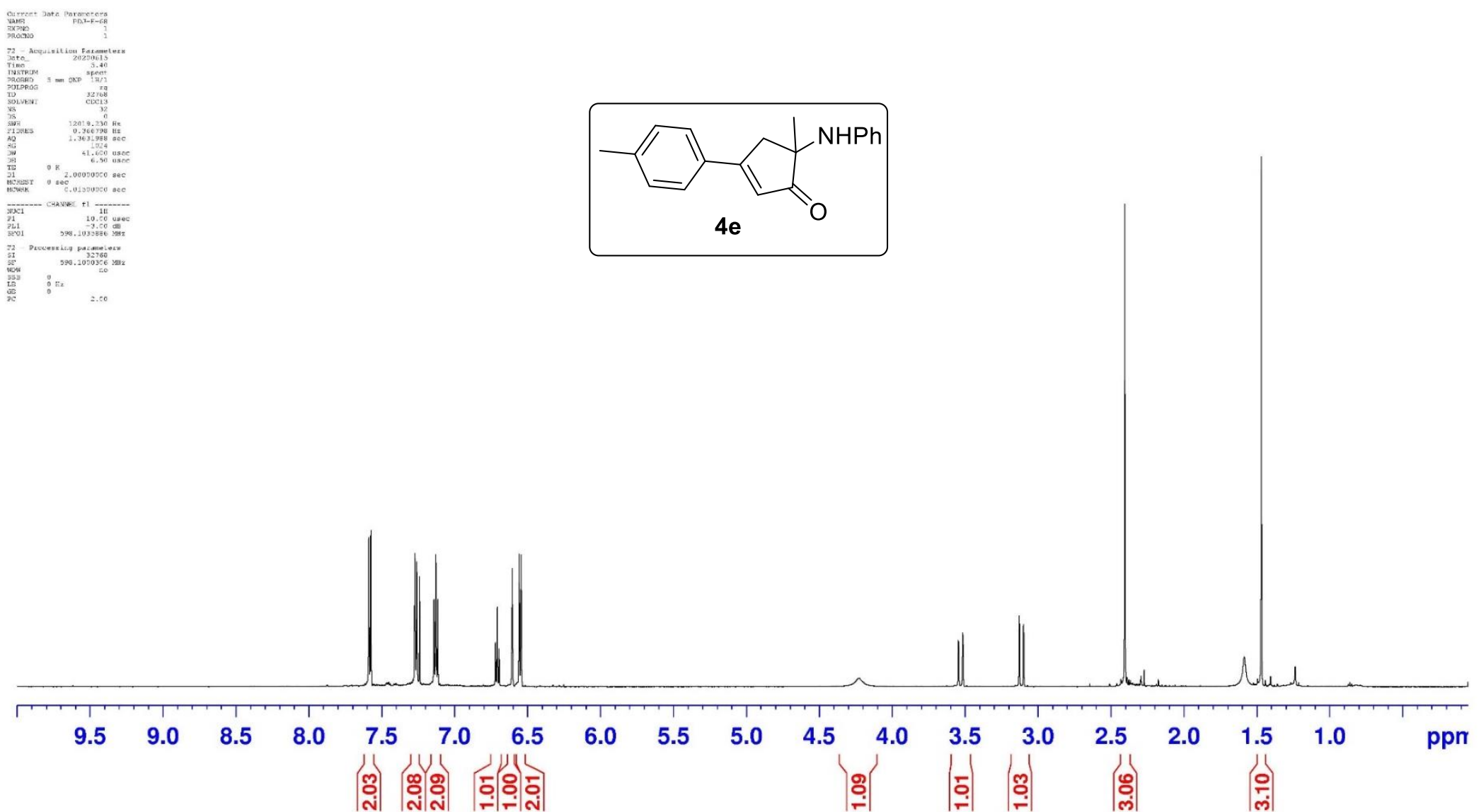

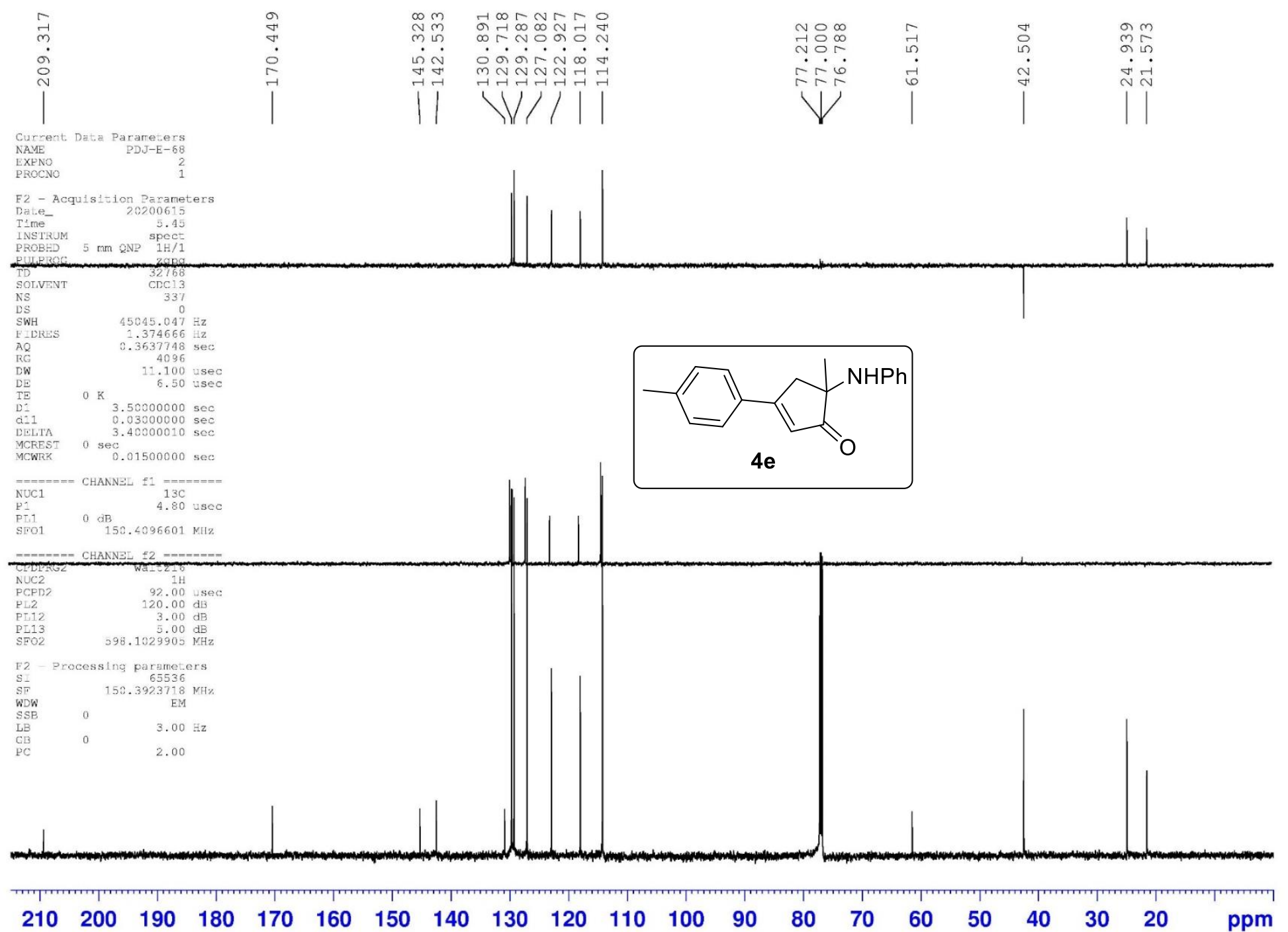


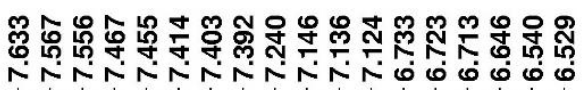

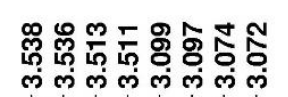

نั0

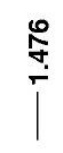
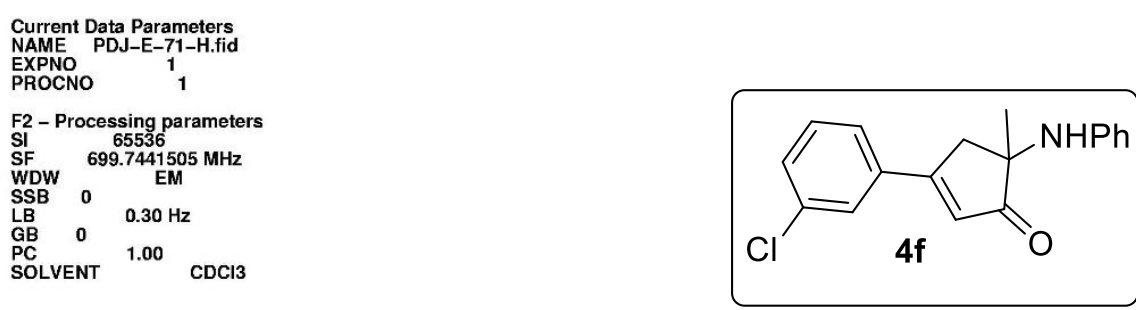

$\begin{array}{llll}\text { PC } & 0 & \\ \text { SOLVENT } & 1.00 \quad \text { CDCI3 } & \end{array}$

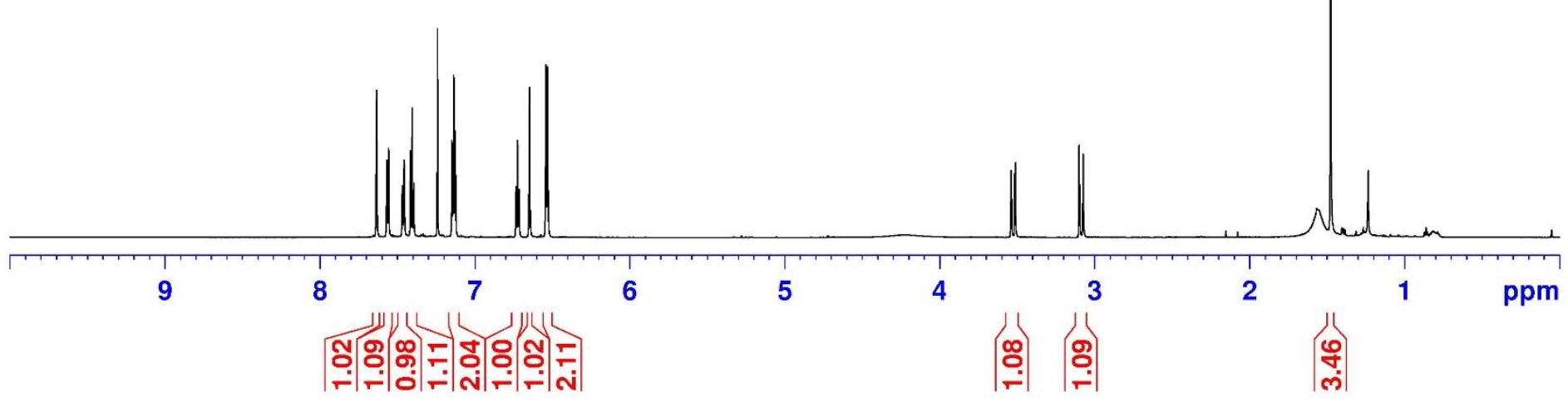




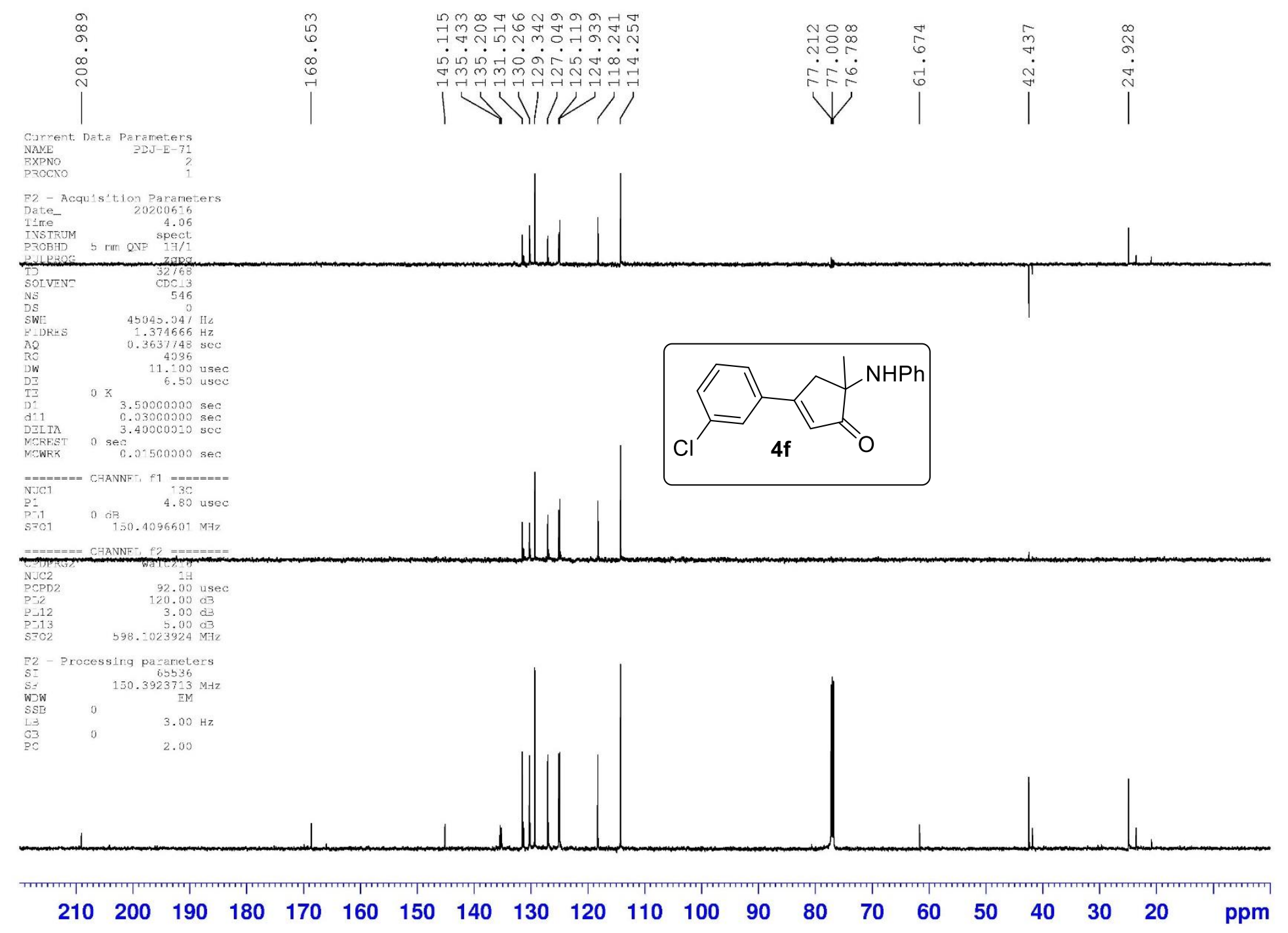




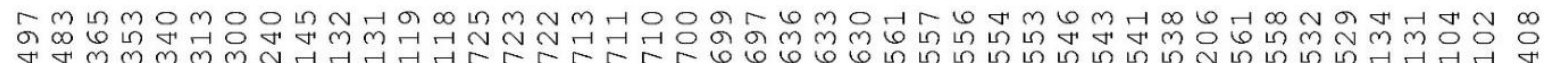

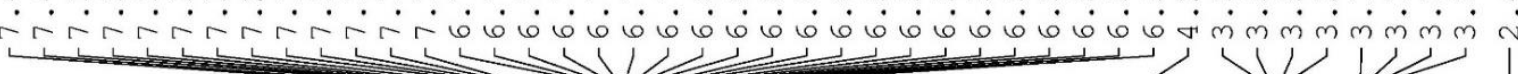
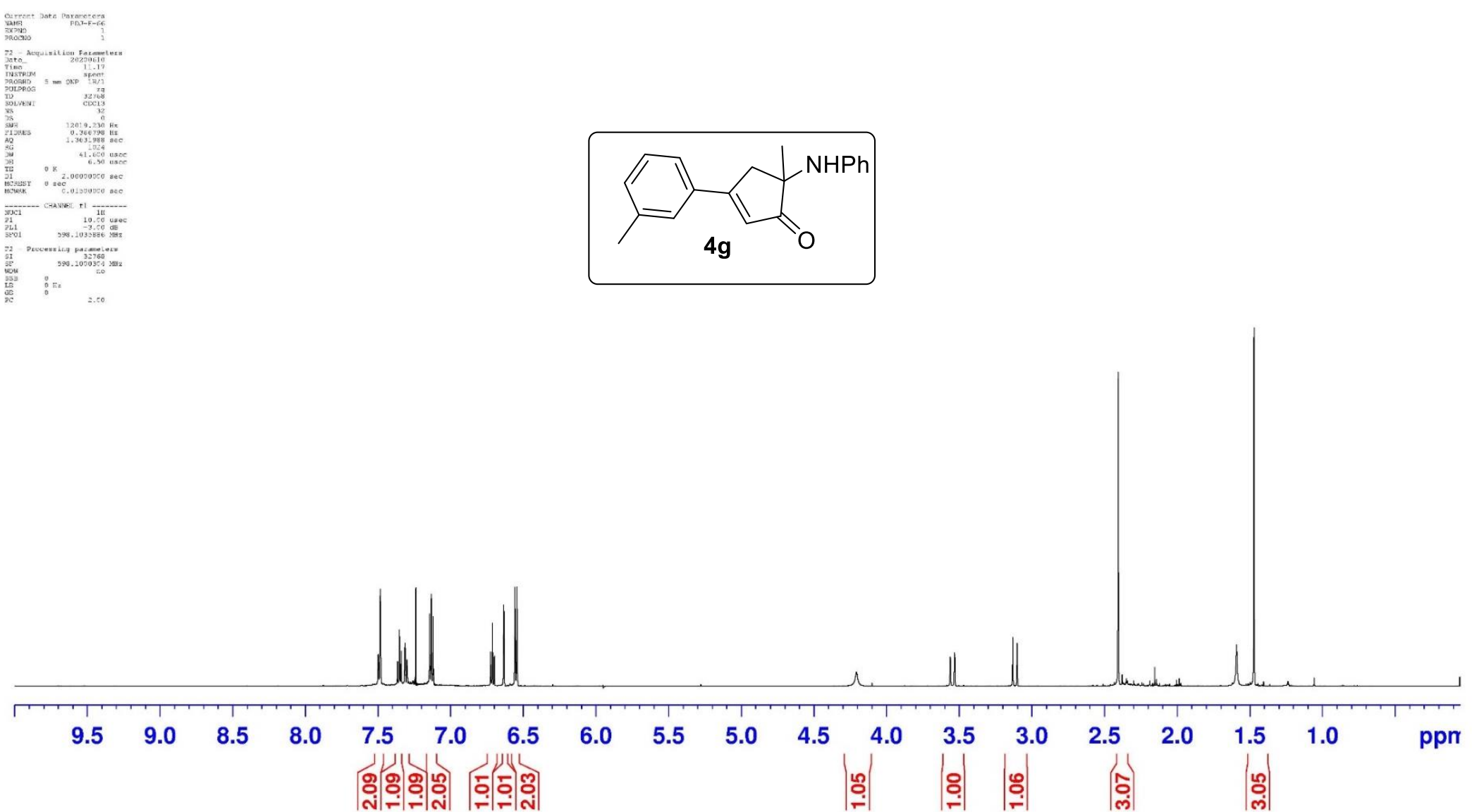


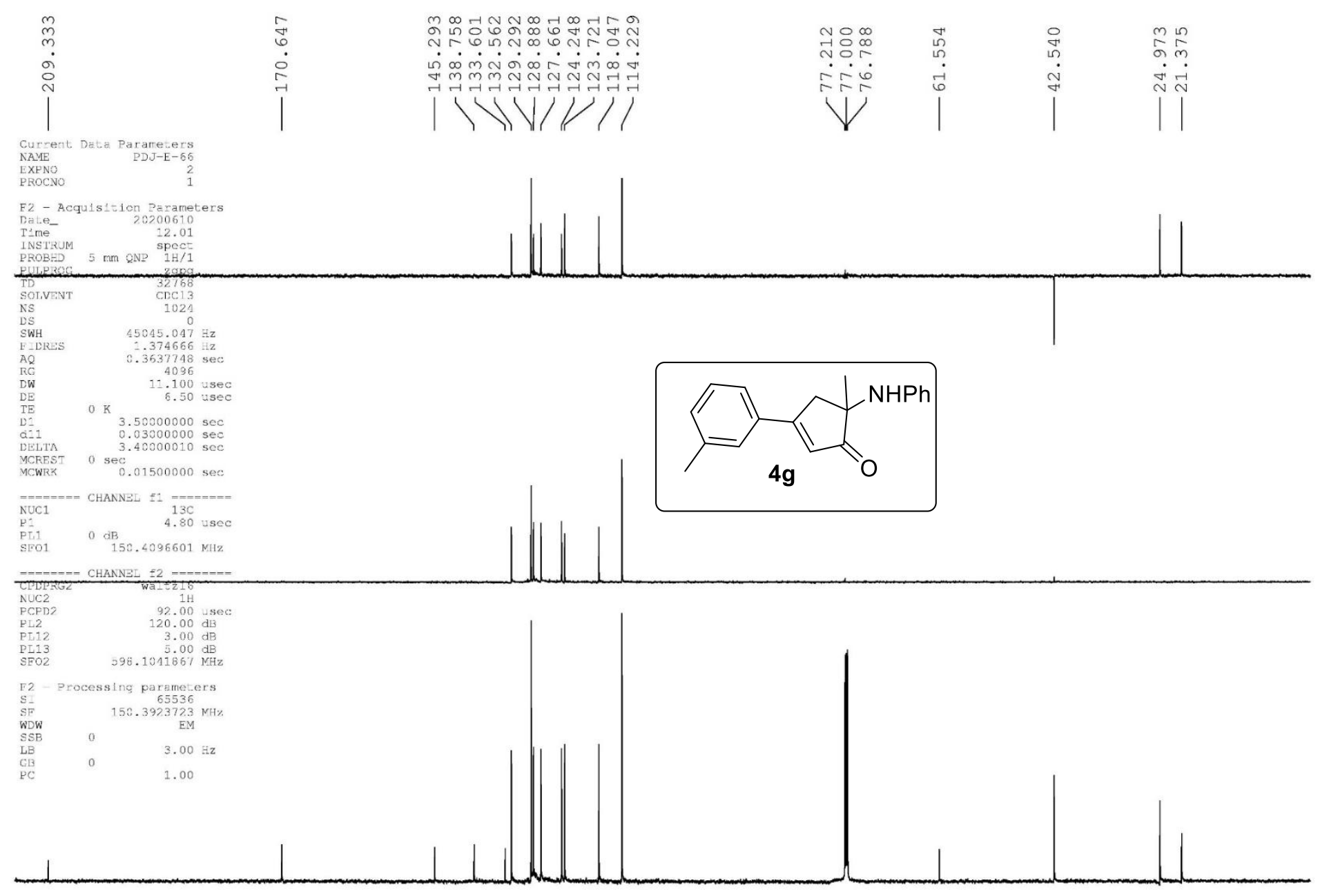

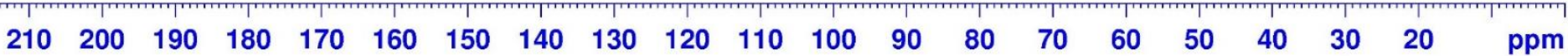




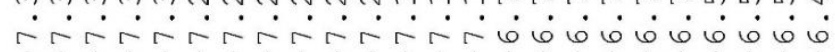

1.
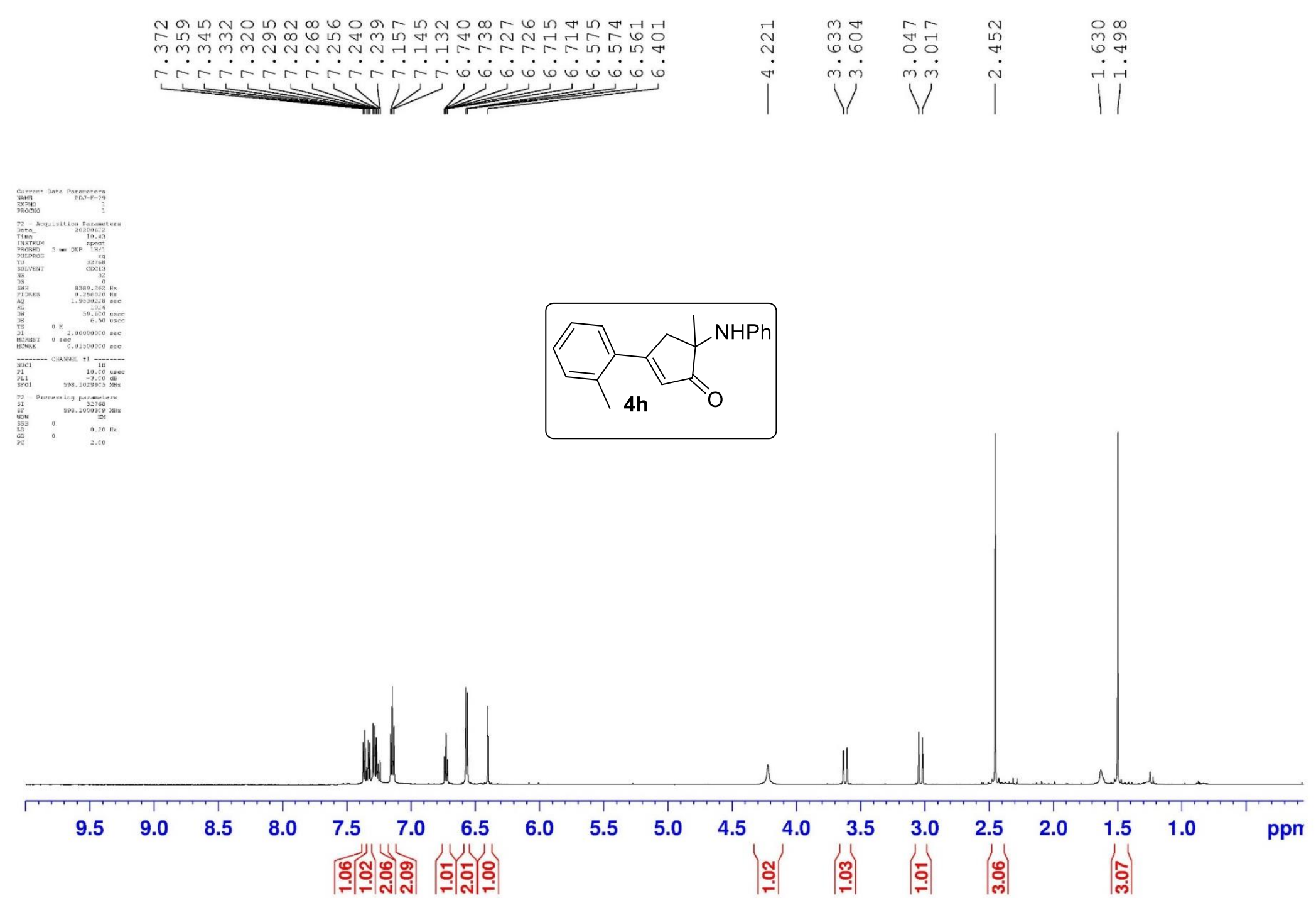


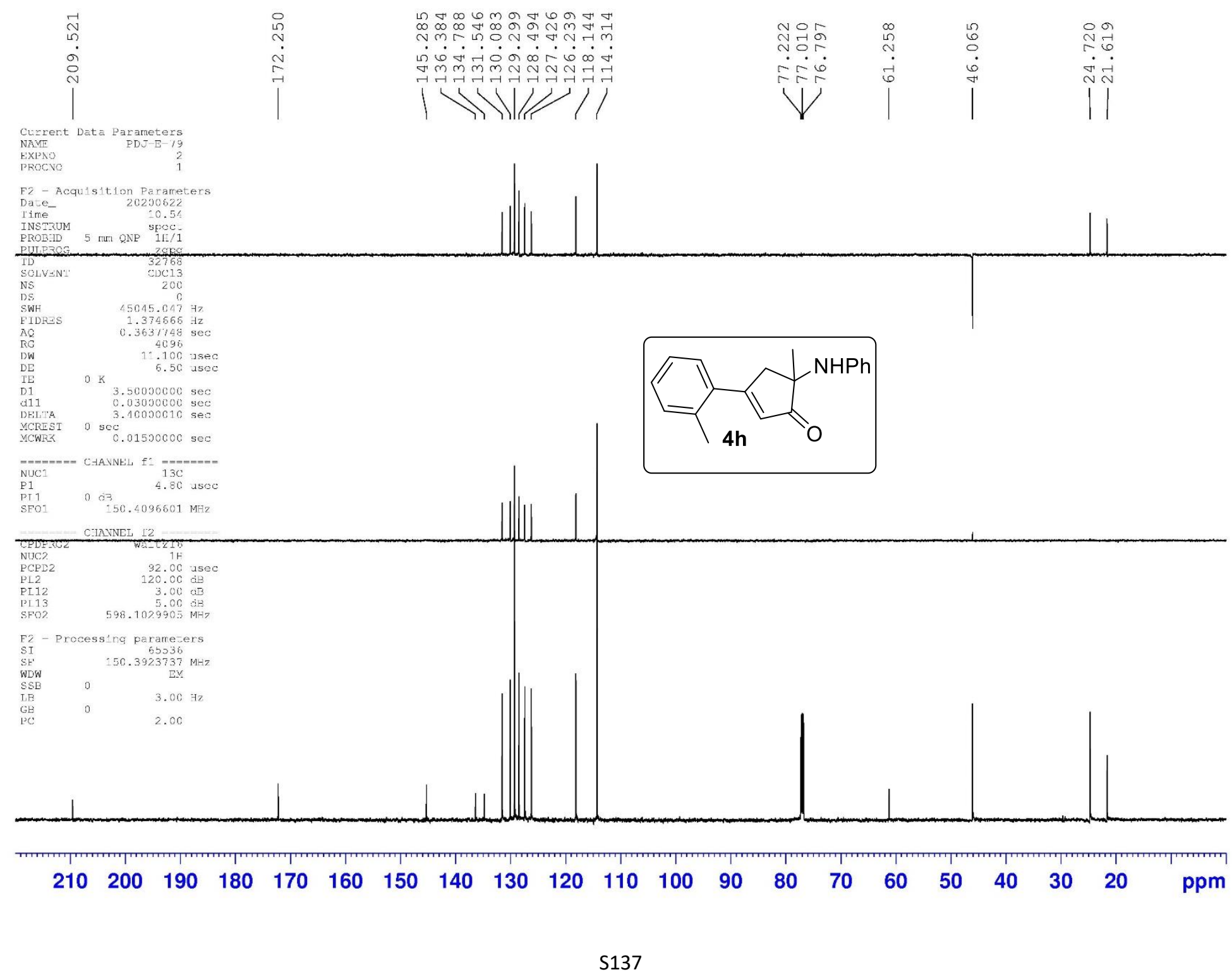




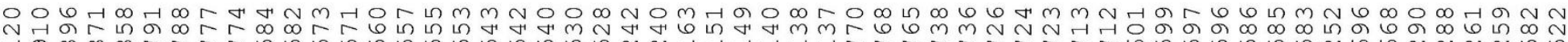
n

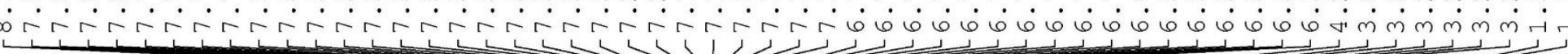
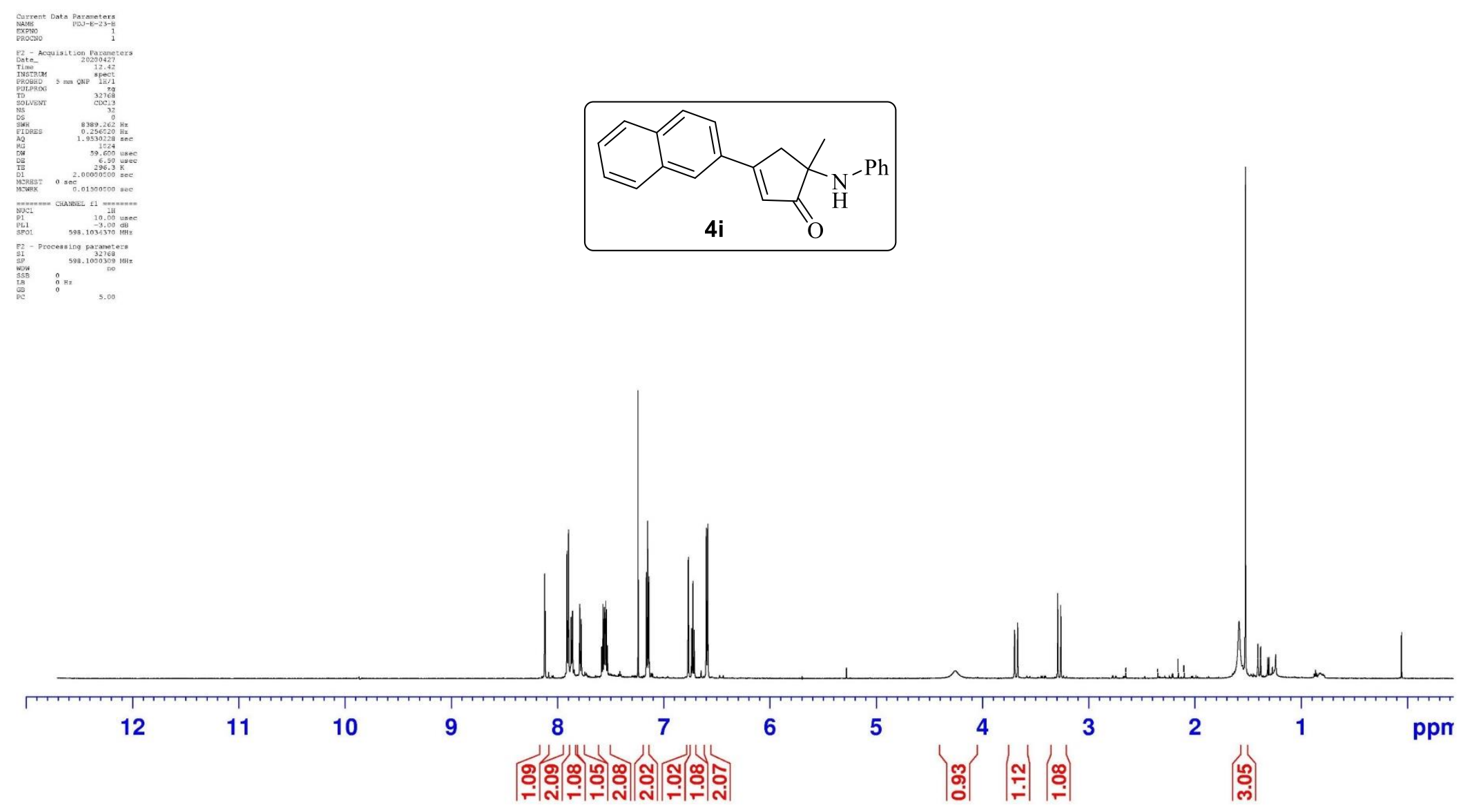


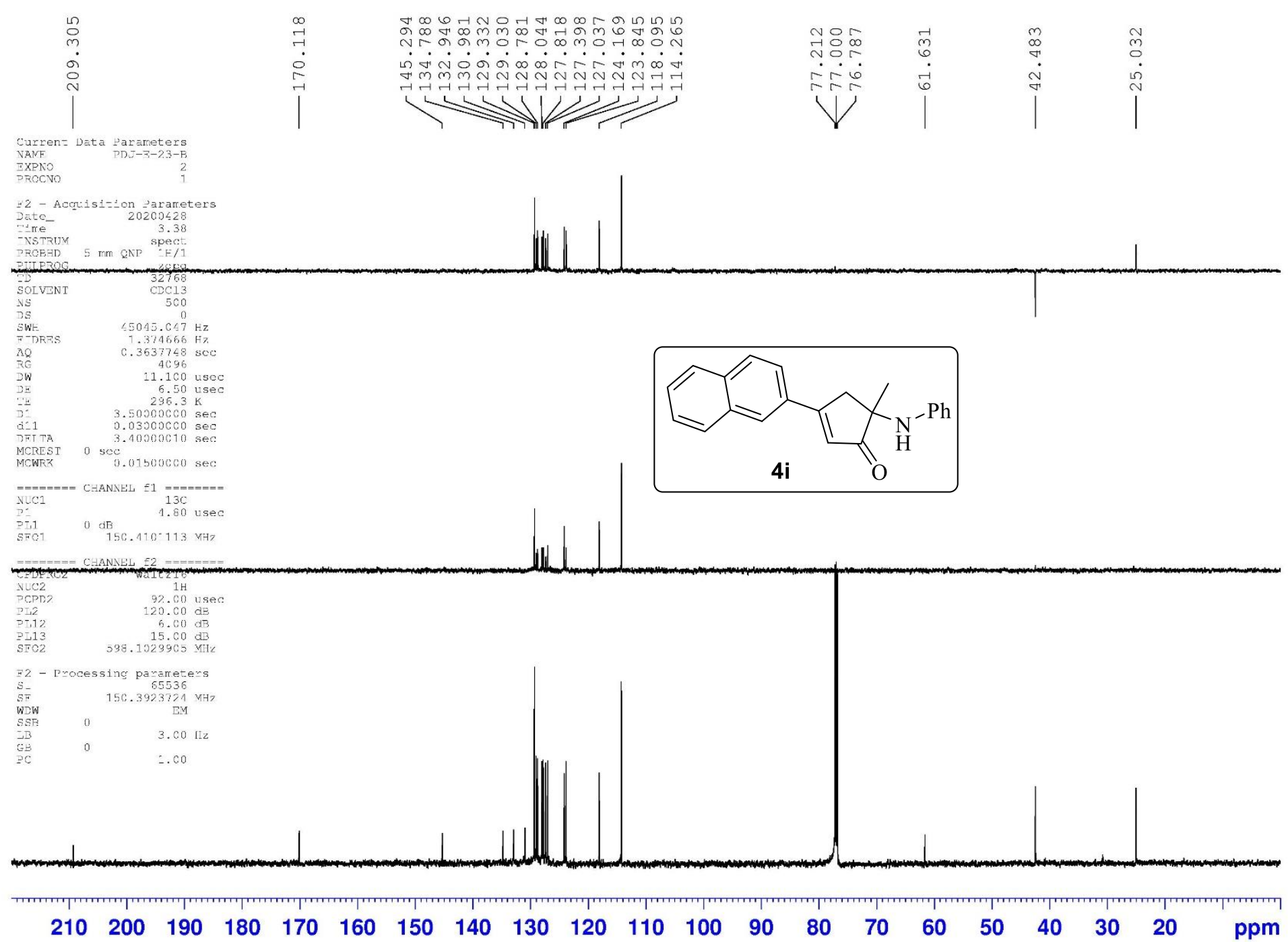




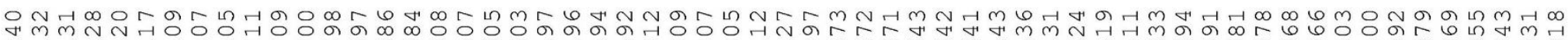

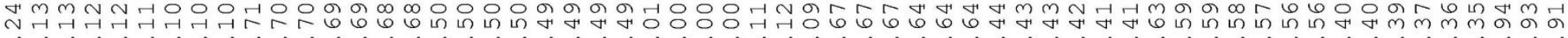

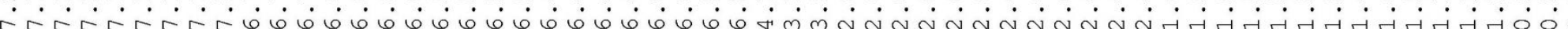

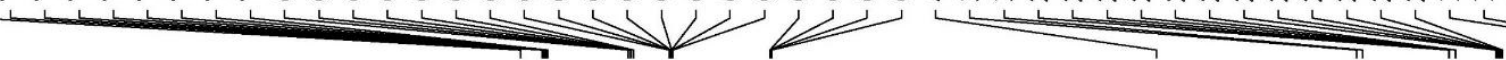
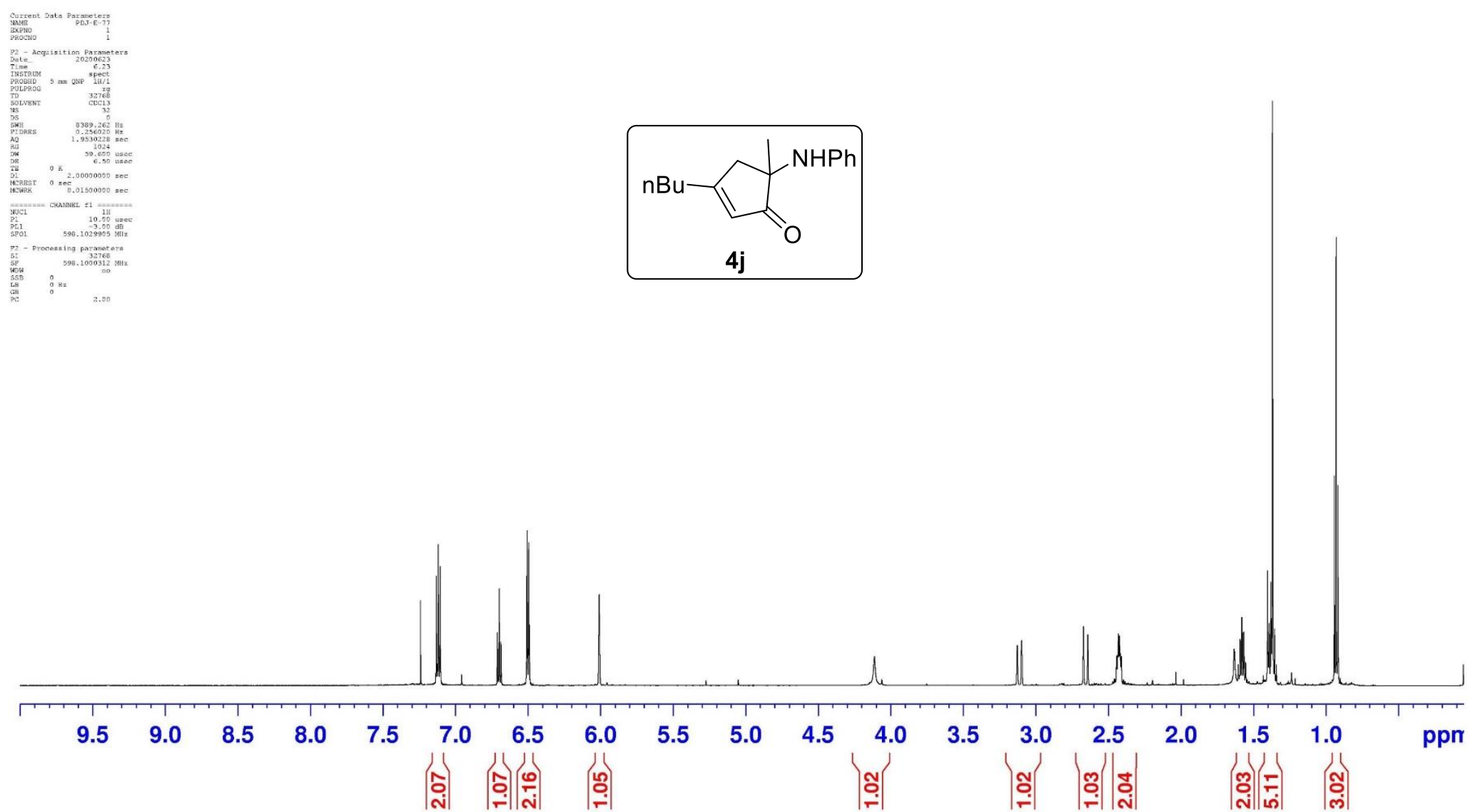


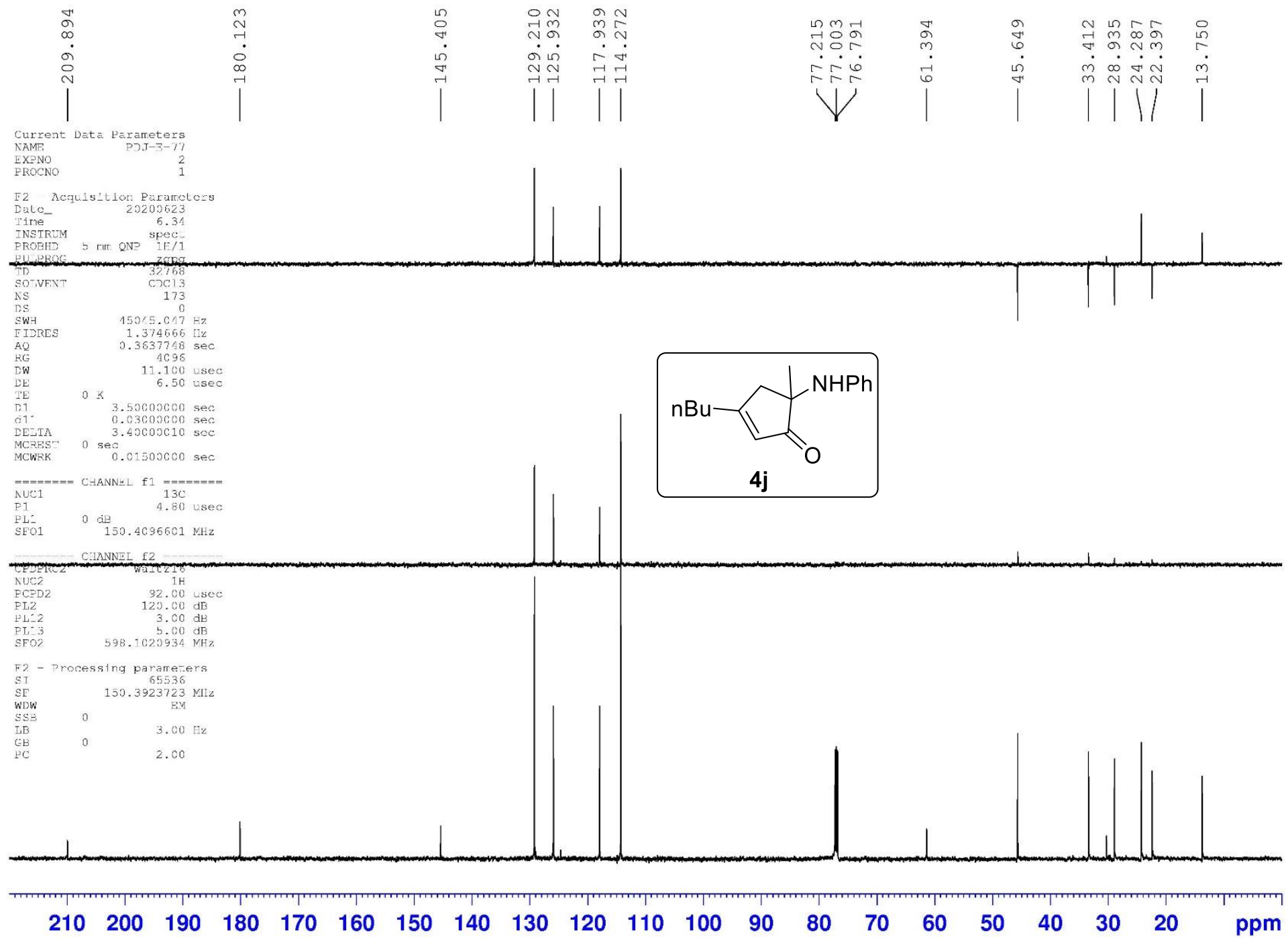




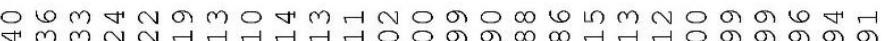

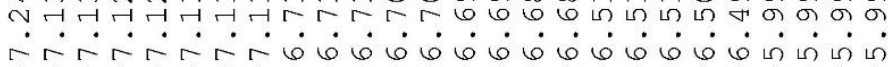

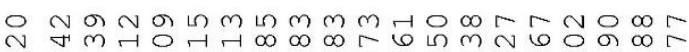
국두. $\dot{\sim} \dot{m} \dot{m} \dot{\sim} \dot{\sim} \dot{\sim} \dot{\sim} \dot{\sim} \dot{\sim} \dot{\sim} \dot{\sim} \dot{\sim} \dot{\sim} \dot{r} \dot{-}$
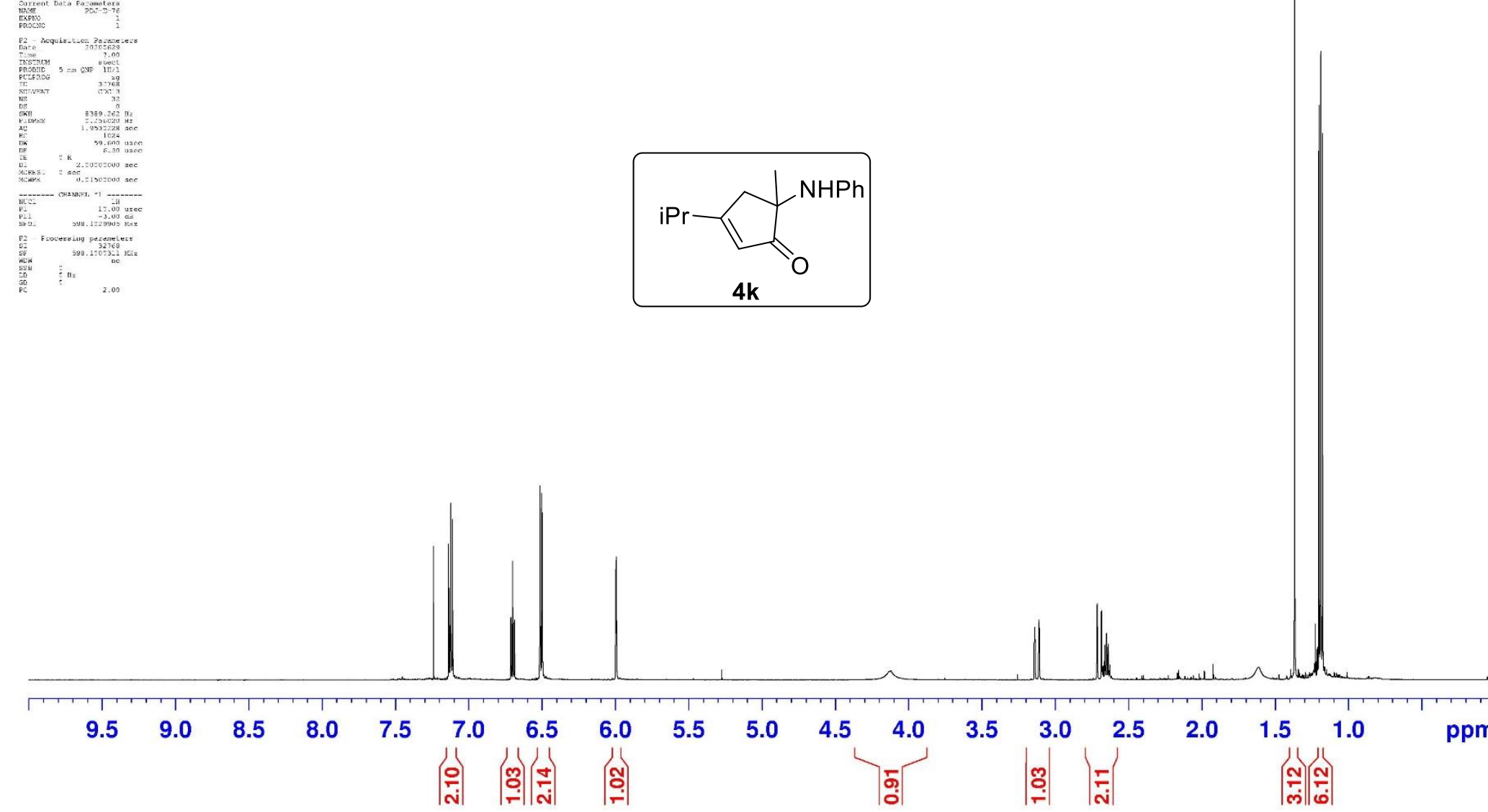


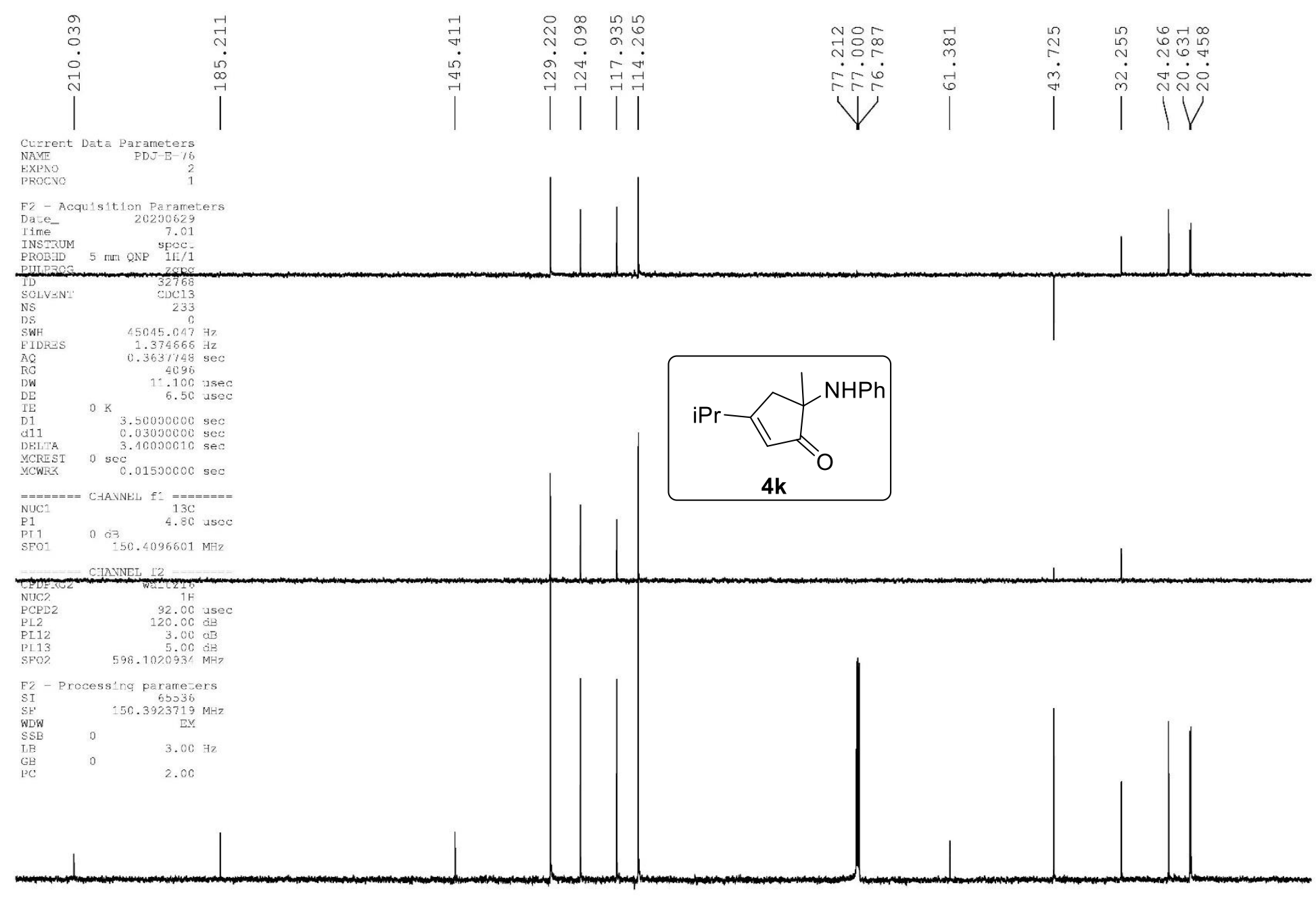

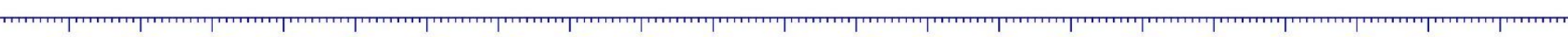
$\begin{array}{lllllllllllllllllllll}210 & 200 & 190 & 180 & 170 & 160 & 150 & 140 & 130 & 120 & 110 & 100 & 90 & 80 & 70 & 60 & 50 & 40 & 30 & 20 & \text { ppm }\end{array}$ 

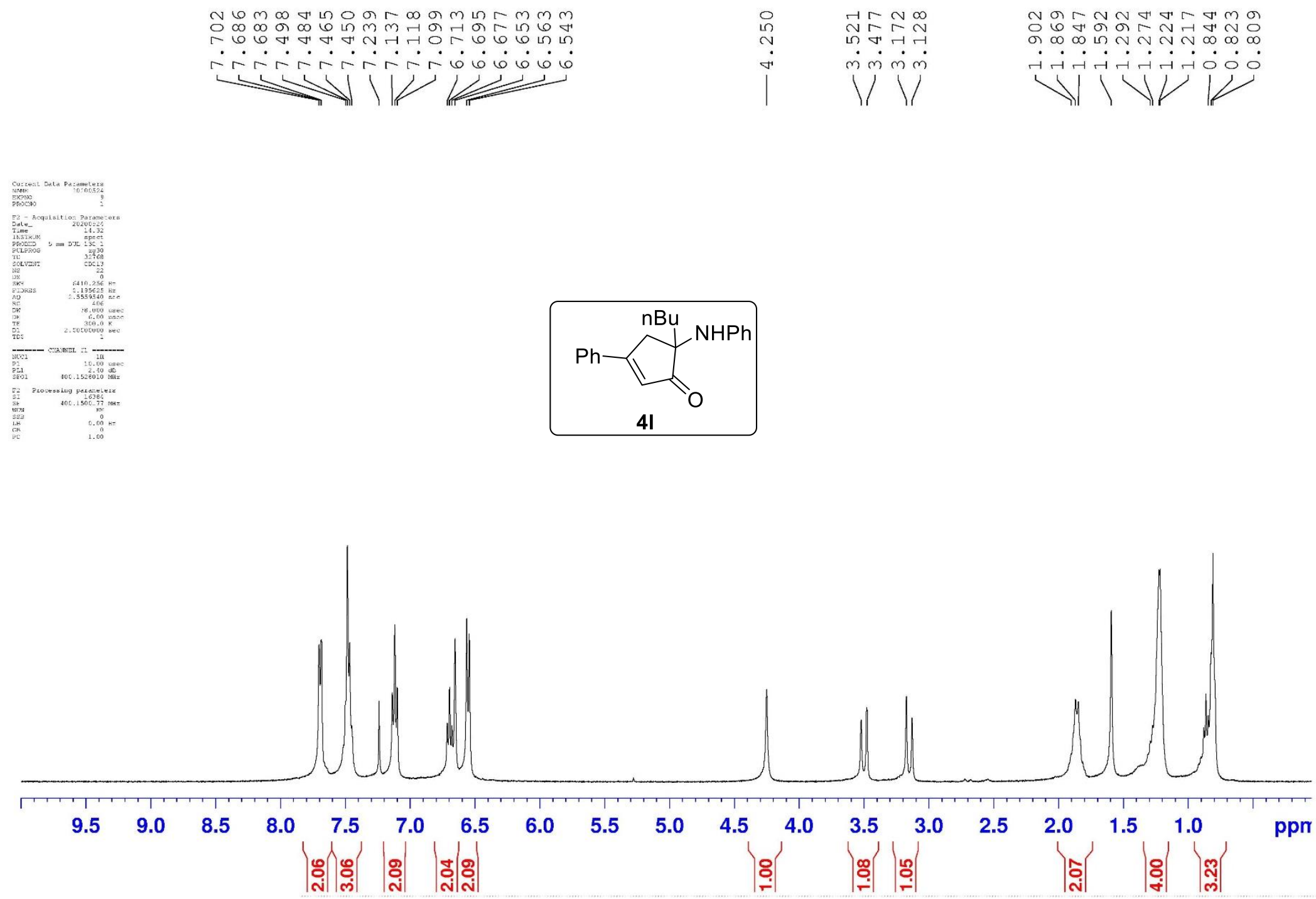


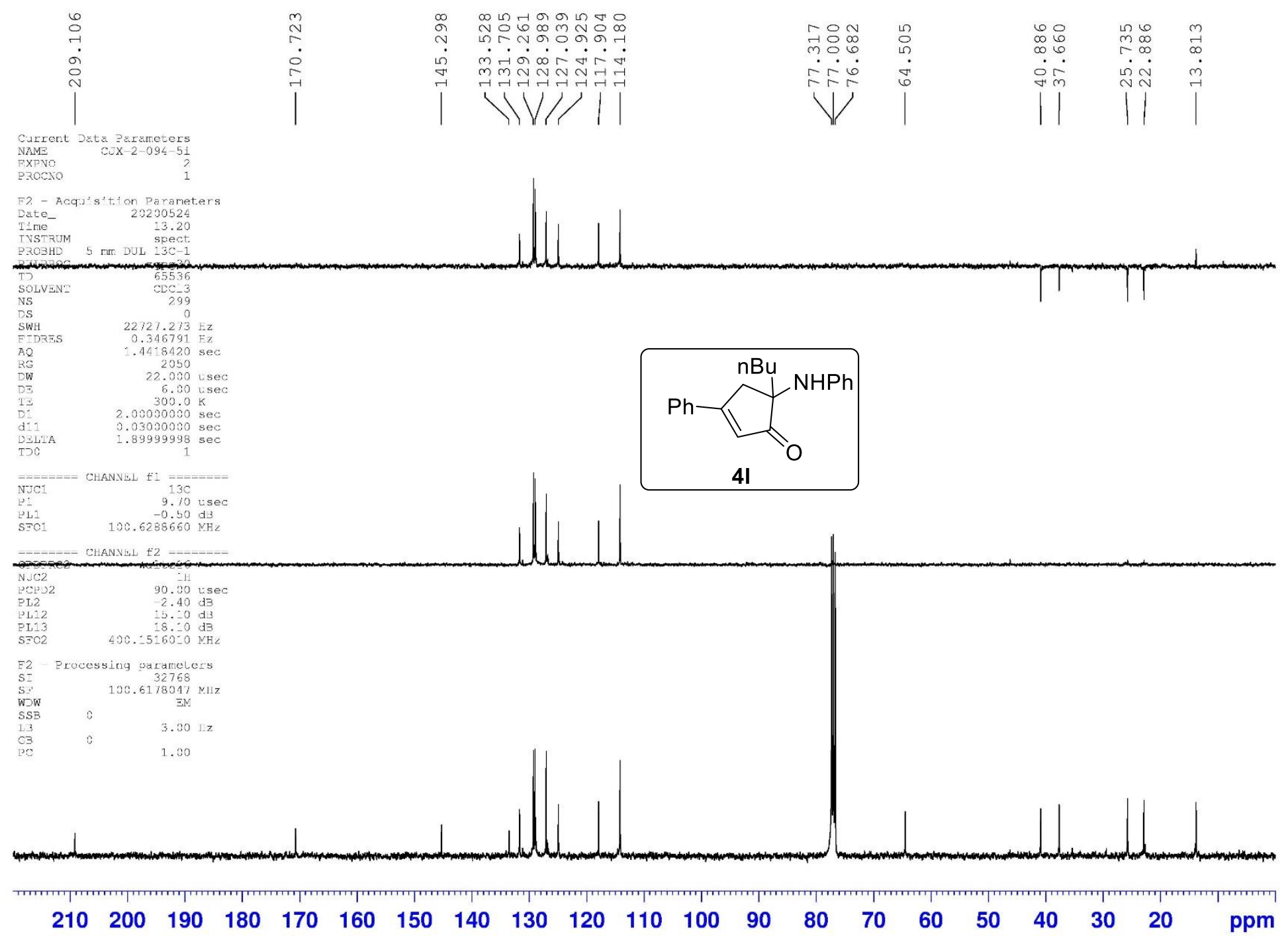



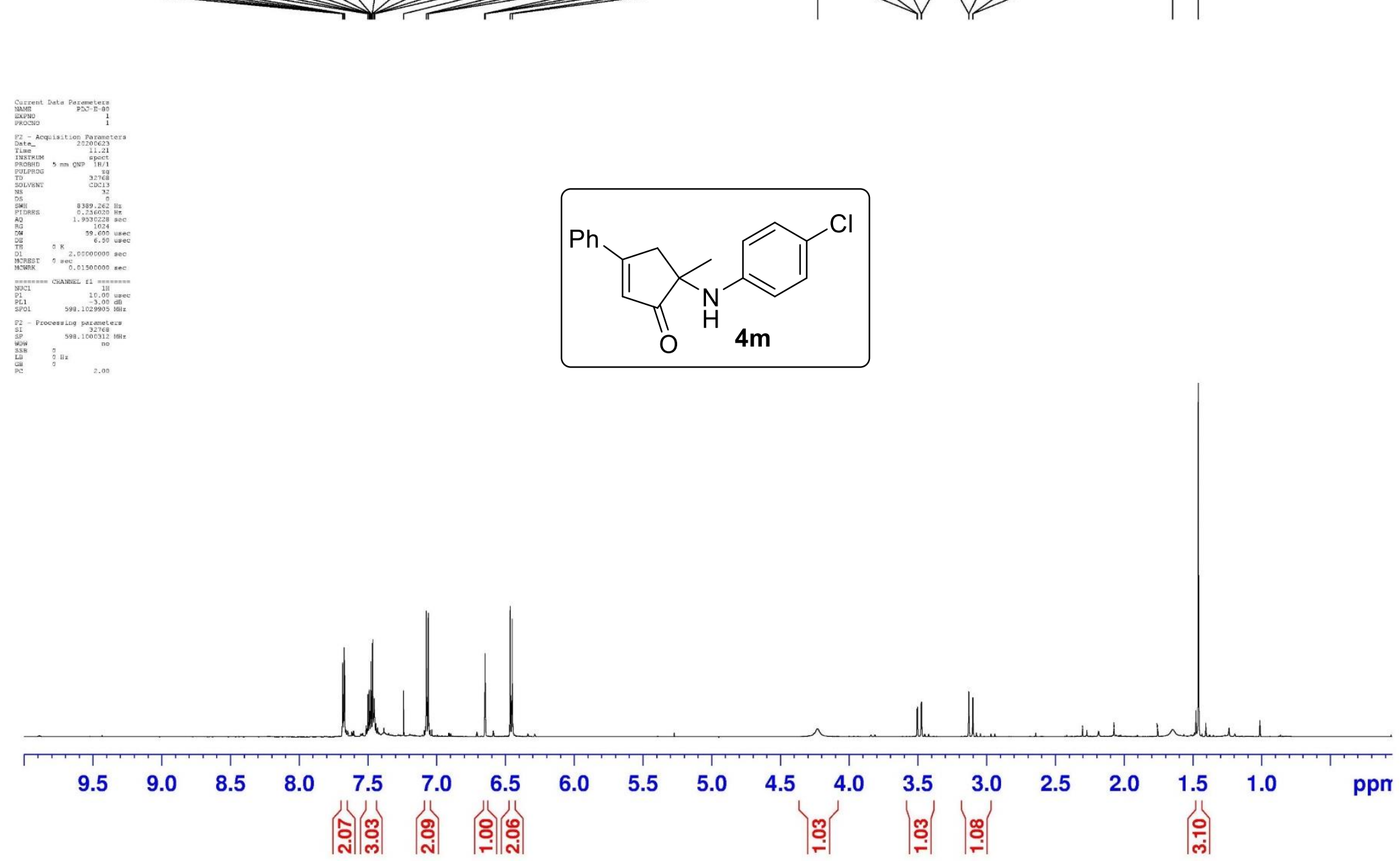


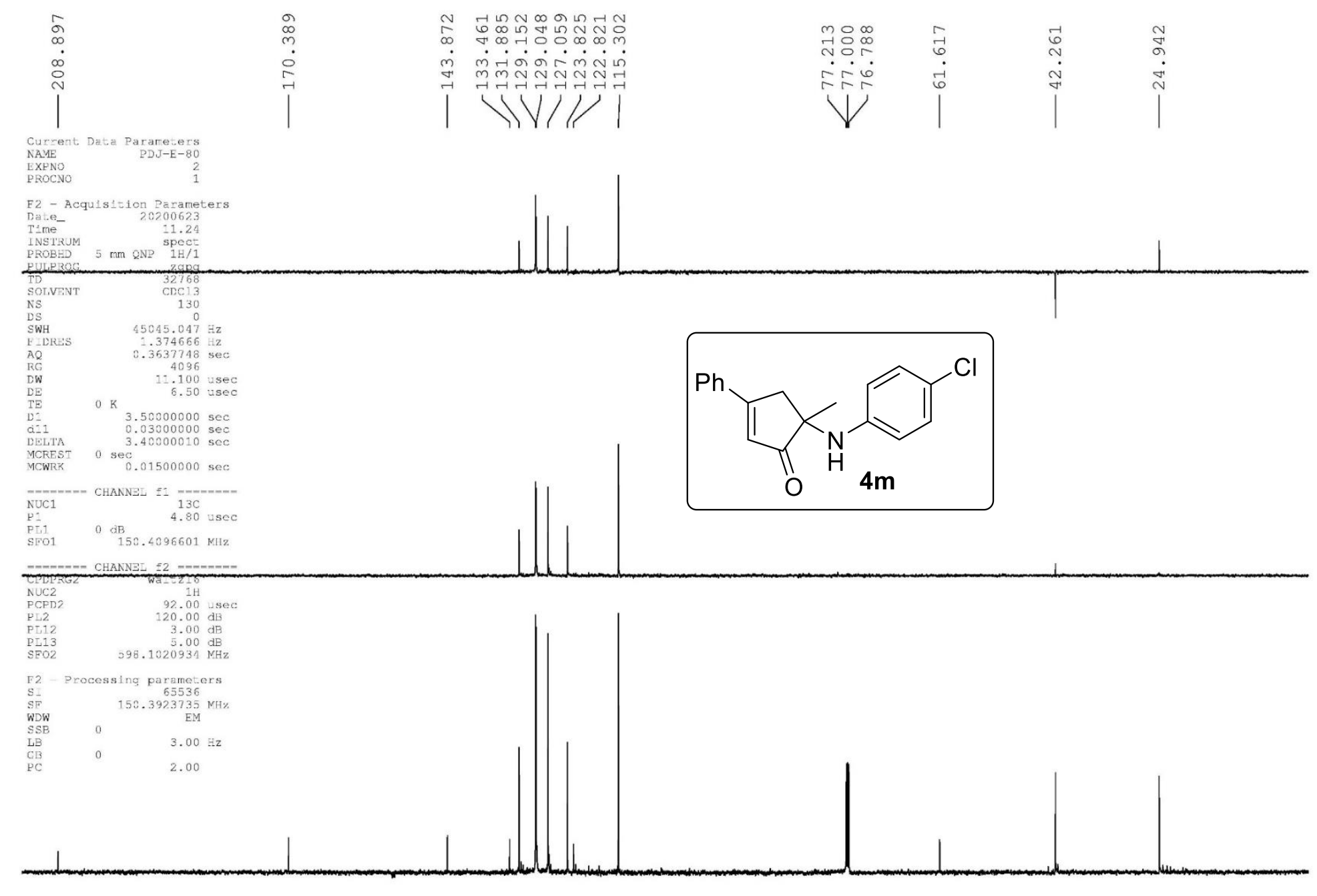

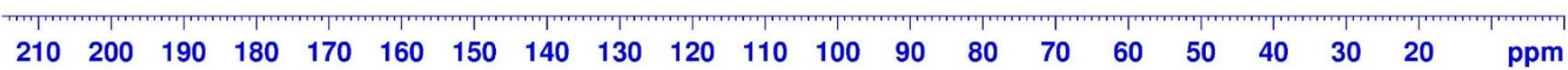




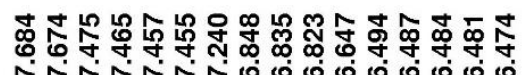 \\ तrrintiogosologo \\ $\cdots N$}

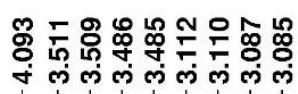

$\stackrel{8}{\stackrel{8}{8}}$

Current Data Parameters
NAME PDJE- -86 -H.fid

EXPNO
PROCNO

F2 - Processing parameters

SF $699.7441500 \mathrm{MHz}$

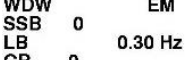

$\begin{array}{llll} & \\ \text { SC } & 0 & 1.00 & \\ \text { SOLVENT } & & \text { CDC13 }\end{array}$
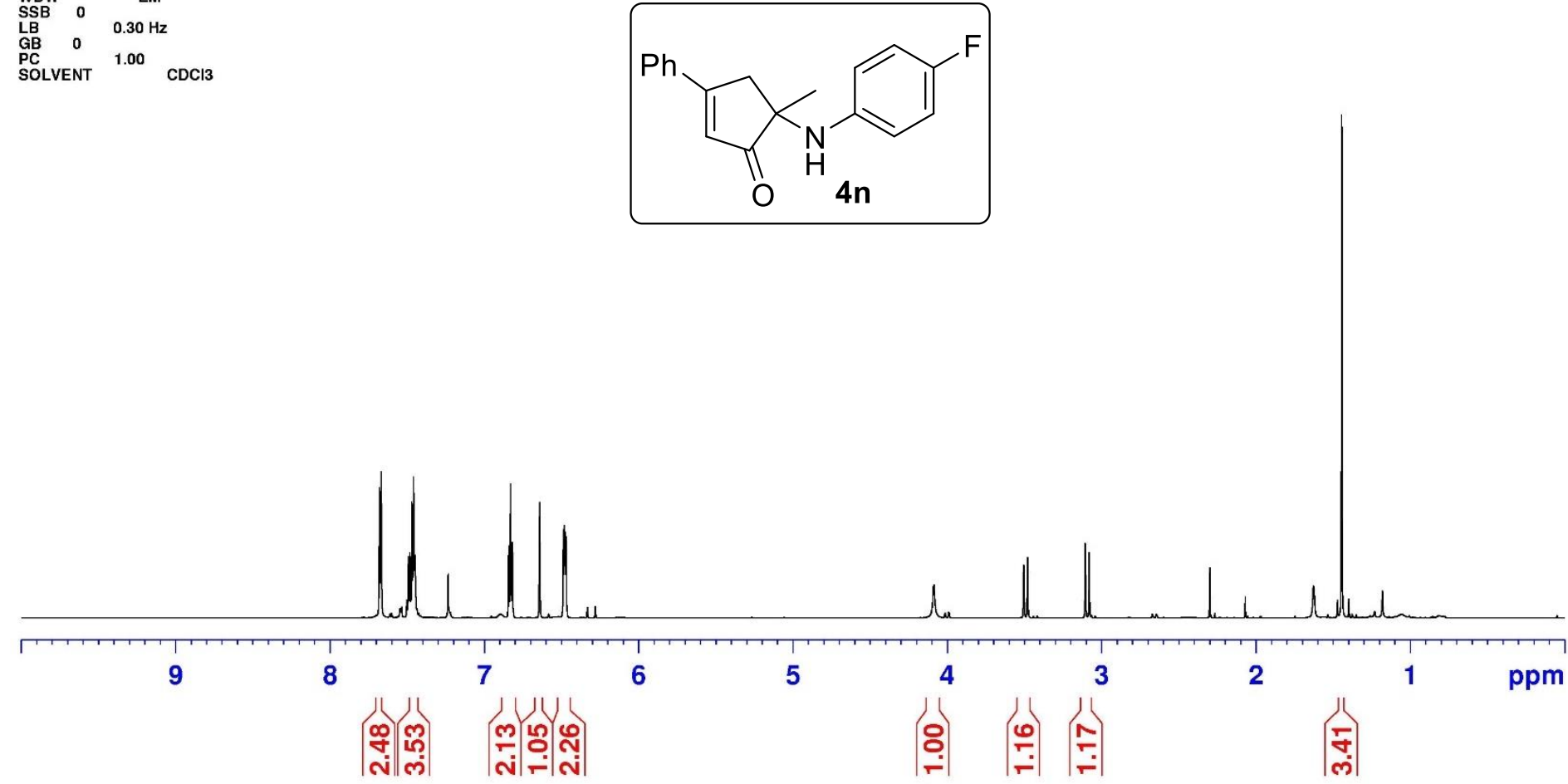

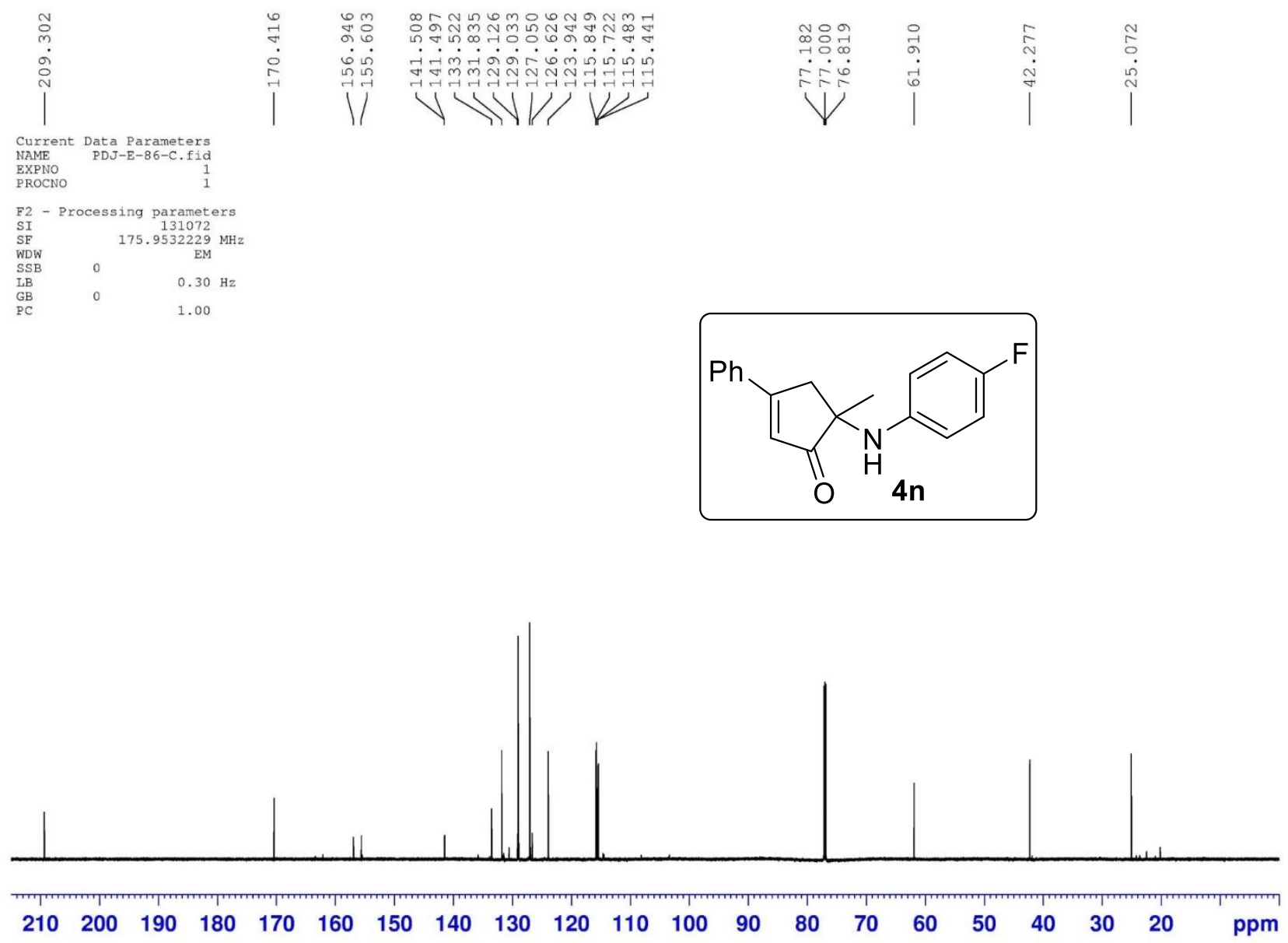

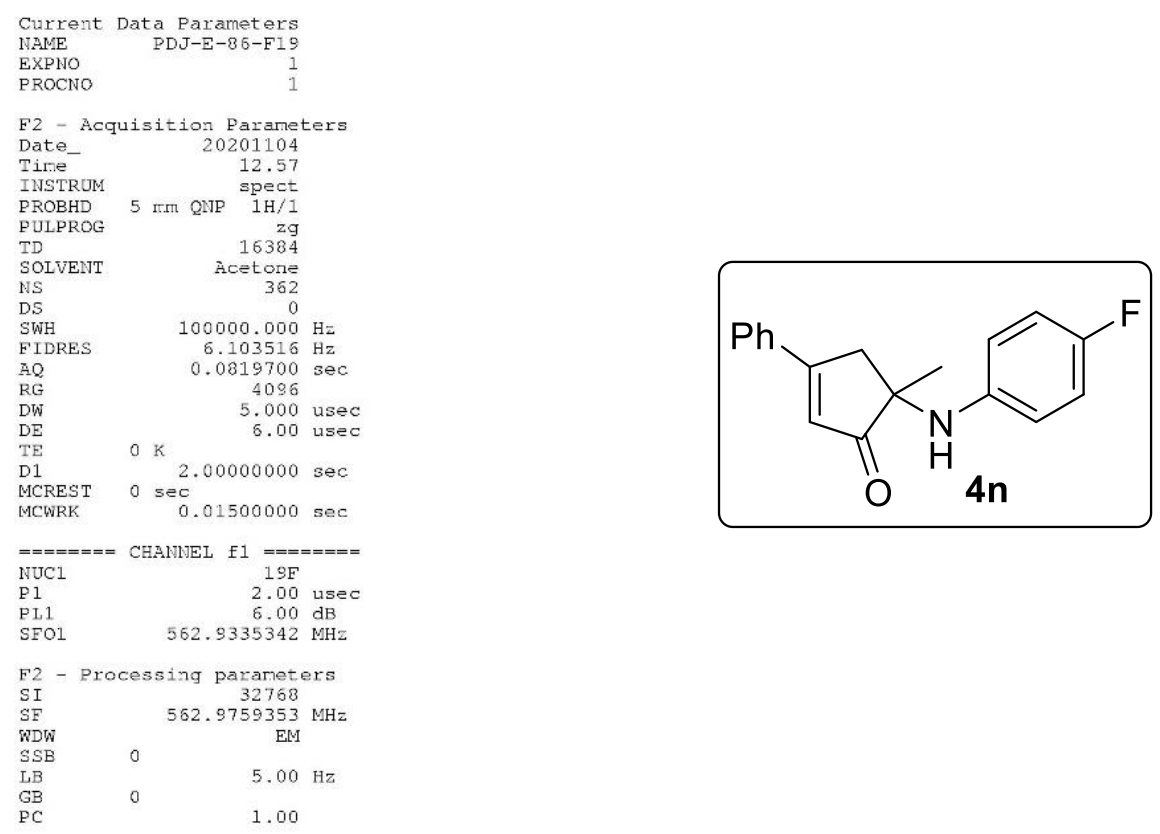

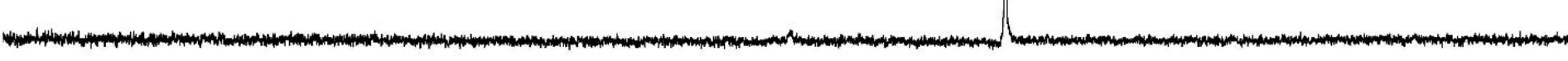

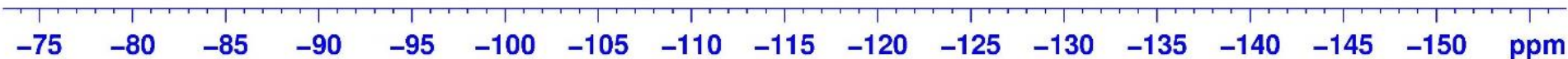



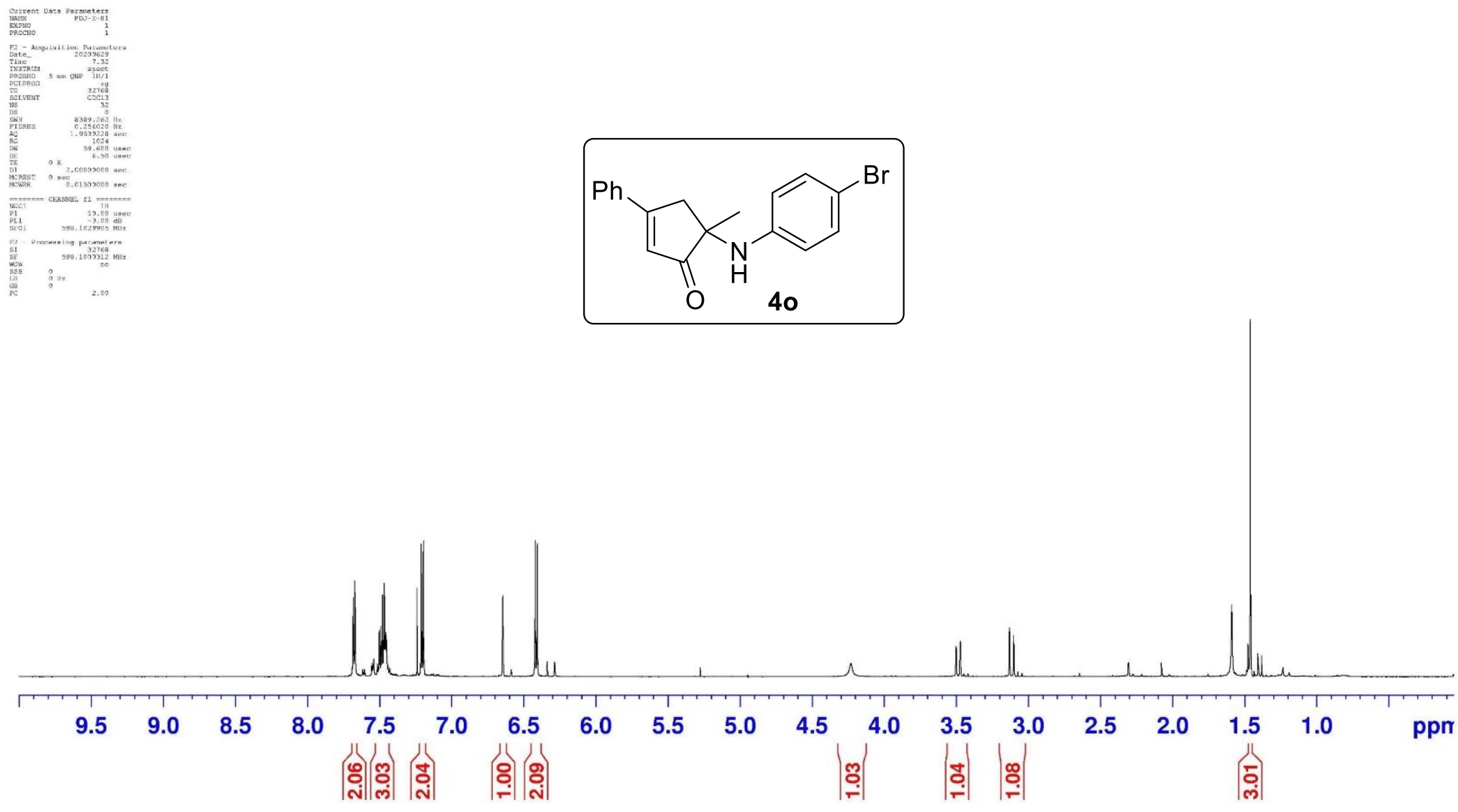


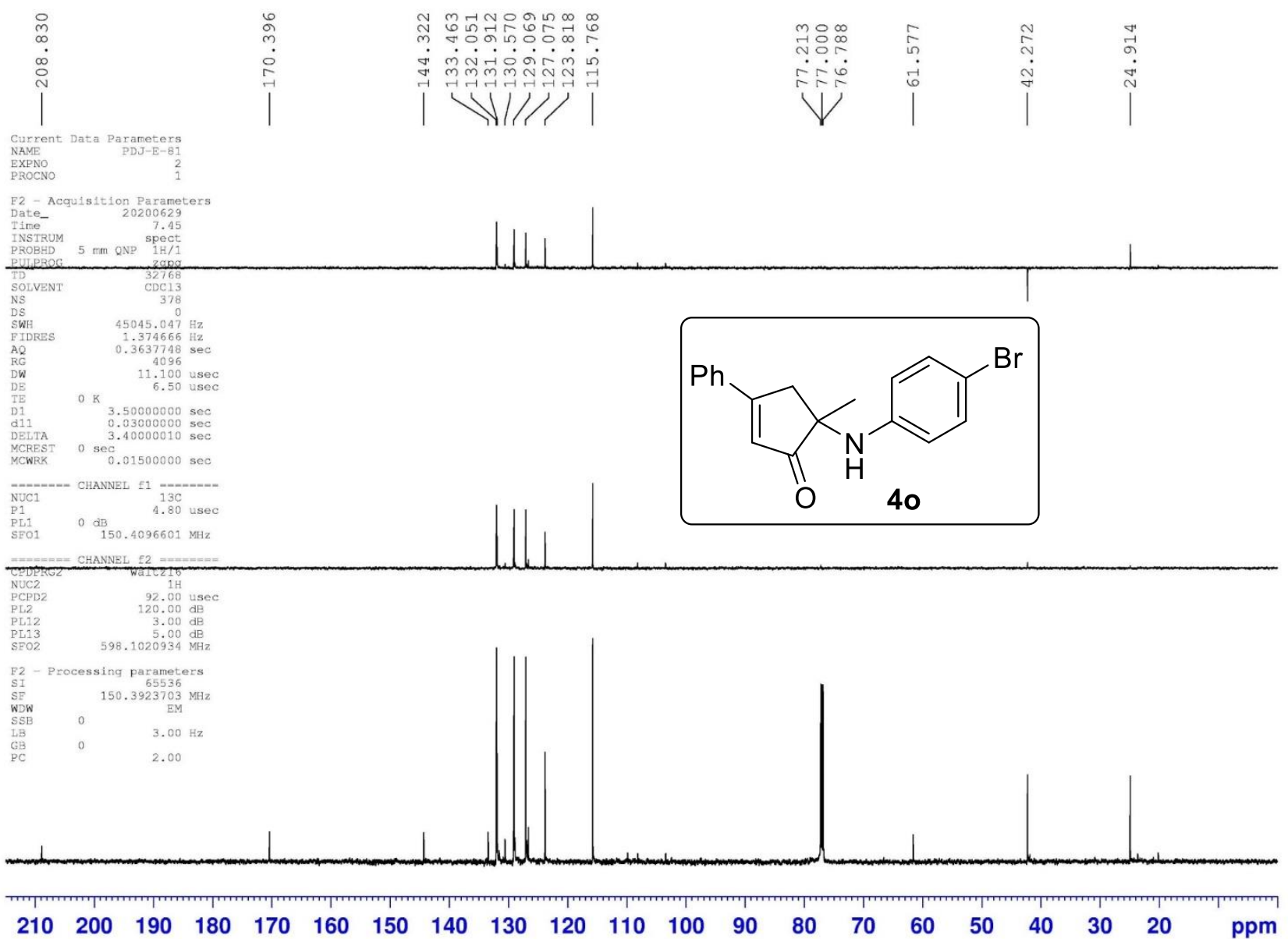




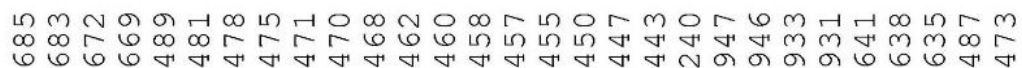

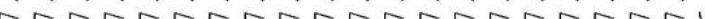

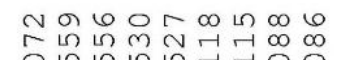

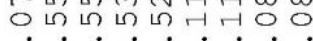

$\ddot{n} \dot{n} \dot{m} \dot{m} \dot{m} \dot{m}$

긍

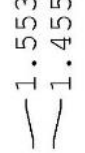

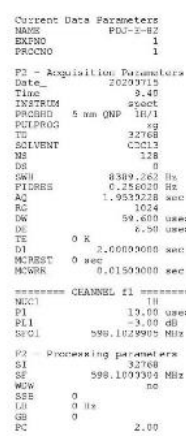
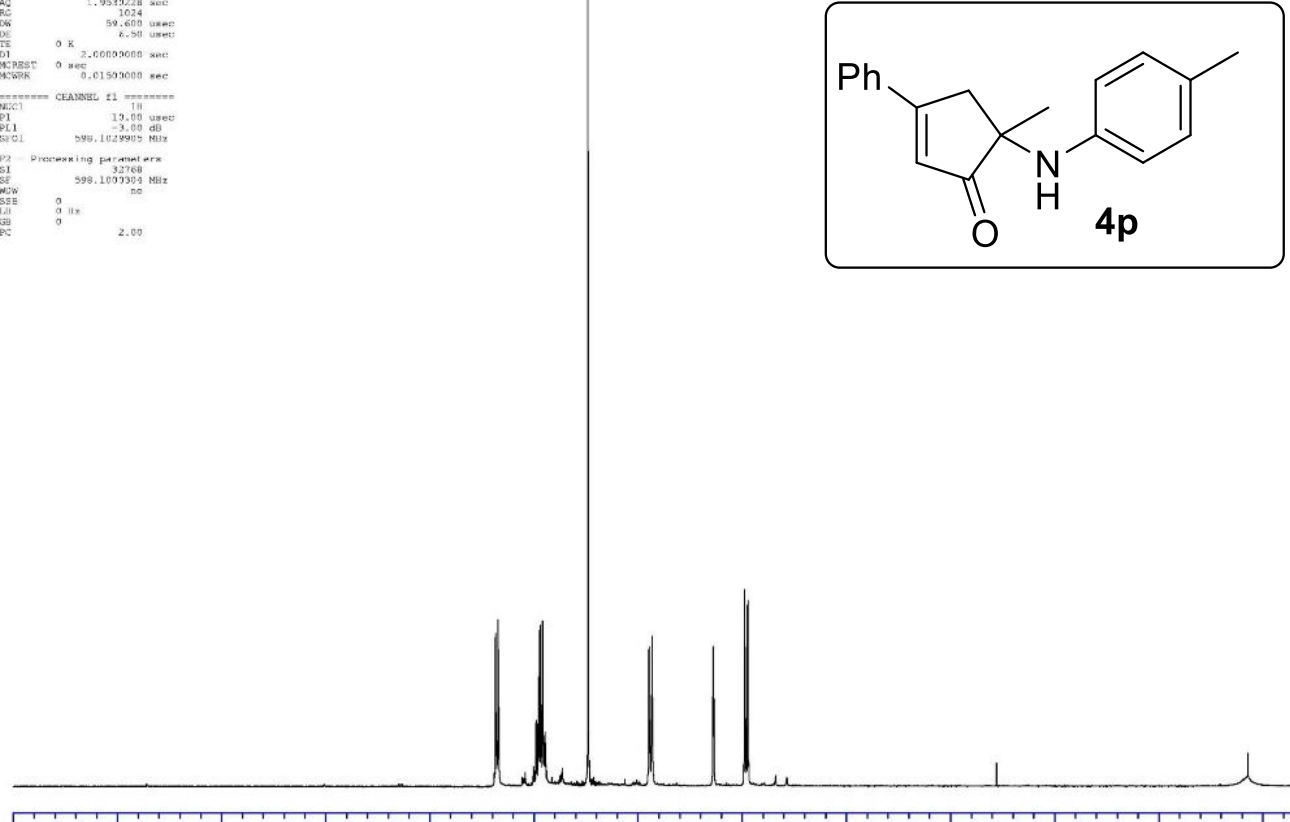

$9.5 \quad 9.0$

$\begin{array}{lll}7.5 & 7.0 & 6.5\end{array}$

6.05 .5

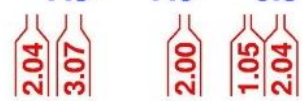

5.0
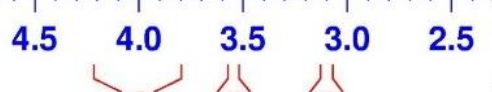

뿌

苗 


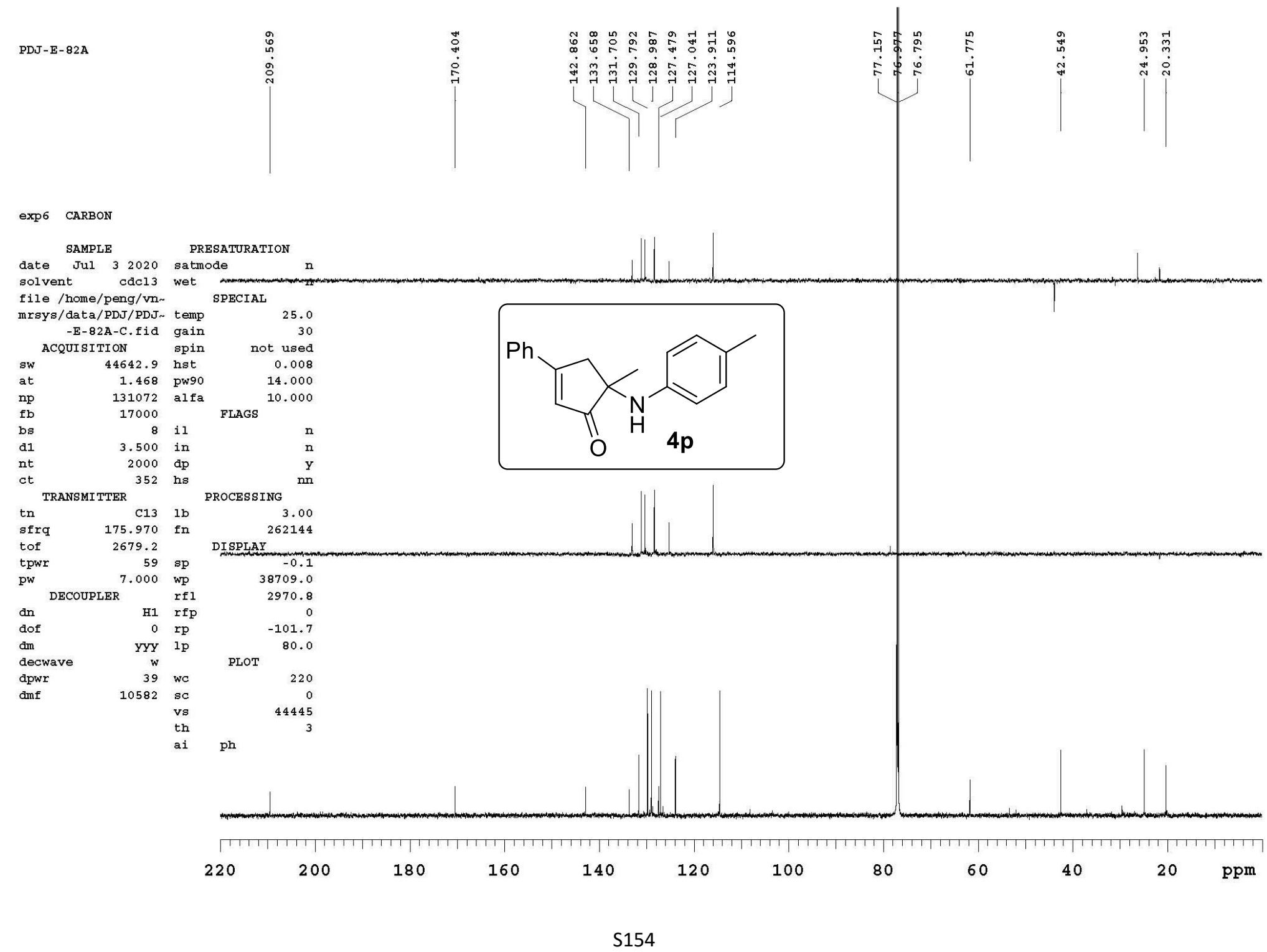




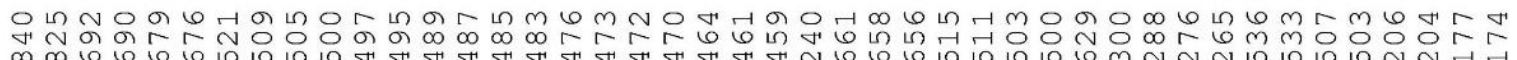

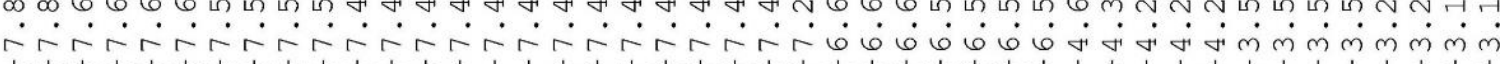

r
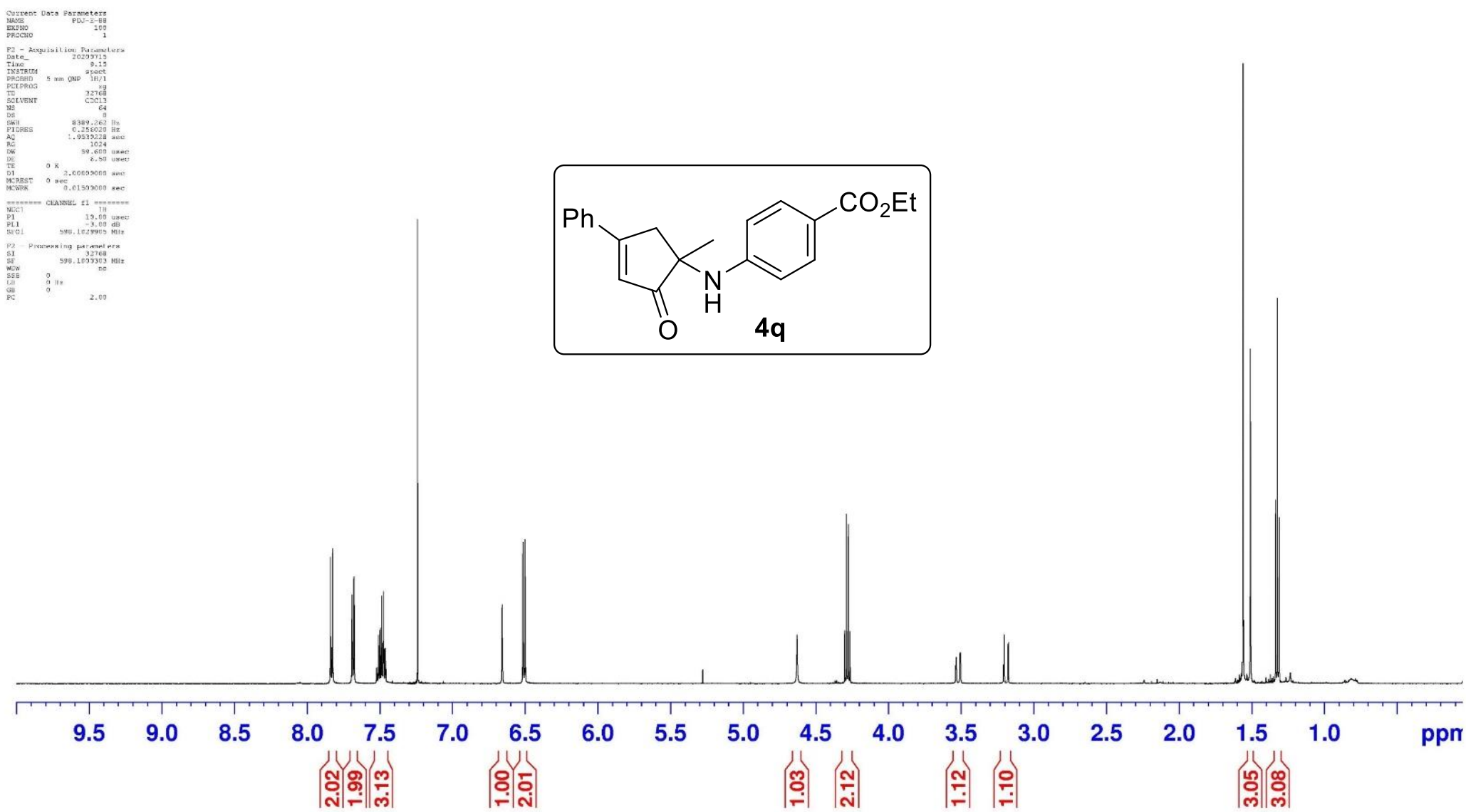


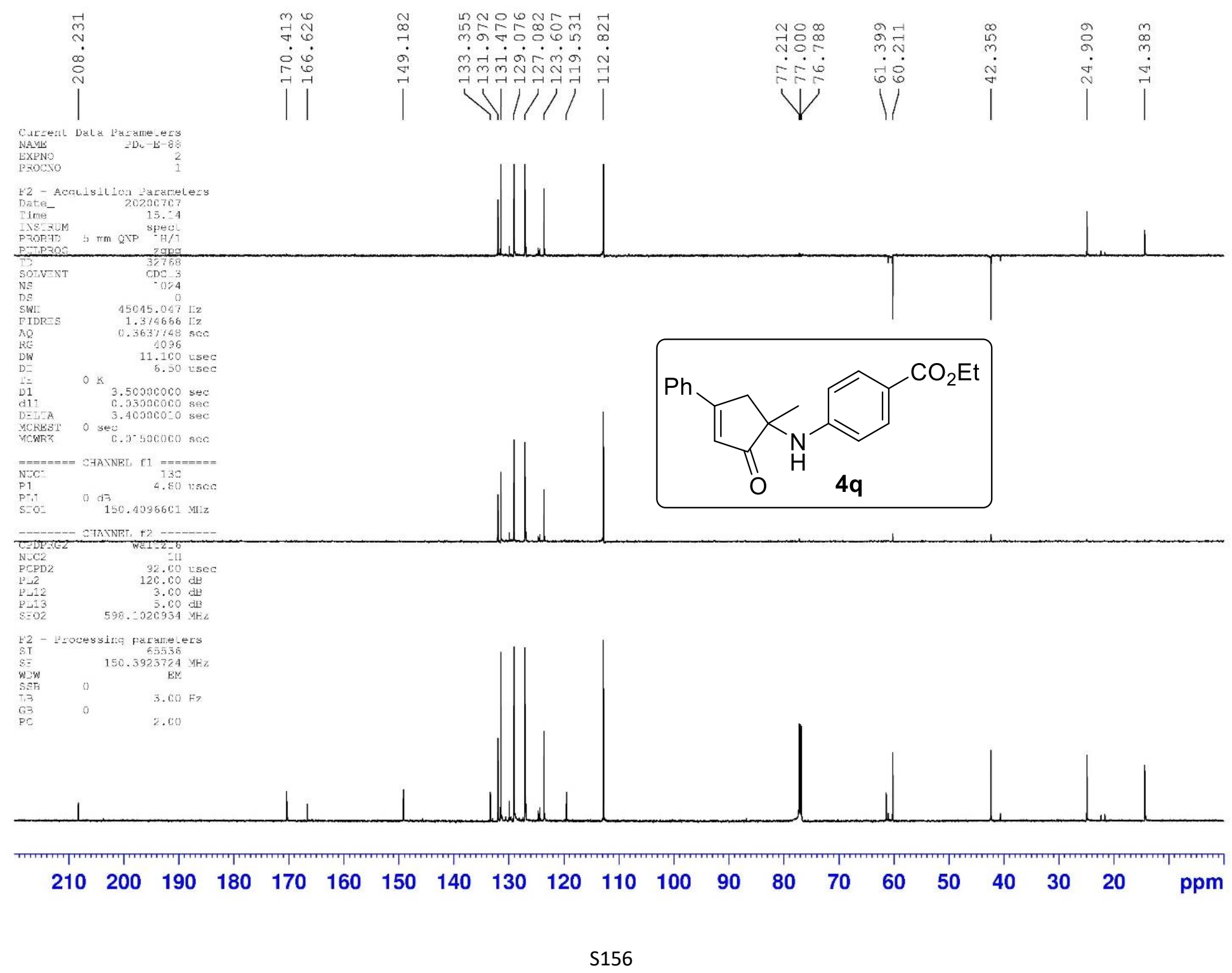




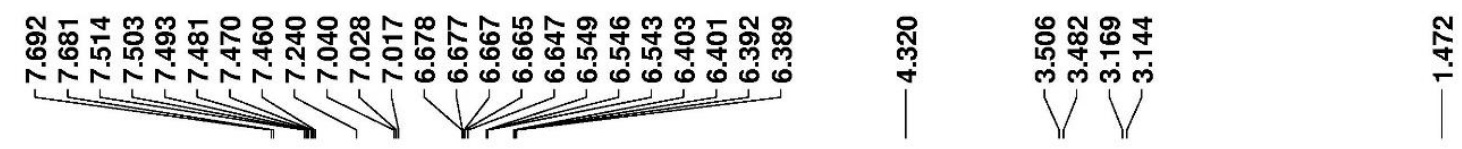
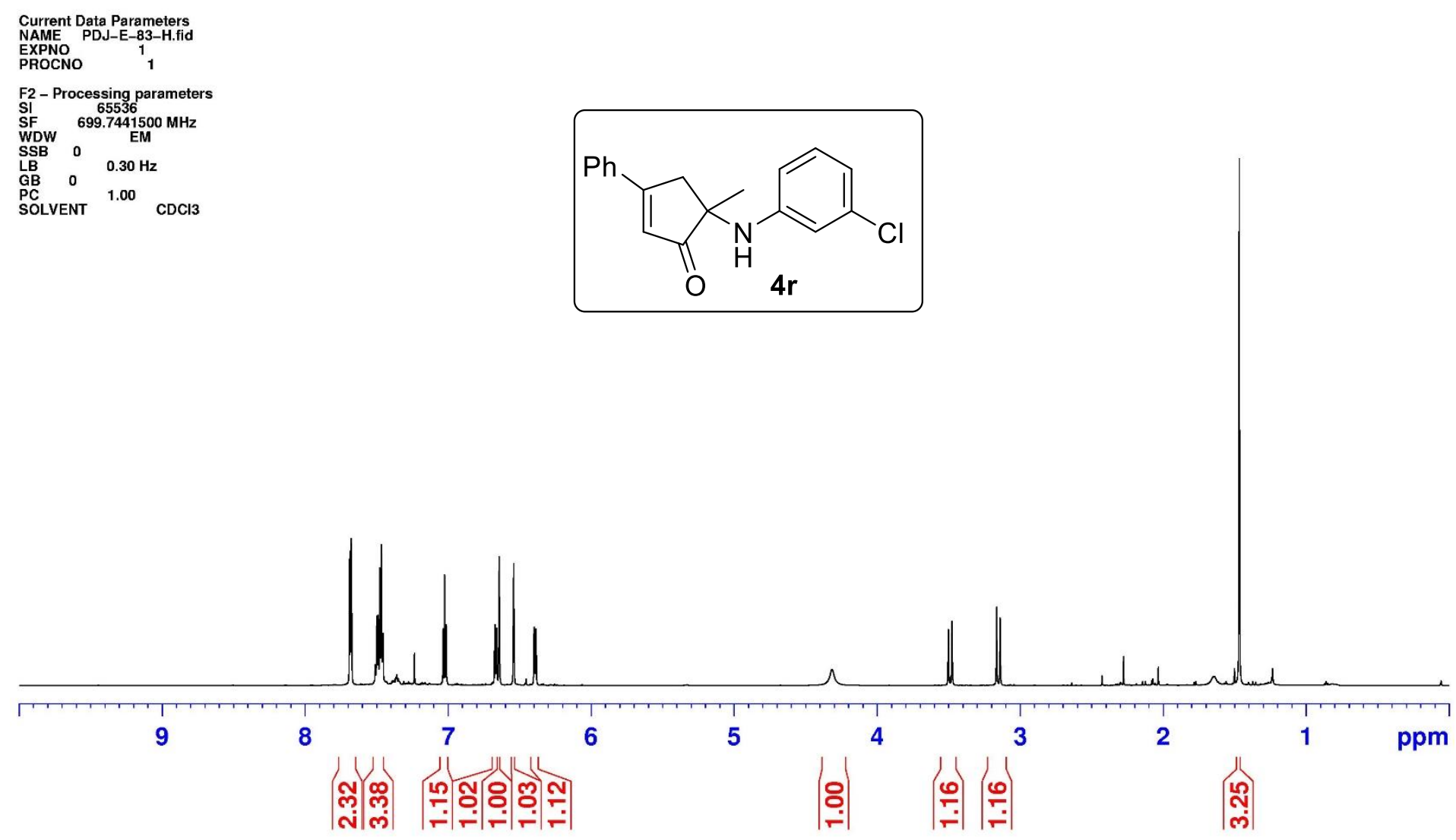


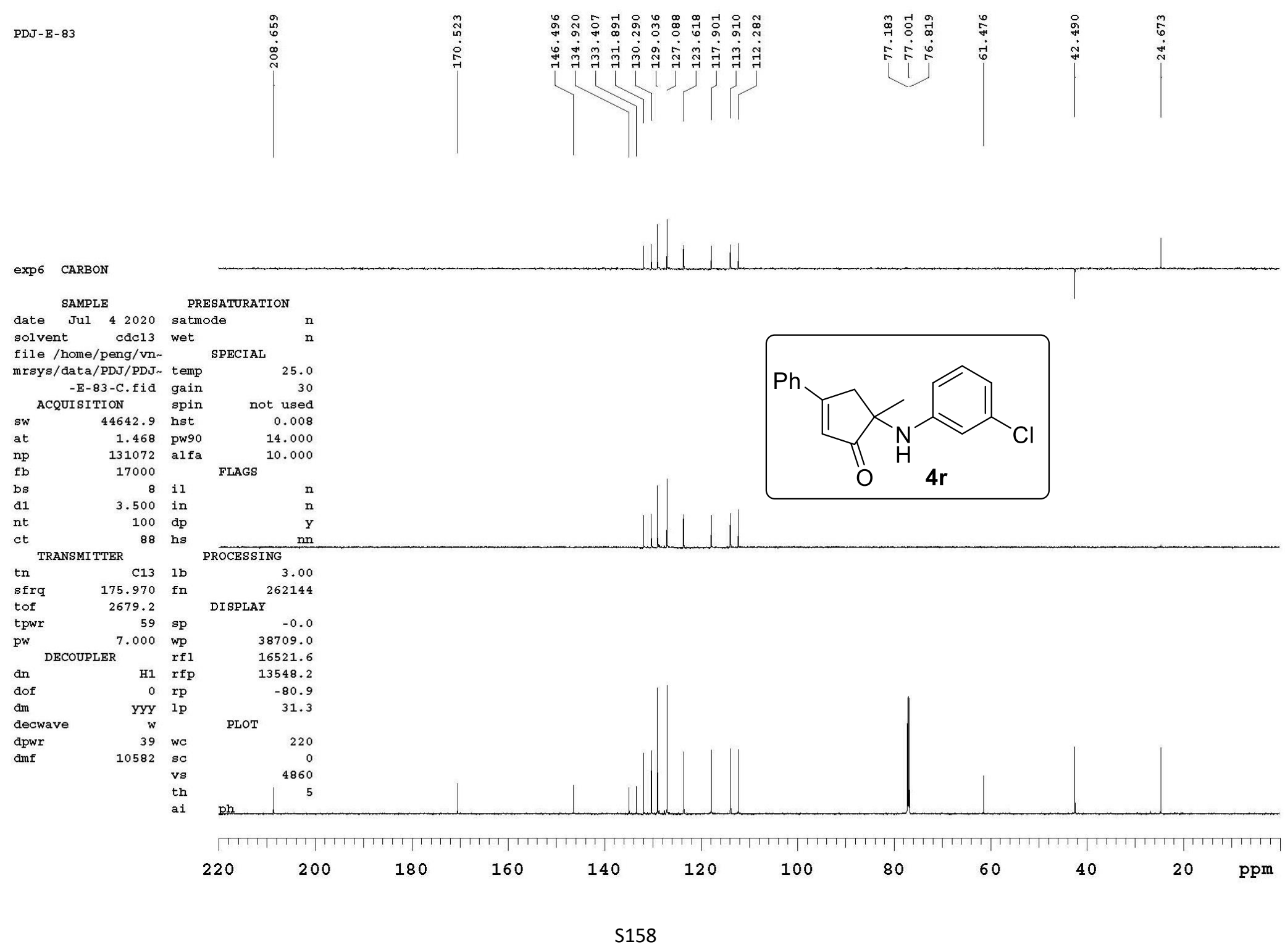




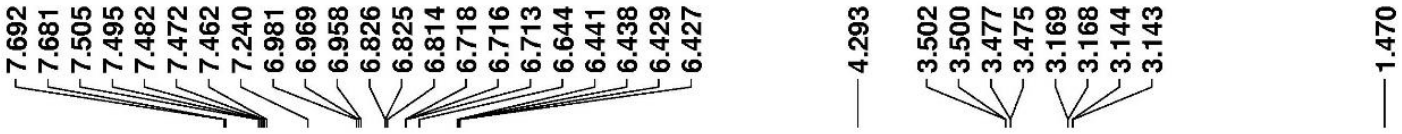
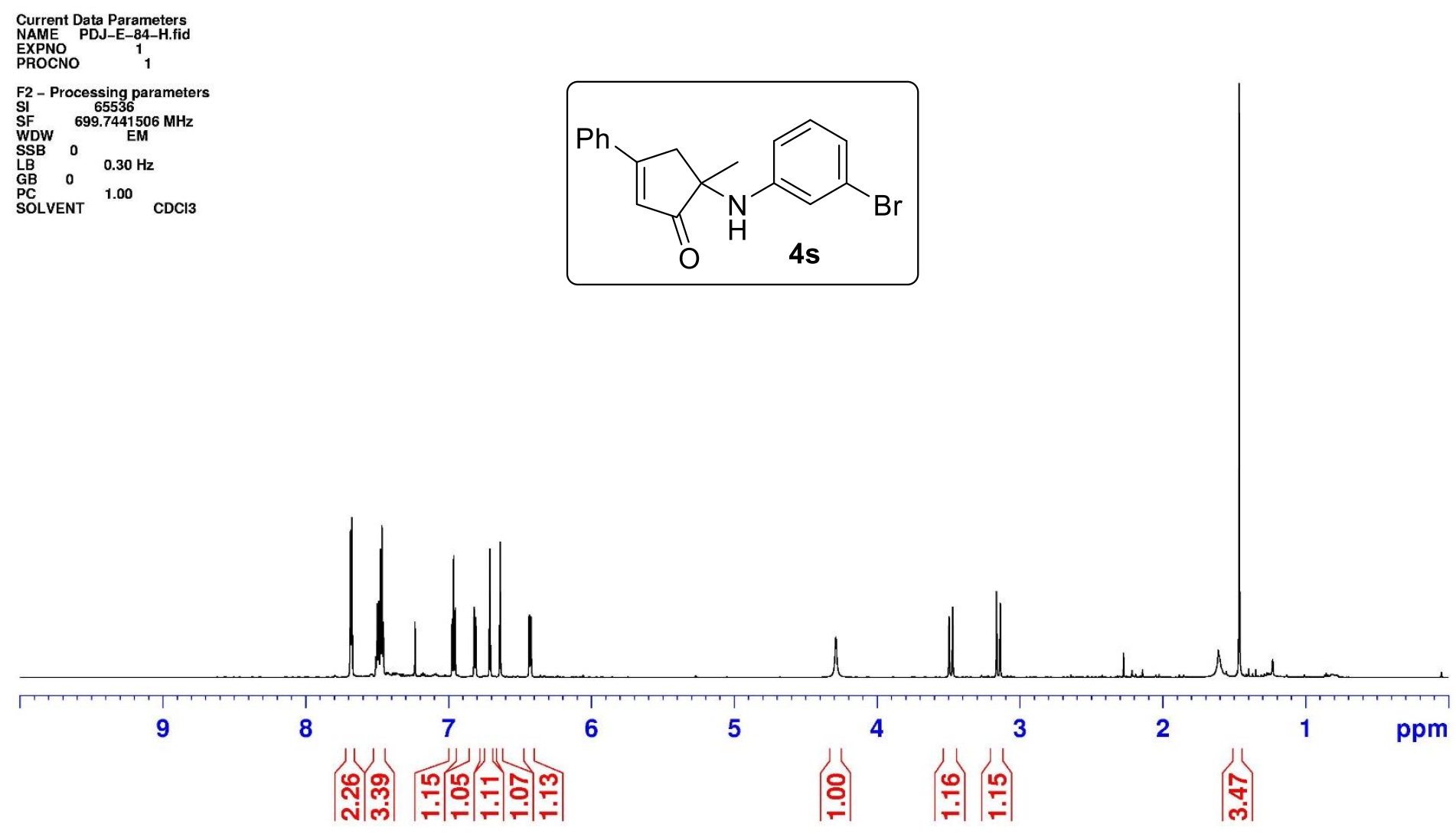


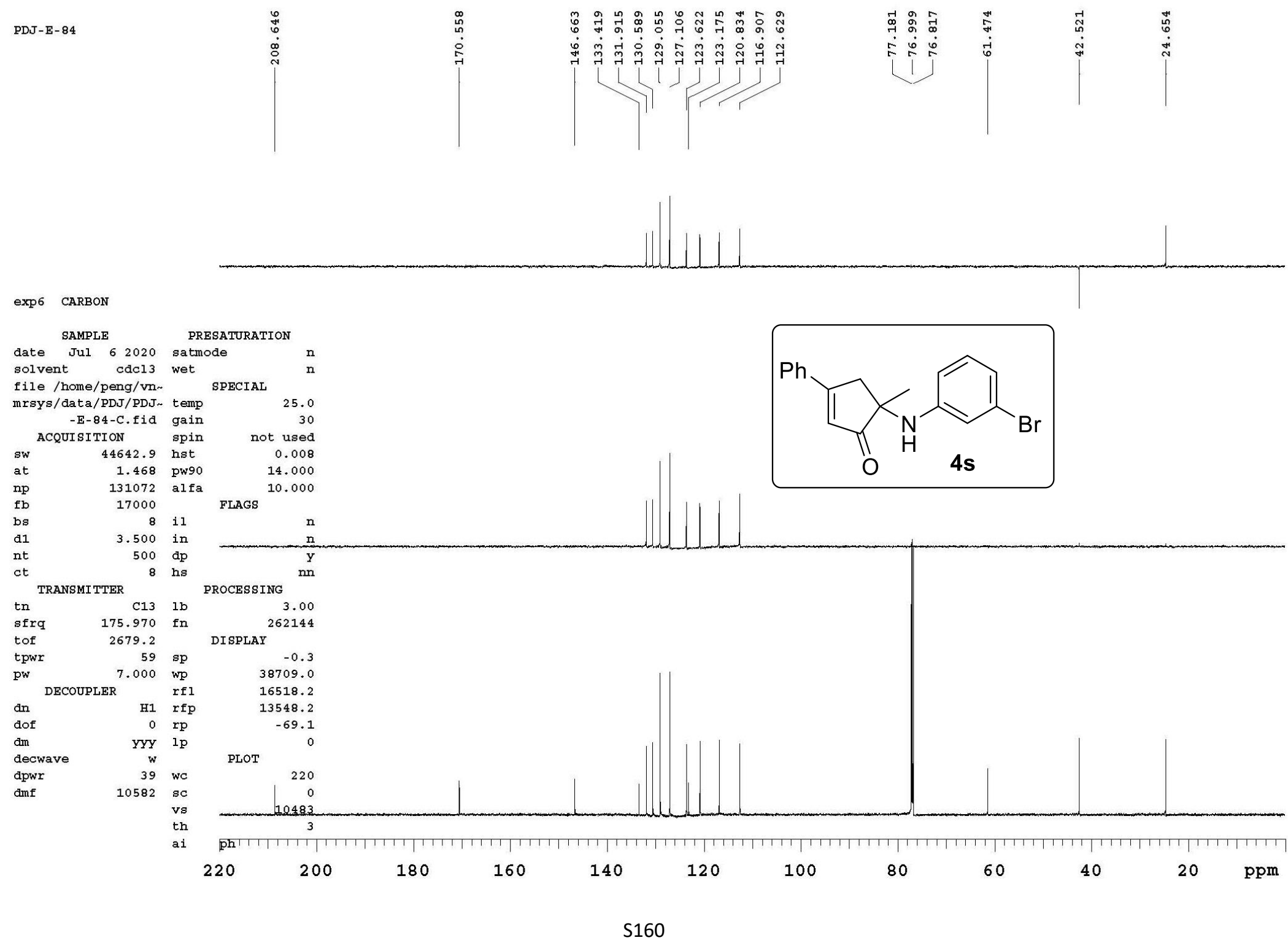




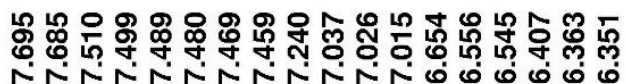 \\ 31 \\ |}

Current Data Parameters

PROCNO

F2 - Processing parameters

$\begin{array}{ll}\mathrm{SI} & 65536 \\ \mathrm{SF} & 699.7441499 \mathrm{MHz}\end{array}$

$\begin{array}{lll}S S B & 0 & 0.30 \mathrm{~Hz}\end{array}$

$\begin{array}{lll}\mathrm{GB} & 0 & \mathbf{1 . 0 0}\end{array}$
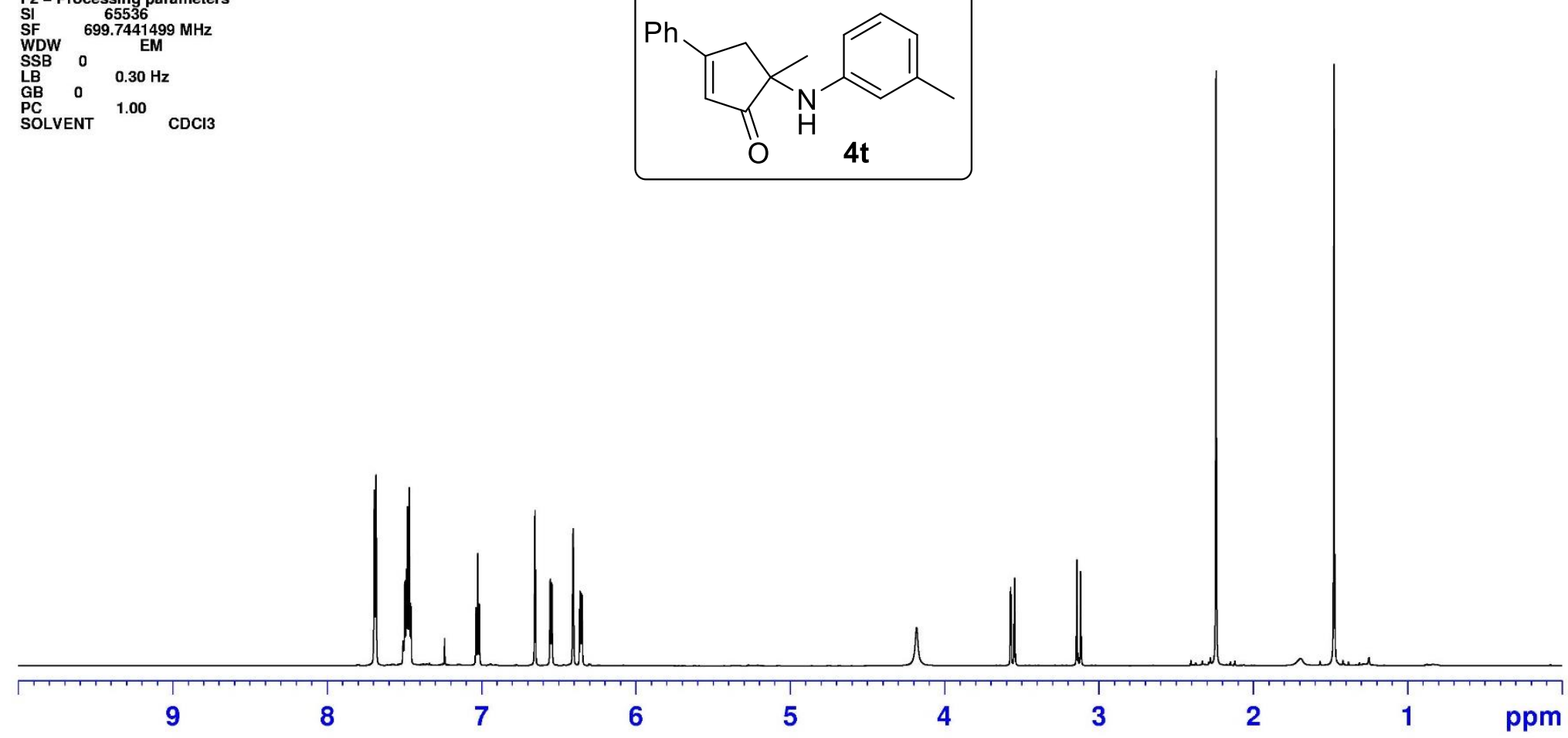


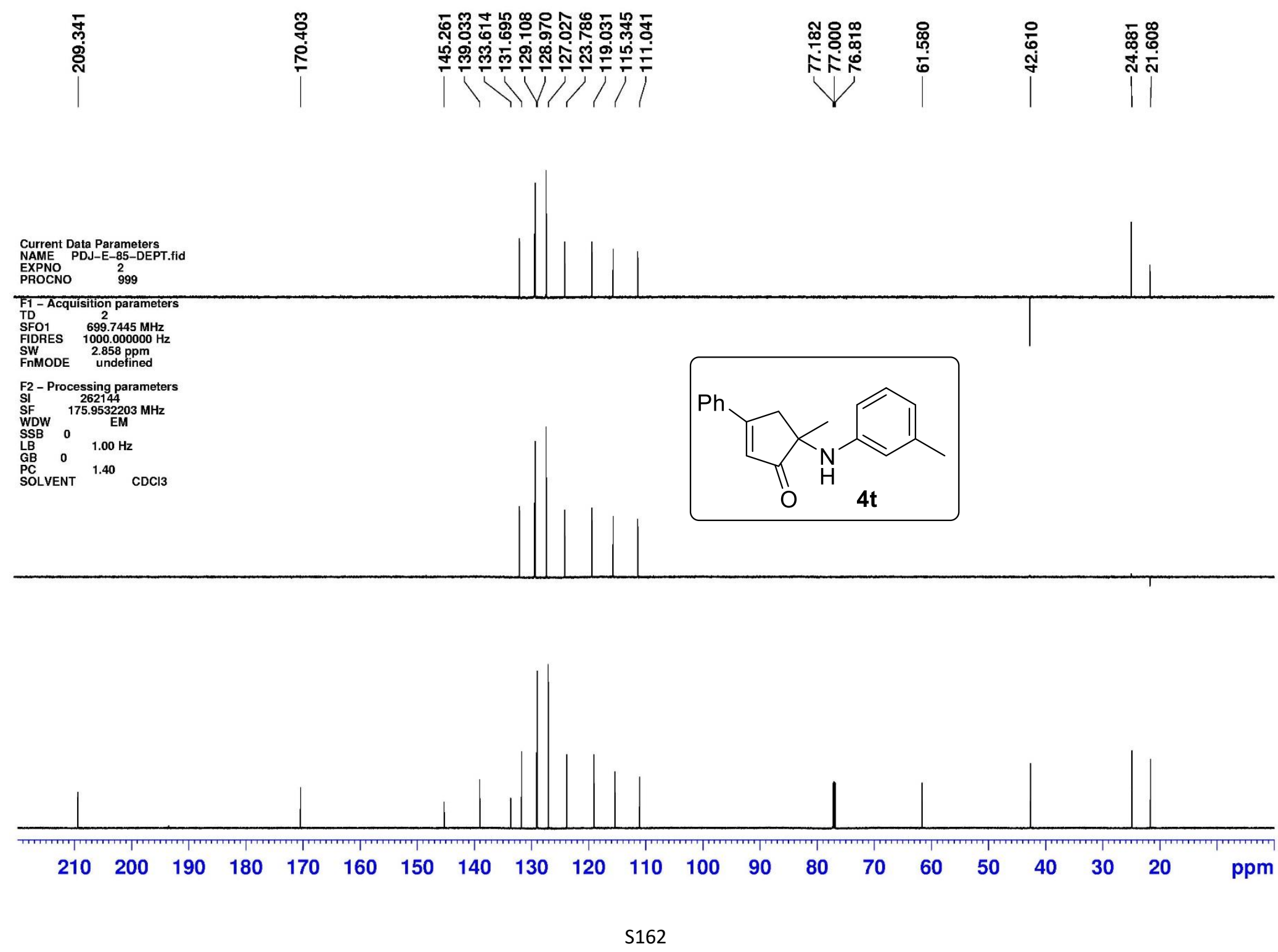




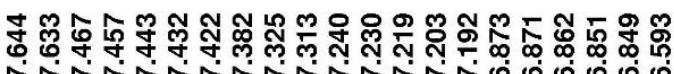

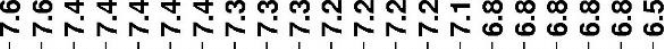

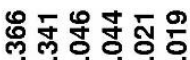

लं लिं लिल

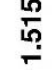

Current Data Parameters

NEPNO
PROCNO

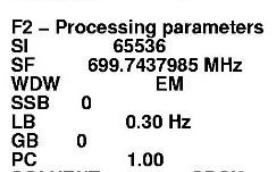

$\begin{array}{llll} & \\ \text { PC } & 0 & 0 & \\ \text { SOLVENT } & 1.00 \quad \text { CDC13 }\end{array}$
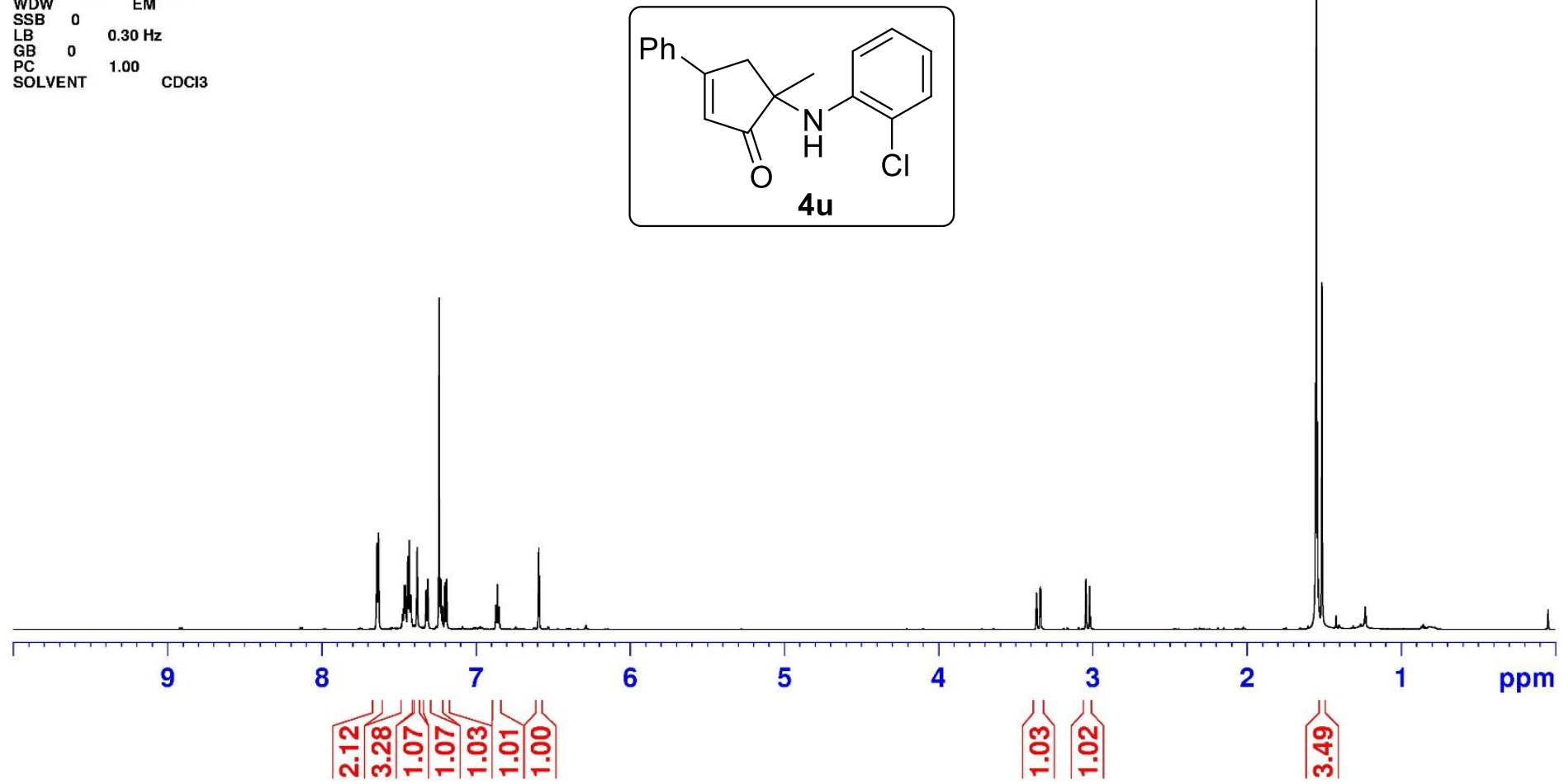


|

Current Data Parameters

EXPNO ${ }_{1}$

F2 - Processing parameters

SI 131072

WDW 0 EM

$0.30 \mathrm{H}$

$\begin{array}{llll}\text { PC } & 0 & \\ \text { SOLVENT } & 1.00 \quad \text { CDC13 }\end{array}$
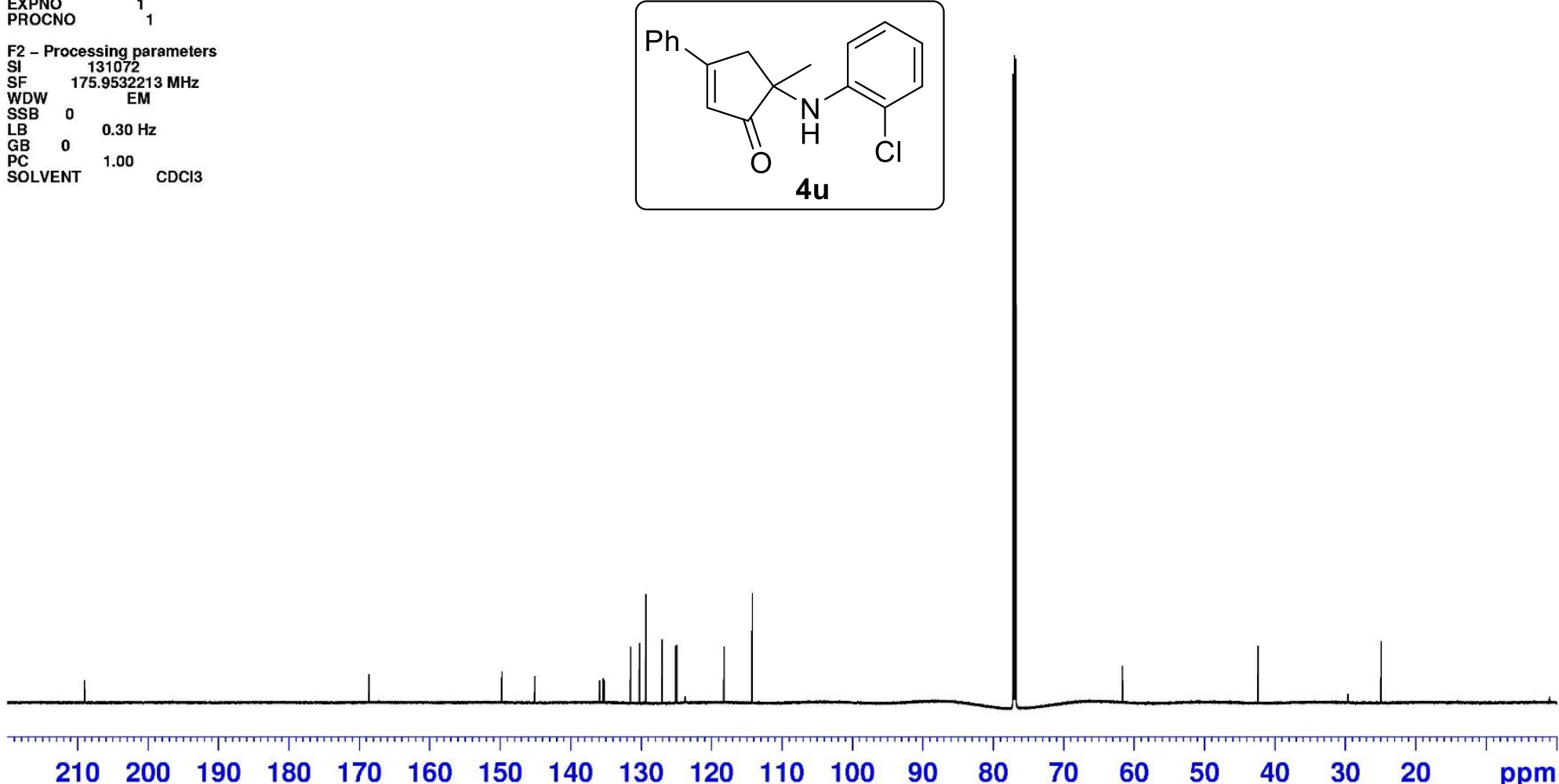


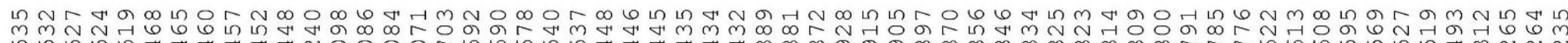

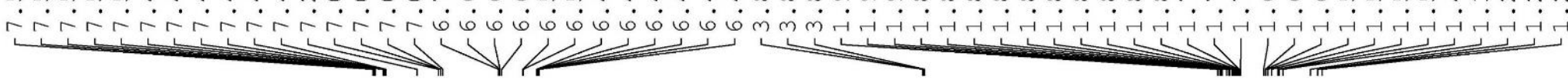
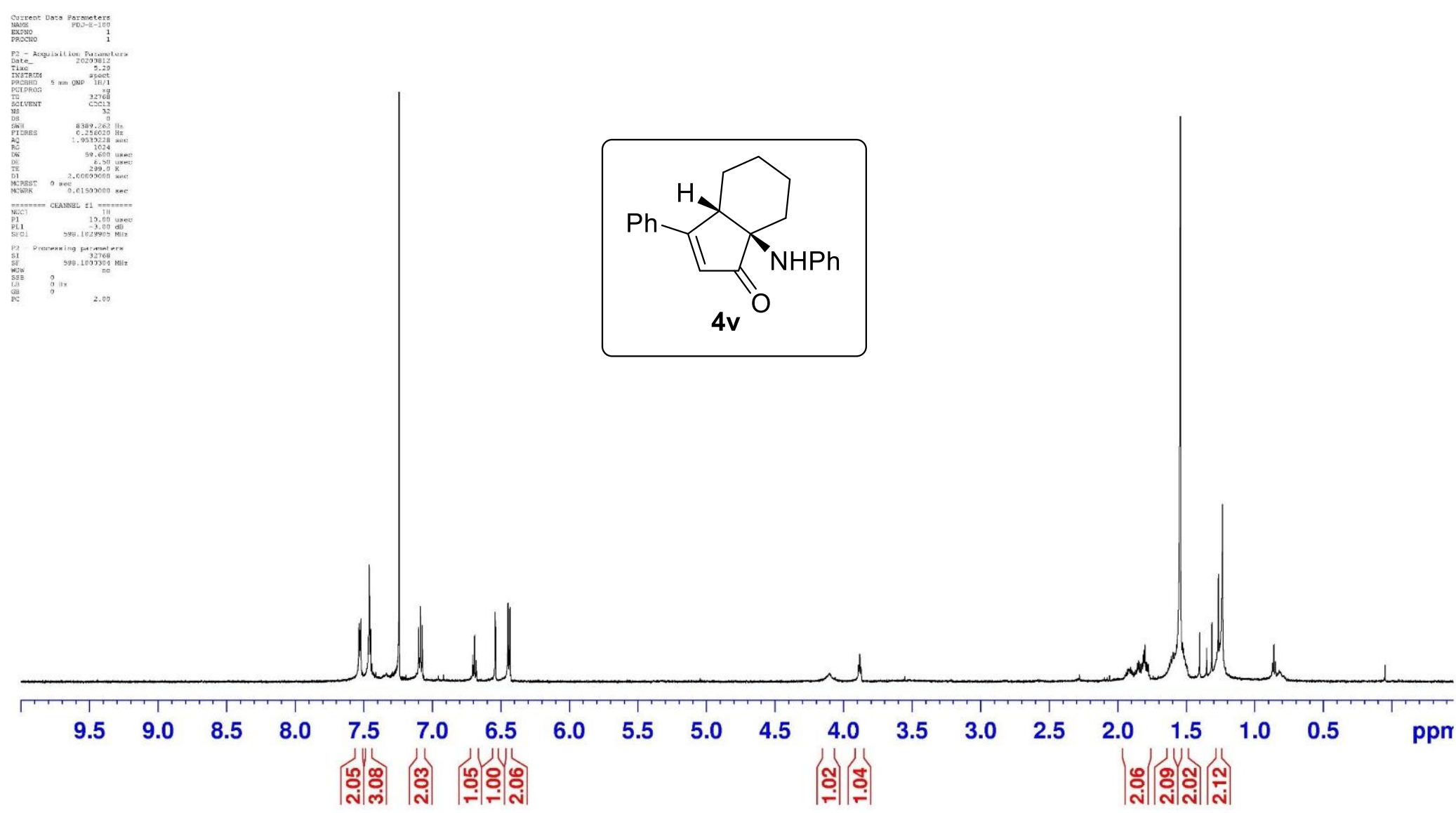


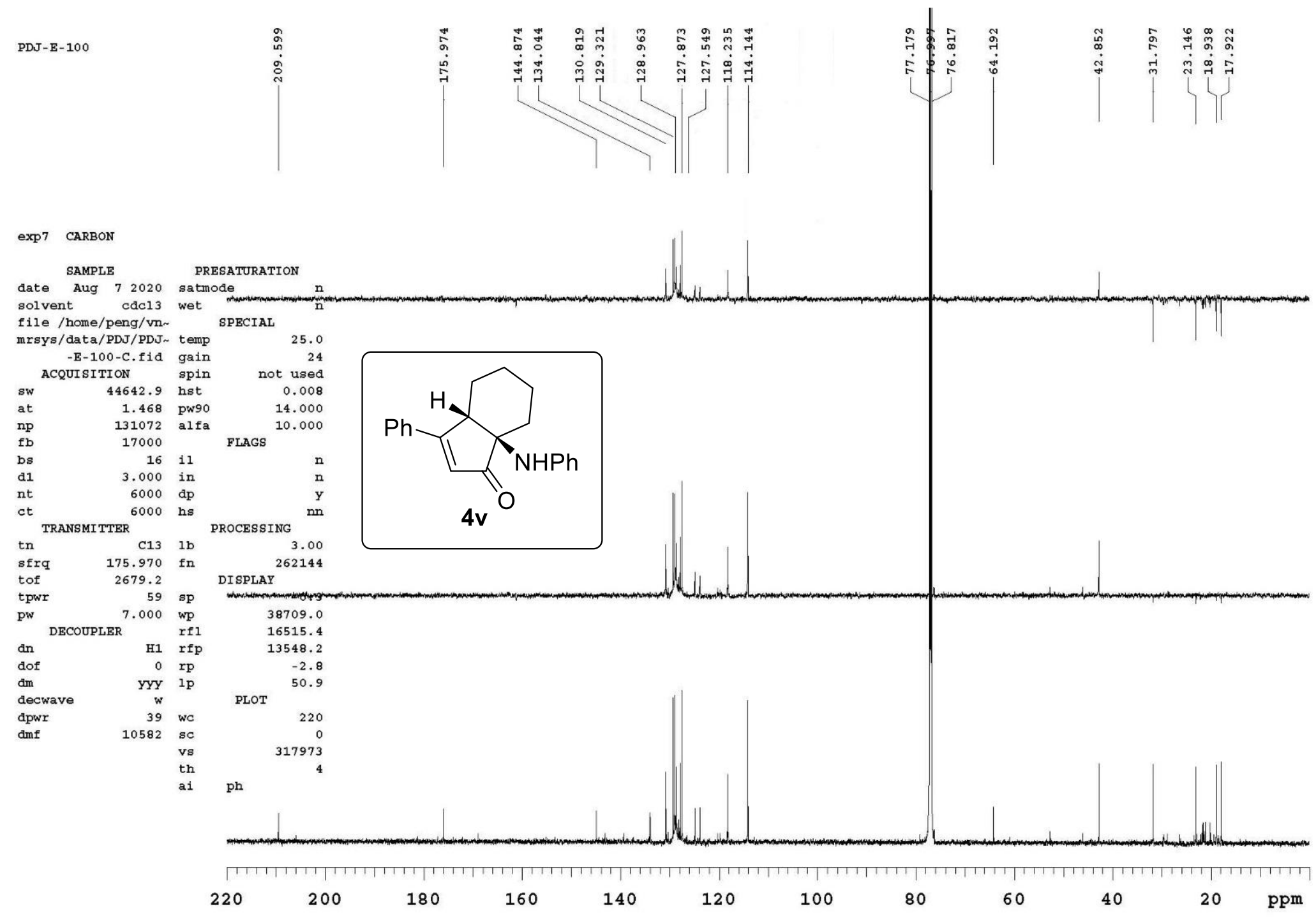




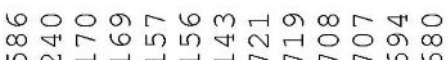

$\therefore r i r r i b \dot{\theta} \dot{\theta} \dot{0}$

$\underbrace{2}$

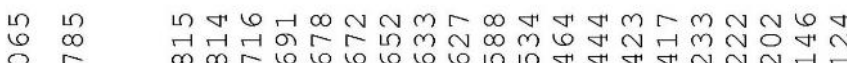

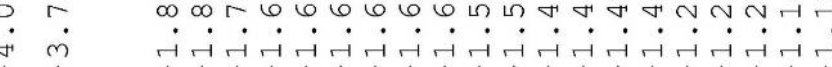

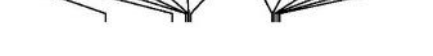

됴|
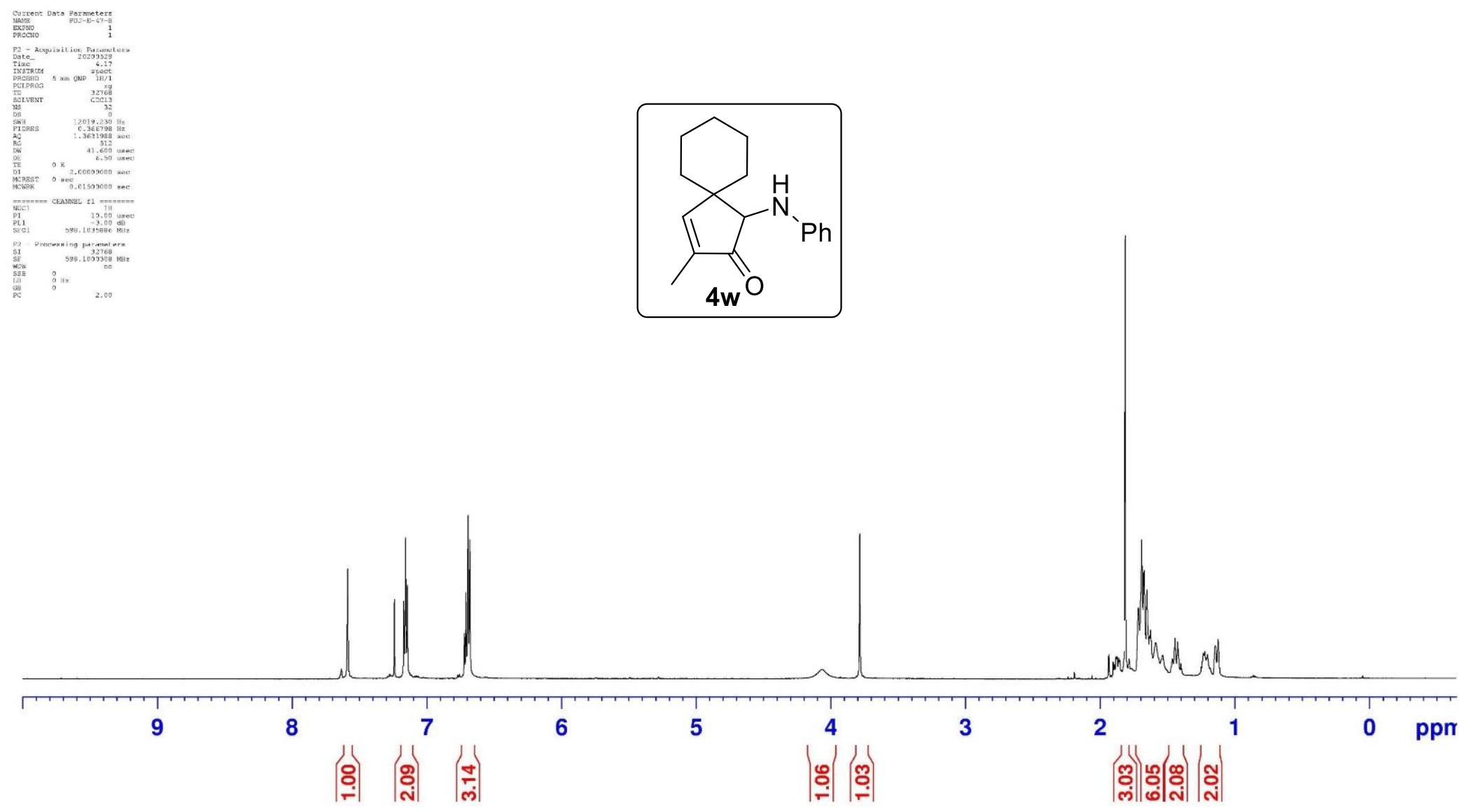


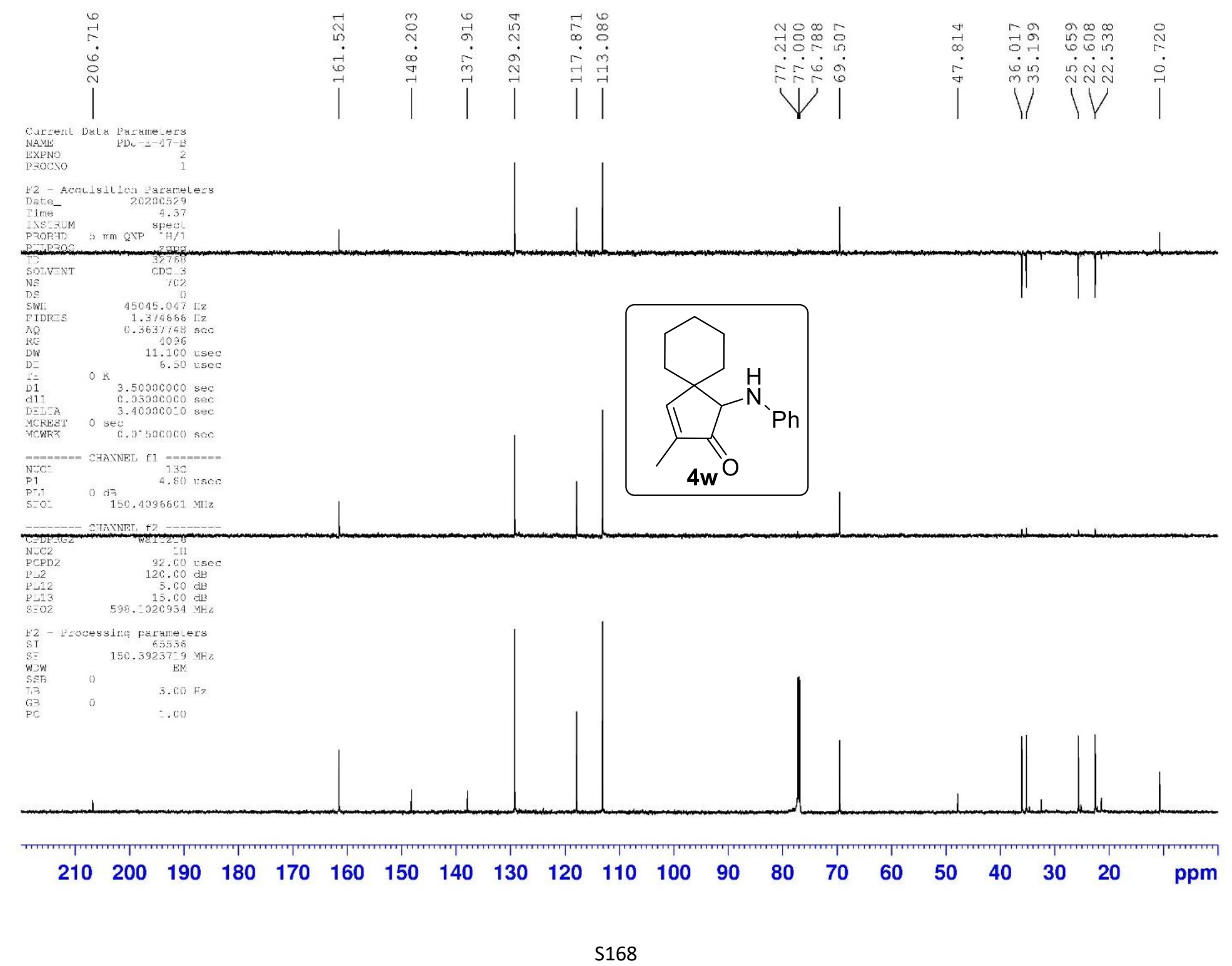



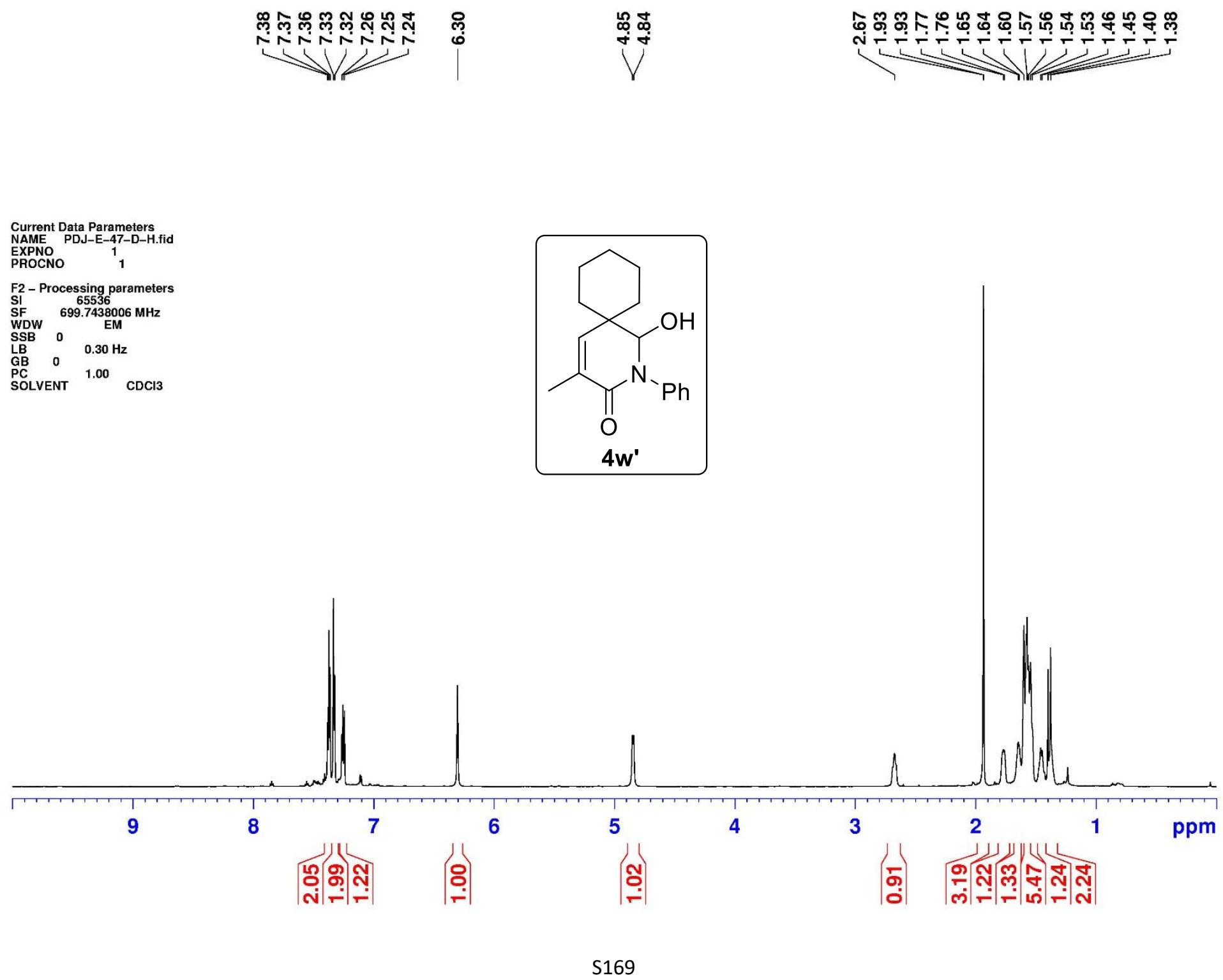


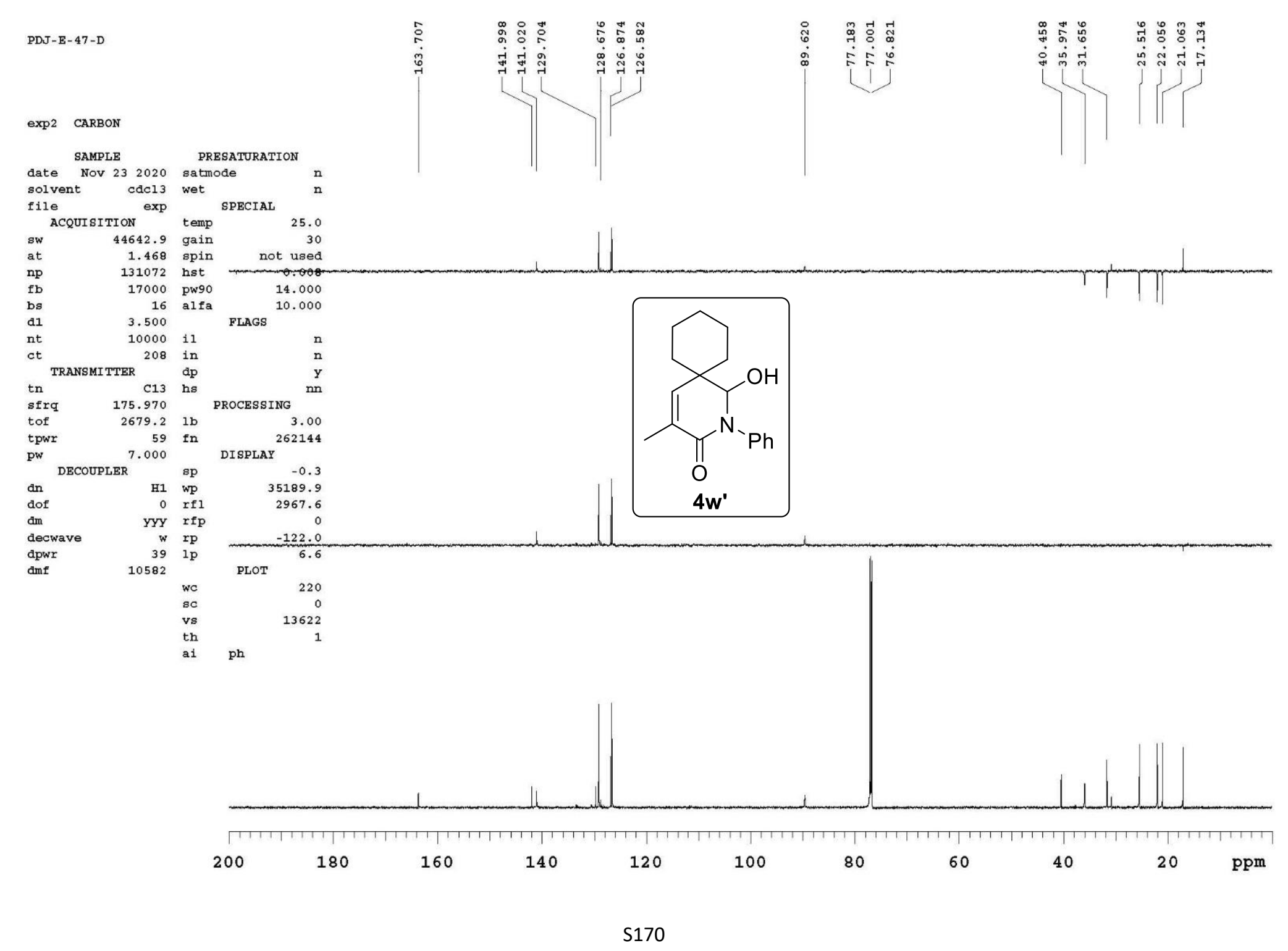



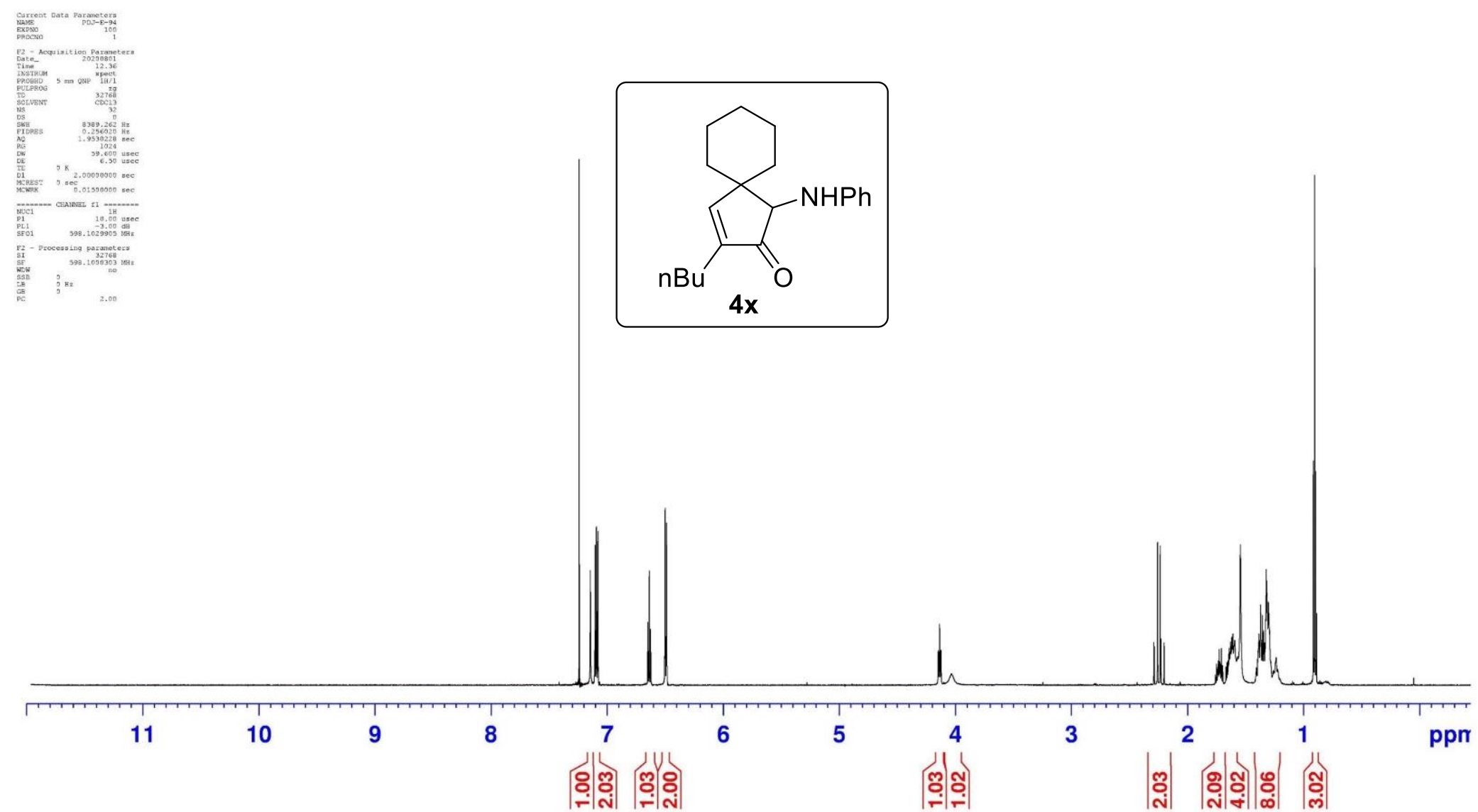


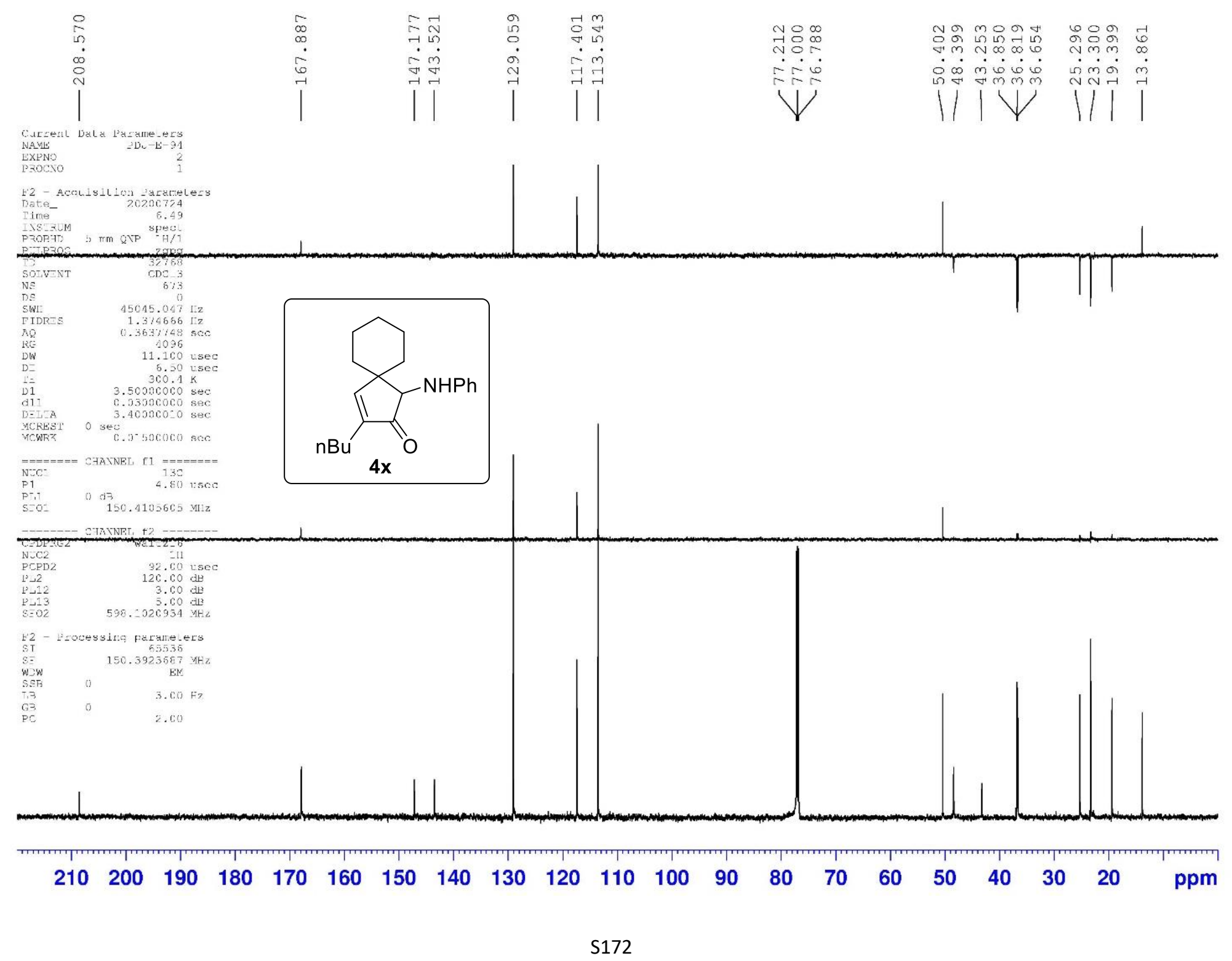




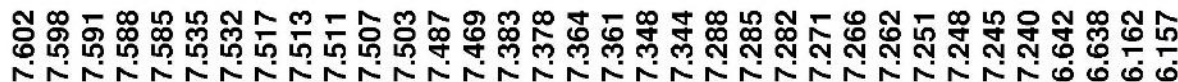
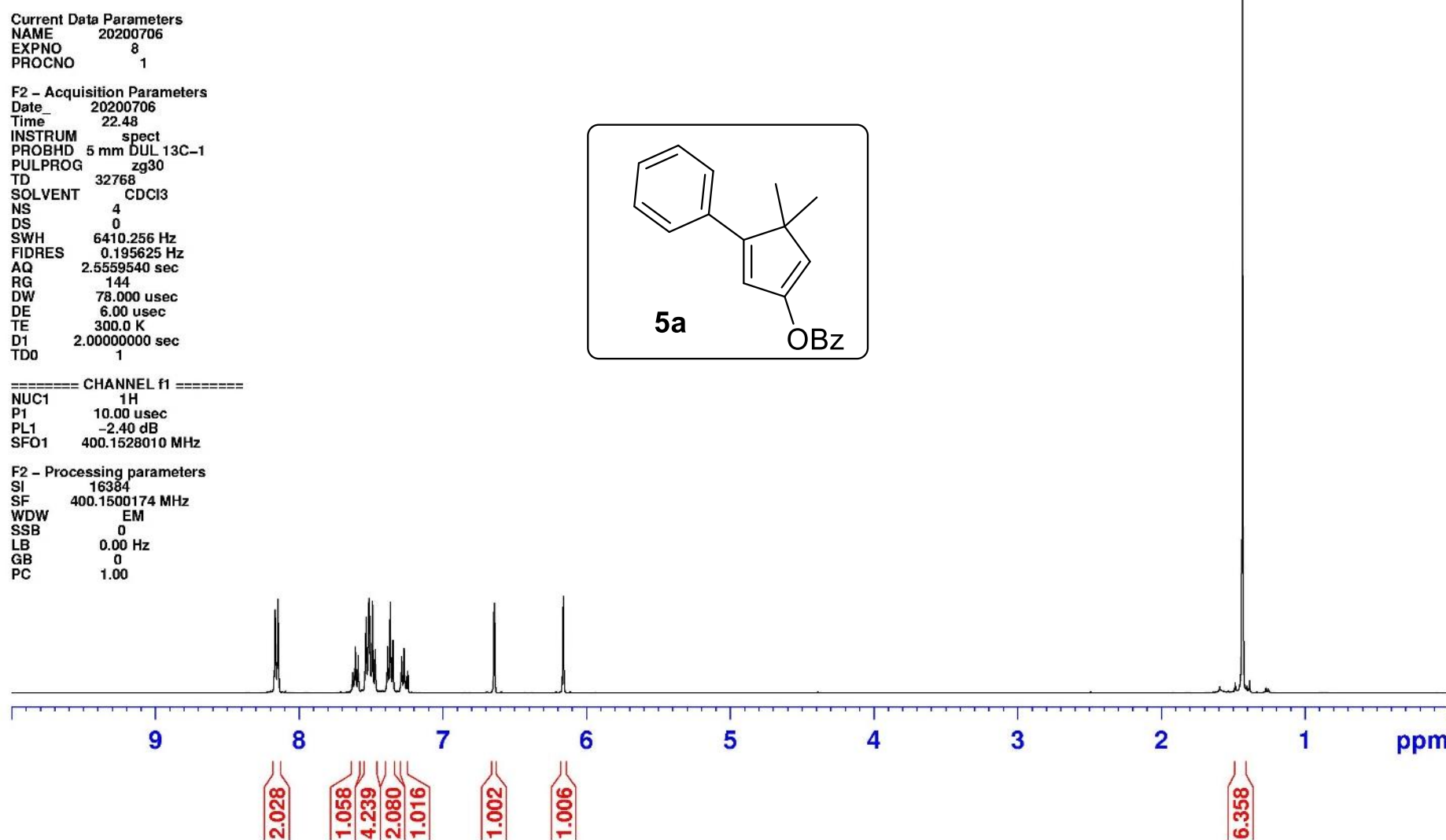

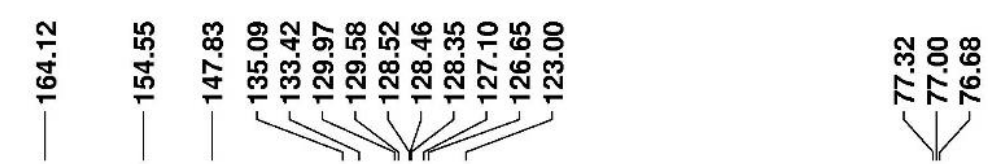

Current Data Parameters
NAME 20200706

$\begin{array}{lr}\text { NAME } & 20200706 \\ \text { EXPNO } & 9 \\ \text { PROCNO } & 1\end{array}$

F2 - Acquisition Parameters
Date- 2020006
Time
22.51

IIMSTRUM
spect
PROBHD 5 m 5 DUL 13C-1

TDDPROG 65536 zgpa

SOLVENT CDC13

$\begin{array}{lr}\text { SWH } & 22727.273 \mathrm{~Hz} \\ \text { FIDRES } & 0.346791 \mathrm{~Hz}\end{array}$

$A Q \quad 1.4418420 \mathrm{sec}$

$\begin{array}{lr}\text { AG } & 2050 \\ \text { DW } & 22.000 \text { use } \\ \text { DE } & 600 \text { usec } \\ \text { TE } & 300.0 \mathrm{~K}\end{array}$

$\begin{array}{ll}\text { DE } & 300.0 \mathrm{~K} \\ \text { D1 } & 2.0000000 \mathrm{sec} \\ \text { d11 } & 0.03000000 \mathrm{sec}\end{array}$

$\begin{array}{ll}\text { d11 } & 2.003000000 \mathrm{sec} \\ \text { DELTA } & 1.8999998 \mathrm{sec}\end{array}$

$=======$ CHANNEL $\mathrm{f1}=======$

$\begin{array}{cc}\text { NUC1 } & 13 \mathrm{C} \\ \mathrm{P} 1 & 9.70 \text { usec }\end{array}$

$\begin{array}{ll}\text { PL1 } & -0.50 \mathrm{~dB} \\ \mathrm{SFO} 1 & \mathbf{1 0 0 . 6 2 8 8 6 6 0} \mathrm{MHz}\end{array}$

CPDPRG2 $\underset{\text { Waltz16 }}{\text { CHANEL } 12======}$

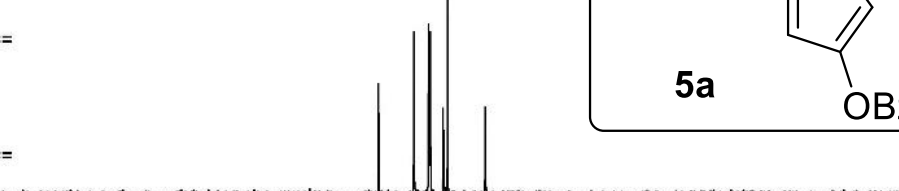

$\begin{array}{lc}\mathrm{PLPD} & -2.00 \mathrm{dsec} \\ \mathrm{PL2} & -2.40 \mathrm{~dB} \\ \mathrm{PL12} & 15.10 \mathrm{~dB} \\ \mathrm{PL13} & 18.10 \mathrm{~dB} \\ \mathrm{SFO2} & 400.1516010 \mathrm{MHz}\end{array}$

F2 - Processing parameters

$\begin{array}{lc}\text { SI } & 32768 \\ \text { SF } & 100.6178042 \mathrm{MHz} \\ \text { WDW } & 0 \\ \text { SSB } & 0 \\ \text { LB } & 3.00 \mathrm{~Hz} \\ \text { GB } & 0 \\ \text { PC } & 1.00\end{array}$

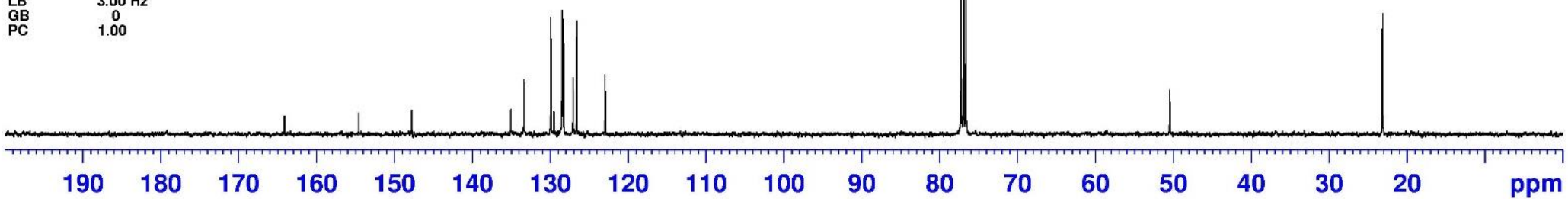




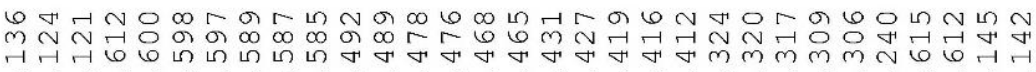

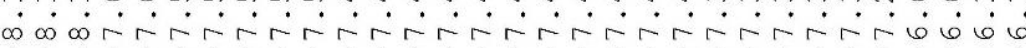

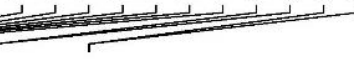
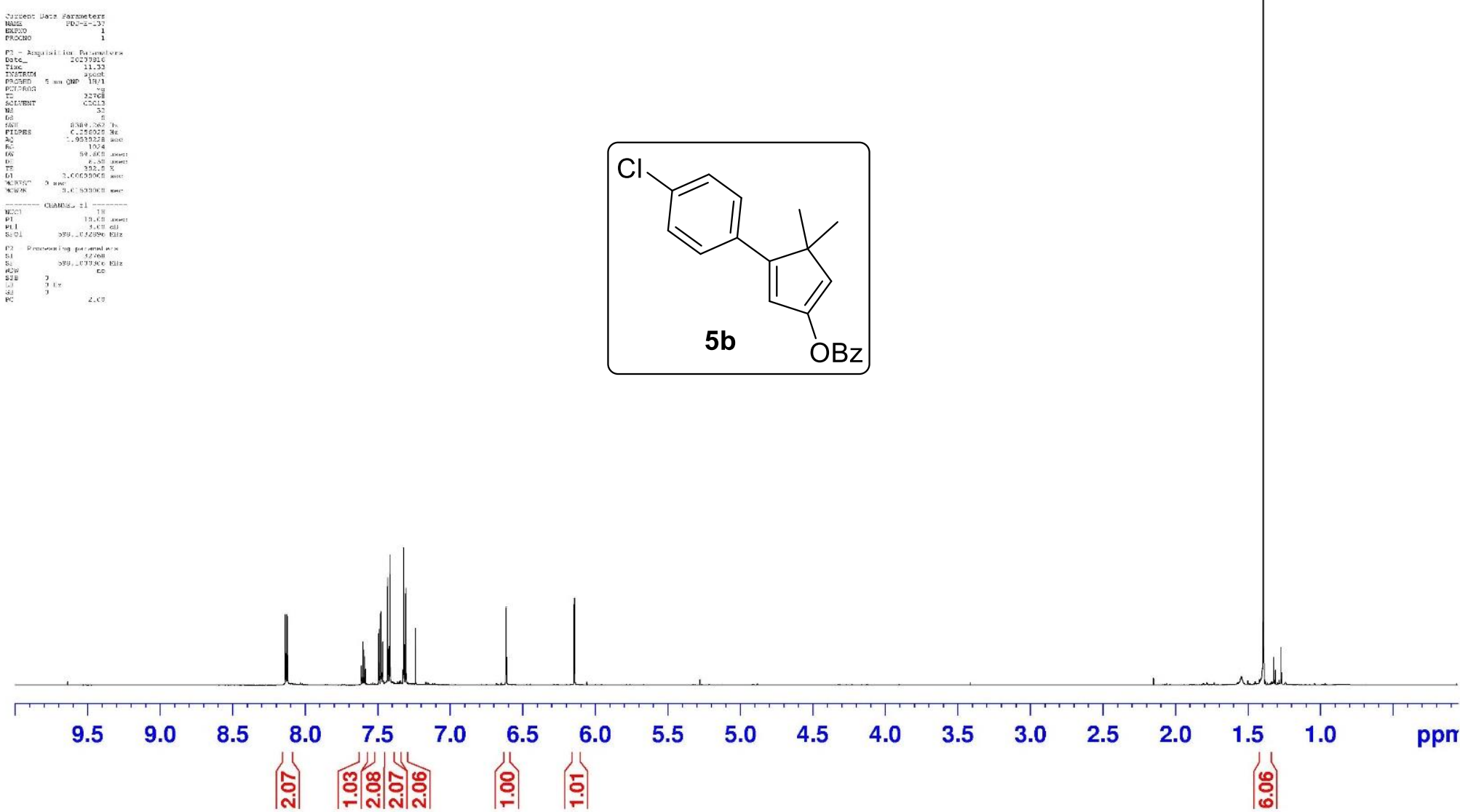


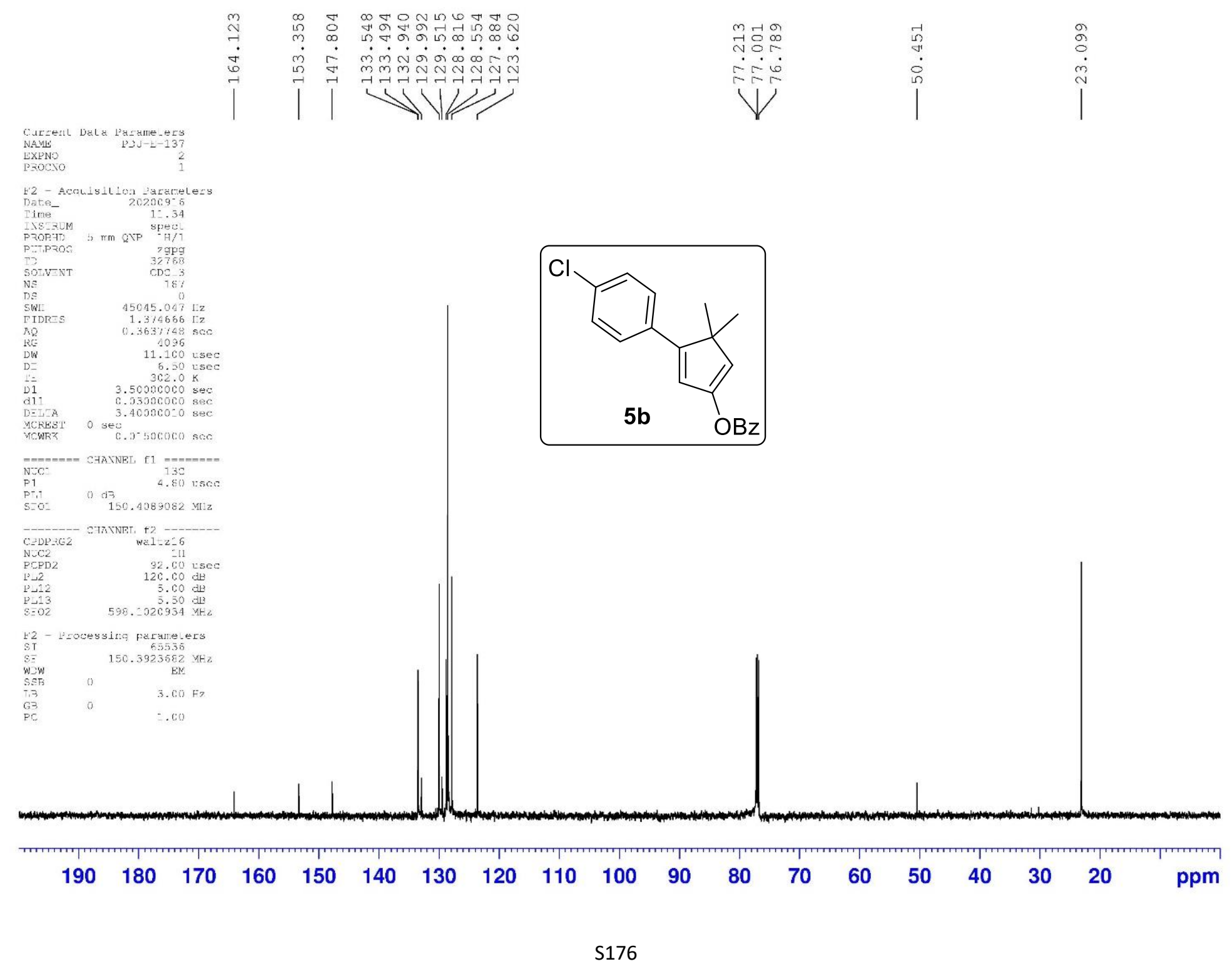




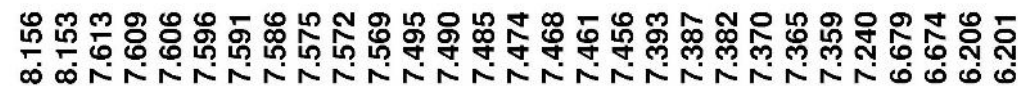

Current Data Parameters

NAME
EXPNO
PROCNO

F2-Acquisition Parameter

Time- 2020017

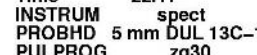

TD
SOLVENT

$\begin{array}{lll}\mathrm{NS} & 10 \\ \mathrm{NS} & 0 \\ \mathrm{DS} & 040.256 \mathrm{~Hz} \\ \mathrm{SWH} & 641.256 \mathrm{H}\end{array}$

$\begin{array}{lll}\text { FIDRES } & \begin{array}{l}0.19562525 \mathrm{~Hz} \\ \text { AQ }\end{array} \\ 2.5559540 \mathrm{sec}\end{array}$

$\begin{array}{ll}\mathrm{AG} & 2.559 .250 \mathrm{sec} \\ \mathrm{RW} & 78.000 \mathrm{usec} \\ \mathrm{DW} & 6.000 \mathrm{sec}\end{array}$

$\begin{array}{ccc}6.00 \mathrm{usec} \\ \mathrm{TE} & 30.0 \mathrm{~K} \\ \mathrm{TE} & 2.0000000 \mathrm{sec}\end{array}$

D1 2.00000000

NUC1 $==$ CHANNEL $\mathrm{f1}======$

$\begin{array}{ll}\text { P1 } & 10.00 \text { usec } \\ \text { PL1 } & -2.40 \mathrm{~dB} \\ \text { SFO1 } & 400.1528010 \mathrm{MHz}\end{array}$

F2 - Processing parameters

$\begin{array}{ll}\text { SI } & 16384 \\ \text { WDW } & 400.1500169 \mathrm{MHz}\end{array}$

$\begin{array}{lll}\text { WDW } & & \\ \text { SSB } & 0 \\ \text { LB } & 0 \\ \text { GB } & 0 & \text { Hz }\end{array}$

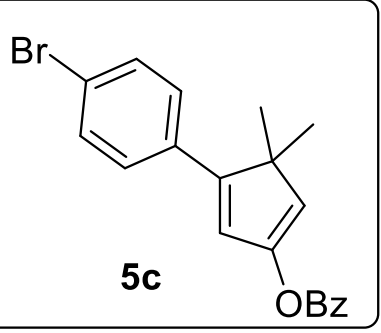

$\mathrm{PC} \quad 1.00$

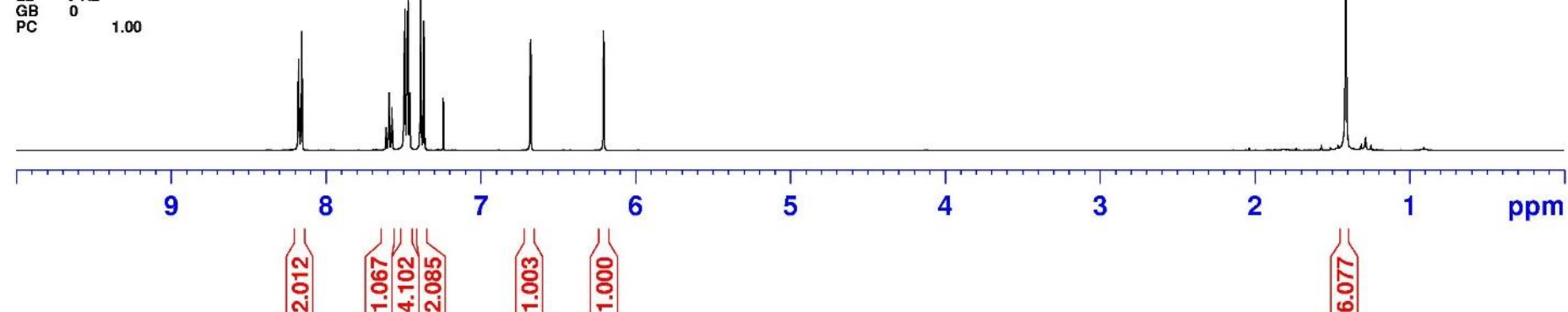




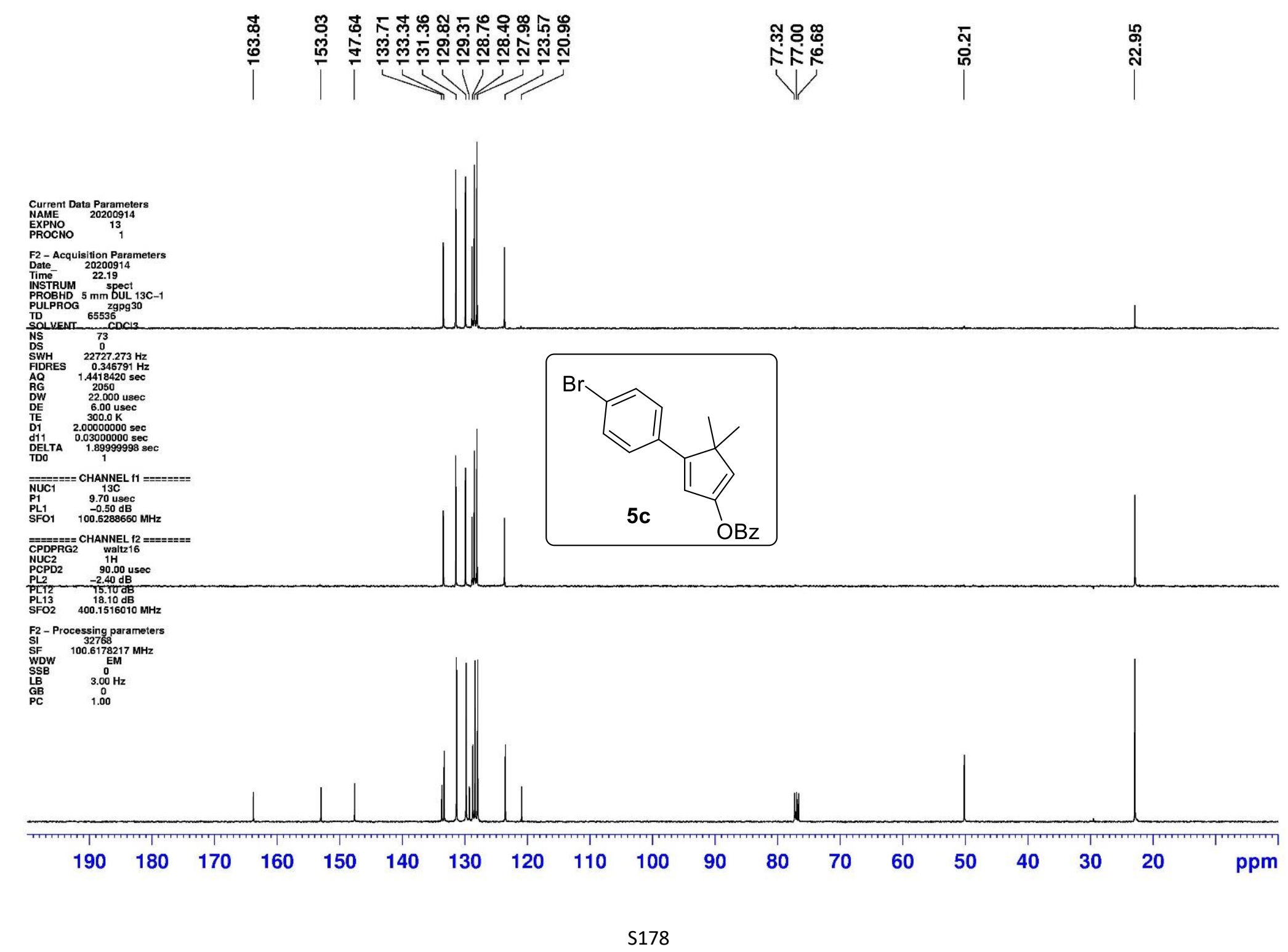




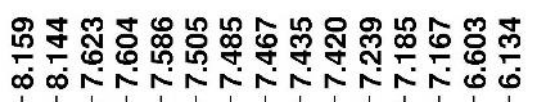

|
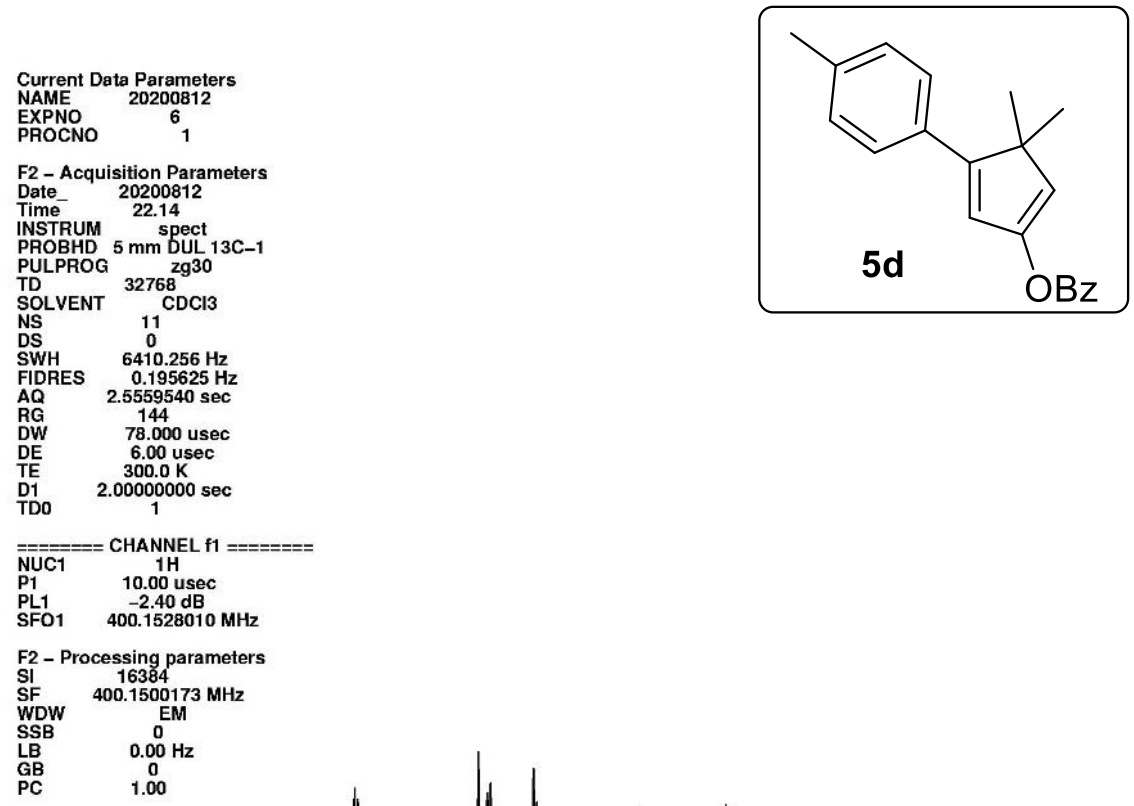

PULPROG

$\begin{array}{ll}\text { TD } & 32768 \\ \text { SOLVENT } & \mathrm{CDCl} 3\end{array}$

OBz

$$
\begin{aligned}
& \text { FIDRES } \quad \begin{array}{l}
0.1956256 \mathrm{~Hz} \\
\text { Hz }
\end{array} \\
& \begin{array}{ll}
\mathrm{RG} & 1.54 \\
\mathrm{DW} & 78.000 \mathrm{usec}
\end{array} \\
& =\text { CHANNEL } f 1====
\end{aligned}
$$

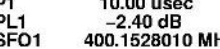

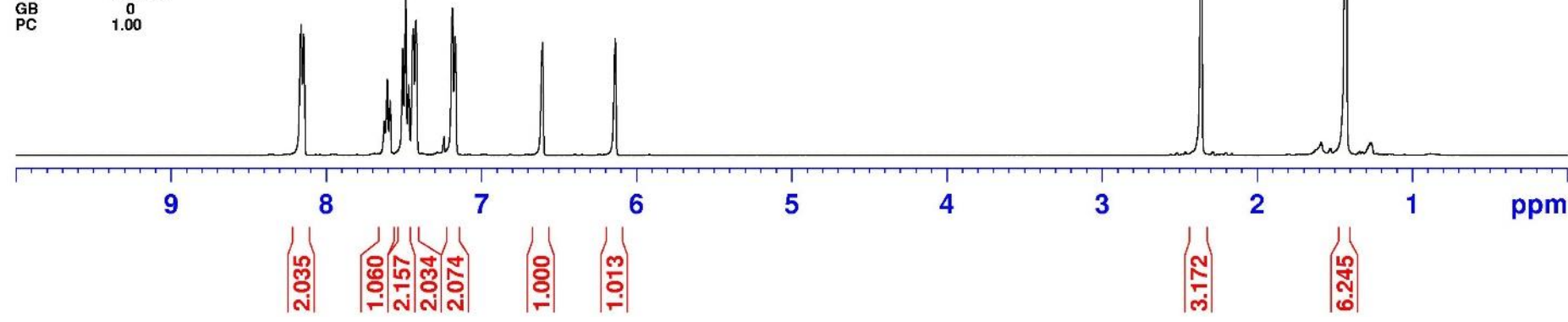



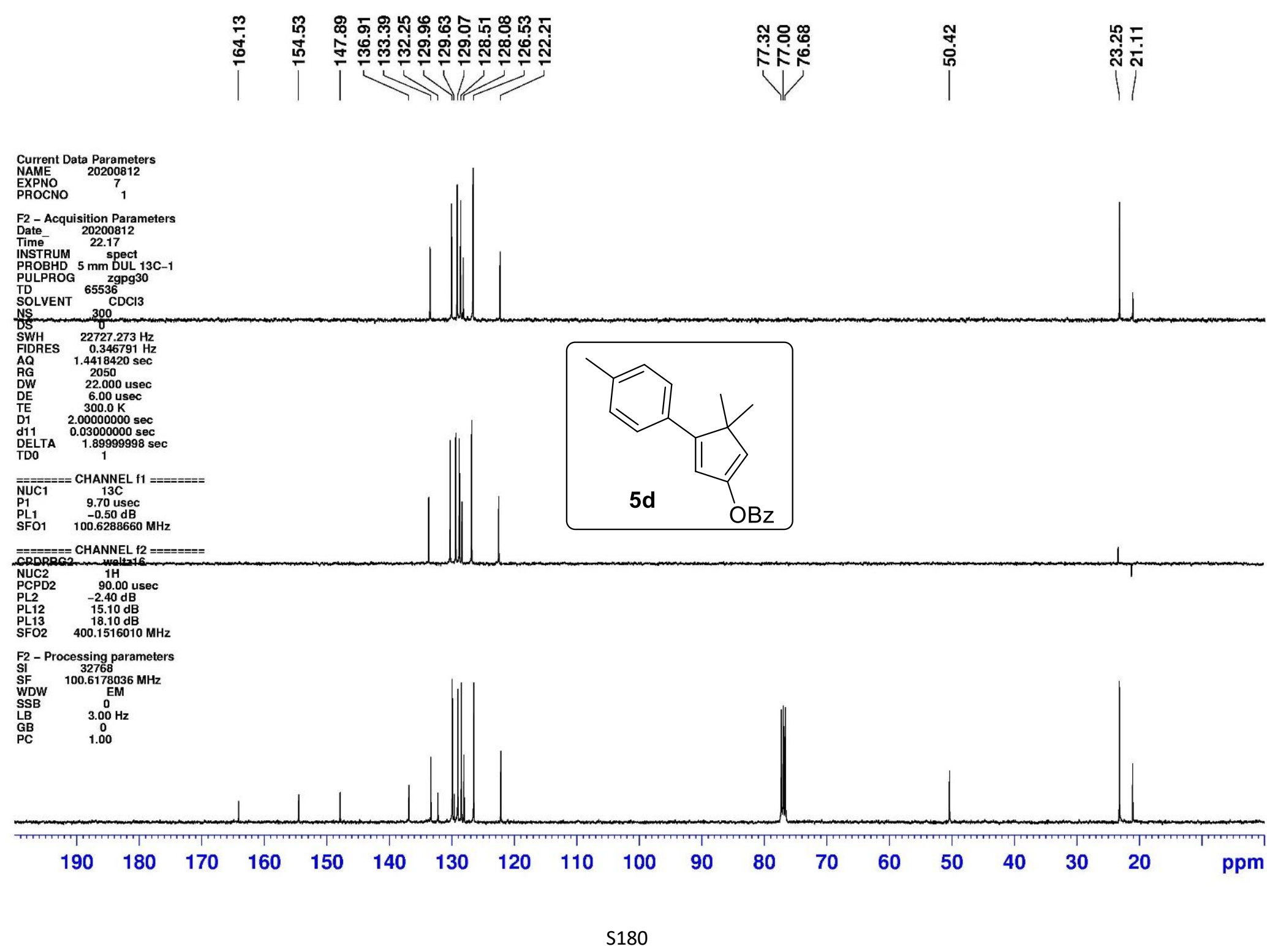


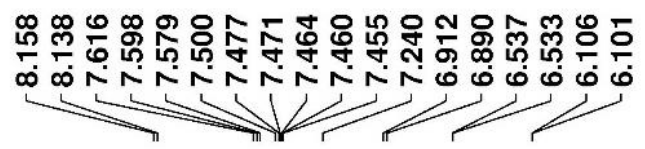

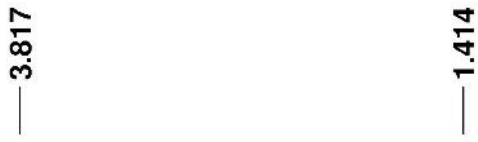

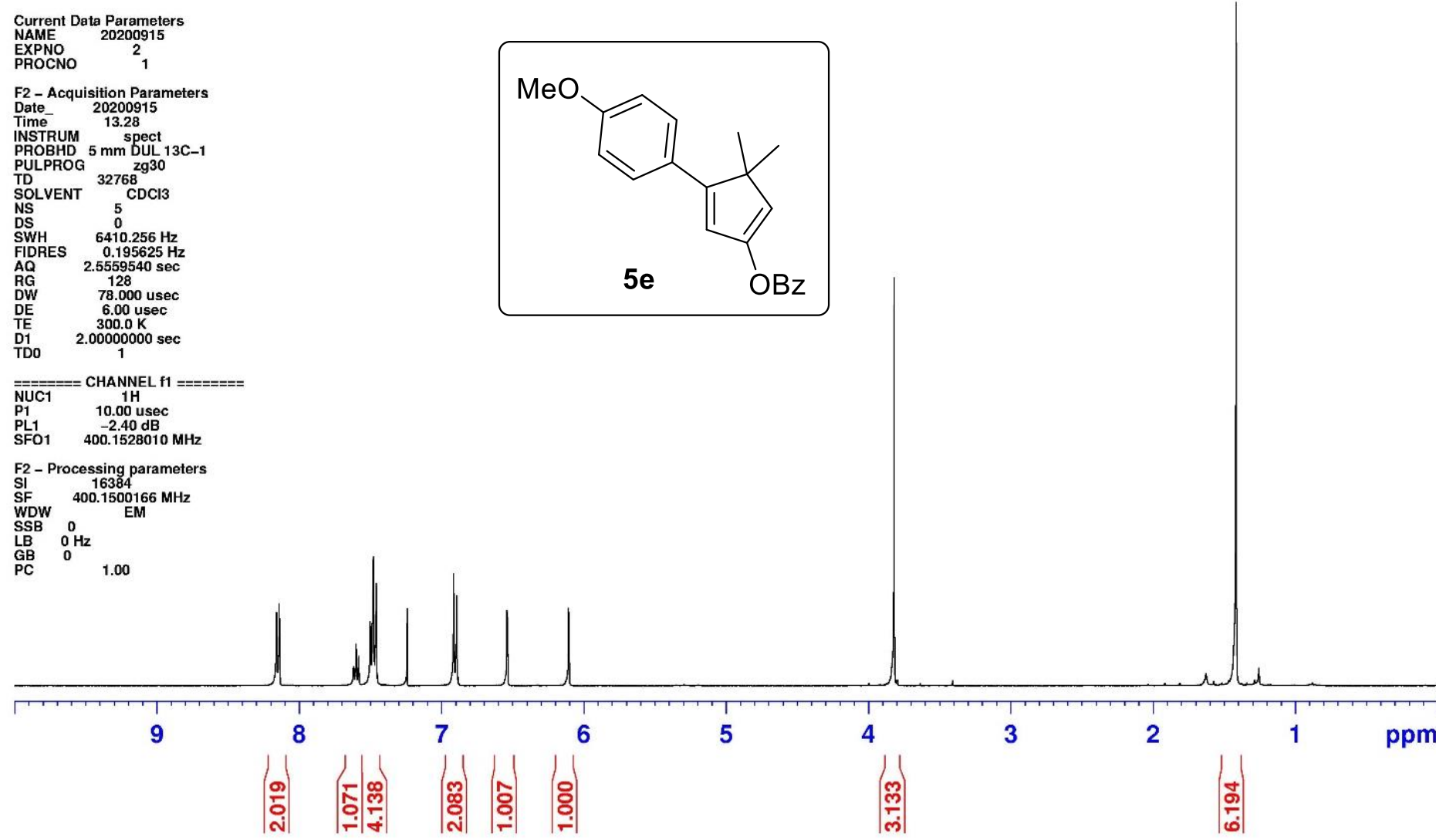




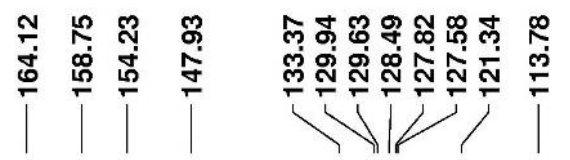

స్ల్

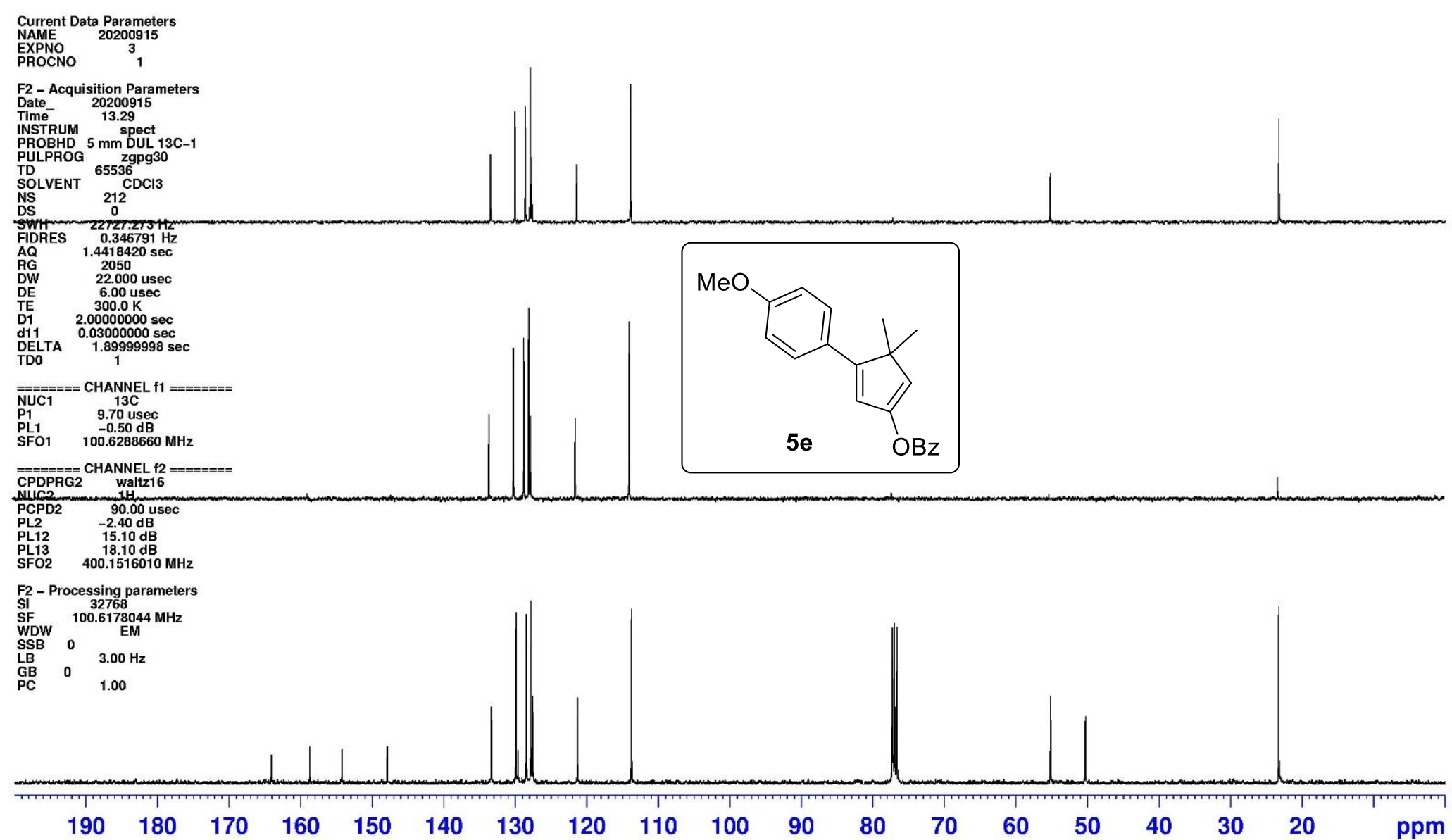




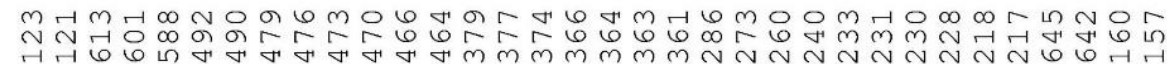

ㄱ.

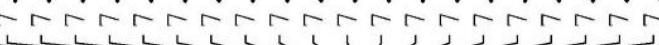

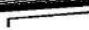
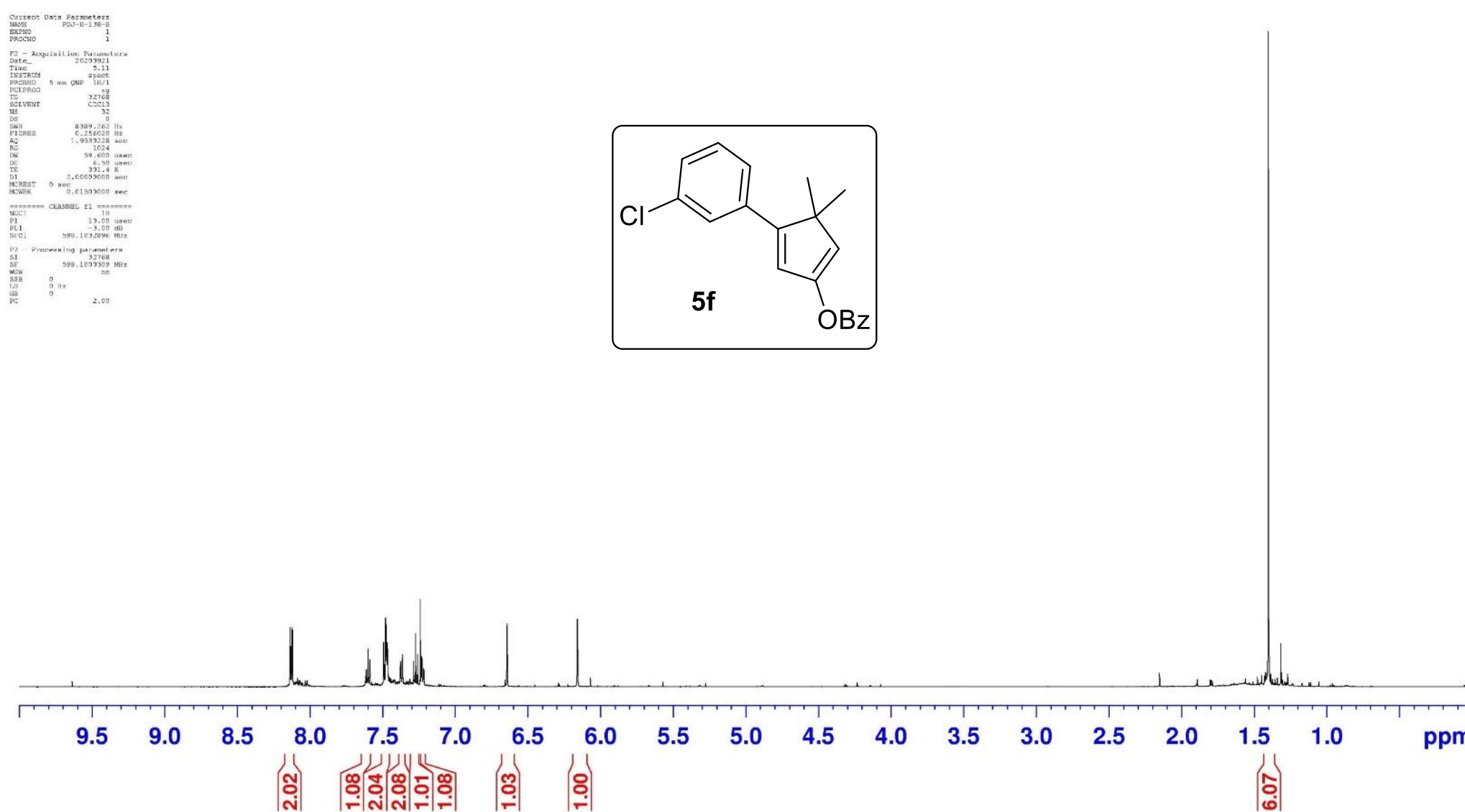


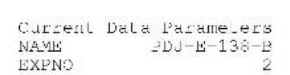
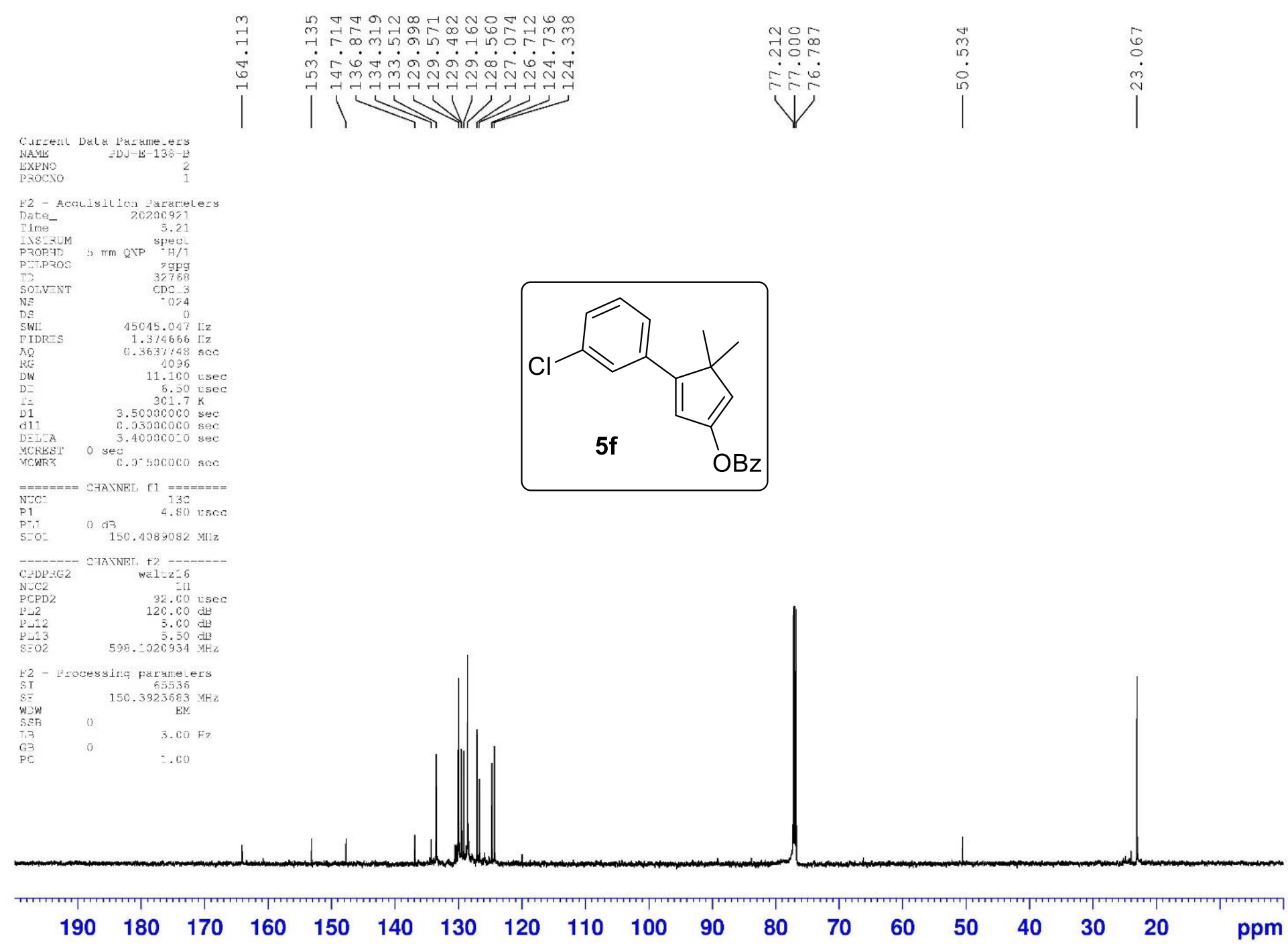


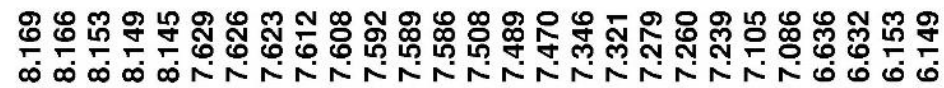
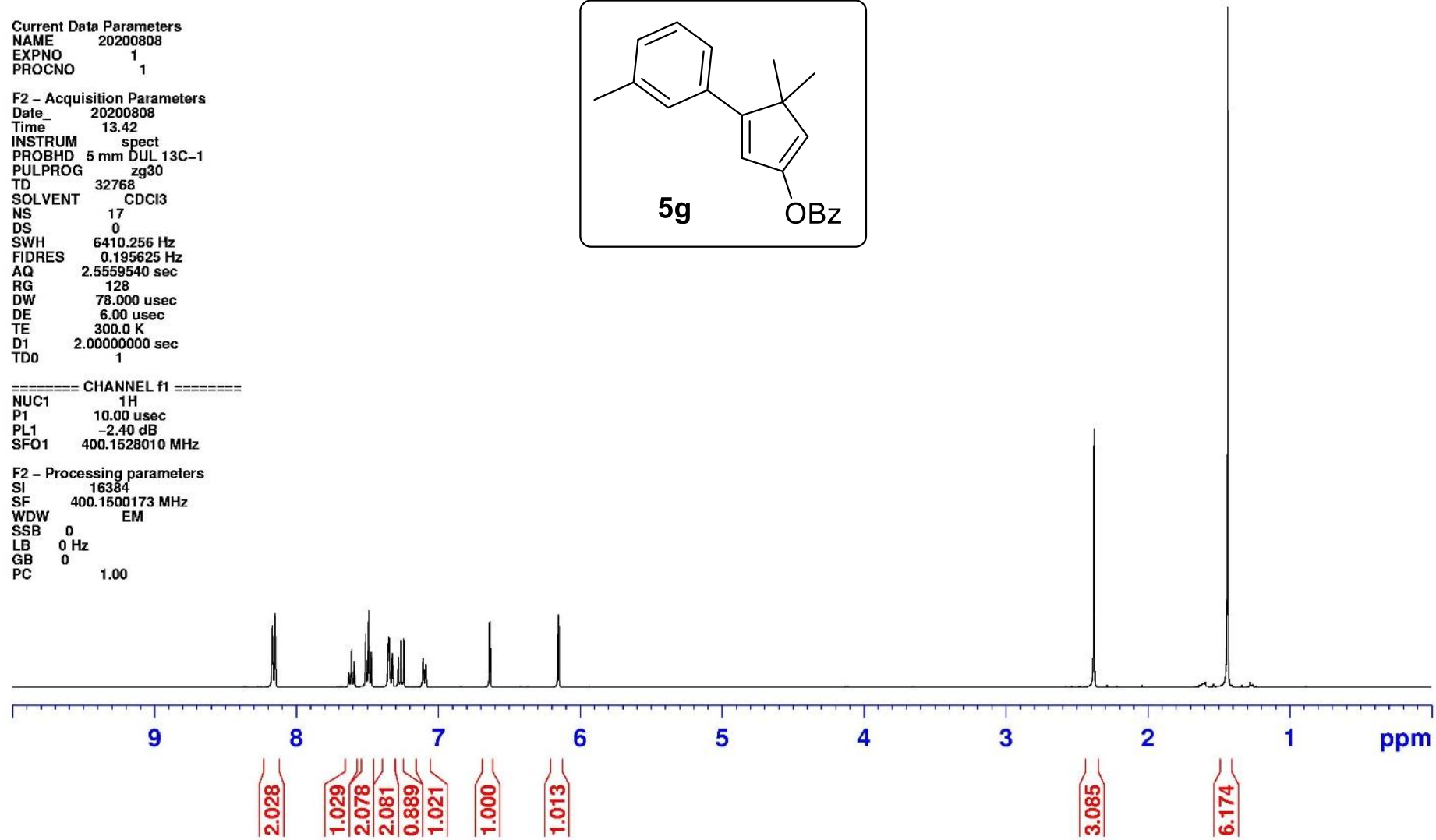

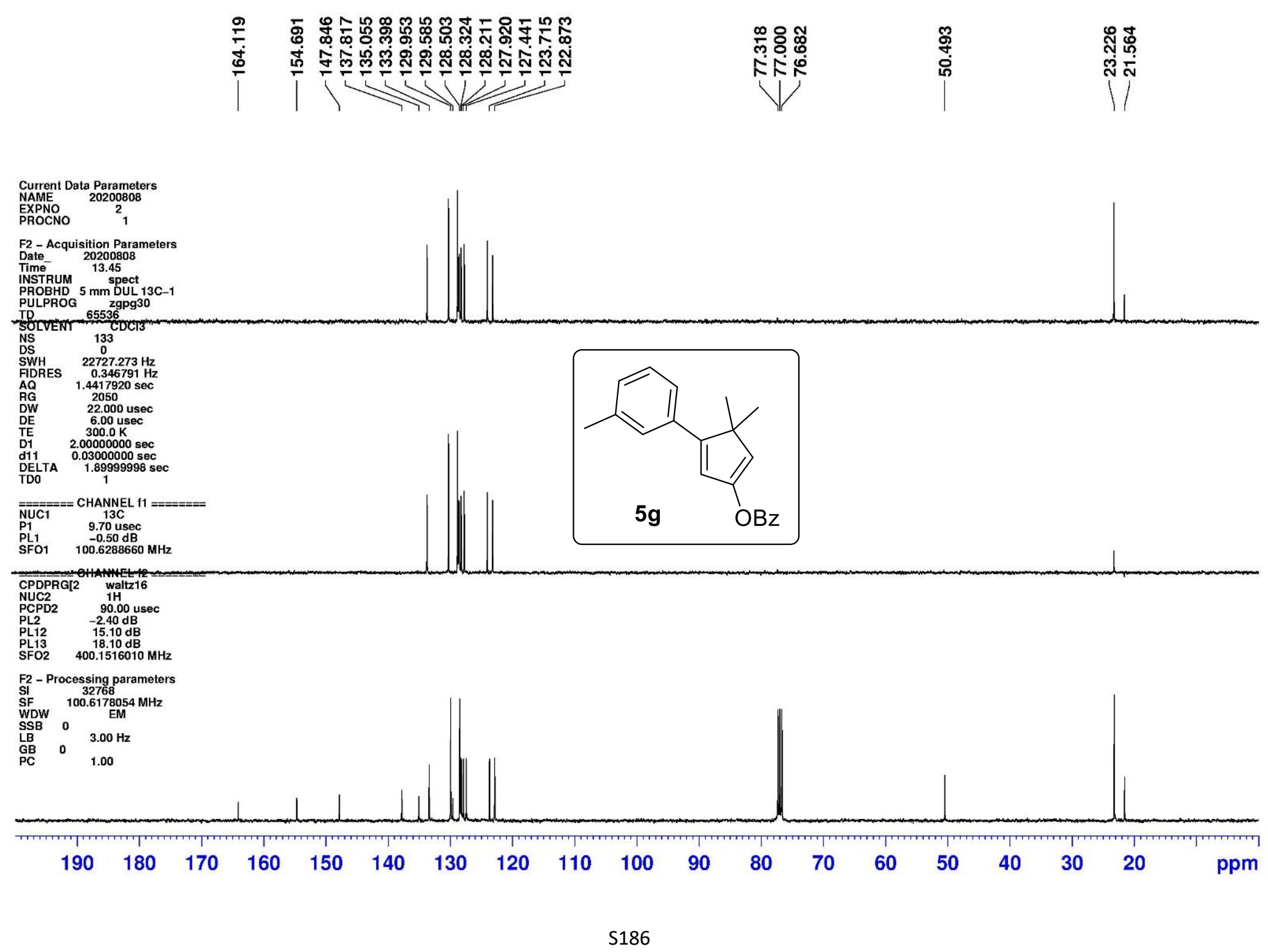
$\underbrace{\infty}$

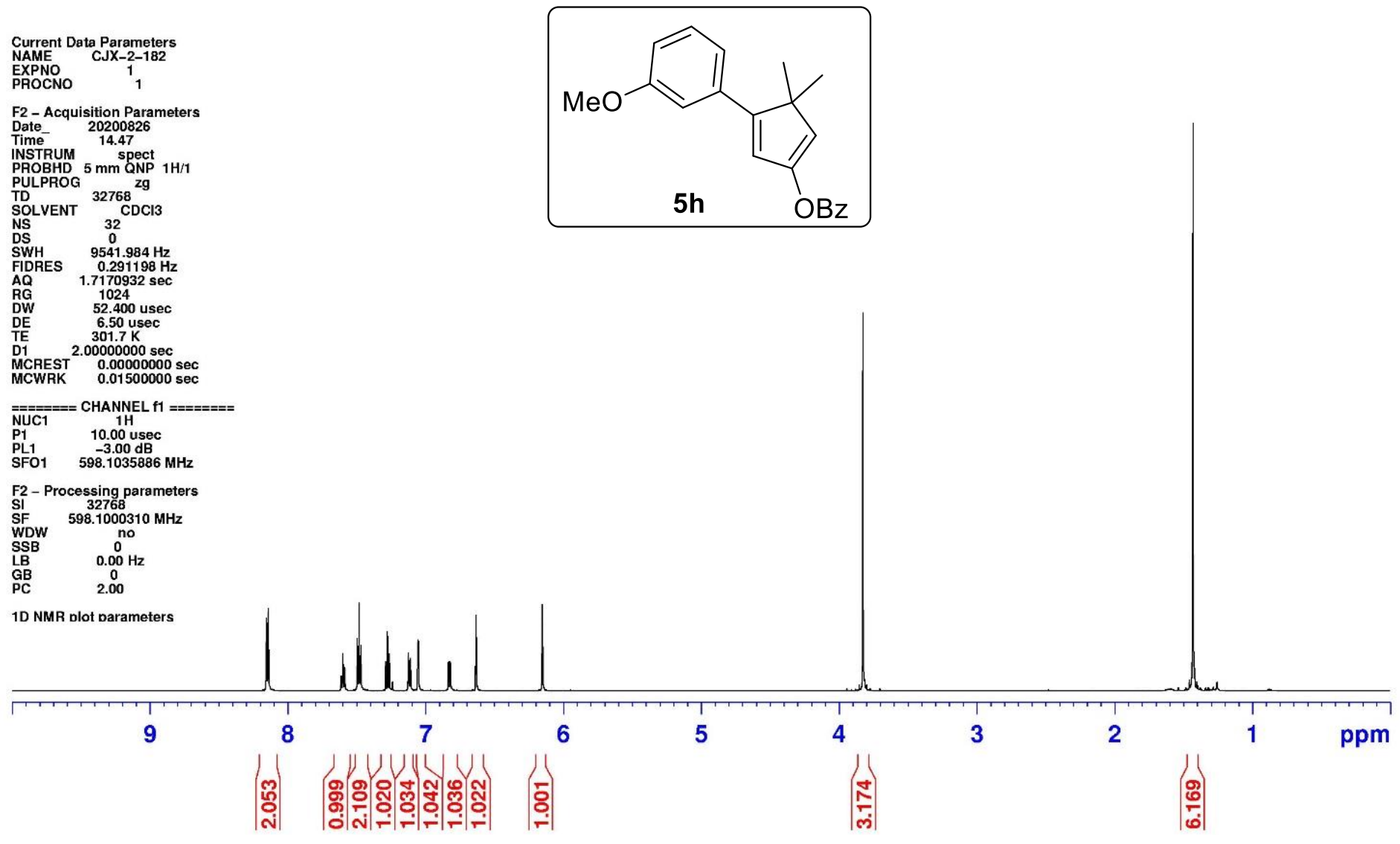



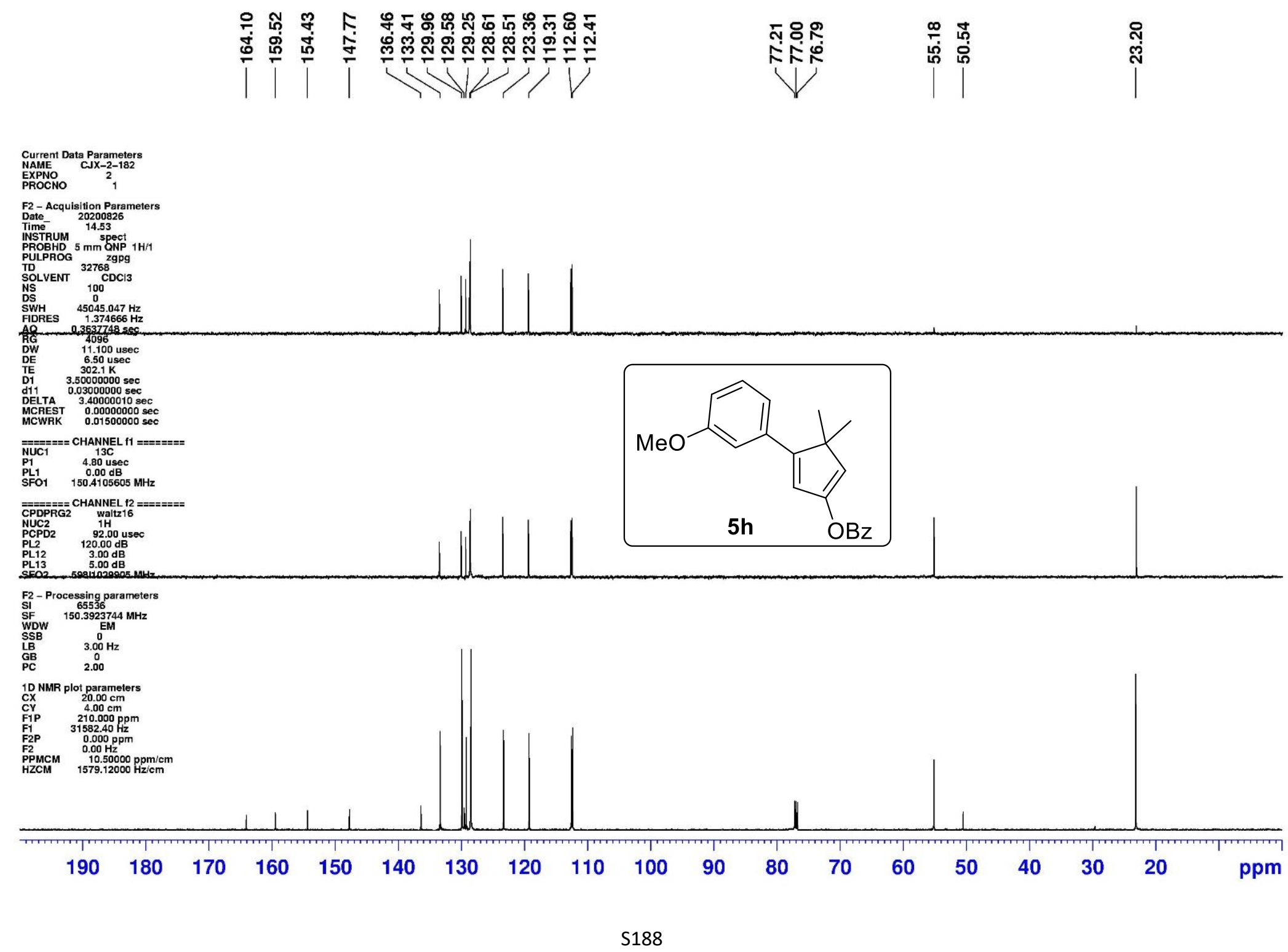

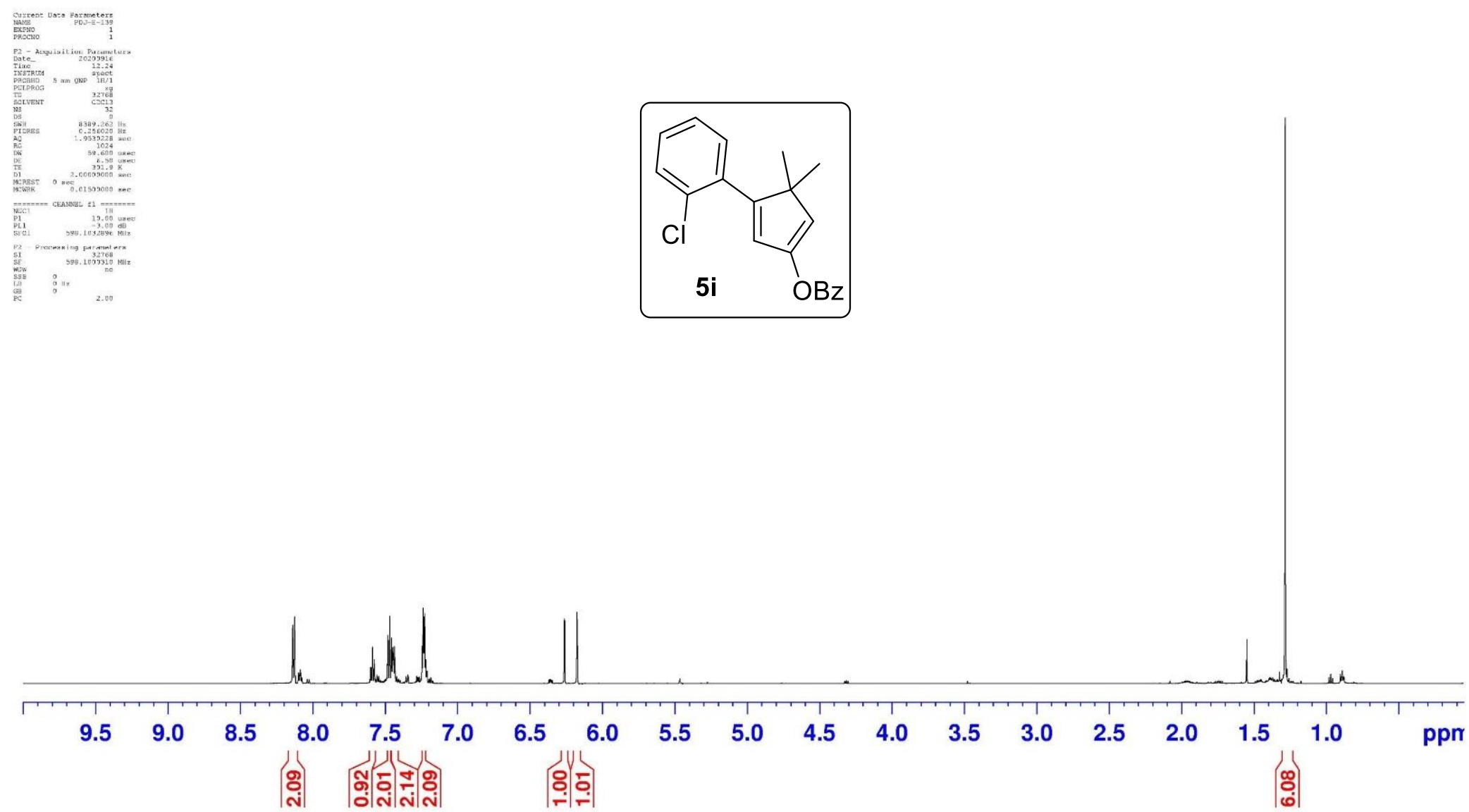


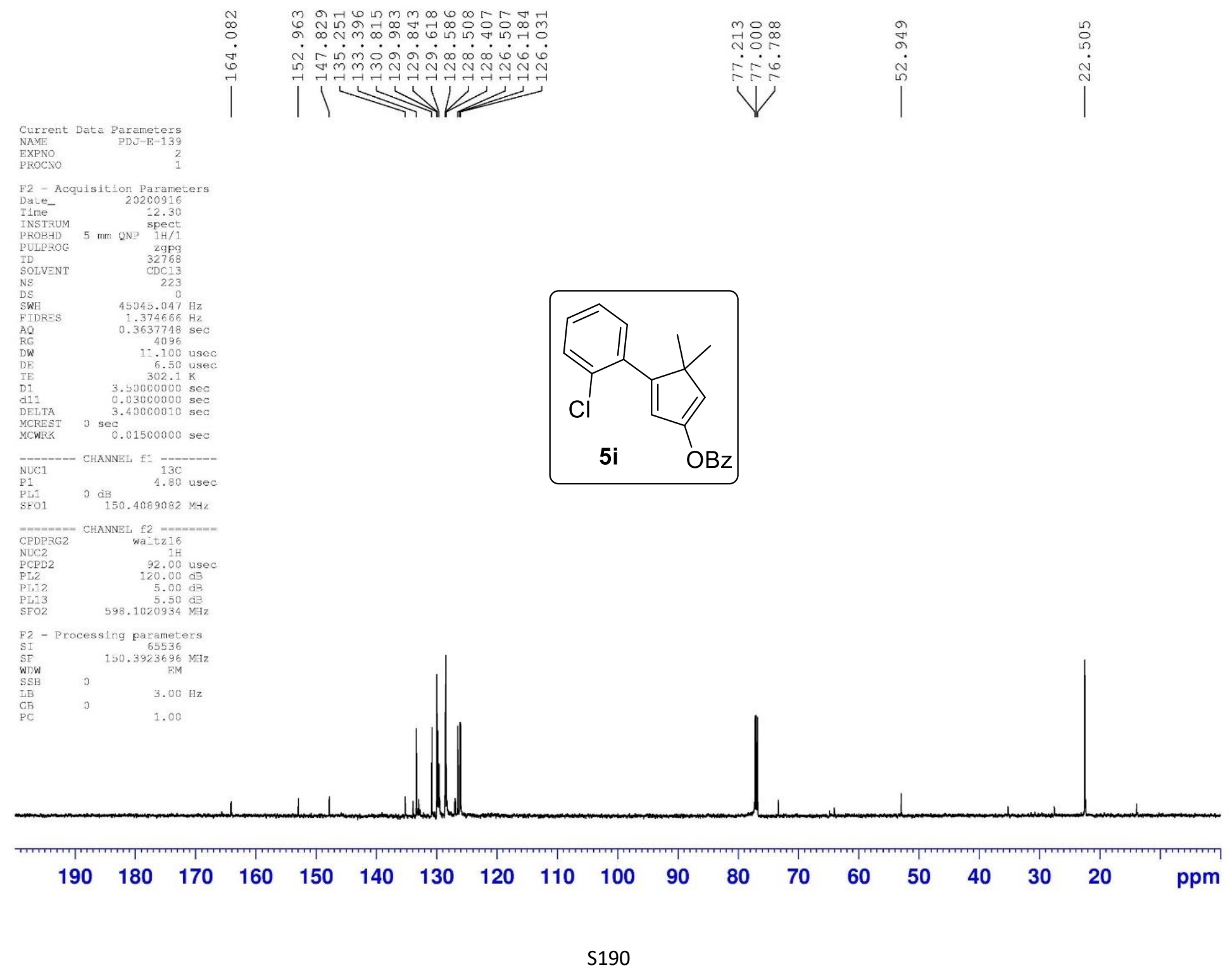




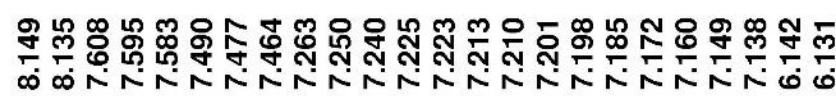

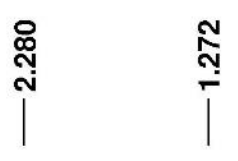
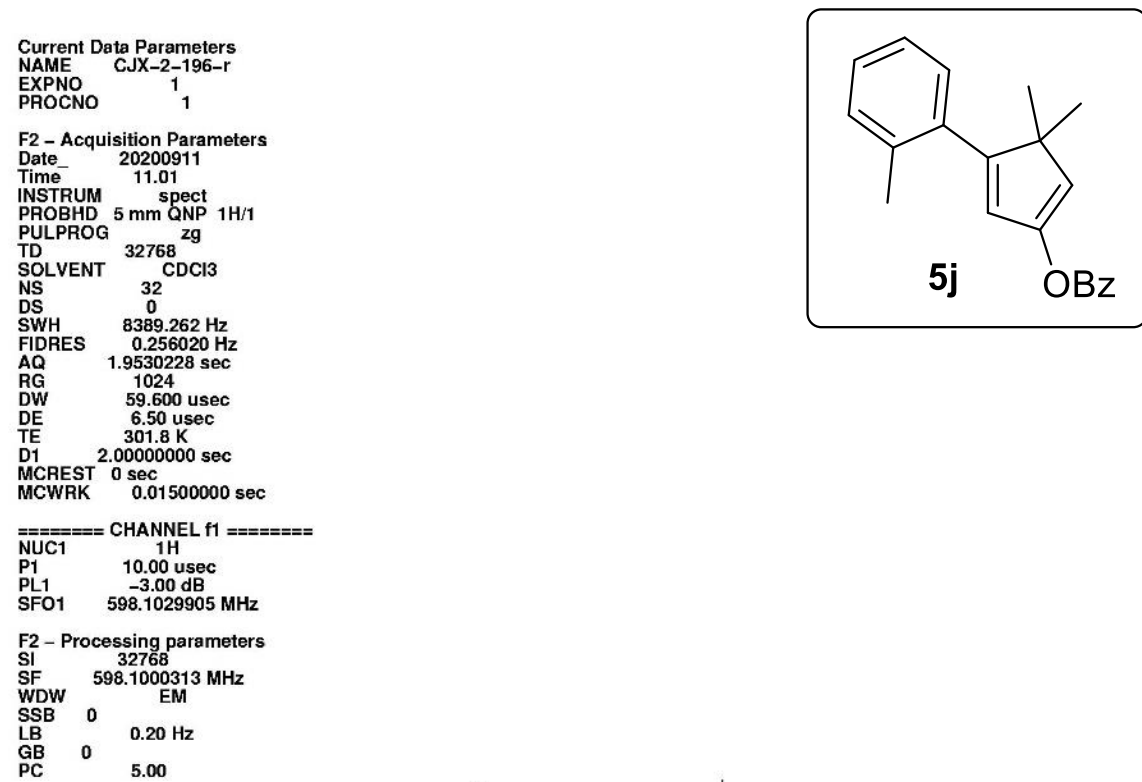

5.00

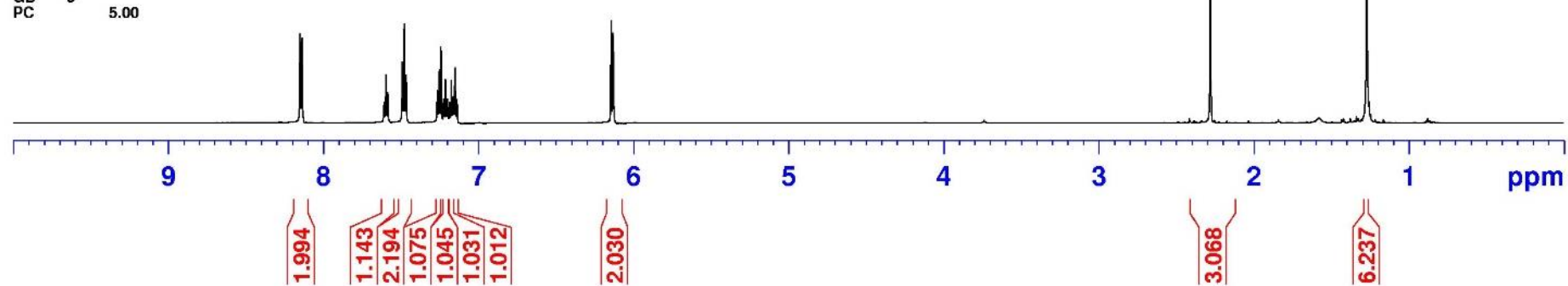



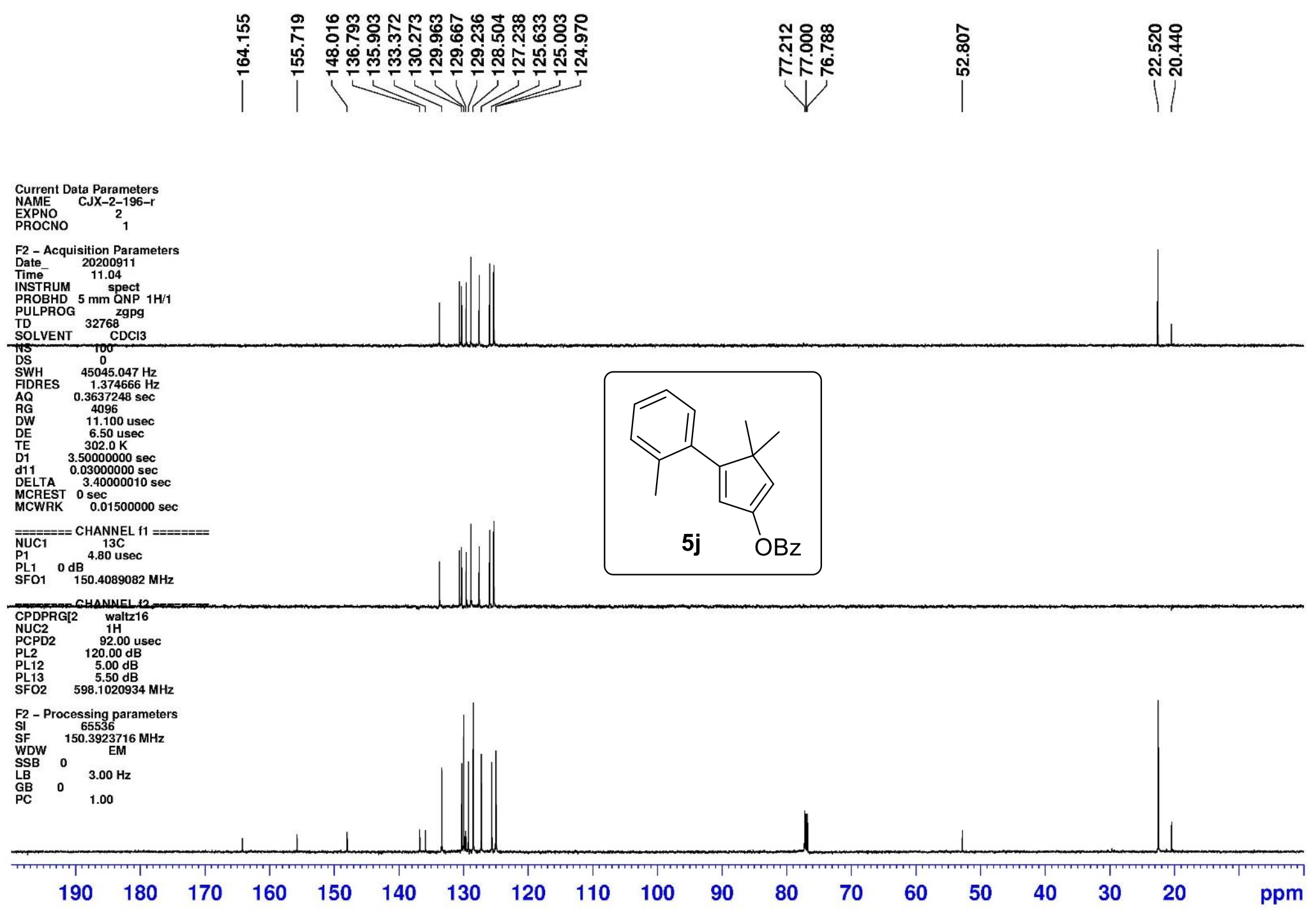


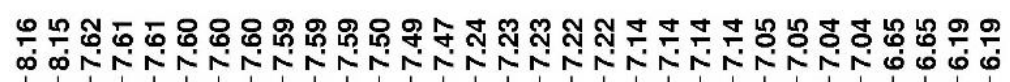

Current Data Parameters
NAME CJX-2-202

PXPNO

$$
\begin{aligned}
& \text { F2- Acquisition Parameters } \\
& \text { Daie- } \quad 2020.091
\end{aligned}
$$
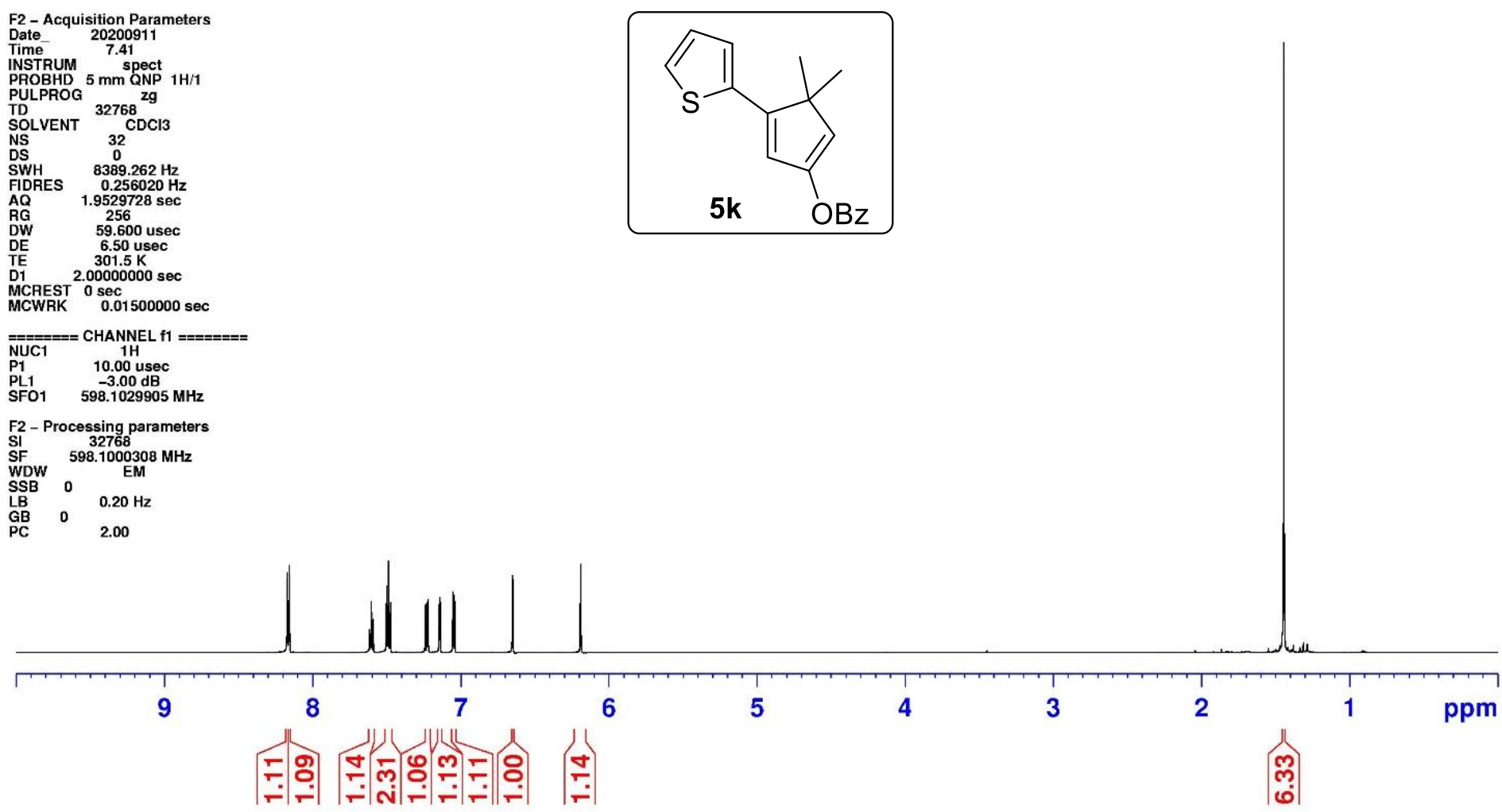

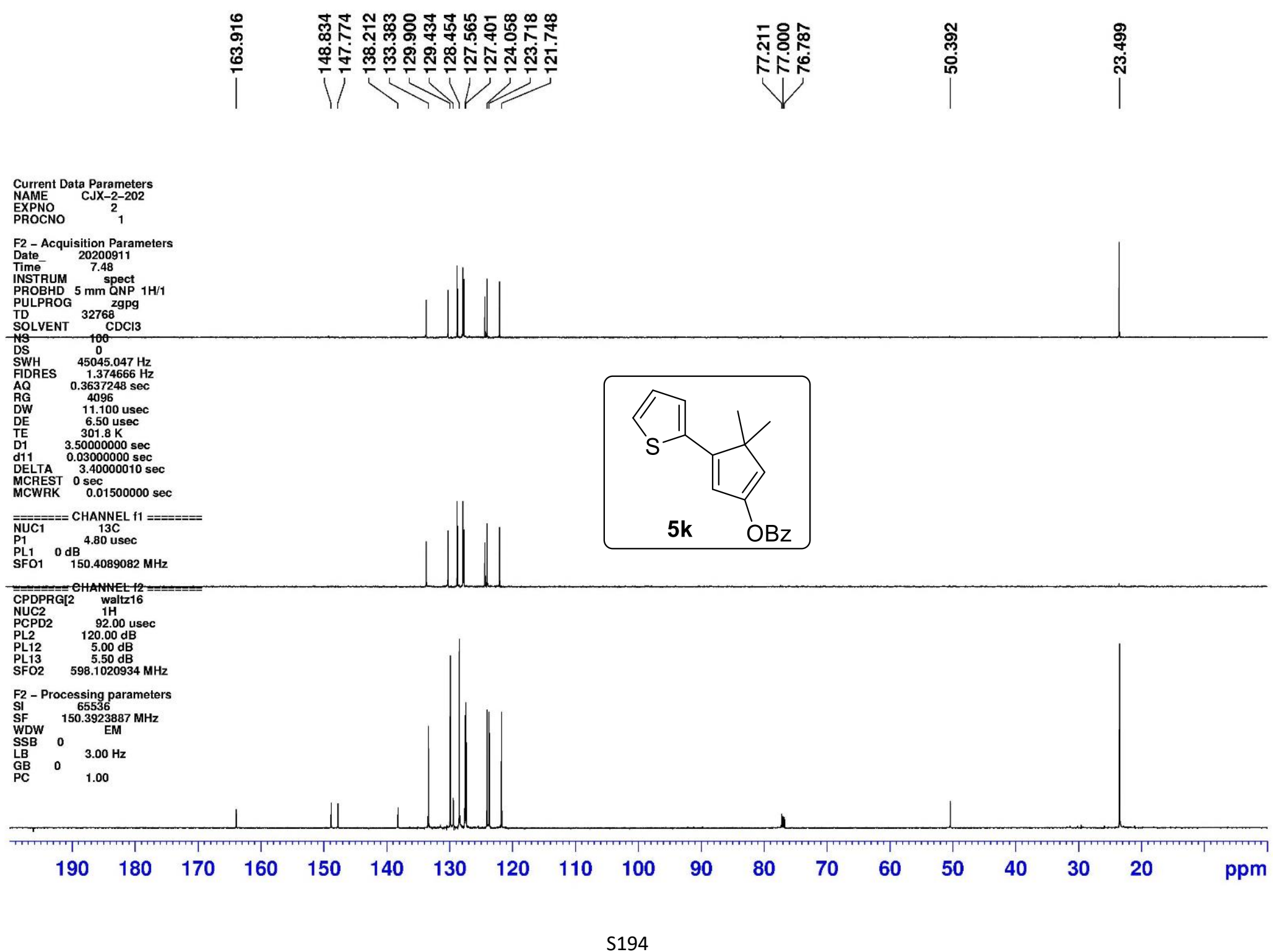


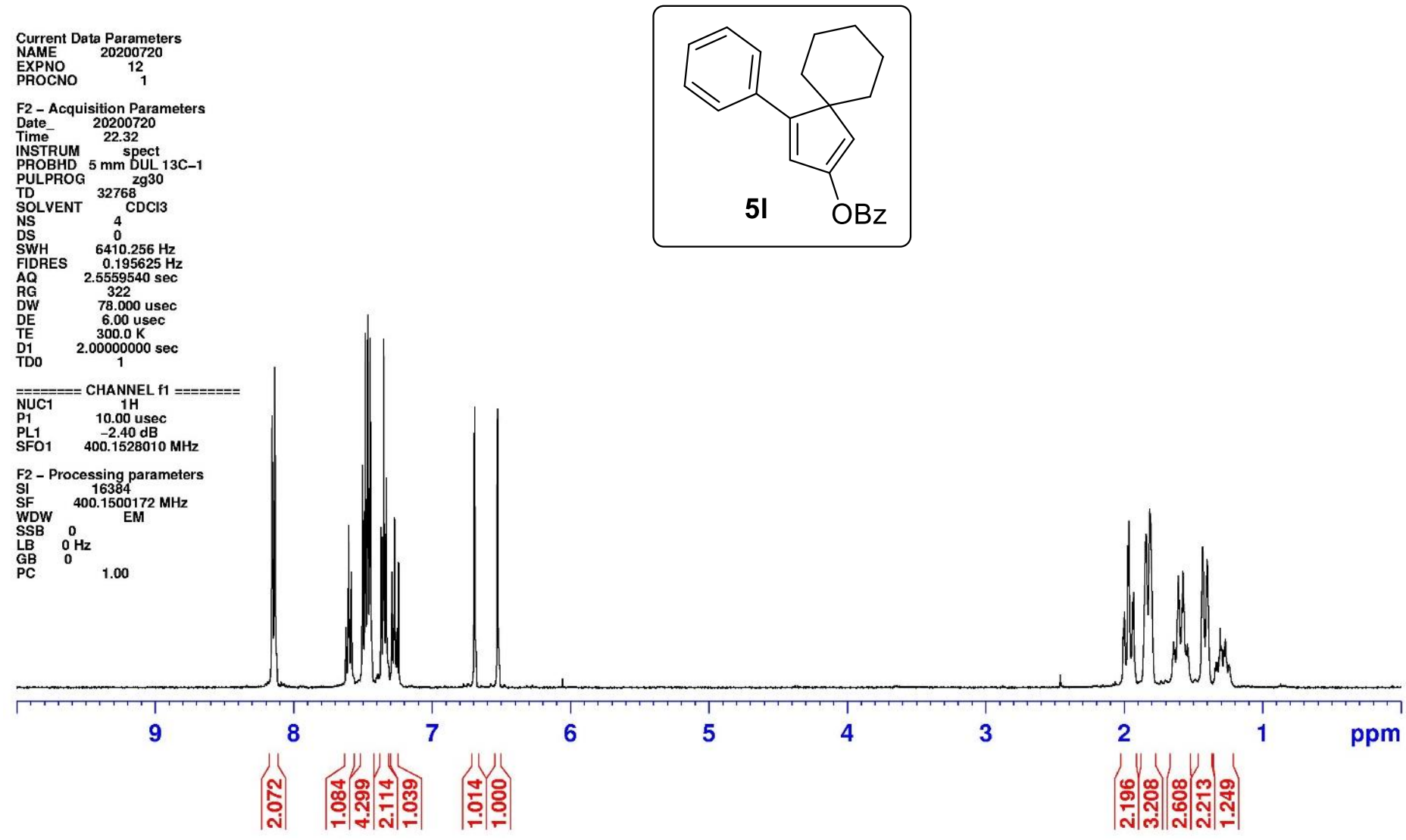



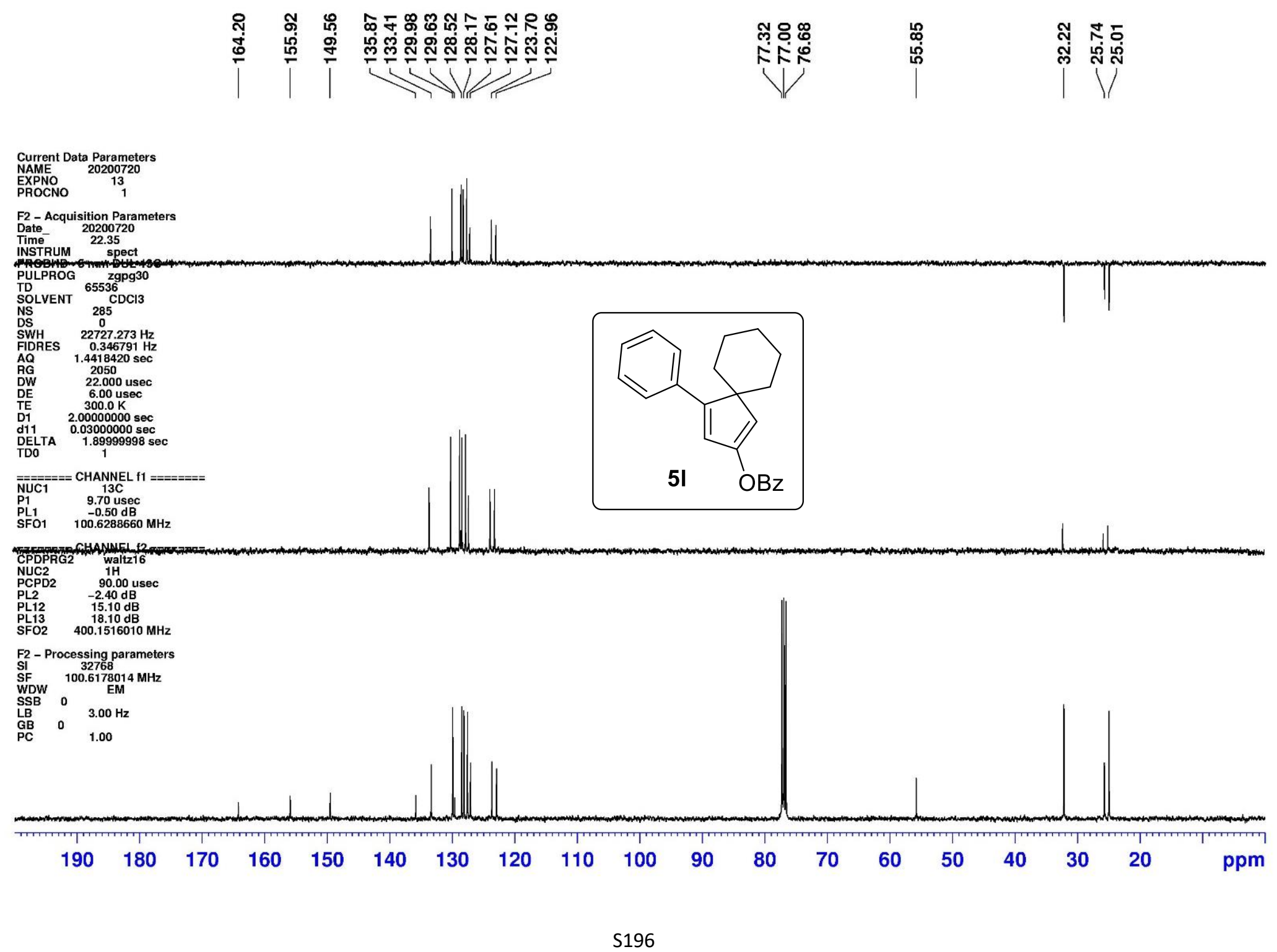


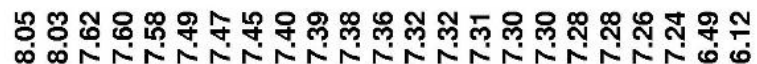

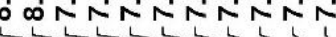
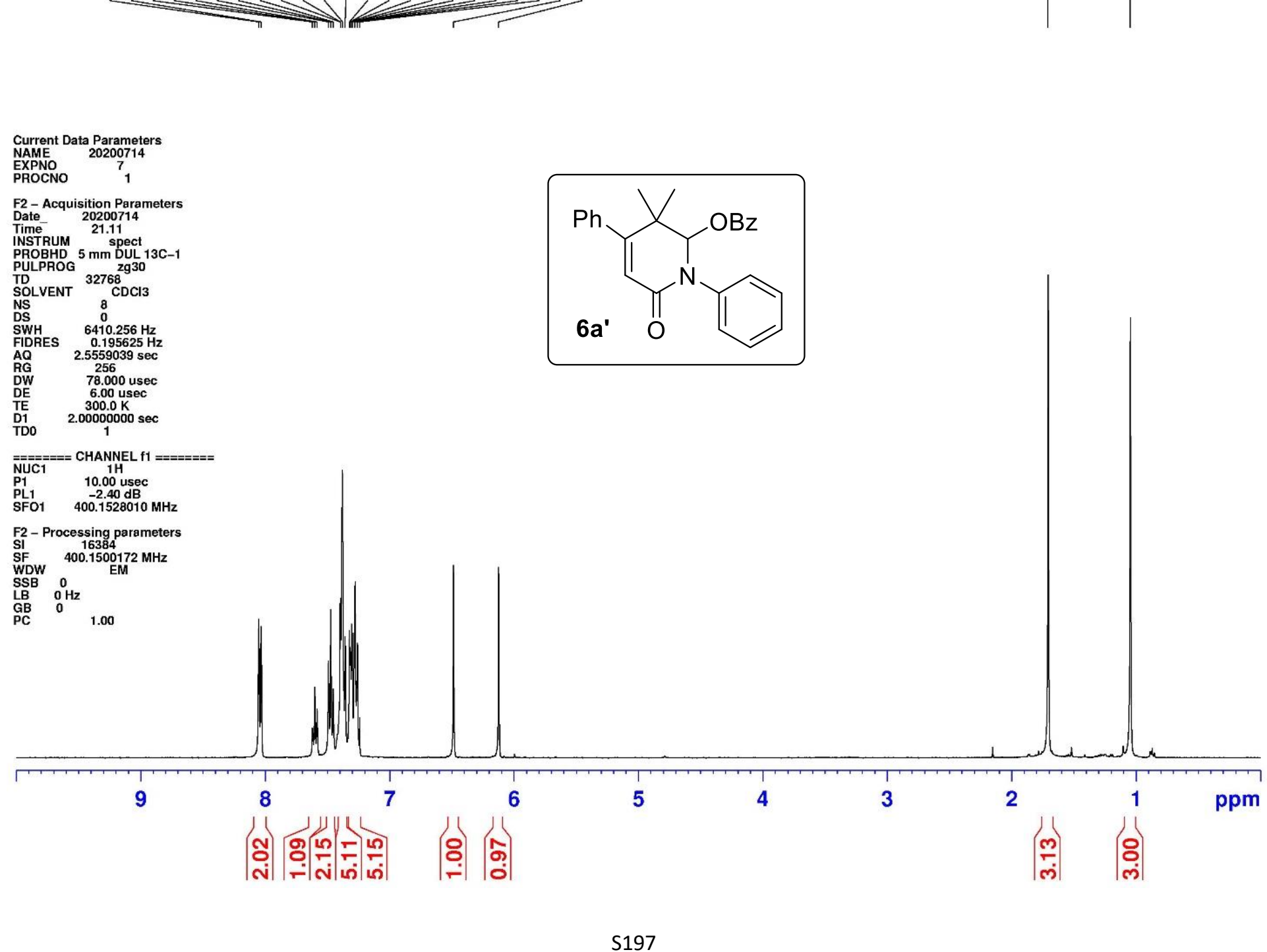


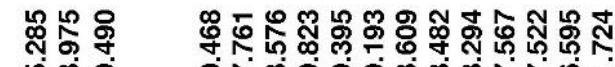

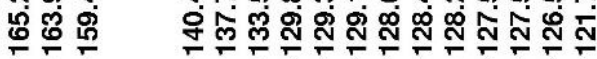

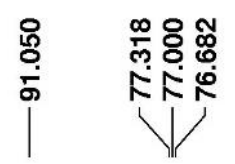 \\ స}

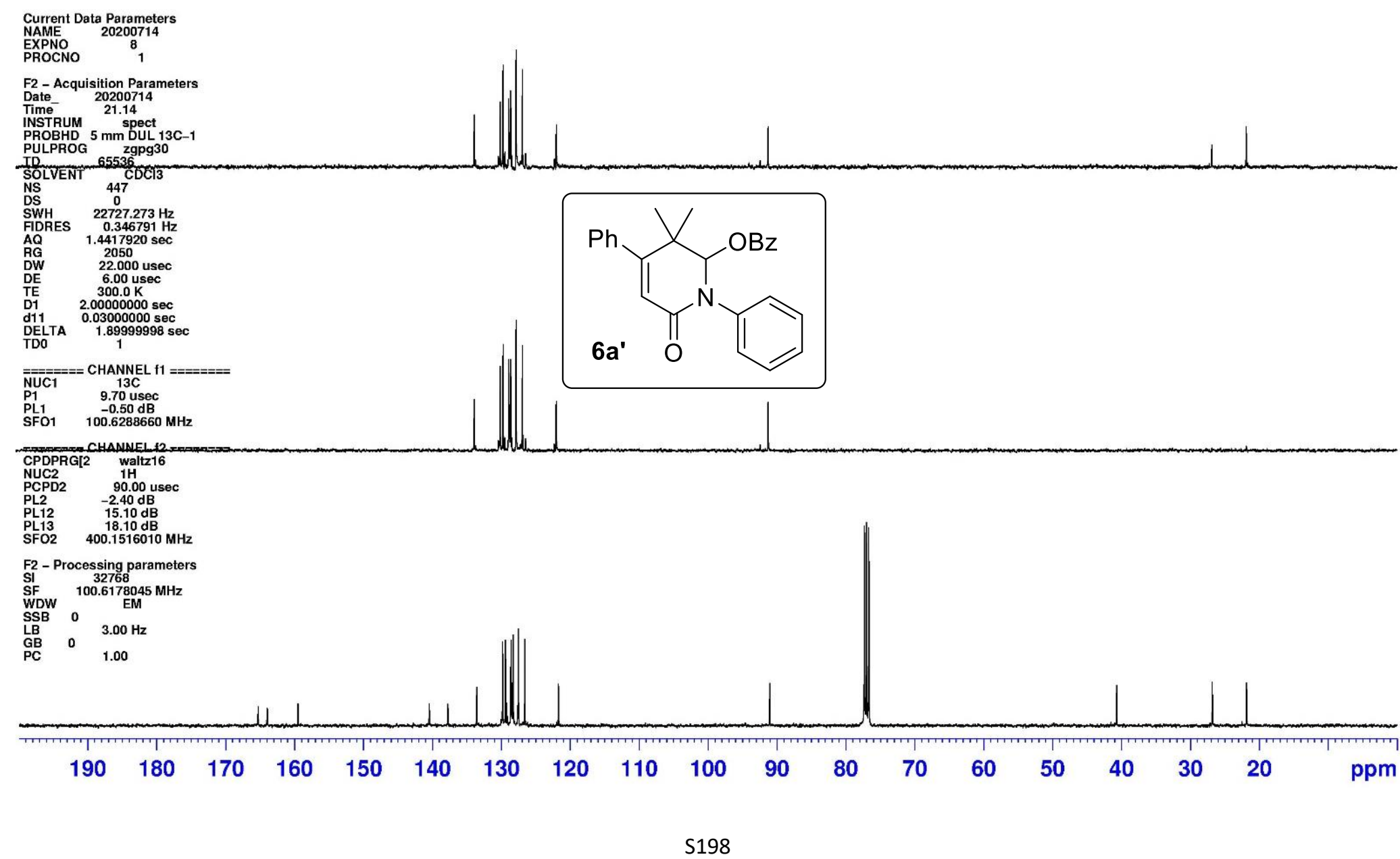




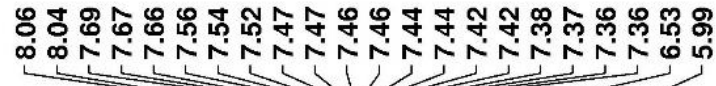

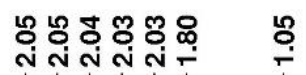

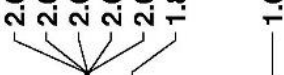

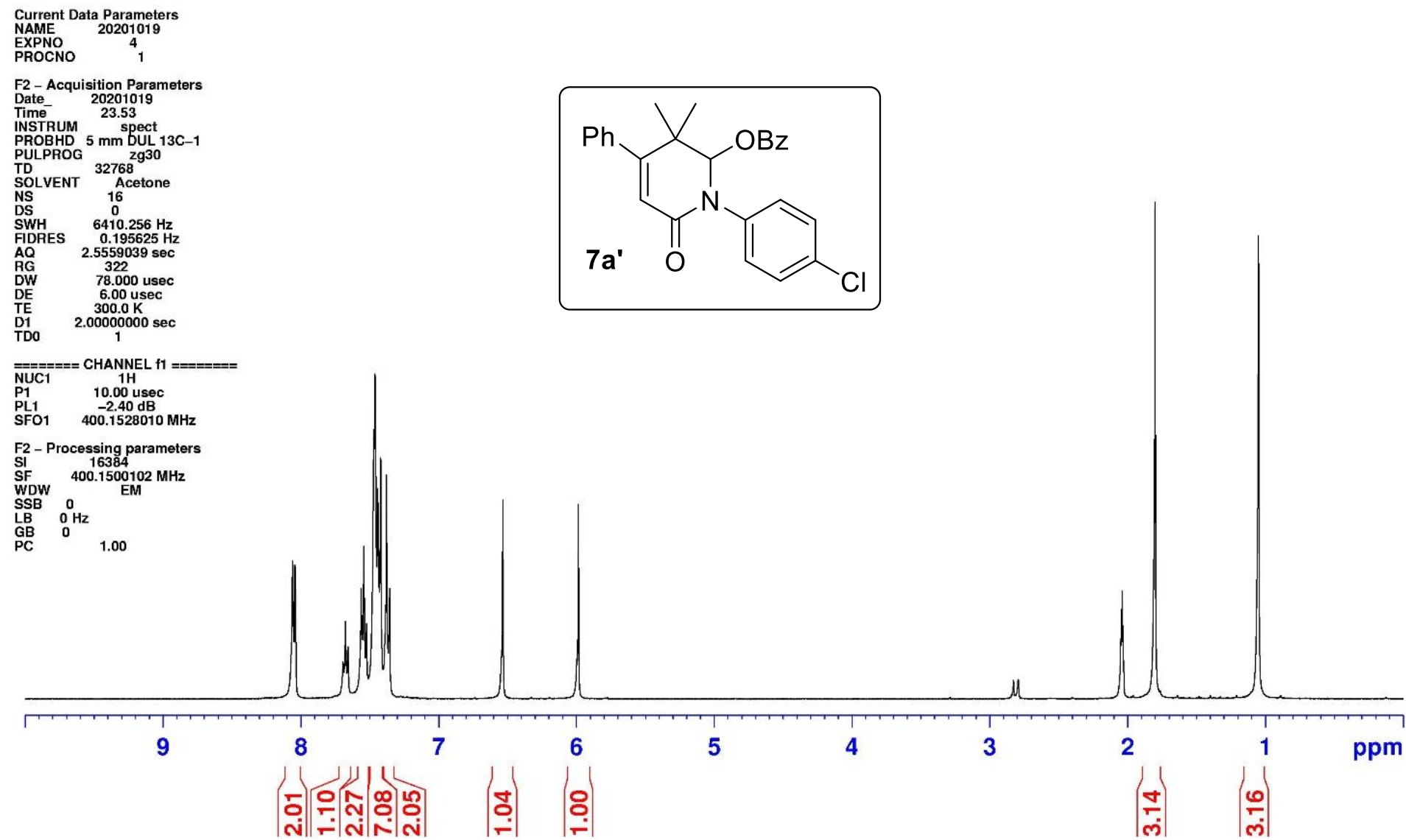




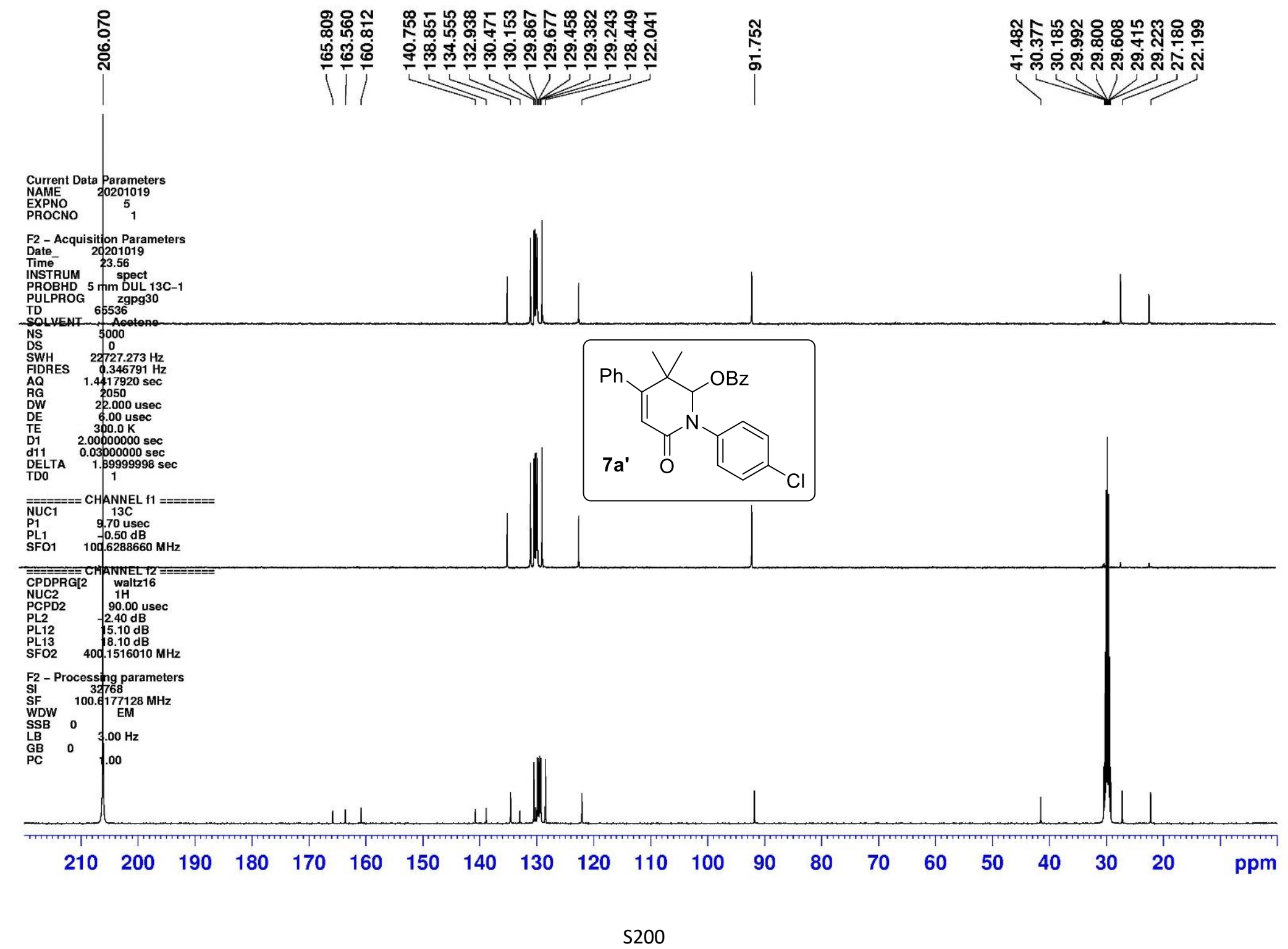



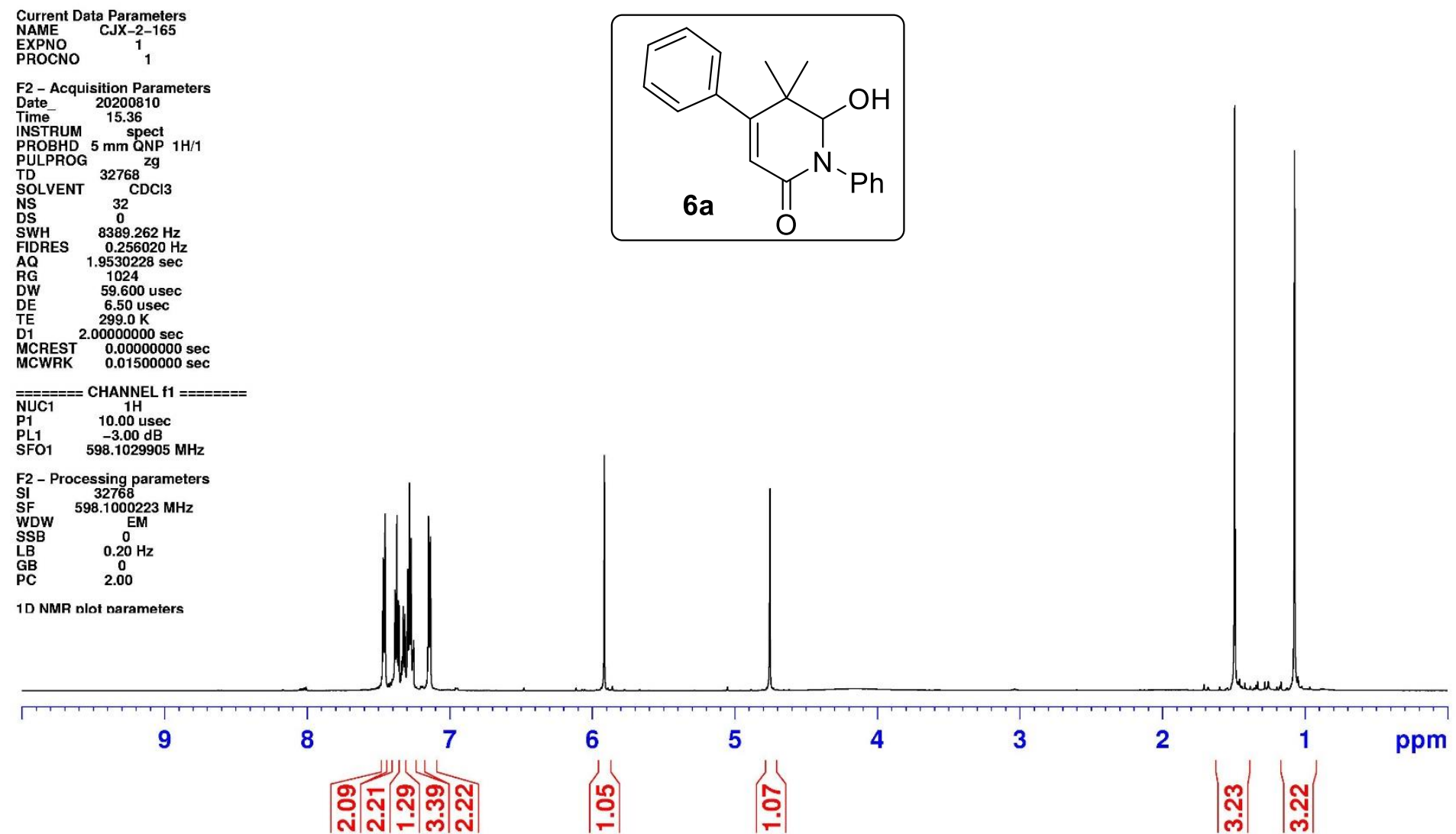


|

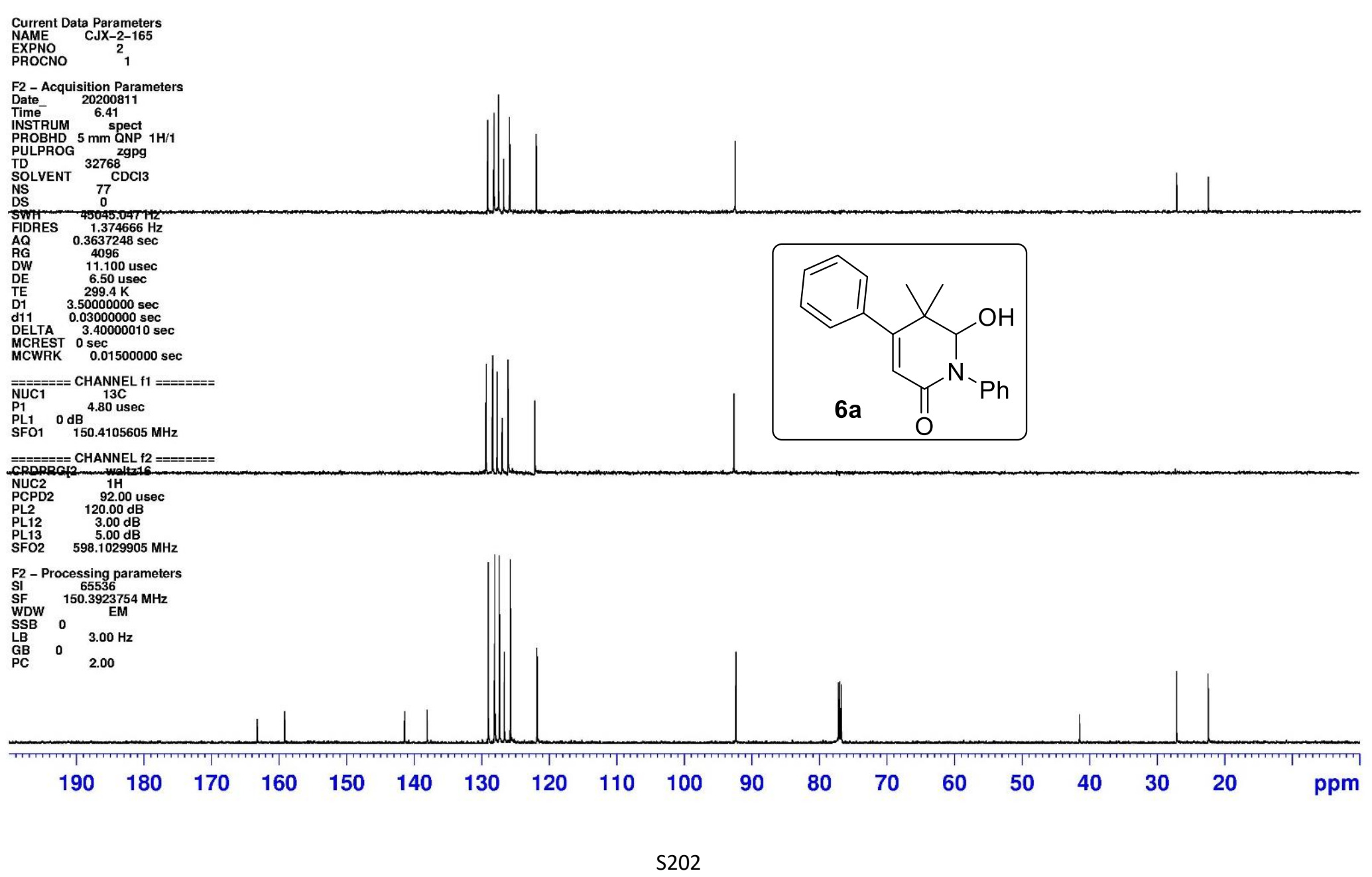



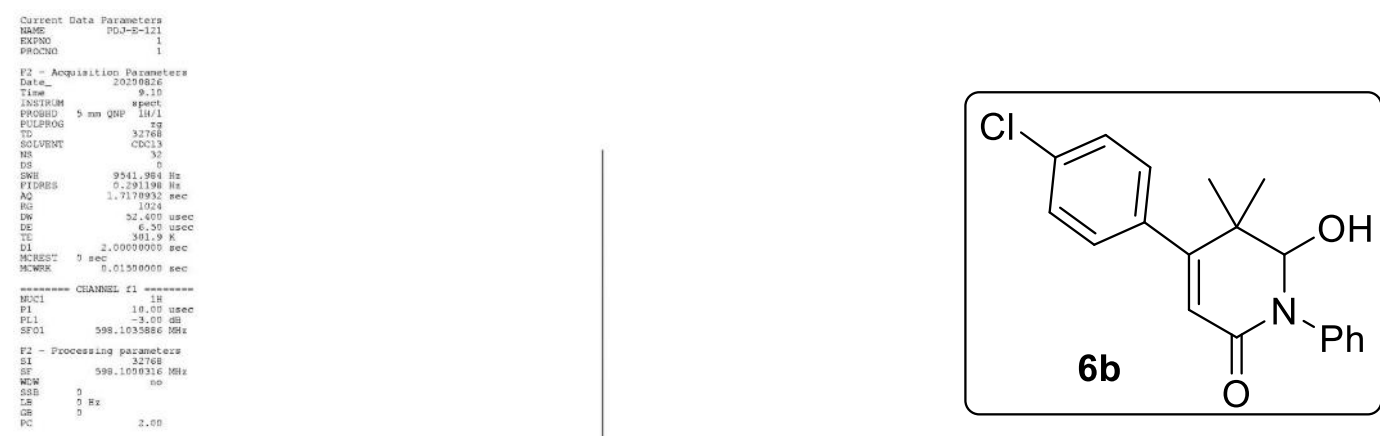

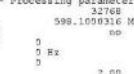

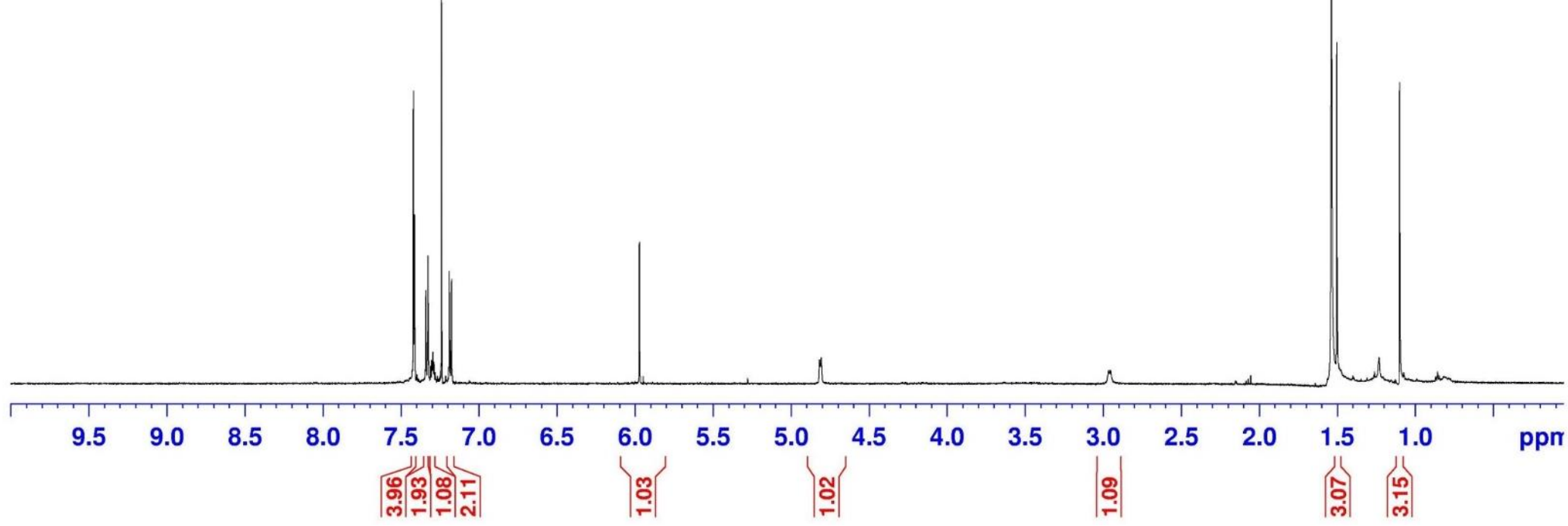




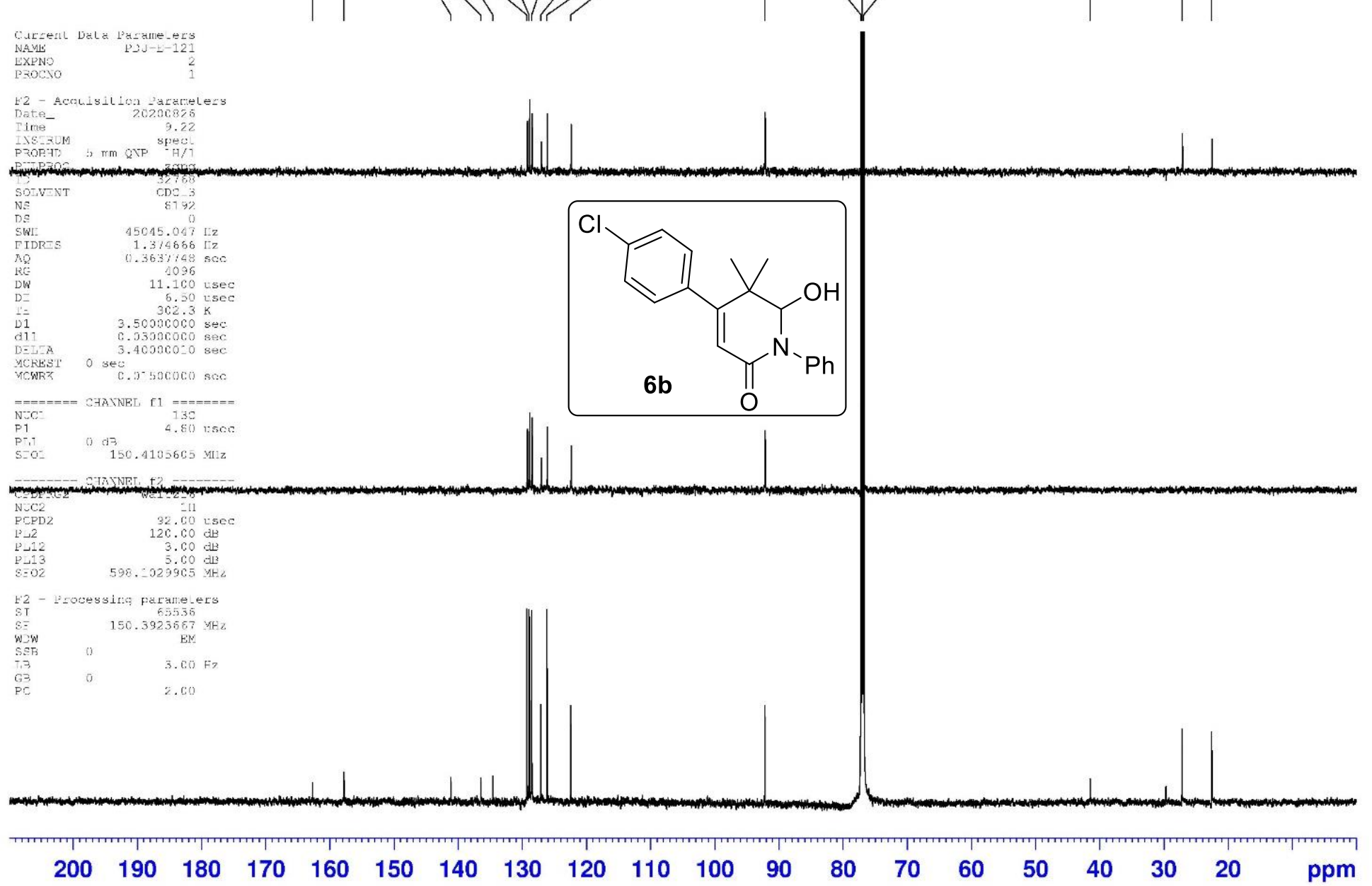



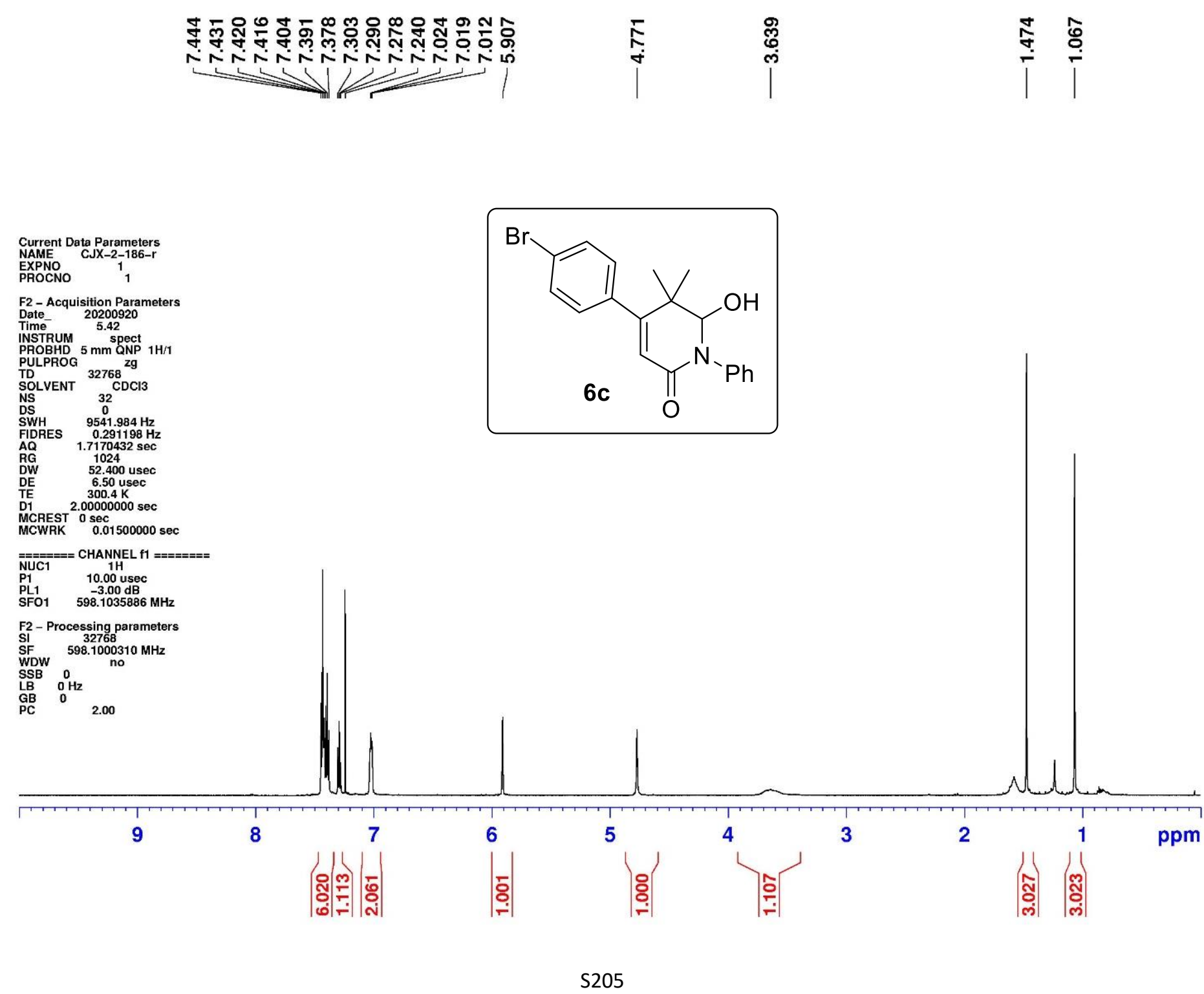
|

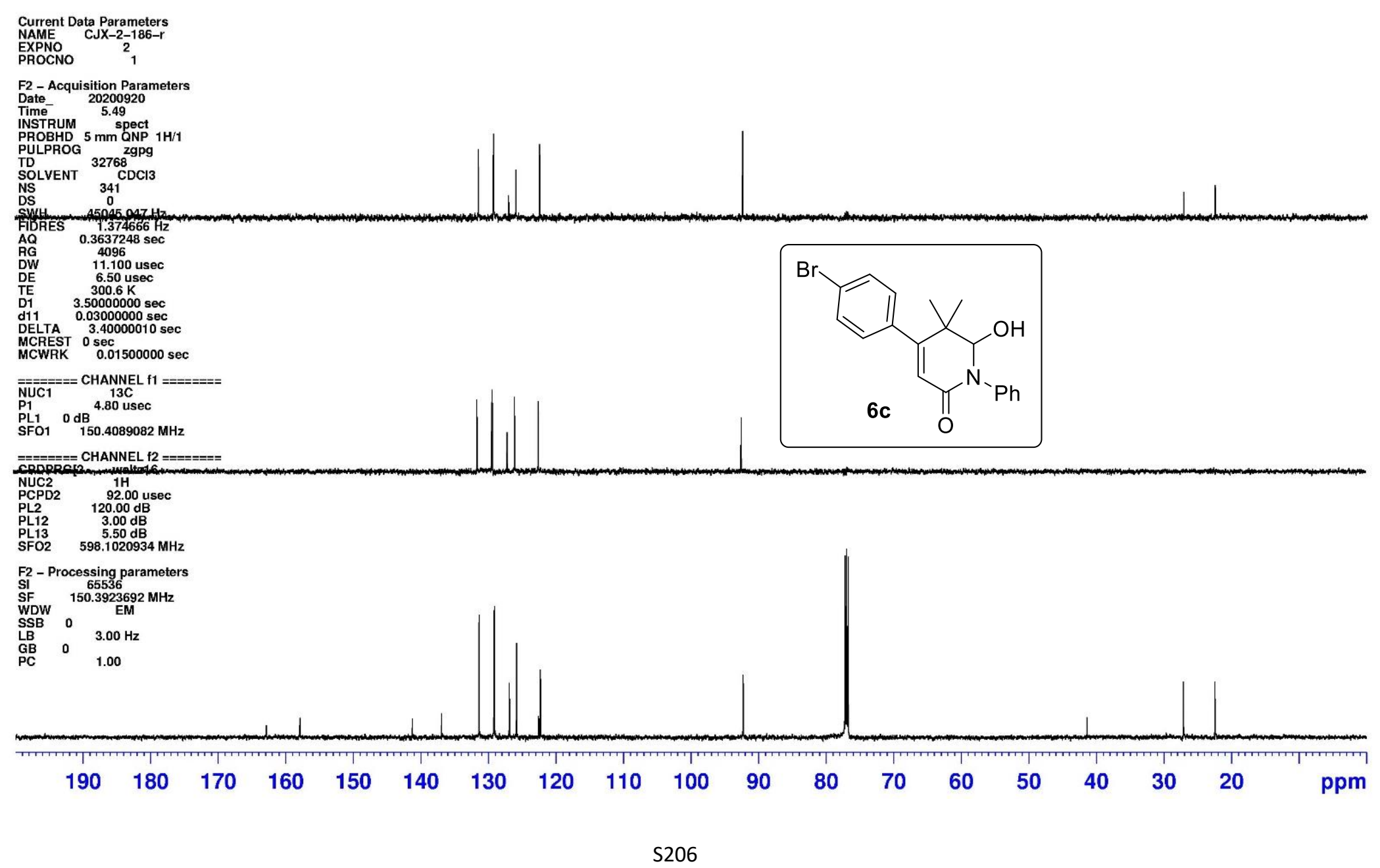




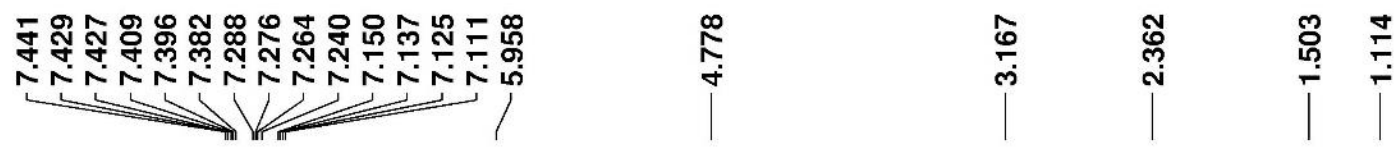

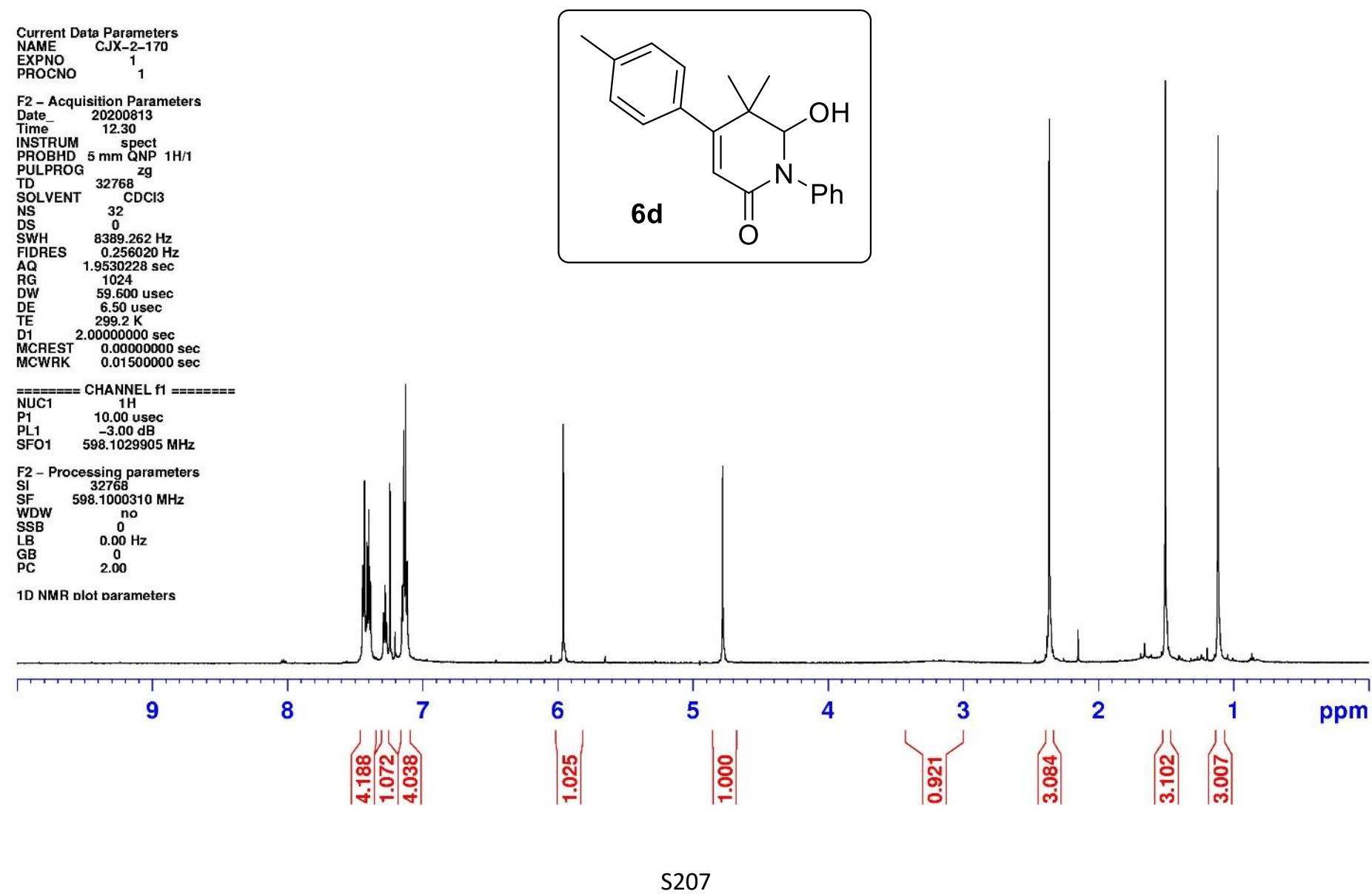




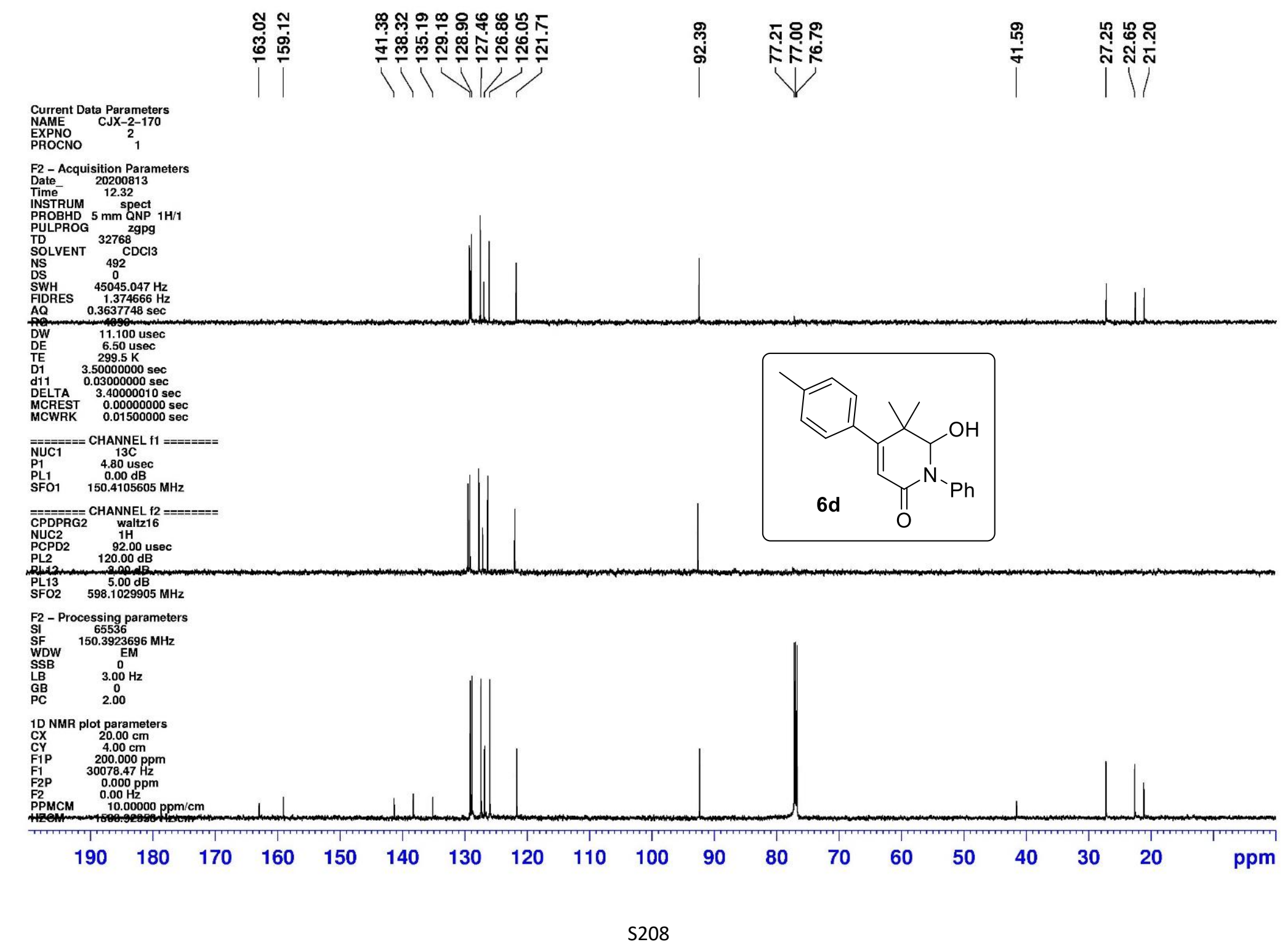




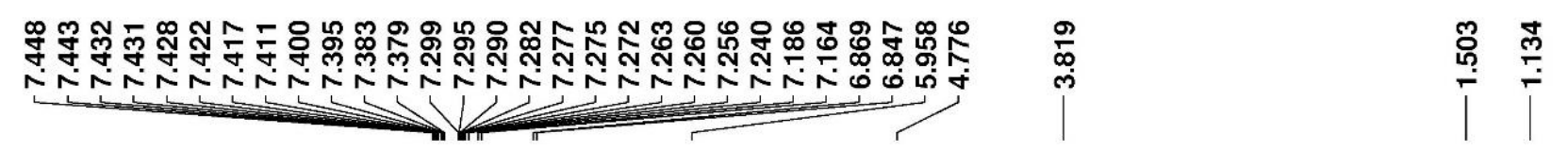

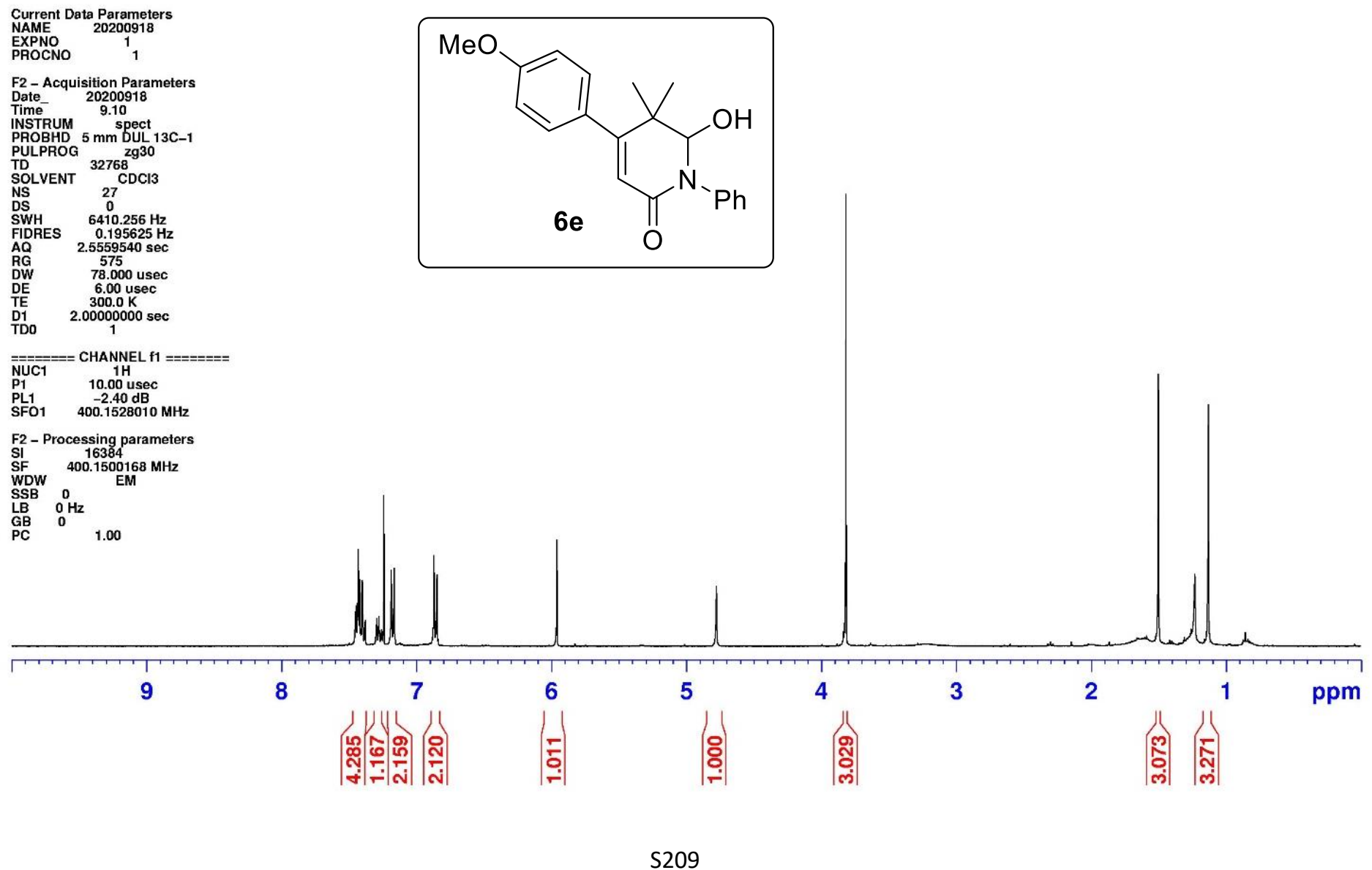



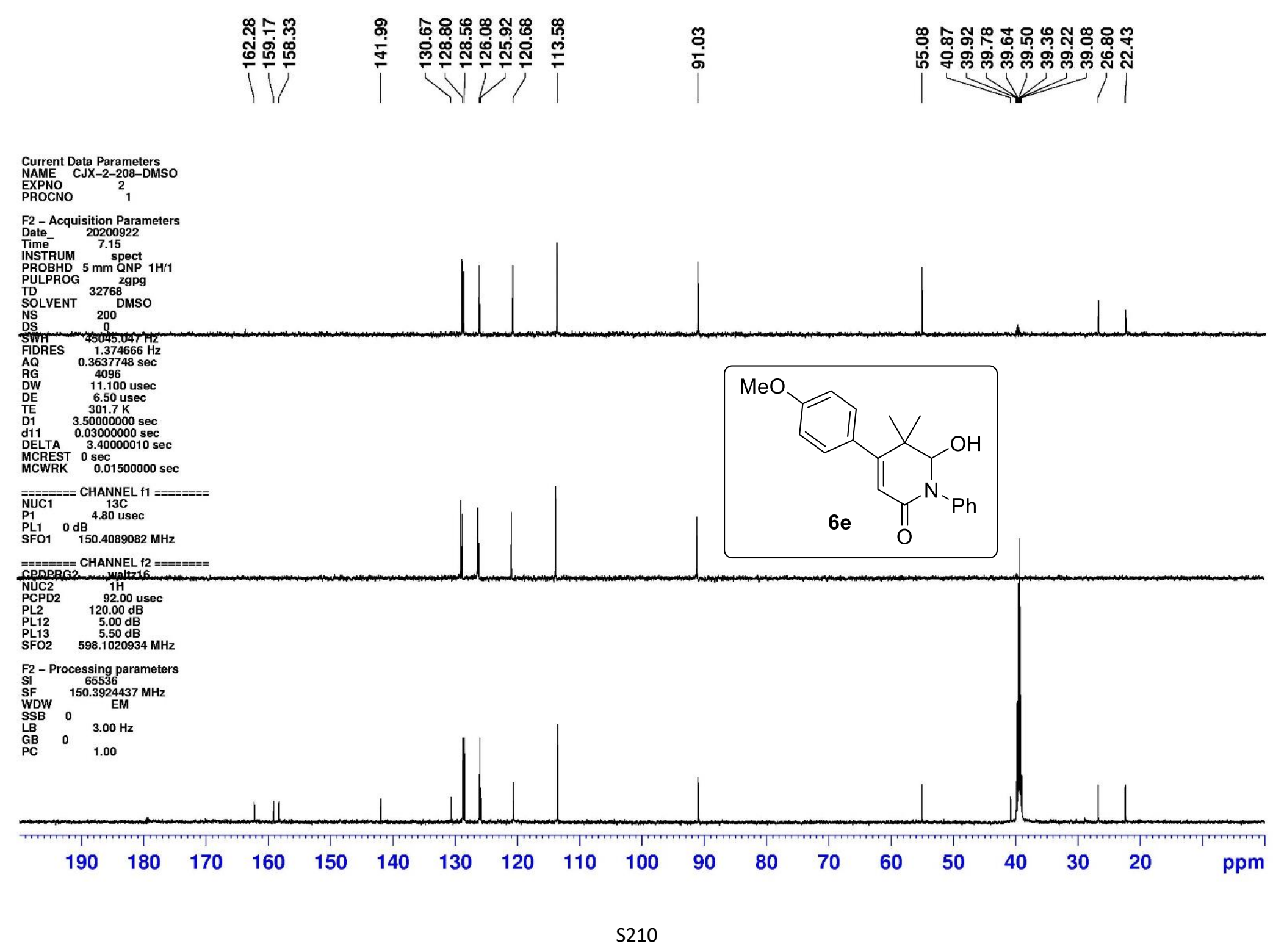

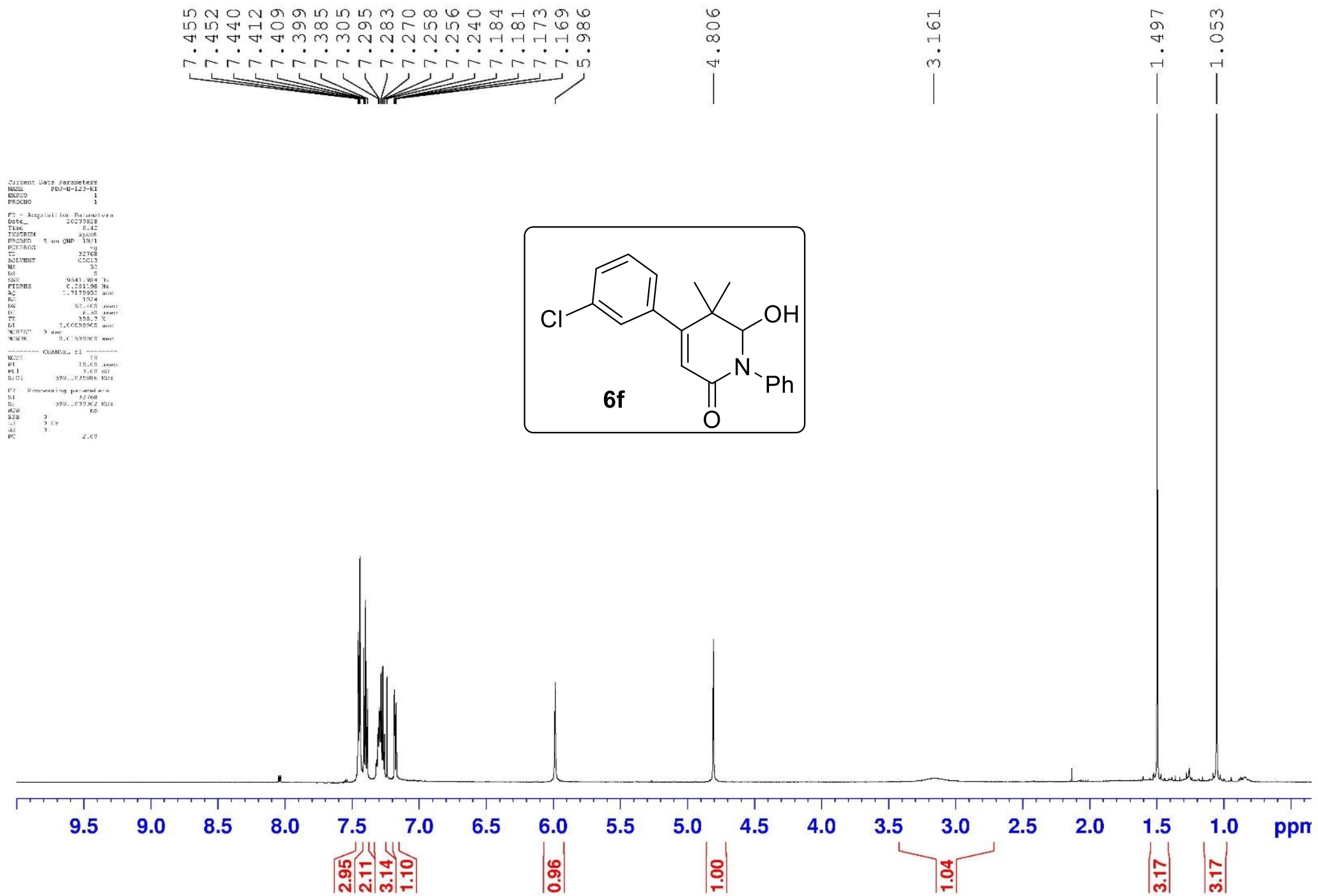


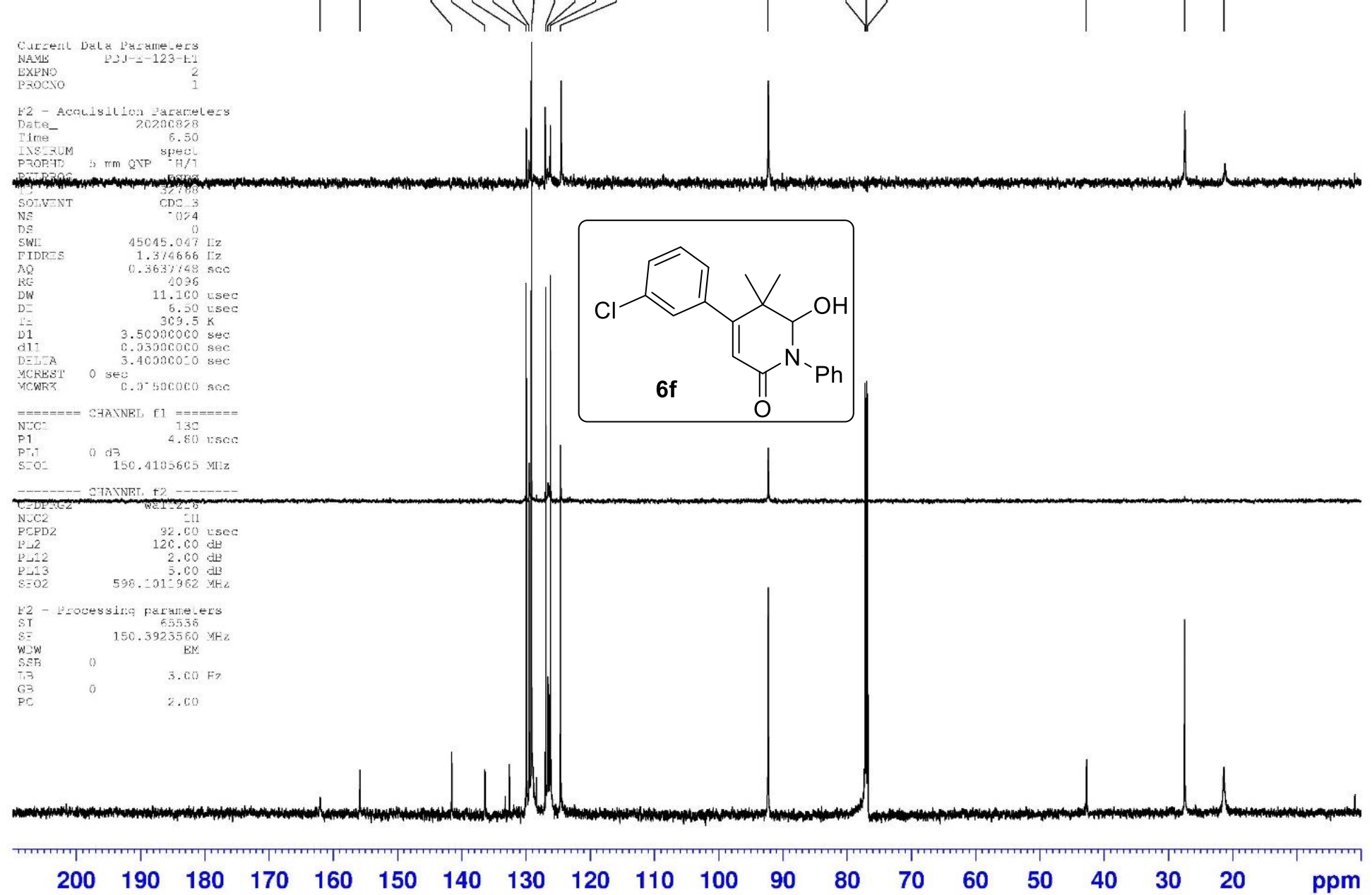




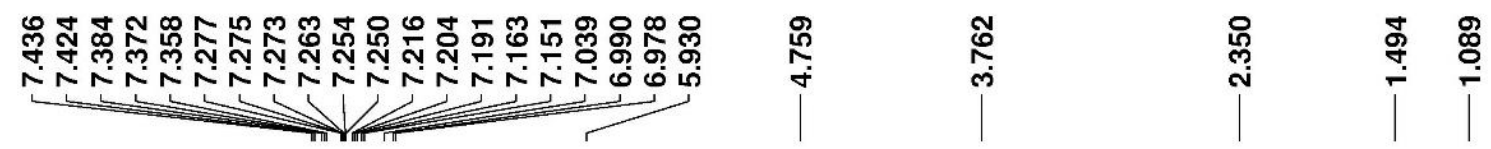

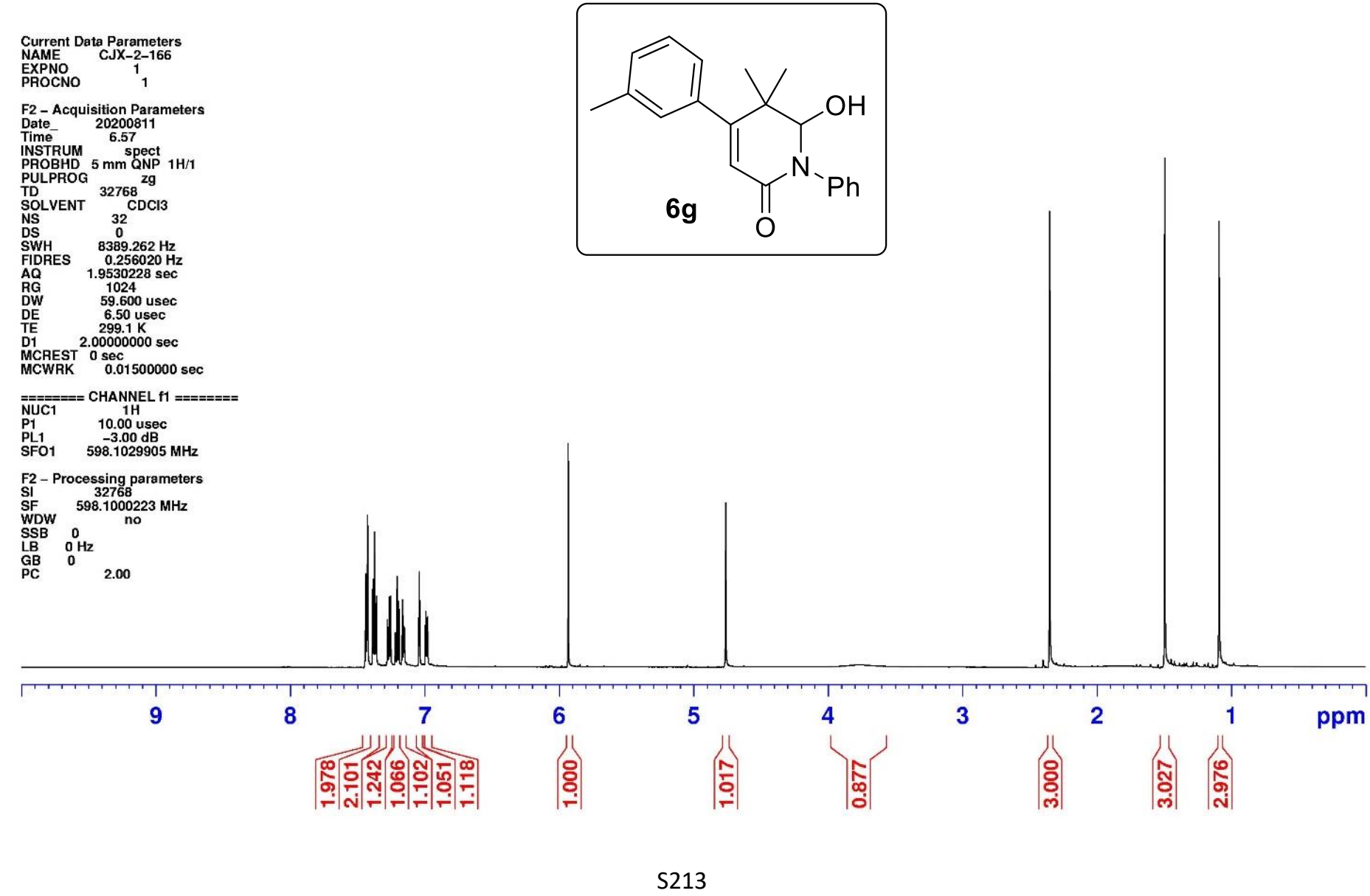



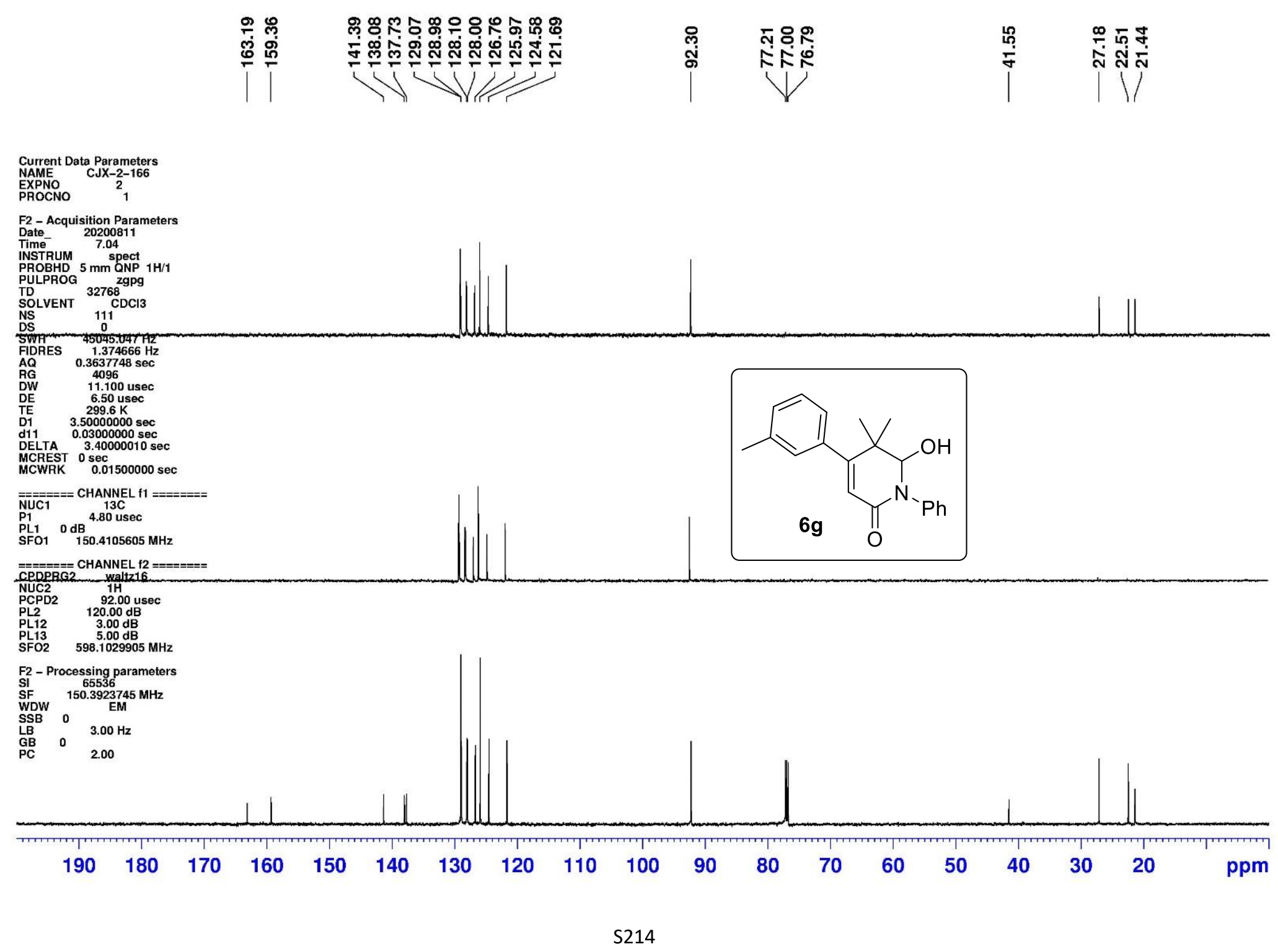


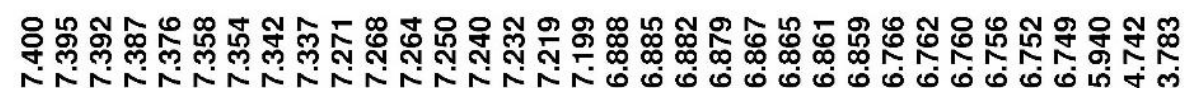

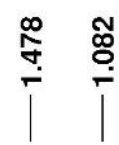
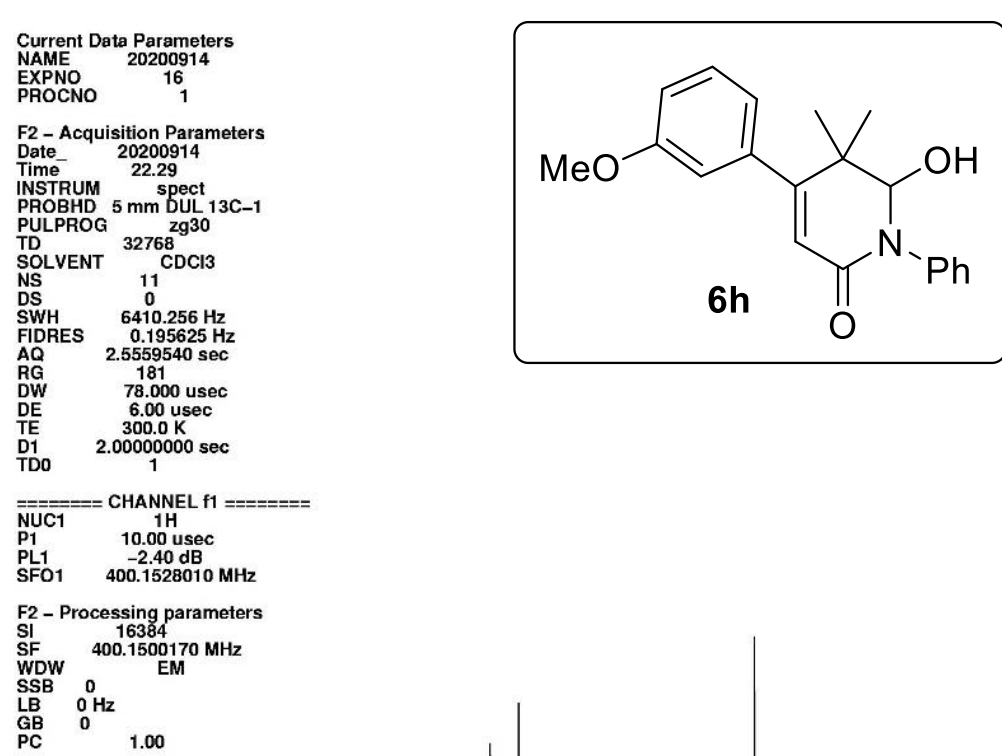

$\mathrm{PC} \quad 1.00$

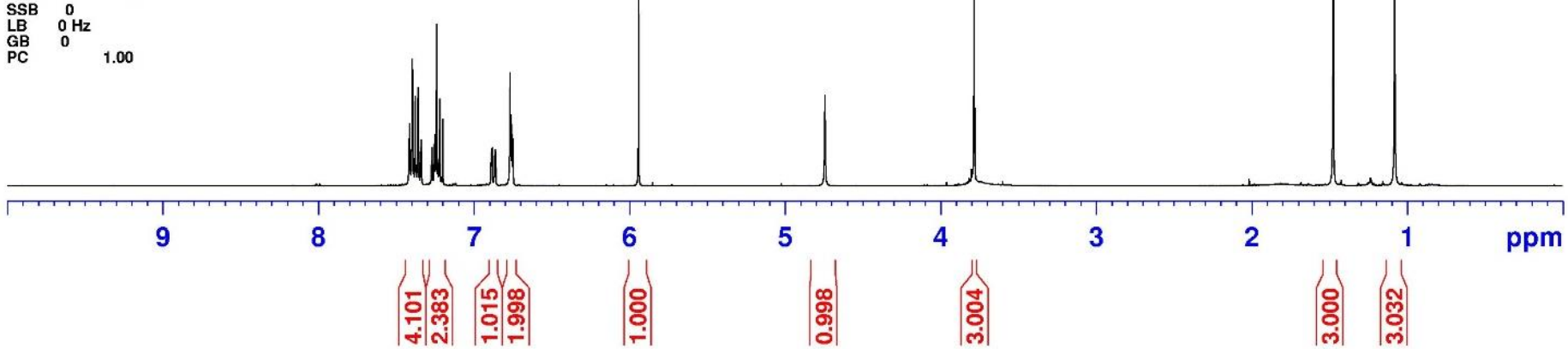




|V

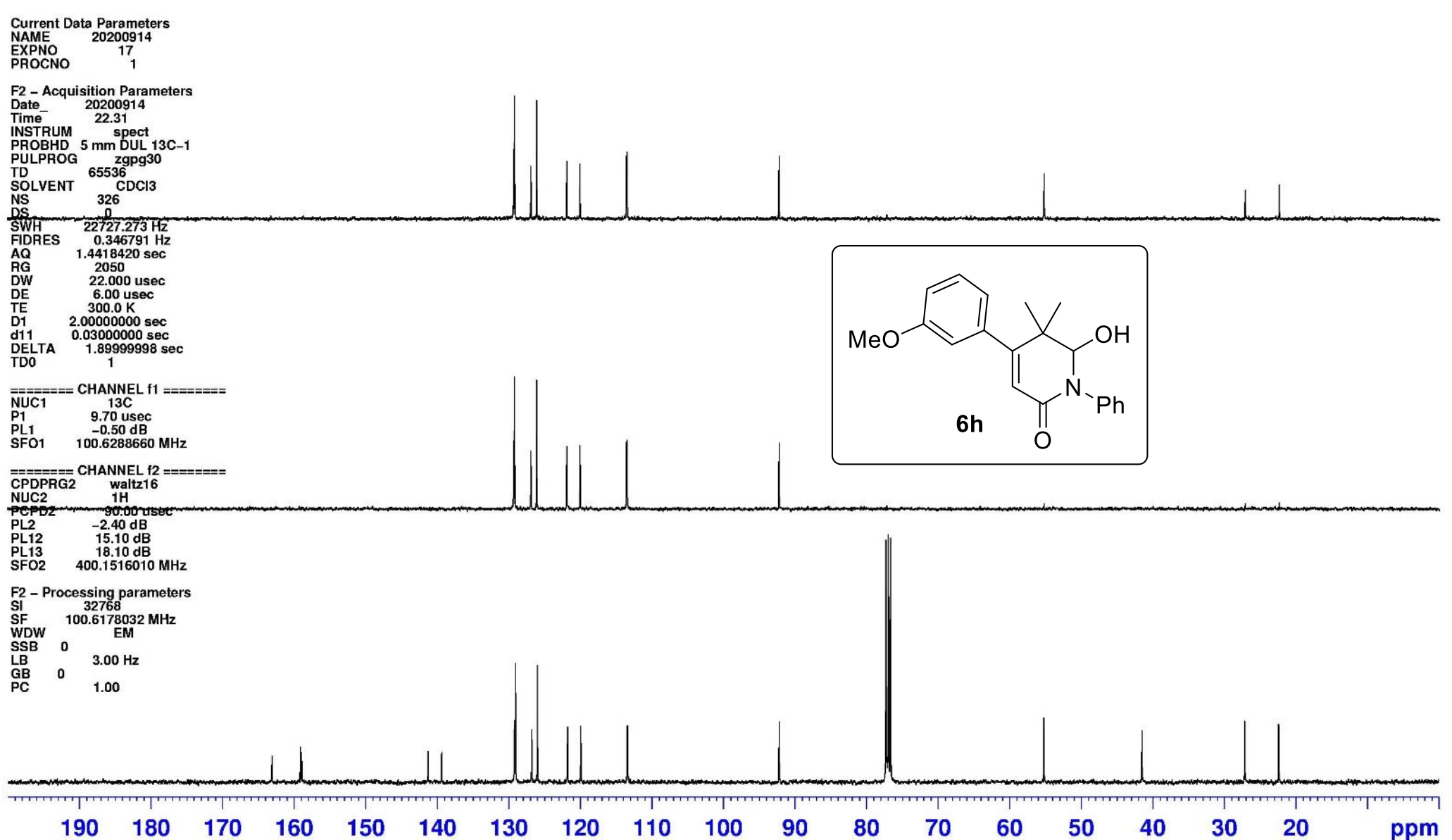




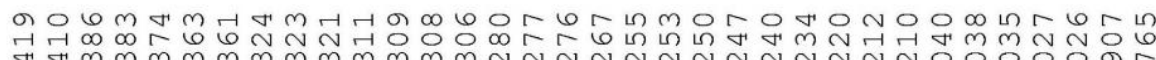

न न

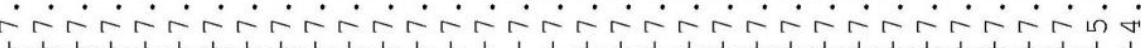

$\longrightarrow$
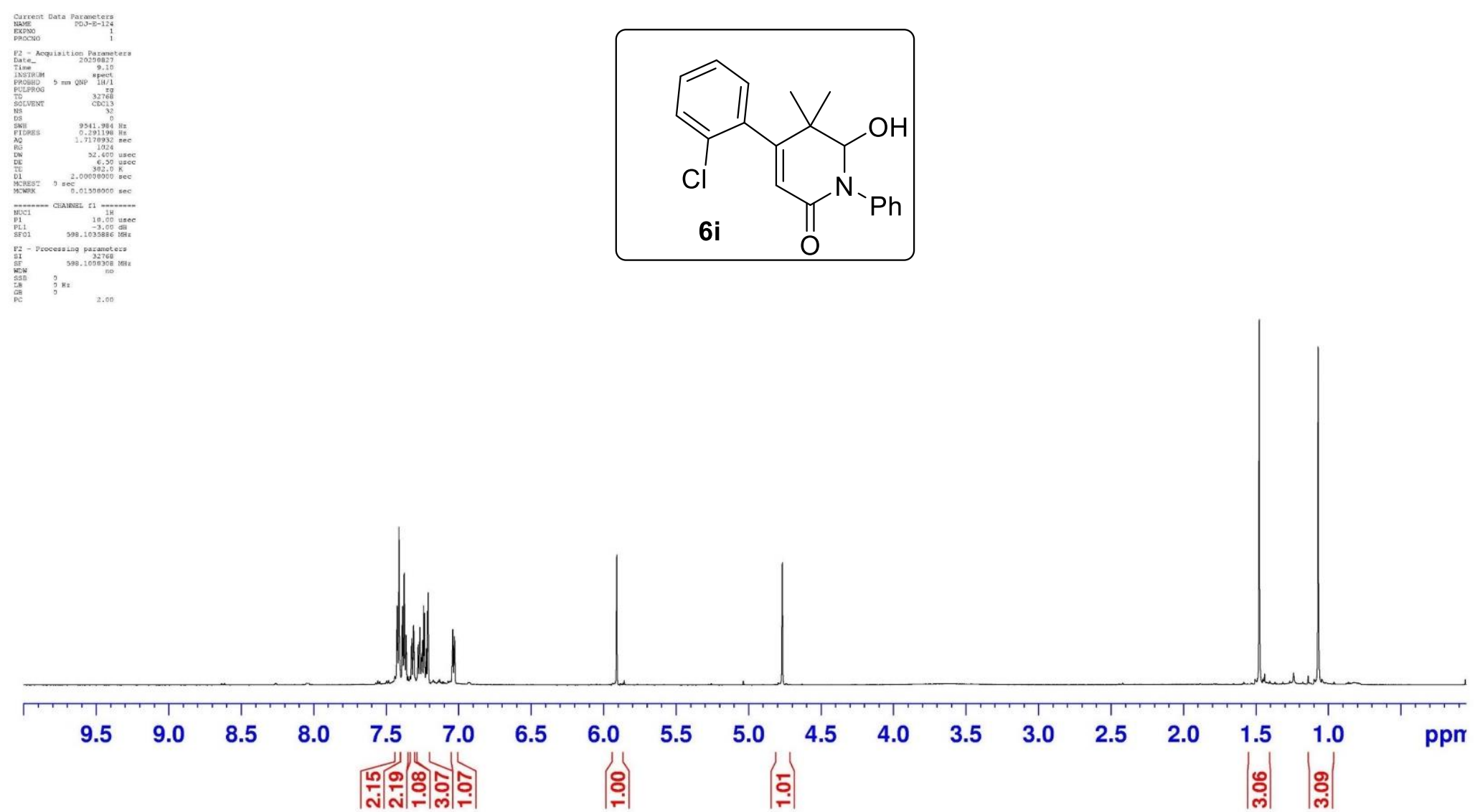


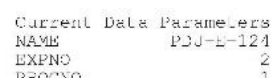
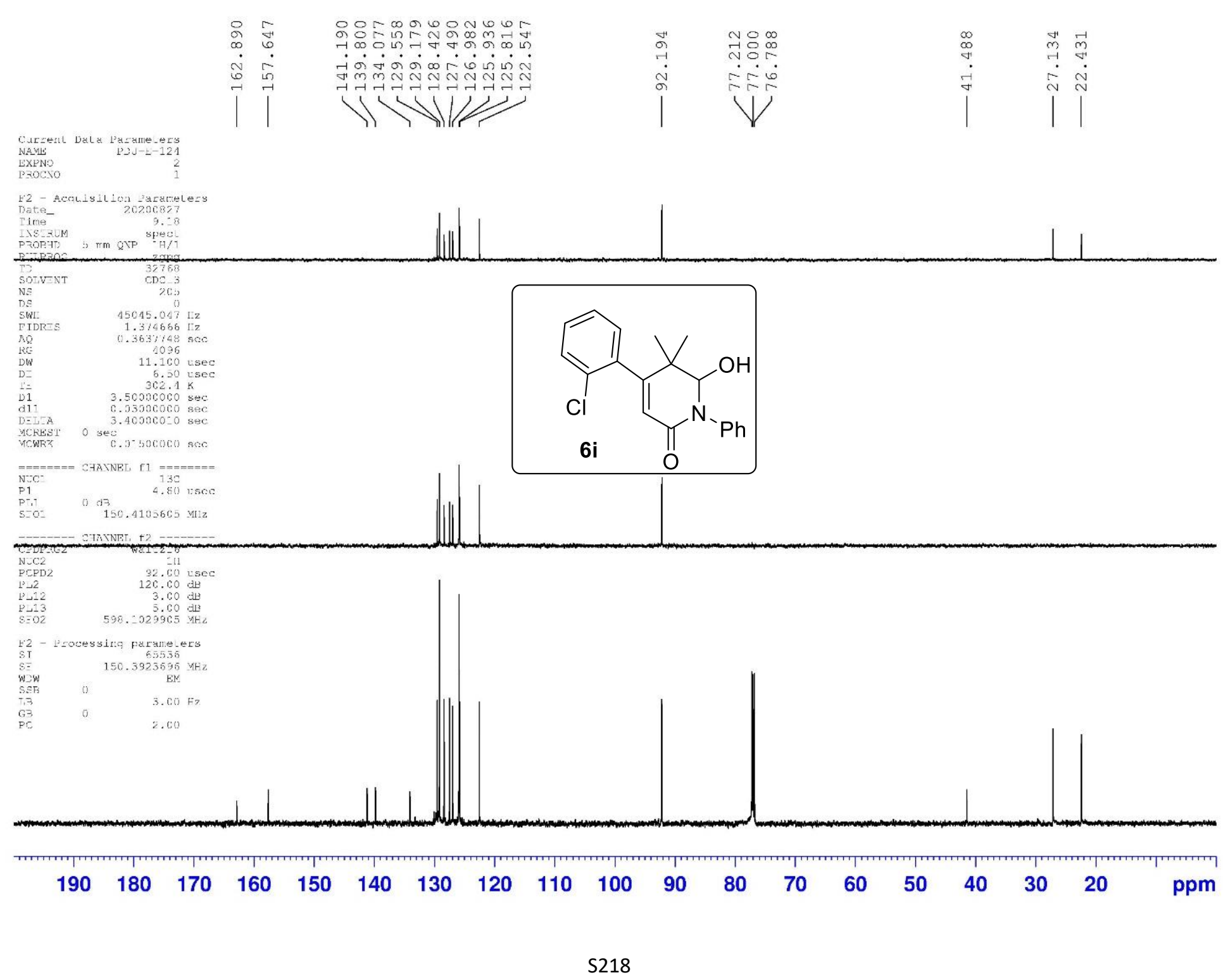


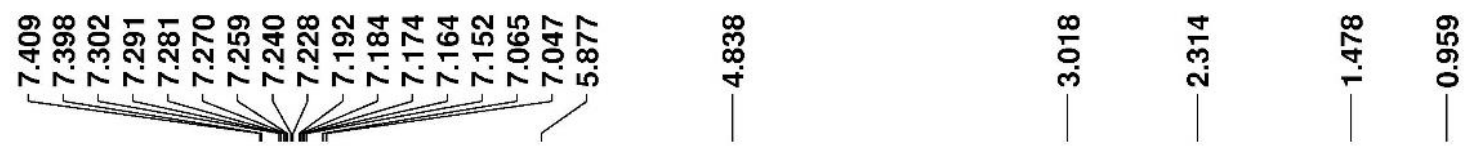

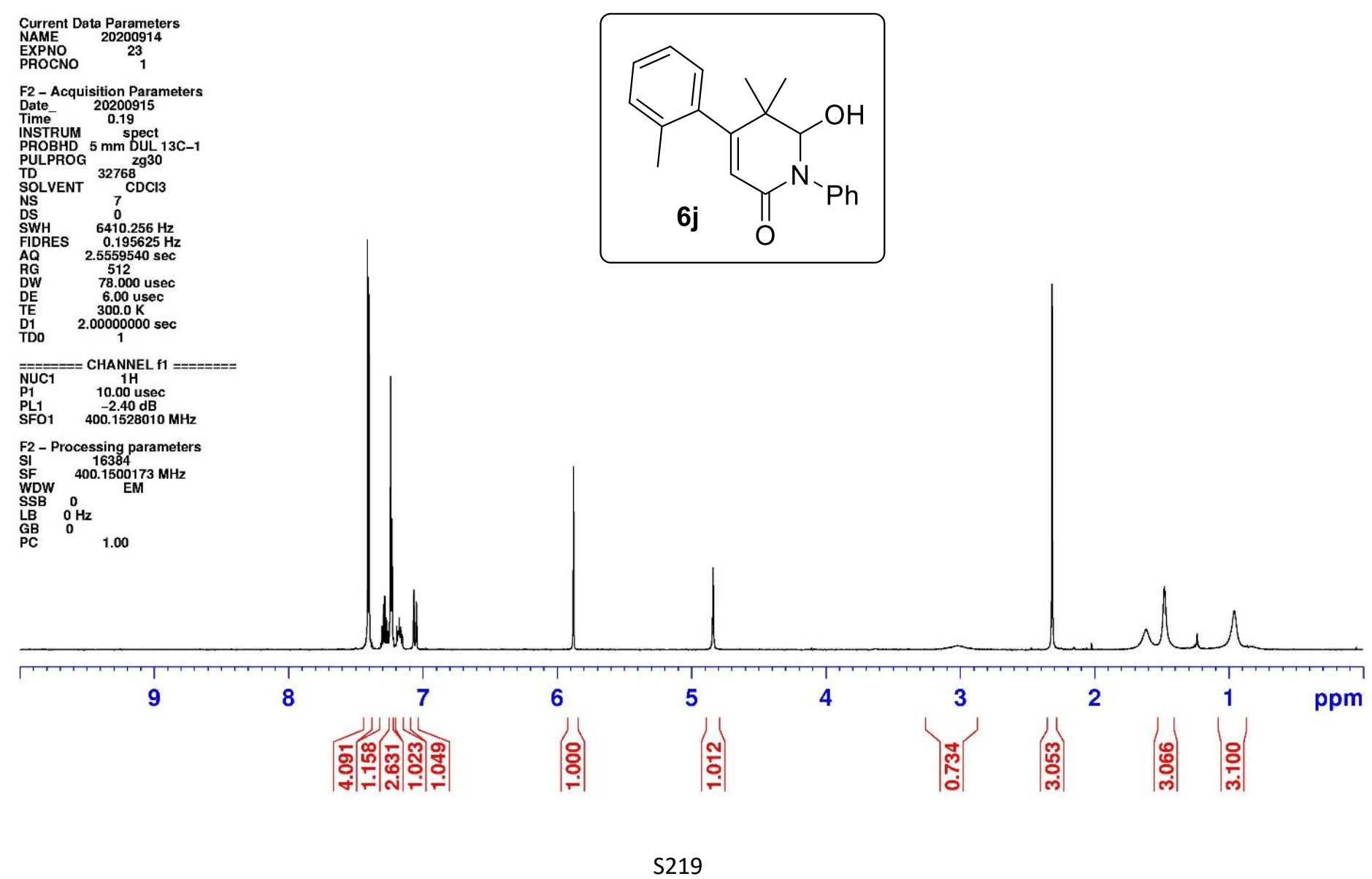




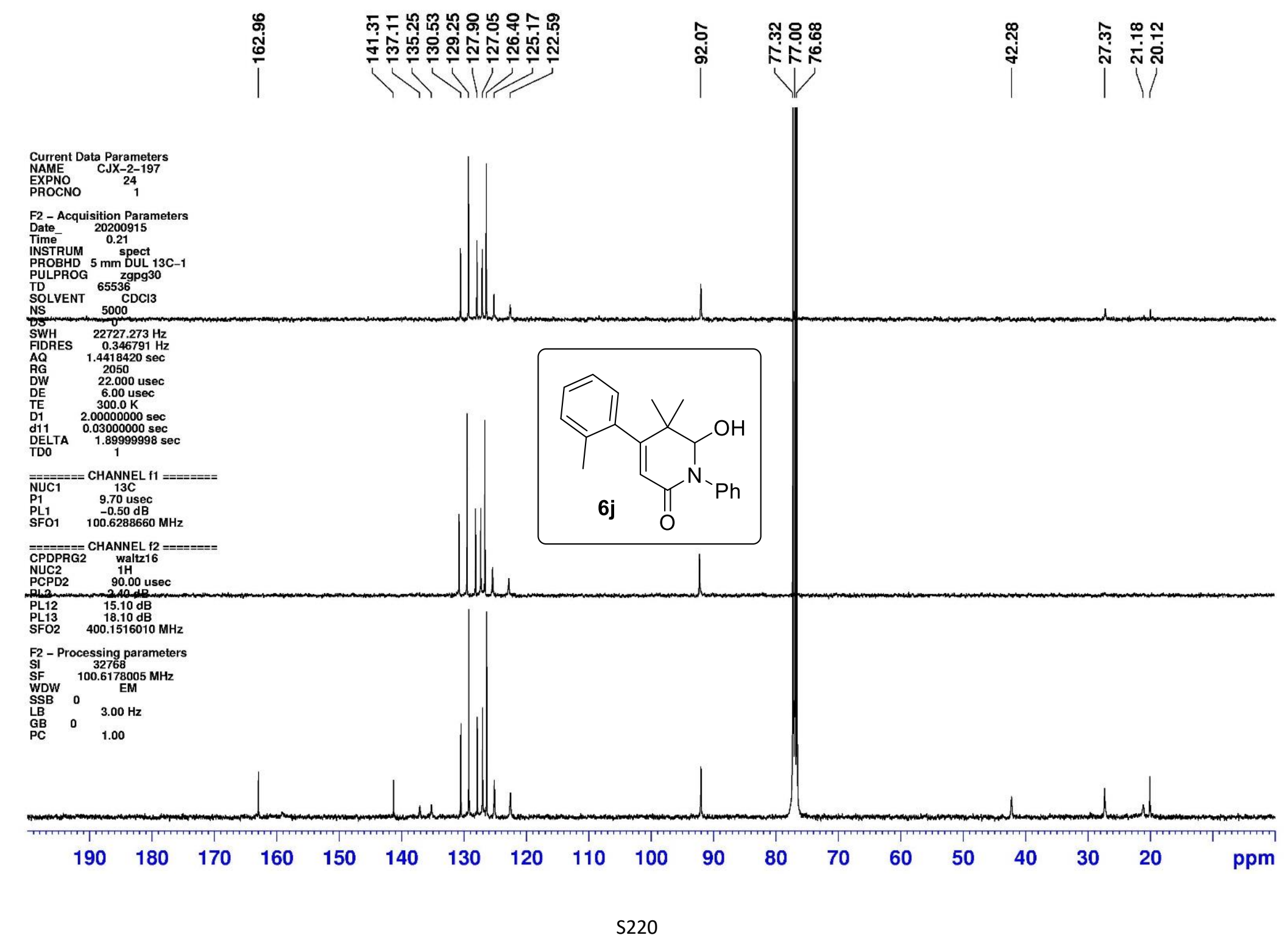




\section{PROCNO}

F2- Acquisition Parameters
Date_ 20200921

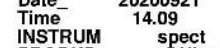

PHOBDD $5 \mathrm{~mm} \mathrm{mLL}_{2930} 13 \mathrm{C}_{-1}$

PULPROG
SOLVENT ${ }^{32768} \mathrm{CDCl}^{2 \mathrm{~g} 30}$
NS

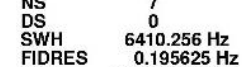

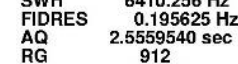

$\begin{array}{lrl}\mathrm{RG} & 912 \mathrm{ugc} \\ \mathrm{RW} & 78.000 \text { usec } \\ \mathrm{DW} & 6.00 \mathrm{usec} \\ \mathrm{DE} & 300.0 \mathrm{~K}\end{array}$

$\begin{array}{lll}\text { TE } & 3.000 \mathrm{~K} \\ \text { T1 } & 2.00000000 \mathrm{sec} \\ \text { TDo } & 1\end{array}$

$\overline{\text { NuC } 1}=$ CHANNEL $11====$

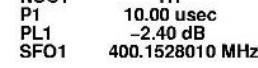

F2 - Processing parameters

$\begin{array}{lrl}\text { SI } & 16384 \\ \text { SF } & 40.1500172 \mathrm{MHz} \\ \text { WWW } & \text { EM }\end{array}$

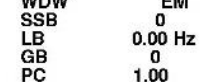

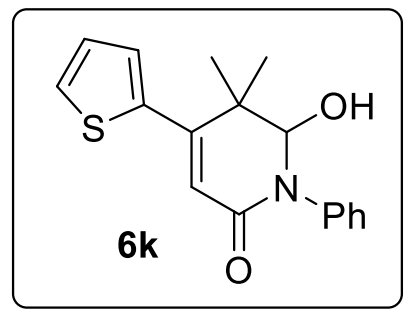

Uhlll

9

8

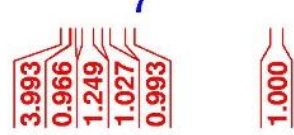

6

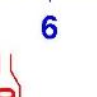

5

$\mid$

4

年

S221 


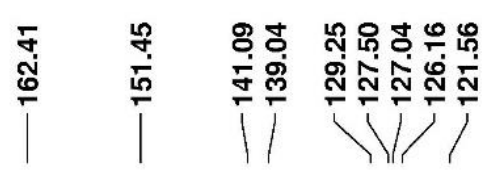

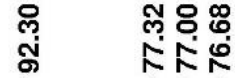

|

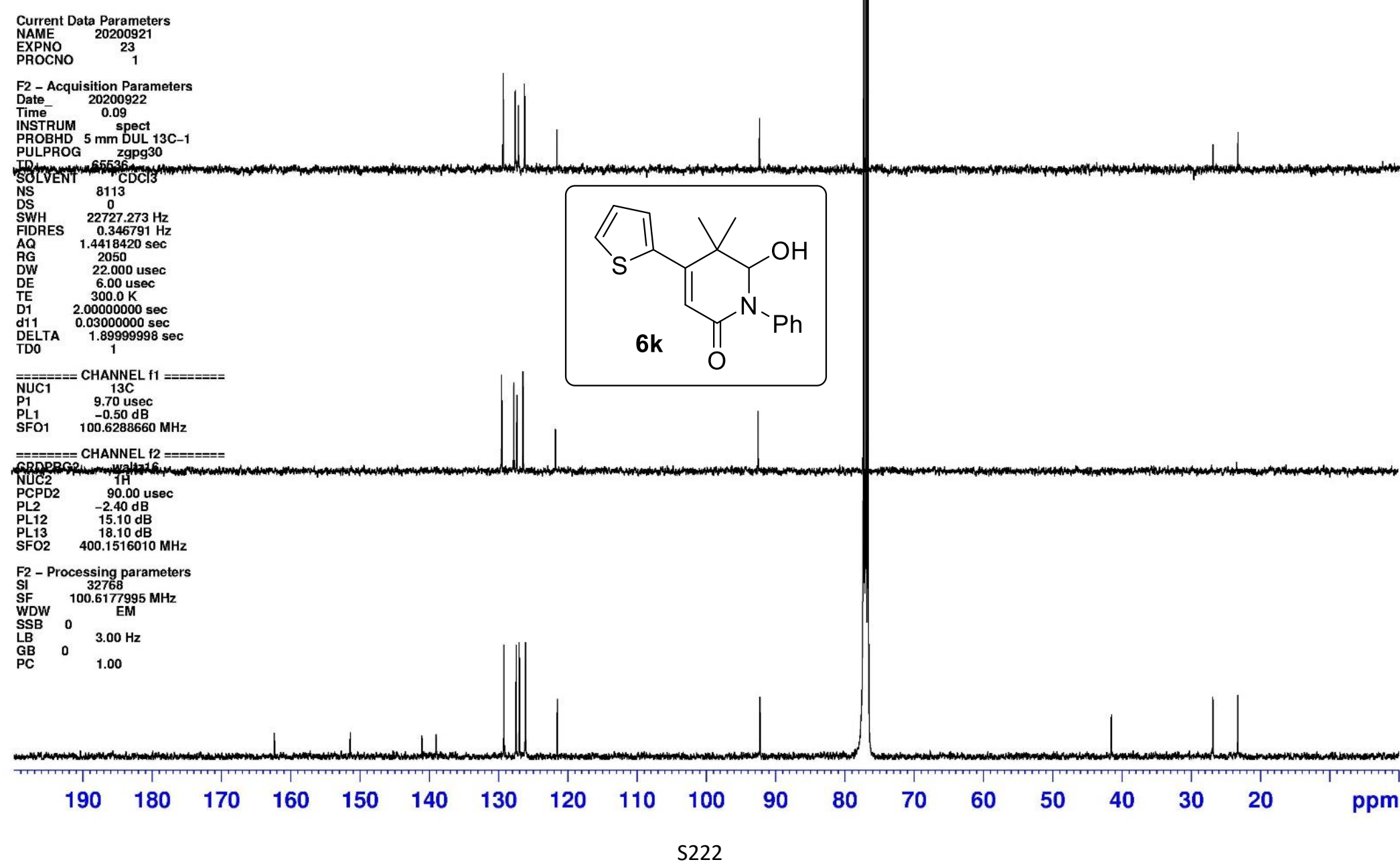




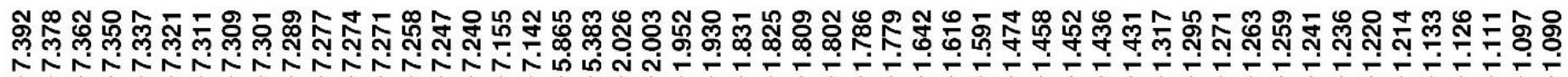

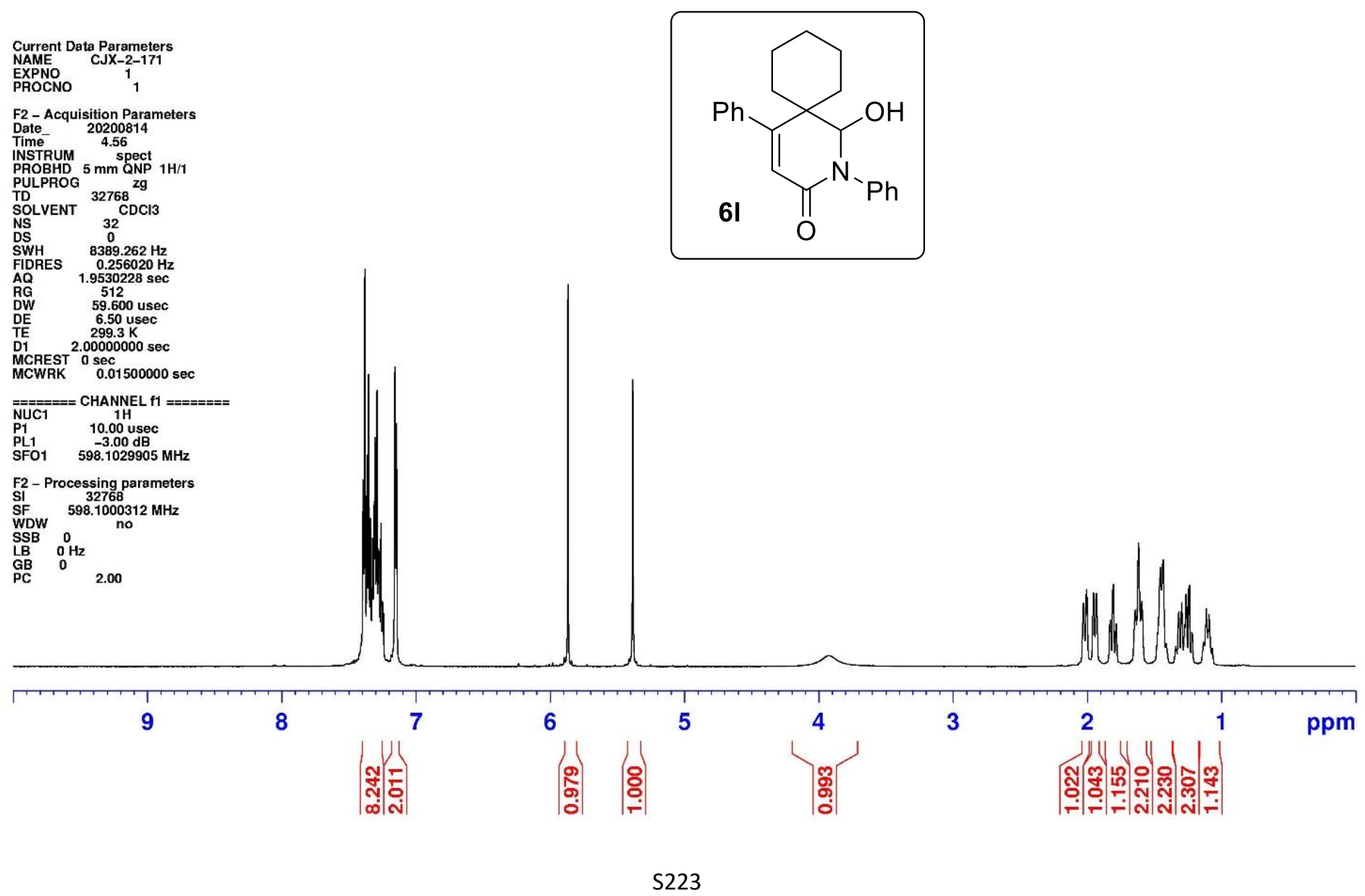




$\prod^{\circ}$

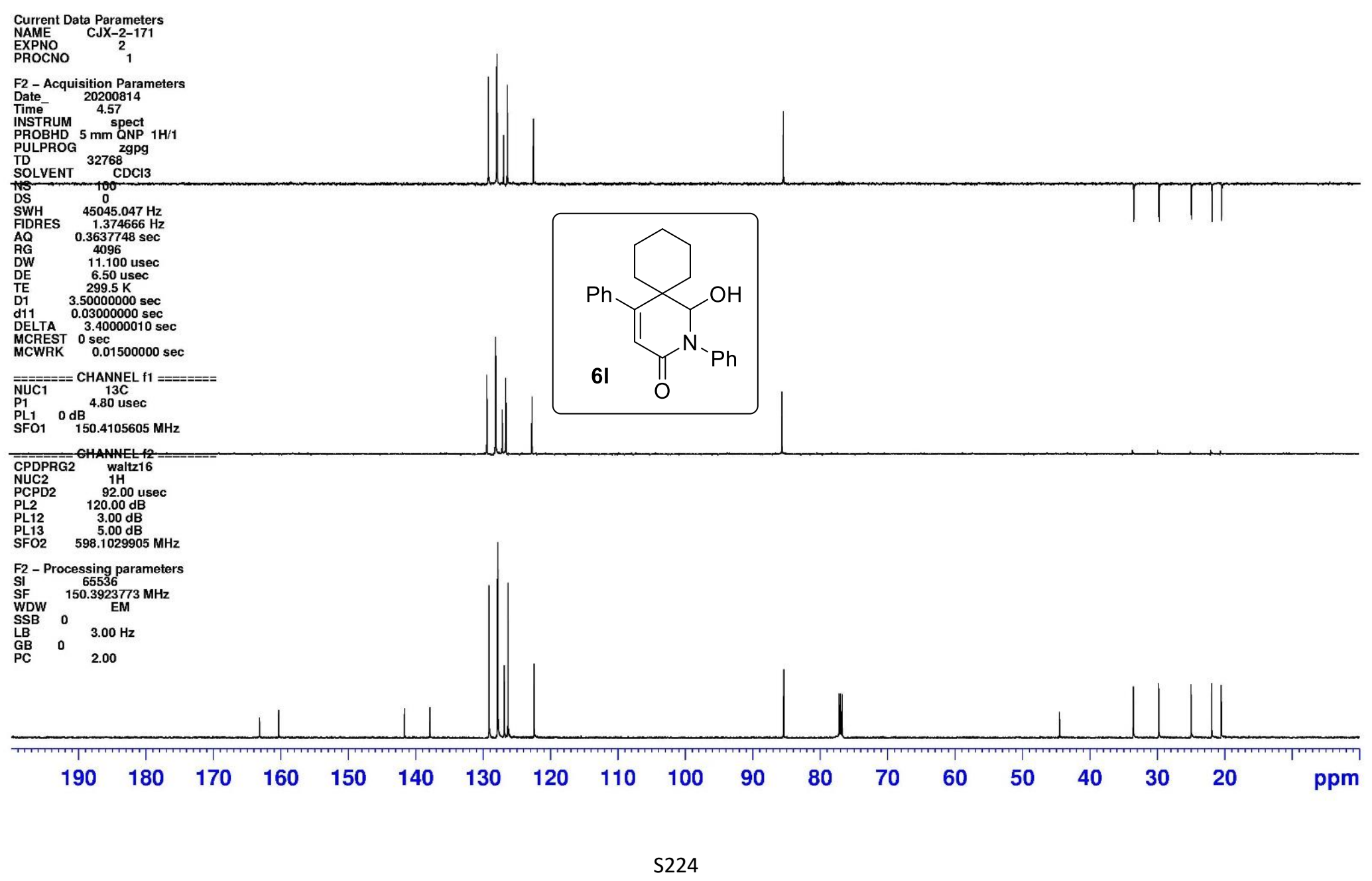



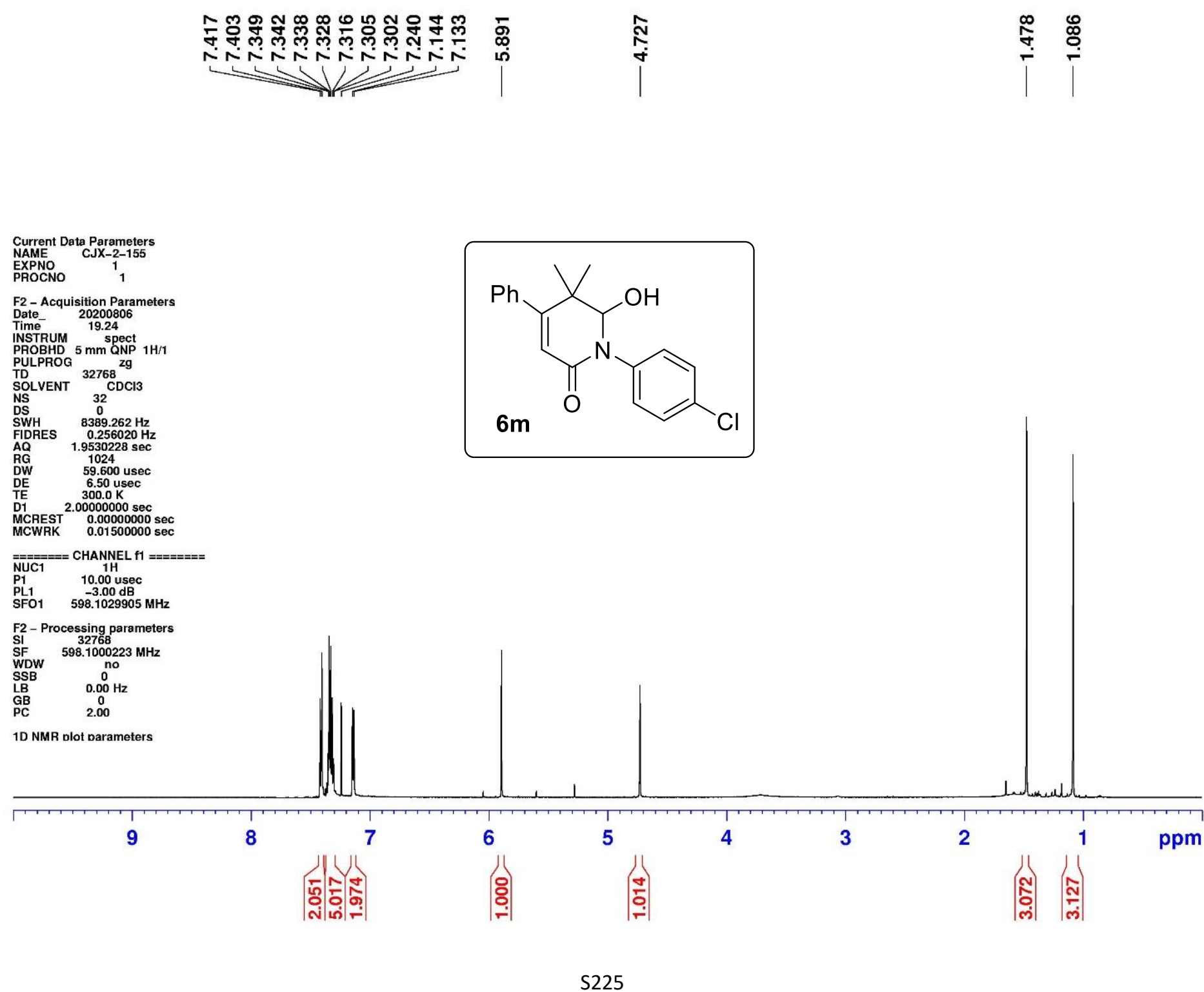


||

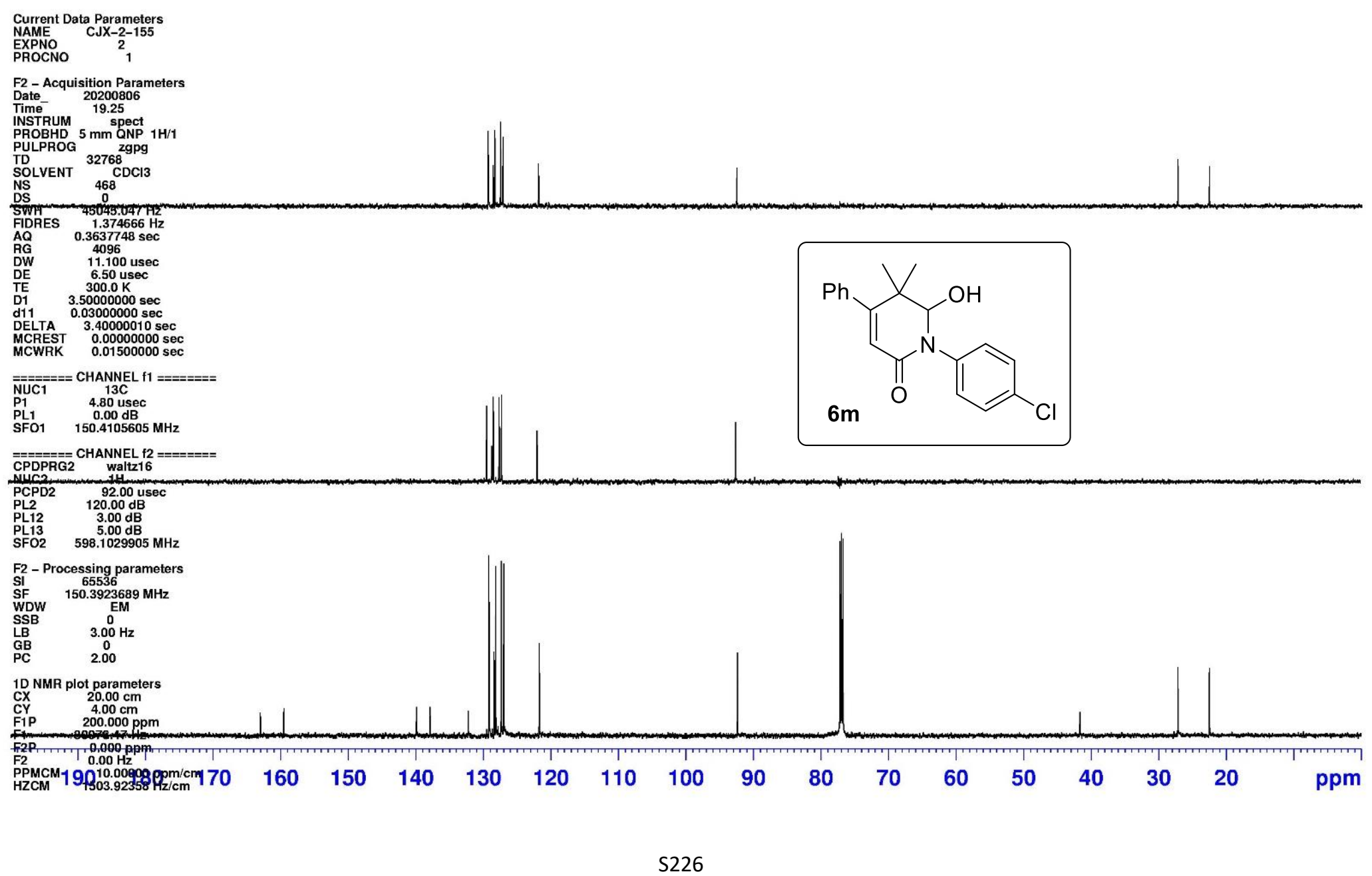




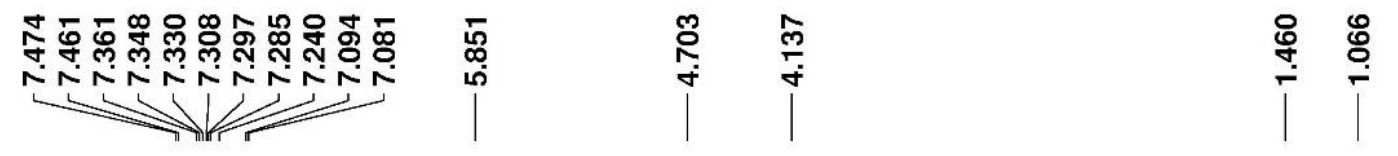

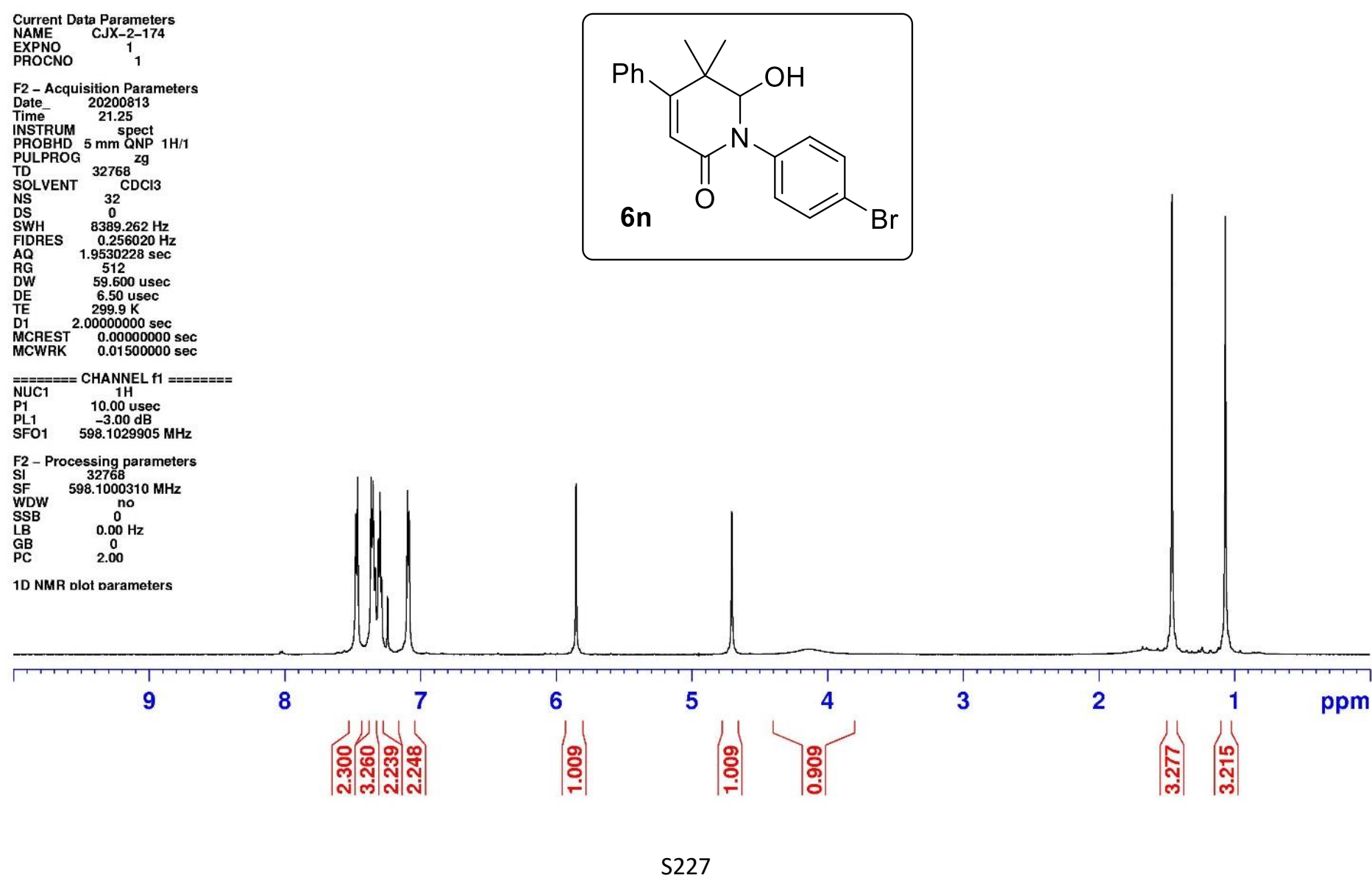




|广

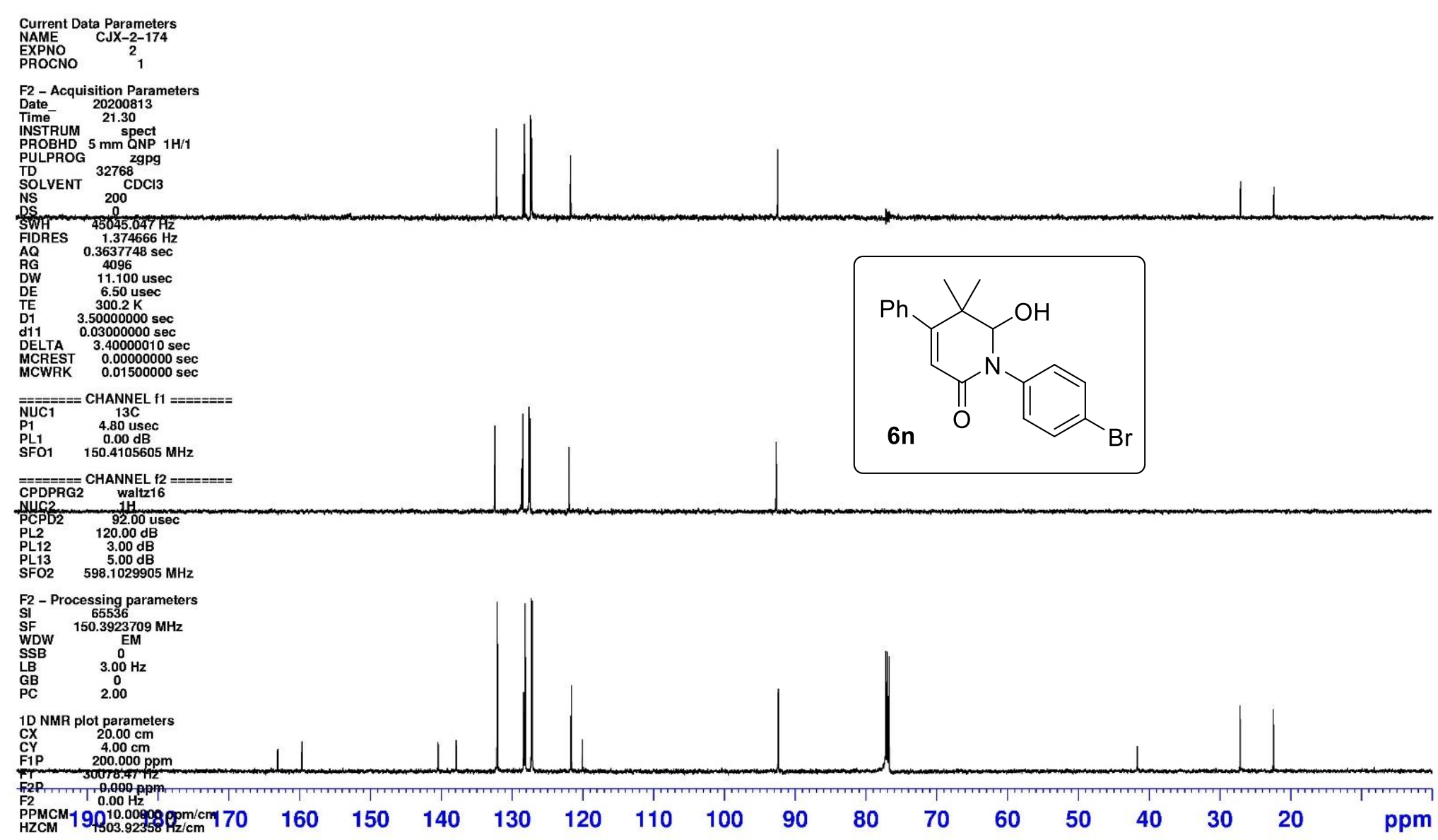




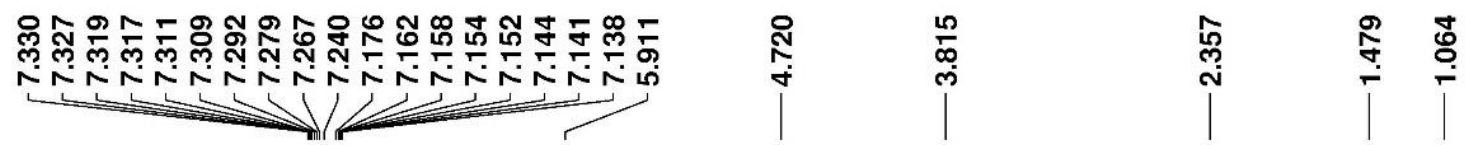

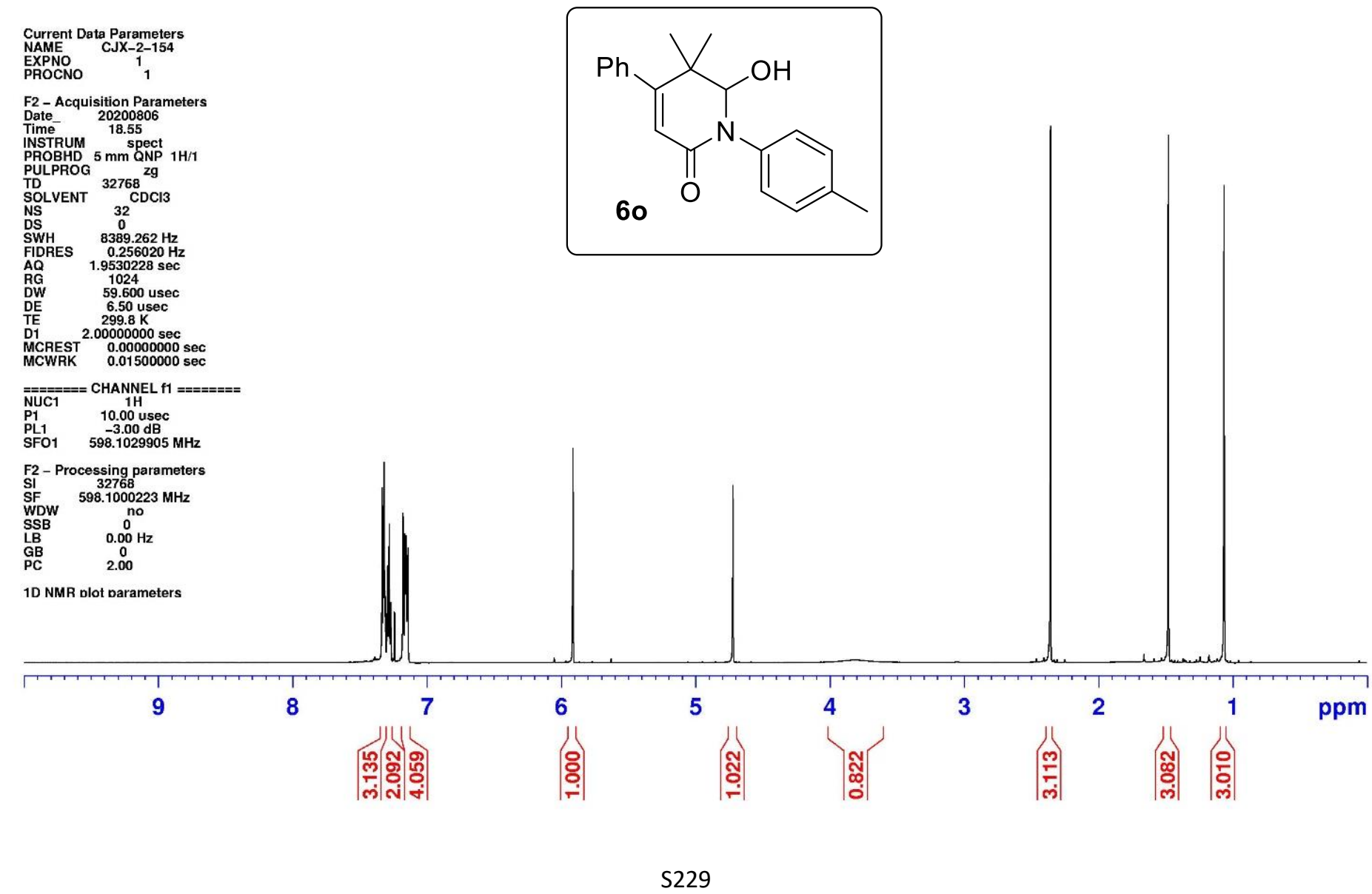



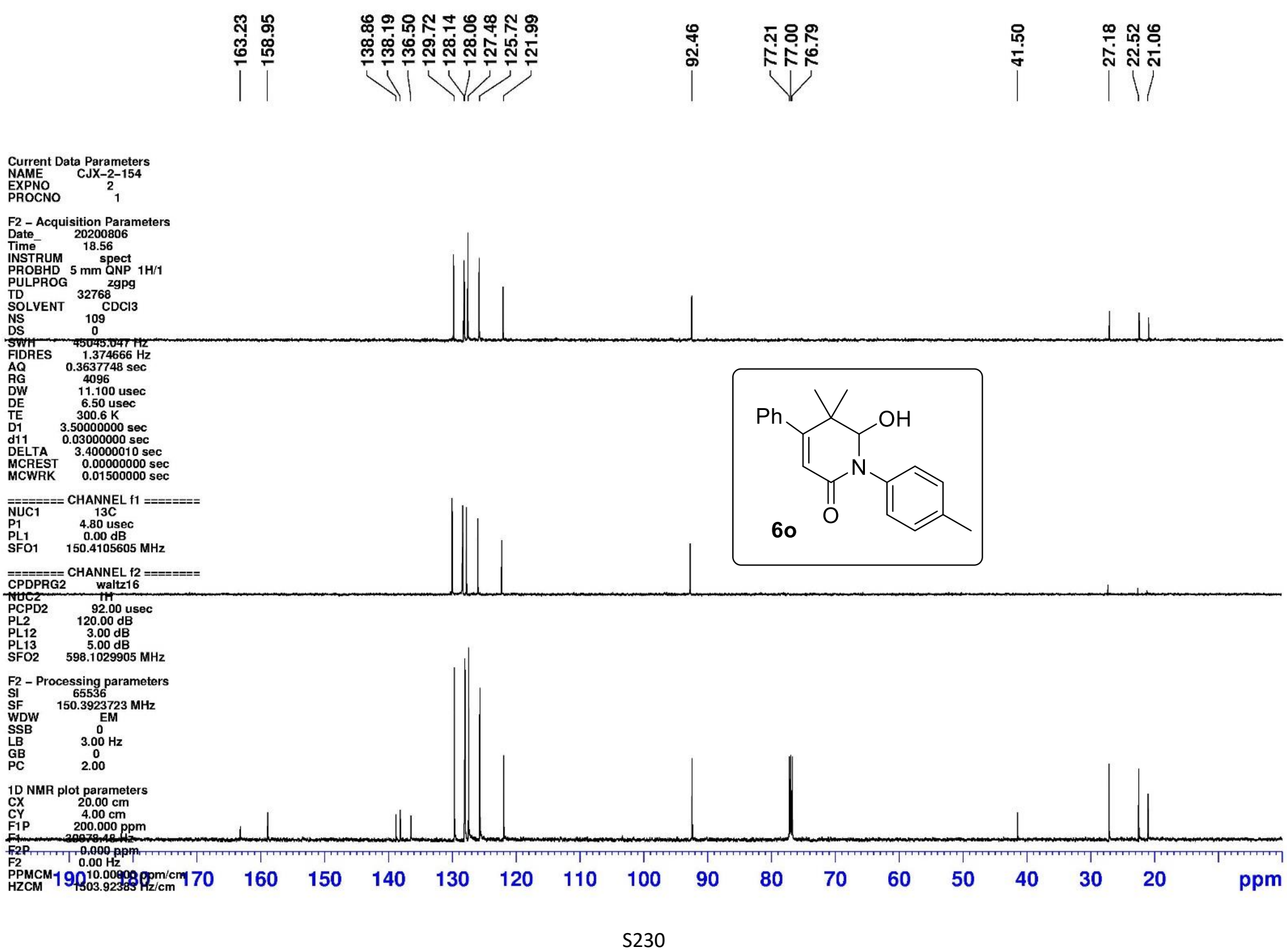


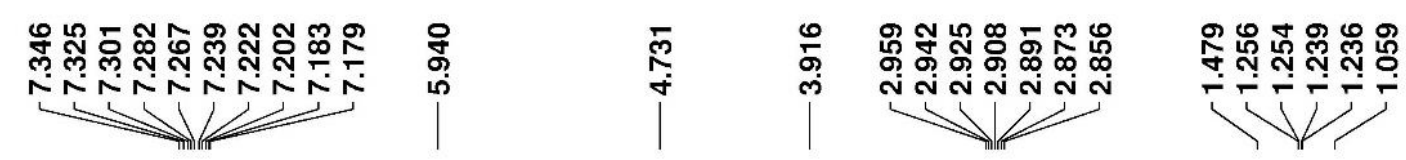

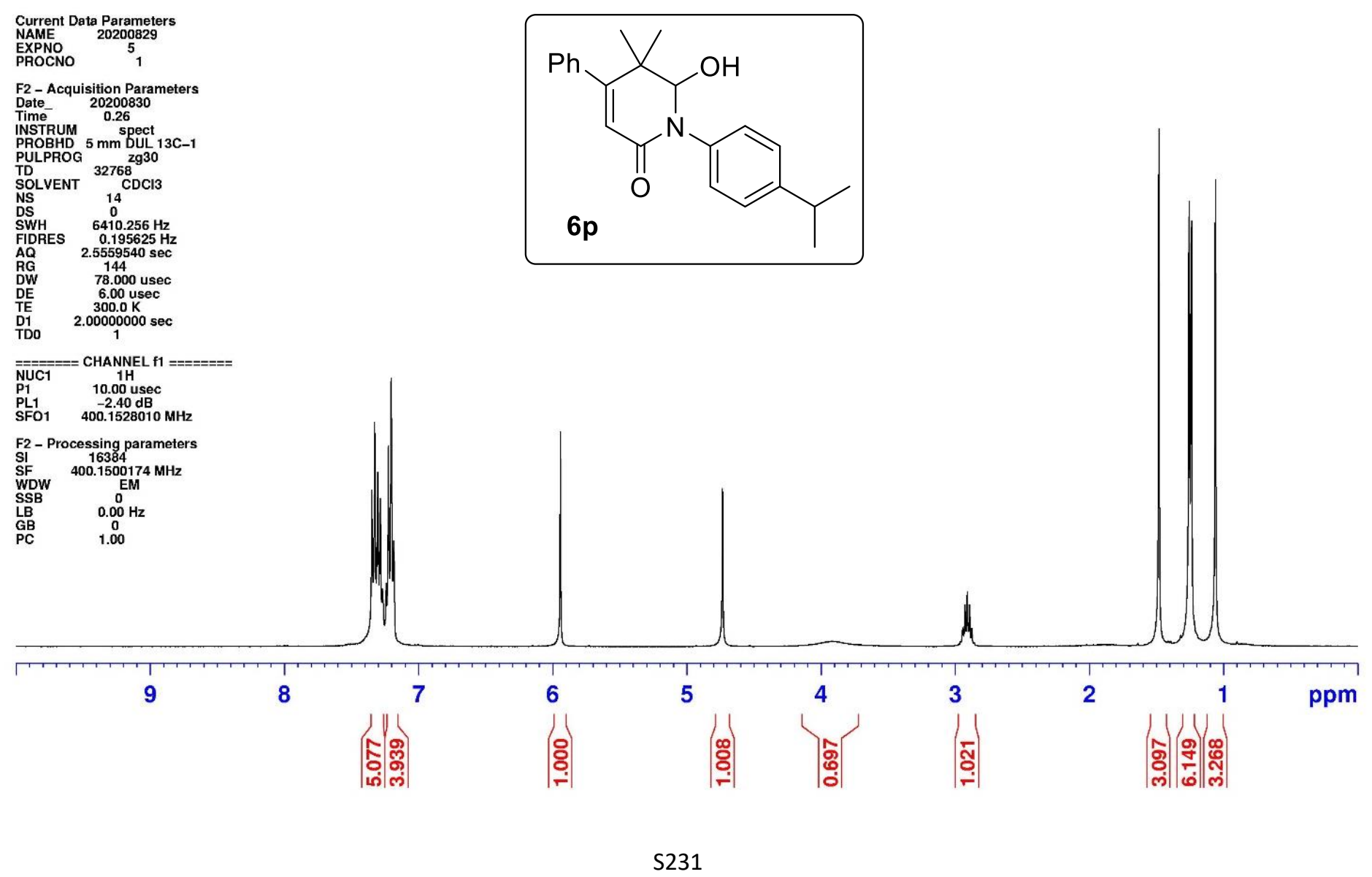



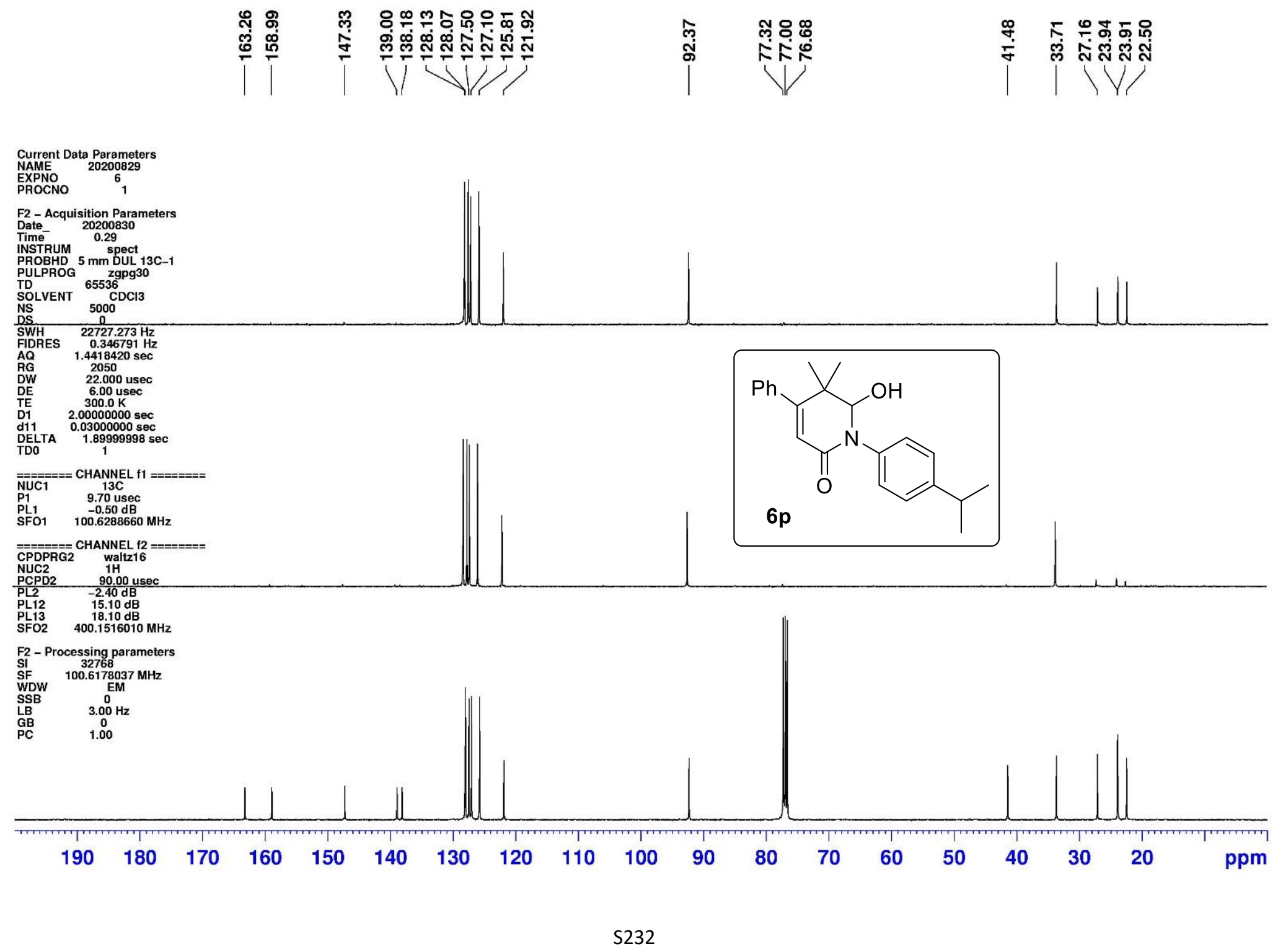


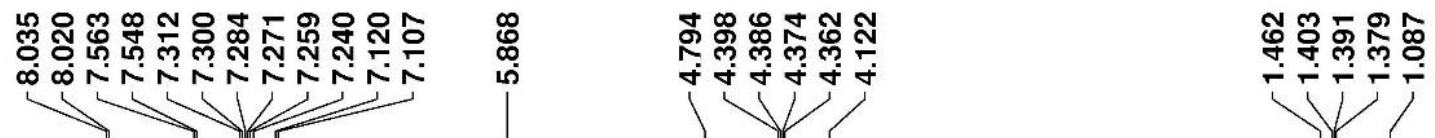

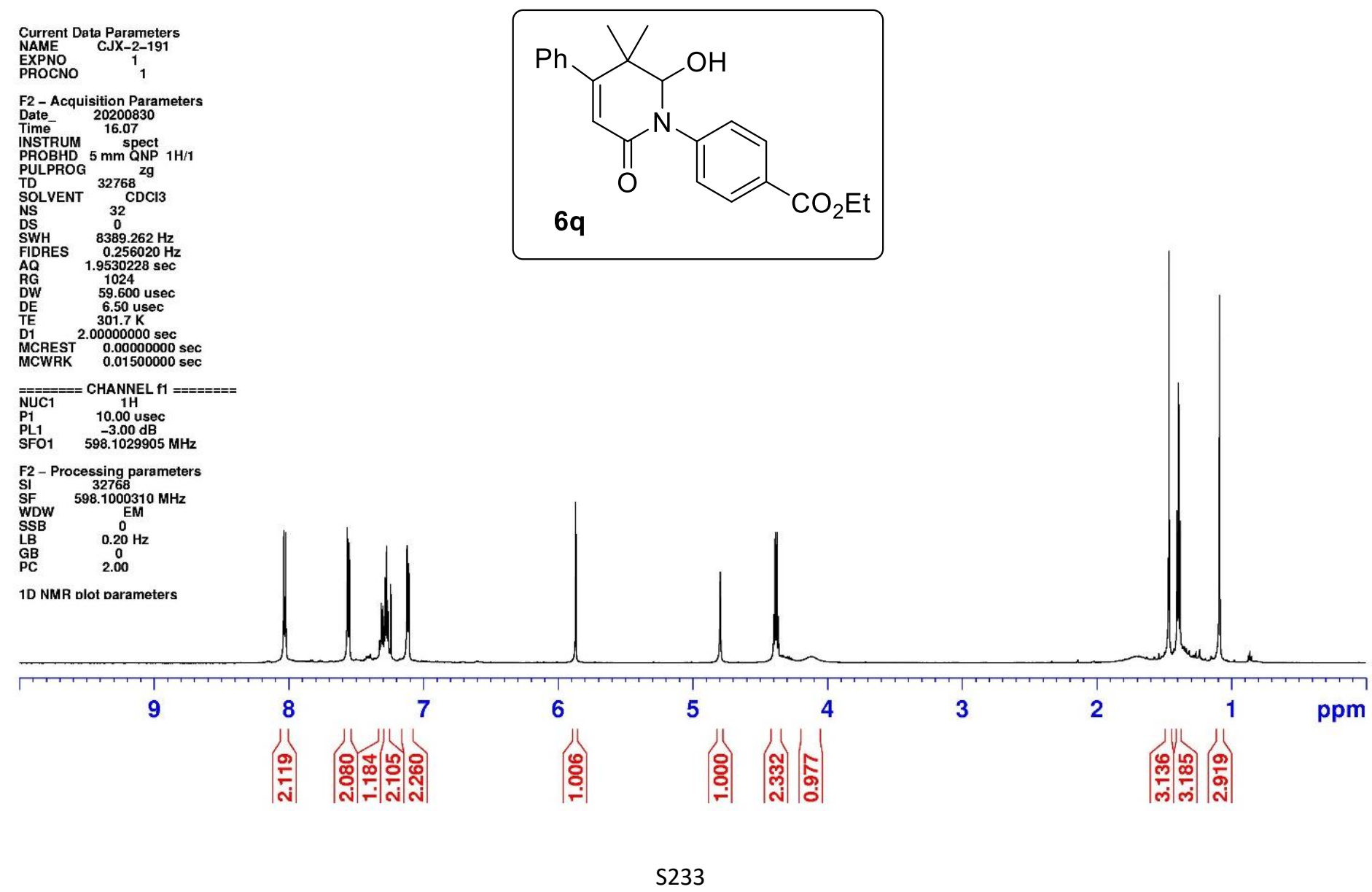




\section{||}

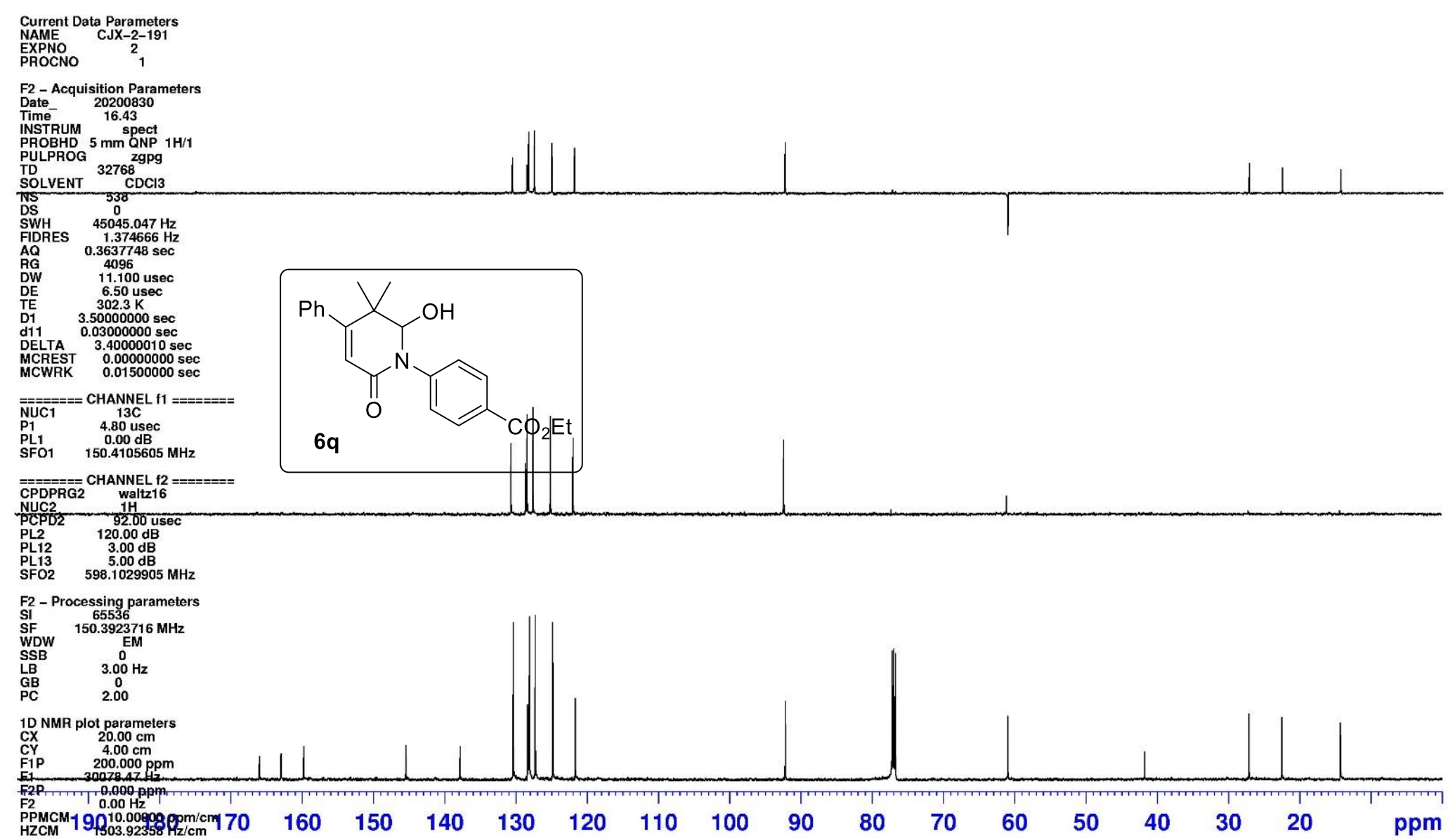




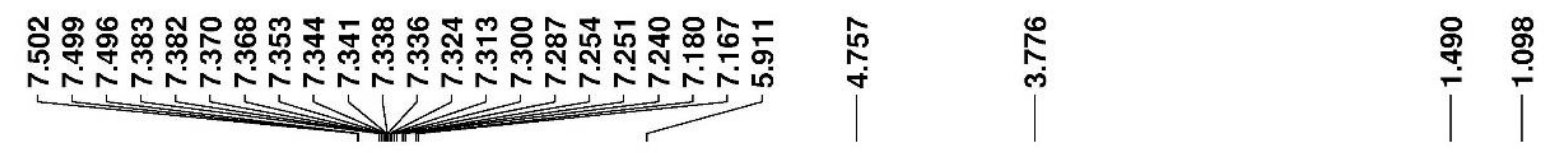

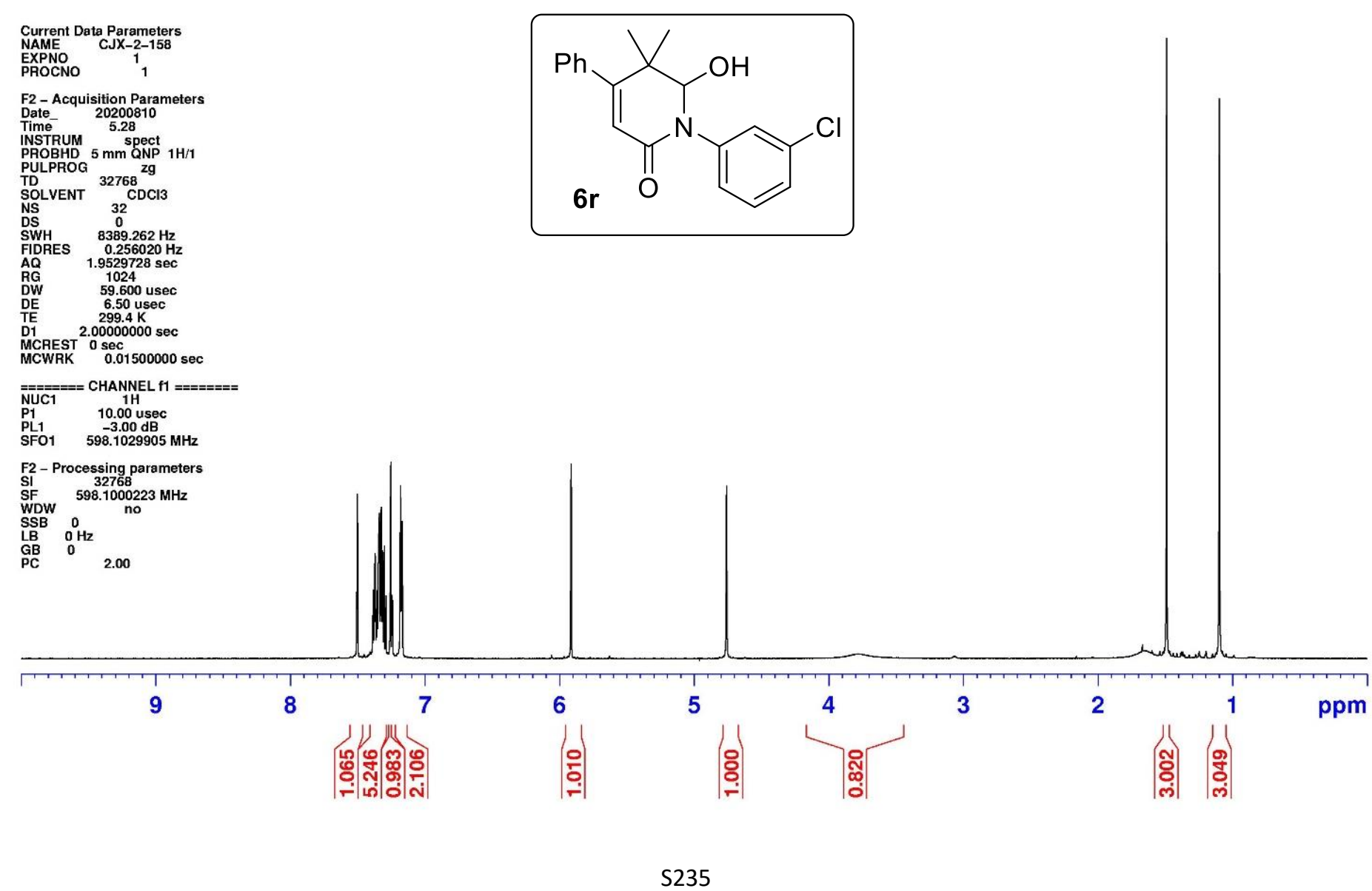




|广

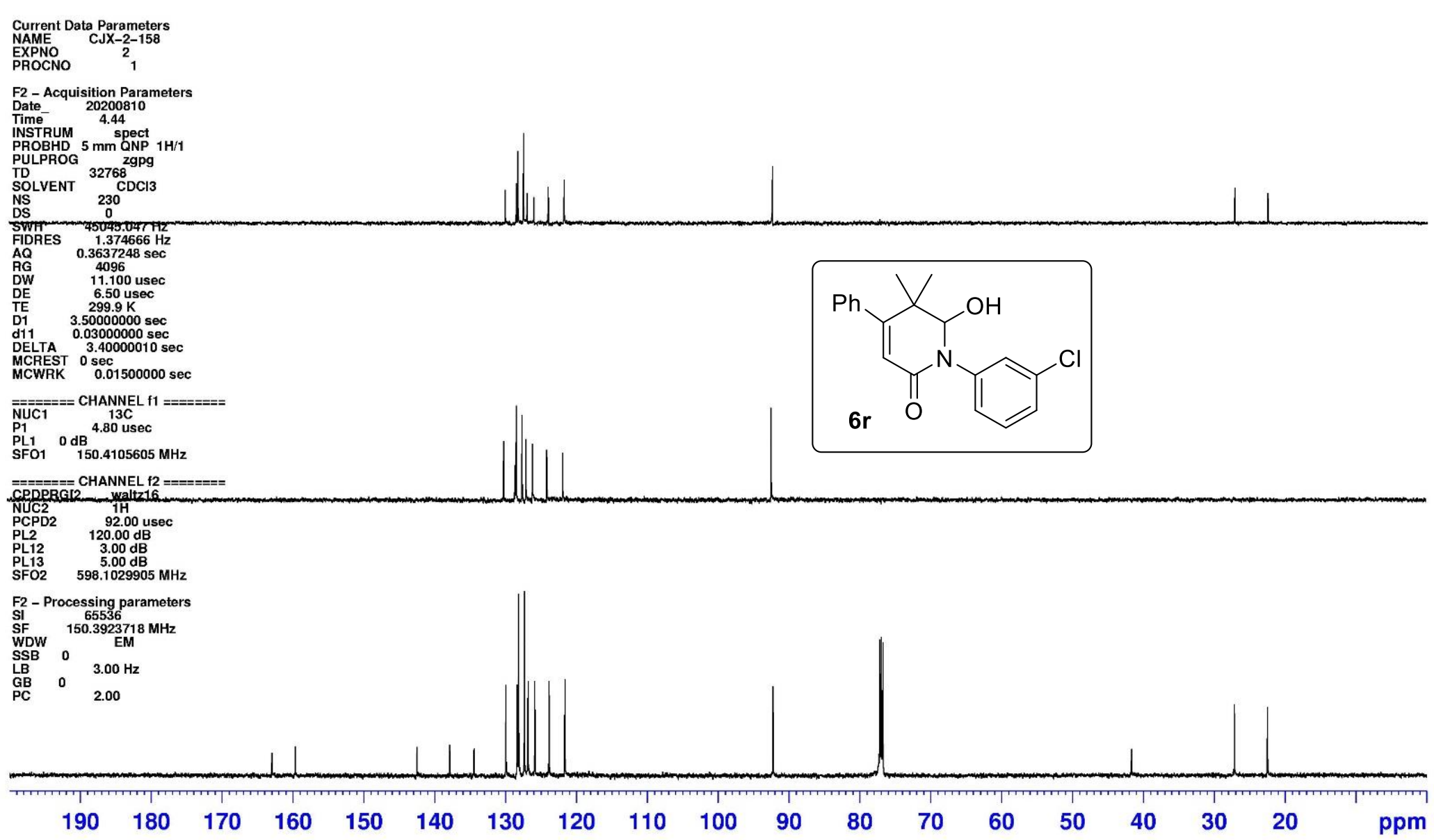



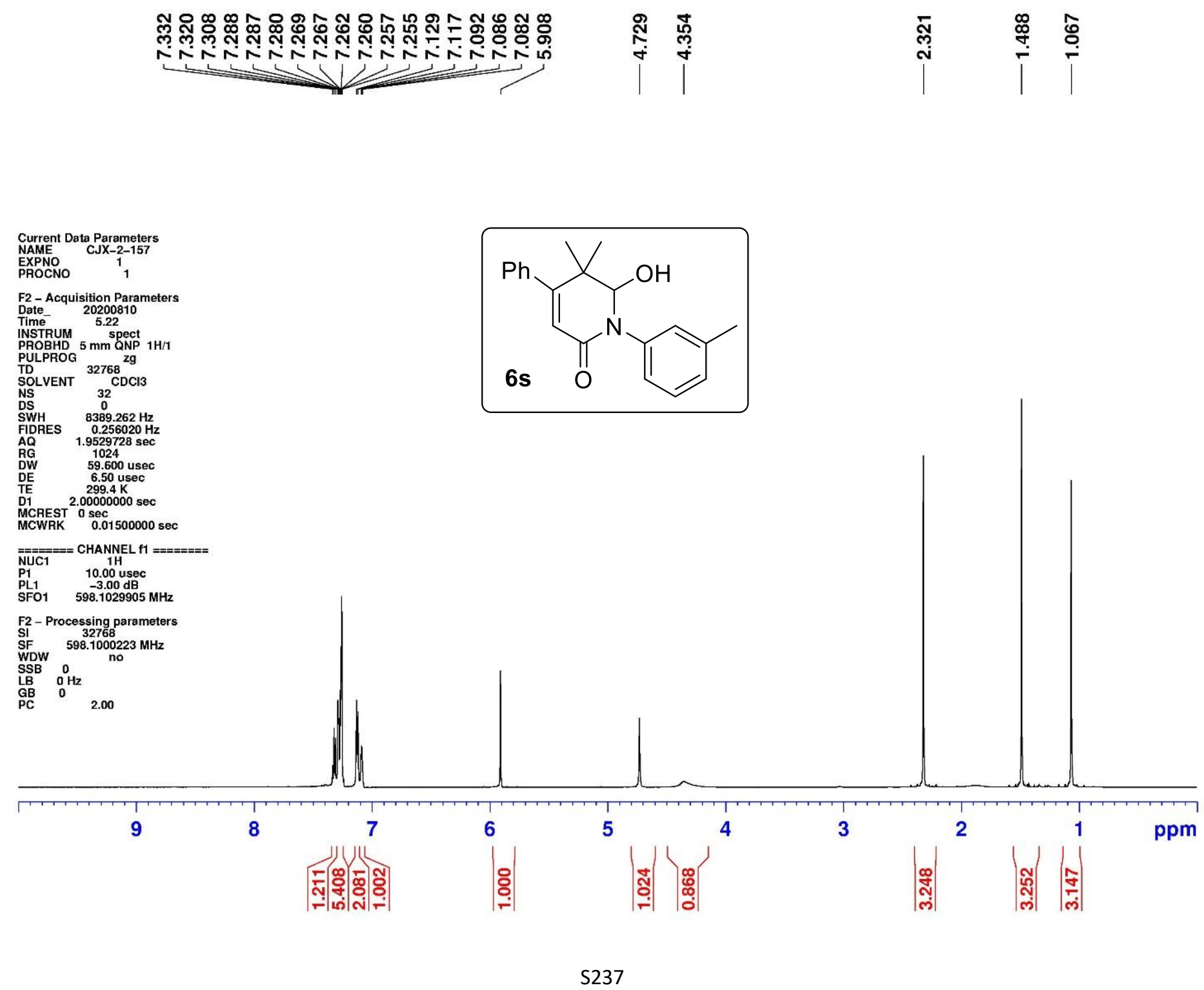

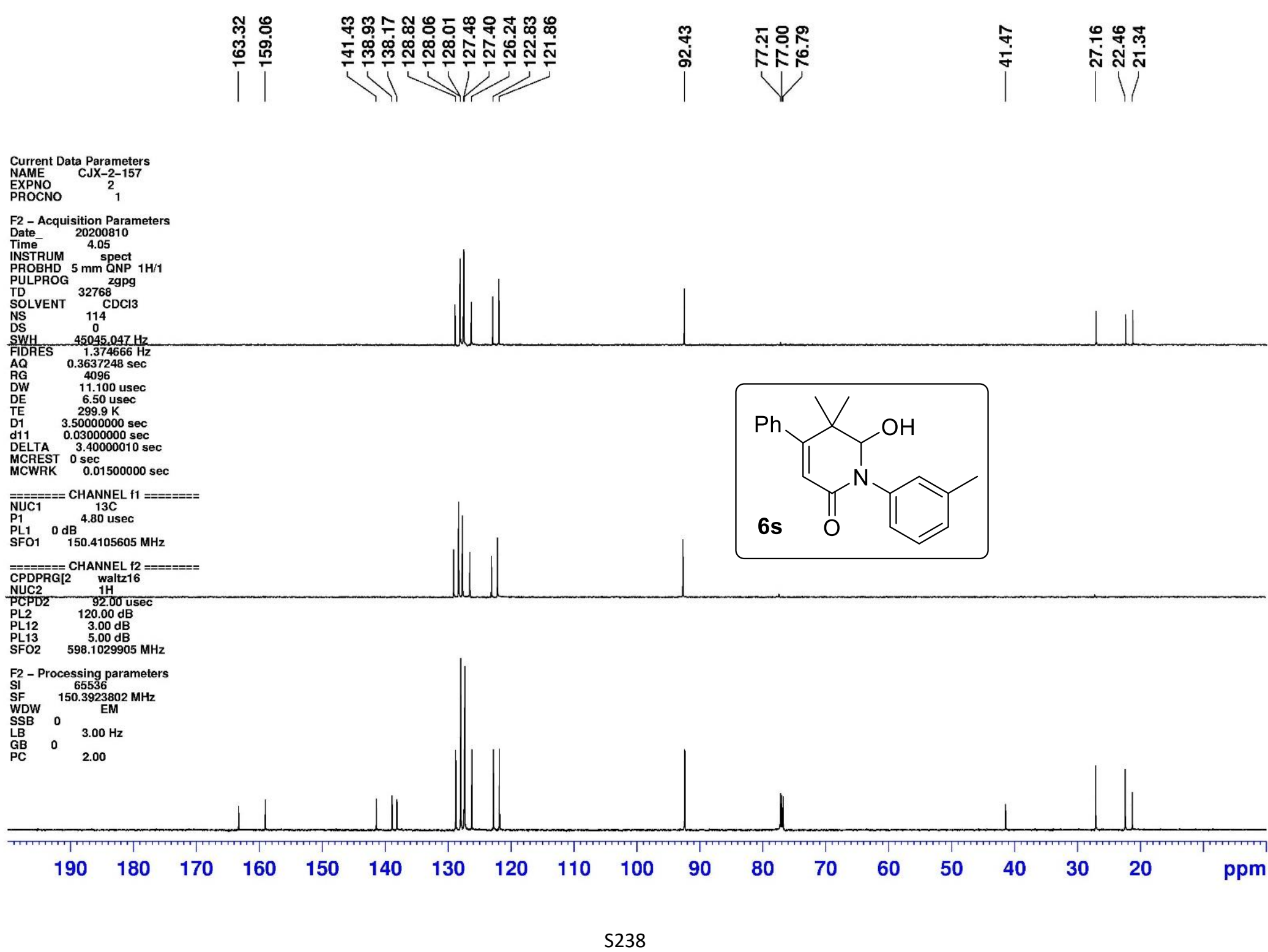


|רוֹ |

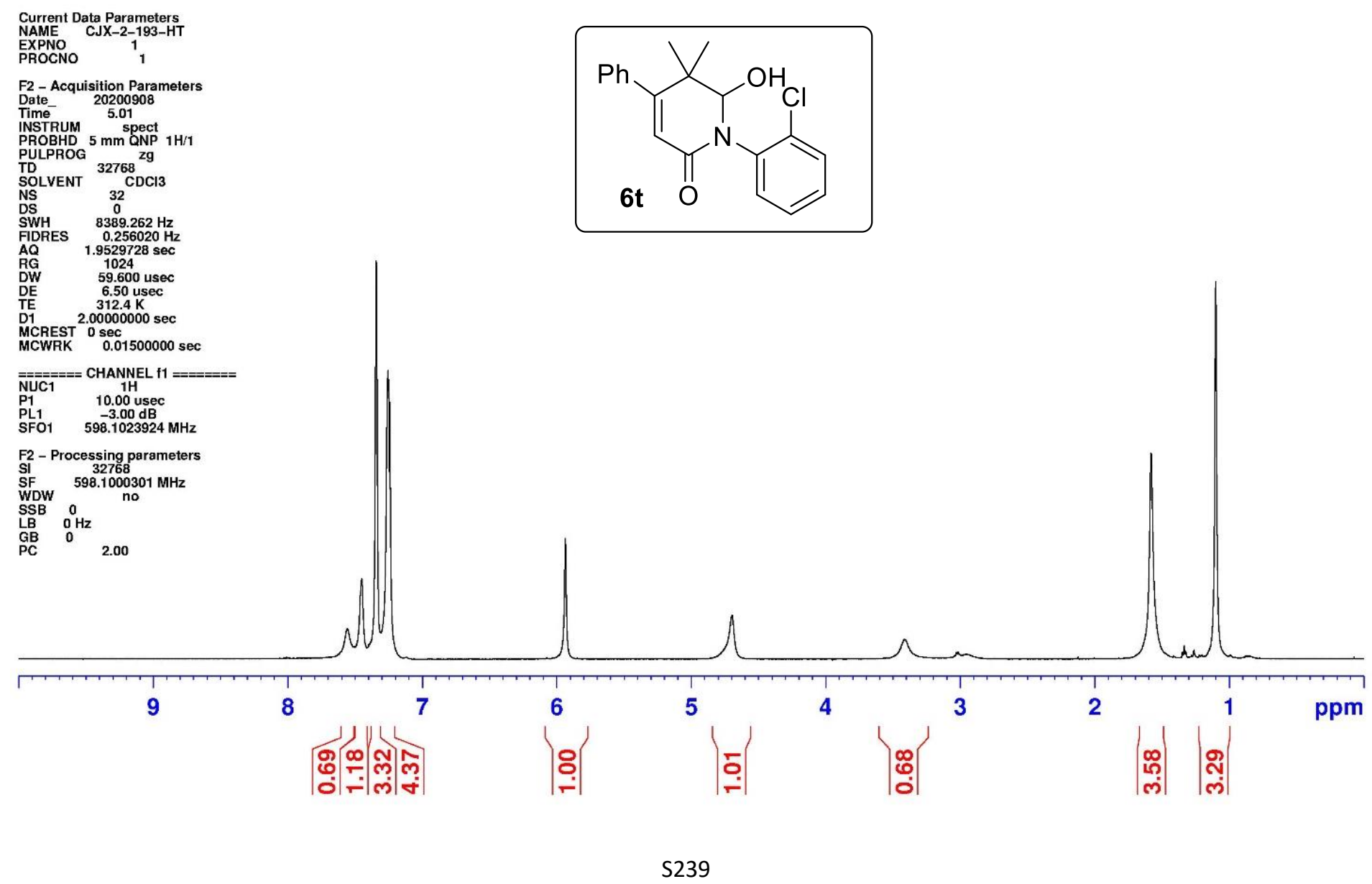




|

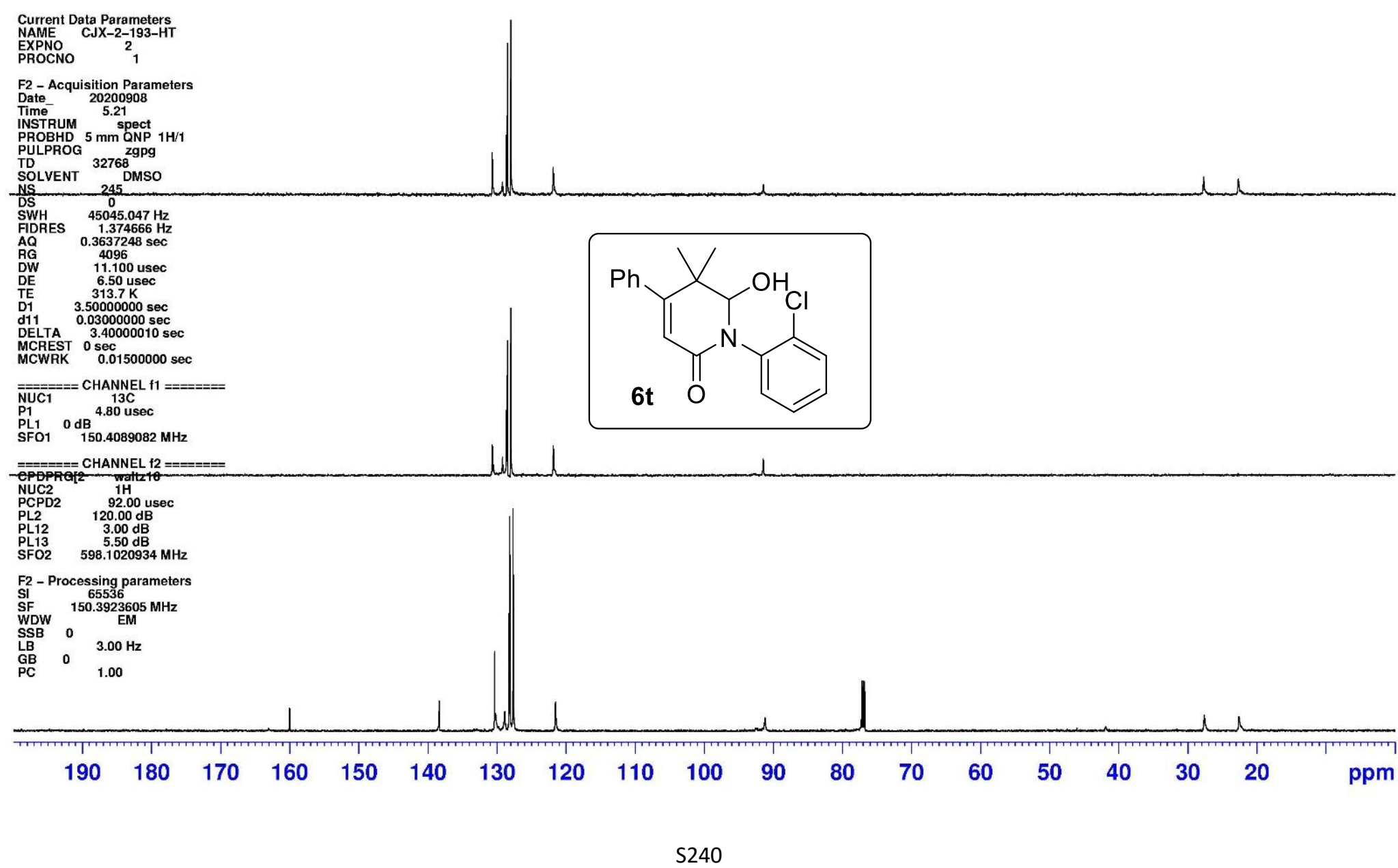



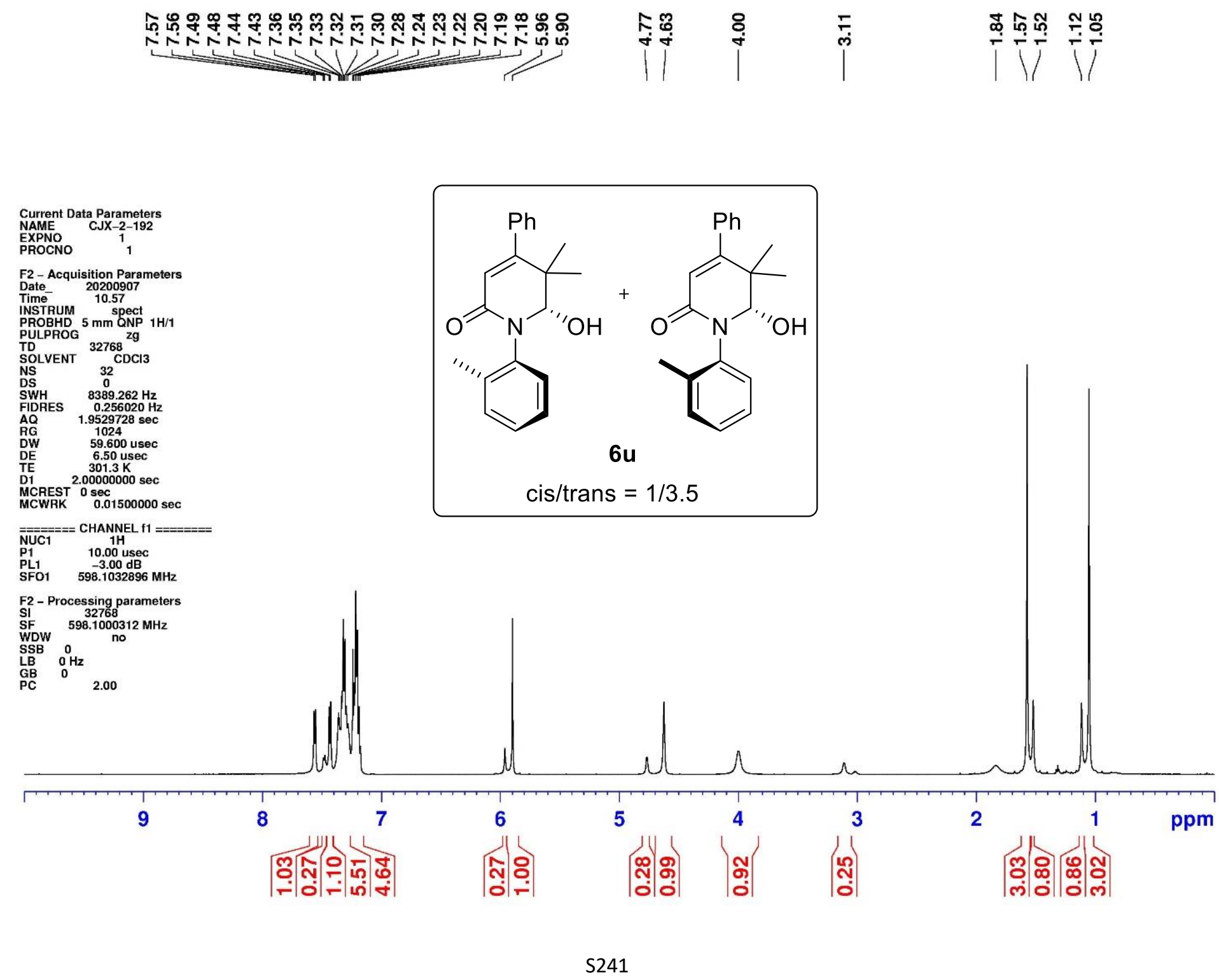

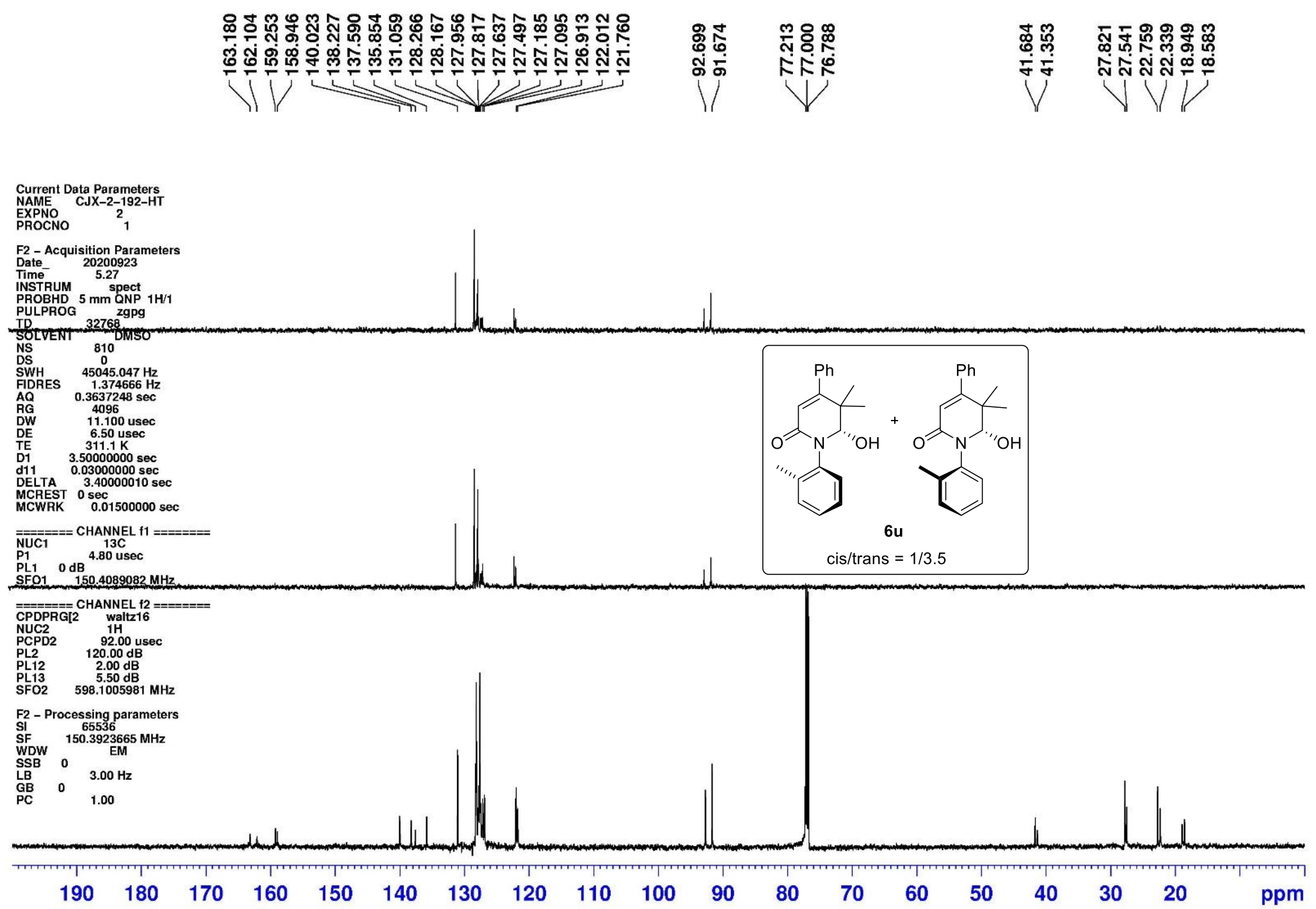


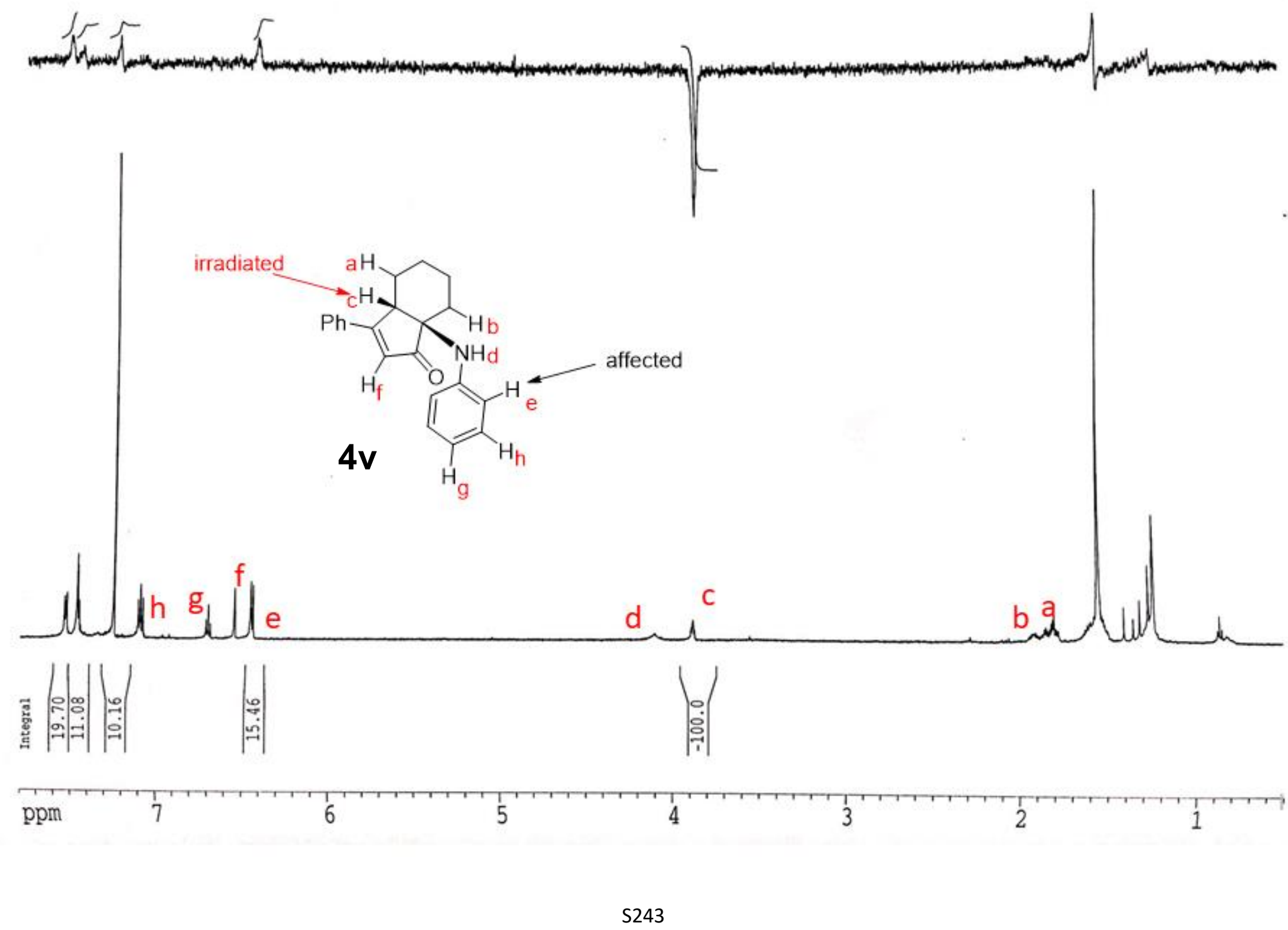




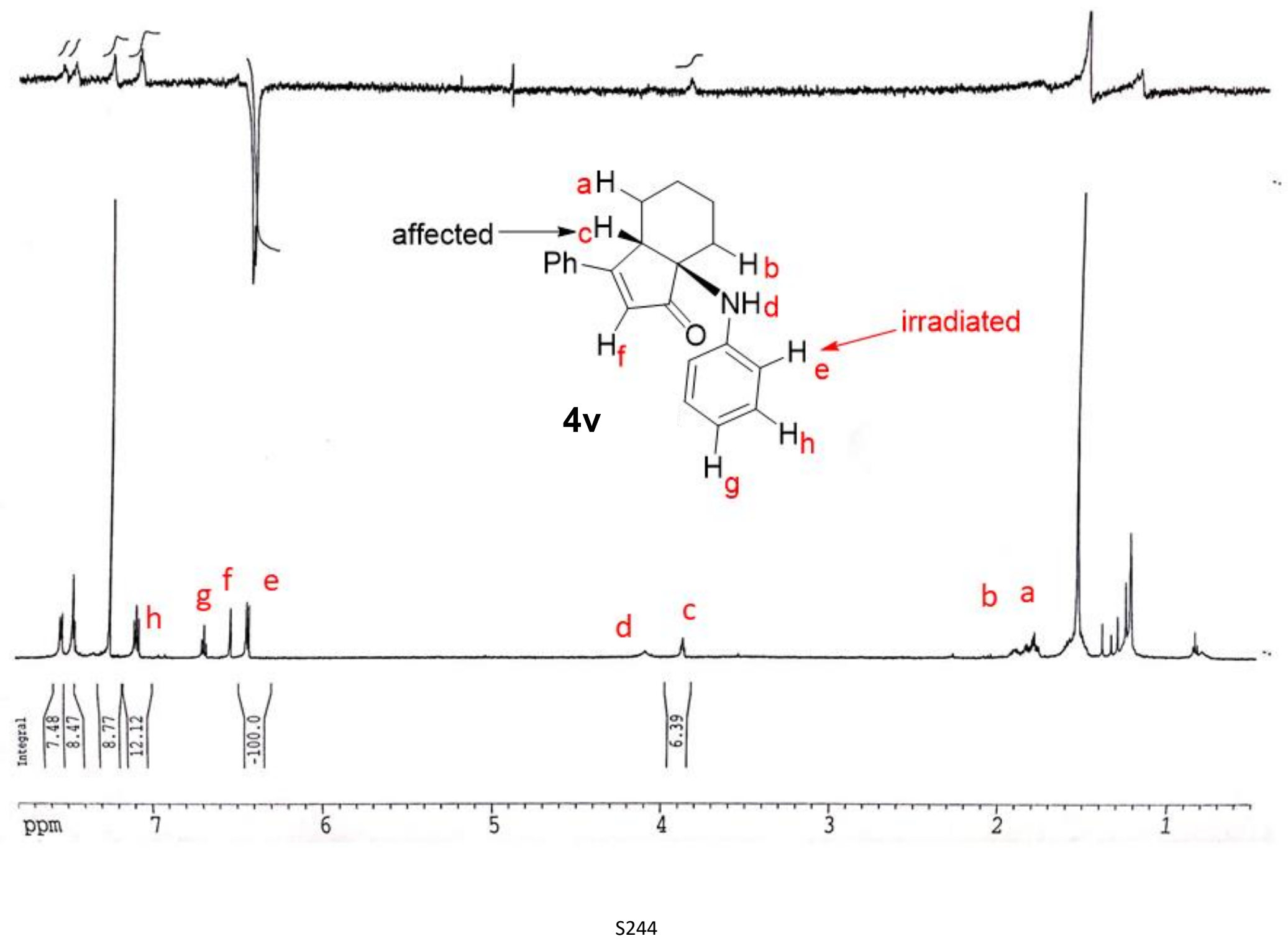

